\title{
De novo asymmetric synthesis of mannopeptimycin-E, novobiocin and methymycin analogues
}

\author{
Sanjeeva Rao Guppi \\ West Virginia University
}

Follow this and additional works at: https://researchrepository.wvu.edu/etd

\section{Recommended Citation}

Guppi, Sanjeeva Rao, "De novo asymmetric synthesis of mannopeptimycin-E, novobiocin and methymycin analogues" (2007). Graduate Theses, Dissertations, and Problem Reports. 4304.

https://researchrepository.wvu.edu/etd/4304

This Dissertation is protected by copyright and/or related rights. It has been brought to you by the The Research Repository @ WVU with permission from the rights-holder(s). You are free to use this Dissertation in any way that is permitted by the copyright and related rights legislation that applies to your use. For other uses you must obtain permission from the rights-holder(s) directly, unless additional rights are indicated by a Creative Commons license in the record and/ or on the work itself. This Dissertation has been accepted for inclusion in WVU Graduate Theses, Dissertations, and Problem Reports collection by an authorized administrator of The Research Repository @ WVU.

For more information, please contact researchrepository@mail.wvu.edu. 
De Novo Asymmetric Synthesis of Mannopeptimycin-E, Novobiocin and Methymycin Analogues

\title{
Sanjeeva Rao Guppi
}

\author{
Dissertation Submitted to the \\ Eberly College of Arts and Sciences \\ at West Virginia University \\ in Partial Fulfillment of the Requirements \\ for the Degree of \\ Doctor of Philosophy \\ In \\ Organic Chemistry
}

George A. O'Doherty, Ph.D., Chair

Kung K. Wang, Ph.D.

Björn C. Söderberg, Ph.D.

John Penn, Ph.D.

Robert K. Griffith, Ph.D.

C. Eugene Bennett Department of Chemistry

Morgantown, West Virginia

2007

Keywords: Mannopeptimycin-E, Novobiocin and Methymycin

Copyright 2007 Sanjeeva Rao Guppi 


\section{ABSTRACT \\ De Novo Asymmetric Synthesis of Mannopeptimycin-E, Novobiocin and Methymycin Analogues}

\section{Sanjeeva Rao Guppi}

The carbohydrate portion of the natural products plays a crucial role in biology, such as target binding, solubility, tissue targeting, and membrane transportation. The O'Doherty group use a de novo methodology to build the desired functionality and stereochemistry within each sugar, in contrast to the traditional approach using known sugar isomers as starting materials. Recently, we have developed a practical and highly diastereoselective palladium catalyzed glycosylation reaction to control the stereochemistry at the anomeric center. Continuing, our investigations on the utility of this strategy, we turned our attention to the syntheses of the bioactive carbohydrate-based natural product analogues. The targets we chose are mannopeptimycin-E, novobiocin, methymycin, ribofuranose-adenosine and coumarin glycosides. All of which we felt were amenable to medicinal SAR studies.

Mannopeptimicins are cyclic hexapeptides glycosylated with a disaccharide side chain, which possesses potent antibacterial activity against gram-positive bacteria with good activity against drug resistant (MDR) bacteria. An enantioselective syntheses of the manno-disaccharide fragments of mannopeptimycin-E and its nine analogues were been achieved in 7-10 steps and 35-40\% overall yield via an iterative palladium-glycosylation strategy. Key to the success of this approach was the ease with which the $C-4$ isovalerate group was introduced, and the high diastereoselectivity of the palladium-catalyzed glycosylation and bis-dihydroxylation reactions.

Recently novobiocin was shown to inhibit Hsp90 protein, which is a promising target for development of cancer chemotherapeutics. We developed a practical 7-10 steps diastereoselective route for the syntheses of nine different analogues of three aglycones all in good yields. This route relies on an alternative pyranol palladium-catalyzed glycosylation reaction, diastereoselective dihydroxylation, regioselective carbamate installation strategy. Currently, the nine-novobiocin analogues are being tested against different cancer cell lines in Prof. Blagg's labs.

Methymycin is a 12-membered macrolactone, an important class of antibiotics used to treat infections caused by Gram-positive bacteria. We have developed divergent and highly enantioselective route to eight various amino/azido/dideoxy methymycin analogues. The key to the success of this method is the iterative use of the palladium-catalyzed glycosylation reaction, Luche reduction and Myers' reductive rearrangement. Currently, a small library of methymycin analogues was under testing on glycosyltransferase assays in Prof. Hung-Wen Liu labs. 


\section{DEDICATED TO}

My wife, Supraja Guppi, my mother Parvathi, my father Lakshmi Narayana and my grand mother Easwaramma. 


\section{ACKNOWLEDGEMENT}

First of all, I would like to give my sincere gratitude to my advisor, Dr. George A. O'Doherty, for his great support, patience, and encouragement throughout my graduate studies. His mentorship was essential to the completion of this dissertation.

My thanks also go to Dr. Kung Wang, Dr. Björn C. Söderberg, Dr. John Penn and Dr. Robert K. Griffith for their valuable comments that improved the contents of this dissertation.

I am especially thankful to Dr. Md. M. Ahmed and Dr. Satheesh Babu Ravula, who has taught me a lot of neat chemistry tricks and helped me during my research. I would also like to thank the former and present group members, Dr. Joseph Dougherty, Dr. Dennis Tomcik, Dong Gao, Miaosheng Li, Matt Mortensen, Haibing Guo, Maoquan Zhou, Mingde Shan, Philip Harsh, Xiaomei Yu, WenJun Xin, Bulan Wu and Yalan Xing for their help and company and the entire faculty, staff, and graduate students for their friendship and help in a variety of ways.

I would also like to thank Dr. Novruz Akhmedov for his kind and friendly help during NMR experiments and my fellow Brent Reschke for his help in finding the mass spectra of the research samples. I am especially glad and thankful to all my friends, especially I. B. Prasad, Chitti Prasad, Y. Srinivas, Srinivas Reyya, Ganesh Alpuri and Raja Vetsa for their support.

Financial support from the National Institute of Health, C. Eugene Bennett Department of Chemistry at West Virginia University, the National Science Foundation, NSF-EPSCoR (0314742) for a $600 \mathrm{MHz}$ NMR at WVU are gratefully acknowledged.

Finally, and most importantly, I would like to thank my parents for their love and support for my education through the past many years. 


\section{TABLE OF CONTENTS}

Title Page

Abstract $\quad$ ii

$\begin{array}{ll}\text { Dedications } & \text { iii }\end{array}$

Acknowledgement iv

Table of Contents $\quad$ v

List of Figures $\quad$ vii

List of Tables $\quad$ vii

List of Abbreviations $\quad$ vii

Chapter 1. Studies towards palladium-catalyzed glycosylation reaction and syntheses of biologically important glycosides

1.1. Introduction 1

1.2. De novo syntheses of various hexoses 2

1.3. De novo syntheses of coumarin and flavonol glycosides 5

1.4. De novo asymmetric synthesis of homoadenosine. 10

1.5. Summary 14

\section{Chapter 2. Asymmetric synthesis and medicinal chemistry studies of} novobiocin analogues

2.1. Introduction and biological activity to novobiocin analogues 15

2.2. Previous approaches to novobiocin analogues 16

2.3. Project description and goals 21 


\section{Chapter 3. Enantioselective synthesis of mannopeptimycin-E analogues}

3.1. Introduction to mannopeptimycins

3.2. Previous approach to mannopeptimycin analogues

3.4. Syntheses of key fragment of mannoeptimycin-E and its analogues

3.5. Asymmetric syntheses of aza-analogues of mannopeptimycin-E disaccharide portion

3.6. Syntheses of serine and threonine analogues of mannopeptimycin-E disaccharide portion

3.7. Towards asymmetric synthesis of mannopeptimycin-E analogue disaccharide portion

\section{Chapter 4. Syntheses and biosynthetic studies of methymycin analogues}




\section{Chapter 5. Experimental section}

5.1. Instrumentation, materials and manipulation 66

5.2. References and notes 215

\section{List of Figures}

Figure 1. Adenosines and hexopyranosyl nucleosides. 11

$\begin{array}{lr}\text { Figure 2. Naturally occurring aminocoumarin antibiotics. } & 15\end{array}$

Figure 3. The DHN1 and DHN2 are selective Hsp90 inhibitors 19

Figure 4. Structure of mannopeptimycin-E III-1E. 31

Figure 5. 12-membered macrolactones antibiotics. 53

Figure 6. The reverse glycosyl transferase assay using HPLC

chromatogram analysis on our small library of eight compounds. 61

\section{List of Tables}

Table 1. Syntheses of various coumarin glycosides and their yields 7

Table 2. Syntheses of various manno-glycosides and their yields $\quad 10$

Table 3. Activities of mannopeptimycins. 32

\section{List of Abbreviations}

Ac

$\mathrm{AD}$

$\mathrm{Bn}$
Acetyl

Asymmetric dihydroxylation

Benzyl 


\begin{tabular}{|c|c|}
\hline Boc & $N$-tert-butoxycarbonyl \\
\hline BOP & Benzotriazolyloxytris(dimethylamino)phosphonium hexa \\
\hline $\mathrm{bp}$ & Boiling point \\
\hline $\mathrm{Bu}$ & Butyl \\
\hline $\mathrm{BuLi}$ & $n$-Butyllithium \\
\hline calc & Calculated \\
\hline CSA & Camphorsulfonic acid \\
\hline $\mathrm{d}$ & Doublet \\
\hline DBA & trans,trans-dibenzylideneacetone \\
\hline DCC & Dicyclohexylcarbodiimide \\
\hline de & Diastereomeric excess \\
\hline DEAD & Diethyl azodicarboxylate \\
\hline DHQ & Dihydroquinine \\
\hline DHQD & Dihydroquinidine \\
\hline DIBAL-H & Diisobutylaluminum hydride \\
\hline DIEPA & Diisopropylethylamine \\
\hline DMAP & 4-Dimethylaminopyridine \\
\hline DMAP & 4-Dimethylaminopyridine \\
\hline DMDO & Dimethyldioxirane \\
\hline DMF & $N, N$-Dimethylformamide \\
\hline DMP & Dess-Martin periodinane \\
\hline
\end{tabular}




\begin{tabular}{|c|c|}
\hline DMSO & Dimethyl sulfoxide \\
\hline ee & Enatiomeric excess \\
\hline EI & Electron impact \\
\hline Et & Ethyl \\
\hline EtOAc & Ethyl acetate \\
\hline & 2-(1H-Benzotriazole-1-yl)-1,1,3,3-tetramethyluronium \\
\hline HBTU & hexafluorophosphate \\
\hline HKR & Hydrolytic kinetic resolution \\
\hline HMPA & Hexamethylphosphoramide \\
\hline HPLC & High Performance Liquid Chromatography \\
\hline HRMS & High resolution mass spectrum \\
\hline IR & Infrared \\
\hline KHMDS & Potassium hexamethyldisilazide \\
\hline $\mathrm{LAH}$ & Lithium aluminum hydride \\
\hline LDA & Lithium diisopropylamide \\
\hline LHMDS & Lithium hexamethyldisilazide \\
\hline $\mathrm{mCPBA}$ & $m$-Chloroperbenzoic acid \\
\hline $\mathrm{Me}$ & Methyl \\
\hline $\mathrm{MHz}$ & megahertz \\
\hline mmol & millimole(s) \\
\hline MS & Mass spectrum \\
\hline
\end{tabular}




\section{Chapter 1}

\section{Studies towards Palladium-Catalyzed Glycosylation Reaction and Syntheses of Biologically Important Glycosides.}

\subsection{Introduction.}

Recently, it has been shown that the carbohydrate portion of the natural products play a crucial role in its biological activities, such as target binding, solubility, tissue targeting, and membrane transportation. ${ }^{1}$ For example, the corresponding aglycons of natural products are often devoid of activity. Since the initial discovery of biologically active glycosylated natural products, medicinal chemists have desired the ability to change the carbohydrate structures of natural products to reveal the role the carbohydrate plays. Although nature uses a diverse array of carbohydrate structures in these natural products only a limited number of sugar isomers (glucose, galactose and mannose) are provided in accessible quantities for SAR studies, eventually affecting the ability of chemists to install rare/unnatural sugar isomers. Undoubtedly, synthetic alternative methods are required to address this medicinal chemistry need.

An alternative method would be to not use the carbohydrate based starting materials and prepare the desired carbohydrate functionality and chirality from simple achiral starting materials. ${ }^{2,3,4}$ This de novo methodology should allow the medicinal chemist to install a much broader range of carbohydrate structures amenable for SARstudies. This new approach is in stark contrast to using known sugar isomers as starting materials where variability is narrow and extensive the protecting group chemistry is required. The term "de novo" approach is used to illustrate the way we built the desired functionality and stereochemistry within each sugar, in contrast to the traditional 
carbohydrate approach, which start with known sugars. Herein we describe our efforts towards the development of this methodology and its ongoing application towards the synthesis of various carbohydrate structural motifs.

\subsection{De Novo Syntheses of Various Hexose.}

Over the past years, significant efforts have been made toward the development of new synthetic routes to monosaccharides. ${ }^{3}$ Of particular interest is the de novo synthesis of these sugar isomers (i.e., from simple achiral starting materials using asymmetric catalysis). While there are many uses of the term "de novo" in carbohydrate chemistry, ${ }^{5}$ herein the term de novo asymmetric syntheses refer to the use of asymmetric catalysis for the asymmetric synthesis of carbohydrates from achiral compounds (vide infra).

The de novo enantioselective synthesis of the various hexoses stands as a challenge to asymmetric catalysis. ${ }^{3}$ Despite some seminal efforts toward the hexoses, notably by Masamune/Sharpless (epoxidation), ${ }^{2}$ Danishefsky (Diels-Alder), ${ }^{6}$ Johnson/Hudlicky (enzymatic desymmetrization) ${ }^{4}$ and Wong/Sharpless (osmium/enzyme), ${ }^{7}$ a practical, non-enzymatic route does not exist to all the hexoses. ${ }^{8}$ Recently this task has also been taken up by others like MacMillan (iterative aldol strategy) ${ }^{9}$ and White (allylic oxidation) ${ }^{10}$.

For the last ten years O'Doherty group have endeavored to develop practical methods for the de novo synthesis of the hexoses. Our group has resulted in the discovery of two orthogonal approaches to pyrano-hexoses with variable $C$-6 substitution as $-\mathrm{CH}_{3} /-\mathrm{CH}_{2} \mathrm{OH}$.

Our first generation approach started with furfuraldehyde I-1, which when exposed to the Petersen olefination conditions $\left(\mathrm{TMSCH}_{2} \mathrm{MgCl} / 1 \mathrm{~N} \mathrm{HCl}\right)$ produced 
vinylfuran I-2 (Scheme 1). We derived the furfuryl alcohol stereochemistry in either enantiomeric form $\mathbf{I}-\mathbf{3}$ and $\mathbf{I}-\mathbf{4}$ by means of the Sharpless asymmetric dihydroxylation $($ AD-mix- $\alpha / \beta)$ of vinyl furan $\mathbf{I}-2{ }^{11}$

Scheme 1. O'Doherty group's first-generation approach to furfuryl alcohols.

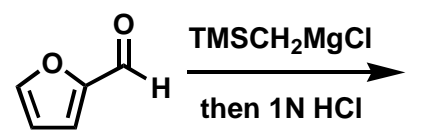

I-1

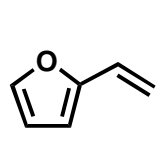

I-2

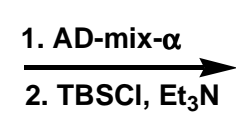

$90 \%$

AD-mix- $\alpha$ : (DHQ) $)_{2}$ PHAL<smiles>OC(CO[Sb])c1ccco1</smiles>

I-3

Generated as a $2 \mathrm{M}$ solution in $\mathbf{E t}_{2} \mathbf{O}$

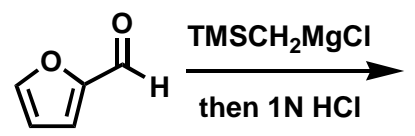

I-2

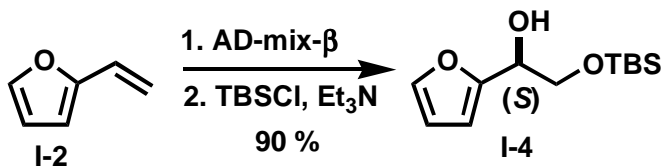

Generated as a $2 \mathrm{M}$ solution in $\mathbf{E t}_{2} \mathbf{O}$

AD-mix- $\beta$ : (DHQD) $)_{2}$ PHAL

Because the enantioselectivity of dihydroxylation step was too low ( $90 \%$ ee), we looked for a better approach and settled on the Noyori reduction of acylfurans (Scheme 2). ${ }^{12}$ This improved approach requires the preparation of the acylketone $\mathbf{I - 9}$ in three steps from glycolic acid I-5 via a 2-lithiofuran addition to the TBS-protected amide I-7 in 70\% overall yield. The furyl alcohols $\mathbf{I - 3 / I - 4}$ were enantioselectively prepared from Noyori asymmetric reduction of furyl ketone $\mathbf{I}-\mathbf{9}$ catalyst $\mathbf{I}-\mathbf{1 0 a} / \mathbf{I}-10 b$ in good yields (85-90\%) and excellent enantioselectivity (>95\% ee). ${ }^{13}$

Scheme 2. O'Doherty group's improved approach to furyl alcohols via Noyori reduction. 


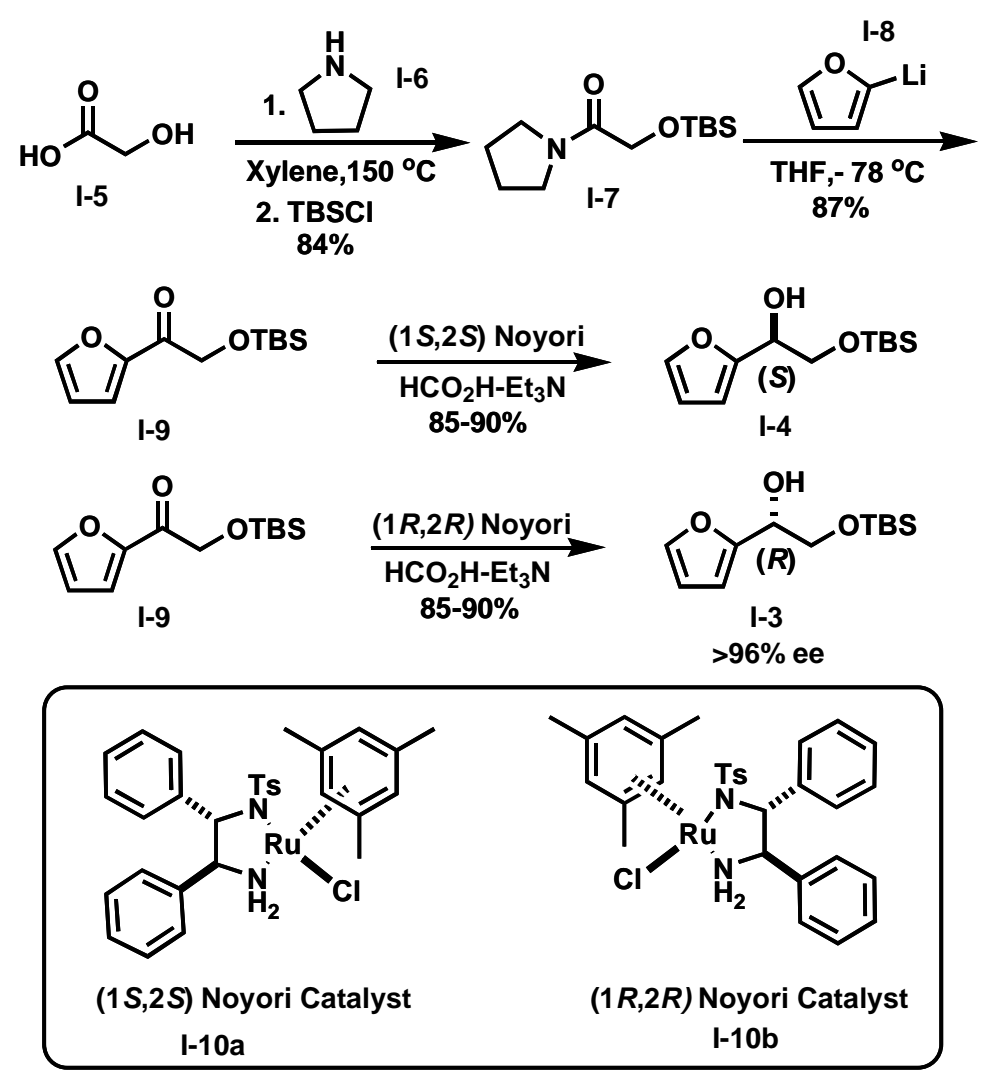

The synthesis of pyrano-hexoses relies on the use of the Achmatowicz rearrangement ${ }^{14}$ (Scheme 4 for mechanism), which is an oxidative rearrangement of furfuryl alcohols I-3/I-4 to hemiacetals I-11/I-12. Thus, treatment of furyl alcohols I-3/I4 with NBS in aqueous THF gave hemiacetals I-11/I-12 in very good yields (Scheme 3). We have succeeded in developing a short route that is flexible enough for the syntheses of four possible diastereomeric hexoses I-13, I-14, I-15 and I-16 by selectively acylation with Boc anhydride in high yields (86-88\%). Depending on the reaction temperature, the acylation step can selectively give the $\alpha$-Boc pyranones $\mathbf{I}-\mathbf{1 3}$ and $\mathbf{I}-\mathbf{1 5}$ at $\left(-78{ }^{\circ} \mathrm{C}\right)$ or a $1: 1$ ratio of $\alpha / \beta$-Boc pyranones at room temperature. 
Scheme 3. Syntheses of pyranones.
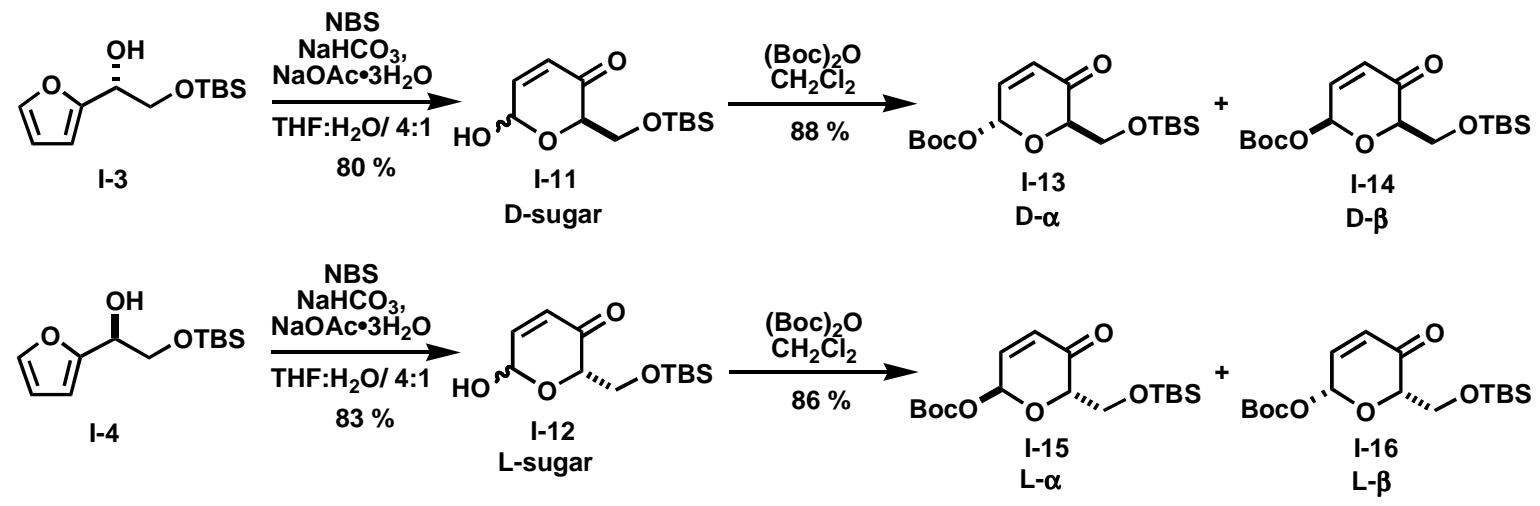

\begin{tabular}{rrrr|}
$-78^{\circ} \mathrm{C}$ & 20 & $:$ & 1 \\
$0^{\circ} \mathrm{C}$ & 1 & $:$ & 1 \\
\hline
\end{tabular}

Scheme 4. Achmatowicz reaction and its mechanism.

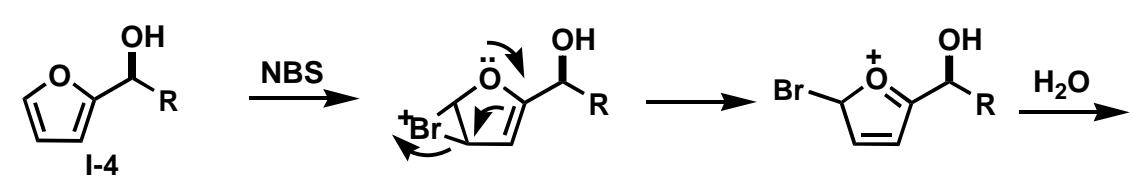
$\mathrm{R}=\mathrm{CH}_{3} / \mathrm{CH}_{2} \mathrm{OTBS}$

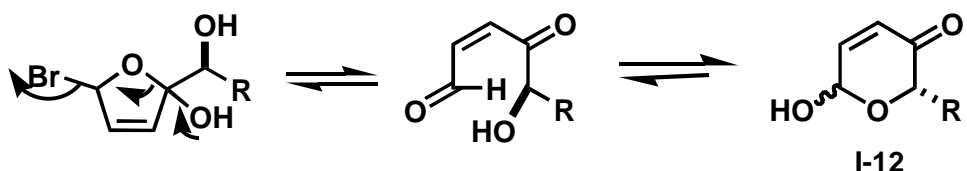

\subsection{De Novo Synthesis of Coumarin and Flavonol Glycosides.}

A related family of interesting natural products are the coumarin and flavonol glycosides, which are used for the treatments of digestive disordered, bronchitis, and inflammation in the traditional medicine. ${ }^{15,16,17}$ These active coumarin and flavonol glycosides attracted our attention towards the synthesis of several manno-glycoside analogues of these phenols, flavones and coumarins glycosides using our recently developed palladium catalyzed glycosylation reaction. 
Recently, we have developed a practical and highly diastereoselective palladium catalyzed glycosylation reaction (coupling of $\mathbf{I - 1 5}$ and $\mathbf{R O H}$ to give I-17) to control the stereochemistry at the anomeric center (Scheme 4). We have also demonstrated the practical utility of this reaction for the de novo synthesis of oligosaccharides. ${ }^{18}$

Scheme 4. Pd-catalyzed reaction and its mechanism.

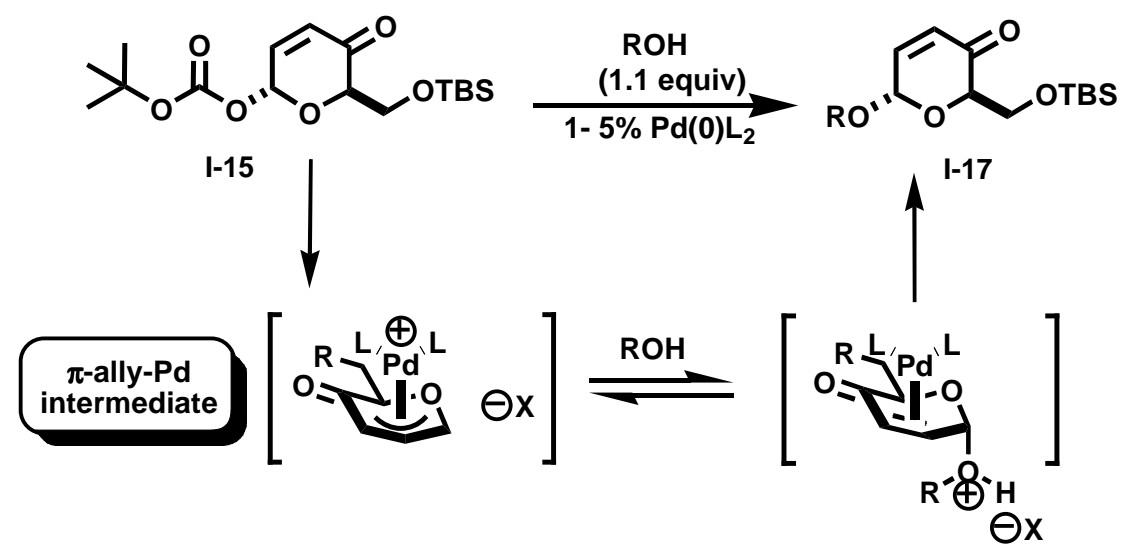

Herein, we describe our results for the de novo synthesis of $\alpha$-and $\beta$ - linked coumarin and flavonol glycosides using this palladium catalyzed glycosylation reaction. In addition, the coumarin and flavone glycosylation products were transformed into manno-glycosides using a simple reduction/oxidation sequence.

Since our approach is quite mild and equally amenable to the glycosyl transfer of either a $\alpha$-and $\beta$-linked glycosides. Initially we subjected several substituted phenols to the diastereoselective glycosylation reaction and gave excellent results. ${ }^{19}$ With these primary results at hand, we next explored the glycosylation reaction of the coumarins. The palladium-catalyzed glycosylation of coumarin I-18 with $\alpha / \beta$-pyranones I-15/I-16 gave the respective $\alpha / \beta$-glycosides $\mathbf{I - 1 9} \alpha / \mathbf{I}-\mathbf{1 9} \beta$ as a single diastereomers in good yields. 
Scheme 5. Syntheses of coumarin glycosides.
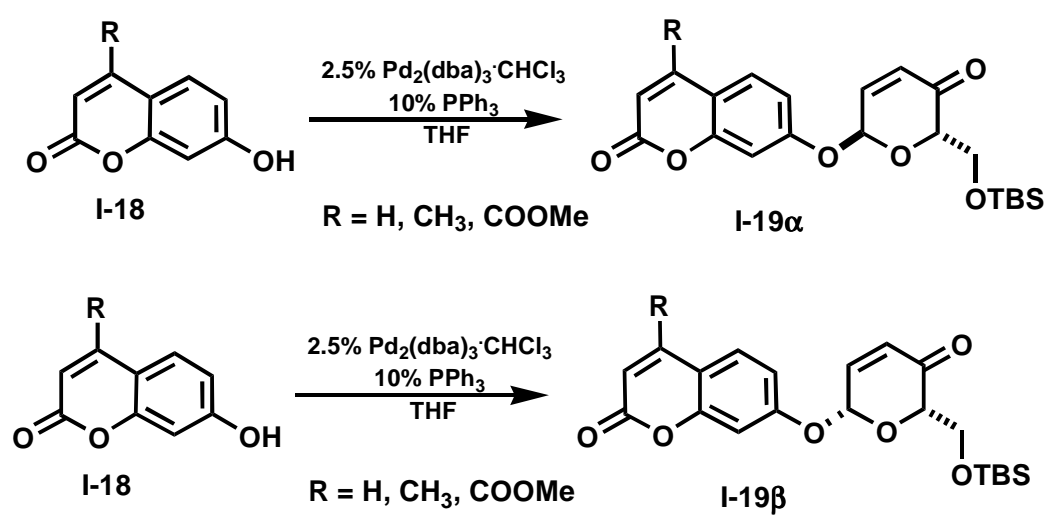

The glycosylation reaction worked well with good yields for phenols and simple alcohols ${ }^{19}$ but not promising yields for coumarin and flavanol aglycones. A change in solvent from $\mathrm{CH}_{2} \mathrm{Cl}_{2}$ to THF for glycosylation of coumarins and flavone remarkably improved the yields. The yields of various coumarin $\alpha / \beta$-glycosides shown in the table 1.

Table 1. Syntheses of various coumarin glycosides and their yields. 

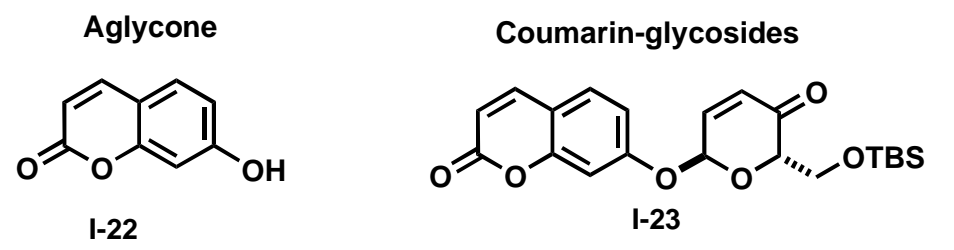

Yield \%

$92 \%$

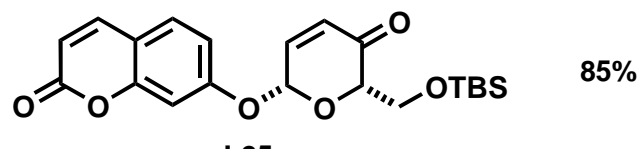<smiles>Cc1cc(=O)oc2cc(O)ccc12</smiles><smiles>Cc1cc(=O)oc2cc(O[C@@H]3C=CC(=O)[C@@H](CO[Sb])O3)ccc12</smiles>

$88 \%$<smiles>Cc1cc(=O)oc2cc(O[C@@H]3C=CC(=O)[C@@H](C[OH2+])O3)ccc12</smiles>

$78 \%$<smiles>COC(=O)c1cc(=O)oc2cc(O)ccc12</smiles><smiles>[B-]OC[C@H]1O[C@H](Oc2ccc3c(C(=O)OC)cc(=O)oc3c2)C=CC1=O</smiles>

$76 \%$

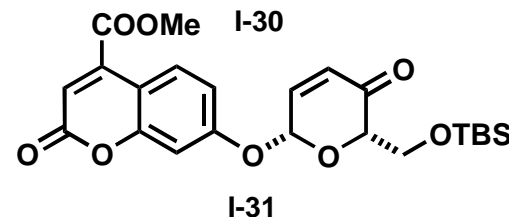

$70 \%$

We next turned our attention towards the preparation of $\alpha$-and $\beta$-flavanolglycosides. Thus, using the same Pd-catalyzed glycosylation conditions $(2.5 \mathrm{~mol} \%$ $\left.\operatorname{Pd}(0) / 10 \% \mathrm{PPh}_{3}, \mathrm{THF}\right)$, the $\alpha-/ \beta$-pyranones $\mathbf{I}-\mathbf{1 5} / \mathbf{I}-16$ were coupled with flavanol providing $\alpha / \beta$ - glycosides $\mathbf{I}-33 \alpha / \mathbf{I}-33 \beta$ respectively. The yields for flavanol glycosides were shown in Scheme 6.

Scheme 6. Syntheses of flavone glycosides. 


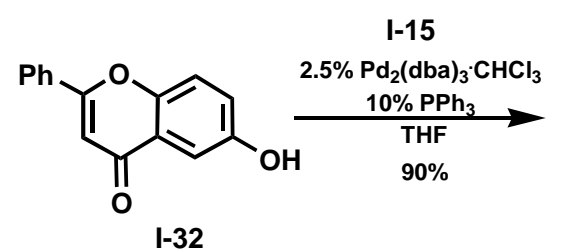<smiles>CC(C)(C)CO[C@H]1O[C@H](Oc2ccc3oc(-c4ccccc4)cc(=O)c3c2)C=CC1=O</smiles>
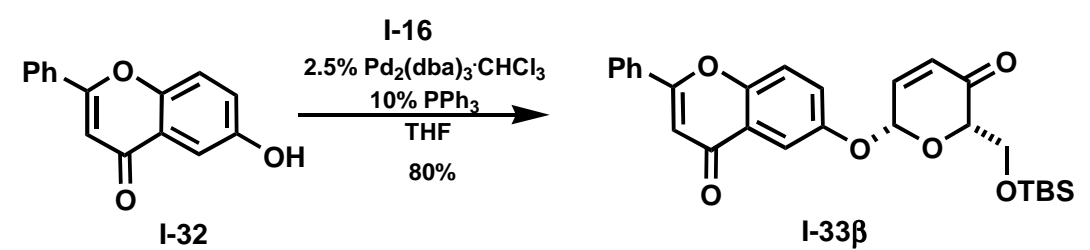

The flavonol and coumarin $\alpha$-glycoside products were easily converted into Lhexo-pyranoses by a two-step reduction/oxidation sequence. Thus by exposure of the glycosylated products to $\mathrm{NaBH}_{4}$ at $-78{ }^{\circ} \mathrm{C}$ in $\mathrm{CH}_{2} \mathrm{Cl}_{2}$ and $\mathrm{CH}_{3} \mathrm{OH}$ (Scheme 7) gave good yields (78-96\%) of equatorial alcohols I-41a-d with complete stereocontrol (Table 2). Similarly, the resulting allylic alcohols I-41a-d could be diastereoselectively oxidized to the manno-triols I-42a-d in excellent yields (84-92\%) with complete stereocontrol under the Upjohn condition $\left(\mathrm{OsO}_{4} / \mathrm{NMO}\right){ }^{20}$ The yields for reduction and dihydroxylation steps were all high and are summerized in table 2.

Scheme 7. Syntheses of various manno-sugars.

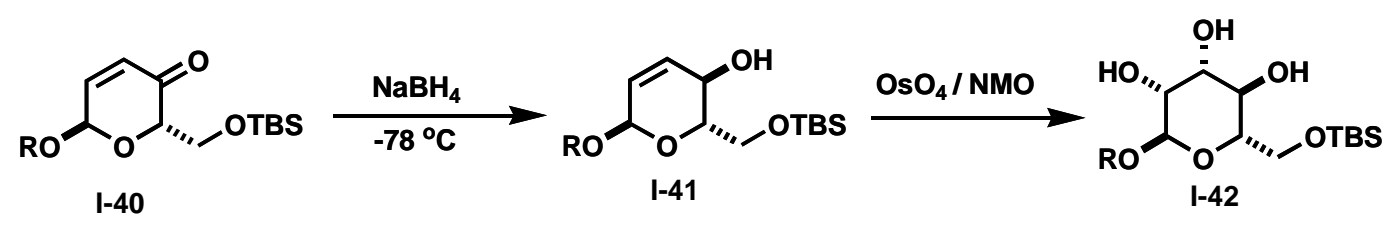

Table 2 Syntheses of various manno-glycosides and their yields 
Reduction yields<smiles>O=c1ccc2ccc(O[C@@H]3C=C[C@H](O)[C@@H](C[OH2+])O3)cc2o1</smiles>

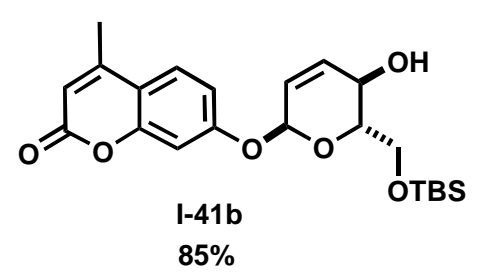<smiles></smiles>

Dihydroxylation yields<smiles>O=c1ccc2ccc(OC3O[C@H](CO[Sb])[C@@H](O)[C@H](O)[C@H]3O)cc2o1</smiles><smiles>CO[Sb](=O)(=O)OC[C@H]1O[C@@H](Oc2ccc3c(C)cc(=O)oc3c2)[C@H](O)[C@@H](O)[C@@H]1O</smiles><smiles>CC(C)(C)O[Sb](C)(C)(C)(C)C</smiles>

\subsection{De Novo Asymmetric Synthesis of Homoadenosine.}

\section{4.a. Introduction.}

The hexopyranosyl nucleosides make up a large and varied class of natural products (e.g., blasticidin, ${ }^{21}$ gougerotin, ${ }^{22}$ hikizimycin, ${ }^{23}$ mildiomycin, ${ }^{24}$ the bagougeramines, ${ }^{25} \mathrm{SF}-2140,{ }^{26}$ the pentopyranines, ${ }^{27}$ and miharamycin ${ }^{28}$ ). In addition to the intriguing structures, they also possess distinct biological activities. ${ }^{29}$ Inspired by these natural products, chemists have made two hexopyranosyl nucleosides 2-deoxy- $\beta$-Dribo-hexopyranose adenosine (I-51 $)^{30}$ and 2,3-dideoxy- $\beta$-D-ribo-hexopyranose adenosine (I-52). ${ }^{31}$ These homologous nucleosides of adenosine and deoxyadenosine possess obvious structural and configurational similarities to the corresponding ribofuranose adenosines I-53 and I-54 (Figure 1).

\footnotetext{
${ }^{\dagger}$ Reproduced with permission from Org. Lett. 2006, 8, 293-296. Copyright 2006, with permission from American Chemical Society.
} 
Figure 1. Adenosines and hexopyranosyl nucleosides.
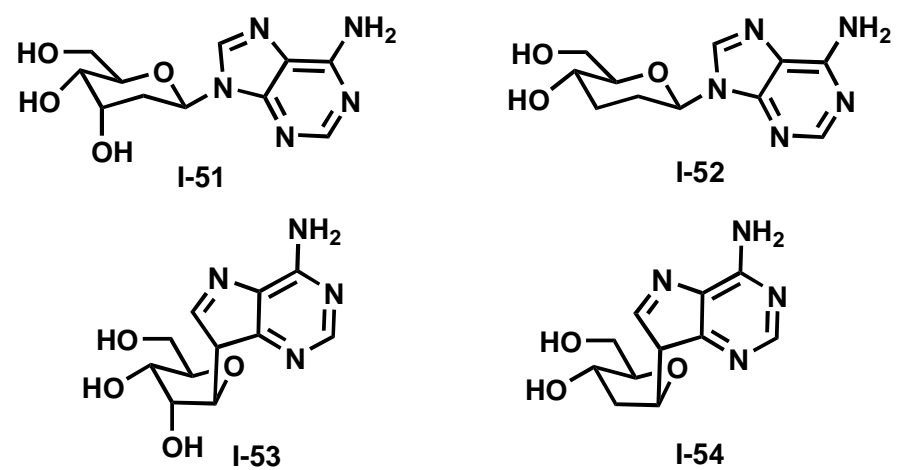

Biological studies of these ring-expanded analogues (I-51 and I-52) have led to the discovery of several pyrano-nucleotide analogues with both antitumor and antiviral activity. $^{32}$

\section{4.b. Project Description and Goals.}

While there has been significant synthetic effort toward the synthesis of adenosine analogues, ${ }^{29}$ we were interested in preparing pyrano-analogues of this class of compounds from an achiral starting material using enantioselective catalysis to set the asymmetry (de novo synthesis). In addition, we were interested in a synthesis that allows for the diastereoselective installation of the base at $C-1$. To test the breadth of this methodology, we set out to prepare various analogues of pyranose adenosines (I-51 and I-52). To accomplish this goal, we needed to extend the palladium glycosylation reaction to nitrogen nucleophiles (e.g., benzimidazole and purines). ${ }^{33}$ Herein, we describe our successful efforts to prepare the homoadenosine analogues (ent)- $\mathbf{I - 5 1}$ and (ent)- I-52. ${ }^{34}$

Scheme 8. Pd-catalyzed $N$-glycosylation. 


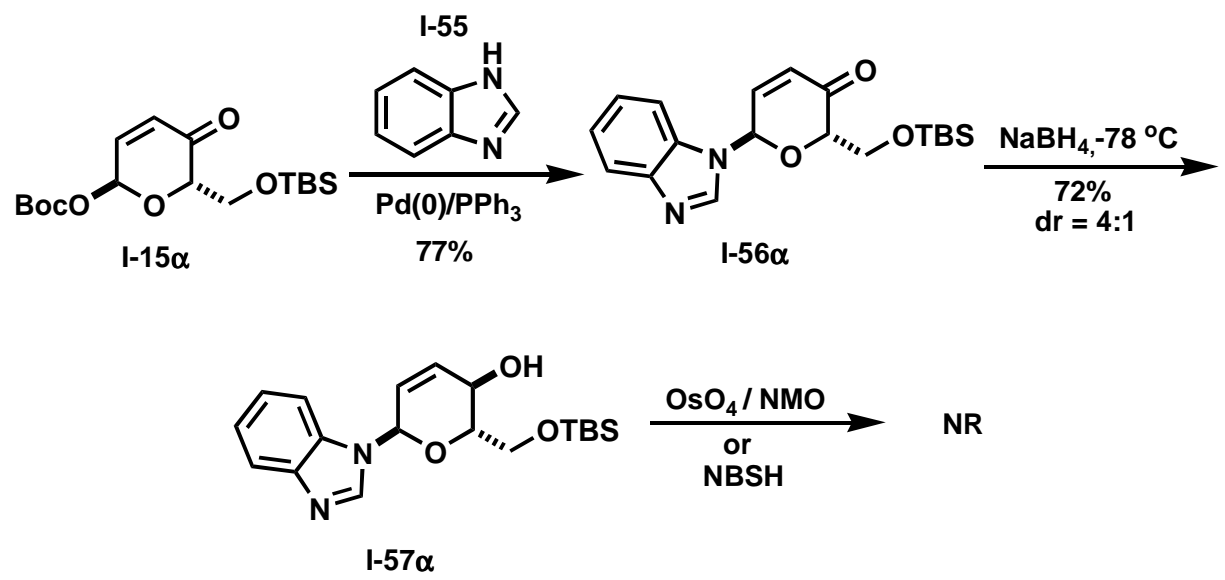

To test the strategy, we carried out a model study using benzimidazole I-55 and pyranone I-15 $\boldsymbol{\alpha}$. The Pd-catalyzed $N$-glycosylation of benzimidazole I-55 and pyranone I-15 $\alpha$ successfully gave the desired glycosylated pyranone I-56 $\alpha$ in good yield with complete stereocontrol (Scheme 8).

Our initial attempts at post-glycosylation modification of the $\mathrm{N}$-glycoside with the $\alpha$-anomers were not encouraging. The pyranone $\mathbf{I}-\mathbf{5 6} \alpha$ was readily reduced to give the $C$ 4 allylic alcohol I-57 $\boldsymbol{\alpha}$. This reduction, however, occurred with less stereocontrol at $C-4$ (4:1) as compared to $\mathrm{NaBH}_{4}$ reduction of pyranone with $C$-1 $\alpha$-oxygen substituents. ${ }^{35}$ The post-glycosylation chemistry for these $N$-glycosides diverged with the $O$-glycosides during our attempts to modify the double bond of pyran I-57 $\boldsymbol{\alpha}$. To our surprise, all attempts to either dihydroxylate or reduce $\mathbf{I - 5 7} \boldsymbol{\alpha}$ were unsuccessful (Scheme 8).

Scheme 9. Pd-catalyzed $N$-glycosylation: Synthesis of $N$-glycosylated sugars. 


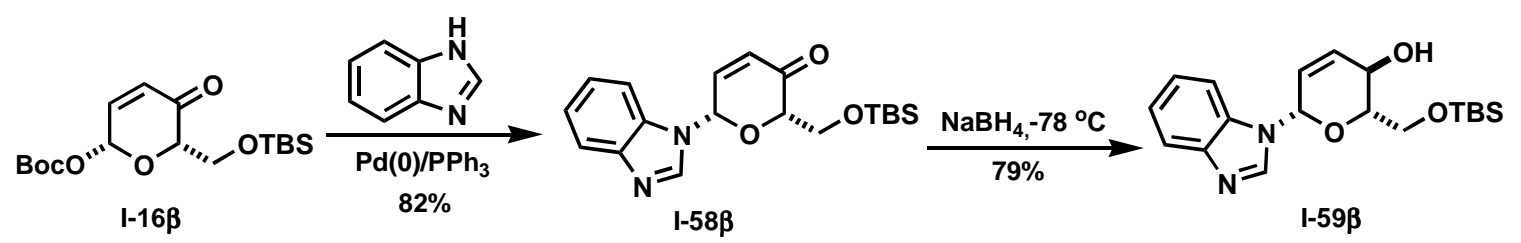

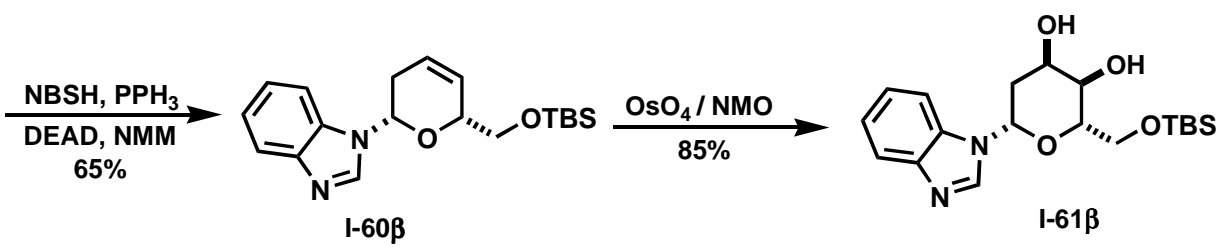

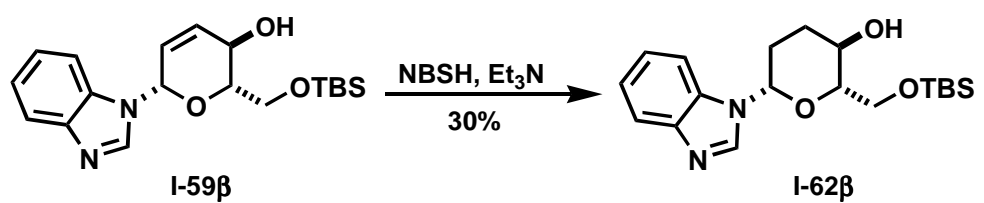

To our relief, our difficulties with the $\alpha$-anomer did not occur with the $\beta$-isomers (Schemes 9). The Pd-catalyzed $N$-glycosylation of benzimidazole I-55 and pyranone I$16 \boldsymbol{\beta}$ successfully gave the desired glycosylated pyranone I-58 $\beta$ in good yield. Reduction of the pyranone $\mathbf{I - 5 8} \boldsymbol{\beta}$ using $\mathrm{NaBH}_{4}$ at $-78{ }^{\circ} \mathrm{C}$ gave exclusively allylic alcohol $\mathbf{I}-\mathbf{5 9} \boldsymbol{\beta}$. Subjecting alcohol I-59 $\beta$ to Myers' reductive 1,3-transposition condition ${ }^{36}$ (NBSH, $\mathrm{PPh}_{3} / \mathrm{DEAD}, \mathrm{NMM},-30{ }^{\circ} \mathrm{C}$ to $\mathrm{rt}$ ) provided the rearranged olefin I-60 $\beta$ in good yield (65\%). Dihydroxylation of I-60 $\beta$ using the Upjohn conditions $\left(\mathrm{OsO}_{4} / \mathrm{NMO}\right)^{20}$ gave the diol I-61 $\boldsymbol{\beta}$ in $85 \%$ yield. In contrast to the oxidation chemistry, I-59 $\boldsymbol{\beta}$ could also be reduced, although not in high yield. Thus, exposing I-60 $\boldsymbol{\beta}$ to excess diimide precursor $\left(\mathrm{NBSH} / \mathrm{Et}_{3} \mathrm{~N}\right)$ gave the 2,3-dideoxypyranose $\mathbf{I}-\mathbf{6 2} \boldsymbol{\beta}$ in low yield $(30 \%)$ but with good recovery of starting material (50\%). While the yield of I-62 $\beta$ was low, this procedure was superior to traditional hydrogenation $\left(\mathrm{H}_{2}, 5 \% \mathrm{Pd} / \mathrm{C}\right.$ in $\left.\mathrm{MeOH}\right)$, which occurred with complete hydrogenolysis of the $C$-1 benzimidazole. These successful model studies inspired us to synthesize the adenosine analogues. ${ }^{34}$ 
Scheme 10. Enantioselective synthesis of homoadenosine and 2'-eoxy-homoadenosine.
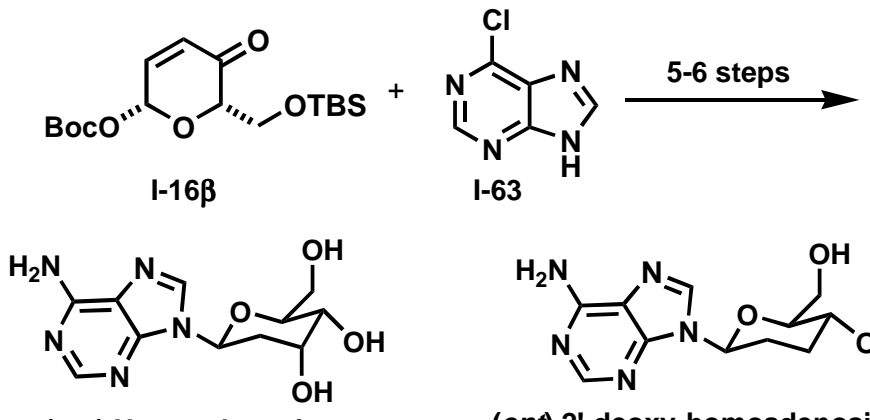

(ent) Homoadenosine

I-51

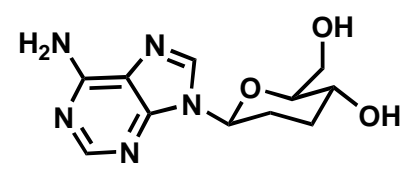

(ent) 2'-deoxy-homoadenosine

I-52

Our synthesis commenced with the coupling of Boc-protected pyranone I-16 $\beta$ and commercially available 6-chloropurine I-63. Further subjection to the similar transformations used in model study gave homeadenosine (ent)-I-51 and 2'deoxyhomoadenosine (ent)-I-52 in good yields.

\subsection{Summary.}

In conclusion, we have applied our practical palladium-catalyzed $O$-glycosylation reaction for the syntheses of phenol, flavonol and coumarin glycosides. Using our threestep protocol we have demonstrated the syntheses of coumarin and flavonol L-mannopyranoses with excellent stereocontrol and high yields.

We have also developed highly enantio- and diastereoselective procedure for the preparation of hexopyranose adenosine analogues using palladium-catalyzed $\mathrm{N}$ glycosylation reaction in five to six steps from Boc-protected pyranone. The synthesis of other potential analogues and evaluation of the biological activity of these compounds are ongoing. 


\section{Chapter 2}

\section{Asymmetric Synthesis and Medicinal Chemistry Studies of Novobiocin}

\section{Analogues.}

\subsection{Introduction and biological activity to novobiocin analogues}

The Aminocoumarin antibiotics, like novobiocin II-1, clorobiocin II-2 and Coumermycin A1 II-3 (Figure 2) are secondary metabolites isolated from several Streptomyces strains and show potent activity against Gram-positive bacteria. ${ }^{37}$ These coumarin antibiotics share a key structural core of a 3-acetamido-4-hydroxy-coumarin and a rare-sugar fragment both of which play critical role in biological activities. These coumarin antibiotics bind to type II topoisomerases and DNA-gyrase B subunit and inhibit the enzyme catalyzed hydrolysis of ATP. ${ }^{38}$

Figure 2. Naturally occurring aminocoumarin antibiotics.

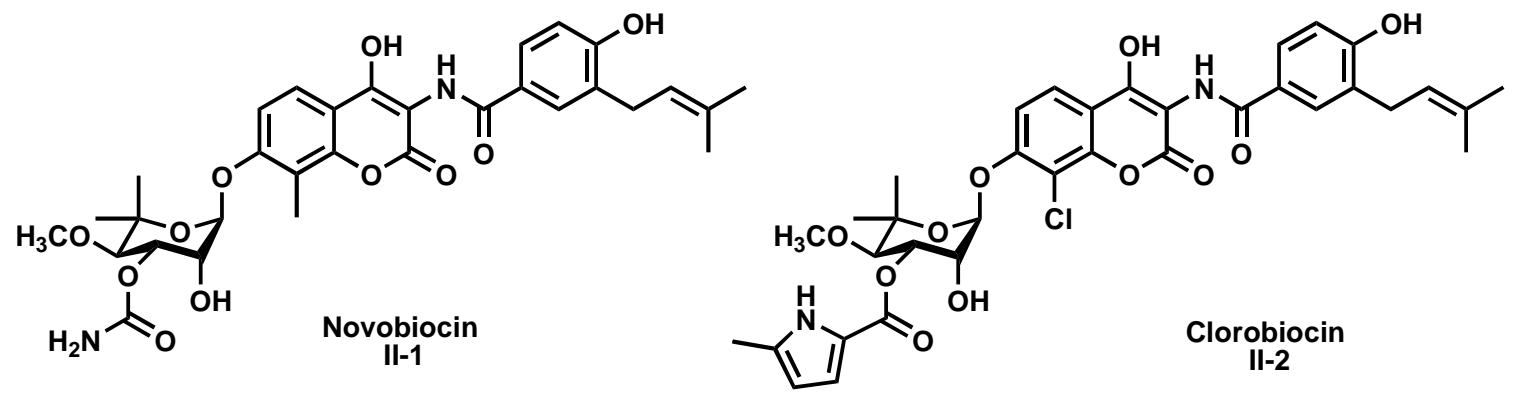

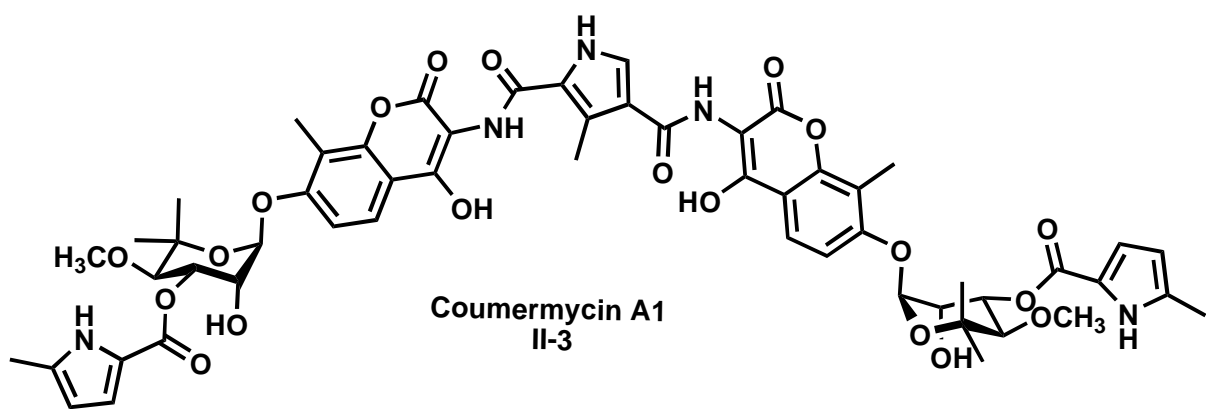


Recently Novobiocin was shown to inhibit Hsp90 through C-terminal ATP binding site, which is a promising target for development of cancer chemotherapeutics (i.e., antitumor agents capable of inhibiting all six hallmarks of cancer by restraining Hsp90 protein folding machinery). ${ }^{39}$ This promising biological activity and interesting structural features have inspired researchers to synthesize Novobiocin analogues.

\subsection{Previous approaches to novobiocin analogues.}

\section{2.a. Photolabile novobiocin analogues.}

Blagg et al. reported a convergent synthesis of four photolabile Novobiocin analogues in $2004 .^{40}$ Their synthesis started with the known compound 4,7-dihydroxy-8methyl-2H-1-benzopyran-2-one II-4, ${ }^{41}$ which when treated with diazonium salt II-5 gave the corresponding diazocoumarin derivative II-6 (Scheme 11). This diazaphenyl group serves as an amine protecting group in II-6. Previously Blagg had prepared 3-sulphone coumarin analogues, which failed to selectively glycosylate ( 7 vs 4 phenolic positions) under typical Schmidt glycosylation condition. ${ }^{42}$

Scheme 11. Synthesis of aminocoumarins.<smiles>Cc1c(O)ccc2c(O)cc(=O)oc12</smiles>

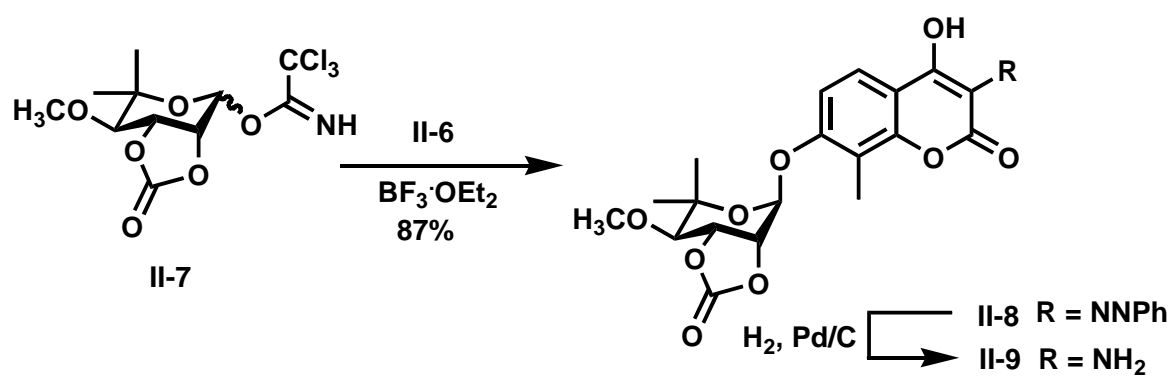


In contrast when the diazocoumarin II-6 was treated with trichloroacetimidate II7 in the presence of $\mathrm{BF}_{3} \cdot \mathrm{OEt}_{2}$, it produced the 7-noviose diazocoumarin derivative $\mathbf{I I - 8}$ in good yield. Diazacoumarin II-8 was deprotected by using hydrogenation conditions afford to 3-aminocoumarin II-9. Due to the instability of II-9 to purification conditions, crude II-9 was subjected to DCC conditions with 3-azido or 4-azidobenzoic acids yielding the desired amides II-10 and II-13 (Scheme 12). Previously Vaterlaus had studied the carbonate ring opening reaction to regioselectively form the desired carbamates. $^{43}$

Scheme 12. Synthesis of photolabile novobiocin analogues.

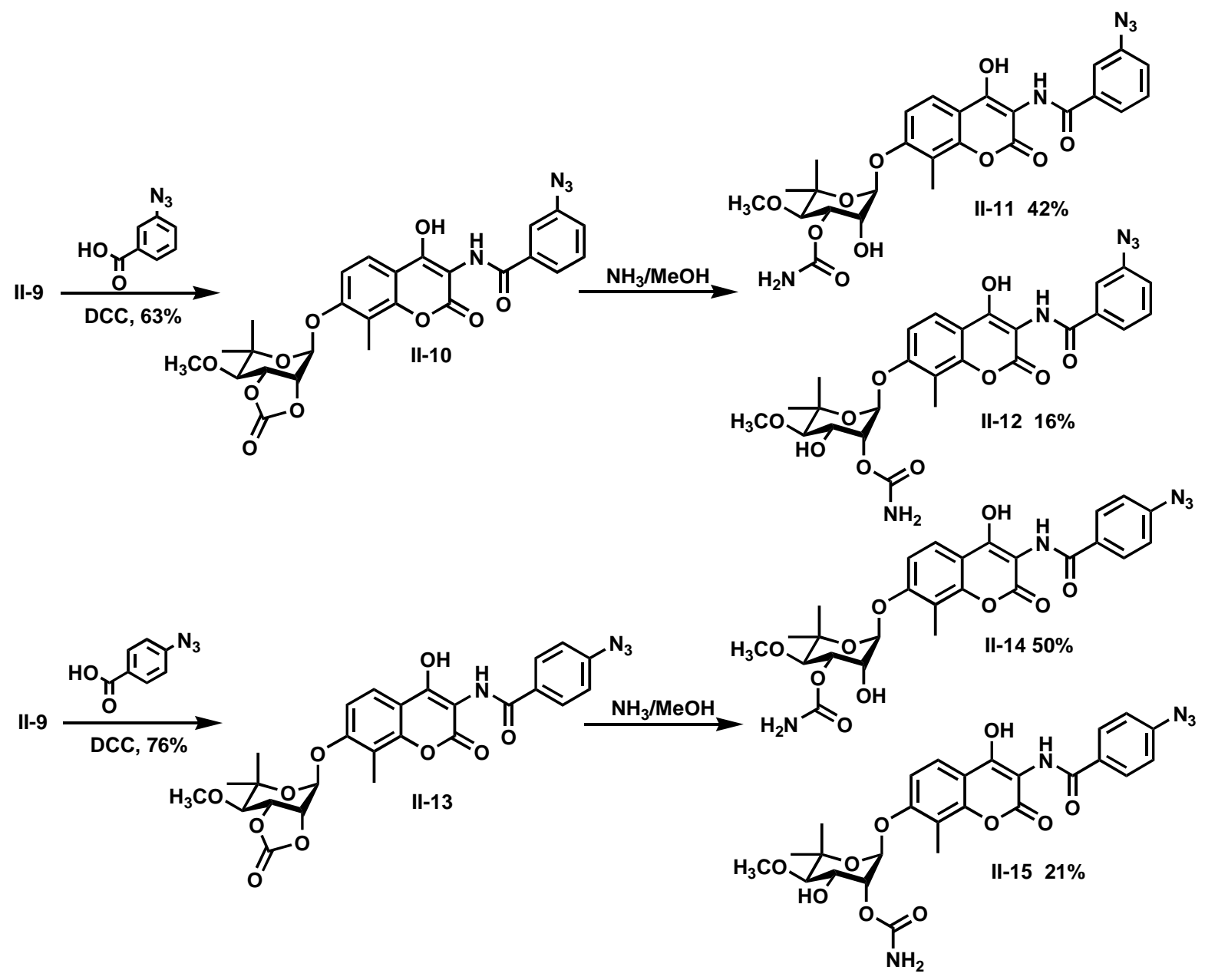


After careful optimization, Prof. Blagg group came up with a general methanolic ammonia (room temperature) conditions to prepare 3-carbamate noviose products II-11 and II-14 from amides II-10 and II-13 resulted in good yields respectively. Even under these optimized conditions the ring opening selectivities were less than perfect. Thus, significant amounts of the 2-carbamate isomers II-12 and II-15 were also isolated as minor products.

\section{2.b. Hsp90 inhibitors}

With success achieved in the initial synthesis of photolabile novobiocin analogues, Blagg turns to the preparation of novobiocin libraries with various coumarin substitution (Scheme 13). In 2005, Blagg et al. reported the Hsp90 inhibitors identified from a library of novobiocin analogues. ${ }^{44}$ They started the library syntheses with a range of coumarins (II-A - I-E). These II-A - II-E coumarins were coupled with trichloroacetimidate-noviose carbonate II-7 in the presence of $\mathrm{BF}_{3} \cdot \mathrm{OEt}_{2}$ to yield five coumarin- carbonates (II-A1 - II-E1). These carbonates II-A1 - II-E1 were subjected to methanolic ammonia (ring opening conditions) to provide 2'-carbamoyl II-A2-II-E2, 3'carbamoyl II-A3-II-E3, and des-carbamoyl compounds II-A4-II-E4 in good yields.

Scheme 13. Syntheses of novobiocin analogues. 
<smiles>CC(=O)Nc1cc2ccc(O)cc2oc1=O</smiles>

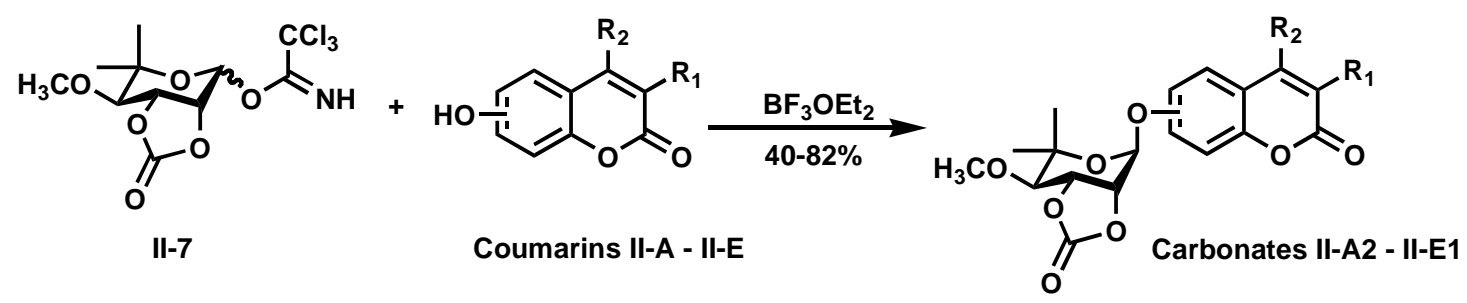

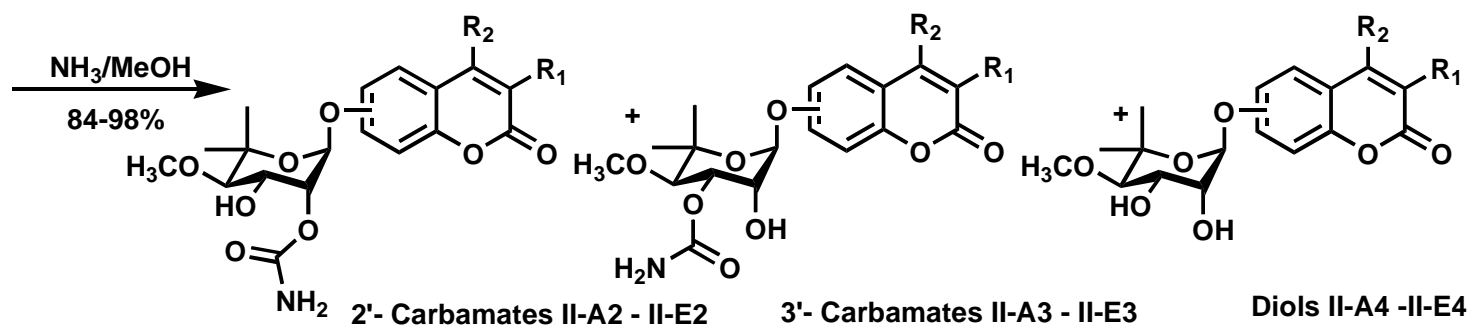

All novobiocin analogues in Scheme 3 were tested against Hsp90 client protein by incubating with $\mathrm{SKBr} 3$ breast cancer cells at a concentration of $100 \mu \mathrm{M}$. Westernblot analysis of protein lysates showed that analogue II-A4 (Figure 2) was the most active compound.

In 2006, Blagg et al. reported two more novobiocin analogues DHN1 and DHN2 (Figure 3), which are evaluated against Hsp90 and both analogues were significantly more potent than the natural product novobiocin. ${ }^{45}$ The DHN2 analogue proved to be more active than DHN1.

Figure 3. The DHN1 and DHN2 are selective Hsp90 inhibitors. 

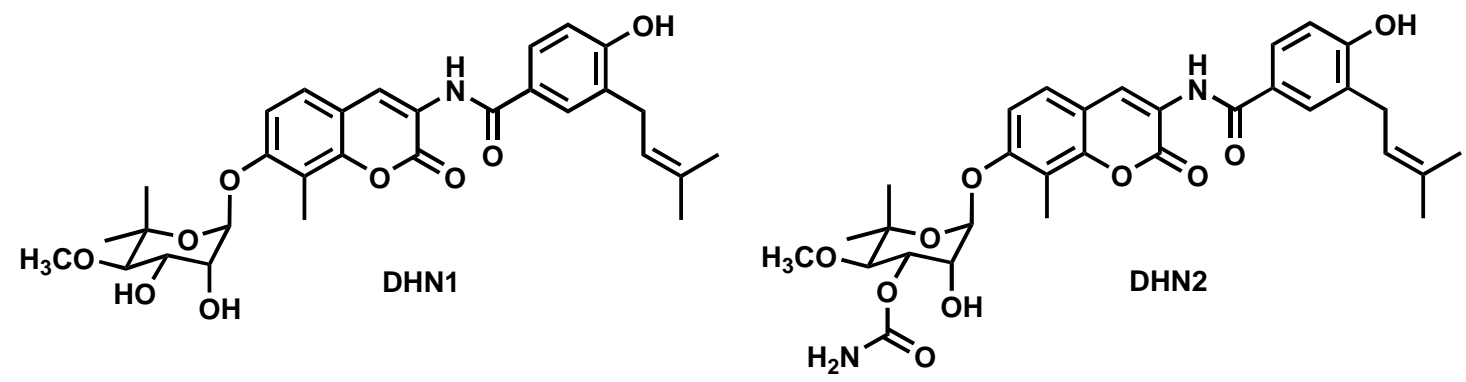

\section{2.c. Biosynthesis and Glycorandomization of novobiocin analogues}

Prof. Thorson's group also investigated the synthesis of Novobiocin analogues using a biosynthetic method. They called their approach in-vitro glycorandomization (IVG). ${ }^{46}$ IVG was used for the biosynthesis of sugar libraries of various complex natural products. ${ }^{47}$ In this study they have found a novobiocin specific glycosyl transferase enzyme NovM and screened it for activity with four aglycon coumarin analogues and 40 sugar nucleotides. With these optimized procedure they used the NovM enzyme for the glycosylation of three nucleotides (II-16, II-20 and II-22) with the novobiocin aglycon II-18 to afford three new coumarin antibiotics (Scheme 14). Out of three novobiocin analogues, compound II-19 showed the best antibiotic activity (MIC $5 \mu \mathrm{g}$ $\mathrm{mL}^{-1}$ ) compared with novobiocin II-1 (MIC $0.06 \mu \mathrm{g} \mathrm{mL}^{-1}$ ) and was consistent with novobiocin II-1 mechanism of action. ${ }^{2}$ 
Scheme 14. Biosynthesis of new coumarin antibiotics.<smiles>CC(C)=CCc1cc(C(=O)Nc2c(O)c3ccc(OC4OC(C)[C@H](O)[C@H](O)[C@H]4O)c(C)c3oc2=O)ccc1O</smiles>

Presented herein are two different approaches to glycosylated coumarin antibiotic analogues of the novobiocin. These approaches allowed for the preparation of analogues containing aminocoumarin core with various substituted deoxysugars. Interestingly, each group used modular synthesis with advanced building blocks and successfully addressed the key issues of both synthetic selectivity and biological activity.

\subsection{Project Description and Goals}

Since inhibitors of $\mathrm{Hsp} 90$ are considered to be novel target for anticancer therapeutics, we decided to prepare a small library of novobiocin analogues using our palladium-coupling chemistry. In collaboration with Prof. Blagg's lab, we planned to test our compounds on different cancer cell lines. This we felt was an excellent opportunity 
to test our various novobiocin analogue compounds. A continuing theme of using asymmetric catalysis to improve the synthetic efficiency and atom economy of carbohydrate synthesis. The retrosynthetic analysis for our synthesis of novobiocin analogues is shown in Scheme 15. Several coumarin aglycones were coupled with pyranone ester II-30 using our Pd-catalyzed glycosylation as the key reaction. ${ }^{48}$ The pyranone ester II-30 can be constructed from commercially available starting material acylfuran II-24.

Scheme 15. Retrosynthesis of novobiocin analogues.

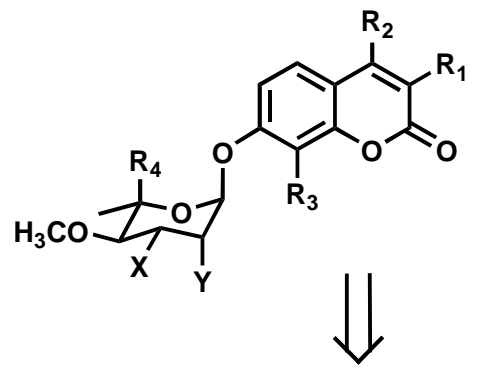

$\mathrm{R}_{1}=\mathrm{H}, \mathrm{NHCOCH}_{3}, \mathrm{NHCO}\left(\mathrm{C}_{12} \mathrm{H}_{13} \mathrm{O}\right)$

$\mathrm{R}_{2}=\mathrm{H}, \mathrm{CH}_{3}$

$\mathrm{R}_{3}=\mathrm{H}, \mathrm{CH}_{3}$

$\mathbf{R}_{4}=\mathbf{H}$

$\mathrm{X}=\mathrm{OCONH}_{2}, \mathrm{OH}, \mathrm{H}$

$\mathrm{Y}=\mathrm{OH}, \mathrm{H}$

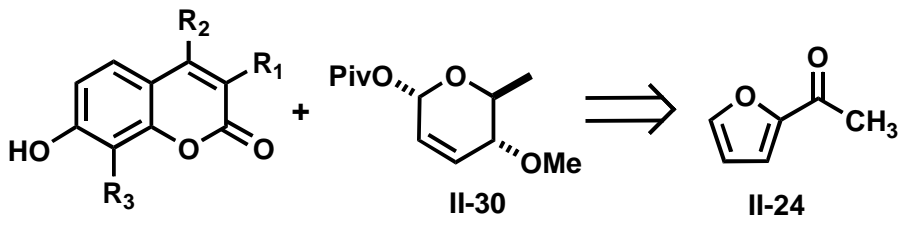

\subsection{Synthesis of Novobiocin Analogues}

In this approach the pyranone asymmetry was derived by the use of a Noyori asymmetric hydrogenation of acylfuran II-24 to yield furan alcohol II-25. ${ }^{49}$ Pyranone II27 can easily be prepared from furan alcohol II-25 by an Achmatowicz rearrangement, followed by hemiacetal protection with Boc anhydride. The more reactive axial anomeric alcohols can be acylated selectively $(>20: 1, \alpha: \beta)$ at $-78{ }^{\circ} \mathrm{C}$. Alternatively at room temperature, (1.3:1) mixture of anomers can be produced with excellent enantiomeric excess $>96 \%\left(\right.$ Scheme 16). ${ }^{50}$ 
Scheme 16. Synthesis of Boc-Pyranone.

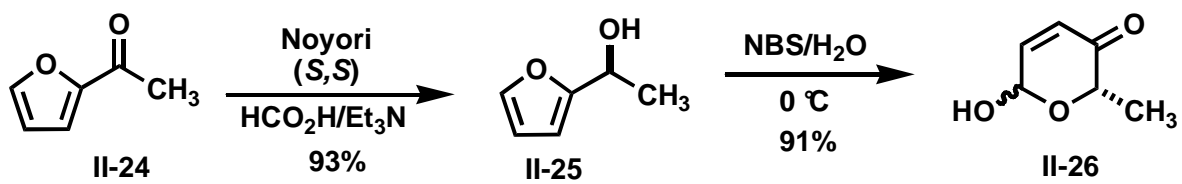<smiles>C[C@H]1O[C@@H](O)C=CC1=O</smiles>

II-26

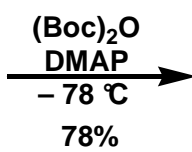

$78 \%$

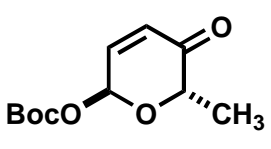

II-27

We next turned our attention toward the preparation of manno-sugar linked coumarins. The glycosylation of 7-hydroxy 4-methyl coumarin alcohol II-28 with the $\alpha$ L-pyranone II-27 in the presence of $\left(5 \mathrm{~mol} \% \mathrm{Pd}(0) / 10 \% \mathrm{PPh}_{3}\right)$ to form the glycosylated pyranone II-29 in $84 \%$ yield as a single diastereomer (Scheme 17). Pyranone II-29 was subjected to Luche conditions ${ }^{51}\left(\mathrm{NaBH}_{4} / \mathrm{CeCl}_{3},-78^{\circ} \mathrm{C}\right)$ to reduce the ketone but unfortunately it fails to produce the desired allylic alcohol.

Scheme 17. Synthesis of glycosylated pyranol.<smiles>CC1OC(OC(C)(C)C)C=CC1=O</smiles>

II-27

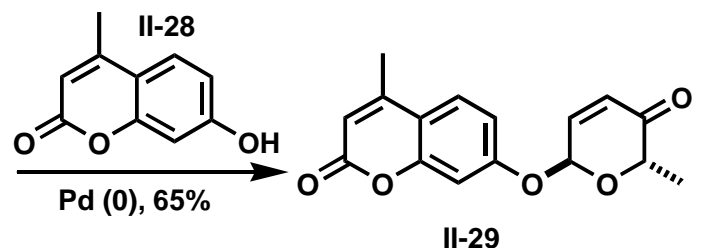<smiles>Cc1cc(=O)oc2cc(OC3C=CC(=O)[C@H](C)O3)ccc12</smiles>

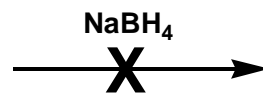

N. R.

II-29

After investigating with different reaction conditions in the reduction step, we modified our approach by shifting the glycosylation step to after the reduction and methylation steps (Scheme 18). In this regard, the protecting group of pyranone was changed from carbonate (Boc) to Piv-ester due stability reasons during the reduction step. 
Thus, hemiacetal II-26 were subjected to $\mathrm{PivCl}$ to give Piv-protected enone II-30 in excellent yield. The ketone of pyranone II-30 was diastereoselectively reduced under Luche conditions forming the equatorial allylic alcohol in excellent yield (90\%) followed by alkylation of allylic alcohol as methyl ether in neutral conditions $\left(\mathrm{Ag}_{2} \mathrm{O}, \mathrm{MeI},\right)$ to give methyl ether II-32 in $85 \%$ yield. We next explored the Pd-catalyzed glycosylation by subjecting allylic pivalate II-32 to coumarin II-28 under glycosylation conditions (5 mol $\left.\% \operatorname{Pd}(0) / 10 \% \mathrm{PPh}_{3}\right)$, which afforded glycosylated coumarin II-33 with complete stereocontrol in $80 \%$ yield.

Scheme 18. Synthesis of Glycosylated Coumarin
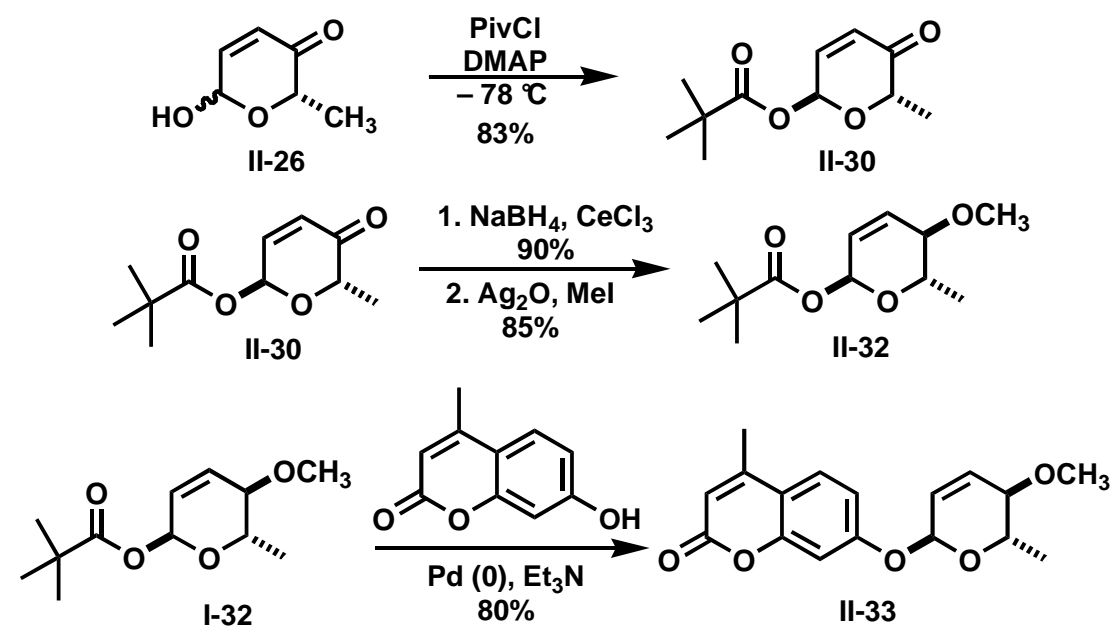

After successful completion of the glycosylation step, we move forward with dihydroxylation of II-33 using the Upjohn conditions ${ }^{20}\left(\mathrm{OsO}_{4} / \mathrm{NMO}\right)$, which gave exclusively the diol II-34 in $85 \%$ yield (Scheme 19). Then we regioselectively protected the $C$-2 axial alcohol of II-34 via ortho ester formation, and selective hydrolysis to provide acetate II-35 in $90 \%$ yield. ${ }^{52}$ Finally the $C-5$ des methyl noviose synthesis was completed by subjecting the free equatorial alcohol with chlorosulfonyl isocynate, 
followed by hydrolysis with $\mathrm{K}_{2} \mathrm{CO}_{3} / \mathrm{MeOH}$ gave $C 3$-carbamoyl analogue II-36 in good yield (80\% two steps). ${ }^{53}$ Alternatively the dideoxy sugar analogue was prepared in excellent yields by exposing the allylic ether II-33 to excess diimide precursor and base (93\% yield). ${ }^{54}$ Thus with this successful model study, we have synthesized three novobiocin analogues II-34, II-36 and II-37.

Scheme 19. Synthesis of des-methyl-novobiocin analogues II-34, II-36 and II-37.

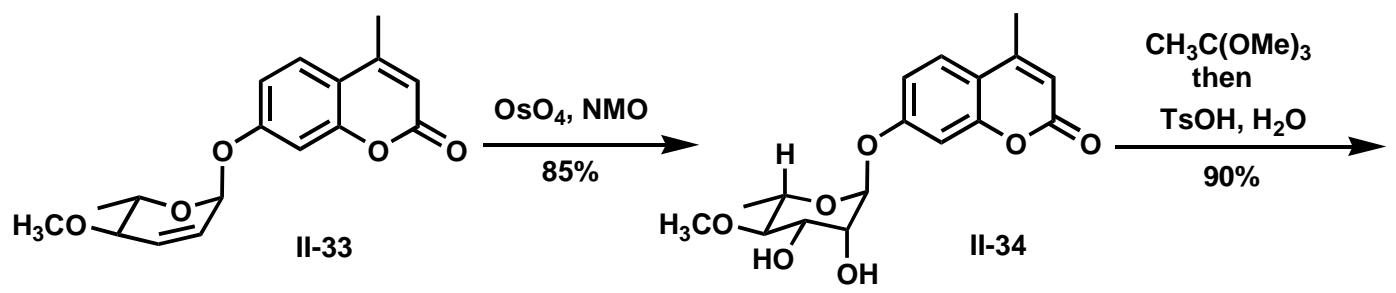

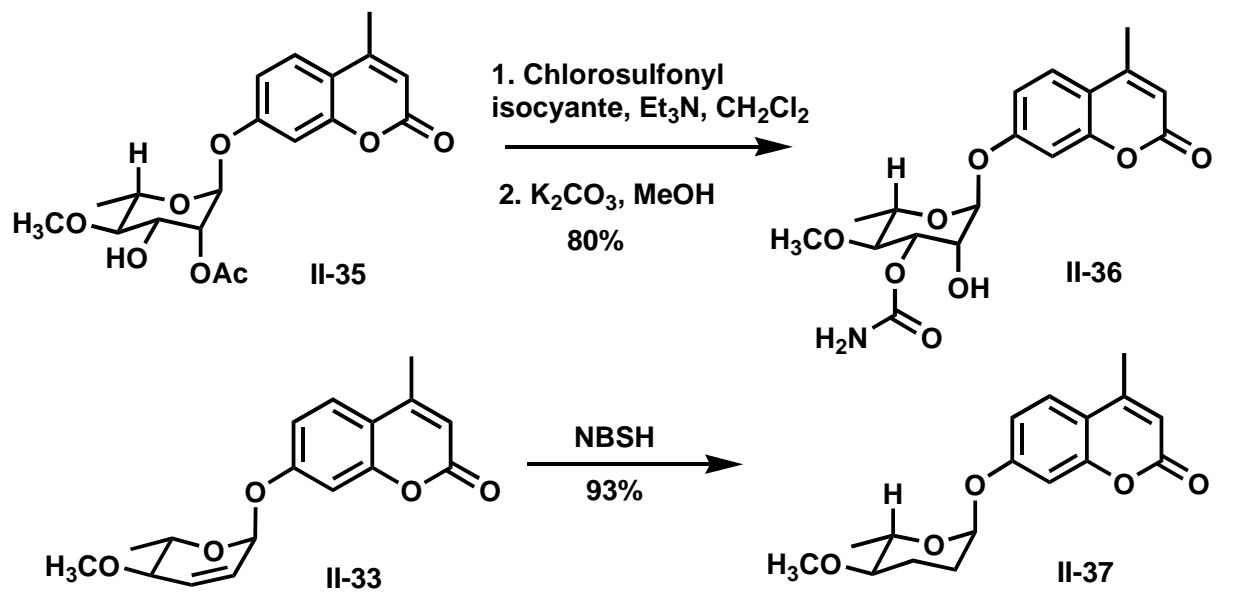

Encouraged by the above results, we prepared a set of six analogues using the above methodology with two biologically promising aglycones II-38 \& II-44 provided by Prof. Blagg research group (Scheme 20). Once again a Pd-catalyzed glycosylation of allylic pivalate II-32 with the acetamido coumarin II-38, followed by series of postglycosylation transformations gave three different analogues II-40, II-41 and II-43 with similar yields comparing with simple coumarin analogues (Scheme 20 \& 21). 
In this vein, we next explored the Pd-catalyzed glycosylation by subjecting allylic pivalate II-32 to coumarin II-38 under glycosylation conditions (5 mol\% $\left.\mathrm{Pd}(0) / 10 \% \mathrm{PPh}_{3}\right)$, which afforded glycosylated coumarin II-39 in $80 \%$ yield (Scheme 10$)$. Dihydroxylation of II-39 using the Upjohn conditions ${ }^{20}\left(\mathrm{OsO}_{4} / \mathrm{NMO}\right)$, which gave exclusively the diol II-40 in $85 \%$ yield. Alternatively the dideoxy sugar analogue II-41 was prepared in excellent yields by exposing the allylic ether II-39 to excess diimide precursor and base (95\% yield).

Scheme 20. Syntheses of des-novobiocin analogues II-40 and II-41.
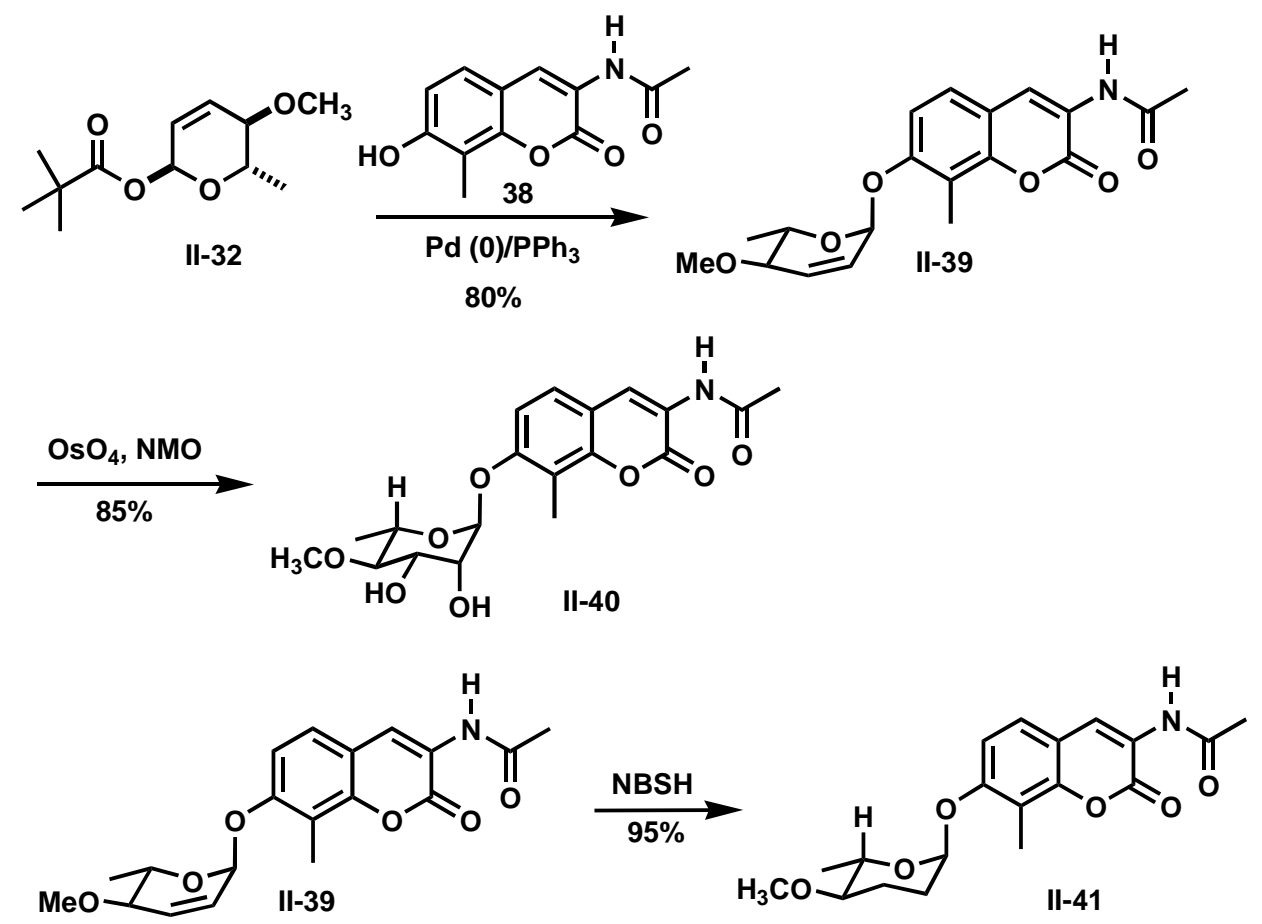

Then we regioselectively protected the $C-2$ axial alcohol of II-40 via ortho ester formation, and selective hydrolysis to provide acetate II-42 in 91\% yield (Scheme 21). Finally the $C-5$ des methyl novobiose synthesis was completed by subjecting the free equatorial alcohol with chlorosulfonyl isocynate, followed by hydrolysis with $\mathrm{K}_{2} \mathrm{CO}_{3} / \mathrm{MeOH}$ gave $C$-3-carbamoyl analogue II-43 in good yield (83\% two steps). 
Scheme 21. Synthesis of des-novobiocin analogue II-43.
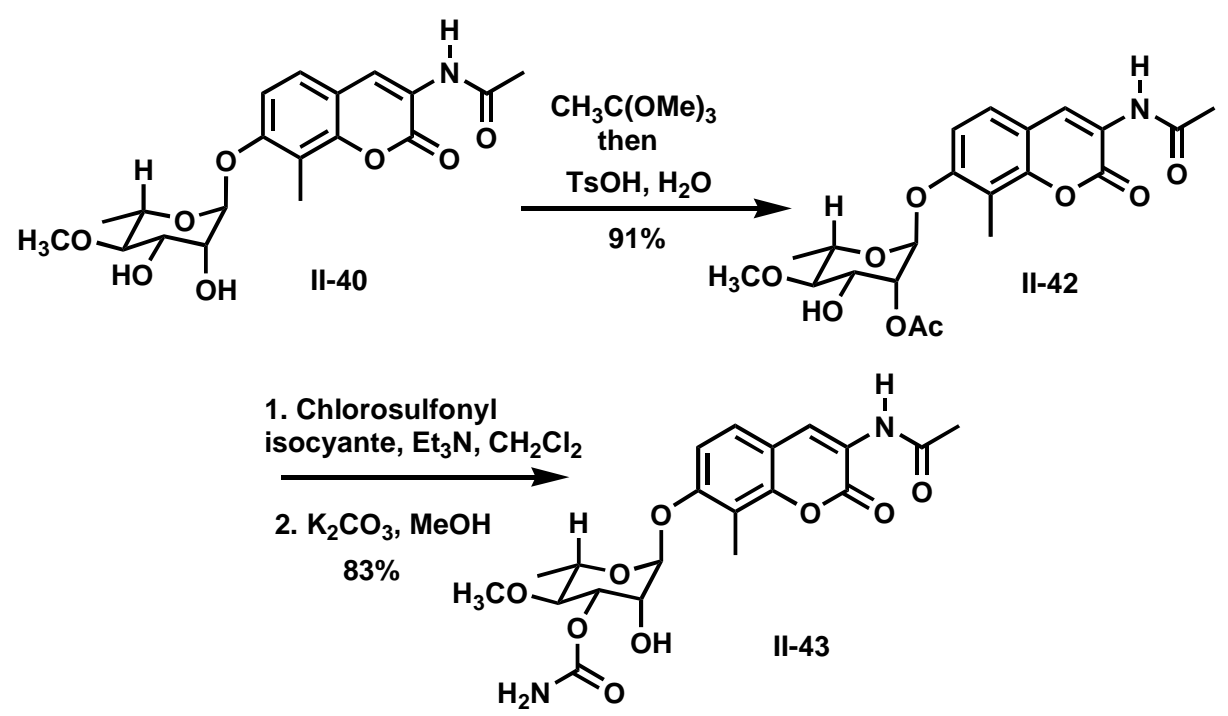

Using the same chemistry, we explored the Pd-catalyzed glycosylation by subjecting allylic pivalate II-32 to coumarin II-44 under glycosylation conditions (5 mol\% $\left.\mathrm{Pd}(0) / 10 \% \mathrm{PPh}_{3}\right)$ gave glycosylated coumarin II-45 in $85 \%$ yield (Scheme 22).

Dihydroxylation of II-45 using the Upjohn conditions ( $\left.\mathrm{OsO}_{4} / \mathrm{NMO}\right)$ gave exclusively the diol II-40 in $86 \%$ yield. Alternatively the dideoxy sugar analogue II-47 was prepared in excellent yields by exposing the allylic ether II-45 to excess diimide precursor and $\mathrm{Et}_{3} \mathrm{~N}$ (96\% yield).

Then again we regioselectively protected the $C$-2 axial alcohol of II-46 via ortho ester formation, and selective hydrolysis to provide acetate II-48 in $90 \%$ yield (Scheme 23). Finally the $C-5$ des methyl novobiose synthesis was completed by subjecting the free equatorial alcohol with chlorosulfonyl isocynate, followed by hydrolysis with $\mathrm{K}_{2} \mathrm{CO}_{3} / \mathrm{MeOH}$ gave $C$-3-carbamoyl analogue II-49 in good yield (81\% two steps). After successful syntheses of a small library of nine novobiocin analogues, they were sent for activity testing at Blagg's lab against different cancer cell lines. 
Scheme 22. Syntheses of des-novobiocin analogue II-46 \& II-47.

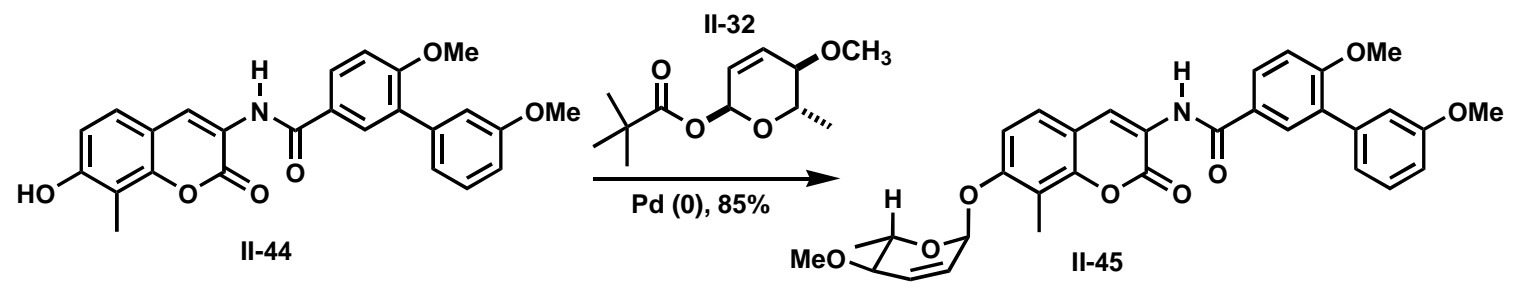

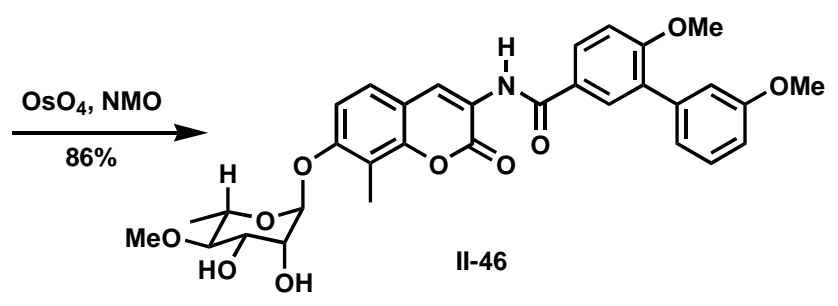<smiles>CCC1OC2CC(Oc3ccc4cc(NC(=O)c5ccc(OC)c(-c6cccc(OC)c6)c5)c(=O)oc4c3C)OC2C1OC</smiles>

Scheme 23. Synthesis of des-novobiocin analogue II-49.

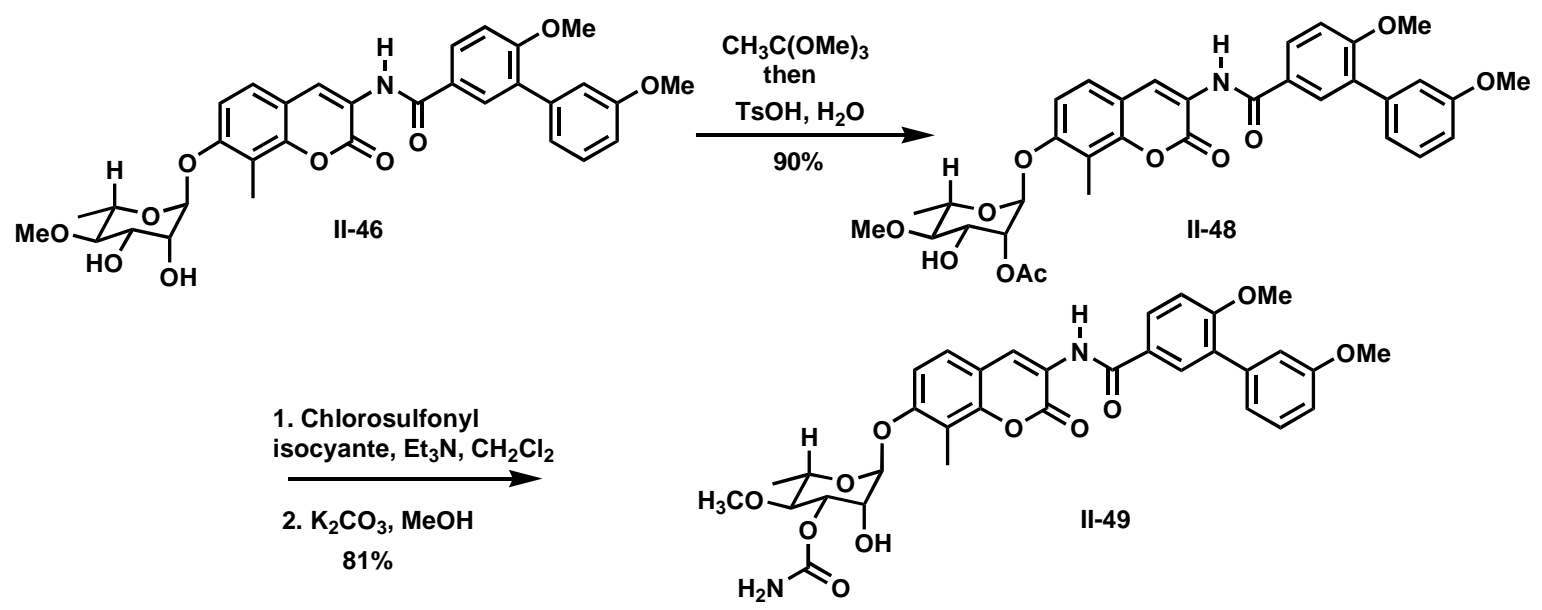

We next investigated the removal of the second methyl group at the $C-5$ center of the sugar position of the molecule. In this connection we have started building di-des- 
methyl novobiocin analogues. Our synthetic strategy (Scheme 24) started by taking furyl alcohol II-50 treating with NBS in aqueous THF (Achmatowicz reaction) gave hemiacetal II-51 in $85 \%$ yield.

Scheme 24. Synthesis of des- $C$-5-methy-novobiocin analogue II-55.<smiles>CC(C)(C)C(=O)OC1C=CC(=O)CO1</smiles><smiles>CC(C)(C)C(=O)OC1C=CC(O)CO1</smiles>

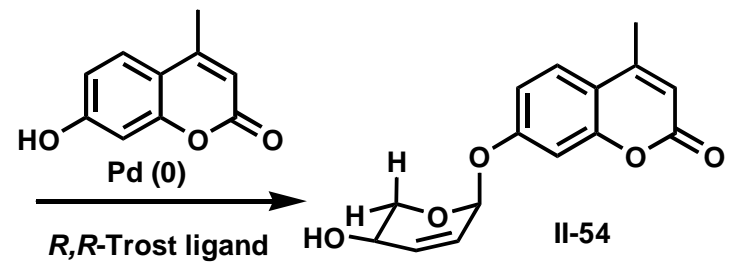<smiles>Cc1cc(=O)oc2cc(OC3OC4C=CC3C(O)C4O)ccc12</smiles><smiles>Cc1cc(=O)oc2cc(OC3OC(O)C(O)C3O)ccc12</smiles>

\begin{tabular}{|c|c|c|}
\hline $\mathrm{Pd}(0)$ glycosylation & $\% \mathrm{ee}^{*}$ & $\%$ yield \\
\hline $\mathrm{PPh}_{3}, 0^{\circ} \mathrm{C}, \mathrm{THF}$ & 0 & $85 \%$ \\
\hline $\begin{array}{l}R, R-\mathrm{Trost} \text { ligand, } \\
0^{\circ} \mathrm{C}, \mathrm{THF}\end{array}$ & 60 & $75 \%$ \\
\hline
\end{tabular}

* measured by HPLC

Acylation of hemiacetal II-51 with PivCl provided the Piv protected pyranone II52 in good yield (80\%). A reduction of pyranone II-52 under Luche conditions gave racemic allylic alcohol II-53 in $86 \%$ yield as a single diastereomer. Using 5 mol\% palladium $/ R, R$ Trost ligand, allylic alcohol II-53 was coupled with coumarin alcohol providing glycoside II-54 in $75 \%$ yield with $60 \%$ ee where as racemic material could be 
prepared using $\mathrm{Ph}_{3} \mathrm{P}$ as the ligand. Exposing allylic alcohol II-54 to the Upjohn conditions $^{20}\left(\mathrm{OsO}_{4} / \mathrm{NMO}\right)$ gave the diol $\mathbf{I I - 5 5}$ in good yield $(85 \%)$.

\subsection{Summary}

In conclusion, we developed a practical 7 to 10 step diastereoselective route for the syntheses of nine different analogues of three aglycones all in good yields. This route relies on an alternative pyranol palladium-catalyzed glycosylation reaction, diastereoselective dihydroxylation, regioselective carbamate installation strategy. Currently, the nine-novobiocin analogues are being tested against different cancer cell lines in Prof. Blagg's labs. 


\section{Chapter 3}

\section{Enantioselective Synthesis of Mannopeptimycin-E analogues. ${ }^{\dagger}$}

\subsection{Introduction to mannopeptimycins.}

The continuing emergence of bacterial resistance to traditional antibiotics has inspired a never-ending search for new antibiotics. ${ }^{55}$ The five mannopeptimycins (III1A-E) were isolated from the fermentation broths of Streptomyces hygroscopicus LLAC98 and related mutant strains. ${ }^{56}$ The key structural features of the mannopeptimycins are a cyclic hexapeptide core with alternating D- and L-amino acids, three of which are rare. Two of the amino acids ( $\beta$-D-hydroxyenuricididine and D-tyrosine) are glycosylated with mannose sugars. The glycosylated amino acids are an $N$-glycosylated $\beta$ hydroxyenuricididine with an $\alpha$-mannose and an $O$-glycosylated tyrosine with a $\alpha$ - $(1,4-$ linked)-bis-manno-pyranosyl pyranoside.

Figure 4. Structure of mannopeptimycin-E III-1E.

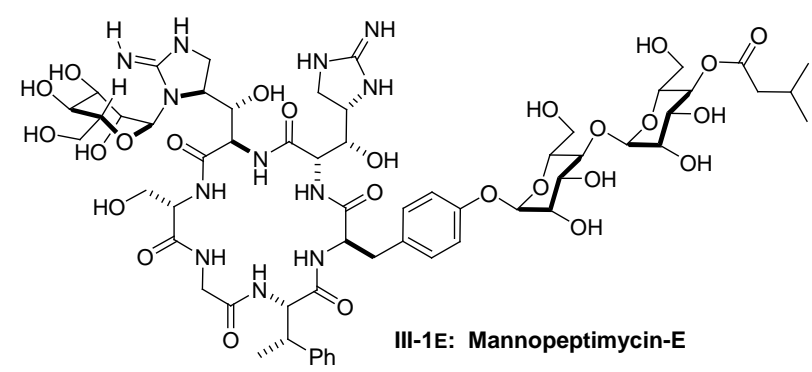

The unique structure and unprecedented biological activity have inspired both biological $^{57}$ and synthetic studies ${ }^{58}$ from labs at Wyeth Pharmaceuticals. Among the mannopeptimycins, mannopeptimycin-E (III-1E, Figure 4) was reported as the most

\footnotetext{
${ }^{\dagger}$ Reproduced with permission from Org. Lett. 2006, 8, 1605-1608. Copyright 2006, with permission from American Chemical Society.
} 
active member against methicillin-resistant staphylococci and vancomycin-resistant enterococci (Table 3). ${ }^{59}$

Table 3. Activities of mannopeptimycins.

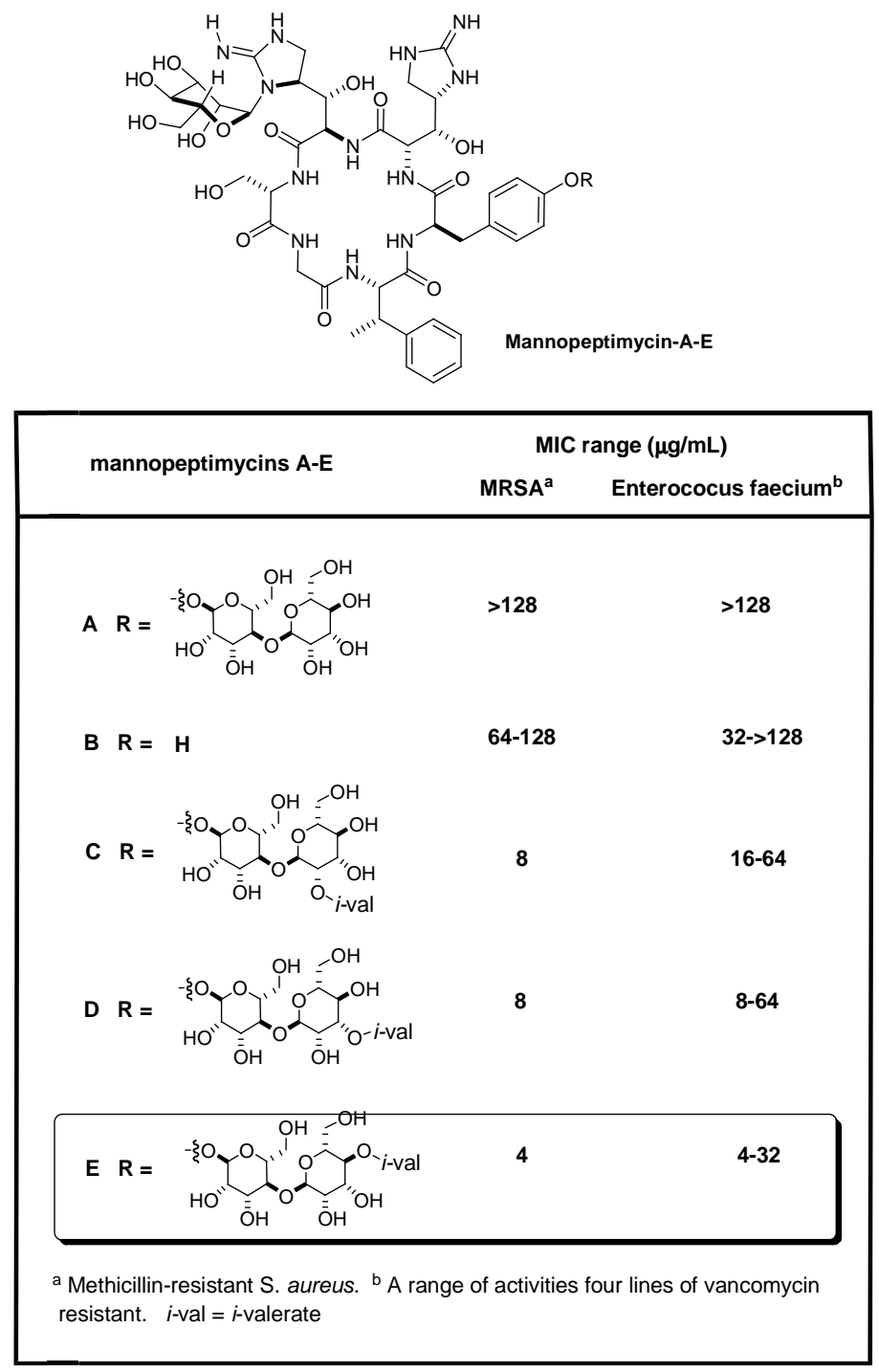

A particularly interesting aspect of the SAR for the mannopeptimycins is how the specific placement of the isovalerate group on the bis-manno-disaccharide correlates with its antibacterial activity. It has been shown that $C-4$ isovalerate substitution on the terminal mannose leads to a substantial increase in antibacterial potency. For instance, 
mannopeptimycins-C and -D, which have $C-2$ and $C-3$ isovalerate groups, respectively, have reduced activity, whereas mannopeptimycins-A and -B, which lack isovalerate substitution, have even lower activity (Table 3). ${ }^{59}$

\subsection{Previous Approaches to Mannopeptimycin Analogues.}

\section{2.a. Synthesis of Mannopeptimycin derivatives}

The crisis associated with the capability of organisms to develop resistance mechanisms to antibiotics has fueled the continuous search for new antibacterial agents. ${ }^{55}$ The mannopeptimycins, a tris-manno-glycosylated class of cyclic hexapeptides with alternating $\mathrm{D}$ and L-amino acids, were isolated as part of this mission. Of the mannopeptimycins, mannopeptimycin-E (III-1E) (Figure 4) is the most active member, displaying activity against methicillin-resistant staphylococci and vancomycinresistant enterococci. ${ }^{56}$ In an effort to identify compounds with improved activity and SAR studies, chemists at $\mathrm{Wyeth}^{58}$ have synthesized several derivatives of mannopeptimycins. Retrosynthetically, they envisioned that the ketal derivative III-4 could be prepared from fully protected linear hexapeptide III-5 using solid-phase chemistry and anticipated to cyclize the hexapeptide with the amide bond formation between the L-serine and unhindered glycine. Free amine of compound III-5 was prepared by using D-tyrosine containing disaccharide III-11 and five commercially available amino acids (Scheme25).

Scheme 25. Retrosynthesis of mannopeptimycin derivative III-4. 

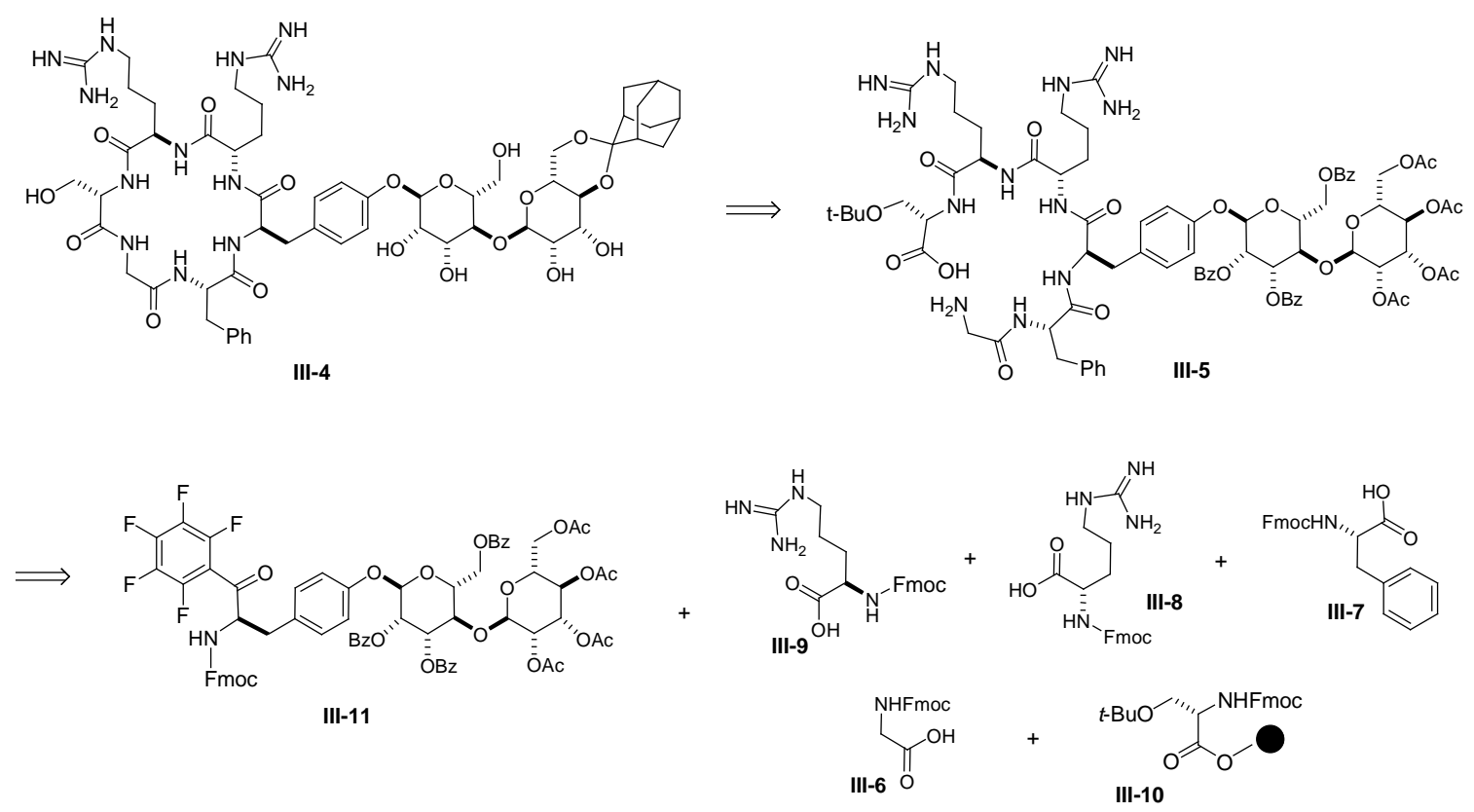

They synthesized the peptide disaccharide derivative III-11 by coupling bis-mannodisaccharide III-13 and protected tyrosine III-12 using $\mathrm{N}$ iodosuccinimide/trimethylsilyltriflate. Compound III-13 was prepared from sugar intermediates III-14 and III-15 (Scheme 26).

Scheme 26. Retrosynthesis of tyrosine-bis-mannose III-11.

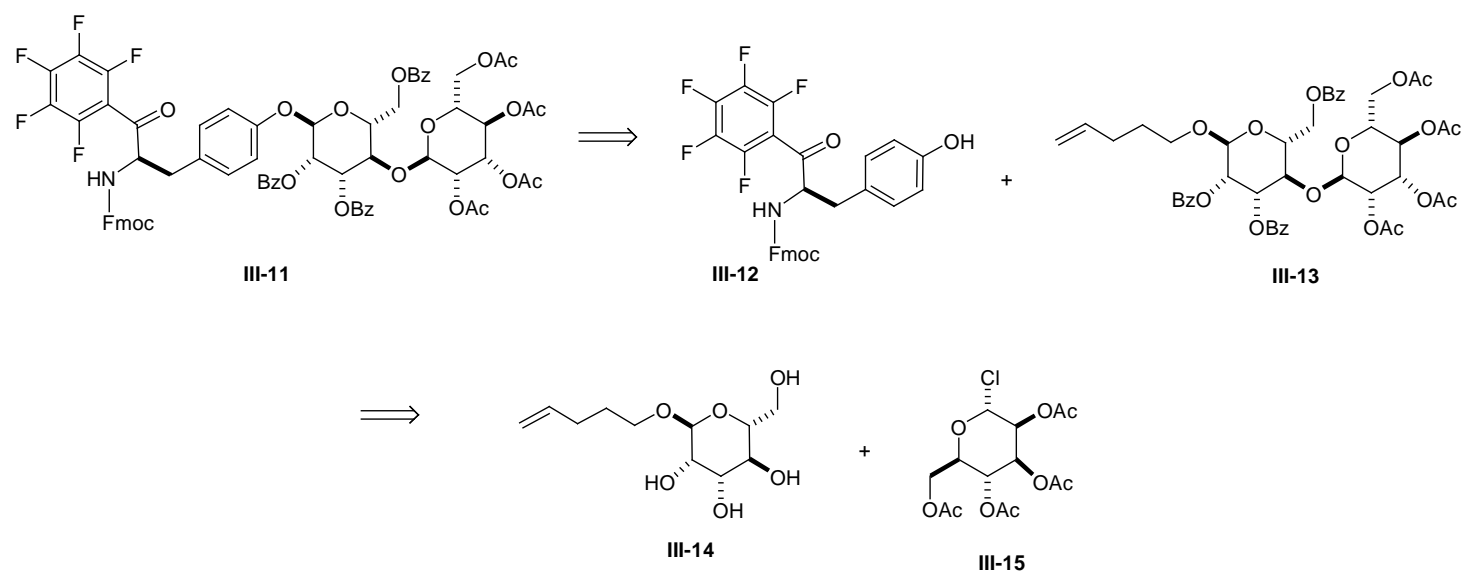




\subsection{Project description and project goals.}

This work confirmed the importance of the $C-4$ isovaleryl group for antibiotic activity. The critical role isovalerate substitution has on the antibacterial activity of the mannopeptimycin-E inspired us to pursue a synthesis of an appropriate $O$-glycosylated D-tyrosine with $C$-4 isovalerate substitution (e.g., III-2a and III-2b, Scheme 27). ${ }^{60}$ In addition to our desire to synthesize and test the mannopeptimycin analogues III-2a and III-2b, we felt that the synthesis of III-2a would serve as part of a model study for our synthesis of the natural product. In addition, the preparation of $\mathbf{I I I - 3 b}$, a fully protected bis-glycosylated tyrosine, would be of use for the synthesis of mannopeptimycin-E. Herein, we report the successful implementation of our palladium-catalyzed glycosylation reaction ${ }^{61,62}$ for the de novo installation of both a D,D- and an L,L-bismanno-disaccharide fragment on a D-tyrosine. The flexibility of the approach is demonstrated by the syntheses of bis-2,3-dideoxy analogues in their D,D- and an L,Lforms. ${ }^{63}$

Scheme 27. Retrosynthesis of mannopeptimycin-E III-1E and its key fragment III-2a. 

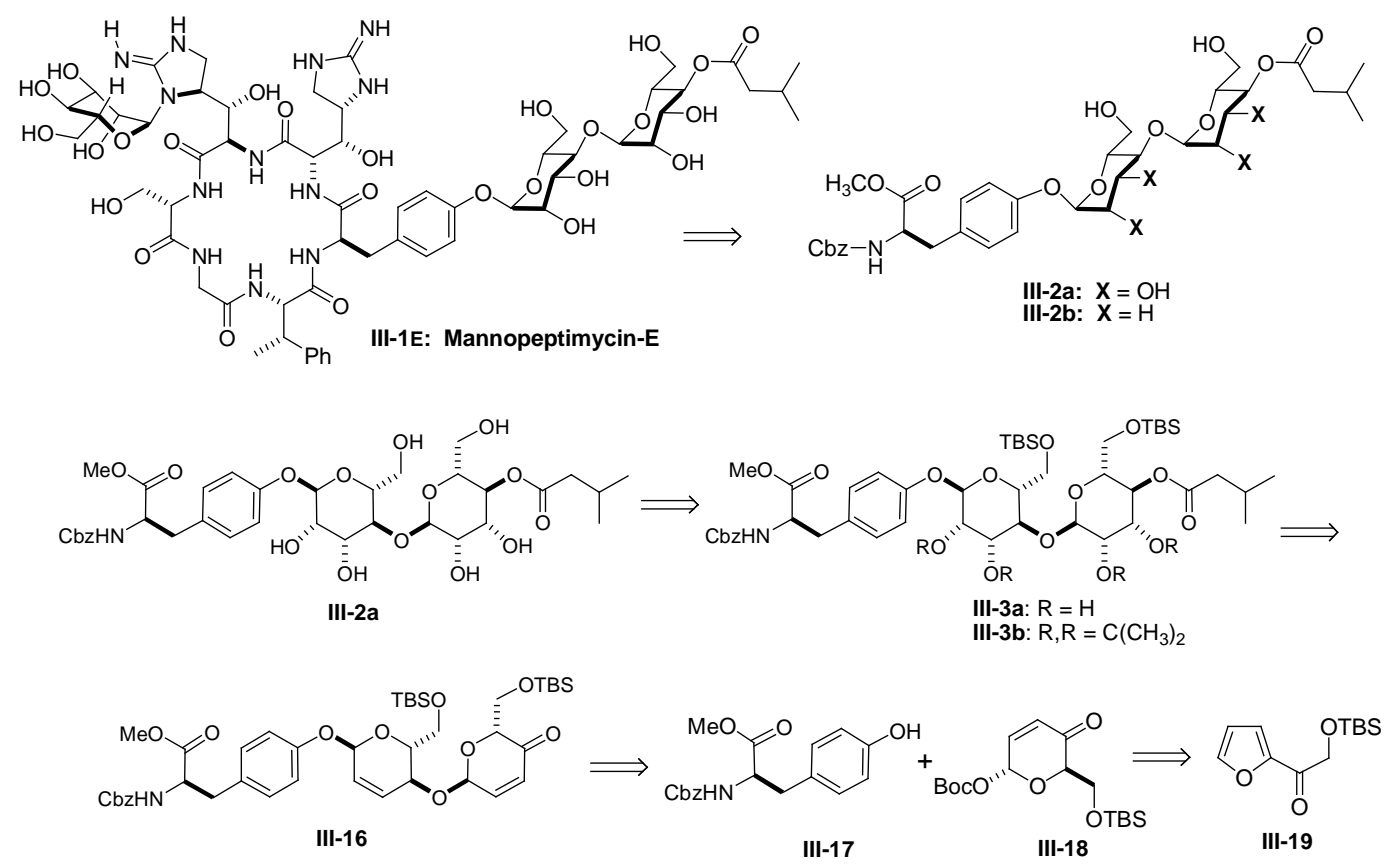

Our retrosynthetic analysis of the disaccharide fragment III-2a and its fully protected variant III-3b is outlined in Scheme 27. We envisioned that the mannostereochemistry in both III-2a and III-3b could be installed by a diastereoselective ketone reduction and a bis-dihydroxylation of a 1,4-linked pyran/pyranone III-16. Similarly, we believed that the pyran/pyranone III-16 could be assembled using a diastereoselective palladium-catalyzed glycosylation of tyrosine III-17. ${ }^{61}$ Recently, we reported a diastereoselective palladium-catalyzed glycosylation reaction that used alcohols as nucleophiles and pyranones such as III-18 as glycosyl donors. Thus, sequential application of our $\mathrm{Pd}(0)$-glycosylation $/ \mathrm{NaBH}_{4}$ reduction/ $\mathrm{Pd}(0)$-glycosylation sequence to tyrosine III-17 and pyranone III-18 was expected to allow for the rapid preparation of III-16. Replacing the above-mentioned bis-dihydroxylation with a bisdiimide reduction might also allow for the preparation of the deoxy analogue III-2b. Previously, we have shown that pyranone III-18 can be prepared in either enantiomeric 
form. Thus, this procedure was expected to allow the incorporation of either D- or Lsugars. ${ }^{64}$

\subsection{Synthesis of key fragment of mannopeptimycin-E and its analogues.}

Our synthesis studies began with the protected D-tyrosine III-17 and pyranone III-18 which, when exposed to $1 \mathrm{~mol} \% \mathrm{Pd}_{2}(\mathrm{dba})_{3} \cdot \mathrm{CHCl}_{3}$ and $4.0 \mathrm{~mol} \%$ of $\mathrm{PPh}_{3}$, underwent a diastereoselective glycosylation with complete $\alpha$-selectivity to afford the pyranone III-20 in 92\% yield. A diastereoselective 1,2-reduction of the enone III-20 occurred, when it was subjected to $\mathrm{NaBH}_{4}$ in $\mathrm{CH}_{2} \mathrm{Cl}_{2} / \mathrm{MeOH}(1: 1)$ at $-24{ }^{\circ} \mathrm{C}$, which afforded allylic alcohol III-21 as a single diastereomer (dr $>20: 1)$. We next investigated the viability of the $C-4$ alcohol in the Pd-catalyzed glycosylation. Exposing allylic alcohol III-21 to a second glycosylation using 1.2 equiv of pyranone III-18 and $1 \mathrm{~mol} \%$ of Pd catalyst $\left(1: 2.5, \mathrm{Pd}_{2}(\mathrm{dba})_{3} . \mathrm{CHCl}_{3} / \mathrm{PPh}_{3}\right)$ afforded the 1,4 -linked- $\alpha$-bis pyranone III16 in good yield (82\%) and virtually complete stereocontrol (Scheme 28).

Scheme 28. De novo synthesis of disaccharide enone III-16.
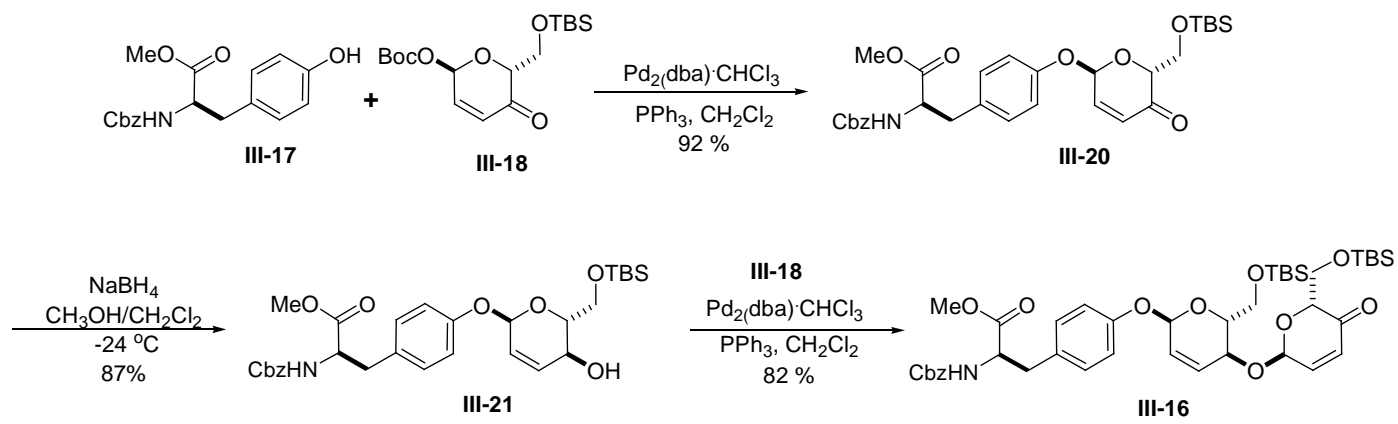

The final post-glycosylation transformation of III-16 is shown in Scheme 29. Treatment of 1,4-linked pyran/pyranone III-16 under the same reduction conditions as before (III-20 to III-21, Scheme 4) gave allylic alcohol III-22 in excellent yield (91\%) and diastereoselectivity (>20:1). The isovalerate group was installed by treating allylic 
alcohol III-22 with isovaleric acid and DCC/DMAP in $\mathrm{CH}_{2} \mathrm{Cl}_{2}$, which provided the $C$-4 isovalerate disaccharide precursor III-23 in excellent yield (96\%). The mannostereochemistry in 3a was diastereoselectively introduced ${ }^{65}$ upon exposure of III-23 to the Upjohn conditions ${ }^{20}\left(\mathrm{OsO}_{4} / \mathrm{NMO}, 85 \%\right)$. Removal of both TBS-ethers was accomplished with $\mathrm{TBAF}\left(0{ }^{\circ} \mathrm{C}\right.$ in $\left.\mathrm{THF}\right)$ affording the $\alpha$-1,4-linked-bis-mannodisaccharide III-2a in good yield (76\%).

Scheme 29. Synthesis of tyrosine Bis-manno-disaccharide III-2a.

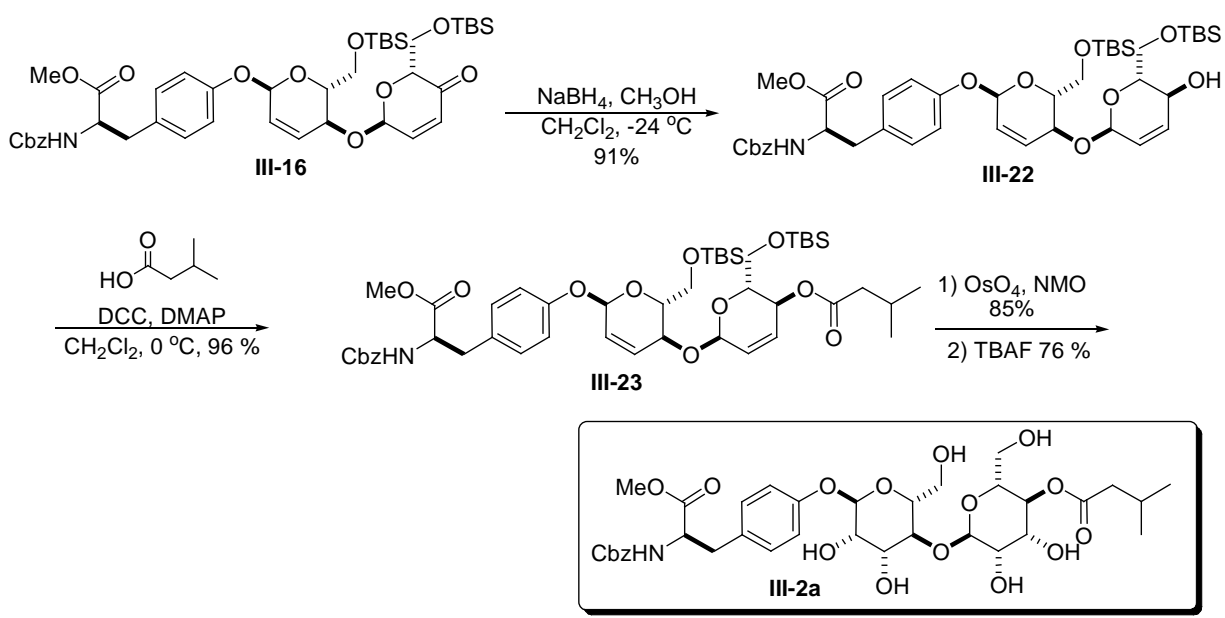

Finally the bis-manno-sugar III-3a could also be converted to the fully protected $\alpha-1,4-$ linked-bis-manno-disaccharide III-3b without any ester migration (Scheme 30). This was easily accomplished by treating a $\mathrm{CH}_{2} \mathrm{Cl}_{2}$ solution of tetraol III-3a with 2,2dimethoxypropane and $10 \mathrm{~mol} \%$ of CSA, which provided the bis-acetonide III-3b in good yield $(80 \%)$.

Scheme 30. Synthesis of fully protected Bis-manno-disaccharide III-3b.

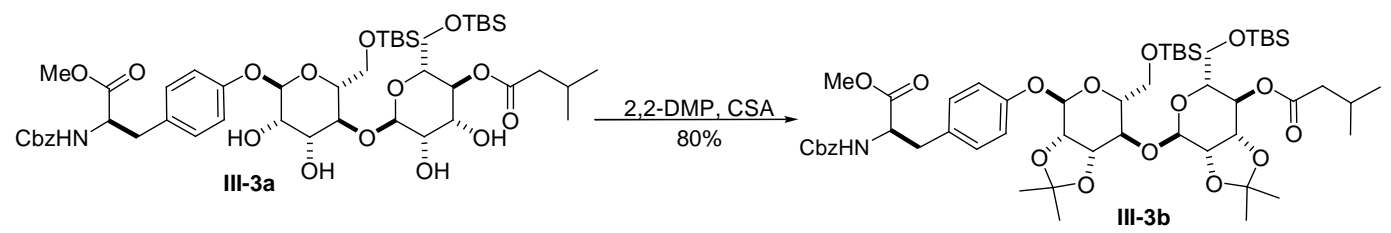


Replacing pyranone III-18 with its L-enantiomer (ent)-III-18 resulted in an equally efficient synthesis of the L,L-bis-manno-disaccahride diastereomer of III-2a, III26 (Scheme 31). Thus, in three analogous steps, D-tyrosine was converted into pyran/pyranone III-24 (69\% overall yield). The L,L-1,4-linked pyran/pyranone III-24 was stereoselectively reduced and acylated to form III-25 in good overall yield (86\%). Once again, two diastereoselective dihydroxylations occurred upon exposure of III-25 to the Upjohn conditions. This bis-dihydroxylation occurred with near perfect stereocontrol, as with the diastereomeric series (cf. Scheme 29). The tetraol product was converted to the unprotected bis-sugar III-26 via a TBS group deprotection (TBAF, 78\%) or to the fully protected diastereomer III-27 by means of an acetonide protection (10 mol \% of CSA/2,2-DMP, 81\%).

Scheme 31. Synthesis of L,L-disaccharide analogues III-26.
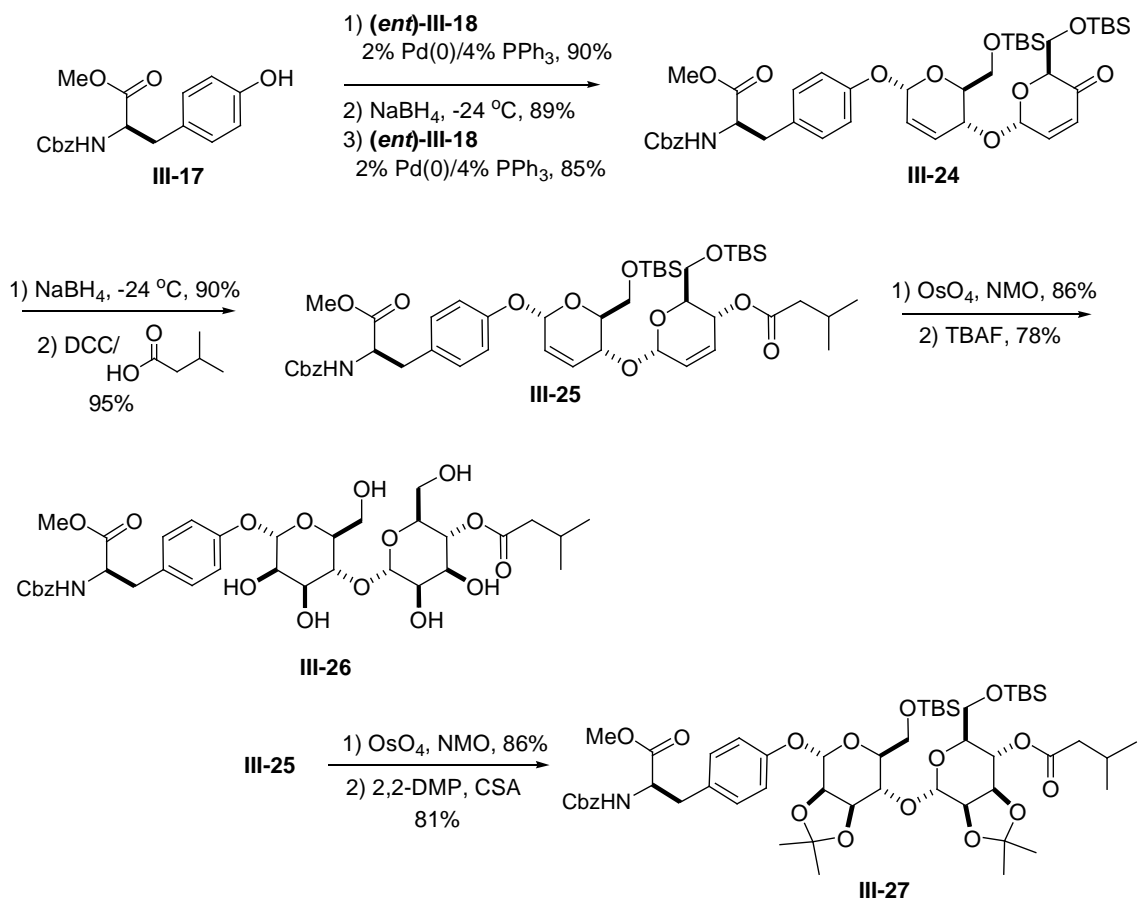
Having synthesized the key disaccharide fragment of mannopeptimycin-E (III-2a and III-3b) along with its L,L-diastereomers (III-26 and III-27), we turned our attention to the preparation of deoxy analogues (Scheme 32). The simplest 2,3-deoxy analogue III28 was obtained by an exhaustive diimide reduction. ${ }^{66}$ Both double bonds of III-23 were reduced using an excess of the diimide precursor $\mathrm{NBSH}$ in $\mathrm{CH}_{2} \mathrm{Cl}_{2}$ to afford the 2,3deoxybis-pyranoside III-28 in nearly quantitative yield (95\%). ${ }^{67}$ Under identical conditions, the diastereomeric L,L-1,4-linked bis bispyran III-25 was reduced to give an excellent yield of the bis-dideoxy analogue III-28 (97\%). ${ }^{67}$

Scheme 32. Synthesis of Bis-2,3-dideoxydisaccharide analogues III-2b and III-28.
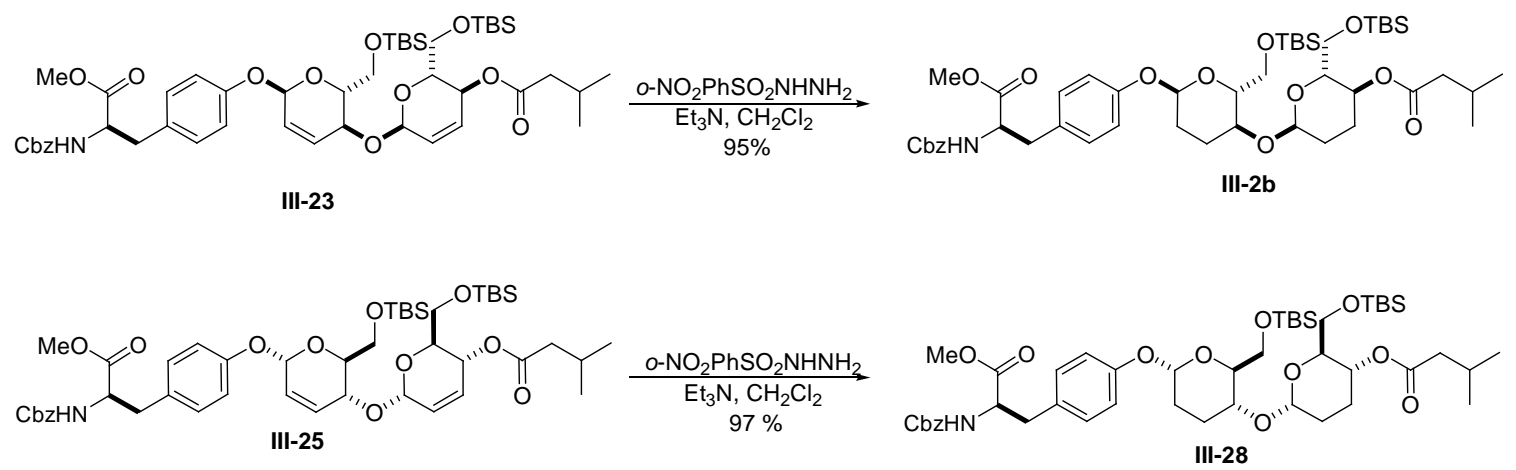

\subsection{Asymmetric synthesis of aza-analogue of mannoeptimycin-E disaccharide portion. ${ }^{\dagger}$}

\section{5.a. Retrosynthetic analysis of aza-analogue of mannopeptimycin-E key fragment.}

In particular, we were interested in the critical role of the $C$-4' isovaleryl group and its specific location played on antibacterial activity. ${ }^{56,59}$ It has been shown that removal or

\footnotetext{
' Reproduced with permission from J. Org. Chem. 2007, 72, 4966-4969. Copyright 2007, with permission from American Chemical Society.
} 
migration of the $C-4$ ' isovalerate substitution on the terminal mannose leads to a substantial decrease in antibacterial potency. ${ }^{56,59}$ Along this vein, we became interested in an aza-analogue III-3 that should be resistant to both hydrolysis and migration (Scheme 33).

Herein, we describe the enantioselective synthesis of the C-4' acylated 1,4manno,manno-4'amino disaccharide analogue III-29 via the iterative use of highly diastereo- and regioselective palladium-catalyzed allylation reactions. Our retrosynthetic analysis of the aza-disaccharide fragment III-29 is outlined in Scheme 33. This route begins with allylic alcohol III-22, which we have previously prepared enroute to the disaccharide III-2, via the sequential application of our $\operatorname{Pd}(0)$-glycosylation/postglycosylation transformations upon a protected tyrosine III-17. ${ }^{61}$ Key to this new approach to the $C$-4' amido analogue III-29 is the regio- and stereoselective conversion of the allylic alcohol III-22 to the allylic azide III-32 by a palladium-catalyzed allylic substitution. ${ }^{68}$ Finally, we envisioned the manno,manno-stereochemistry in III-29 being installed by an azide reduction/acylation and stereoselective bis-dihydroxylation of a 1,4linked-C-4' amino-bis-pyran III-30 ${ }^{69}$ Simply substituting a bis-diimide reduction for the above mentioned bis-dihydroxylation would also allow for the preparation of a deoxyanalogue III-36 (vide infra).

Scheme 33. Retrosynthetic analysis of mannopeptimycin analogue III-29. 


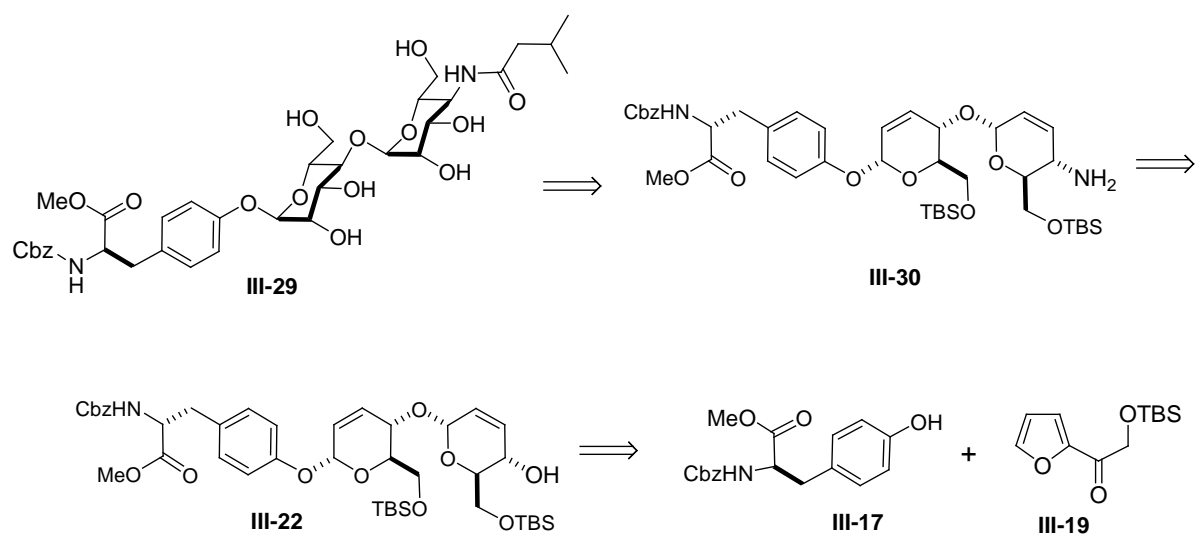

\section{5.b. Synthetic approach of aza-analogue of mannopeptimycin-E key fragment.}

Our divergent approach to the aza-analogue III-29 began with the known bis-pyran III-22 with the conversion of the allylic alcohol portion into an $\pi$-allyl palladium leaving group (Scheme 34). We chose to use the methyl carbonate group as in III-31, which was readily prepared by treating III-22 with methyl chloroformate in excellent yield (90\%). Exposing carbonate III-31 to the conditions developed by Sinou $\left(\mathrm{TMSN}_{3}\right.$, $(\mathrm{Pd}(\text { allyl }) \mathrm{Cl})_{2} / 1,4$-bis(diphenylphosphino)butane) at room temperature afforded a single regio- and stereoisomeric allylic azide III-32 in good yield (88\%). ${ }^{70}$ To avoid problems with allylic rearrangements, the allylic azide III-32 was immediately reduced with $\mathrm{P}(n$ $\mathrm{Bu})_{3} / \mathrm{THF}$ to give allylic amine III-30 $(80 \%){ }^{71}$

Scheme 34. Synthesis of allylic amine III-30.

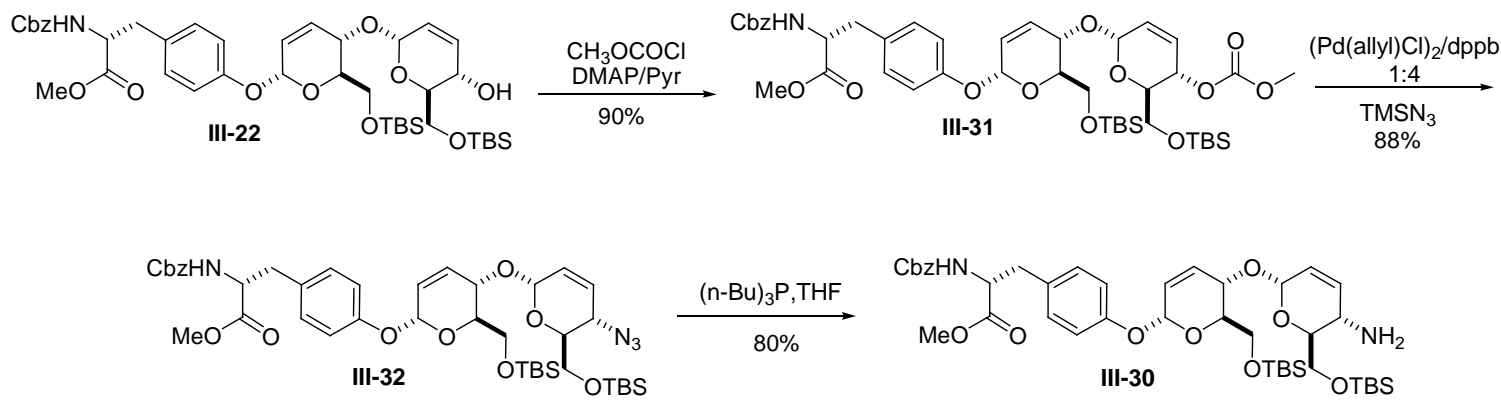


The key isovaleryl group was easily installed by treating allylic amine III-30 with isovaleric acid and DCC/DMAP in $\mathrm{CH}_{2} \mathrm{Cl}_{2}$, which provided the $C$-4 isovaleryl amide disaccharide precursor III-33 in excellent yield (85\%) (Scheme 35). The mannostereochemistry in III-34 was diastereoselectively introduced upon exposure of III-34 to the Upjohn conditions $\left(\mathrm{OsO}_{4} / \mathrm{NMO}, 80 \%\right){ }^{20,62}$ To complete the model system both TBSethers were easily removed with $\mathrm{TBAF}\left(0{ }^{\circ} \mathrm{C}\right.$ in $\left.\mathrm{THF}\right)$ affording the 1,4-linked-bismanno-4'-amido-disaccharide III-29 in good yield (80\%). In addition, the fully protected disaccharide III-35 was also prepared for macrocyclic peptide assembly. This was easily accomplished by converting the bis-manno-C-4'-amido disaccharide III-34 to the bisacetonide III-35 (10 mol \% CSA/2,2-DMP, 80\%).

Scheme 35. Synthesis of tyrosine-bis-manno-amido disaccharide III-29 and III-35.

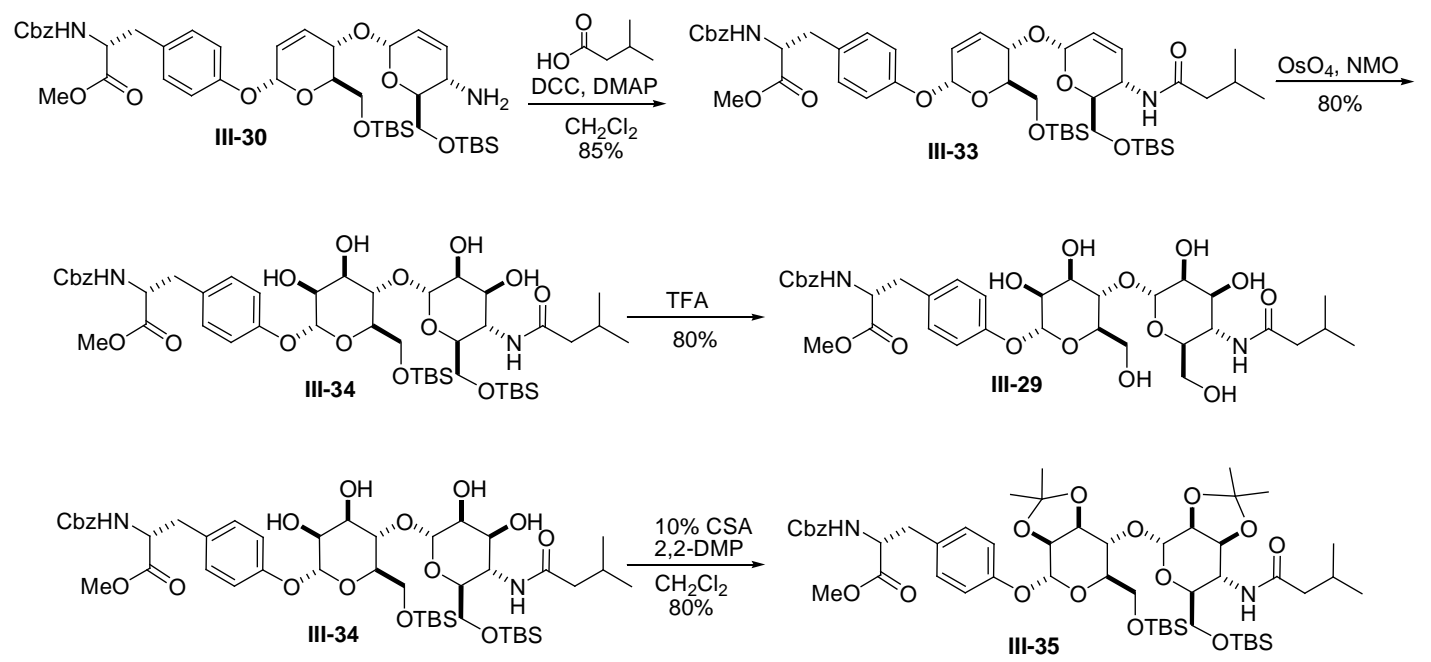

Finally, the 2,3-deoxy analogue III-36 was readily prepared by an exhaustive diimide reduction (Scheme 36). Both double bonds of III-33 were reduced with excess diimide precursor (NBSH) in $\mathrm{CH}_{2} \mathrm{Cl}_{2}$ affording the 2,3-deoxy-C4'-amido bis-pyranoside III-36 in excellent yield $(95 \%){ }^{66}$ 
Scheme 36. Synthesis of bis-2,3-dideoxyamido disaccharide analogue III-36.

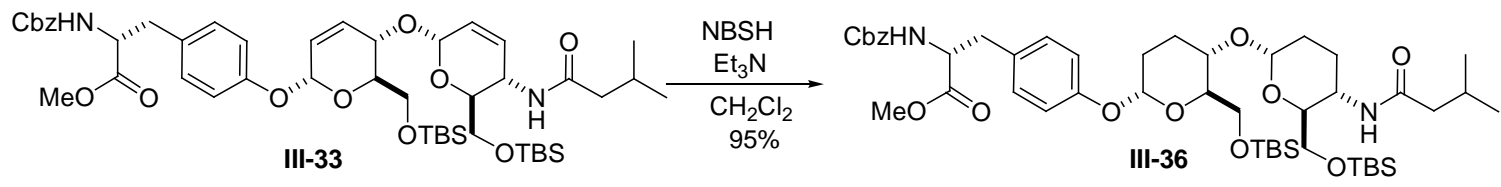

\subsection{Syntheses of Serine and threonine analogue of mannopeptimycin-E key \\ fragment.}

After successfully making the key disaccharide fragment of mannopeptimycin E and its aza-analogues, we turned our attention to synthesize a small library of analogues of the disaccharide fragment of mannopeptimycin-E. In this vein, we replaced pyranone III-18 with its L-enantiomer (ent)-III-18 resulted in an equally efficient synthesis of the serine-L,L-bis-manno-sugar analogues III-41, III-43 (Scheme 37). Thus, in three analogous steps, D-serine was converted into pyran/pyranone III-39 in $67 \%$ overall yield. The L,L-1,4-linked pyran/pyranone III-39 was stereoselectively reduced and acylated to form III-40 in good overall yield (77\%). Once again, two diastereoselective dihydroxylations occurred upon exposure of III-25 to the Upjohn conditions. This bisdihydroxylation occurred with near perfect stereocontrol, as with the diastereomeric series (cf. Scheme 29). The tetraol product was converted to the unprotected bis-sugar III-41 via a TBS group deprotection (TBAF, 78\%) or to the fully protected diastereomer III-42 by means of an acetonide protection (10 mol \% of CSA/2,2-DMP, 75\%). Simply substituting a bis-diimide reduction for the above mentioned bis-dihydroxylation on compound III-40 will allow for the preparation of a deoxy-analogue III-36 with excellent yield $(94 \%)$. 
Scheme 37. Syntheses of serine analogues of mannopeptimycin-E key fragment III-41 and III-43.

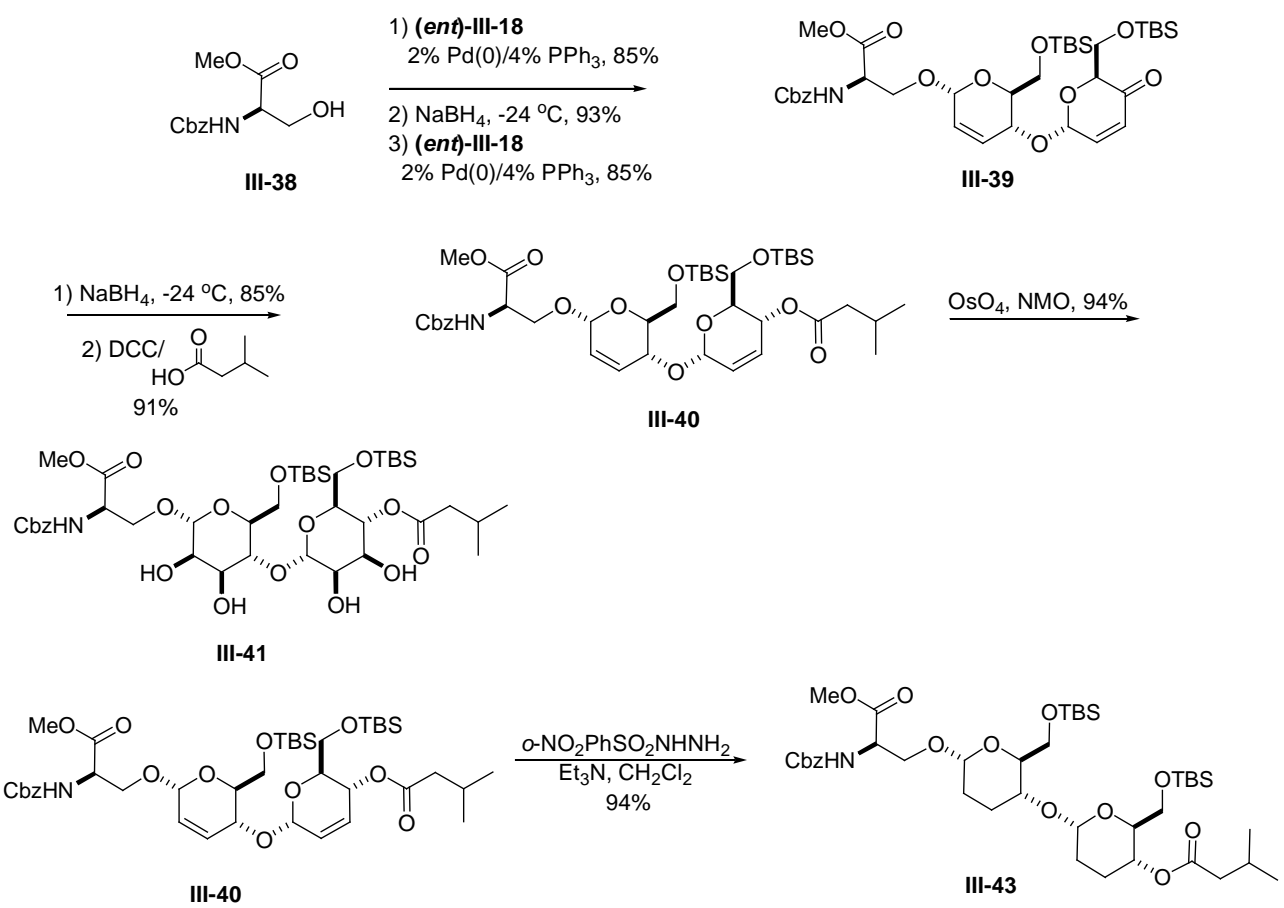

Because of our motivation to test similar compounds as antibiotics, we synthesized threonine-L,L-bis-manno-sugar analogues III-47, III-49 by using Lpyranone (ent)-III-18 and D-threonine III-40 in an equally efficient manner (Scheme 38). Thus, in three analogous steps, D-threonine was converted into pyran/pyranone III45 in 70\% overall yield. The L,L-1,4-linked pyran/pyranone III-45 was stereoselectively reduced and acylated to form III-46 in good overall yield (84\%). Once again, two diastereoselective dihydroxylations occurred upon exposure of III-46 to the Upjohn conditions. This bis-dihydroxylation occurred with near perfect stereocontrol, as with the diastereomeric series (cf. Scheme 29). The tetraol product was converted to the unprotected bis-sugar III-47 via a TBS group deprotection (TBAF, 75\%) or to the fully protected diastereomer III-48 by means of an acetonide protection (10 mol \% of 
CSA/2,2-DMP, 78\%). Simply substituting a bis-diimide reduction for the above mentioned bis-dihydroxylation on compound III-46 will allow for the preparation of a deoxy-analogue III-49 with excellent yield (96\%).

Scheme 38. Synthesis of threonine analogues of mannopeptimycin-E key fragment III-47 and III-49.
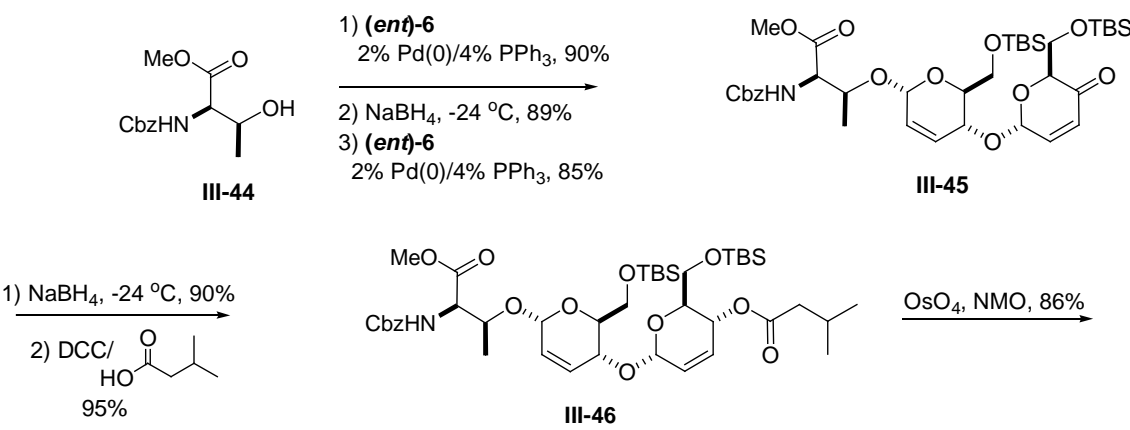

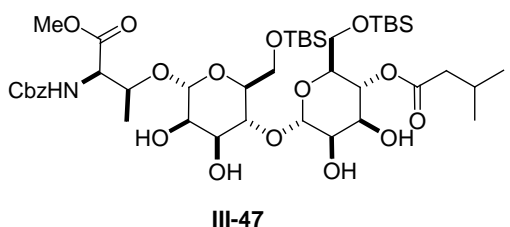

III-47

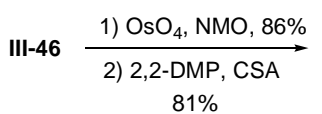
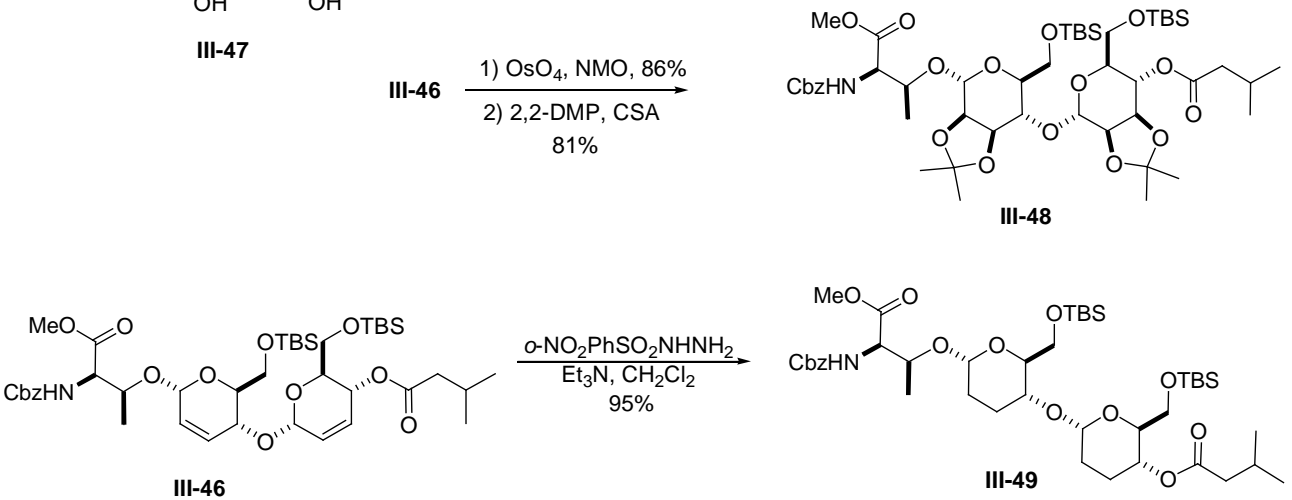

\subsection{Towards asymmetric synthesis of Mannoeptimycin-E analogue.}

The key portion of mannopeptimycin-E and its analogues have been prepared successfully with our de novo approach towards an idea of activity testing. Further, we turned our attention in developing a strategy to check the compatibility of carbohydrate approach with peptide sequence. In this vein, our proposed retrosynthetic approach was 
shown in Scheme 39. Mannopeptimycin analogue III-50 could be assambled from two linear tripeptide intermediates III-51 and III-52 using HBTU coupling conditions (Scheme 39). ${ }^{72}$

Scheme 39. Retrosynthesis of mannopeptimycin-E analogue III-50.

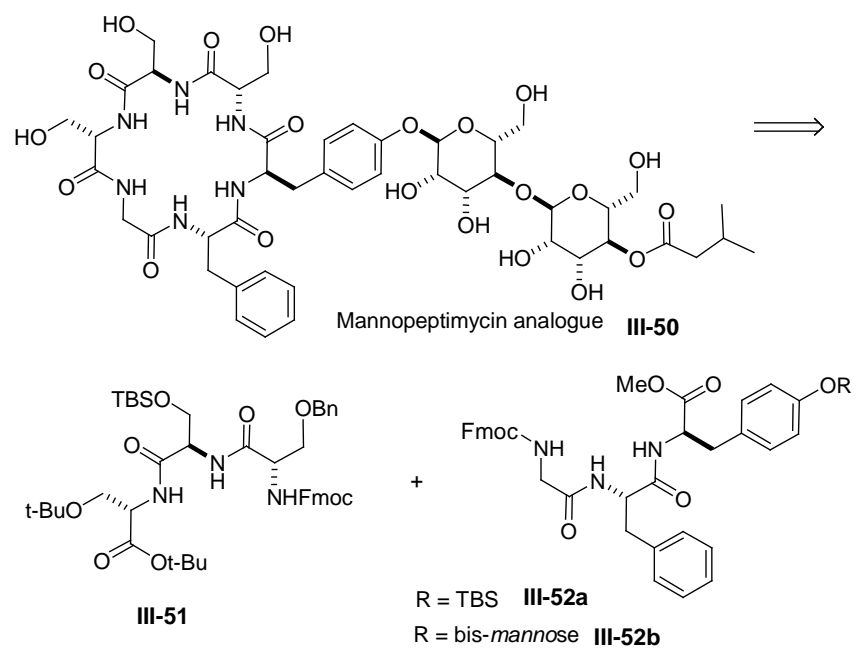

Tripeptide III-51 could be synthesized from D-/L-serine. We envisioned the other tripeptide III-52a could be prepared from coupling of three synthetic peptides glycine, phenylalanine and D-tyrosine (Scheme 40). A related procedure will be used to prepare the $O$-bismannose containing diastereomeric tripeptide $\mathbf{I I I - 5 2 b . ~}$

Scheme 40. Retrosynthesis of tripeptides III-51 and III-52a/b. 

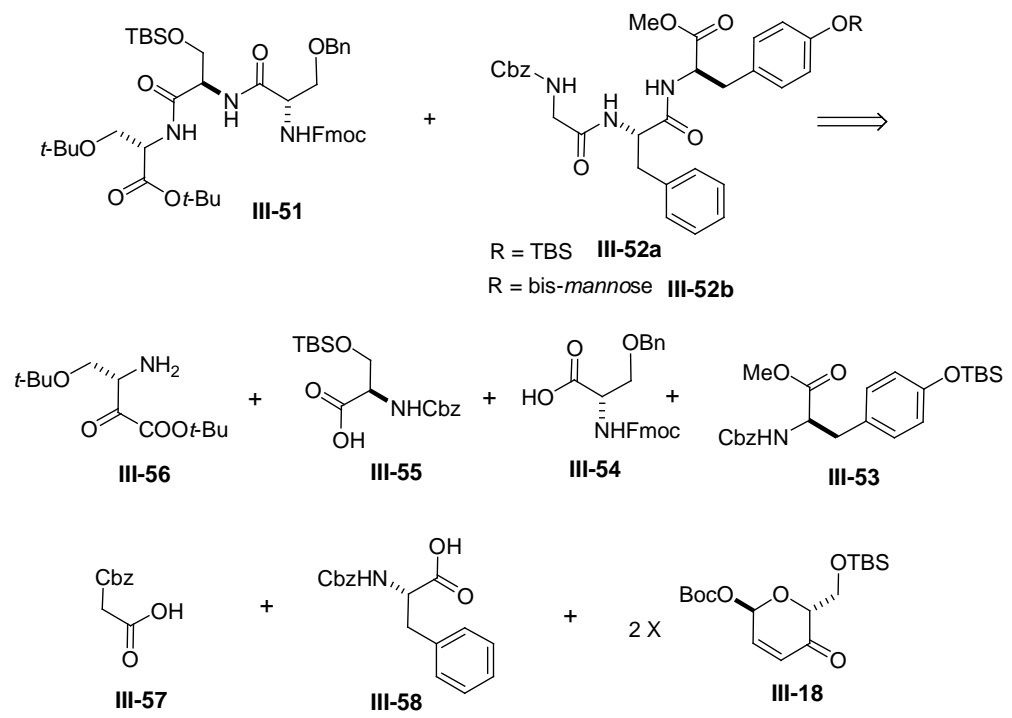

Outlined in Scheme 41 is the synthetic approach of L-ser-D-ser-L-ser tripeptide III-63. Our synthesis started with the protection of the primary alcohol of D-serine III-59 as TBS ether III-60, followed by hydrolysis of methyl ester with $\mathrm{NaOH} / \mathrm{MeOH}$, which gave acid III-55 in excellent yield (85\%). Peptide III-55 was coupled with L-ser- $\mathrm{NH}_{2}$ III-56 using HBTU in presence of $\mathrm{Et}_{3} \mathrm{~N}$ affording dipeptide III-61 in $83 \%$ yield. Exposing dipeptide III-61 under catalytic hydrogenolysis condition $\left(\mathrm{Pd} / \mathrm{C}, \mathrm{H}_{2}, \mathrm{MeOH}\right)$ afforded the dipeptide amine III-62 in good yield (90\%). Tripeptide III-63 was prepared by HBTU-mediated coupling between dipeptide amine III-62 and L-serine-acid III-54 in good yield $(85 \%) .^{72}$

Scheme 41. Synthesis of tripeptide III-63.
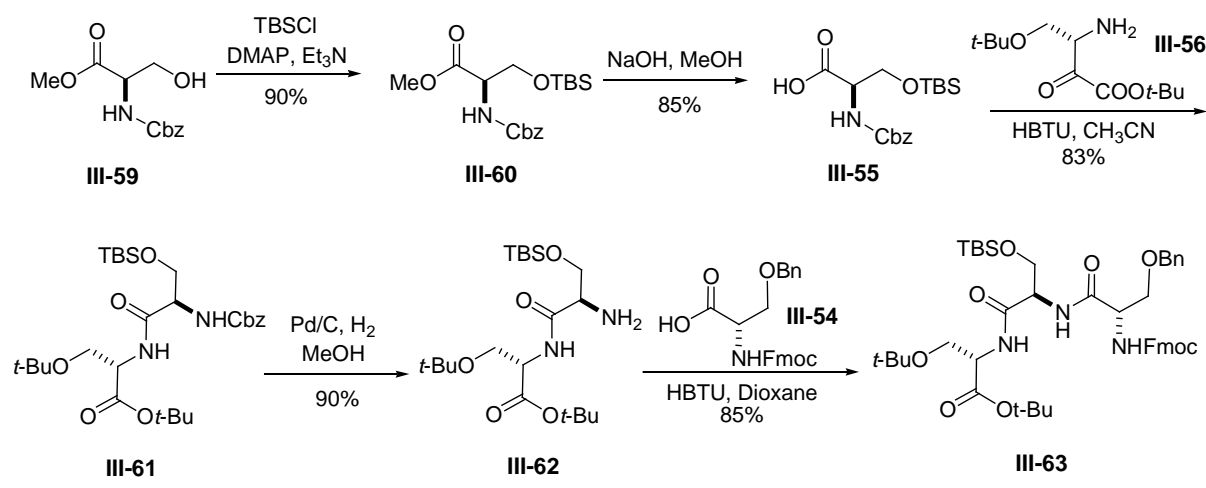
Having synthesized the above tripeptide fragment, we turned our attention to the preparation of Gly-L-Phe-D-Tyr tripeptide III-52a (Scheme 42). Treatment of Cbz-PheOH III-58 with trimethylsilyl chloride in presence of methanol gave methy ester III-64. Subsequent treatment of compound III-64 under hydrolysis condition $\left(\mathrm{Pd} / \mathrm{C}, \mathrm{H}_{2}, \mathrm{MeOH}\right)$ afforded amine III-65 in excellent yield (90\%). Coupling of amine III-65 with Cbz-GlyOH III-57 using HBTU gave dipeptide III-66 in $85 \%$ yield. Further, treatment of dipeptide III-66 under hydrolysis conditions ( $\mathrm{LiOH} / \mathrm{MeOH})$ gave carboxylic acid III-67 in $78 \%$ yield.

Tyrosine amine precursor III-69 can be derived from commercially available Dtyrosine III-68 using four synthetic steps of selective protections and deprotections. Dtyrosine III-68 was protected as methyl ester with $\mathrm{MeOH} / \mathrm{SOCl}_{2}$, followed by protection of the amino group as its Cbz derivative under standard conditions afforded phenol III68a in $95 \%$ yield for two steps. ${ }^{73}$ Subsequent $O$-silylation of phenol III-68a with $\mathrm{TBSCl} / \mathrm{Et}_{3} \mathrm{~N}$ gave fully protected tyrosine III-53 in good yield (87\%). Selective deprotection of Cbz group of compound III-53 using catalytic hydrogenation $\left(\mathrm{Pd} / \mathrm{C}, \mathrm{H}_{2}\right.$, MeOH) gave the desired amine III-69 in good yield (89\%). Treatment of dipeptide acid III-67 with amine III-69 using HBTU gave tripeptide III-52a in 80\% yield.

Scheme 42. Synthesis of tripeptide III-52a. 

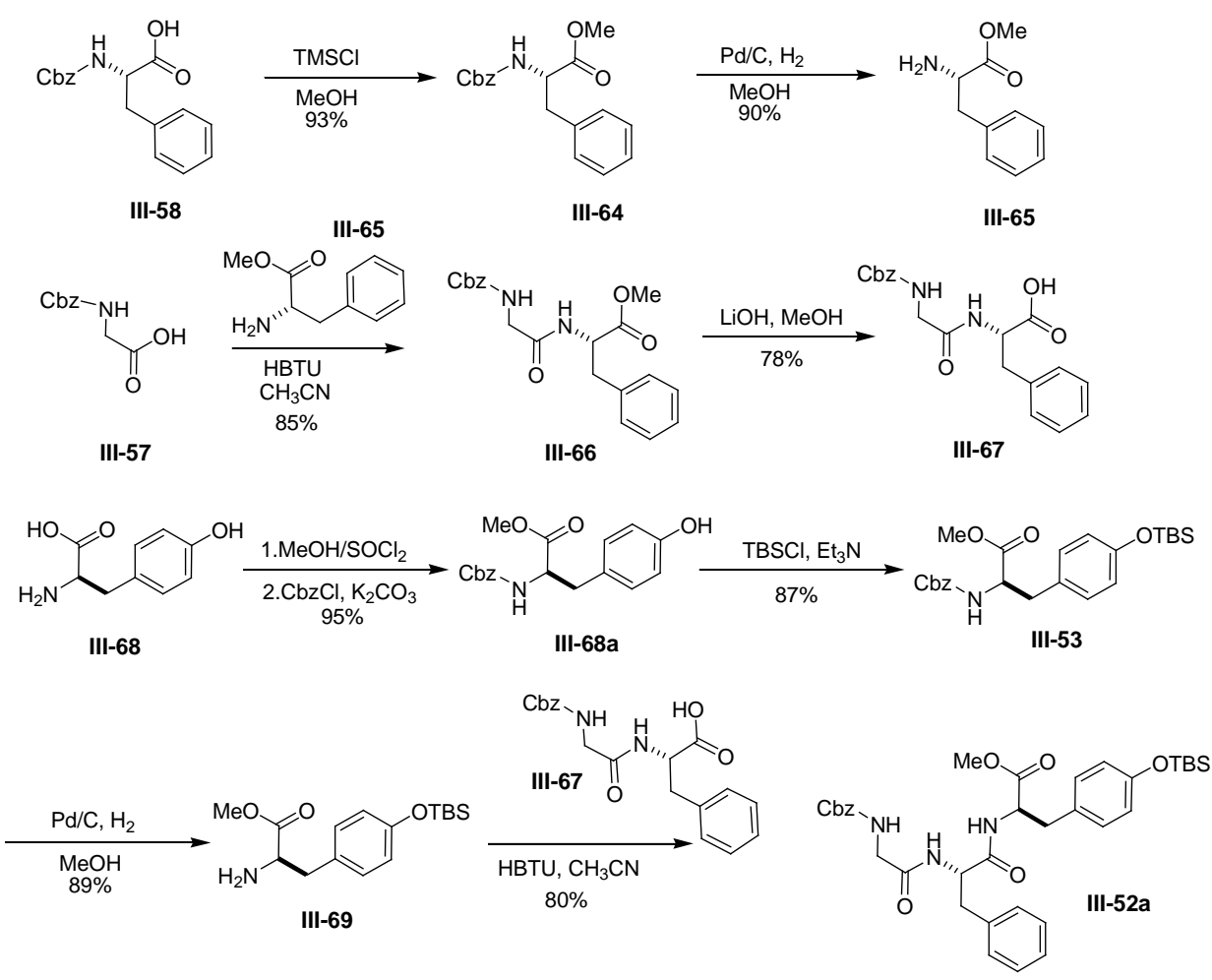

Having successfully synthesized the Gly-L-Phe-D-Tyr tripeptide in good yield, the next goal was to synthesize the $O$-bis-manno-tripeptide III-52b using similar procedures (Scheme 43). Thus, we subjected tyrosine-disaccharide fragment III-3b to catalytic hydrogenation $\left(\mathrm{Pd} / \mathrm{C}, \mathrm{H}_{2}, \mathrm{MeOH}\right)$, which afforded the Bis-manno-Tyr-amine III-70 in $75 \%$ yield. There was some concern about deprotection of TBS-ether during the hydrogenation step, but this turned out not to be the case neutral condition and lower reaction times were maintained. Under HBTU coupling conditions, the amine III-70 and the carboxylic acid III-67 gave desired $O$-bis-manno-tripeptide III-52b in good yield as single diastereomer $(75 \%)$.

Scheme 43. Synthesis of $O$-bis-manno-tripeptide III-52b. 

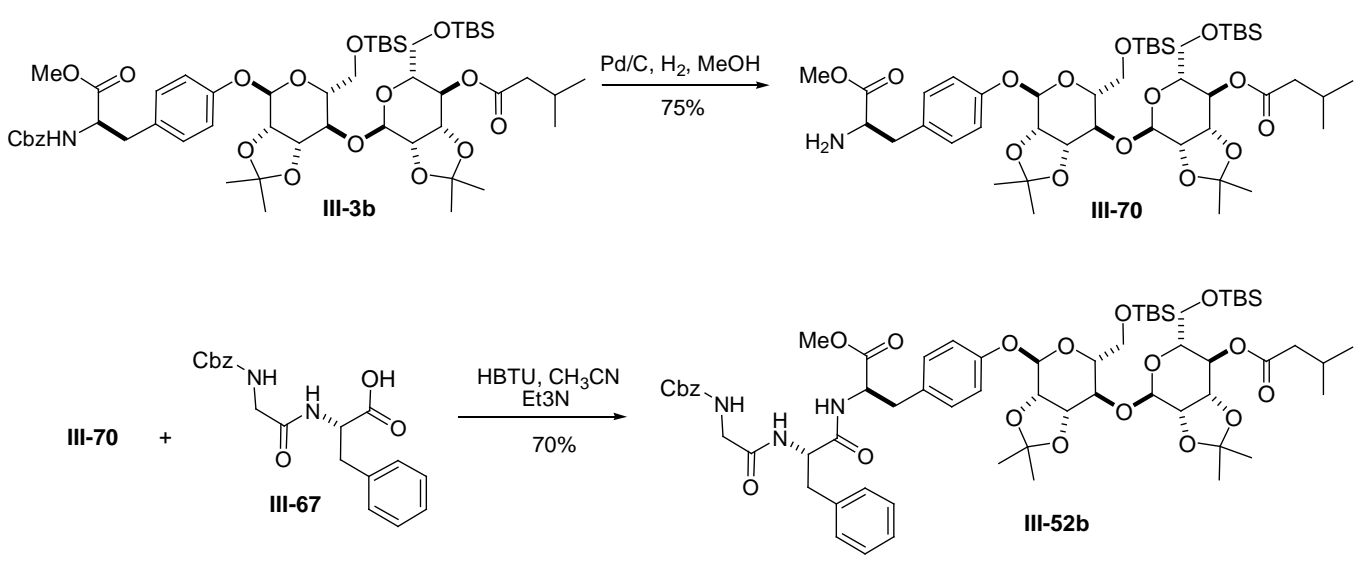

Completion of the synthesis of mannopeptimycin-E analogue III-50 requires selective removal of protecting groups, followed by two coupling steps and global deprotection from the two advanced tripeptide intermediates III-63 and III-52b (Scheme 44). These efforts are ongoing in the group.

Scheme 44. Synthesis of mannopeptimycin-E analogue III-50.

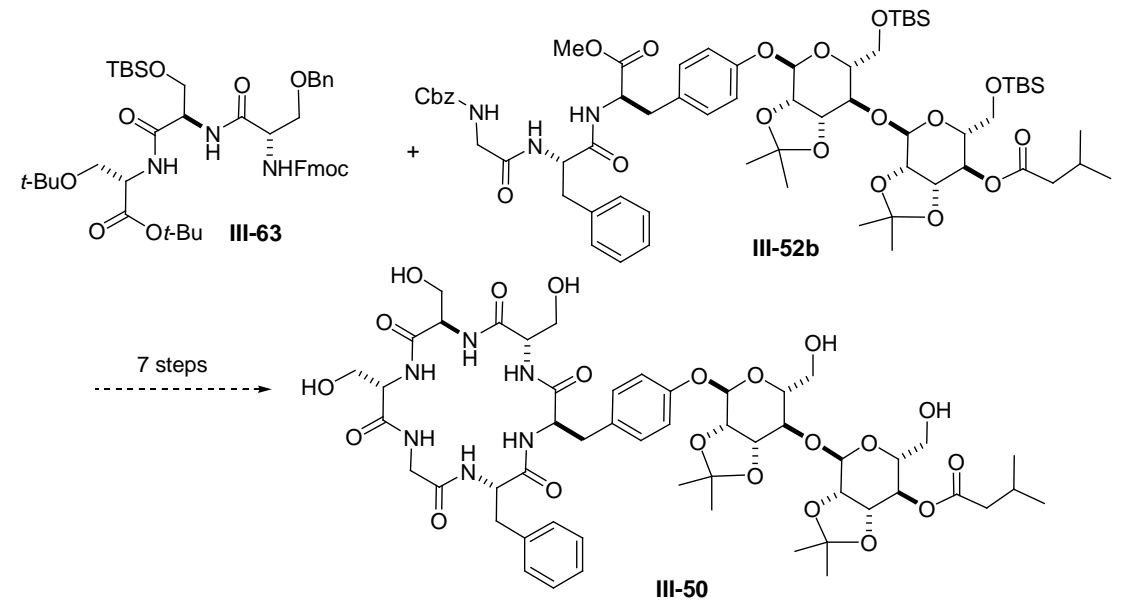

\subsection{Summary}

In conclusion, an enantioselective synthesis of the manno-disaccharide fragments of mannopeptimycin-E and its five analogues were been achieved in seven steps and 35$40 \%$ overall yields from D-tyrosine via an iterative palladium-glycosylation strategy. Key 
to the success of this approach was the ease with which the $C$-4 isovalerate group was introduced, and the high diastereoselectivity of the palladium-catalyzed glycosylation and bis-dihydroxylation reactions. The use of this methodology gave two 1,4-linked-bismanno-4'-amido-disaccharide analogues of mannopeptimycin-E, which have been synthesized in 10 steps with $21 \%$ overall yield from D-tyrosine via a palladium-catalyzed azide allylation reaction for the stereoselective installation of the $C$ - 4 isovaleramide group. We have synthesized the two advanced intermediate tripeptides using our de novo and traditional peptide chemistry. Further studies on the completion of synthesis of mannopeptimycin analogue III-50 and their subsequent biological investigation will be reported in due course. 


\section{Chapter 4}

\section{Syntheses and Biosynthetic Studies of Methymycin Analogues.}

\subsection{Introduction to 12-membered lactone methymycin}

The 12-membered macrolactones are an important class of antibiotics used to treat infections caused by Gram-positive bacteria. ${ }^{74}$ Streptomyces venezuelae ATCC 15439 produces these 12-membered ring polyketides methymycin IV-1, 10deoxymethynolide IV-2 and neomethymycin IV-3, (Figure 5). ${ }^{75}$ These polyketides contain a lactone aglycone attached to rare deoxy amino sugar as an important component. The biological activities of these secondary metabolites are dramatically decreases when the sugar is removed. Therefore modifications of these critical deoxy amino sugar substituents are important in order to generate new macrolide antibiotics with promising biological activity. ${ }^{76}$

Figure 5. 12-membered macrolactones antibiotics.
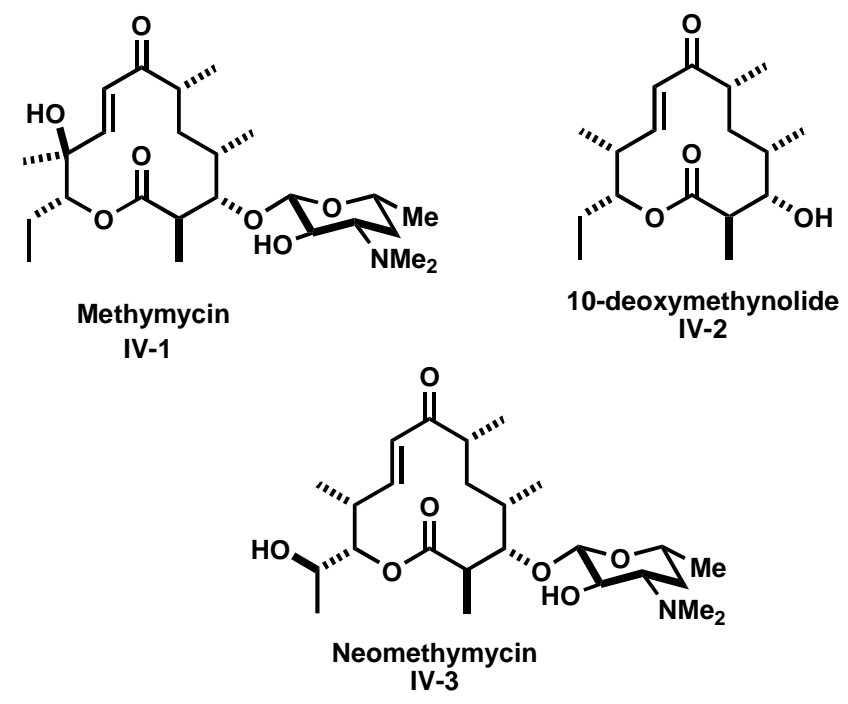

A method for modifying natural products by combining existing biosynthetic genes from diverse allied bacteria to create a pathway for producing unnatural products 
known as combinatorial biosynthesis, which has recently become a promising method for the development of new class of antibiotics. The macrolide antibiotic biosynthetic pathways from different species of Streptomyces has led to genetic manipulations to combine genes from various pathways for the biosynthesis of new hybrid macrolides with promising bioactivity. ${ }^{77}$

There is a significant interest in the possibility of using combinatorial biosynthesis to generate new macrolactones attached with new deoxy sugar moieties. Recent studies on the flexibility of glycosyltransferases in accepting non-native sugars as substrate for attachment to specific macrolactones has made these enzymes an important tool for combinatorial biosynthesis of novel compounds with altered glycosylation patterns. ${ }^{78}$ It is assumed that this research based on a combinatorial biosynthetic strategy will facilitate the development of new novel antibiotics.

\subsection{Previous Approaches to Methymycin Analogues}

\section{2.a. Biosynthesis of methymycin and neomethymycin via glycosylation using DesVII/DesVIII mutants.}

Hung-Wen Liu et al. have been making significant progress in using genetic and biochemical methods to develop new class of macrolide antibiotics using glycosyltransferase as their key biosynthetic tool. ${ }^{79}$ They have used different enzyme gene clusters ranging from desI-desVIII of Streptomyces venezuelae involved in the biosyntheses of 12-membered macrolide antibiotics like methymycin IV-1 and neomethymycin IV-3 (Scheme 45). These polyketides mainly contain the single 3dimethylamino-3,4,6-trideoxy sugar, desoamine, and different identified gene clusters, 
represent a model system to probe the feasibility of making new macrolides containing modified sugar components in vivo. ${ }^{80}$

Scheme 45. Biosynthesis of methymycin IV-1 and neomethymycin IV-3.

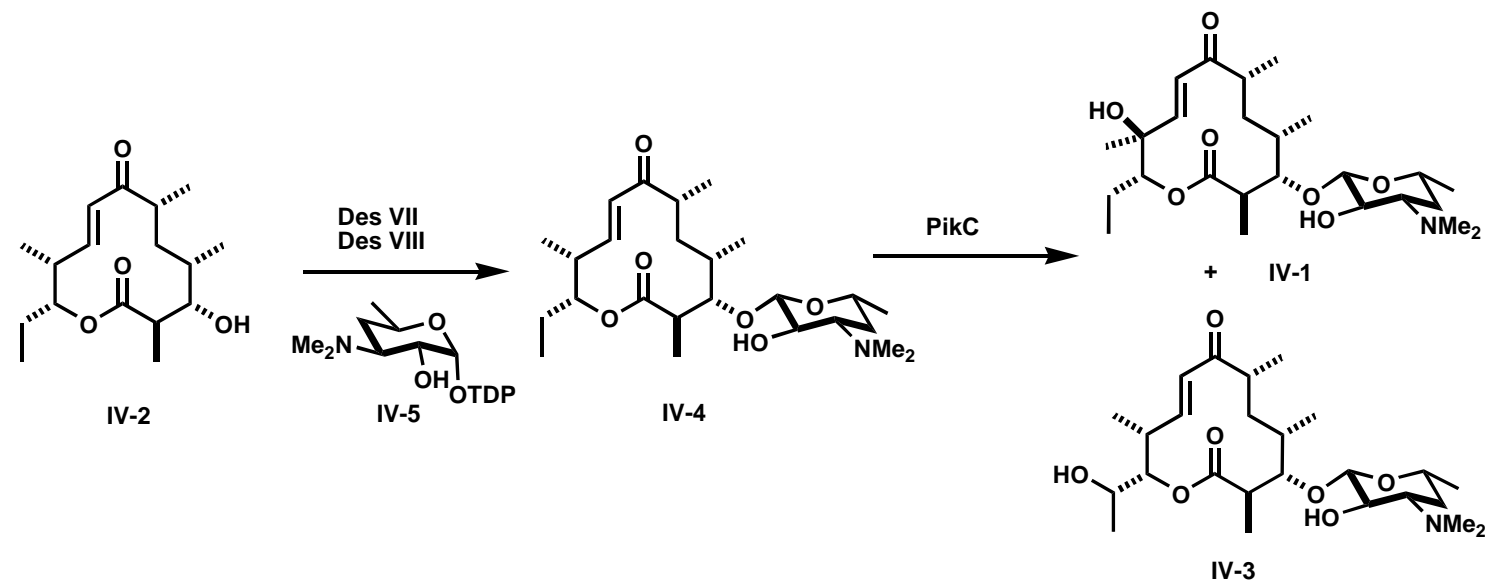

As shown in Scheme 45, they tried to couple the sugar donor TDP-D-deosamine IV-5, and the aglycone acceptor 10-deoxymethynolide IV-2 with the enzyme pair DesVII to get 10-deoxy methymycin IV-4. Unfortunately no products were detected. After exploring various conditions like solvent system/pH range/temperature, then surprisingly discovered that Des VIII as a necessary component in presence of enzyme DesVII at higher $\mathrm{pH}$ to produce the desired compound 10-deoxy methymycin IV-4. Compound IV4 was further modified by another enzyme PikC. They showed the catalytic properties of DesVII/Des VIII pair towards biosynthesis of various donor and acceptor substrates for the development of in vitro glycosylation of macrolides and other classes of natural products. $^{80}$

\subsection{Project Description and Goals}

Since the deoxyaminosugar portion of macrolides is essential for their antimicrobial activity, ${ }^{81}$ their modification hold promise as a valuable approach towards 
preparing new macrolide antibiotics with improved biological properties. ${ }^{82}$ Many research groups have reported the use of modified biosynthetic pathways for the production of new glycosylated antibiotics; ${ }^{83}$ however their use of complex sugar nucleotide intermediates and low-moderate yields leave room for improvement of macrolide antibiotics.

The main goal of this project is to develop a diastereoselective route to install different sugar motifs onto complex antibiotic aglycones from simple achiral starting material. In this connection our research group has initiated collaboration with Prof. Hung-Wen Liu's labs and developed the de novo approach, which would allow for facile synthesis of various methymycin analogues and planned to test our compounds in his labs.

Scheme 46. Retrosynthesis of methymycin analogues.

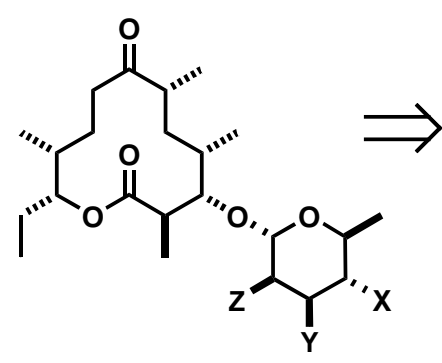

Methymycin L/D-Analogoues $\mathrm{X}=\mathrm{OH}$ or $\mathrm{N}_{3}$ or $\mathrm{NH}_{2}(R / S)$ $\mathrm{Y}=\mathrm{OH}(R / S)$ or $\mathrm{H}$ $\mathrm{Z}=\mathrm{OH}$ or $\mathrm{H}$

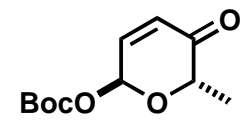

$\alpha$-L-enone IV-6<smiles>CC1O[C@@H](OC(C)(C)C)C=CC1=O</smiles>
$\alpha$-D-enone IV-27<smiles>C=CC=CC(=O)OC1C=CC(C)OC1O[C@H](C)C(C)C(=O)O[C@H](CC)[C@@H](C)CCC(=O)C(C)C</smiles><smiles>CC[C@H](OC(=O)C(C)[C@H](O)[C@@H](C)C[C@@H](C)C(=O)/C=C/C(C)C)C(C)O</smiles>

10-Deoxy methynolide IV-2<smiles>CC1OC(OC(C)(C)C)C=CC1=O</smiles>

IV-6 
Our retrosynthetic analysis for the synthesis of methymycin analogues is shown in Scheme 46. Our plan was to develop a method for the installation of various amino and deoxy sugars onto 10-deoxymethynolide IV-2 using our Pd-glycosylation/post glycosylation modification. ${ }^{84}$ As previously discussed, all the required pyranone stereoisomers IV-6, IV-6a, IV-27 and IV-21 can be prepared by employing an enantioselective Noyori $R, R / S, S$ reduction ${ }^{85}$ of acyl furan $\mathbf{I V - 6 b}$, followed by Achmatowicz oxidation, and diastereoselective carbonate formation. ${ }^{86}$

\subsection{Synthesis of Methymycin analogues}

Our synthetic strategy starts with a selective reduction of the double bond in 10deoxymethynolide IV-2 by subjecting with excess diimide precursor ${ }^{87}$ which gave the desired dihydro aglycone IV-7 in 95\% yield (Scheme 47). Aglycone IV-7 underwent a diastereoselective palladium-catalyzed glycosylation with of $\alpha$-Boc-pyranone IV-6 producing $\alpha$-glycoside IV-8 as a single diastereomer and in good yield (86\%). Luche reduction ${ }^{88}$ of enone IV-8 gave the equatorial allylic alcohol IV-9 in $82 \%$ yield. Diimide reduction of allylic alcohol IV-9 with excess triethylamine and $O$-nitrophenylsulfonyl hydrazide produced dideoxy analogue IV-10 in excellent yield (90\%).

Scheme 47. Synthesis of methymycin analogue IV-10 and IV-11. 


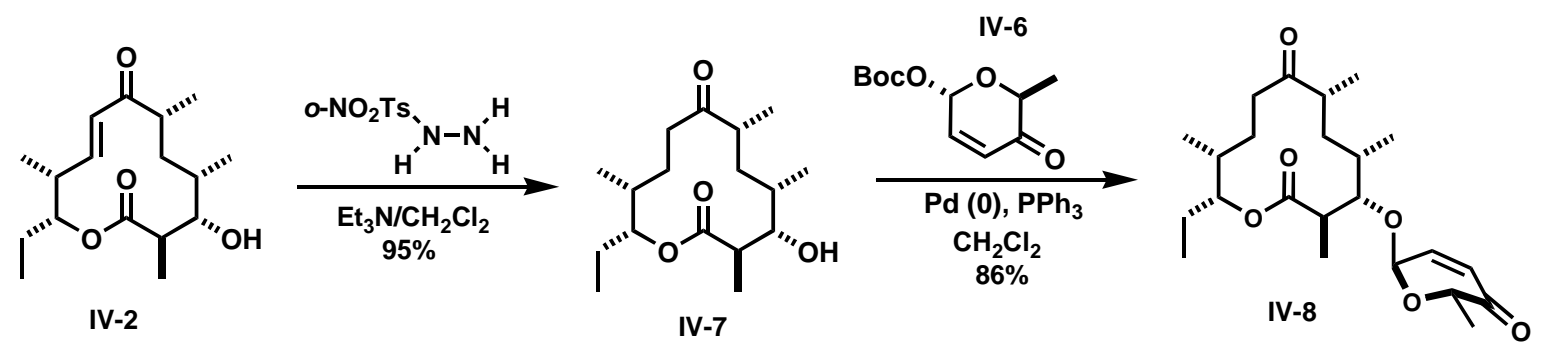

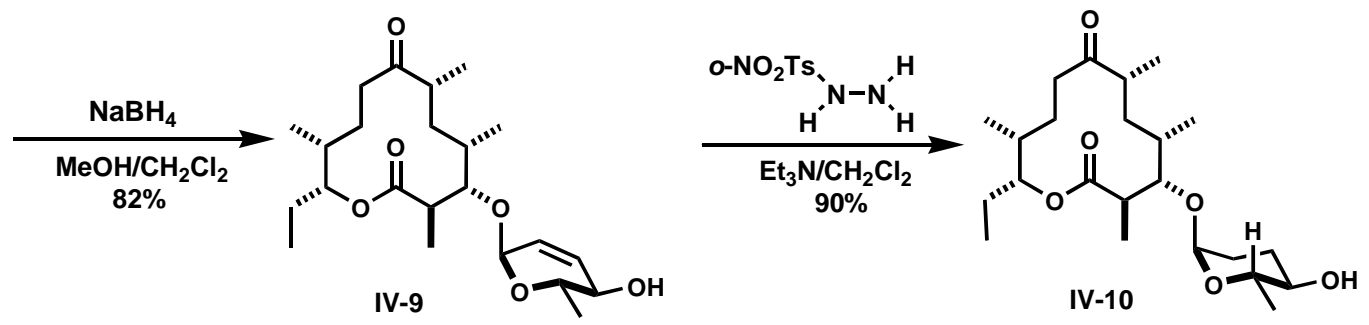

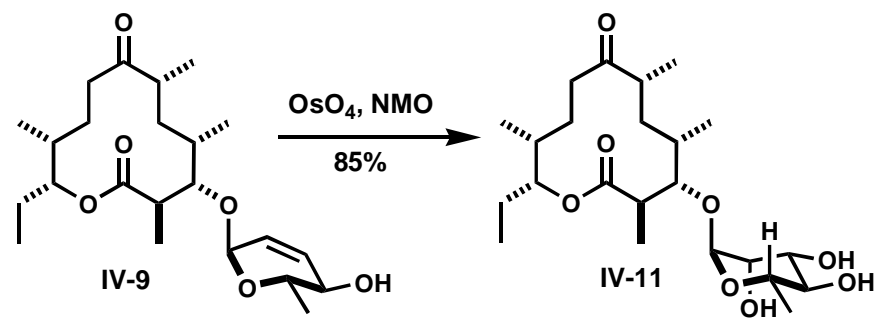

The rhamnose-sugar analogue IV-11 was achieved by diastereoselective dihydroxylation using Upjohn conditions ${ }^{20}\left(\mathrm{OsO}_{4} / \mathrm{NMO}\right)$ in $85 \%$ yield (Scheme 47$)$.

We next investigated methods for construction of various $C$-4-amino/azido sugar analogues (Scheme 48). To do this, we converted the allylic alcohol IV-9 into a methyl carbonate leaving group by reaction with methyl chloroformate to form $C$-4-carbonate IV-12 in 70\% yield.

Scheme 48. Synthesis of methymycin analogues IV-14, IV-15 and IV-16. 

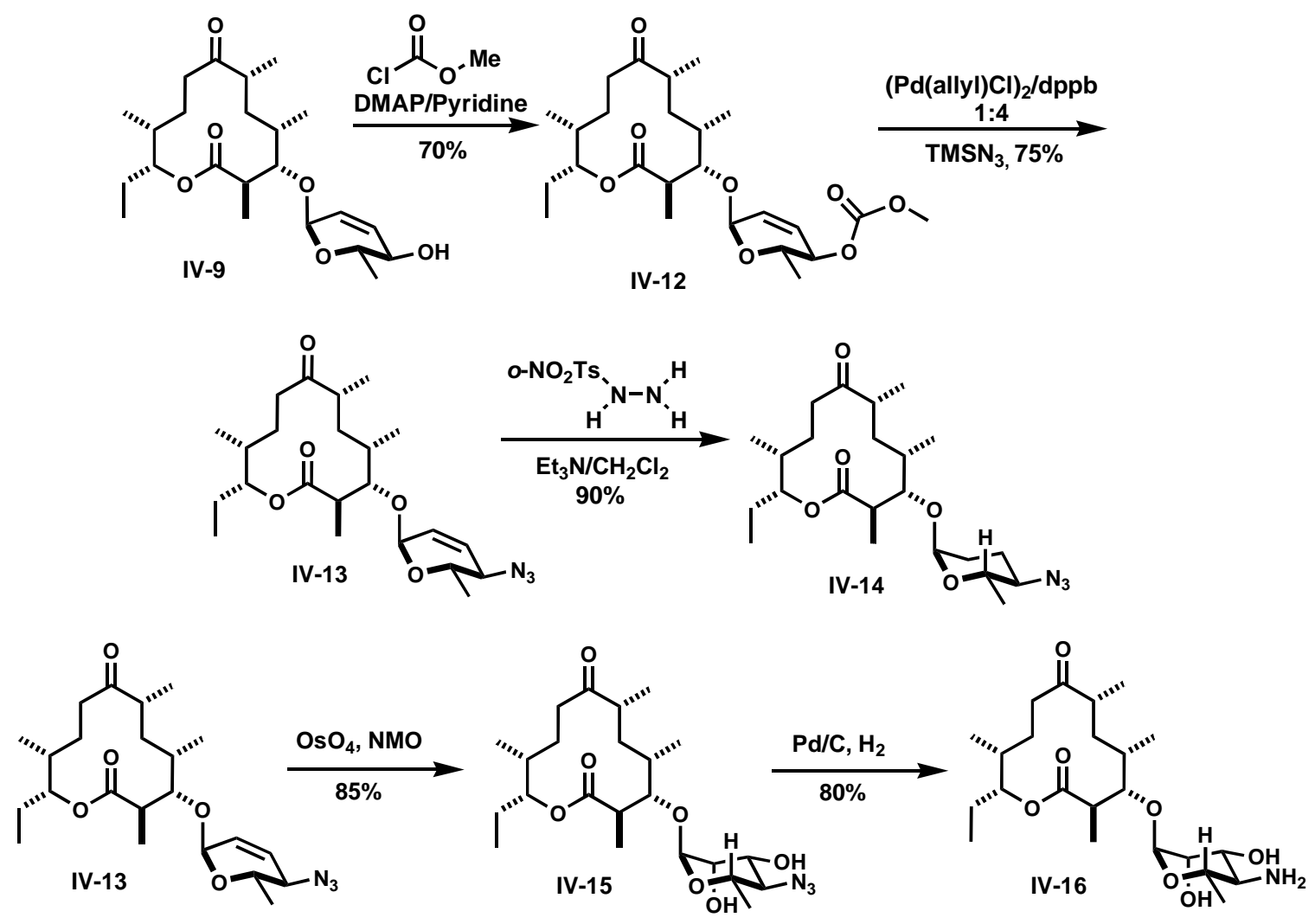

Exposing carbonate IV-12 to the Sinou conditions ${ }^{70}\left(\mathrm{TMSN}_{3},\left(\mathrm{Pd}(\mathrm{allyl}) \mathrm{Cl}_{2} / 1,4-\right.\right.$ bis(diphenylphosphino)butane) afforded a single regio-, and stereoisomeric allylic azide IV- IV-13 in 75\% yield. As before, diimide reduction of allylic azide IV-13 gave 2,3 dideoxy analogue IV-14 in 90\% yield. Once again, a diastereoselective dihydroxylation $\left(\mathrm{OsO}_{4} / \mathrm{NMO}\right)$, followed by reduction of azide $\left(\mathrm{Pd} / \mathrm{C}, \mathrm{H}_{2}, \mathrm{MeOH}\right)$ produced aminomannose analogue IV-16 in 80\% yield via rhamno-azide IV-15 in 85\%.

Hydrogenolysis $\left(\mathrm{Pd} / \mathrm{C}, \mathrm{H}_{2}, \mathrm{MeOH}\right)$ of allylic azide IV-13 gave dideoxy amino sugar analogue IV-17 via one-pot reduction of both azide and allylic double bond in 82\% yield (Scheme 49).

With successful syntheses of amino-and deoxy-rhamnose sugar analogues of methymycin, next explored the synthesis of inverted amino sugar analogue IV-17 at the C-4-position (Scheme 49). 
Scheme 49. Synthesis of dideoxy-amino analogues IV-17 and IV-20.
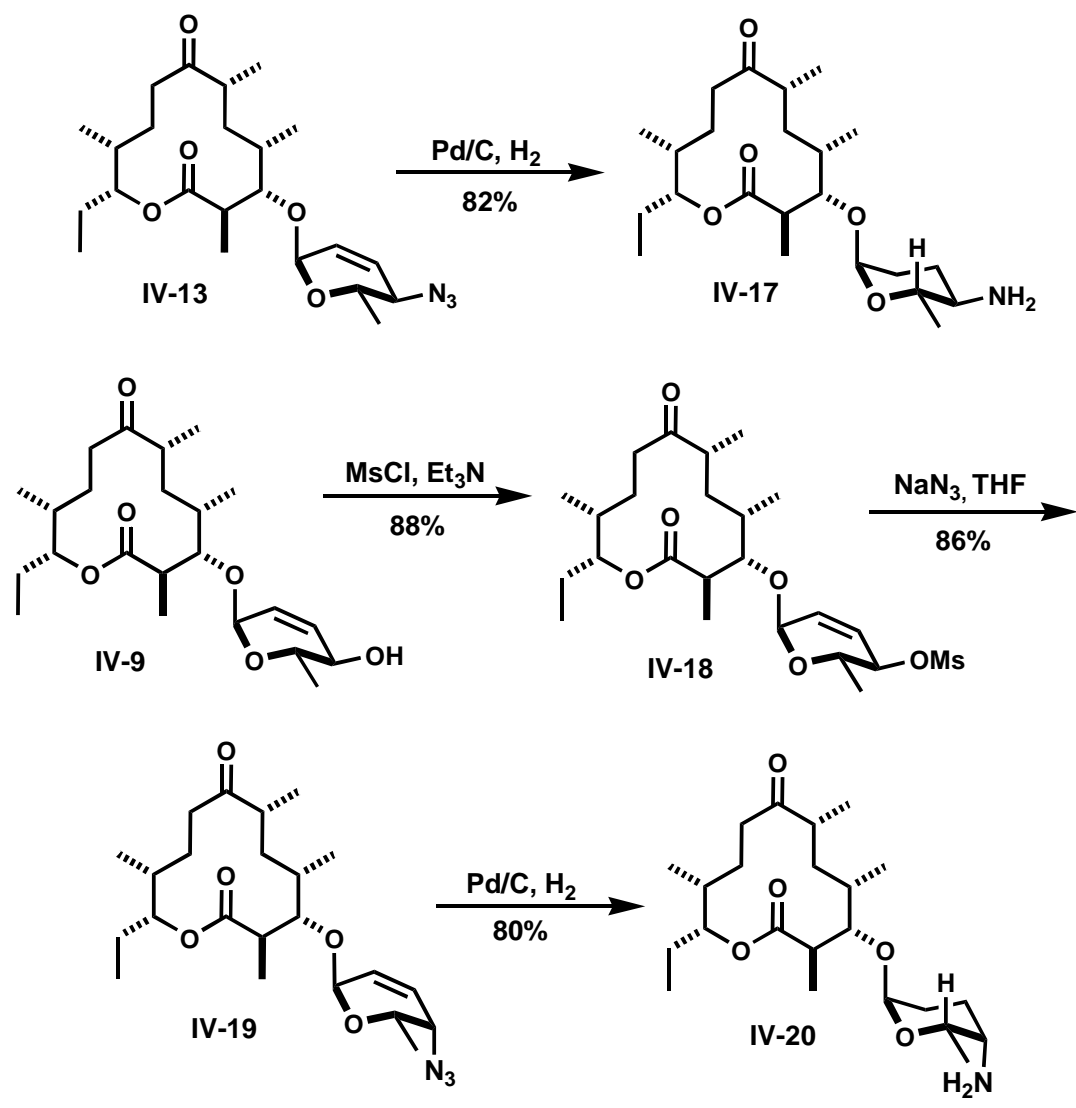

When carbonate IV-12 was subjected to Mitsunobu conditions using TMS azide as the nucleophile, no desired $C$-4-azido compound IV-19 was formed. We next turned to a two step $\mathrm{S}_{\mathrm{N}} 2$ reaction route. Thus allylic alcohol IV-9 was converted into mesylate IV$18\left(\mathrm{MsCl} / \mathrm{Et}_{3} \mathrm{~N}\right)$ in excellent yield $88 \%$, followed by treating IV-18 with $\mathrm{NaN}_{3} / \mathrm{THF}$ to give the inverted $C$-4-azido compound IV-19 in $86 \%$ yield. Reduction of the $C$-4-azido group and allylic double bond in compound IV-19 under hydrogenolysis conditions $\left(\mathrm{Pd} / \mathrm{C}, \mathrm{H}_{2}, \mathrm{MeOH}\right)$ gave $C$-4-deoxy-amino analogue of methymycin IV-20 in excellent yield $80 \%$.

Figure 6. The reverse glycosyl transferase assay using HPLC chromatogram analysis on our small library of eight compounds. 


\section{Reactions with narbonolide}

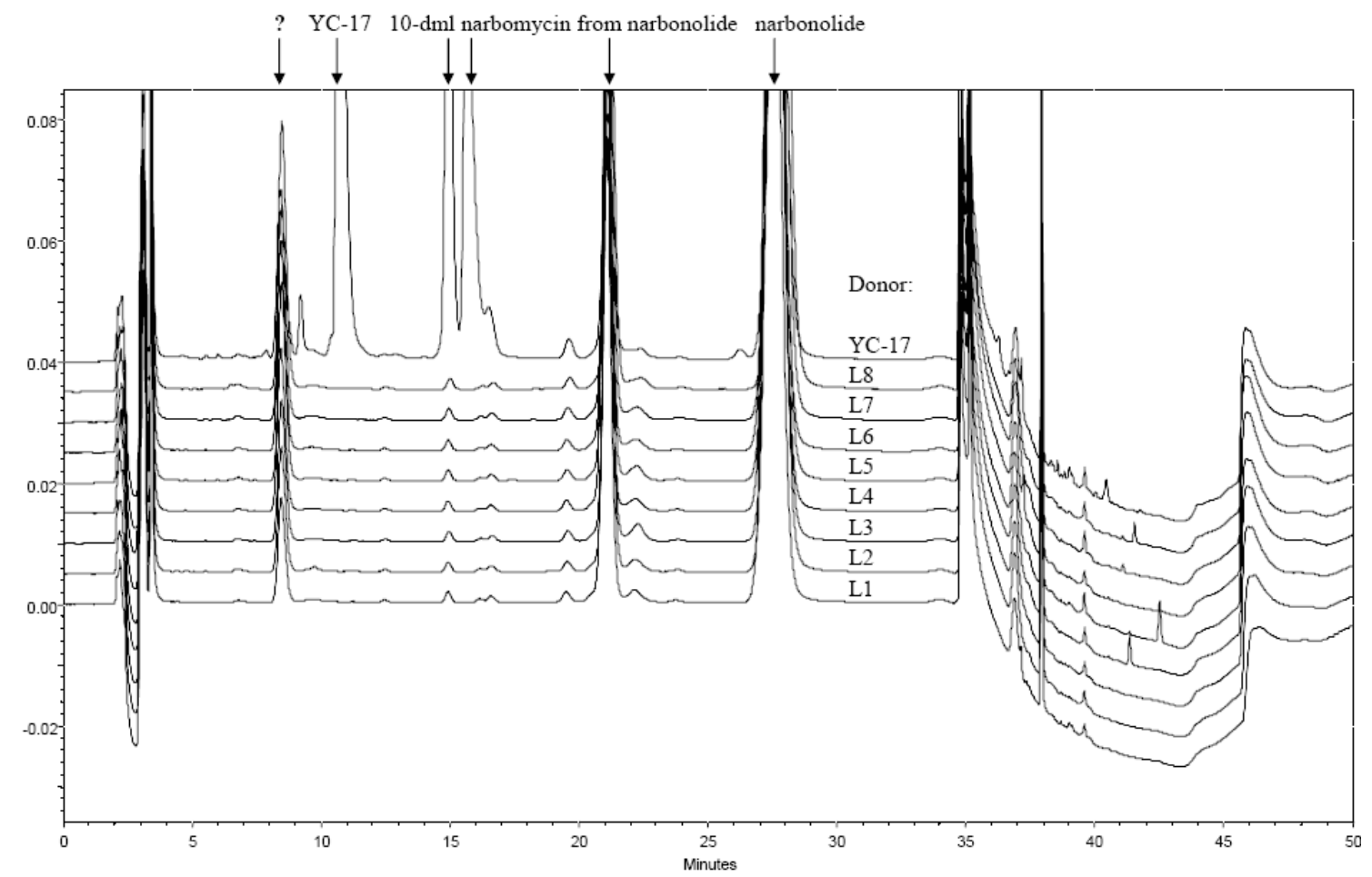

Reactions with tylactone

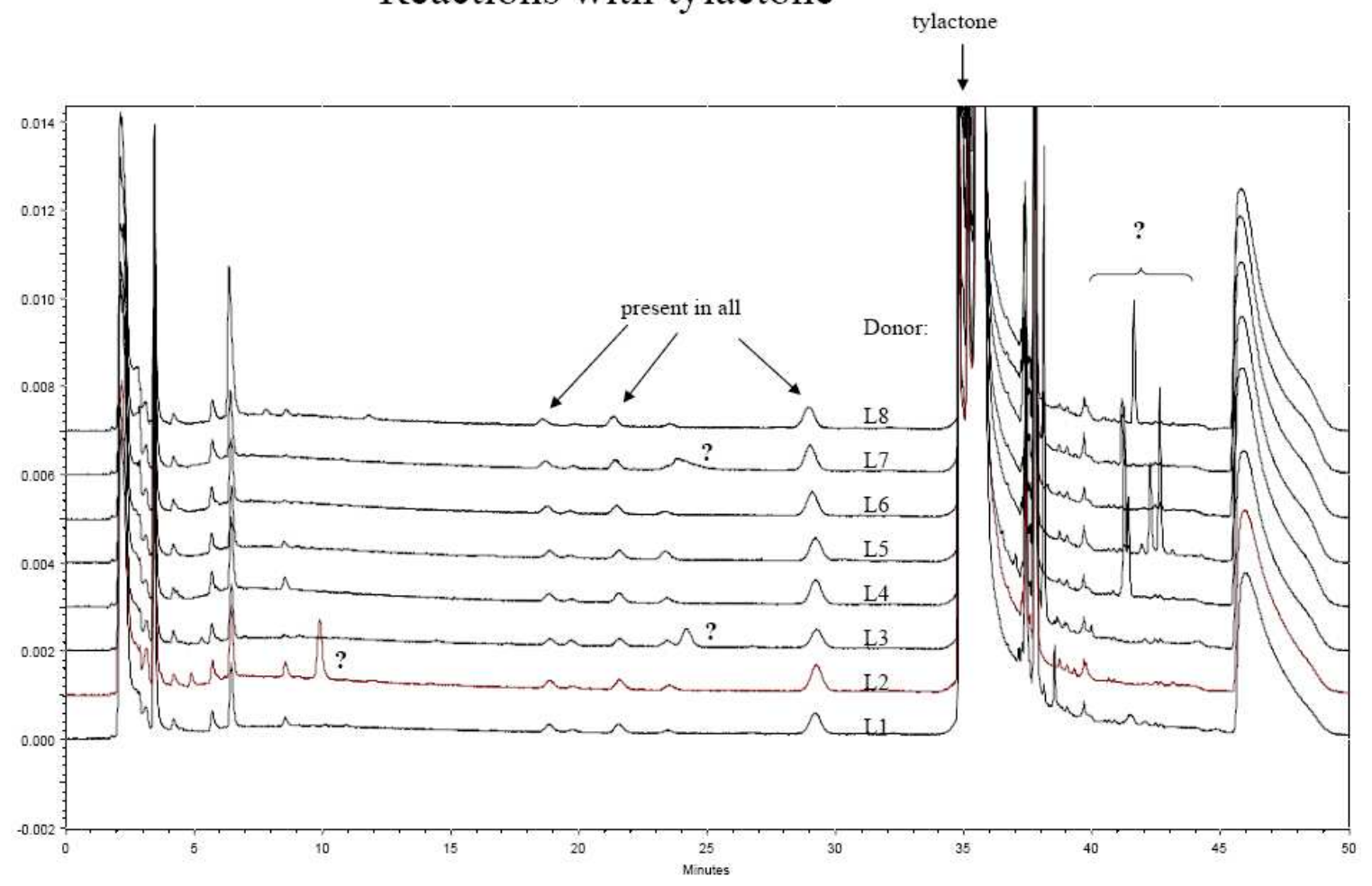




\section{Reactions with $10-\mathrm{dml}$}

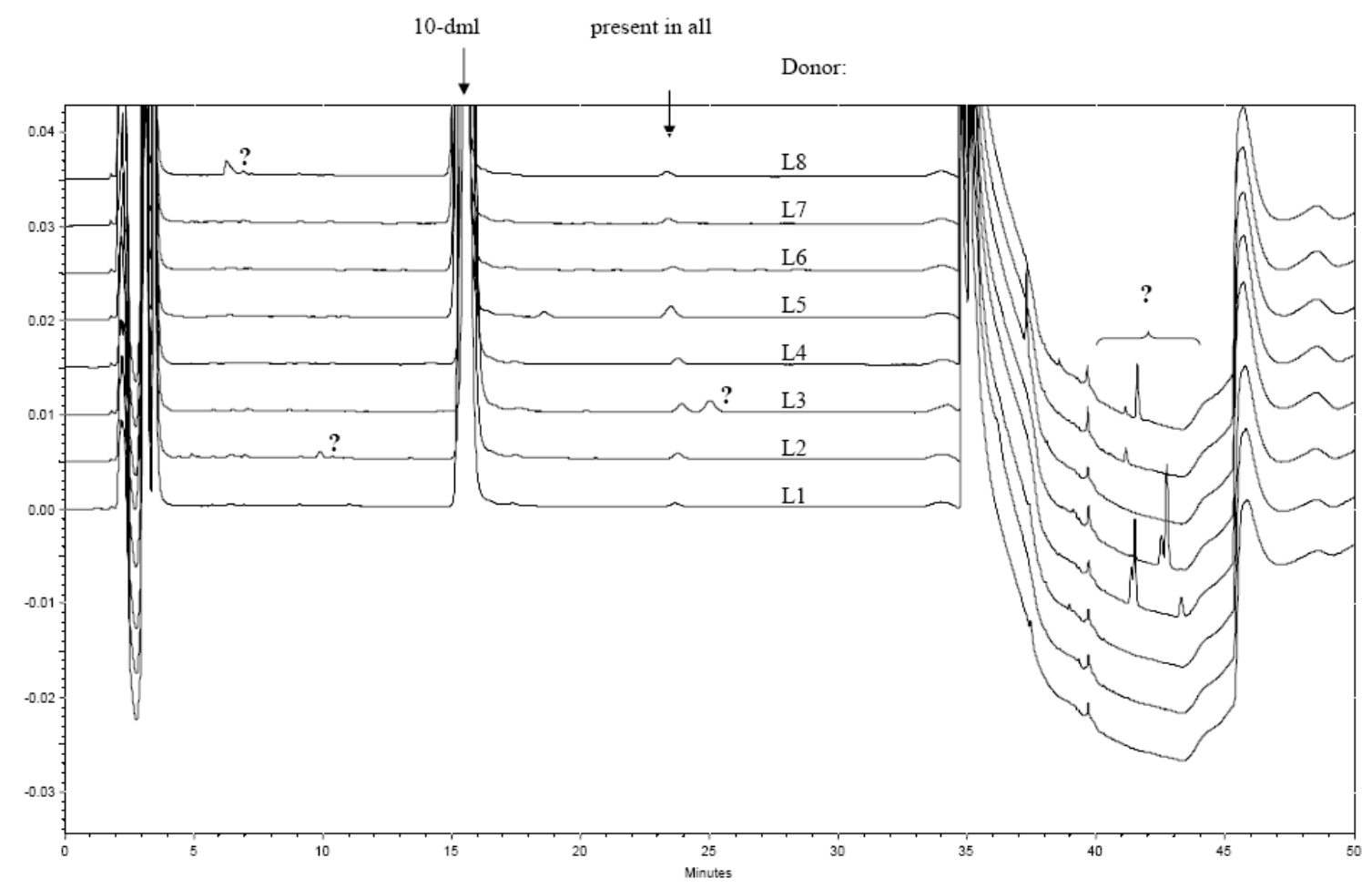

TLC assay of reverse reaction:

Lx means standard of compound 10d-Lx

$\mathrm{X}$ is reaction of the same compound

Aglycone product spots are shown with arrows

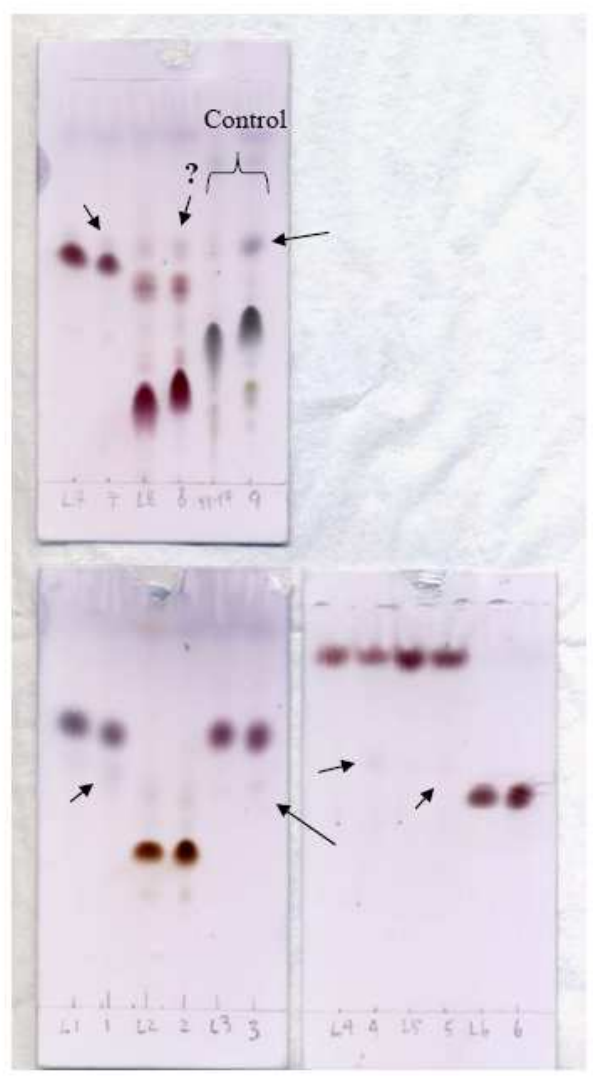


Prof. Hung-Wen Liu group had tested all eight compounds (Compound IV-9, IV10, IV-11, IV-13, IV-14, IV-15, IV-16, IV-17) as sugar donors in the reactions with three different acceptors: 10-deoxymethynolide, narbonolide, and tylactone (Figure 6). They ran assays from $5 \mathrm{~h}$ to overnight and analyzed the reactions with narbonolide by HPLC and TLC, and two other sets by HPLC. Then they did the reverse assay only (no aglycone acceptor added, excess of TDP) just to see if the enzyme recognizes the substrates. By TLC assay, it appears that IV-9, IV-10, IV-13, IV-14, IV-17 are deglycosylated, and IV-11, IV-15, IV-16 are not. These initial results imply that some (IV9, IV-10, IV-13, IV-14, IV-17) sugars are hydrolyzed but not transformed to the new substrate, which implies L-analogs are not recognized well. There may be better chance of success with D-analogs since they see up to $30 \%$ transfer with natural sugar. Thus we plan to investigate the syntheses $\beta$-D-sugar methymycin analogues.

In this connection, we next investigated the synthesis of 2,6 dideoxy $\beta$-D-allosugar analogue IV-25 of methymycin (Scheme 50). The methodology developed by our group for the synthesis of Digitoxin and its analogues has been used in the synthesis of 2,6 dideoxy $\beta$-D-allo-sugar. Thus taking 10-dideoxymethynolide IV-7 and $\beta$-D-pyranone IV-21 subjecting to palladium-catalyzed glycosylation gave the $\beta$-D-glycoside IV-22 as single diastereomer and in good yield (90\%). Luche reduction of ketone IV-22 provided a mixture of diastereomeric allylic alcohols IV-23 in $83 \%$ yield.

Scheme 50. Synthesis of allose-sugar analogue IV-25. 
<smiles>CC[C@H](C)[C@H](OC(=O)C(C)[C@H](C)O)[C@@H](C)CCC(=O)C(C)C</smiles>

IV-7

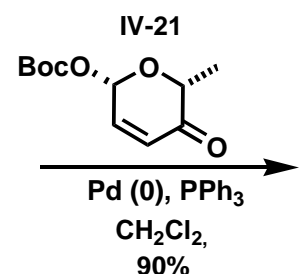

$90 \%$

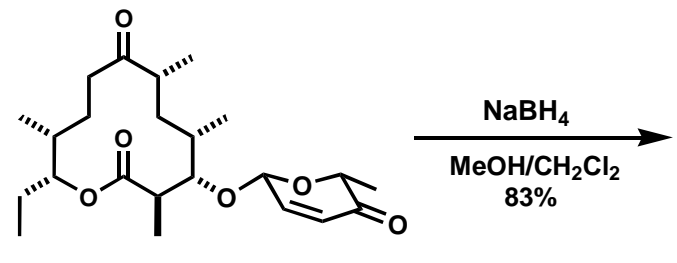

IV-22

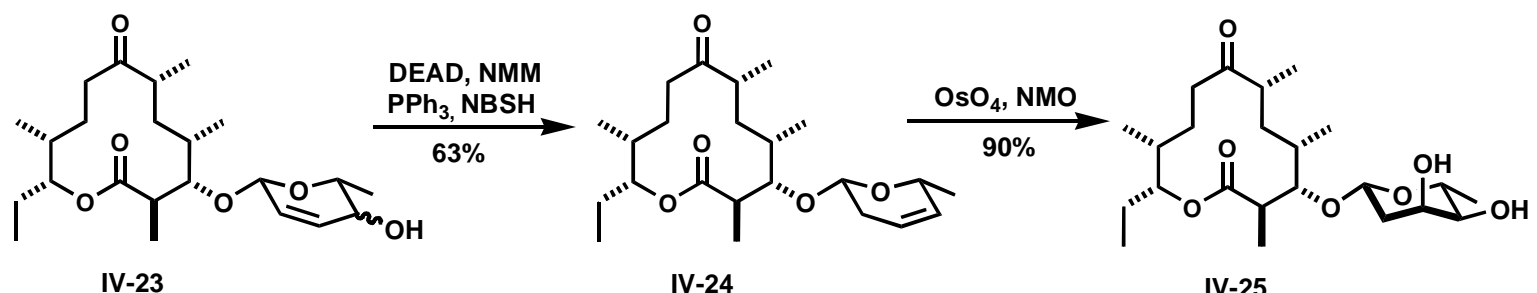

Scheme 51. Mechanism of Myers' reductive rearrangement.<smiles>CC[C@H](OC(=O)[C@H](C)[C@H](OC1C=C[C@@H](O)C(C)O1)[C@@H](C)C[C@@H](C)C(=O)CCC(C)C)[C@@H](C)O</smiles>

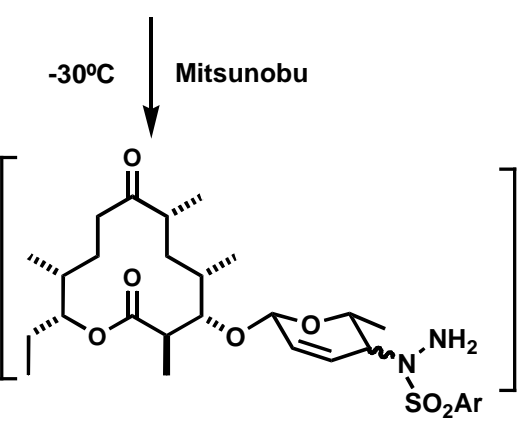

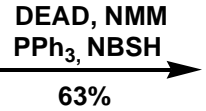<smiles>CC[C@H](OC(=O)C(C)[C@H](OC1CC=CC(C)O1)[C@@H](C)C[C@@H](C)C(=O)CC[C@@H](C)C(N)=O)C(C)C</smiles>

sigmatropic elimination

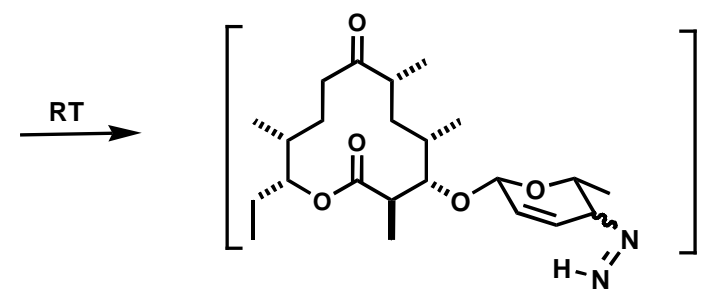

Further, exposing the mixture of allylic alcohols IV- 23 to the Myers' reductive rearrangement conditions ${ }^{89}\left(\mathrm{NBSH}, \mathrm{Ph}_{3} / \mathrm{DEAD}, \mathrm{NMM},-30{ }^{\circ} \mathrm{C}\right.$ to $\left.\mathrm{rt}\right)$ provided olefin $\mathbf{I V}$ 24 in moderate yield (63\%) (Scheme 51). Finally, dihydroxylation of olefin IV-24 using the Upjohn conditions ( $\left.\mathrm{OsO}_{4} / \mathrm{NMO}\right)$ gave exclusively the 2,6 dideoxy allose-sugar analogue IV-25 in $90 \%$ yield.

Encouraged by the above results, we next investigated our palladium-catalyzed glycosylation reaction to methymycin aglycone IV-26 with $\alpha$-D-pyranone IV-27. Our 
strategy worked well and gave single regioselective glycosylated product IV-28 in 75\% yield with the free tertiary alcohol left unreacted (Scheme 52). The structure of IV-28 was confirmed using 1D NOE difference, COSY and heterocorrelation HETCOR experiments.

Scheme 52. Synthesis of methynolide- $\alpha$-D-glycoside IV-28.

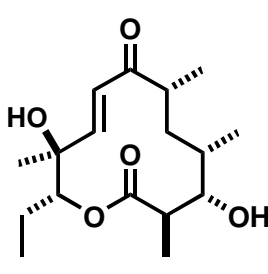

IV-26

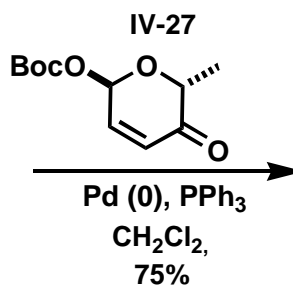

$75 \%$

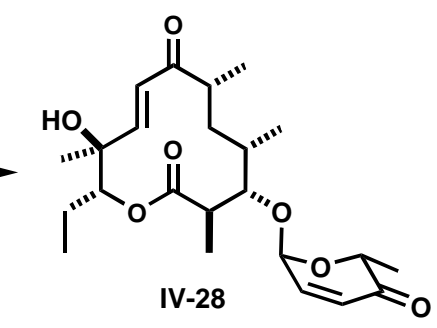

\subsection{Summary}

In conclusion, a divergent and highly enantioselective route to eight various amino/azido/dideoxy methymycin analogues have been developed. The key to the success of this method is the iterative use of the palladium-catalyzed glycosylation reaction, Luche reduction/Myers' reductive rearrangement, diastereoselective dihydroxylation, and regioselective reductions. This unique application of our $\mathrm{Pd}$ catalyzed glycosylation efficiently prepares a challenging and important $\alpha / \beta$-glycoside target. Currently, a small library of methymycin analogues was under testing on glycosyltransferase assays in Prof. Hung-Wen Liu labs. 


\section{Chapter 5}

\section{Experimental Section.}

\section{General Methods and materials.}

General Methods and materials: ${ }^{1} \mathrm{H}$ and ${ }^{13} \mathrm{C}$ spectra were recorded on Joel 270 and Varian 600 spectrometers. Chemical shifts were reported relative to internal tetramethylsilane $(\delta 0.00+)$ or $\mathrm{CDCl}_{3}\left(\delta\right.$ 7.26) or $\mathrm{CD}_{3} \mathrm{OD}\left(\delta\right.$ 4.87) for ${ }^{1} \mathrm{H}$ and $\mathrm{CDCl}_{3}(\delta$ 77.1) or $\mathrm{CD}_{3} \mathrm{OD}(\delta 49.15)$ for ${ }^{13} \mathrm{C}$. Optical rotations were measured with a Jasco DIP-370 digital polarimeter in the solvent specified. Infrared (IR) spectra were obtained on a prospect MIDAC FT-IR spectrometer. Flash column chromatography was performed on ICN reagent 60 (60-200 mesh) silica gel. Analytical thin-layer chromatography was performed with precoated glass-backed plates (Whatman K6F 60^, F254) and visualized by quenching of fluorescence and by charring after treatment with $p$-anisaldehyde or phosphomolybdic acid or potassium permanganate stain. $R f$ values were obtained by elution in the stated solvent ratios (v/v). Ether, THF, methylene chloride and triethylamine were dried by passing through activated alumina ( 8 x 14 mesh) column with nitrogen gas pressure. Commercial reagents were used without purification unless otherwise noted. Air and/or moisture-sensitive reactions were carried out under an atmosphere of argon/nitrogen using oven/flamed-dried glassware and standard syringe/septa techniques.

\section{1-(2'-Furyl)-2-trimethylsilanylethan-1-ol (I-2a). ${ }^{14}$}

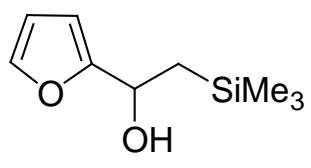


Magnesium turnings (10.1 g, $0.415 \mathrm{~mol})$ were placed in a $1 \mathrm{~L}-3$-neck round bottom flask and a condenser along with a side arm addition funnel were attached. The apparatus was flame dried (3x), each time flushing with nitrogen. Chloromethyltrimethylsilane (42.4 g, $0.346 \mathrm{~mol})$ in $200 \mathrm{~mL}$ of $\mathrm{Et}_{2} \mathrm{O}$ were added slowly to the dry magnesium. After the addition, the solution was refluxed for $1 \mathrm{~h}$. Freshly distilled furfural $(25.0 \mathrm{~mL}, 0.302$ mol) and $300 \mathrm{~mL}$ of $\mathrm{Et}_{2} \mathrm{O}$ were added slowly to the Grignard reagent at $0{ }^{\circ} \mathrm{C}$ and the solution was stirred for $3 \mathrm{~h}$ at $0{ }^{\circ} \mathrm{C}$ and $9 \mathrm{~h}$ at room temp. The reaction was quenched with $200 \mathrm{~mL}$ of sat. aq. $\mathrm{NH}_{4} \mathrm{Cl}$ and extracted $(3 \times 100 \mathrm{~mL})$ with $\mathrm{Et}_{2} \mathrm{O}$. The organic layer was washed with satd aq $\mathrm{NaHCO}_{3}(2 \times 50 \mathrm{~mL})$, brine $(2 \times 50 \mathrm{~mL})$, dried $\left(\mathrm{Na}_{2} \mathrm{SO}_{4}\right)$, and concentrated under reduced pressure to give 1-(2'-furyl)-2-trimethylsilanylethan-1-ol I2a in $90 \%$ yield, $50.1 \mathrm{~g}(0.272 \mathrm{~mol}): R_{f}\left(30 \% \mathrm{Et}_{2} \mathrm{O} /\right.$ hexanes $)=0.58$; IR (thin film, $\left.\mathrm{cm}^{-1}\right)$ 3390, 2950, 2895, 1655, 1505, 1250; ${ }^{1} \mathrm{H}$ NMR $\left(270 \mathrm{MHz}, \mathrm{CDCl}_{3}\right) \delta 7.28(\mathrm{dd}, J=1.8,0.7$ Hz, 1H), $6.24(\mathrm{dd}, J=3.1,1.8 \mathrm{~Hz}, 1 \mathrm{H}), 6.13(\mathrm{~d}, J=3.3 \mathrm{~Hz}, 1 \mathrm{H}), 4.77(\mathrm{dd}, J=8.8,6.9$ Hz, 1H), $3.13(\mathrm{bs}, 1 \mathrm{H}), 1.28(\mathrm{dd}, J=14.1,8.8 \mathrm{~Hz}, 1 \mathrm{H}), 1.23(\mathrm{dd}, J=14.1,6.8 \mathrm{~Hz}, 1 \mathrm{H}),-$ $0.10(\mathrm{~s}, 9 \mathrm{H}) ;{ }^{13} \mathrm{C} \mathrm{NMR}\left(67.5 \mathrm{MHz}, \mathrm{CDCl}_{3}\right) \delta 157.7,141.6,110.1,105.5,65.6,24.8,-1.4$ (3C); CIHRMS Calcd for $\left[\mathrm{C}_{9} \mathrm{H}_{16} \mathrm{O}_{2} \mathrm{Si}^{+}\right.$: 184.0920 . Found 184.0905.

\section{1-(2'-Furyl)-ethan-1R, 2-diol (I-2b). ${ }^{14}$}

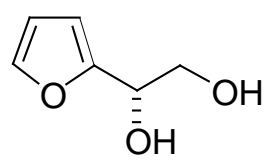

The $\beta$-hydroxy silane I-2a ( $44.2 \mathrm{~g}, 0.240 \mathrm{~mol})$ and $120 \mathrm{~mL}$ of $\mathrm{Et}_{2} \mathrm{O}$ were added to a 500 $\mathrm{mL}$ round bottom flask followed by addition of $120 \mathrm{~mL}$ of $1 \mathrm{M} \mathrm{HCl}$ and the solution was stirred for $1 \mathrm{~h}$. Phases were separated and the aq layer was extracted $(2 \times 50 \mathrm{~mL})$ with 
$\mathrm{Et}_{2} \mathrm{O}$ and combined with the organic layer. The organic layer was washed $(2 \times 50 \mathrm{~mL})$ with sat. aq. $\mathrm{NaHCO}_{3}$ and added to a solution of $300 \mathrm{~mL}$ of $t-\mathrm{BuOH}, 750 \mathrm{~mL}$ of $\mathrm{H}_{2} \mathrm{O}, 50$ $\mathrm{g}$ of $\mathrm{AD}-\mathrm{mix}-\alpha, 133 \mathrm{~g}$ of $\mathrm{K}_{3} \mathrm{Fe}(\mathrm{CN})_{6}$, and $56 \mathrm{~g}$ of $\mathrm{K}_{2} \mathrm{CO}_{3}$ at $0{ }^{\circ} \mathrm{C}$. The solution was vigorously stirred with a mechanical stirrer for $12 \mathrm{~h}$ at $0{ }^{\circ} \mathrm{C}$. The reaction was slowly quenched with $(500 \mathrm{~mL})$ satd aq $\mathrm{Na}_{2} \mathrm{SO}_{3}$. The phases were separated and the aqueous layer was extracted $(6 \times 100 \mathrm{~mL})$ with EtOAc. The organic layer was washed with satd aq $\mathrm{NaHCO}_{3}(2 \times 100 \mathrm{~mL})$, brine $(2 \times 100 \mathrm{~mL})$, dried $\left(\mathrm{Na}_{2} \mathrm{SO}_{4}\right)$, and concentrated under reduced pressure. The crude product was purified by silica gel flash chromatography eluting with $40 \% \mathrm{Et}_{2} \mathrm{O} /$ hexanes to yield 1-(2'-furyl)-ethan-1R,2-diol I-2b $25.0 \mathrm{~g}(0.195$ mol, 85\%): $R_{f}\left(50 \% \mathrm{Et}_{2} \mathrm{O} /\right.$ hexanes $)=0.37 ;[\alpha]^{21}{ }_{\mathrm{D}}=+32.0\left(c\right.$ 2.17, $\left.\mathrm{CH}_{2} \mathrm{Cl}_{2}\right) ; \mathrm{IR}$ (thin film, $\left.\mathrm{cm}^{-1}\right) 3390,2933,2881,1684,1505,1464,1228 ;{ }^{1} \mathrm{H}$ NMR $\left(270 \mathrm{MHz}, \mathrm{CDCl}_{3}\right) \delta$ $7.30(\mathrm{dd}, J=1.8,0.6 \mathrm{~Hz}, 1 \mathrm{H}), 6.27(\mathrm{dd}, J=4.0,1.8 \mathrm{~Hz}, 1 \mathrm{H}), 6.23(\mathrm{dd}, J=4.0,0.6 \mathrm{~Hz}$, $1 \mathrm{H}), 4.71(\mathrm{t}, J=5.9 \mathrm{~Hz}, 1 \mathrm{H}), 4.54(\mathrm{bs}, 2 \mathrm{H}), 3.74(\mathrm{~d}, J=5.9 \mathrm{~Hz}, 2 \mathrm{H}) ;{ }^{13} \mathrm{C}$ NMR $(67.5$ $\left.\mathrm{MHz}, \mathrm{CDCl}_{3}\right) \delta 153.9,142.3,110.5,107.0,68.4,65.0$; CIHRMS Calcd for $\left[\mathrm{C}_{6} \mathrm{H}_{8} \mathrm{O}_{3}+\right.$ $\left.\mathrm{NH}_{4}\right]^{+}$: 146.0817. Found 146.0822; Anal. Calcd for $\mathrm{C}_{6} \mathrm{H}_{8} \mathrm{O}_{3}:$ C, 56.23; H, 6.30. Found: C, 56.04; H, 6.20.

\section{1-(2'-Furyl)-2-tert-butyldimethylsilanyloxyethan-1-R-ol (I-3). ${ }^{14}$}

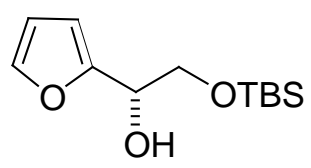

Diol 1-(2'-furyl)-ethan-1R,2-diol I-2b (0.986 g, $7.70 \mathrm{mmol}), 15 \mathrm{~mL}$ of $\mathrm{CH}_{2} \mathrm{Cl}_{2}$, and 5.5 $\mathrm{mL}$ of $\mathrm{Et}_{3} \mathrm{~N}$ were added to a round bottom flask and cooled to $0{ }^{\circ} \mathrm{C}$. A catalytic amount (50 mg, $0.41 \mathrm{mmol}$ ) of DMAP was added followed by addition of tert-butyldimethylsilyl chloride $(1.19 \mathrm{~g}, 7.89 \mathrm{mmol})$ and the solution was stirred at $0{ }^{\circ} \mathrm{C}$ for $6 \mathrm{~h}$. The reaction 
was quenched with $1 \mathrm{M} \mathrm{NaHSO}_{4}$ and extracted $(3 \times 25 \mathrm{~mL})$ with $\mathrm{Et}_{2} \mathrm{O}$, washed with satd aq $\mathrm{NaHCO}_{3}(2 \times 20 \mathrm{~mL})$, and dried $\left(\mathrm{Na}_{2} \mathrm{SO}_{4}\right)$. The crude product was purified by silica gel flash chromatography eluting with $25 \% \mathrm{Et}_{2} \mathrm{O} /$ hexanes to yield 1-(2'-furyl)-2-tertbutyldimethylsilanyloxyethan-1R-ol $\quad \mathbf{I - 3} \quad \mathbf{1 . 6 9} \mathrm{g} \quad(6.97 \quad \mathrm{mmol}, \quad 91 \%): \quad R_{f} \quad(30 \%$ $\mathrm{Et}_{2} \mathrm{O} /$ hexanes $)=0.55 ;[\alpha]^{21}{ }_{\mathrm{D}}=+15.9\left(c 1.37, \mathrm{CH}_{2} \mathrm{Cl}_{2}\right)$; IR (thin film, $\left.\mathrm{cm}^{-1}\right)$ 3447, 2954, 2930, 2884, 2857, 1471, 1463, 1361; ${ }^{1} \mathrm{H}$ NMR $\left(270 \mathrm{MHz}, \mathrm{CDCl}_{3}\right) \delta 7.37(\mathrm{dd}, J=1.8,0.9$ $\mathrm{Hz}, 1 \mathrm{H}), 6.35(\mathrm{dd}, J=3.3,1.8 \mathrm{~Hz}, 1 \mathrm{H}), 6.33(\mathrm{dd}, J=3.3,0.9 \mathrm{~Hz}, 1 \mathrm{H}), 4.75(\mathrm{dd}, J=6.4$, $4.6 \mathrm{~Hz}, 1 \mathrm{H}), 3.86(\mathrm{dd}, J=10.1,4.6 \mathrm{~Hz}, 1 \mathrm{H}), 3.85$ (dd, $J=10.1,6.7 \mathrm{~Hz}, 1 \mathrm{H}), 3.04$ (bs, $1 \mathrm{H}), 0.90(\mathrm{~s}, 9 \mathrm{H}), 0.07(\mathrm{~s}, 6 \mathrm{H}) ;{ }^{13} \mathrm{C} \mathrm{NMR}\left(67.5 \mathrm{MHz}, \mathrm{CDCl}_{3}\right) \delta$ 154.0, 142.0, 110.3, 107.1, 68.5, 65.9, 26.0 (3C), 18.4, -5.3 (2C); CIHRMS Calcd for $\left[\left(\mathrm{C}_{12} \mathrm{H}_{22} \mathrm{O}_{3} \mathrm{Si}\right)-\mathrm{H}_{2} \mathrm{O}\right]^{+}$: 225.1310. Found 225.1296; Anal. Calcd for C, 59.47; H, 9.16. Found C, 59.80; H, 9.37.

6-Hydroxy-(2R)-2-tert-butyldimethylsilanyloxymethyl-2H-pyran-3-(6H)-one (I-12). ${ }^{14}$<smiles>O=C1C=C[C@@H](O)O[C@@H]1C[Os+3]</smiles>

Compound 1-(2'-furyl)-2-tert-butyldimethylsilanyloxyethan-1R-ol $\quad$ I-4 $(1.69 \mathrm{~g}, \quad 6.97$ mmol), $12 \mathrm{~mL}$ of $\mathrm{THF}$, and $3 \mathrm{~mL}$ of $\mathrm{H}_{2} \mathrm{O}$ were added to a round bottom flask and cooled to $0{ }^{\circ} \mathrm{C}$. Solid $\mathrm{NaHCO}_{3}(1.17 \mathrm{~g}, 13.9 \mathrm{mmol}), \mathrm{NaOAc} \cdot 3 \mathrm{H}_{2} \mathrm{O}(0.950 \mathrm{~g}, 6.98 \mathrm{mmol})$, and NBS (1.24 g, $6.97 \mathrm{mmol})$ were added to the solution and the mixture was stirred for $1 \mathrm{~h}$ at $0{ }^{\circ} \mathrm{C}$. The reaction was quenched with satd aq $\mathrm{NaHCO}_{3}(15 \mathrm{~mL})$, extracted $(3 \times 25$ $\mathrm{mL})$ with $\mathrm{Et}_{2} \mathrm{O}$, dried $\left(\mathrm{Na}_{2} \mathrm{SO}_{4}\right)$, concentrated under reduced pressure and purified by silica gel chromatography eluting with $20 \%$ EtOAc/hexanes to give 6-hydroxy-(2R)-2tert-butyldimethylsilanyloxymethyl-2H-pyran-3-(6H)-one I-12 $1.71 \mathrm{~g}(6.62 \mathrm{mmol}, 95 \%)$ : 
$R_{f}\left(40 \% \mathrm{Et}_{2} \mathrm{O} /\right.$ hexanes $)=0.40 ;$ IR $\left(\right.$ thin film, $\left.\mathrm{cm}^{-1}\right) 3388,2951,2929,2884,2858,1699$, 1464,$1256 ;{ }^{1} \mathrm{H}$ NMR $\left(270 \mathrm{MHz}, \mathrm{CDCl}_{3}\right)$ major isomer $\delta 6.93(\mathrm{dd}, J=10.3,3.3 \mathrm{~Hz}, 1 \mathrm{H})$, $6.12(\mathrm{dd}, J=10.4,0.6 \mathrm{~Hz}, 1 \mathrm{H}), 5.79(\mathrm{dd}, J=5.1,3.1 \mathrm{~Hz}, 1 \mathrm{H}), 4.59(\mathrm{dd}, J=5.0,2.8 \mathrm{~Hz}$, 1H), $4.02(\mathrm{dd}, J=11.2,5 \mathrm{~Hz}, 1 \mathrm{H}), 3.93(\mathrm{dd}, J=11.2,2.0 \mathrm{~Hz}, 1 \mathrm{H}), 0.87(\mathrm{~s}, 9 \mathrm{H}), 0.07$ (s, $3 \mathrm{H}), 0.06(\mathrm{~s}, 3 \mathrm{H}) ;{ }^{13} \mathrm{C} \mathrm{NMR}\left(67.5 \mathrm{MHz}, \mathrm{CDCl}_{3}\right)$ major isomer $\delta$ 194.9, 145.9, 128.1, 88.1, 76.7, 63.5, 25.8 (3C), 18.5, -5.2, -5.3; CIHRMS Calcd for $\left[\left(\mathrm{C}_{12} \mathrm{H}_{22} \mathrm{O}_{4} \mathrm{Si}\right)+\mathrm{H}\right]^{+}$: 259.1366. Found 259.1366; Anal. Calcd for C, 55.79; H, 8.59. Found C, 55.86; H, 8.45.

Carbonic acid tert-butyl ester 6-(tert-butyl-dimethyl-silanyloxymethyl)-5-oxo-5,6dihydro-2H-pyran-2-yl ester (I-15). ${ }^{14}$

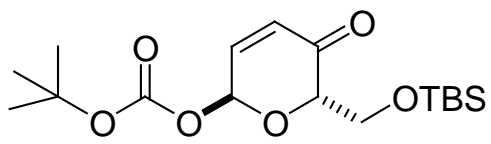

6-Hydroxy-(2R)-2-tert-butyldimethylsilanyloxymethyl-2H-pyran-3-(6H)-one I-12 (2.58 $\mathrm{g}, 10 \mathrm{mmol})$ was dissolved in $\mathrm{CH}_{2} \mathrm{Cl}_{2}(8 \mathrm{~mL})$ and the solution was cooled to $-78{ }^{\circ} \mathrm{C}$. A $\mathrm{CH}_{2} \mathrm{Cl}_{2}(2 \mathrm{~mL})$ solution of $(\mathrm{Boc})_{2} \mathrm{O}(2.61 \mathrm{~g}, 12 \mathrm{mmol})$ and a catalytic amount of DMAP (122 mg, $1 \mu \mathrm{mol}$ ) was added to the reaction mixture. The reaction was stirred for $1 \mathrm{~h}$ at $78{ }^{\circ} \mathrm{C}$. The reaction was quenched with $50 \mathrm{~mL}$ of satd. aq $\mathrm{NaHCO}_{3}$, extracted $(3 \times 50$ $\mathrm{mL}$ ) with $\mathrm{Et}_{2} \mathrm{O}$, dried $\left(\mathrm{Na}_{2} \mathrm{SO}_{4}\right)$, and concentrated under reduced pressure. The crude product was purified using silica gel flash chromatography eluting with $6 \%$ EtOAc/hexanes to give $2.93 \mathrm{~g}(8.20 \mathrm{mmol}, 82 \%)$ of carbonic acid tert-butyl ester 6-(tertbutyl-dimethyl-silanyloxymethyl)-5-oxo-5,6-dihydro-2H-pyran-2-yl ester I-15: $R_{f}(20 \%$ $\mathrm{Et}_{2} \mathrm{O} /$ hexanes $)=0.70 ;[\alpha]^{21} \mathrm{D}=+47.7\left(\mathrm{c}=1.5, \mathrm{CH}_{2} \mathrm{Cl}_{2}\right) ; \mathrm{IR}\left(\right.$ thin film, $\left.\mathrm{cm}^{-1}\right) 2956,2932$, $2858,1754,1703,1472,1371,1277,1257 ;{ }^{1} \mathrm{H} \mathrm{NMR}\left(270 \mathrm{MHz}, \mathrm{CDCl}_{3}\right) \delta 6.88(\mathrm{dd}, J=$ 
$10.2,3.7 \mathrm{~Hz}, 1 \mathrm{H}), 6.45(\mathrm{~d}, J=3.5 \mathrm{~Hz}, 1 \mathrm{H}), 6.23(\mathrm{~d}, J=10.2 \mathrm{~Hz}, 1 \mathrm{H}), 4.54(\mathrm{dd}, J=3.5$, $3.3 \mathrm{~Hz}, 1 \mathrm{H}), 4.05(\mathrm{~d}, J=3.5 \mathrm{~Hz}, 1 \mathrm{H}), 4.03(\mathrm{~d}, J=3.5 \mathrm{~Hz}, 1 \mathrm{H}), 1.51(\mathrm{~s}, 9 \mathrm{H}), 0.84(\mathrm{~s}, 9 \mathrm{H})$, 0.05 (s, 3H), $0.04(\mathrm{~s}, 3 \mathrm{H}) ;{ }^{13} \mathrm{C} \mathrm{NMR}\left(67.5 \mathrm{MHz}, \mathrm{CDCl}_{3}\right) \delta$ 193.6, 151.7, 141.4, 129.2, 89.1, 83.5, 77.7, 62.6, 27.6 (3C), 25.8 (3C), 18.2, -5.3 (2C); CIHRMS Calcd for $\left[\mathrm{C}_{17} \mathrm{H}_{30} \mathrm{O}_{6} \mathrm{Si}+\mathrm{Na}\right]^{+}:$381.1716. Found 381.1716.

Carbonic acid tert-butyl ester 6-(tert-butyl-dimethyl-silanyloxymethyl)-5-oxo-5,6dihydro-2H-pyran-2-yl ester (I-16). ${ }^{14}$

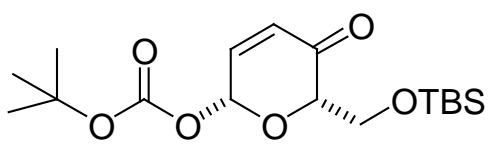

6-Hydroxy-(2R)-2-tert-butyldimethylsilanyloxymethyl-2H-pyran-3-(6H)-one I-12 (2.58 $\mathrm{g}, 10 \mathrm{mmol})$ was dissolved in $\mathrm{CH}_{2} \mathrm{Cl}_{2}(8 \mathrm{~mL})$ and the solution was cooled to $0{ }^{\circ} \mathrm{C}$. A $\mathrm{CH}_{2} \mathrm{Cl}_{2}(2 \mathrm{~mL})$ solution of $\left.(\mathrm{BOC})\right)_{2} \mathrm{O}(2.61 \mathrm{~g}, 12 \mathrm{mmol})$ and a catalytic amount of DMAP (122 mg, $1 \mu \mathrm{mol}$ ) was added to the reaction mixture. The reaction was stirred for $1 \mathrm{~h}$ at 0 ${ }^{\circ} \mathrm{C}$. The reaction was quenched with $50 \mathrm{~mL}$ of satd. aq $\mathrm{NaHCO}_{3}$, extracted $(3 \times 50 \mathrm{~mL})$ with $\mathrm{Et}_{2} \mathrm{O}$, dried $\left(\mathrm{Na}_{2} \mathrm{SO}_{4}\right)$, and concentrated under reduced pressure. The crude product was purified using silica gel flash chromatography eluting with 6\% EtOAc/hexanes to give $2.57 \mathrm{~g}(7.17 \mathrm{mmol}, 72 \%)$ of two diastereomers of carbonic acid tert-butyl ester 6(tert-butyl-dimethyl-silanyloxymethyl)-5-oxo-5,6-dihydro-2H-pyran-2-yl ester $\mathbf{6 c \alpha}$ and I-16 in $1: 1$ ratio $R_{f}\left(20 \% \mathrm{Et}_{2} \mathrm{O} /\right.$ hexanes $)=0.70 ;[\alpha]^{21}{ }_{\mathrm{D}}=-32.27\left(\mathrm{c}=1.5, \mathrm{CH}_{2} \mathrm{Cl}_{2}\right) ; \mathrm{IR}$ (thin film, $\left.\mathrm{cm}^{-1}\right) 2926,1742,1680,1283,1252,1162,1066 ;{ }^{1} \mathrm{H}$ NMR $(270 \mathrm{MHz}$, $\left.\mathrm{CDCl}_{3}\right) \delta 6.86(\mathrm{dd}, J=10.4,2.7 \mathrm{~Hz}, 1 \mathrm{H}), 6.40(\mathrm{dd}, J=2.7,1.3 \mathrm{~Hz}, 1 \mathrm{H}), 6.25(\mathrm{dd}, J=$ $10.4,1.2 \mathrm{~Hz}, 1 \mathrm{H}), 4.33(\mathrm{dd}, J=5.9,3.7 \mathrm{~Hz}, 1 \mathrm{H}), 4.00(\mathrm{dd}, J=11.2,5.7 \mathrm{~Hz}, 1 \mathrm{H}), 3.94$ 
(dd, $J=11.0,3.7 \mathrm{~Hz}, 1 \mathrm{H}), 1.51(\mathrm{~s}, 9 \mathrm{H}), 0.85(\mathrm{~s}, 9 \mathrm{H}), 0.05(\mathrm{~s}, 3 \mathrm{H}), 0.04(\mathrm{~s}, 3 \mathrm{H}) ;{ }^{13} \mathrm{C} \mathrm{NMR}$ $\left(67.5 \mathrm{MHz}, \mathrm{CDCl}_{3}\right) \delta 193.6,152.0,142.6,129.2,89.2,83.4,80.7,64.4,27.7$ (3C), 25.8 (3C), 18.3, -5.4 (2C); CIHRMS Calcd for $\left[\mathrm{C}_{17} \mathrm{H}_{30} \mathrm{O}_{6} \mathrm{Si}+\mathrm{Na}\right]^{+}$: 381.1716. Found 381.1714 .

\section{6-Benzyloxy-2-(tert-butyl-dimethyl-silanyloxymethyl)-6H-pyran-3-one (I-17).}

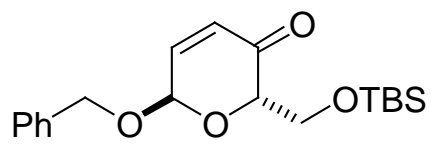

A $\mathrm{CH}_{2} \mathrm{Cl}_{2}(3.2 \mathrm{~mL})$ solution of compound $\mathbf{I}-15(1.5 \mathrm{~g}, 4.189 \mathrm{mmol})$ and benzyl alcohol (543 mg, $5.027 \mathrm{mmol}$ ) was cooled to $0{ }^{\circ} \mathrm{C}$. $\quad \mathrm{A} \mathrm{CH}_{2} \mathrm{Cl}_{2}(1 \mathrm{~mL})$ solution of $\mathrm{Pd}_{2}(\mathrm{DBA})_{3}{ }^{-}$ ${ }^{\cdot} \mathrm{CHCl}_{3}(54 \mathrm{mg}, 2.5 \mathrm{~mol} \%)$ and $\mathrm{PPh}_{3}(43 \mathrm{mg}, 10 \mathrm{~mol} \%$ ) was added to the reaction mixture at $0{ }^{\circ} \mathrm{C}$. The reaction mixture was stirred at $0{ }^{\circ} \mathrm{C}$ for 1 hour. The reaction mixture was quenched with $5 \mathrm{~mL}$ of satd aq $\mathrm{NaHCO}_{3}$, extracted $(3 \times 5 \mathrm{~mL})$ with $\mathrm{Et}_{2} \mathrm{O}$, dried $\left(\mathrm{Na}_{2} \mathrm{SO}_{4}\right)$, and concentrated under reduced pressure. The crude product was purified using silica gel flash chromatography eluting with $6 \%$ EtOAc/hexanes to give $1.29 \mathrm{~g}$ $(3.72 \mathrm{mmol}, 89 \%)$ of I-17: $R_{f}(20 \%$ EtOAc/hexanes $)=0.56 ;[\alpha]^{21}=+27.5(\mathrm{c}=1.5$, $\mathrm{CH}_{2} \mathrm{Cl}_{2}$ ); IR (thin film, $\mathrm{cm}^{-1}$ ) 2929, 2359, 1699, 1253, 1133, 1037, 836; ${ }^{1} \mathrm{H}$ NMR (270 $\left.\mathrm{MHz}, \mathrm{CDCl}_{3}\right) \delta 7.34(\mathrm{~m}, 5 \mathrm{H}), 6.86(\mathrm{dd}, J=10.2,3.3 \mathrm{~Hz}, 1 \mathrm{H}), 6.10(\mathrm{~d}, J=10.2 \mathrm{~Hz}, 1 \mathrm{H})$, $5.40(\mathrm{~d}, J=3.3 \mathrm{~Hz}, 1 \mathrm{H}), 4.87(\mathrm{~d}, J=11.6 \mathrm{~Hz}, 1 \mathrm{H}), 4.67(\mathrm{~d}, J=11.6 \mathrm{~Hz}, 1 \mathrm{H}), 4.50(\mathrm{dd}, J$ $=4.5,2.9 \mathrm{~Hz}, 1 \mathrm{H}), 4.04(\mathrm{~m}, 2 \mathrm{H}), 0.88(\mathrm{~s}, 9 \mathrm{H}), 0.08(\mathrm{~s}, 6 \mathrm{H}) ;{ }^{13} \mathrm{C} \mathrm{NMR}(67.5 \mathrm{MHz}$, $\left.\mathrm{CDCl}_{3}\right) \delta 194.6,143.8,136.9,128.5,128.2(2 \mathrm{C}), 128.0(2 \mathrm{C}), 92.0,76.1,70.4,62.4,25.8$ (3C), 18.2, -5.3 (2C); CIHRMS Calcd for $\left[\mathrm{C}_{19} \mathrm{H}_{28} \mathrm{O}_{4} \mathrm{Si}+\mathrm{H}\right]^{+}:$349.1835. Found 349.1819. 
6-Benzyloxy-2-(tert-butyl-dimethyl-silanyloxymethyl)-3,6-dihydro-2H-pyran-3-ol (I17b).<smiles>OC1C=CC(OCc2ccccc2)O[C@@H]1C[SeH3-]</smiles>

A $\mathrm{CH}_{2} \mathrm{Cl}_{2}(2.86 \mathrm{~mL})$ solution of compound $\mathbf{I - 1 7}(1 \mathrm{~g}, 2.86 \mathrm{mmol})$ and $\mathrm{MeOH}(2.8 \mathrm{~mL})$ was cooled to $-78{ }^{\circ} \mathrm{C}$. $\mathrm{NaBH}_{4}(108 \mathrm{mg}, 2.86 \mathrm{mmol})$ was added and the reaction mixture was stirred at $-78^{\circ} \mathrm{C}$ for 3 hours. The reaction mixture was diluted with ether $(10 \mathrm{~mL})$ and was quenched with $5 \mathrm{~mL}$ of satd aq $\mathrm{NaHCO}_{3}$, extracted $(3 \times 5 \mathrm{~mL})$ with $\mathrm{Et}_{2} \mathrm{O}$, dried $\left(\mathrm{Na}_{2} \mathrm{SO}_{4}\right)$, and concentrated under reduced pressure. The crude product was purified using silica gel flash chromatography eluting with 20\% EtOAc/hexanes to give $903 \mathrm{mg}$ $(2.58 \mathrm{mmol}, 90 \%)$ of I-17b: $R_{f}(40 \%$ EtOAc/hexanes $)=0.32 ;[\alpha]^{21}{ }_{\mathrm{D}}=-31.0 \quad(\mathrm{c}=1$, $\mathrm{CH}_{2} \mathrm{Cl}_{2}$ ); IR (thin film, $\mathrm{cm}^{-1}$ ) 3442, 2929, 1462, 1255, 1043, 838; ${ }^{1} \mathrm{H}$ NMR $(270 \mathrm{MHz}$, $\left.\mathrm{CDCl}_{3}\right) \delta 7.35(\mathrm{~m}, 5 \mathrm{H}), 5.93(\mathrm{dd}, J=10.2,1.1 \mathrm{~Hz}, 1 \mathrm{H}), 5.77(\mathrm{ddd}, J=10.2,2.5,2.3 \mathrm{~Hz}$, $1 \mathrm{H}), 5.74(\mathrm{dd}, J=2.5,2.3 \mathrm{~Hz}, 1 \mathrm{H}), 5.04(\mathrm{dd}, J=2.5,1.9 \mathrm{~Hz}, 1 \mathrm{H}), 4.77(\mathrm{~d}, J=11.8 \mathrm{~Hz}$, 1H), $4.59(\mathrm{~d}, J=11.8 \mathrm{~Hz}, 1 \mathrm{H}), 4.18(\mathrm{~m}, 1 \mathrm{H}), 3.80(\mathrm{dd}, J=8.5,3.9 \mathrm{~Hz}, 1 \mathrm{H}), 3.74(\mathrm{~m}$, $1 \mathrm{H}), 2.88(\mathrm{~d}, J=3.7 \mathrm{~Hz}, 1 \mathrm{H}), 0.92(\mathrm{~s}, 9 \mathrm{H}), 0.10(\mathrm{~s}, 6 \mathrm{H}) ;{ }^{13} \mathrm{C} \mathrm{NMR}\left(67.5 \mathrm{MHz}, \mathrm{CDCl}_{3}\right) \delta$ 138.0, 132.9, 128.3 (2C), 127.9 (2C), 127.6, 125.7, 93.4, 70.1, 70.0, 67.0, 65.0, $25.8(3 \mathrm{C})$, 18.2, -5.4, 5.5; CIHRMS Calcd for $\left[\mathrm{C}_{19} \mathrm{H}_{30} \mathrm{O}_{4} \mathrm{SiNa}^{+}\right]$: 373.1811 Found 373.1805.

\section{2-Benzyloxy-6-(tert-butyl-dimethyl-silanyloxymethyl)-tetrahydro-pyran-3,4,5-triol} (I-17c).<smiles>CC(C)(C)[O+]C[C@H]1OC(OCc2ccccc2)[C@H](O)[C@H](O)C1O</smiles> 
To a $t$-butanol, acetone $(0.3 \mathrm{~mL}, 1: 1,1 \mathrm{M})$ solution of allyl alcohol $\mathbf{I - 1 7 b}(100 \mathrm{mg}, 0.285$ mmol) at $0{ }^{\circ} \mathrm{C}$ was added a solution of $(50 \% \mathrm{w} / \mathrm{v})$ of $\mathrm{N}$-methyl morpholine $\mathrm{N}$-oxide / water $(0.1 \mathrm{~mL})$. Crystalline $\mathrm{OsO}_{4}(0.5 \mathrm{mg}, 1 \mathrm{~mol} \%)$ was added and the reaction was stirred for $12 \mathrm{~h}$. The reaction mixture was concentrated and was pipetted directly on to a silica gel column $(1 \mathrm{~cm} \times 4$ ") $)$ using a small amount of $\mathrm{CH}_{2} \mathrm{Cl}_{2}(0.6 \mathrm{~mL})$ in three portions. Impurities were eluted with ether and the product was eluted with $\mathrm{MeOH} / \mathrm{ether}$ (2:98 to 4:96). Pure fractions were combined and concentrated to afford triol I-17c $91 \mathrm{mg}(0.238$ mmol, 84\%): $R_{f}(70 \%$ EtOAc/hexanes $)=0.32 ;[\alpha]^{21}=-50.1\left(\mathrm{c}=2, \mathrm{CH}_{2} \mathrm{Cl}_{2}\right) ; \mathrm{IR}($ thin film, $\left.\mathrm{cm}^{-1}\right) 3406,2929,1460,1254,1098,838 ;{ }^{1} \mathrm{H}$ NMR $\left(270 \mathrm{MHz}, \mathrm{CDCl}_{3}\right) \delta 7.31(\mathrm{~m}$, 5H), $4.86(\mathrm{~s}, 1 \mathrm{H}), 4.70(\mathrm{~d}, J=11.8 \mathrm{~Hz}, 1 \mathrm{H}), 4.47(\mathrm{~d}, J=11.8 \mathrm{~Hz}, 1 \mathrm{H}), 4.27(\mathrm{~m}, 1 \mathrm{H}), 4.02$ $(\mathrm{m}, 1 \mathrm{H}), 3.91-3.61(\mathrm{~m}, 7 \mathrm{H}), 0.89(\mathrm{~s}, 9 \mathrm{H}), 0.07(\mathrm{~s}, 6 \mathrm{H}) ;{ }^{13} \mathrm{C} \mathrm{NMR}\left(67.5 \mathrm{MHz}, \mathrm{CDCl}_{3}\right) \delta$ 137.2, 128.3 (2C), 127.9 (2C), 127.7, 98.7, 71.7, 70.4, 69.6, 68.8, 67.5, 64.2, 25.8 (3C), 18.2, -5.3, 5.4; CIHRMS Calcd for $\left[\mathrm{C}_{19} \mathrm{H}_{32} \mathrm{O}_{6} \mathrm{SiNa}^{+}\right]$: 407.1866 Found 407.1882.

\section{7-[6-(tert-Butyl-dimethyl-silanyloxymethyl)-5-oxo-5,6-dihydro-2H-pyran-2-yloxy]-} chromen-2-one (I-25).<smiles>O=C1C=C[C@@H](Oc2ccc3ccc(=O)oc3c2)O[C@@H]1C[SeH2]</smiles>

A THF (0.3 mL) solution of compound I-16 (100 mg, $0.28 \mathrm{mmol})$ and 7-hydroxy cumarin (90 mg, $0.56 \mathrm{mmol})$ was cooled to $0{ }^{\circ} \mathrm{C}$. A THF $(0.3 \mathrm{~mL})$ solution of $\mathrm{Pd}_{2}(\mathrm{DBA})_{3}{ }^{\circ} \mathrm{CHCl}_{3}$ (7.2 $\mathrm{mg}, 2.5 \mathrm{~mol} \%)$ and $\mathrm{PPh}_{3}(7.3 \mathrm{mg}, 10 \mathrm{~mol} \%)$ was added to the reaction mixture at 0 ${ }^{\circ} \mathrm{C}$. The reaction mixture was stirred at $0{ }^{\circ} \mathrm{C}$ for 1 hour. The reaction mixture was quenched with $2 \mathrm{~mL}$ of satd aq $\mathrm{NaHCO}_{3}$, extracted $(3 \times 5 \mathrm{~mL})$ with $\mathrm{Et}_{2} \mathrm{O}$, dried 
$\left(\mathrm{Na}_{2} \mathrm{SO}_{4}\right)$, and concentrated under reduced pressure. The crude product was purified using silica gel flash chromatography eluting with $15-20 \%$ EtOAc/hexanes to give $\mathbf{I}-25$ $75 \mathrm{mg}(0.18 \mathrm{mmol}, 65 \%)$ of $10 \mathrm{~b} \alpha: R_{f}(20 \%$ EtOAc/hexanes $)=0.36 ;[\alpha]^{21} \mathrm{D}=129.9(\mathrm{c}=$ 0.7, $\mathrm{CH}_{2} \mathrm{Cl}_{2}$ ); IR (thin film, $\mathrm{cm}^{-1}$ ) 2927, 1735, 1732, 1696, 1231, 1125, 836; ${ }^{1} \mathrm{H}$ NMR $\left(600 \mathrm{MHz}, \mathrm{CD}_{3} \mathrm{OD}\right) \delta 7.83(\mathrm{~d}, J=9.6 \mathrm{~Hz}, 1 \mathrm{H}), 7.50(\mathrm{~d}, J=9.6 \mathrm{~Hz}, 1 \mathrm{H}), 7.11(\mathrm{dd}, J=$ 10.2, $2.4 \mathrm{~Hz}, 1 \mathrm{H}), 7.10(\mathrm{~d}, J=2.4 \mathrm{~Hz}, 1 \mathrm{H}), 7.04(\mathrm{dd}, J=9.0,1.8 \mathrm{~Hz}, 1 \mathrm{H}), 6.24($ br s, 1H), $6.23(\mathrm{~d}, 2.4 \mathrm{~Hz}, 1 \mathrm{H}), 6.21(\mathrm{dd}, J=2.4,1.2 \mathrm{~Hz}, 1 \mathrm{H}), 4.39$ (dd, $J=6.6,3.0 \mathrm{~Hz}, 1 \mathrm{H})$, $3.95(\mathrm{dd}, J=11.4,7.2 \mathrm{~Hz}, 1 \mathrm{H}), 3.88(\mathrm{dd}, J=10.8,3.0 \mathrm{~Hz}, 1 \mathrm{H}), 0.80$ (s, 9H), -0.09 (s,

$3 \mathrm{H}),-0.16(\mathrm{~s}, 3 \mathrm{H}) ;{ }^{13} \mathrm{C}$ NMR $\left(150 \mathrm{MHz}, \mathrm{CD}_{3} \mathrm{OD}\right) \delta 198.4,195.3,162.9,161.4,145.8$, $145.4,130.4,129.8,115.4(2 \mathrm{C}), 114.4,105.1,94.0,81.7,64.9,26.1(3 \mathrm{C}), 19.0,-5.3,-5.5$; CIHRMS Calcd for $\left[\mathrm{C}_{21} \mathrm{H}_{26} \mathrm{O}_{6} \mathrm{Si}+\mathrm{Na}\right]^{+}: 425.1390$. Found 425.1420.

\section{7-[6-(tert-Butyl-dimethyl-silanyloxymethyl)-5-oxo-5,6-dihydro-2H-pyran-2-yloxy]-} chromen-2-one (I-23).<smiles>O=C1C=CC(Oc2ccc3ccc(=O)oc3c2)O[C@@H]1C[SeH2]</smiles>

A THF (0.3 mL) solution of compound I-15 (100 mg, $0.28 \mathrm{mmol})$ and 7-hydroxy cumarin (90 mg, $0.56 \mathrm{mmol})$ was cooled to $0{ }^{\circ} \mathrm{C}$. A THF $(0.3 \mathrm{~mL})$ solution of $\mathrm{Pd}_{2}(\mathrm{DBA})_{3}{ }^{\circ} \mathrm{CHCl}_{3}$ (7.2 $\mathrm{mg}, 2.5 \mathrm{~mol} \%)$ and $\mathrm{PPh}_{3}(7.3 \mathrm{mg}, 10 \mathrm{~mol} \%)$ was added to the reaction mixture at 0 ${ }^{\circ} \mathrm{C}$. The reaction mixture was stirred at $0{ }^{\circ} \mathrm{C}$ for 1 hour. The reaction mixture was quenched with $2 \mathrm{~mL}$ of satd aq $\mathrm{NaHCO}_{3}$, extracted $(3 \times 5 \mathrm{~mL})$ with $\mathrm{Et}_{2} \mathrm{O}$, dried $\left(\mathrm{Na}_{2} \mathrm{SO}_{4}\right)$, and concentrated under reduced pressure. The crude product was purified using silica gel flash chromatography eluting with 15-20\% EtOAc/hexanes to give $75 \mathrm{mg}$ 
$(0.18 \mathrm{mmol}, 65 \%)$ of I-23: $R_{f}(20 \%$ EtOAc/hexanes $)=0.36 ;[\alpha]^{21}{ }_{\mathrm{D}}=-96.10(\mathrm{c}=0.7$, $\mathrm{CH}_{2} \mathrm{Cl}_{2}$ ); IR (thin film, $\mathrm{cm}^{-1}$ ) 2927, 1736, 1702, 1615, 1231, 1125, 836; ${ }^{1} \mathrm{H}$ NMR (600 $\left.\mathrm{MHz}, \mathrm{CD}_{3} \mathrm{OD}\right) \delta 7.64(\mathrm{~d}, J=9.6 \mathrm{~Hz}, 1 \mathrm{H}), 7.40(\mathrm{~d}, J=9.0 \mathrm{~Hz}, 1 \mathrm{H}), 7.14(\mathrm{~d}, J=1.8 \mathrm{~Hz}$, $1 \mathrm{H}), 7.06(\mathrm{dd}, J=9.0,2.4 \mathrm{~Hz}, 1 \mathrm{H}), 7.02(\mathrm{dd}, J=10.2,3.0 \mathrm{~Hz}, 1 \mathrm{H}), 6.30(\mathrm{~d}, J=9.01 \mathrm{H})$, $6.29(\mathrm{~d}, J=10.2 \mathrm{~Hz}, 1 \mathrm{H}), 6.09(\mathrm{~d}, J=3.0 \mathrm{~Hz}, 1 \mathrm{H}), 4.50(\mathrm{dd}, J=4.8,3.0 \mathrm{~Hz}, 1 \mathrm{H}), 4.05$ (dd, $J=12.0,4.2 \mathrm{~Hz}, 1 \mathrm{H}), 3.03(\mathrm{dd}, J=12.0,3.0 \mathrm{~Hz}, 1 \mathrm{H}), 0.71(\mathrm{~s}, 9 \mathrm{H}), 0.04(\mathrm{~s}, 3 \mathrm{H}), 0.03$ (s, 3H); ${ }^{13} \mathrm{C}$ NMR $\left(150 \mathrm{MHz}, \mathrm{CD}_{3} \mathrm{OD}\right) \delta 193.8,160.8,159.7,155.4,143.0,141.7,129.6$, 128.8, 114.2, 114.0, 113.8(2C), 104.4, 91.7, 62.5, 25.7(3C), 18.1, -5.4(2C); CIHRMS Calcd for $\left[\mathrm{C}_{21} \mathrm{H}_{26} \mathrm{O}_{6} \mathrm{Si}+\mathrm{Na}\right]^{+}$: 425.1390. Found 425.1373.

\section{7-[6-(tert-Butyl-dimethyl-silanyloxymethyl)-5-hydroxy-5,6-dihydro-2H-pyran-2- yloxy]-chromen-2-one (I-41a).}

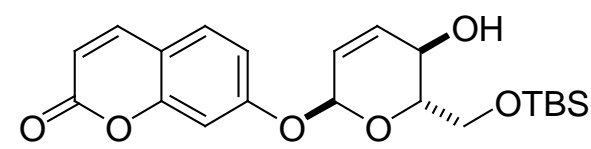

A THF (0.2 mL) solution of compound I-23 (100 mg, $0.24 \mathrm{mmol})$ and $\mathrm{MeOH}(0.2 \mathrm{~mL})$ was cooled to $-78{ }^{\circ} \mathrm{C}$. $\mathrm{NaBH}_{4}(9.20 \mathrm{mg}, 0.24 \mathrm{mmol})$ was added and the reaction mixture was stirred at $-78^{\circ} \mathrm{C}$ for 3 hours. The reaction mixture was diluted with ether $(5 \mathrm{~mL})$ and was quenched with $5 \mathrm{~mL}$ of satd aq $\mathrm{NaHCO}_{3}$, extracted $(2 \times 5 \mathrm{~mL})$ with $\mathrm{Et}_{2} \mathrm{O}$, dried $\left(\mathrm{Na}_{2} \mathrm{SO}_{4}\right)$, and concentrated under reduced pressure. The crude product was purified using silica gel flash chromatography eluting with $20 \%$ EtOAc/hexanes to give $90 \mathrm{mg}$ $(0.21 \mathrm{mmol}, 90.0 \%)$ of I-41a: $R_{f}(30 \%$ EtOAc/hexanes $)=0.42 ;[\alpha]^{21}{ }_{\mathrm{D}}=-203.7 \quad(\mathrm{c}=1$, $\mathrm{CH}_{3} \mathrm{OH}$ ); IR (thin film, $\mathrm{cm}^{-1}$ ) 3557, 2954, 1733, 1615, 1118, 835; ${ }^{1} \mathrm{H}$ NMR $(270 \mathrm{MHz}$, $\left.\mathrm{CDCl}_{3}\right) \delta 7.64(\mathrm{~d}, J=9.6 \mathrm{~Hz}, 1 \mathrm{H}), 7.38(\mathrm{~d}, J=8.4 \mathrm{~Hz}, 1 \mathrm{H}), 7.07(\mathrm{~d}, J=2.4 \mathrm{~Hz}, 1 \mathrm{H})$, 
$6.97(\mathrm{dd}, J=9.0,2.4 \mathrm{~Hz}, 1 \mathrm{H}), 6.28(\mathrm{~d}, J=9.0 \mathrm{~Hz}, 1 \mathrm{H}), 6.13(\mathrm{dd}, J=10.2,2.4 \mathrm{~Hz}, 1 \mathrm{H})$, $5.90(\mathrm{ddd}, J=10.2,2.4,2.4 \mathrm{~Hz}, 1 \mathrm{H}), 5.70(\mathrm{~d}, J=2.4 \mathrm{~Hz}, 1 \mathrm{H}), 4.30(\mathrm{ddd}, J=5.4,3.0,1.2$ $\mathrm{Hz}, 1 \mathrm{H}), 3.88(\mathrm{dd}, J=9.6,5.4 \mathrm{~Hz}, 1 \mathrm{H}), 3.80(\mathrm{dd}, J=9.0,4.2 \mathrm{~Hz}, 1 \mathrm{H}), 3.75(\mathrm{dd}, J=10.2$, $6.6 \mathrm{~Hz}, 1 \mathrm{H}), 3.10(\mathrm{~d}, J=3.6 \mathrm{~Hz}, 1 \mathrm{H}), 0.87(\mathrm{~s}, 9 \mathrm{H}), 0.09$ (s, 3H), $0.06(\mathrm{~s}, 3 \mathrm{H}) ;{ }^{13} \mathrm{C} \mathrm{NMR}$ $\left(67.5 \mathrm{MHz}, \mathrm{CDCl}_{3}\right) \delta 161.2,160.3,155.5,143.3,134.5,128.7,123.9,114.1,113.7$, 113.4, 104.1, 92.6, 71.0, 66.5,65.0, 25.7(3C), 18.1, -5.5, -5.6; CIHRMS Calcd for $\left[\mathrm{C}_{21} \mathrm{H}_{28} \mathrm{O}_{6} \mathrm{Si}+\mathrm{Na}\right]^{+}:$427.1547. Found 427.1538.

\section{7-[6-(tert-Butyl-dimethyl-silanyloxymethyl)-5-oxo-5,6-dihydro-2H-pyran-2-yloxy]-} chromen-2-one (I-42a).

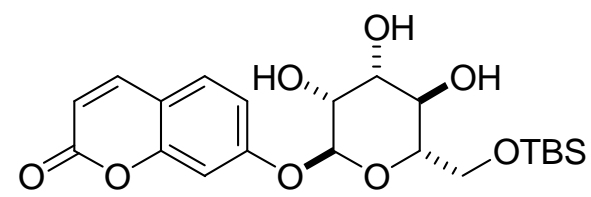

To a $t$-butanol, acetone $(0.4 \mathrm{~mL}, 1: 1,1 \mathrm{M})$ solution of allyl alcohol I-41a $(160 \mathrm{mg}, 0.363$ mmol) at $0{ }^{\circ} \mathrm{C}$ was added a solution of $(50 \% \mathrm{w} / \mathrm{v})$ of $\mathrm{N}$-methyl morpholine $\mathrm{N}$-oxide / water $(0.2 \mathrm{~mL})$. Crystalline $\mathrm{OsO}_{4}(1.0 \mathrm{mg}, 1 \mathrm{~mol} \%)$ was added and the reaction was stirred for $12 \mathrm{~h}$. The reaction mixture was concentrated and was pipetted directly on to a silica gel column using a small amount of $\mathrm{CH}_{2} \mathrm{Cl}_{2}(0.6 \mathrm{~mL})$ in three portions. Impurities were eluted with ether and the product was eluted with $\mathrm{MeOH} / \mathrm{ether} \mathrm{(2:98} \mathrm{to} \mathrm{4:96).} \mathrm{Pure}$ fractions were combined and concentrated to afford triol I-42a $145 \mathrm{mg}(0.329 \mathrm{mmol}$, $84 \%): R_{f}(50 \%$ EtOAc/hexanes $)=0.29 ;[\alpha]^{21} \mathrm{D}=-63.4\left(\mathrm{c}=2, \mathrm{CH}_{2} \mathrm{Cl}_{2}\right) ; \mathrm{IR}$ (thin film, $\left.\mathrm{cm}^{-1}\right) 3449,3420,2955,1742,1618,1129,836 ;{ }^{1} \mathrm{H}$ NMR $\left(600 \mathrm{MHz}, \mathrm{CD}_{3} \mathrm{OD}\right) \delta 7.91(\mathrm{~d}$, $J=9.6 \mathrm{~Hz}, 1 \mathrm{H}$ ), $7.57(\mathrm{~d}, J=9.0 \mathrm{~Hz}, 1 \mathrm{H}), 7.16(\mathrm{br} \mathrm{s}, 1 \mathrm{H}), 7.09(\mathrm{~d}, J=7.8 \mathrm{~Hz}, 1 \mathrm{H}), 6.30$ (d, $J=10.2 \mathrm{~Hz}, 1 \mathrm{H}), 5.60$ (br s, $1 \mathrm{H}$ ), 4.05 (br s, $1 \mathrm{H}), 3.95$ (d, $J=11.4 \mathrm{~Hz}, 1 \mathrm{H}), 3.90$ (dd, 
$J=9.0,3.0 \mathrm{~Hz}, 1 \mathrm{H}), 3.75(\mathrm{dd}, J=10.8,6.0 \mathrm{~Hz}, 1 \mathrm{H}), 3.65(\mathrm{dd}, J=10.2,9.0 \mathrm{~Hz}, 1 \mathrm{H}), 3.33$

(m, 3H), $0.78(\mathrm{~s}, 9 \mathrm{H}), 0.03(\mathrm{~s}, 3 \mathrm{H}), 0.01(\mathrm{~s}, 3 \mathrm{H}) ;{ }^{13} \mathrm{C} \mathrm{NMR}\left(150 \mathrm{MHz}, \mathrm{CD}_{3} \mathrm{OD}\right) \delta 163.2$,

161.0, 156.8, 145.7, 130.5, 115.6, 115.3, 114.3, 105.1, 100.0, 76.6, 72.5, 71.6, 68.6, 64.4,

26.4 (3C), 19.1, -5.0, -5.1; CIHRMS Calcd for $\left[\mathrm{C}_{21} \mathrm{H}_{30} \mathrm{O}_{8} \mathrm{Si}+\mathrm{Na}\right]^{+}$: 461.1602. Found 461.1578 .

7-[6-(tert-Butyl-dimethyl-silanyloxymethyl)-5-oxo-5,6-dihydro-4-methyl-2H-pyran-

2-yloxy]-chromen-2-one (I-27).

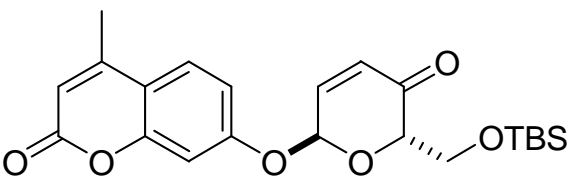

A THF ( $0.3 \mathrm{~mL})$ solution of compound $\mathbf{I}-15$ (100 $\mathrm{mg}, 0.28 \mathrm{mmol})$ and 7-hydroxy cumarin (98.6 mg, $0.56 \mathrm{mmol})$ was cooled to $0{ }^{\circ} \mathrm{C}$. A THF $(0.3 \mathrm{~mL})$ solution of $\mathrm{Pd}_{2}(\mathrm{DBA})_{3} \mathrm{CHCl}_{3}$ (7.2 $\mathrm{mg}, 2.5 \mathrm{~mol} \%)$ and $\mathrm{PPh}_{3}(7.3 \mathrm{mg}, 10 \mathrm{~mol} \%)$ was added to the reaction mixture at 0 ${ }^{\circ} \mathrm{C}$. The reaction mixture was stirred at $0{ }^{\circ} \mathrm{C}$ for 1 hour. The reaction mixture was quenched with $2 \mathrm{~mL}$ of satd aq $\mathrm{NaHCO}_{3}$, extracted $(3 \times 5 \mathrm{~mL})$ with $\mathrm{Et}_{2} \mathrm{O}$, dried $\left(\mathrm{Na}_{2} \mathrm{SO}_{4}\right)$, and concentrated under reduced pressure. The crude product was purified using silica gel flash chromatography eluting with $15 \%$ EtOAc/hexanes to give $98 \mathrm{mg}$ $(0.23 \mathrm{mmol}, 82 \%)$ of I-27: $R_{f}(30 \%$ EtOAc/hexanes $)=0.50 ;[\alpha]^{21}{ }_{\mathrm{D}}=-75.4(\mathrm{c}=0.7$, $\mathrm{CH}_{2} \mathrm{Cl}_{2}$ ); IR (thin film, $\mathrm{cm}^{-1}$ ) 2928, 1726, 1700, 1611, 1262, 1134, 834; ${ }^{1} \mathrm{H}$ NMR (600 $\left.\mathrm{MHz}, \mathrm{CDCl}_{3}\right) \delta 7.52(\mathrm{~d}, J=2.4 \mathrm{~Hz}, 1 \mathrm{H}), 7.13(\mathrm{~d}, J=2.4 \mathrm{~Hz}, 1 \mathrm{H}), 7.07(\mathrm{dd}, J=9.0,2.4$ Hz, 1H), $7.02(\mathrm{dd}, J=9.6,3.6 \mathrm{~Hz}, 1 \mathrm{H}), 6.28(\mathrm{~d}, J=10.8 \mathrm{~Hz}, 1 \mathrm{H}), 6.17(\mathrm{~d}, J=1.2 \mathrm{~Hz}$, $1 \mathrm{H}), 6.10(\mathrm{~d}, J=3.6 \mathrm{~Hz}, 1 \mathrm{H}), 4.50(\mathrm{dd}, J=4.8,2.4 \mathrm{~Hz}, 1 \mathrm{H}), 4.05(\mathrm{dd}, J=11.4,4.8 \mathrm{~Hz}$, $1 \mathrm{H}), 4.02(\mathrm{dd}, J=12.0,3.0 \mathrm{~Hz}, 1 \mathrm{H}), 2.40(\mathrm{~d}, J=1.2 \mathrm{~Hz}, 3 \mathrm{H}), 0.81(\mathrm{~s}, 9 \mathrm{H}), 0.04(\mathrm{~s}, 3 \mathrm{H})$, 
$0.03(\mathrm{~s}, 3 \mathrm{H}) ;{ }^{13} \mathrm{C}$ NMR $\left(67.5 \mathrm{MHz}, \mathrm{CDCl}_{3}\right) \delta 193.8,160.9,159.6,154.8,152.1,141.7$, 129.2(2C), 125.6, 115.1, 113.5, 112.9, 104.4, 91.7, 62.5, 25.7(3C), 18.6, 18.2, -5.4, -5.5; CIHRMS Calcd for $\left[\mathrm{C}_{22} \mathrm{H}_{28} \mathrm{O}_{6} \mathrm{Si}+\mathrm{Na}\right]^{+}:$439.1547. Found 439.1535.

7-[6-(tert-Butyl-dimethyl-silanyloxymethyl)-5-oxo-5,6-dihydro-4-methyl2H-pyran-2yloxy]-chromen-2-one (I-28).

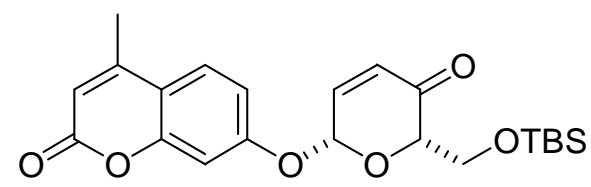

A THF (0.3 mL) solution of compound I-16 (100 mg, $0.28 \mathrm{mmol})$ and 7-hydroxy cumarin (98.6 mg, $0.56 \mathrm{mmol})$ was cooled to $0{ }^{\circ} \mathrm{C}$. A THF $(0.3 \mathrm{~mL})$ solution of $\mathrm{Pd}_{2}(\mathrm{DBA})_{3}{ }_{3} \mathrm{CHCl}_{3}$ (7.2 $\mathrm{mg}, 2.5 \mathrm{~mol} \%)$ and $\mathrm{PPh}_{3}(7.3 \mathrm{mg}, 10 \mathrm{~mol} \%)$ was added to the reaction mixture at 0 ${ }^{\circ} \mathrm{C}$. The reaction mixture was stirred at $0{ }^{\circ} \mathrm{C}$ for 1 hour. The reaction mixture was quenched with $2 \mathrm{~mL}$ of satd aq $\mathrm{NaHCO}_{3}$, extracted $(3 \times 5 \mathrm{~mL})$ with $\mathrm{Et}_{2} \mathrm{O}$, dried $\left(\mathrm{Na}_{2} \mathrm{SO}_{4}\right)$, and concentrated under reduced pressure. The crude product was purified using silica gel flash chromatography eluting with 20-25\% EtOAc/hexanes to give $73 \mathrm{mg}$ $(0.17 \mathrm{mmol}, 61 \%)$ of I-28: $R_{f}(30 \%$ EtOAc/hexanes $)=0.40 ;[\alpha]^{21}{ }_{\mathrm{D}}=-49(\mathrm{c}=0.7$, $\mathrm{CH}_{2} \mathrm{Cl}_{2}$ ); IR (thin film, $\mathrm{cm}^{-1}$ ) 2930, 1703, 1732, 1614, 1256, 1118, 836; ${ }^{1} \mathrm{H}$ NMR (600 $\left.\mathrm{MHz}, \mathrm{CDCl}_{3}\right) \delta 7.61(\mathrm{~d}, J=9.0 \mathrm{~Hz}, 1 \mathrm{H}), 7.01(\mathrm{dd}, J=10.2,3.0 \mathrm{~Hz}, 1 \mathrm{H}), 6.84(\mathrm{dd}, J=$ 9.0, 2.4 Hz, 1H), $7.72(\mathrm{~d}, J=3.0 \mathrm{~Hz}, 1 \mathrm{H}), 6.12(\mathrm{dd}, J=1.2,1.2 \mathrm{~Hz}, 1 \mathrm{H}), 6.09(\mathrm{~d} J=10.8$ $\mathrm{Hz}, 1 \mathrm{H}), 5.24(\mathrm{dd}, J=3.6,1.2 \mathrm{~Hz}, 1 \mathrm{H}), 4.42(\mathrm{dd}, J=4.8,2.4 \mathrm{~Hz}, 1 \mathrm{H}), 4.08(\mathrm{dd}, J=10.8$, $4.8 \mathrm{~Hz}, 1 \mathrm{H}), 4.02(\mathrm{dd}, J=11.4,2.4 \mathrm{~Hz}, 1 \mathrm{H}), 2.44(\mathrm{~d}, J=1.2 \mathrm{~Hz}, 3 \mathrm{H}), 0.90(\mathrm{~s}, 9 \mathrm{H}), 0.09$ (s, 3H), 0.08 (s, 3H); ${ }^{13} \mathrm{C}$ NMR (150 MHz, $\left.\mathrm{CDCl}_{3}\right) \delta$ 196.6, 163.8, 162.9, 156.5, 155.9, 
146.1, 128.6, 127.4, 114.3, 113.8, 111.2, 103.4, 95.4, 77.1, 63.8, 26.3 (3C), 19.1, 18.6, 5.2, -5.3; CIHRMS Calcd for $\left[\mathrm{C}_{22} \mathrm{H}_{28} \mathrm{O}_{6} \mathrm{Si}+\mathrm{Na}\right]^{+}$: 439.1547. Found 439.1554 .

\section{7-[6-(tert-Butyl-dimethyl-silanyloxymethyl)-5-hydroxy-5,6-dihydro-4-methyl-2H-} pyran-2-yloxy]-chromen-2-one (I-41b).

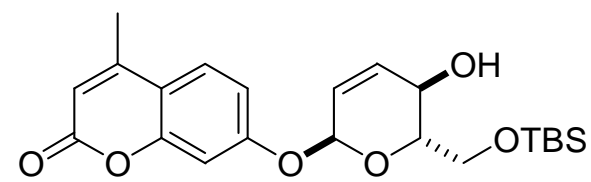

A THF $(0.2 \mathrm{~mL})$ solution of compound $\mathbf{I}-28(100 \mathrm{mg}, 0.23 \mathrm{mmol})$ and $\mathrm{MeOH}(0.2 \mathrm{~mL})$ was cooled to $-78{ }^{\circ} \mathrm{C} . \mathrm{NaBH}_{4}(8.70 \mathrm{mg}, 0.23 \mathrm{mmol})$ was added and the reaction mixture was stirred at $-78^{\circ} \mathrm{C}$ for 3 hours. The reaction mixture was diluted with ether $(5 \mathrm{~mL})$ and was quenched with $5 \mathrm{~mL}$ of satd aq $\mathrm{NaHCO}_{3}$, extracted $(2 \times 5 \mathrm{~mL})$ with $\mathrm{Et}_{2} \mathrm{O}$, dried $\left(\mathrm{Na}_{2} \mathrm{SO}_{4}\right)$, and concentrated under reduced pressure. The crude product was purified using silica gel flash chromatography eluting with 50\% EtOAc/hexanes to give $73 \mathrm{mg}$ $(0.17 \mathrm{mmol}, 73.0 \%)$ of $\mathbf{I}-41 \mathbf{b}: R_{f}(30 \%$ EtOAc/hexanes $)=0.40 ;[\alpha]^{21}{ }_{\mathrm{D}}=-110.2 \quad(\mathrm{c}=1$, $\mathrm{CH}_{3} \mathrm{OH}$ ); IR (thin film, $\left.\mathrm{cm}^{-1}\right)$ 3425, 2926, 1710, 1611, 1121, 835; ${ }^{1} \mathrm{H}$ NMR (600 MHz, $\left.\mathrm{CDCl}_{3}\right) \delta 7.50(\mathrm{~d}, J=8.4 \mathrm{~Hz}, 1 \mathrm{H}), 7.07(\mathrm{~d}, J=2.4 \mathrm{~Hz}, 1 \mathrm{H}), 6.99(\mathrm{dd}, J=9.0,2.4 \mathrm{~Hz}$, 1H), $6.14(\mathrm{~m}, 2 \mathrm{H}), 5.89$ (ddd, $J=10.2,2.4,2.4 \mathrm{~Hz}, 1 \mathrm{H}), 5.70(\mathrm{~m}, 1 \mathrm{H}), 4.31$ (dd, $J=8.4$, $1.2 \mathrm{~Hz}, 1 \mathrm{H}$ ), $3.88(\mathrm{dd}, J=9.6,4.8 \mathrm{~Hz}, 1 \mathrm{H}), 3.81(\mathrm{ddd}, J=7.2,4.8,4.8 \mathrm{~Hz}, 1 \mathrm{H}), 3.75$ (dd, $J=9.6,7.2 \mathrm{~Hz}, 1 \mathrm{H}), 3.03(\mathrm{~d}, J=3.0 \mathrm{~Hz}, 1 \mathrm{H}) 2.04(\mathrm{~d}, J=0.6 \mathrm{~Hz}, 3 \mathrm{H}), 0.87(\mathrm{~s}, 9 \mathrm{H})$, 0.09 (s, 3H), $0.06(\mathrm{~s}, 3 \mathrm{H}) ;{ }^{13} \mathrm{C}$ NMR $\left(67.5 \mathrm{MHz}, \mathrm{CDCl}_{3}\right) \delta 161.2,160.1,154.9,152.4$ $134.4,125.4,123.9,114.5,113.7,112.4,104.1,92.5,70.8,66.8,65.2,25.7(3 \mathrm{C}), 18.7$, 18.1, -5.5, -5.6; CIHRMS Calcd for $\left[\mathrm{C}_{22} \mathrm{H}_{30} \mathrm{O}_{6} \mathrm{Si}+\mathrm{Na}\right]^{+}$: 441.1703. Found 441.1725. 


\section{7-[6-(tert-Butyl-dimethyl-silanyloxymethyl)-5-oxo-5,6-dihydro-4-methyl-2H-pyran-}

\section{2-yloxy]- chromen-2-one (I-42b).}

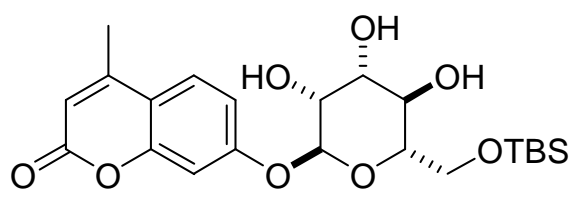

To a $t$-butanol, acetone $(0.4 \mathrm{~mL}, 1: 1,1 \mathrm{M})$ solution of allyl alcohol I-41b $(160 \mathrm{mg}, 0.373$ mmol) at $0{ }^{\circ} \mathrm{C}$ was added a solution of $(50 \% \mathrm{w} / \mathrm{v})$ of $\mathrm{N}$-methyl morpholine $\mathrm{N}$-oxide / water $(0.2 \mathrm{~mL})$. Crystalline $\mathrm{OsO}_{4}(1.0 \mathrm{mg}, 1 \mathrm{~mol} \%)$ was added and the reaction was stirred for $12 \mathrm{~h}$. The reaction mixture was concentrated and was pipetted directly on to a silica gel column $\left(1 \mathrm{~cm} \times 4\right.$ ") using a small amount of $\mathrm{CH}_{2} \mathrm{Cl}_{2}(0.6 \mathrm{~mL})$ in three portions. Impurities were eluted with ether and the product was eluted with $\mathrm{MeOH} /$ ether (2:98 to 4:96). Pure fractions were combined and concentrated to afford triol I-42b $145 \mathrm{mg}$ $(0.329 \mathrm{mmol}, 84 \%): R_{f} \quad(50: 49: 1 \mathrm{EtOAc} /$ hexanes$/ \mathrm{methanol})=0.35 ;[\alpha]^{21}{ }_{\mathrm{D}}=-84(\mathrm{c}=2$ $\mathrm{CH}_{2} \mathrm{Cl}_{2}$ ); IR (thin film, $\mathrm{cm}^{-1}$ ) 3406, 2927, 1729, 1613, 1068, 836; ${ }^{1} \mathrm{H}$ NMR $(600 \mathrm{MHz}$, $\left.\mathrm{CDCl}_{3}\right) \delta 7.52(\mathrm{~d}, J=9.0 \mathrm{~Hz}, 1 \mathrm{H}), 7.05(\mathrm{~d}, J=2.4 \mathrm{~Hz}, 1 \mathrm{H}), 6.99(\mathrm{dd}, J=8.4,2.4 \mathrm{~Hz}$, $1 \mathrm{H}), 6.18(\mathrm{~d}, J=1.2 \mathrm{~Hz}, 1 \mathrm{H}), 5.62(\mathrm{~d}, J=1.2 \mathrm{~Hz}, 1 \mathrm{H}), 4.17(\mathrm{~d}, J=2.4 \mathrm{~Hz}, 1 \mathrm{H}), 4.07$ (ddd, $J=9.0,3.6,2.4 \mathrm{~Hz}, 1 \mathrm{H}), 3.94(\mathrm{dd}, J=9.6,9.0 \mathrm{~Hz}, 1 \mathrm{H}), 3.87$ (dd, $J=10.2,4.8$ $\mathrm{Hz}, 1 \mathrm{H}), 3.79(\mathrm{dd}, J=10.2,6.6 \mathrm{~Hz}, 1 \mathrm{H}), 3.65(\mathrm{dddd}, J=10.8,6.6,4.8,4.8 \mathrm{~Hz}, 1 \mathrm{H})$, $3.36(\mathrm{~s}, ? \mathrm{H}), 2.79$ (d, $J=3.6 \mathrm{~Hz}, 1 \mathrm{H}), 2.67(\mathrm{~d}, J=3.0 \mathrm{~Hz}, 1 \mathrm{H}), 2.40$ (d, $J=0.6 \mathrm{~Hz}, 3 \mathrm{H})$, $0.86(\mathrm{~s}, 9 \mathrm{H}), 0.07(\mathrm{~s}, 3 \mathrm{H}), 0.05(\mathrm{~s}, 3 \mathrm{H}) ;{ }^{13} \mathrm{C} \mathrm{NMR}\left(150 \mathrm{MHz}, \mathrm{CDCl}_{3}\right) \delta 161.1,158.7$ $154.8,152.4,125.6,114.8,113.3,112.7,104.0,97.6,76.5,71.2,71.1,69.7,64.6,25.7$ (3C), 18.6, 18.1, -5.5, -5.6 ; CIHRMS Calcd for $\left[\mathrm{C}_{22} \mathrm{H}_{32} \mathrm{O}_{8} \mathrm{Si}+\mathrm{Na}\right]^{+}$: 475.1758. Found 475.1778 . 


\section{Methyl-7-((2S,6S)-6-((tert-butyldimethylsilyloxy)methyl)-5-oxo-5,6-dihydro-2H-}

pyran-2-yloxy)-2-oxo-2H-chromene-4-carboxylate (I-30).

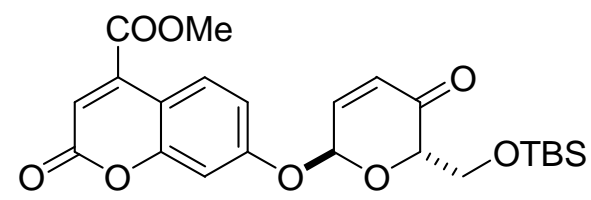

A THF (2.0 mL) solution of compound I-15 (400 mg, $1.11 \mathrm{mmol})$ and 7-hydroxy cumarin (492.0 mg, $2.22 \mathrm{mmol})$ was cooled to $0{ }^{\circ} \mathrm{C}$. A THF $(1.0 \mathrm{~mL})$ solution of $\mathrm{Pd}_{2}(\mathrm{DBA})_{3^{-}}$ $\mathrm{CHCl}_{3}(28.7 \mathrm{mg}, 2.5 \mathrm{~mol} \%)$ and $\mathrm{PPh}_{3}(29.0 \mathrm{mg}, 10 \mathrm{~mol} \%)$ was added to the reaction mixture at $0{ }^{\circ} \mathrm{C}$. The reaction mixture was stirred at $0{ }^{\circ} \mathrm{C}$ for 1 hour. The reaction mixture was quenched with $5 \mathrm{~mL}$ of satd aq $\mathrm{NaHCO}_{3}$, extracted $(3 \times 10 \mathrm{~mL})$ with $\mathrm{Et}_{2} \mathrm{O}$, dried $\left(\mathrm{Na}_{2} \mathrm{SO}_{4}\right)$, and concentrated under reduced pressure. The crude product was purified using silica gel flash chromatography eluting with 30-50\% EtOAc/hexanes to give $300 \mathrm{mg}(0.64 \mathrm{mmol}, 57 \%)$ of I-30: $R_{f}(20 \%$ EtOAc/hexanes $)=0.37 ;[\alpha]^{21}{ }_{\mathrm{D}}=-68.1$ $\left(\mathrm{c}=0.7, \mathrm{CH}_{2} \mathrm{Cl}_{2}\right.$ ); IR (thin film, $\mathrm{cm}^{-1}$ ) $2927,1737,1703,1613,1263,1135,837 ;{ }^{1} \mathrm{H} \mathrm{NMR}$ $\left(600 \mathrm{MHz}, \mathrm{CD}_{3} \mathrm{OD}\right) \delta 7.59(\mathrm{~d}, J=9.0 \mathrm{~Hz}, 1 \mathrm{H}), 7.14(\mathrm{~d}, J=3.0 \mathrm{~Hz}, 1 \mathrm{H}), 7.08(\mathrm{dd}, J=$ 9.6, $2.4 \mathrm{~Hz}, 1 \mathrm{H}), 6.26(\mathrm{~d}, J=1.8 \mathrm{~Hz} 1 \mathrm{H}), 6.23(\mathrm{~d}, J=7.2 \mathrm{~Hz}, 1 \mathrm{H}), 6.21(\mathrm{~s}, 1 \mathrm{H}), 4.47$ (dd, $J=4.8,2.4 \mathrm{~Hz}, 1 \mathrm{H}), 3.99(\mathrm{~m}, 1 \mathrm{H}), 3.98(\mathrm{dd}, J=12,4.2 \mathrm{~Hz}, 1 \mathrm{H}), 3.94(\mathrm{dd}, J=12.0$, $3.0 \mathrm{~Hz}, 1 \mathrm{H}), 3.66(\mathrm{~s}, 3 \mathrm{H}), 0.75(\mathrm{~s}, 9 \mathrm{H}),-0.01(\mathrm{~s}, 3 \mathrm{H}),-0.02(\mathrm{~s}, 3 \mathrm{H}) ;{ }^{13} \mathrm{C} \mathrm{NMR}(150 \mathrm{MHz}$, $\left.\mathrm{CD}_{3} \mathrm{OD}\right) \delta 195.5,171.1,162.6,161.4,150.9,143.9,129.7,127.7,127.5,115.3,115.2$, 105.4, 92.4, 78.0, 63.5, 53.0, 38.1, 26.3 (3C), 19.0, -5.2, -5.3; CIHRMS Calcd for $\left[\mathrm{C}_{23} \mathrm{H}_{28} \mathrm{O}_{8} \mathrm{SiCH}_{2} \mathrm{Na}\right]^{+}$: 497.1602. Found 497.1621.

\section{Methyl-7-((2R,6S)-6-((tert-butyldimethylsilyloxy)methyl)-5-oxo-5,6-dihydro-2H-} pyran-2-yloxy)-2-oxo-2H-chromene-4-carboxylate (I-31). 


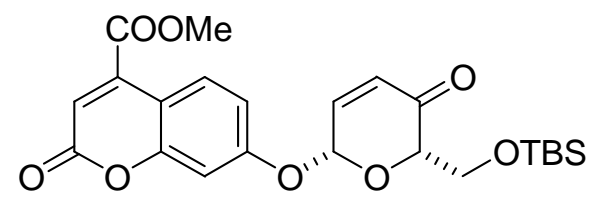

A THF (0.1 mL) solution of compound I-16 (30 mg, $0.096 \mathrm{mmol})$ and $\mathrm{MeOH}(0.1 \mathrm{~mL})$ was cooled to $-78{ }^{\circ} \mathrm{C}$. $\mathrm{NaBH}_{4}(3.36 \mathrm{mg}, 0.096 \mathrm{mmol})$ was added and the reaction mixture was stirred at $-78^{\circ} \mathrm{C}$ for 3 hours. The reaction mixture was diluted with ether $(3 \mathrm{~mL})$ and was quenched with $2 \mathrm{~mL}$ of satd aq $\mathrm{NaHCO}_{3}$, extracted $(2 \times 5 \mathrm{~mL})$ with $\mathrm{Et}_{2} \mathrm{O}$, dried $\left(\mathrm{Na}_{2} \mathrm{SO}_{4}\right)$, and concentrated under reduced pressure. The crude product was purified using silica gel flash chromatography eluting with 100\% EtOAc/hexanes to give $39 \mathrm{mg}$ $(0.083 \mathrm{mmol}, 85 \%)$ of I-31: $R_{f}(30 \%$ EtOAc/hexanes $)=0.31 ;[\alpha]^{21}{ }_{\mathrm{D}}=+10, C 1$, $\mathrm{CH}_{2} \mathrm{Cl}_{2}$ ); IR (thin film, $\mathrm{cm}^{-1}$ ) 3384, 3034, 2975, 1656, 1634, 1047, 879; ${ }^{1} \mathrm{H}$ NMR (600 $\left.\mathrm{MHz}, \mathrm{CDCl}_{3}\right) \quad 7.56(\mathrm{~d}, J=9.6 \mathrm{~Hz}, 1 \mathrm{H}), 7.09(\mathrm{dd}, J=10.8,1.8 \mathrm{~Hz}, 1 \mathrm{H}), 6.96(\mathrm{~d}, J=1.8$ $\mathrm{Hz}, 1 \mathrm{H}), 6.85$ (dd, $J=9.0,2.4 \mathrm{~Hz}, 1 \mathrm{H}), 6.47$ (br s, 1H), 6.21 (ddd, $J=10.2,8.4,1.8 \mathrm{~Hz}$, 1H), $5.05(\mathrm{~m}, 1 \mathrm{H}), 4.32(\mathrm{~d}, J=9.0 \mathrm{~Hz}, 1 \mathrm{H}), 4.00(\mathrm{dd}, J=11.4,2.4 \mathrm{~Hz}, 1 \mathrm{H}), 3.83(\mathrm{dd}, J=$ 11.4, 6.6 Hz, 1H), 3.75 (s, 3H), 0.76 (s, 9H), -0.09 (s, 3H), -0.11 (s, 3H); ${ }^{13} \mathrm{C} \mathrm{NMR}(150$ $\left.\mathrm{MHz}, \mathrm{CDCl}_{3}\right) \delta 193.5,169.2,160.7,155.2,149.5,147.5,128.4,125.5,113.8,117.7$, 103.5, 82.2, 73.8, 62.1, 53.1, 50.0, 37.9, 25.6 (3C), 18.1, -5.63, -5.65; CIHRMS Calcd for $\left[\mathrm{C}_{23} \mathrm{H}_{28} \mathrm{O}_{8} \mathrm{SiCH}_{2} \mathrm{Na}\right]^{+}:$497.1602. Found 497.1618

\section{7-((2S,6S)-6-((tert-butyldimethylsilyloxy)methyl)-5-oxo-5,6-dihydro-2H-pyran-2-}

\section{yloxy)-2-phenyl-4H-chromen-4-one (I-33).}<smiles>O=C1C=CC(Oc2ccc3c(=O)cc(-c4ccccc4)oc3c2)O[C@@H]1C[OH2+]</smiles> 
A THF (0.3 mL) solution of compound I-15 (100 mg, $0.28 \mathrm{mmol})$ and 6-hydroxy flavone (133.3 mg, $0.56 \mathrm{mmol})$ was cooled to $0{ }^{\circ} \mathrm{C}$. A THF $(0.3 \mathrm{~mL})$ solution of $\mathrm{Pd}_{2}(\mathrm{DBA})_{3^{-}}$ $\mathrm{CHCl}_{3}(7.2 \mathrm{mg}, 2.5 \mathrm{~mol} \%)$ and $\mathrm{PPh}_{3}(7.3 \mathrm{mg}, 10 \mathrm{~mol} \%)$ was added to the reaction mixture at $0{ }^{\circ} \mathrm{C}$. The reaction mixture was stirred at $0{ }^{\circ} \mathrm{C}$ for 1 hour. The reaction mixture was quenched with $2 \mathrm{~mL}$ of satd aq $\mathrm{NaHCO}_{3}$, extracted $(3 \times 5 \mathrm{~mL})$ with $\mathrm{Et}_{2} \mathrm{O}$, dried $\left(\mathrm{Na}_{2} \mathrm{SO}_{4}\right)$, and concentrated under reduced pressure. The crude product was purified using silica gel flash chromatography eluting with $20 \%$ EtOAc/hexanes to give $120.6 \mathrm{mg}(0.25 \mathrm{mmol}, 90 \%)$ of I-33: $R_{f}(30 \%$ EtOAc/hexanes $)=0.66 ;[\alpha]^{21}{ }_{\mathrm{D}}=-95.1(\mathrm{c}$ $=0.7, \mathrm{CH}_{2} \mathrm{Cl}_{2}$ ); IR (thin film, $\mathrm{cm}^{-1}$ ) 2927, 1701, 1637, 1455, 1255, 1129, 835; ${ }^{1} \mathrm{H} \mathrm{NMR}$ $\left(600 \mathrm{MHz}, \mathrm{CDCl}_{3}\right) \delta 7.95(\mathrm{~d}, J=3.0 \mathrm{~Hz}, 1 \mathrm{H}), 7.91-7.93(\mathrm{~m}, 2 \mathrm{H}), 7.54-7.50(\mathrm{~m}, 4 \mathrm{H}), 7.47$ $(\mathrm{dd}, J=9.0,3.0 \mathrm{~Hz}, 1 \mathrm{H}), 7.04(\mathrm{dd}, J=10.2,3.6 \mathrm{~Hz}, 1 \mathrm{H}), 6.80(\mathrm{~s}, 1 \mathrm{H}), 6.28(\mathrm{~d}, J=10.2$ $\mathrm{Hz}, 1 \mathrm{H}), 6.11(\mathrm{~d}, J=3.6 \mathrm{~Hz}, 1 \mathrm{H}), 4.56(\mathrm{dd}, J=4.2,3.0 \mathrm{~Hz}, 1 \mathrm{H}), 4.06-4.05(\mathrm{~m}, 2 \mathrm{H}), 0.82$ (s, 9H), $0.06(\mathrm{~s}, 3 \mathrm{H}), 0.03(\mathrm{~s}, 3 \mathrm{H}) ;{ }^{13} \mathrm{C} \mathrm{NMR}\left(67.5 \mathrm{MHz}, \mathrm{CDCl}_{3}\right) \delta$ 194.1, 177.8, 163.2, 154.0, 151.9, 142.2, 131.7, 131.5, 129.0(3C), 126.2(2C), 124.7, 124.5(2C), 119.5, 110.8, 106.8, 92.2, 62.6, 25.7(3C), 18.2, -5.4, -5.5; CIHRMS Calcd for $\left[\mathrm{C}_{27} \mathrm{H}_{30} \mathrm{O}_{6} \mathrm{Si}+\mathrm{Na}\right]^{+}$: 501.1703. Found 501.1680.

\section{7-((2S,5R,6S)-6-((tert-butyldimethylsilyloxy)methyl)-5-hydroxy-5,6-dihydro-2H-} pyran-2-yloxy)-2-phenyl-4H-chromen-4-one (I-41c).<smiles>O=c1cc(-c2ccccc2)oc2cc(OC3C=CC(O)[C@H](C[OH2+])O3)ccc12</smiles> 
A THF $(0.2 \mathrm{~mL})$ solution of compound $\mathbf{I}-33(100 \mathrm{mg}, 0.20 \mathrm{mmol})$ and $\mathrm{MeOH}(0.2 \mathrm{~mL})$ was cooled to $-78{ }^{\circ} \mathrm{C}$. $\mathrm{NaBH}_{4}(7.90 \mathrm{mg}, 0.20 \mathrm{mmol})$ was added and the reaction mixture was stirred at $-78^{\circ} \mathrm{C}$ for 3 hours. The reaction mixture was diluted with ether $(5 \mathrm{~mL})$ and was quenched with $5 \mathrm{~mL}$ of satd aq $\mathrm{NaHCO}_{3}$, extracted $(2 \times 5 \mathrm{~mL})$ with $\mathrm{Et}_{2} \mathrm{O}$, dried $\left(\mathrm{Na}_{2} \mathrm{SO}_{4}\right)$, and concentrated under reduced pressure. The crude product was purified using silica gel flash chromatography eluting with $30 \%$ EtOAc/hexanes to give $73 \mathrm{mg}$ $(0.15 \mathrm{mmol}, 76.0 \%)$ of I-41c: $R_{f}(30 \%$ EtOAc/hexanes $)=0.44 ;[\alpha]^{21}=-113.10 \quad(\mathrm{c}=1$, $\mathrm{CH}_{3} \mathrm{OH}$ ); IR (thin film, $\mathrm{cm}^{-1}$ ) 3346, 2956, 1738, 1623, 1073, 831; ${ }^{1} \mathrm{H}$ NMR $(600 \mathrm{MHz}$, $\left.\mathrm{CDCl}_{3}\right) \delta 7.92-7.91(\mathrm{~m}, 2 \mathrm{H}), 7.86(\mathrm{~d}, J=3.0 \mathrm{~Hz}, 1 \mathrm{H}), 7.23-7.50(\mathrm{~m}, 3 \mathrm{H}), 7.40(\mathrm{dd}, J=$ 9.6, 3.0 Hz, 1H), 6.80 (s, 1H), 6.12 (dddd, $J=10.2,3.0,1.2,1.2 \mathrm{~Hz}, 1 \mathrm{H}), 5.91$ (dddd, $J=$ $10.2,4.8,2.4,2.4 \mathrm{~Hz}, 1 \mathrm{H}), 5.74(\mathrm{dd}, J=2.4,1.8 \mathrm{~Hz}, 1 \mathrm{H}), 4.31-4.29(\mathrm{~m}, 1 \mathrm{H}), 4.11$ (dd, $J$ $=14.4,7.2 \mathrm{~Hz}, 1 \mathrm{H}), 3.89(\mathrm{ddd}, J=9.0,5.4,5.4 \mathrm{~Hz}, 1 \mathrm{H}), 3.86(\mathrm{dd}, J=9.0,4.8 \mathrm{~Hz}, 1 \mathrm{H})$, $3.78(\mathrm{dd}, J=9.0,6.6 \mathrm{~Hz}, 1 \mathrm{H}) 3.17(\mathrm{~d}, J=3.0 \mathrm{~Hz}, 1 \mathrm{H}), 0.87(\mathrm{~s}, 9 \mathrm{H}), 0.09(\mathrm{~s}, 3 \mathrm{H}), 0.06$ $(\mathrm{s}, 3 \mathrm{H}) ;{ }^{13} \mathrm{C} \mathrm{NMR}\left(150 \mathrm{MHz}, \mathrm{CDCl}_{3}\right) \delta 178.1,168.2,163.2,1159.5,154.5,151.7,134.1$, $131.8,131.5,129.0,126.2,124.8,124.6,124.4,119.3,110.2,106.8,93.0,70.8,66.9$, 65.3, 25.7(3C), 18.1, -5.5, -5.6; CIHRMS Calcd for $\left[\mathrm{C}_{27} \mathrm{H}_{32} \mathrm{O}_{6} \mathrm{Si}+\mathrm{Na}\right]^{+}:$503.1860. Found 503.1873 .

7-((2S,3R,4R,5R,6S)-6-((tert-butyldimethylsilyloxy)methyl)-3,4,5-trihydroxytetrahydro-2H-pyran-2-yloxy)-2-phenyl-4H-chromen-4-one (I-42c).<smiles>CC(C)[O+]C[C@H]1OC(Oc2ccc3c(=O)cc(-c4ccccc4)oc3c2)[C@H](O)[C@@H](O)C1O</smiles> 
To a $t$-butanol, acetone $(0.4 \mathrm{~mL}, 1: 1,1 \mathrm{M})$ solution of allyl alcohol I-41c $(160 \mathrm{mg}, 0.333$ mmol) at $0{ }^{\circ} \mathrm{C}$ was added a solution of $(50 \% \mathrm{w} / \mathrm{v})$ of $\mathrm{N}$-methyl morpholine $\mathrm{N}$-oxide / water $(0.2 \mathrm{~mL})$. Crystalline $\mathrm{OsO}_{4}(1.0 \mathrm{mg}, 1 \mathrm{~mol} \%)$ was added and the reaction was stirred for $12 \mathrm{~h}$. The reaction mixture was concentrated and was pipetted directly on to a silica gel column $(1 \mathrm{~cm} \times 4$ ") $)$ using a small amount of $\mathrm{CH}_{2} \mathrm{Cl}_{2}(0.6 \mathrm{~mL})$ in three portions. Impurities were eluted with ether and the product was eluted with $\mathrm{MeOH} / \mathrm{ether}(2: 98$ to 4:96). Pure fractions were combined and concentrated to afford triol I-42c $144 \mathrm{mg}$ $(0.279 \mathrm{mmol}, 84 \%): R_{f}(50: 40: 10 \mathrm{EtOAc} / \mathrm{hexanes} / \mathrm{methanol})=0.24 ;[\alpha]^{21}{ }_{\mathrm{D}}=-96.4(\mathrm{c}=$ 2, $\mathrm{CH}_{2} \mathrm{Cl}_{2}$ ); IR (thin film, $\mathrm{cm}^{-1}$ ) 3332, 2947, 1739, 1366, 1120, 978; ${ }^{1} \mathrm{H}$ NMR $(600 \mathrm{MHz}$, $\left.\mathrm{CDCl}_{3}\right) \delta 7.92-7.90(\mathrm{~m}, 2 \mathrm{H}), 7.81(\mathrm{~d}, J=2.4 \mathrm{~Hz}, 1 \mathrm{H}), 7.53-7.51(\mathrm{~m}, 4 \mathrm{H}), 7.41(\mathrm{dd}, J=$ 9.6, 3.0 Hz, 1H), $6.80(\mathrm{~s}, 1 \mathrm{H}), 5.64(\mathrm{~d}, J=1.2 \mathrm{~Hz}, 1 \mathrm{H}), 4.11(\mathrm{dd}, J=3.0,1.8 \mathrm{~Hz}, 1 \mathrm{H})$, $4.00(\mathrm{dd}, J=9.0,3.6 \mathrm{~Hz}, 1 \mathrm{H}), 3.86(\mathrm{dd}, J=6.0,3.0 \mathrm{~Hz}, 1 \mathrm{H}), 3.84(\mathrm{dd}, J=5.4,2.4 \mathrm{~Hz}$, 1H), $3.80(\mathrm{dd}, J=10.8,5.4 \mathrm{~Hz}, 1 \mathrm{H}), 3.65(\mathrm{ddd}, J=9.6,4.8,4.8 \mathrm{~Hz}, 1 \mathrm{H}), 1.80(\mathrm{~d}, 3 \mathrm{H})$, $0.83(\mathrm{~s}, 9 \mathrm{H}), 0.05(\mathrm{~s}, 3 \mathrm{H}), 0.03(\mathrm{~s}, 3 \mathrm{H}) ;{ }^{13} \mathrm{C} \mathrm{NMR}\left(150 \mathrm{MHz}, \mathrm{CDCl}_{3}\right) \delta$ 179.2, 164.6, $154.1,152.2,132.0,131.7,129.2(2 \mathrm{C}), 126.5(2 \mathrm{C}), 125.1,124.3,119.9,109.9,105.9$, 99.0,75.4, 71.4, 70.6, 67.5, 63.3, 25.2 (3C), 18.0, -6.1, -6.2 ; CIHRMS Calcd for $\left[\mathrm{C}_{27} \mathrm{H}_{34} \mathrm{O}_{8} \mathrm{Si}+\mathrm{Na}\right]^{+}:$537.1915. Found 537.1894.

\section{7-((2S,5R,6S)-6-((tert-butyldimethylsilyloxy)methyl)-5-hydroxy-tetrahydro-2H-} pyran-2-yloxy)-2-phenyl-4H-chromen-4-one (I-43).<smiles>O=c1cc(-c2ccccc2)oc2cc(OC3CCC(O)[C@@H](C[OH2+])O3)ccc12</smiles> 
The allyl alcohol compound I-41c (70 mg, $0.15 \mathrm{mmol}), \mathrm{O}-\mathrm{NO}_{2} \mathrm{ArSO}_{2} \mathrm{NHNH}_{2}$ (236.5 mg, $1.16 \mathrm{mmol}$ ) were dissolved in $1.46 \mathrm{~mL}$ of $\mathrm{CH}_{2} \mathrm{Cl}_{2}$ in a round bottom flask and cooled $0{ }^{\circ} \mathrm{C}$ under nitrogen condition then triethylamine $(146.5 \mathrm{mg}, 1.5 \mathrm{mmol})$ was added and the reaction mixture was stirred at $0{ }^{\circ} \mathrm{C}$ for 12 hours and on completion monitored by TLC, reaction mixture is three portions. The crude product was purified using silica gel flash chromatography eluting with 30\% EtOAc/hexanes to give I-43 $56 \mathrm{mg}(0.11 \mathrm{mmol}$, $80 \%): R_{f}(30 \%$ EtOAc/Hexane $)=0.44 ;[\alpha]^{21}=-95.30\left(c 2, \mathrm{CH}_{2} \mathrm{Cl}_{2}\right) ;$ IR (thin film, $\mathrm{cm}^{-}$

1) $2985,1737,1372,1252,1097,846 ;{ }^{1} \mathrm{H}$ NMR $\left(600 \mathrm{MHz}, \mathrm{CDCl}_{3}\right) \delta 7.93(\mathrm{~m}, 3 \mathrm{H}), 7.54-$ $7.49(\mathrm{~m}, 5 \mathrm{H}), 6.82(\mathrm{~d}, J=1.8 \mathrm{~Hz}, 1 \mathrm{H}), 5.62(\mathrm{~d}, J=1.8 \mathrm{~Hz}, 1 \mathrm{H}), 3.80(\mathrm{dd}, J=10.2,4.2$ $\mathrm{Hz}, 1 \mathrm{H}), 3.73(\mathrm{dd}, J=16.8,7.8 \mathrm{~Hz}, 1 \mathrm{H}), 3.68(\mathrm{dd}, J=10.2,7.2 \mathrm{~Hz}, 1 \mathrm{H}), 3.66(\mathrm{dd}, J=$ 8.4, 4.2 Hz, 1H), $2.34(\mathrm{dd}, J=7.8,7.2 \mathrm{~Hz}, 1 \mathrm{H}), 2.08-1.97(\mathrm{~m}, 4 \mathrm{H}), 0.85(\mathrm{~s}, 9 \mathrm{H}), 0.06$ (s, $3 \mathrm{H}), 0.03(\mathrm{~s}, 3 \mathrm{H}) ;{ }^{13} \mathrm{C} \mathrm{NMR}\left(150 \mathrm{MHz}, \mathrm{CDCl}_{3}\right) \delta 180.5,165.7,155.8,153.1,133.1$, $133.0,132.9,130.4,127.6,126.3,125.5,124.9,120.9,110.8,107.1,106.8,77.0,66.6$, 64.4, 30.2, 28.1, 26.4(3C), 19.2, -5.0, -5.1; CIHRMS Calcd for $\left[\mathrm{C}_{27} \mathrm{H}_{34} \mathrm{O}_{6} \mathrm{SiNa}^{+}\right]$: 505.2016. Found 505.2025.

7-((2R,6S)-6-((tert-butyldimethylsilyloxy)methyl)-5-oxo-5,6-dihydro-2H-pyran-2yloxy)-2-phenyl-4H-chromen-4-one (I-33).<smiles>O=C1C=C[C@@H](Oc2ccc3c(=O)cc(-c4ccccc4)oc3c2)O[C@@H]1C[OH2+]</smiles>

A THF (0.2 mL) solution of compound I-15 (50 mg, $0.14 \mathrm{mmol})$ and 6-hydroxy flavone (65.3 mg, $0.28 \mathrm{mmol})$ was cooled to $0{ }^{\circ} \mathrm{C}$. A THF $(0.2 \mathrm{~mL})$ solution of $\operatorname{Pd}_{2}(\mathrm{DBA})_{3} \cdot \mathrm{CHCl}_{3}$ 
(3.6 mg, $2.5 \mathrm{~mol} \%$ ) and $\mathrm{PPh}_{3}(3.6 \mathrm{mg}, 10 \mathrm{~mol} \%$ ) was added to the reaction mixture at 0 ${ }^{\circ} \mathrm{C}$. The reaction mixture was stirred at $0{ }^{\circ} \mathrm{C}$ for 1 hour. The reaction mixture was quenched with $2 \mathrm{~mL}$ of satd aq $\mathrm{NaHCO}_{3}$, extracted $(3 \times 5 \mathrm{~mL})$ with $\mathrm{Et}_{2} \mathrm{O}$, dried $\left(\mathrm{Na}_{2} \mathrm{SO}_{4}\right)$, and concentrated under reduced pressure. The crude product was purified using silica gel flash chromatography eluting with 20\% EtOAc/hexanes to give $52.5 \mathrm{mg}$ $(0.11 \mathrm{mmol}, 80 \%)$ of I-33: $R_{f}(30 \%$ EtOAc/hexanes $)=0.60 ;[\alpha]^{21}{ }_{\mathrm{D}}=70.1(\mathrm{c}=1.0$, $\mathrm{CH}_{2} \mathrm{Cl}_{2}$ ); IR (thin film, $\mathrm{cm}^{-1}$ ) 2929, 1721, 1642, 1456, 1251, 1121, 839; ${ }^{1} \mathrm{H}$ NMR (600 $\left.\mathrm{MHz}, \mathrm{CD}_{3} \mathrm{OD}\right) \delta 8.04(\mathrm{~d}, J=1.8 \mathrm{~Hz}, 1 \mathrm{H}), 8.03(\mathrm{~d}, J=1.8 \mathrm{~Hz}, 1 \mathrm{H}), 7.91(\mathrm{~d}, J=3.0 \mathrm{~Hz}$, $1 \mathrm{H}), 7.71(\mathrm{~d}, J=9.0 \mathrm{~Hz}, 1 \mathrm{H}), 7.60-7.54(\mathrm{~m}, 5 \mathrm{H}), 7.19(\mathrm{dd}, J=10.2,3.6 \mathrm{~Hz}, 1 \mathrm{H}), 6.90(\mathrm{br}$ $\mathrm{S}, 1 \mathrm{H}), 6.25(\mathrm{dd}, J=12.6,10.2 \mathrm{~Hz}, 1 \mathrm{H}), 4.55(\mathrm{dd}, J=4.8,2.4 \mathrm{~Hz}, 1 \mathrm{H}), 4.05(\mathrm{dd}, J=$ 11.4, $4.8 \mathrm{~Hz}, 1 \mathrm{H}), 4.01(\mathrm{dd}, J=11.4,2.4 \mathrm{~Hz}, 1 \mathrm{H}), 0.78$ (s, 9H), 0.03 (s, 3H), 0.01 (s, 3H);

${ }^{13} \mathrm{C}$ NMR (150 MHz, $\left.\mathrm{CD}_{3} \mathrm{OD}\right) \delta 195.4,179.4,164.4,155.5,150.6,144.8,143.2,131.9$, 131.7 (2C), 129.1, 129.0 (2C), 127.5 (2C), 123.6, 119.5, 107.6, 105.5, 76.0, 62.6, 25.1 (3C), 17.9, -6.4 (2C); CIHRMS Calcd for $\left[\mathrm{C}_{27} \mathrm{H}_{30} \mathrm{O}_{6} \mathrm{SiNa}\right]^{+}$: 501.1703. Found 501.1700.

\section{(2S,6S)-6-(1'H-benzimidazol-1'-yl)-2-(tert-butyl-dimethyl-silanyloxymethyl)-2H-} pyran-3(6H)-one (I-58 $\beta)$ :

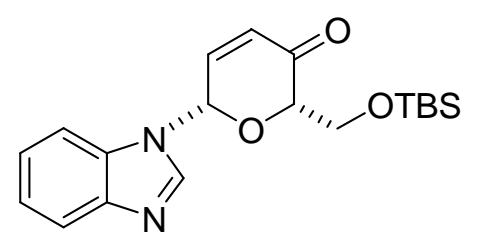

A $\mathrm{CH}_{2} \mathrm{Cl}_{2}(1.0 \mathrm{~mL})$ solution of pyranone $\mathbf{I - 1 6} \boldsymbol{\beta}(358 \mathrm{mg}, 1.00 \mathrm{mmol})$ and benzimadazole (141.7 mg, $1.20 \mathrm{mmol})$ was cooled to $0{ }^{\circ} \mathrm{C}$. $\mathrm{A} \mathrm{CH}_{2} \mathrm{Cl}_{2}(1.0 \mathrm{~mL})$ solution of $\mathrm{Pd}_{2}(\mathrm{DBA})_{3^{-}}$ $\mathrm{CHCl}_{3}(25.8 \mathrm{mg}, 2.5 \mathrm{~mol} \%)$ and $\mathrm{PPh}_{3}(26.2 \mathrm{mg}, 10 \mathrm{~mol} \%)$ was added to the reaction 
mixture at $0{ }^{\circ} \mathrm{C}$. The reaction mixture was stirred at $0{ }^{\circ} \mathrm{C}$ for $30 \mathrm{~min}$. The reaction mixture was quenched with $5 \mathrm{~mL}$ of saturated aq $\mathrm{NaHCO}_{3}$, extracted $(3 \times 5 \mathrm{~mL})$ with $\mathrm{Et}_{2} \mathrm{O}$, dried $\left(\mathrm{Na}_{2} \mathrm{SO}_{4}\right)$, and concentrated under reduced pressure. The crude product was purified using silica gel flash chromatography eluting with 50:40:10 EtOAc/hexanes/methanol to give (2S,6S)-6-(1'H-benzo[d]imidazol-1'-yl)-2-(tert-butyldimethyl-silanyloxymethyl)-2H-pyran-3(6H)-one $\mathbf{I - 5 8} \boldsymbol{\beta}(250 \mathrm{mg}, 1.00 \mathrm{mmol}, 82 \%)$ as viscous oil. $R_{f}(50 \%$ EtOAc/hexanes $)=0.20 ;[\alpha]_{\mathrm{D}}^{26}=-11\left(c=1.0, \mathrm{CH}_{2} \mathrm{Cl}_{2}\right) ; \mathrm{IR}$ (thin film, $\left.\mathrm{cm}^{-1}\right) 2854,1702,1606,1458,1251,1070,834 ;{ }^{1} \mathrm{H}$ NMR $\left(600 \mathrm{MHz}, \mathrm{CDCl}_{3,3} \delta 8.00\right.$ (s, 1H), $7.82(\mathrm{dd}, J=7.2,1.8 \mathrm{~Hz}, 1 \mathrm{H}), 7.54(\mathrm{dd}, J=7.2,1.8 \mathrm{~Hz}, 1 \mathrm{H}), 7.32(\mathrm{ddd}, J=7.2$, 7.2, $1.2 \mathrm{~Hz}, 1 \mathrm{H}), 7.30$ (ddd, $J=7.2,7.2,1.2 \mathrm{~Hz}, 1 \mathrm{H}), 7.07(\mathrm{dd}, J=10.2,1.8 \mathrm{~Hz}, 1 \mathrm{H})$, $6.55(\mathrm{dd}, J=4.2,1.8 \mathrm{~Hz}, 1 \mathrm{H}), 6.46(\mathrm{dd}, J=10.2,1.8 \mathrm{~Hz}, 1 \mathrm{H}), 4.45(\mathrm{dd}, J=3.0,1.8 \mathrm{~Hz}$, $1 \mathrm{H}), 4.44(\mathrm{dd}, J=3.0,1.8 \mathrm{~Hz}, 1 \mathrm{H}), 4.06(\mathrm{dd}, J=6.6,3.0 \mathrm{~Hz}, 1 \mathrm{H}), 0.81(\mathrm{~s}, 9 \mathrm{H}),-0.04(\mathrm{~s}$, $3 \mathrm{H}),-0.05(\mathrm{~s}, 3 \mathrm{H}) ;{ }^{13} \mathrm{C}$ NMR (150 MHz, $\left.\mathrm{DCl}_{3}\right) . \delta 192.5,145.0,144.0,141.5,132.5$, 131.0, 123.7, 123.1, 120.6, 111.1, 81.4, 78.7, 62.2, 25.7 (3C), 18.2, -5.4, -5.5; CIHRMS Calcd for $\left[\mathrm{C}_{19} \mathrm{H}_{26} \mathrm{~N}_{2} \mathrm{O}_{3} \mathrm{SiNa}\right]^{+}$: 381.1604 , Found : 381.1599 .

(2S,3R,6S)-6-(1'H-benzo[d]imidazol-1'-yl)-3,6-dihydro-2-(tert-butyl-dimethylsilanyloxymethy)-2H-pyran-3-ol (I-59ß):

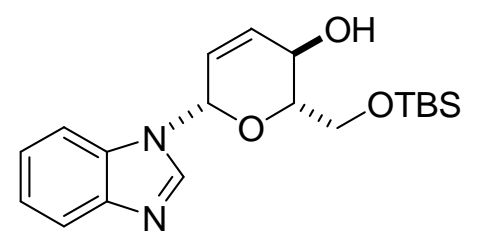

A $\mathrm{CH}_{2} \mathrm{Cl}_{2}(0.5 \mathrm{~mL})$ solution of enone $\mathbf{I - 5 8} \boldsymbol{\beta}(170 \mathrm{mg}, 0.454 \mathrm{mmol})$ and $\mathrm{MeOH}(0.5 \mathrm{~mL})$ was cooled to $-78{ }^{\circ} \mathrm{C}$. $\mathrm{NaBH}_{4}(20.6 \mathrm{mg}, 0.544 \mathrm{mmol})$ was added and the reaction 
mixture was stirred at $-78^{\circ} \mathrm{C}$ for $3 \mathrm{~h}$. The reaction mixture was diluted with ether $(5 \mathrm{~mL})$ and was quenched with $5 \mathrm{~mL}$ of saturated aq $\mathrm{NaHCO}_{3}$, extracted $(3 \times 5 \mathrm{~mL})$ with $\mathrm{Et}_{2} \mathrm{O}$, dried $\left(\mathrm{Na}_{2} \mathrm{SO}_{4}\right)$, and concentrated under reduced pressure. The crude product was purified using silica gel flash chromatography eluting with 50:45:5 EtOAc/hexanes/methanol to give (2S,3R,6S)-6-(1'H-benzo[d]imidazol-1'-yl)-3,6dihydro-2-(tert-butyl-dimethyl-silanyloxymethy)-2H-pyran-3-ol $\quad \mathbf{I}-\mathbf{5 9} \boldsymbol{\beta}(135 \mathrm{mg}, \quad 0.37$ mmol, 79\%) as viscous oil. $\mathrm{R}_{\mathrm{f}}(50 \%$ EtOAc/hexanes $)=0.20 ;[\alpha]_{\mathrm{D}}^{26}=-0.23(\mathrm{c}=1.0$, $\mathrm{CH}_{2} \mathrm{Cl}_{2}$ ); IR (thin film, $\mathrm{cm}^{-1}$ ) 3192, 2928, 2856, 1459, 1119, 1087; ${ }^{1} \mathrm{H}$ NMR (600 MHz, $\left.\mathrm{CDCl}_{3}\right) \delta 7.98(\mathrm{~s}, 1 \mathrm{H}), 7.79(\mathrm{dd}, J=6.6,2.4 \mathrm{~Hz}, 1 \mathrm{H}), 7.46(\mathrm{dd}, J=6.6,1.8 \mathrm{~Hz}, 1 \mathrm{H}), 7.29$ (ddd, $J=7.2,6.6,2.4 \mathrm{~Hz}, 1 \mathrm{H}), 7.28(\mathrm{ddd}, J=6.6,6.6,1.8 \mathrm{~Hz}, 1 \mathrm{H}), 6.29(\mathrm{dd}, J=2.4,2.4$ $\mathrm{Hz}, 1 \mathrm{H}), 6.27(\mathrm{dd}, J=2.4,1.8 \mathrm{~Hz}, 1 \mathrm{H}), 5.94(\mathrm{ddd}, J=9.6,3.0,2.4 \mathrm{~Hz}, 1 \mathrm{H}), 4.52(\mathrm{dd}, J=$ 8.4, $2.4 \mathrm{~Hz}, 1 \mathrm{H}), 3.97(\mathrm{dd}, J=10.2,4.8 \mathrm{~Hz}, 1 \mathrm{H}), 3.84(\mathrm{ddd}, J=11.4,5.4,4.2 \mathrm{~Hz}, 1 \mathrm{H})$, $3.77(\mathrm{dd}, J=10.2,7.8 \mathrm{~Hz}, 1 \mathrm{H}), 3.33(\mathrm{~s}, 1 \mathrm{H}), 0.89(\mathrm{~s}, 9 \mathrm{H}), 0.08(\mathrm{~s}, 3 \mathrm{H}), 0.07(\mathrm{~s}, 3 \mathrm{H}) ;{ }^{13} \mathrm{C}$ NMR (67.5 MHz, $\left.\mathrm{CDCl}_{3}\right) \delta 138.0,133.0,128.3(2 \mathrm{C}), 127.9$ (2C), 127.6, 125.7, 93.4, 70.4, 70.0, 66.7, 65.1, $25.8(3 \mathrm{C}), 18.2,-5.4,-5.5$; CIHRMS Calcd for $\left[\mathrm{C}_{19} \mathrm{H}_{28} \mathrm{~N}_{2} \mathrm{O}_{3} \mathrm{SiH}\right]^{+}$: 361.1942, Found : 361.1984 .

((2R,6S)-6-(1'H-benzo[d]imidazol-1'-yl)-5,6-dihydro-2H-pyran-2-yl)

tert-butyldimethyl-silanyloxymethane (I-60ß).

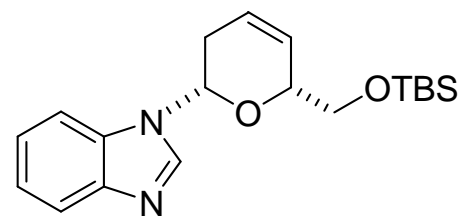

A flask was charged with dry N-methyl morpholine ( NMM ) $25 \mathrm{ml}$, triphenyl phosphine (459 mg, $1.172 \mathrm{mmol}$ ) and was cooled to $-30{ }^{\circ} \mathrm{C}$ under $\mathrm{Ar}$ atmosphere. 
Diethylazodicarboxylate $(251.4 \mu \mathrm{l}, 1.597 \mathrm{mmol})$ was added and the reaction was stirred for $5 \mathrm{~min}$, allylic alcohol $\mathbf{I}-\mathbf{5 9} \boldsymbol{\beta}(200 \mathrm{mg}, 0.531 \mathrm{mmol})$ was added in a $1 \mathrm{M}$ solution of NMM and the reaction mixture was stirred for $10 \mathrm{~min}$, followed by addition of onitrobenzenesulfonyl hydrazide (NBSH) $(323.5 \mathrm{mg}, 1.593 \mathrm{mmol})$. The reaction was stirred at $-30{ }^{\circ} \mathrm{C}$ for $2 \mathrm{~h}$ and was monitored by TLC, upon consumption of starting material, warm up to room temperature and stirred for another $4 \mathrm{~h}$. The reaction mixture was diluted with ether $(10 \mathrm{~mL})$ and was quenched with $5 \mathrm{~mL}$ of satd. aq $\mathrm{NaHCO}_{3}$, extracted $(3 \times 5 \mathrm{~mL})$ with $\mathrm{Et}_{2} \mathrm{O}$, dried $\left(\mathrm{Na}_{2} \mathrm{SO}_{4}\right)$, and concentrated under reduced pressure. The crude product was purified using silica gel flash chromatography eluting with $50 \%$ EtOAc/hexanes to give $\left((2 \mathrm{R}, 6 \mathrm{~S})-6-\left(1^{\prime} \mathrm{H}-\right.\right.$ benzo[d]imidazol-1'-yl)-5,6-dihydro2H-pyran-2-yl) tert-butyl-dimethyl-silanyloxymethane I-60ß (119 mg, $0.345 \mathrm{mmol}, 65 \%$ ) as viscous oil. $R_{f}(50: 49: 1 \%$ EtOAc/hexanes $/$ methanol $)=0.35 ;[\alpha]_{\mathrm{D}}^{26}=+72 \quad(c=1.0$, $\mathrm{CH}_{2} \mathrm{Cl}_{2}$ ); IR (thin film, $\mathrm{cm}^{-1}$ ) 3296, 2929, 1721, 1066, 835; ${ }^{1} \mathrm{H}$ NMR (600 MHz, $\left.\mathrm{CDCl}_{3}\right) \delta$ $8.04(\mathrm{~m}, 1 \mathrm{H}), 7.79(\mathrm{dd}, J=7.8,1.8 \mathrm{~Hz}, 1 \mathrm{H}), 7.50(\mathrm{dd}, J=7.2,2.4 \mathrm{~Hz}, 1 \mathrm{H}), 7.28(\mathrm{ddd}, J=$ 7.2, 7.2, $1.2 \mathrm{~Hz}, 1 \mathrm{H}), 7.27$ (ddd, $J=7.2,7.2,1.2 \mathrm{~Hz}, 1 \mathrm{H}), 5.96(\mathrm{dd}, J=10.2,6.0 \mathrm{~Hz}, 1 \mathrm{H})$, $5.92(\mathrm{dd}, J=10.2,2.4 \mathrm{~Hz}, 1 \mathrm{H}), 5.77(\mathrm{dd}, J=9.6,3.0 \mathrm{~Hz}, 1 \mathrm{H}), 4.56(\mathrm{br} \mathrm{s}, 1 \mathrm{H}), 3.75(\mathrm{dd}, J$ $=9.6,6.0 \mathrm{~Hz}, 1 \mathrm{H}), 3.61(\mathrm{dd}, J=9.6,6.0 \mathrm{~Hz}, 1 \mathrm{H}), 2.90(\mathrm{ddd}, J=10.8,10.2,3.0 \mathrm{~Hz}, 1 \mathrm{H})$, 2.50 (ddd, $J=10.8,9.6,3.0 \mathrm{~Hz}, 1 \mathrm{H}), 0.86(\mathrm{~s}, 9 \mathrm{H}), 0.02(\mathrm{~s}, 3 \mathrm{H}),-0.002(\mathrm{~s}, 3 \mathrm{H}) ;{ }^{13} \mathrm{C} \mathrm{NMR}$ $\left(150 \mathrm{MHz}, \mathrm{CDCl}_{3}\right) \delta 140.3,127.8,123.5,123.2(2 \mathrm{C}), 122.9,122.7,120.2,111.0,80.3$, 77.4, 65.3, 30.4, 25.7(3C), 18.2, -3.5, -5.4; CIHRMS Calcd for $\left[\mathrm{C}_{19} \mathrm{H}_{28} \mathrm{~N}_{2} \mathrm{O}_{2} \mathrm{SiNa}^{+}\right]$: 367.1812, Found : 367.1829 . 


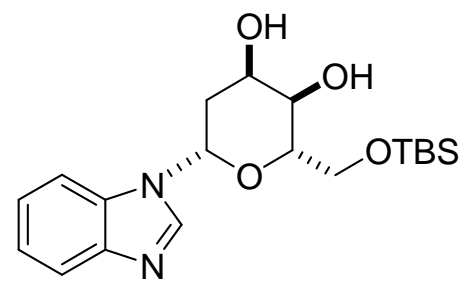

To a solution of olefin $\mathbf{I - 6 0 \beta}(80 \mathrm{mg}, 0.110 \mathrm{mmol})$ in $t$-butanol/ acetone $(0.4 \mathrm{~mL}, 1: 1$, $1 \mathrm{M})$ at $0{ }^{\circ} \mathrm{C}$ was added a solution of $(50 \% \mathrm{w} / \mathrm{v})$ of $\mathrm{N}$-methyl morpholine $\mathrm{N}$-oxide / water $(0.12 \mathrm{~mL})$ and crystalline $\mathrm{OsO}_{4}(0.6 \mathrm{mg}, 1 \mathrm{~mol} \%)$ was added. The reaction mixture was stirred for 12 hours and the reaction was quenched with adding EtOAC and satd. $\mathrm{NaHCO}_{3}$. The organic layer was separated and concentrated. It was purified by a silica gel column using $5 \%$ methaol/EtOAc. Pure fractions were combined and concentrated to afford $\quad(2 S, 3 R, 4 R, 6 S)-6-\left(1^{\prime} H\right.$-benzo[d]imidazol-1'-yl)-tetrahydro-2-(tert-butyldimethyl-silanyloxymethy)-2H-pyran-3,4-diol I-61 $\beta$ (36 $\mathrm{mg}, 0.094 \mathrm{mmol}, 85 \%$ ) as viscous oil. $\mathrm{R}_{\mathrm{f}}(10 \%$ methanol $/ 40 \%$ EtOAc/50hexanes $)=0.40 ;[\alpha]_{\mathrm{D}}^{26}=+22(c=1.2$, $\mathrm{CH}_{2} \mathrm{Cl}_{2}$ ); IR (thin film, cm-1) 3166, 2928, 1615, 1065, 777; 1H NMR (600 MHz, $\left.\mathrm{CDCl}_{3}\right) \delta 8.29(\mathrm{~s}, 1 \mathrm{H}), 7.67(\mathrm{dd}, J=7.6,1.8 \mathrm{~Hz}, 1 \mathrm{H}), 7.66(\mathrm{dd}, J=7.6,1.8 \mathrm{~Hz}, 1 \mathrm{H}), 7.30$ (ddd, $J=7.2,7.2,1.8 \mathrm{~Hz}, 1 \mathrm{H}), 7.28(\mathrm{ddd}, J=7.2,7.2,1.8 \mathrm{~Hz}, 1 \mathrm{H}), 6.09(\mathrm{dd}, J=11.4,1.8$ $\mathrm{Hz}, 1 \mathrm{H}), 4.27(\mathrm{dd}, J=5.4,3.6 \mathrm{~Hz}, 1 \mathrm{H}), 4.03(\mathrm{dd}, J=10.2,5.2 \mathrm{~Hz}, 1 \mathrm{H}), 3.97(\mathrm{dd}, J=$ 11.4, $2.4 \mathrm{~Hz}, 1 \mathrm{H}), 3.85(\mathrm{dd}, J=11.4,5.4 \mathrm{~Hz}, 1 \mathrm{H}), 3.72(\mathrm{dd}, J=10.2,3.6 \mathrm{~Hz}, 1 \mathrm{H}), 2.52$ (ddd, $J=13.2,11.4,2.4 \mathrm{~Hz}, 1 \mathrm{H}), 2.29(\mathrm{ddd}, J=13.2,3.6,2.4 \mathrm{~Hz}, 1 \mathrm{H}), 0.85(\mathrm{~s}, 9 \mathrm{H}),-$ $0.02(\mathrm{~s}, 3 \mathrm{H}),-0.05(\mathrm{~s}, 3 \mathrm{H}) ; 13 \mathrm{C} \mathrm{NMR}\left(150 \mathrm{MHz}, \mathrm{CDCl}_{3}\right) \delta 144.4,142.4,134.4,124.6$, 
124.1 (2C), 120.3 (2C), 113.1, 80.3, 78.0, 68.8, 66.3, 64.6, 38.2, 26.5, 19.3, -4.9, -5.0;

CIHRMS Calcd for $\left[\mathrm{C}_{19} \mathrm{H}_{30} \mathrm{~N}_{2} \mathrm{O}_{4} \mathrm{SiNa}^{+}\right]$: 401.1867, Found: 401.1870 .

\section{$(2 S, 3 R, 6 S)-6-\left(1^{\prime} H-b e n z o[d]\right.$ imidazol-1'-yl)-tetrahydro-2-(tert-butyl-dimethyl-}

\section{silanyloxymethyl)-2H-pyran-3-ol (I-62 $\beta)$.}

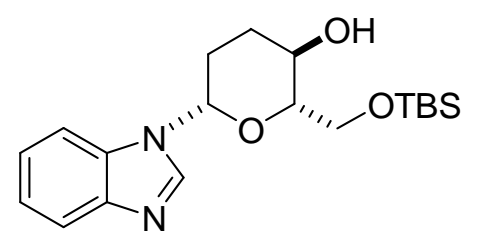

The allyl alcohol $\mathbf{I}-59 \boldsymbol{\beta}$ (200 mg, $0.53 \mathrm{mmol}), o-\mathrm{NO}_{2} \mathrm{ArSO}_{2} \mathrm{NHNH}_{2}$ (863 mg, 4.25 mmol) were dissolved in $5.0 \mathrm{~mL}$ of $\mathrm{CH}_{2} \mathrm{Cl}_{2}$ in a round bottom flask and cooled $0{ }^{\circ} \mathrm{C}$ under nitrogen condition then triethylamine $(760 \mu \mathrm{l}, 5.31 \mathrm{mmol})$ was added and the reaction mixture was stirred at $0{ }^{\circ} \mathrm{C}$ for 12 hours and on completion monitored by TLC. The reaction mixture was diluted with ether $(10 \mathrm{~mL})$ and was quenched with $5 \mathrm{~mL}$ of saturated $\mathrm{NaHCO}_{3}$ and aqueous layers were extracted $(3 \times 10 \mathrm{~mL})$ with $\mathrm{Et}_{2} \mathrm{O}$, dried over sodium sulphate and concentrated under reduced pressure. The crude product was purified using silica gel flash chromatography eluting with 50:45:5 EtOAc/hexanes/methanol to give (2S,3R,6S)-6-(1'H-benzo[d]imidazol-1'-yl)-tetrahydro2-(tert-butyl-dimethyl-silanyloxymethyl)-2H-pyran-3-ol I-62 3 (60 mg, 0.16 mmol, $30 \%$ )

as viscous oil. $R_{f}(50 \%$ EtOAc/Hexane $)=0.25 ;[\alpha]_{\mathrm{D}}^{26}=+0.23\left(c 1.0, \mathrm{CH}_{2} \mathrm{Cl}_{2}\right)$; IR (thin film, $\left.\mathrm{cm}^{-1}\right) 3195,2952,2928,1459,1252,1237,1085,922,835 ;{ }^{1} \mathrm{H}$ NMR $(600 \mathrm{MHz}$, $\left.\mathrm{CDCl}_{3}\right) \delta 8.0(\mathrm{~s}, 1 \mathrm{H}), 7.79(\mathrm{dd}, J=6.6,2.4 \mathrm{~Hz}, 1 \mathrm{H}), 7.47(\mathrm{dd}, J=6.6,2.4 \mathrm{~Hz}, 1 \mathrm{H}), 7.30$ (ddd, $J=7.2,7.2,1.8 \mathrm{~Hz}, 1 \mathrm{H}), 7.29(\mathrm{ddd}, J=7.2,7.2,1.8 \mathrm{~Hz}, 1 \mathrm{H}), 5.57(\mathrm{dd}, J=10.8,2.4$ Hz, 1H), 3.97 (dd, $J=10.2,4.8 \mathrm{~Hz}, 1 \mathrm{H}) 3.80(\mathrm{dd}, J=10.2,7.8 \mathrm{~Hz}, 1 \mathrm{H}), 3.64(\mathrm{dd}, J=4.8$, 
$4.2 \mathrm{~Hz}, 1 \mathrm{H}), 2.36$ (ddd, $J=12.0,9.0,3.6 \mathrm{~Hz}, 1 \mathrm{H}), 2.28$ (ddd, $J=15.0,9.6,3.6 \mathrm{~Hz}, 1 \mathrm{H}$ ), $2.20(\mathrm{dd}, J=6.6,3.0 \mathrm{~Hz}, 1 \mathrm{H}), 2.18(\mathrm{dd}, J=6.6,3.0 \mathrm{~Hz}, 1 \mathrm{H}), 2.04(\mathrm{~s}, 1 \mathrm{H}), 0.91(\mathrm{~s}, 9 \mathrm{H})$,

0.09 (s, 3H), 0.09 (s, 3H); ${ }^{13} \mathrm{C}$ NMR (150 MHz, $\left.\mathrm{CDCl}_{3}\right) \delta$ 140.1, 123.2, 122.6, 120.5 (2C), 110.6 (2C), 83.0, 79.2, 69.6, 65.9, 30.8, 29.6, 25.7 (3C), 18.1, -5.5, -5.6; CIHRMS Calcd for $\left[\mathrm{C}_{19} \mathrm{H}_{30} \mathrm{~N}_{2} \mathrm{O}_{3} \mathrm{SiNa}\right]^{+}$: 385.1917 , Found : 385.1920 .

(2S,6R)-6-(1'H-benzo[d]imidazol-1'-yl)-2-(tert-butyl-dimethyl-silanyloxymethyl)-2Hpyran-3(6H)-one (I-56 $\alpha)$ :

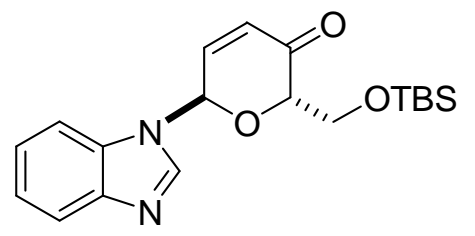

A $\mathrm{CH}_{2} \mathrm{Cl}_{2}(0.8 \mathrm{~mL})$ solution of pyranone $\mathbf{I}-15 \boldsymbol{\alpha}$ (289 $\left.\mathrm{mg}, 0.807 \mathrm{mmol}\right)$ and benzimadazole (114 mg, $1.20 \mathrm{mmol})$ was cooled to $0{ }^{\circ} \mathrm{C} . \quad \mathrm{A} \mathrm{CH}_{2} \mathrm{Cl}_{2}(0.8 \mathrm{~mL})$ solution of $\mathrm{Pd}_{2}(\mathrm{DBA})_{3} \mathrm{CHCl}_{3}(20.8 \mathrm{mg}, 2.5 \mathrm{~mol} \%)$ and $\mathrm{PPh}_{3}(21.1 \mathrm{mg}, 10 \mathrm{~mol} \%)$ was added to the reaction mixture at $0{ }^{\circ} \mathrm{C}$. The reaction mixture was stirred at $0{ }^{\circ} \mathrm{C}$ for $30 \mathrm{~min}$. The reaction mixture was diluted with $5 \mathrm{~mL}$ of satd aq $\mathrm{NaHCO}_{3}$, extracted $(3 \times 5 \mathrm{~mL})$ with $\mathrm{Et}_{2} \mathrm{O}$, dried $\left(\mathrm{Na}_{2} \mathrm{SO}_{4}\right)$, and concentrated under reduced pressure. The crude product was purified using silica gel flash chromatography eluting with 50:45:5 EtOAc/hexanes/methanol to give $(2 S, 6 R)-6-\left(1^{\prime} \mathrm{H}\right.$-benzo[d]imidazol-1'-yl)-2-(tert-butyldimethyl-silanyloxymethyl)-2H-pyran-3(6H)-one $\mathbf{I}-56 \boldsymbol{\alpha}(230 \mathrm{mg}, 0.642 \mathrm{mmol}, 77 \%)$ as viscous oil. $R_{f}(50 \%$ EtOAc/hexanes $)=0.35 ;[\alpha]_{\mathrm{D}}^{26}=-2\left(c=1.0, \mathrm{CH}_{2} \mathrm{Cl}_{2}\right) ; \mathrm{IR}$ (thin film, $\left.\mathrm{cm}^{-1}\right) 3309,2946,1646,1459,1257,1021,744 ;{ }^{1} \mathrm{H}$ NMR $\left(600 \mathrm{MHz}, \mathrm{CDCl}_{3}\right) \delta 7.96(\mathrm{~s}$, 1H), $7.82(\mathrm{dd}, J=7.6,1.8 \mathrm{~Hz}, 1 \mathrm{H}), 7.55(\mathrm{dd}, J=7.2,1.8 \mathrm{~Hz}, 1 \mathrm{H}), 7.34(\mathrm{ddd}, J=6.6,6.6$, 
$1.8 \mathrm{~Hz}, 1 \mathrm{H}), 7.33$ (ddd, $J=7.2,7.2,1.8 \mathrm{~Hz}, 1 \mathrm{H}), 7.16(\mathrm{dd}, J=10.2,2.4 \mathrm{~Hz}, 1 \mathrm{H}), 6.84$ (dd, $J=2.4,1.8 \mathrm{~Hz}, 1 \mathrm{H}), 6.53(\mathrm{dd}, J=10.2,1.8 \mathrm{~Hz}, 1 \mathrm{H}), 4.21(\mathrm{dd}, J=3.6,2.4 \mathrm{~Hz}, 1 \mathrm{H})$, $4.10(\mathrm{dd}, J=11.4,3.6 \mathrm{~Hz}, 1 \mathrm{H}), 4.05(\mathrm{dd}, J=11.4,2.4 \mathrm{~Hz}, 1 \mathrm{H}), 0.85(\mathrm{~s}, 9 \mathrm{H}), 0.07(\mathrm{~s}, 3 \mathrm{H})$, $0.02(\mathrm{~s}, 3 \mathrm{H}) .{ }^{13} \mathrm{C}$ NMR $\left(150 \mathrm{MHz}, \mathrm{CDCl}_{3}\right) \delta 193.4,144.1,142.8,141.3,133.0$, 131.1(2C), 123.8, 123.1, 120.6, 110.6, 78.8, 64.4, 25.7 (3C), 18.1, -5.5, -5.6; CIHRMS Calcd for $\left[\mathrm{C}_{19} \mathrm{H}_{26} \mathrm{~N}_{2} \mathrm{O}_{3} \mathrm{SiNa}\right]^{+}$: 381.1604 . Found : 381.1602 .

((2S,5R,6S)-5,6-dihydro-5-hydroxy-6-methyl-2H-pyran-2-yl pivalate (II-30).

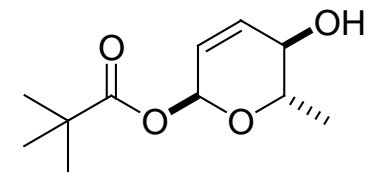

A solution of pyranone $\mathbf{I I - 2 6}(327 \mathrm{mg}, 1.54 \mathrm{mmol})$ in dry $\mathrm{CH}_{2} \mathrm{Cl}_{2}(1.5 \mathrm{~mL})$ and $0.4 \mathrm{M}$ $\mathrm{CeCl}_{3} / \mathrm{MeOH}(1.5 \mathrm{~mL})$ was cooled to $-78{ }^{\circ} \mathrm{C} . \mathrm{NaBH}_{4}(58.4 \mathrm{mg}, 1.54 \mathrm{mmol})$ was added and the reaction mixture was stirred for $4 \mathrm{~h}$ at $-78{ }^{\circ} \mathrm{C}$. The resulting solution was diluted with ether $(10 \mathrm{~mL})$ and was quenched with $5 \mathrm{~mL}$ of saturated $\mathrm{NaHCO}_{3}$, extracted $(3 \times 5$ $\mathrm{mL}$ ) with $\mathrm{Et}_{2} \mathrm{O}$, dried $\left(\mathrm{Na}_{2} \mathrm{SO}_{4}\right)$, and concentrated under reduced pressure. The crude product was purified using silica gel chromatography eluting with $40 \%$ EtOAc/hexane to give enol II-30 (297 mg, $1.34 \mathrm{mmol}, 90 \%$ ) as a white solid, mp: $62.5-64.0^{\circ} \mathrm{C} ; R f=0.30$ $\left(40 \%\right.$ EtOAc/Hexane); $[\alpha]_{\mathrm{D}}^{26}=-28\left(c=1.0, \mathrm{CH}_{2} \mathrm{Cl}_{2}\right) ; \mathrm{IR}\left(\right.$ thin film, $\left.\mathrm{cm}^{-1}\right) 3391,2972$, 2933, 2837, 1612, 1513, 1246, 1029, 999, 819; ${ }^{1} \mathrm{H}$ NMR $\left(600 \mathrm{MHz}, \mathrm{CDCl}_{3}\right) \delta 6.21(\mathrm{dd}, J$ $=2.4,1.8 \mathrm{~Hz}, 1 \mathrm{H}), 6.04(\mathrm{~d}, J=9.6 \mathrm{~Hz}, 1 \mathrm{H}), 5.75(\mathrm{ddd}, J=10.2,2.4,2.4 \mathrm{~Hz}, 1 \mathrm{H}), 3.90(\mathrm{~d}$, $J=8.4 \mathrm{~Hz}, 1 \mathrm{H}), 3.72(\mathrm{~m}, 1 \mathrm{H}), 1.31(\mathrm{~d}, J=6.6 \mathrm{~Hz}, 3 \mathrm{H}), 1.20(\mathrm{~s}, 9 \mathrm{H}) ;{ }^{13} \mathrm{C}$ NMR $(150$ $\left.\mathrm{MHz}, \mathrm{CDCl}_{3}\right) \delta 177.7,134.7,125.1,88.4,70.3,69.2,39.1,27.1$ (3C), 18.0; CIHRMS: Calculated for $\left[\mathrm{C}_{11} \mathrm{H}_{18} \mathrm{O}_{4} \mathrm{Na}^{+}\right]$: 237.1102. Found: 237.1099 
(2S,5R,6S)-5,6-dihydro-5-methoxy-6-methyl-2H-pyran-2-yl pivalate (II-32).

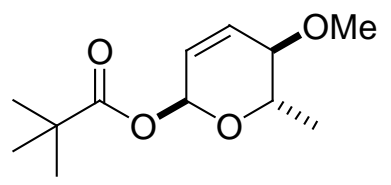

To a mixture of allylic alcohol II-30 (1.7 g, $7.94 \mathrm{mmol})$ and silver (I) oxide $(9.1 \mathrm{~g}, 39.7$ mmol), was added $2.5 \mathrm{ml} \mathrm{CH} 3$. The reaction suspension was allowed to stirred for 2 days under room temperature, passed a celite pad with $100 \mathrm{ml} \mathrm{Et}_{2} \mathrm{O}$, concentrated under reduced pressure and purified using silica gel flash chromatography eluting with pure 57\% EtOAc/Hexane to give alcohol $21(1.6 \mathrm{mg}, 6.98 \mathrm{mmol}, 88 \%)$ alcohol II-32 as white foam; $R f=0.52(30 \%$ EtOAc/Hexane $) ;[\alpha]_{\mathrm{D}}^{26}=-128\left(c=2.0, \mathrm{CH}_{2} \mathrm{Cl}_{2}\right) ; \mathrm{IR}$ (thin film, $\left.\mathrm{cm}^{-1}\right) 2973,2954,2111,1738,1277,1130,1042,921 ;{ }^{1} \mathrm{H}$ NMR $\left(600 \mathrm{MHz}, \mathrm{CDCl}_{3}\right) \delta$ 6.19 (br s, 1H), $6.15(\mathrm{~d}, J=10.2 \mathrm{~Hz}, 1 \mathrm{H}), 5.75(\mathrm{td}, J=10.2,2.4 \mathrm{~Hz}, 1 \mathrm{H}), 3.78$ (ddd, $J=$ 12.0, 9.0, 6.6 Hz, 1H), $3.48(\mathrm{dd}, J=9.0,1.2 \mathrm{~Hz}, 1 \mathrm{H}), 3.42(\mathrm{~s}, 3 \mathrm{H}), 1.29$ (d, $J=6.0, \mathrm{~Hz}$, 3H), $1.18(\mathrm{~s}, 9 \mathrm{H}) ;{ }^{13} \mathrm{C}$ NMR $\left(150 \mathrm{MHz}, \mathrm{CDCl}_{3}\right) \delta 201.4,131.2,124.9,88.3,77.7,68.0$, 56.8, 38.9, 26.9 (3C), 18.1; CIHRMS: Calculated for $\left[\mathrm{C}_{12} \mathrm{H}_{20} \mathrm{O}_{4} \mathrm{Na}^{+}\right]$: 251.1259, Found: 251.1253.

7-((2S,5R,6S)-5,6-dihydro-5-methoxy-6-methyl-2H-pyran-2-yloxy)-4-methyl-2Hchromen-2-one (II-33).

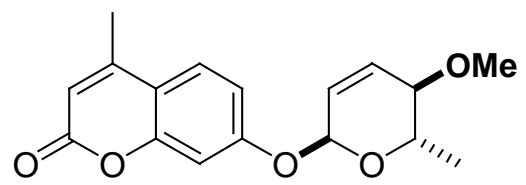

A THF (8 mL) solution of Piv-enol II-32 (953 mg, $4.18 \mathrm{mmol})$ and 4-methyl coumarin alcohol $5(882 \mathrm{mg}, 6.27 \mathrm{mmol})$ was cooled to $0{ }^{\circ} \mathrm{C}$. A THF $(2.0 \mathrm{~mL})$ solution of 
$\mathrm{Pd}_{2}(\mathrm{dba})_{3} \cdot \mathrm{CHCl}_{3}(108 \mathrm{mg}, 2.5 \mathrm{~mol} \%)$ and $\mathrm{PPh}_{3}(110 \mathrm{mg}, 10 \mathrm{~mol} \%)$ was added to the reaction mixture at $0{ }^{\circ} \mathrm{C}$. The reaction mixture was stirred at $0{ }^{\circ} \mathrm{C}$ for 5 hours. The reaction mixture was quenched with $5 \mathrm{~mL}$ of satd. aq. $\mathrm{NaHCO}_{3}$, extracted $(3 \times 5 \mathrm{~mL})$ with $\mathrm{Et}_{2} \mathrm{O}$, dried $\left(\mathrm{Na}_{2} \mathrm{SO}_{4}\right)$, and concentrated under reduced pressure. The crude product was purified using silica gel flash chromatography eluting with $30 \%$ EtOAc/hexanes to give coumarin-enol II-33 (1.07 g, $3.55 \mathrm{mmol}, 85 \%)$ as viscous oil. $R_{f}(20 \%$

EtOAc/hexane $)=0.33 ;[\alpha]_{\mathrm{D}}^{26}=-145\left(c=1.0, \mathrm{CH}_{2} \mathrm{Cl}_{2}\right) ; \mathrm{IR}\left(\right.$ thin film, $\left.\mathrm{cm}^{-1}\right) 2950,2939$, $1731,1613,1389,1134,1067,951 ;{ }^{1} \mathrm{H}$ NMR $\left(600 \mathrm{MHz}, \mathrm{CDCl}_{3}\right) \delta 7.50(\mathrm{~d}, J=9.0 \mathrm{~Hz}$, 1H), $7.08(\mathrm{~d}, J=2.4 \mathrm{~Hz}, 1 \mathrm{H}), 6.98(\mathrm{dd}, J=8.4,2.4 \mathrm{~Hz}, 1 \mathrm{H}), 6.23(\mathrm{~d}, J=10.2 \mathrm{~Hz}, 1 \mathrm{H})$, $6.15(\mathrm{~d}, J=1.2 \mathrm{~Hz}, 1 \mathrm{H}), 5.91$ (ddd, $J=10.2,3.0,1.8 \mathrm{~Hz}, 1 \mathrm{H}), 5.67$ (br s, 1H), 3.87 (ddd, $J=12.6,9.0,6.6 \mathrm{~Hz}, 1 \mathrm{H}), 3.54(\mathrm{dd}, J=9.6,1.2 \mathrm{~Hz}, 1 \mathrm{H}), 3.44(\mathrm{~s}, 3 \mathrm{H}), 2.40(\mathrm{~d}, J=1.2$ $\mathrm{Hz}, 3 \mathrm{H}), 1.26(\mathrm{~d}, J=6.0 \mathrm{~Hz}, 3 \mathrm{H}) ;{ }^{13} \mathrm{C} \mathrm{NMR}\left(150 \mathrm{MHz}, \mathrm{CDCl}_{3}\right) \delta 161.2,160.3,125.9$, $152.4,131.9,125.4,125.0,114.4,113.7,112.4,104.2,93.0,77.7,67.0,56.6,18.6,18.1$; CIHRMS: Calculated for $\left[\mathrm{C}_{17} \mathrm{H}_{18} \mathrm{O}_{5} \mathrm{H}^{+}\right]$: 303.1232, Found: 303.1228 .

\section{4-methyl-7-(tetrahydro-3,4-dihydroxy-5-methoxy-6-methyl-2H-pyran-2-yloxy)-2H-} chromen-2-one (II-34).

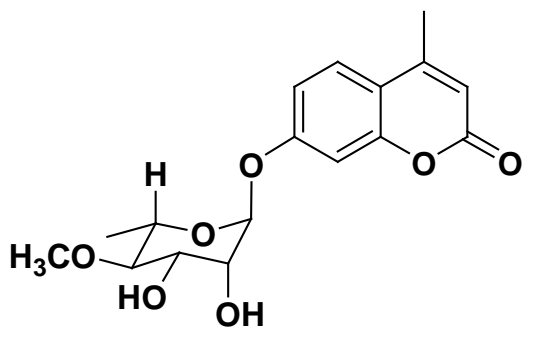

To a $\mathrm{CH}_{2} \mathrm{Cl}_{2}(0.2 \mathrm{~mL})$ solution of diene ester II-33 $(400 \mathrm{mg}, 1.32 \mathrm{mmol})$ at $0{ }^{\circ} \mathrm{C}$ was added a solution of $(50 \% \mathrm{w} / \mathrm{v})$ of $N$-methyl morpholine $N$-oxide / water $(0.7 \mathrm{~mL})$. 
Crystalline $\mathrm{OsO}_{4}(17 \mathrm{mg}, 5 \mathrm{~mol} \%)$ was added and the reaction was stirred for $8 \mathrm{~h}$. The reaction mixture was concentrated and was pipetted directly on to a silica gel column using $\mathrm{CH}_{2} \mathrm{Cl}_{2}(1 \mathrm{~mL})$ in three portions. Impurities were eluted with ether and the product was eluted with $\mathrm{MeOH} / \mathrm{EtOAc} / \mathrm{Hexane}$ (10:40:50). Pure fractions were combined and concentrated to afford diol II-34 (368 mg, $1.09 \mathrm{mmol}, 83 \%)$ as viscous oil. $R_{f}(60 \%$ EtOAc/Hexane $)=0.20 ;[\alpha]_{\mathrm{D}}^{26}=-50\left(c=1, \mathrm{CH}_{2} \mathrm{Cl}_{2}\right) ; \mathrm{IR}\left(\right.$ thin film, $\left.\mathrm{cm}^{-1}\right) 3412,2917$, $2949,1708,1615,1559,1372,1290,1118,1071,849 ;{ }^{1} \mathrm{H} \mathrm{NMR}\left(600 \mathrm{MHz}, \mathrm{CDCl}_{3}\right) \delta$ $7.51(\mathrm{~d}, J=8.4 \mathrm{~Hz}, 1 \mathrm{H}), 7.03(\mathrm{~d}, J=2.4 \mathrm{~Hz}, 1 \mathrm{H}), 6.98(\mathrm{dd}, J=9.0,2.0 \mathrm{~Hz}, 1 \mathrm{H}), 6.15(\mathrm{~d}$, $J=1.2 \mathrm{~Hz}, 1 \mathrm{H}), 5.56(\mathrm{~d}, J=1.2 \mathrm{~Hz}, 1 \mathrm{H}), 4.17(\mathrm{~d}, J=1.8 \mathrm{~Hz}, 1 \mathrm{H}), 4.05(\mathrm{ddd}, J=9.0,4.2$, $4.2 \mathrm{~Hz}, 1 \mathrm{H}$ ), 3.65 (dddd, $J=9.6,6.0,6.0,6.0 \mathrm{~Hz}, 1 \mathrm{H}), 3.58$ (s, $3 \mathrm{H}$ ), 3.19 (dd, $J=9.6,9.6$ Hz, 1H), $3.07(\mathrm{dd}, J=4.2 \mathrm{~Hz}, 1 \mathrm{H}), 2.92(\mathrm{~d}, J=4.8 \mathrm{~Hz}, 1 \mathrm{H}), 2.39(\mathrm{~d}, J=1.2 \mathrm{~Hz}, 3 \mathrm{H})$, $1.27(\mathrm{~s}, 3 \mathrm{H}) ;{ }^{13} \mathrm{C} \mathrm{NMR}\left(150 \mathrm{MHz}, \mathrm{CDCl}_{3}\right) \delta 161.2,158.9,154.8,152.5,125.6,114.7$, 113.2, 112.6, 104.0, 97.5, 82.6, 71.0, 70.6, 68.5, 60.9, 18.6, 17.6; CIHRMS Calcd for $\left[\mathrm{C}_{17} \mathrm{H}_{20} \mathrm{O}_{7} \mathrm{Na}^{+}\right]:$359.1106. Found 359.1102.

\section{4-methyl-7-(tetrahydro-3-hydroxy-4-acetoxy-5-methoxy-6-methyl-2H-pyran-2-} yloxy)-2H-chromen-2-one (II-35).

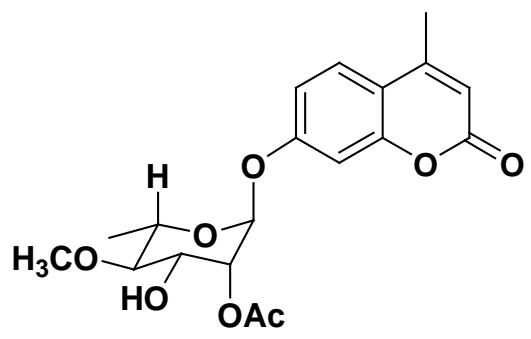

To a solution of diol II-34 $(110 \mathrm{mg}, 0.31 \mathrm{mmol})$ and trimethyl orthoacetate $(1.02 \mathrm{ml}, 8.1$ mmol) mixture in THF $(0.6 \mathrm{ml})$ was added $p$-toluenesulfonic acid monohydrate $(2.6 \mathrm{mg}$, 
$0.02 \mathrm{mmol}$ ), stirring for $0.5 \mathrm{~h}$. The solvent was removed under reduced pressure and

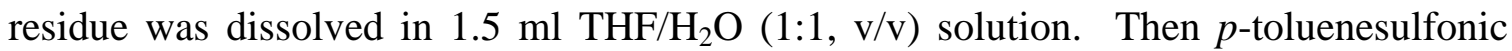
acid (26.3 mg $0.15 \mathrm{mmol}$ ) was added. Stirring was continued until hydrolysis was complete as seen by TLC. The reaction was quenched with adding EtOAc and satd. NaHCO3. The organic layer was separated, dried $\left(\mathrm{Na}_{2} \mathrm{SO}_{4}\right)$, concentrated under reduced pressure and purified by silica gel flash chromatography eluting with 70\% EtOAc/hexane to give acetate $\mathbf{I I - 3 5}(104 \mathrm{mg}, 0.28 \mathrm{mmol}, 90 \%)$ as white foam. $R f=0.35(50 \%$ EtOAc/Hexane); $[\alpha]_{\mathrm{D}}^{26}=-121\left(c=1.0, \mathrm{CH}_{2} \mathrm{Cl}_{2}\right)$; IR (thin film, $\left.\mathrm{cm}^{-1}\right) 3437,2938,1728$, 1611, 1389, 1232, 1067, 976, 850; ${ }^{1} \mathrm{H}$ NMR (600 MHz, $\left.\mathrm{CDCl}_{3}\right) \delta 7.51(\mathrm{~d}, J=9.0 \mathrm{~Hz}$, $1 \mathrm{H}), 7.03(\mathrm{~d}, J=2.4 \mathrm{~Hz}, 1 \mathrm{H}), 6.94(\mathrm{dd}, J=9.0,4.2 \mathrm{~Hz}, 1 \mathrm{H}), 6.17(\mathrm{~d}, J=1.2 \mathrm{~Hz}, 1 \mathrm{H})$, $5.51(\mathrm{~d}, J=1.2 \mathrm{~Hz}, 1 \mathrm{H}), 5.29(\mathrm{dd}, J=3.6,1.8 \mathrm{~Hz}, 1 \mathrm{H}), 4.21(\mathrm{ddd}, J=9.0,5.4,3.6 \mathrm{~Hz}$, 1H), 3.69 (dddd, $J=9.0,6.6,6.6,6.6 \mathrm{~Hz}, 1 \mathrm{H}), 3.60(\mathrm{~s}, 3 \mathrm{H}), 3.15(\mathrm{dd}, J=9.6,9.0 \mathrm{~Hz}, 1 \mathrm{H})$, $2.40(\mathrm{~d}, J=1.8 \mathrm{~Hz}, 3 \mathrm{H}), 2.28(\mathrm{~d}, J=4.8 \mathrm{~Hz}, 1 \mathrm{H}), 2.21(\mathrm{~s}, 3 \mathrm{H}), 1.29(\mathrm{~d}, J=6.6 \mathrm{~Hz}, 3 \mathrm{H})$;

${ }^{13} \mathrm{C}$ NMR $\left(150 \mathrm{MHz}, \mathrm{CDCl}_{3}\right) \delta 170.6,160.9,158.7,154.9,152.1,125.6,114.9,113.2$, 112.8, 104.1, 95.6, 82.9, 71.9, 69.6, 68.7, 61.0, 20.9, 18.6, 17.9; CIHRMS: Calculated for $\left[\mathrm{C}_{19} \mathrm{H}_{22} \mathrm{O}_{8} \mathrm{Na}^{+}\right]:$401.1212, Found: 401.1206 .

\section{2-(7-hydroxy-4-methyl-2H-chromen-2-one)-tetrahydro-3-hydroxy-5-methoxy-6- methyl-2H-pyran-4-yl carbamate (II-36).}




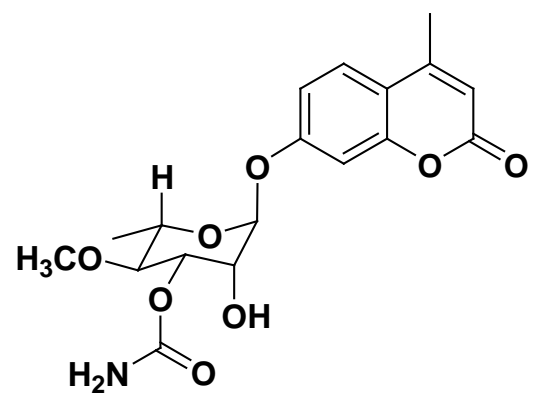

To a $\mathrm{CH}_{2} \mathrm{Cl}_{2}(1.5 \mathrm{~mL}, 0.1 \mathrm{M})$ solution of acetate alcohol $\mathbf{I I - 3 5}(70 \mathrm{mg}, 0.17 \mathrm{mmol})$ at 0 ${ }^{\circ} \mathrm{C}$ was added trichloro acetyl isocyanate $(32 \mu \mathrm{L}, 0.26 \mathrm{mmol})$ and the reaction was stirred for $1 \mathrm{~h}$. The solvent was removed under reduced pressure and residue was dissolved in $2.0 \mathrm{ml} \mathrm{MeOH} / \mathrm{H}_{2} \mathrm{O}(2: 1, \mathrm{v} / \mathrm{v})$ solution. Then potassium carbonate $(72.5 \mathrm{mg} 0.53 \mathrm{mmol})$ was added at $0{ }^{\circ} \mathrm{C}$. Stirring was continued until hydrolysis was complete as seen by TLC. The reaction was quenched with adding EtOAc and satd. NaHCO3. The organic layer was separated, dried $\left(\mathrm{Na}_{2} \mathrm{SO}_{4}\right)$, concentrated under reduced pressure and purified by silica gel flash chromatography eluting with $\mathrm{MeOH} / \mathrm{EtOAc} / \mathrm{Hexane}$ (10:40:50). Pure fractions were combined and concentrated to afford carbamate II-36 (53 mg, $0.14 \mathrm{mmol}$, $80 \%)$ as viscous oil. $R_{f}(50 \%$ EtOAc/Hexane $)=0.25 ;[\alpha]_{\mathrm{D}}^{26}=-44\left(c 0.5, \mathrm{CH}_{2} \mathrm{Cl}_{2}\right) ; \mathrm{IR}$ (thin film, $\mathrm{cm}^{-1}$ ) 3436, 2852, 1708, 1611, 1376, 1103, 1067, 992, 786; ${ }^{1} \mathrm{H}$ NMR (600 $\left.\mathrm{MHz}, \mathrm{CDCl}_{3}\right) \delta \delta 7.68(\mathrm{~d}, J=9.0 \mathrm{~Hz}, 1 \mathrm{H}), 7.07(\mathrm{dd}, J=9.0,2.4 \mathrm{~Hz}, 1 \mathrm{H}), 7.05(\mathrm{~d}, J=4.2$ $\mathrm{Hz}, 1 \mathrm{H}), 6.17(\mathrm{~d}, J=1.2 \mathrm{~Hz}, 1 \mathrm{H}), 5.51(\mathrm{~d}, J=1.8 \mathrm{~Hz}, 1 \mathrm{H}), 5.01(\mathrm{dd}, J=9.6,3.6 \mathrm{~Hz}, 1 \mathrm{H})$, $4.15(\mathrm{dd}, J=3.6,1.8 \mathrm{~Hz}, 1 \mathrm{H}), 3.62(\mathrm{~m}, 1 \mathrm{H}), 3.48(\mathrm{~s}, 3 \mathrm{H}), 3.32(\mathrm{dd}, J=9.0,9.0 \mathrm{~Hz}, 1 \mathrm{H})$, $2.42(\mathrm{~d}, J=1.8 \mathrm{~Hz}, 3 \mathrm{H}), 1.21(\mathrm{~d}, J=6.0 \mathrm{~Hz}, 3 \mathrm{H}) ; \mathrm{NMR}\left(150 \mathrm{MHz}, \mathrm{CDCl}_{3}\right) \delta 163.4$, $160.8,159.1,156.2,155.6,127.6,116.2,115.1,113.1,104.8,99.8,81.5,75.4,70.4,70.2$, 61.1, 18.7, 18.2; CIHRMS Calcd for $\left[\mathrm{C}_{18} \mathrm{H}_{21} \mathrm{NO}_{8} \mathrm{Na}^{+}\right]$: 402.1164. Found 402.1161. 


\section{4-methyl-7-(tetrahydro-5-methoxy-6-methyl-2H-pyran-2-yloxy)-2H-chromen-2-one}

(II-37).

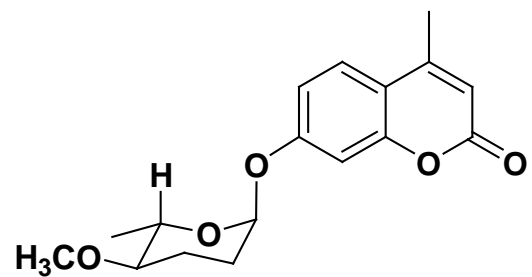

The ene compound II-33 (60 mg, $0.20 \mathrm{mmol})$ and $o-\mathrm{NO}_{2} \mathrm{C}_{6} \mathrm{H}_{4} \mathrm{SO}_{2} \mathrm{NHNH}_{2}(322 \mathrm{mg}, 1.80$ mmol) were dissolved in $2.0 \mathrm{~mL}$ of $\mathrm{CH}_{2} \mathrm{Cl}_{2}$ in a round bottom flask and cooled $0{ }^{\circ} \mathrm{C}$ under nitrogen atmosphere then triethylamine $(288 \mu \mathrm{L}, 2.0 \mathrm{mmol})$ was added and the reaction mixture was stirred at $0{ }^{\circ} \mathrm{C}$ for 12 hours and on completion, as monitored by TLC. The reaction mixture was concentrated and was pipetted directly on to a silica gel column using $\mathrm{CH}_{2} \mathrm{Cl}_{2}(1 \mathrm{~mL})$ in three portions. The crude product was purified using silica gel flash chromatography eluting with $25 \%$ EtOAc/hexanes to give deoxy compound II-37 (54 mg, $0.18 \mathrm{mmol}, 90 \%)$ as viscous oil. $R_{f}(50 \%$ EtOAc/Hexane $)=$ $0.40 ;[\alpha]_{\mathrm{D}}^{26}=-140\left(c 1, \mathrm{CH}_{2} \mathrm{Cl}_{2}\right)$; IR (thin film, $\left.\mathrm{cm}^{-1}\right)$ 2934, 2929, 1729, 1511, 1390, 1104, 1067, 849; ${ }^{1} \mathrm{H}$ NMR (600 MHz, $\left.\mathrm{CDCl}_{3}\right) \delta 7.49(\mathrm{~d}, J=8.4 \mathrm{~Hz}, 1 \mathrm{H}), 7.07(\mathrm{~d}, J=2.4$ Hz, 1H), $6.98(\mathrm{dd}, J=9.0,2.4 \mathrm{~Hz}, 1 \mathrm{H}), 6.14(\mathrm{dd}, J=2.4,1.2 \mathrm{~Hz}, 1 \mathrm{H}), 5.53(\mathrm{dd}, J=3.6$, $1.2 \mathrm{~Hz}, 1 \mathrm{H}), 3.39$ (s, 3H), 3.64 (ddd, $J=12.0,9.6,6.6 \mathrm{~Hz}, 1 \mathrm{H}), 2.93(\mathrm{~m}, 1 \mathrm{H}), 2.39$ (d, $J=$ $1.2 \mathrm{~Hz}, 3 \mathrm{H}), 2.11(\mathrm{~m}, 2 \mathrm{H}), 1.86(\mathrm{~m}, 2 \mathrm{H}), 1.18(\mathrm{~d}, J=6.0 \mathrm{~Hz}, 3 \mathrm{H}) ;{ }^{13} \mathrm{C}$ NMR $(150 \mathrm{MHz}$, $\left.\mathrm{CDCl}_{3}\right) \delta 161.2,159.8,155.0,152.3,125.3,114.2,113.5,112.3,103.9,94.9,80.2,69.2$ 56.5, 29.0, 23.0, 18.6, 18.1; CIHRMS Calcd for $\left[\mathrm{C}_{17} \mathrm{H}_{20} \mathrm{O}_{5} \mathrm{H}^{+}\right]$: 305.1389. Found 305.1384 . 


\section{$\mathrm{N}$-(7-(5,6-dihydro-5-methoxy-6-methyl-2H-pyran-2-yloxy)-8-methyl-2-oxo-2H-}

chromen-3-yl)acetamide (II-39).

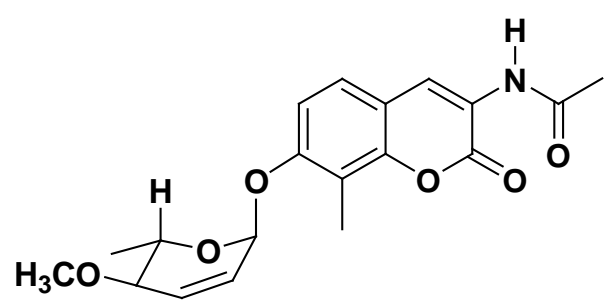

A THF (1.5 mL) solution of Piv-enol II-32 $(115 \mathrm{mg}, 0.50 \mathrm{mmol})$ and acetamido coumarin alcohol $5(128.7 \mathrm{mg}, 0.55 \mathrm{mmol})$ was cooled to $0{ }^{\circ} \mathrm{C}$. A THF $(0.5 \mathrm{~mL})$ solution of $\mathrm{Pd}_{2}(\mathrm{dba})_{3} \cdot \mathrm{CHCl}_{3}(21.4 \mathrm{mg}, 2.5 \mathrm{~mol} \%)$ and $\mathrm{PPh}_{3}(21.8 \mathrm{mg}, 10 \mathrm{~mol} \%)$ was added to the reaction mixture at $0{ }^{\circ} \mathrm{C}$. The reaction mixture was stirred at $0{ }^{\circ} \mathrm{C}$ for 4 hours. The reaction mixture was quenched with $5 \mathrm{~mL}$ of satd. aq. $\mathrm{NaHCO}_{3}$, extracted $(3 \times 5 \mathrm{~mL})$ with $\mathrm{Et}_{2} \mathrm{O}$, dried $\left(\mathrm{Na}_{2} \mathrm{SO}_{4}\right)$, and concentrated under reduced pressure. The crude product was purified using silica gel flash chromatography eluting with $40 \%$ EtOAc/hexanes to give coumarin-enol II-39 (158 g, $0.44 \mathrm{mmol}, 80 \%)$ as viscous oil. $R_{f}(50 \%$ EtOAc/hexane $)=0.373 ;[\alpha]_{\mathrm{D}}^{26}=-132\left(c=1.0, \mathrm{CH}_{2} \mathrm{Cl}_{2}\right) ; \mathrm{IR}\left(\right.$ thin film, $\left.\mathrm{cm}^{-1}\right) 2936$, $1698,1671,1604,1501,1365,1090,1031,976,808 ;{ }^{1} \mathrm{H}$ NMR $\left(600 \mathrm{MHz}, \mathrm{CDCl}_{3}\right) \delta 8.62$ (s, 1H), $7.97(\mathrm{~s}, 1 \mathrm{H}), 7.30(\mathrm{~d}, J=8.4 \mathrm{~Hz}, 1 \mathrm{H}), 7.18(\mathrm{~d}, J=9.0 \mathrm{~Hz}, 1 \mathrm{H}), 6.24(\mathrm{~d}, J=10.2$ Hz, 1H), 5.94 (ddd, $J=7.2,4.8,2.4 \mathrm{~Hz}, 1 \mathrm{H}), 5.65(\mathrm{~d}, J=2.4 \mathrm{~Hz}, 1 \mathrm{H}), 3.92(\mathrm{ddd}, J=$ 12.0, 9.6, $6.0 \mathrm{~Hz}, 1 \mathrm{H}), 3.54(\mathrm{ddd}, J=9.0,3.0,1.8 \mathrm{~Hz}, 1 \mathrm{H}), 3.45$ (s, 3H), 2.30 (s, 3H), $2.22(\mathrm{~s}, 3 \mathrm{H}), 1.29(\mathrm{~d}, J=6.0 \mathrm{~Hz}, 3 \mathrm{H}) ;{ }^{13} \mathrm{C} \mathrm{NMR}\left(150 \mathrm{MHz}, \mathrm{CDCl}_{3}\right) \delta$ 169.1, 159.1, 157.0, 149.0, 131.5, 125.5, 125.4, 124.2, 121.6, 115.5, 114.1, 112.5, 93.6, 77.8, 67.0, 56.7, 24.6, 18.1, 8.3; CIHRMS: Calculated for $\left[\mathrm{C}_{19} \mathrm{H}_{21} \mathrm{O}_{6} \mathrm{Na}^{+}\right]$: 382.1266, Found: 382.1262. 


\section{$N$-(8-methyl-2-oxo-7-(tetrahydro-3,4-dihydroxy-5-methoxy-6-methyl-2H-pyran-2-}

yloxy)-2H-chromen-3-yl)acetamide (II-40).

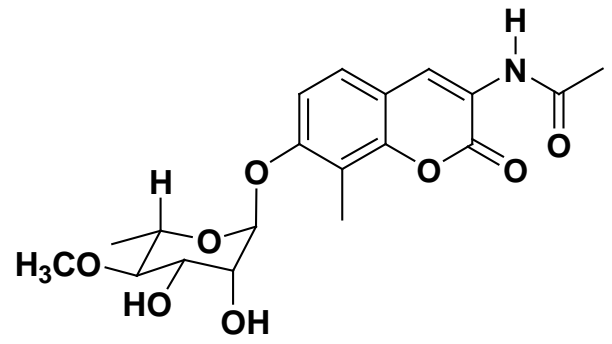

To a $\mathrm{CH}_{2} \mathrm{Cl}_{2}(1.0 \mathrm{~mL})$ solution of ene compound II-39 $(150 \mathrm{mg}, 0.417 \mathrm{mmol})$ at $0{ }^{\circ} \mathrm{C}$ was added a solution of $(50 \% \mathrm{w} / \mathrm{v})$ of $N$-methyl morpholine $N$-oxide / water $(208 \mu \mathrm{L})$. Crystalline $\mathrm{OsO}_{4}(6 \mathrm{mg}, 5 \mathrm{~mol} \%)$ was added and the reaction was stirred for $10 \mathrm{~h}$. The reaction mixture was concentrated and was pipetted directly on to a silica gel column using $\mathrm{CH}_{2} \mathrm{Cl}_{2}(1 \mathrm{~mL})$ in three portions. Impurities were eluted with ether and the product was eluted with EtOAc/Hexane (90:10). Pure fractions were combined and concentrated to afford diol II-40 (148 $\mathrm{mg}, 0.35 \mathrm{mmol}, 85 \%)$ as viscous oil. $R_{f}(100 \%$ EtOAc $)=0.30$; $[\alpha]_{\mathrm{D}}^{26}=-65\left(c=1, \mathrm{CH}_{2} \mathrm{Cl}_{2}\right) ; \mathrm{IR}$ (thin film, $\left.\mathrm{cm}^{-1}\right) 3336,2917,2950,1714,1607,1529$, 1376, 1250, 1120, 980; ${ }^{1} \mathrm{H}$ NMR (600 MHz, $\left.\mathrm{CDCl}_{3}\right) \delta 8.61(\mathrm{~s}, 1 \mathrm{H}), 7.98(\mathrm{~s}, 1 \mathrm{H}), 7.30(\mathrm{~d}$, $J=9.0 \mathrm{~Hz}, 1 \mathrm{H}), 7.15(\mathrm{~d}, J=9.0 \mathrm{~Hz}, 1 \mathrm{H}), 5.57(\mathrm{~d}, J=1.2 \mathrm{~Hz}, 1 \mathrm{H}), 4.21(\mathrm{dd}, J=5.4,3.0$ Hz, 1H), 4.06 (ddd, $J=9.0,8.4,3.0 \mathrm{~Hz}, 1 \mathrm{H}), 3.68(\mathrm{~m}, 1 \mathrm{H}), 3.59$ (s, 3H), 3.19 (dd, $J=$ 9.6, $9.6 \mathrm{~Hz}, 1 \mathrm{H}), 2.59$ (m, 2H), 2.29 (s, 3H), $2.23(\mathrm{~s}, 3 \mathrm{H}), 1.29$ (d, $J=6.0 \mathrm{~Hz}, 3 \mathrm{H}) ;{ }^{13} \mathrm{C}$ NMR $\left(150 \mathrm{MHz}, \mathrm{CDCl}_{3}\right) \delta 161.9,159.0,155.3,149.0,125.6,124.1,121.8,114.7,114.3$, 111.4, 97.4, 82.9, 71.3, 70.8, 68.5, 61.0, 24.6, 18.0, 8.2; CIHRMS Calcd for $\left[\mathrm{C}_{19} \mathrm{H}_{23} \mathrm{NO}_{8} \mathrm{Na}^{+}\right]:$416.1321. Found 416.1317. 


\section{$N$-(8-methyl-2-oxo-7-(tetrahydro-3-acetoxy,4-hydroxy-5-methoxy-6-methyl-2H- pyran-2-yloxy)-2H-chromen-3-yl)acetamide (II-42).}

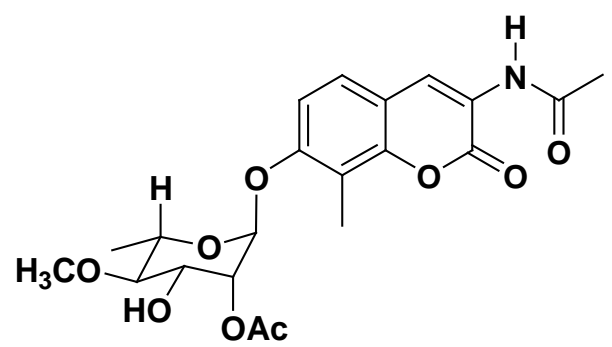

To a solution of diol II-40 ( $80 \mathrm{mg}, 0.20 \mathrm{mmol})$ and trimethyl orthoacetate $(130 \mu \mathrm{L}, 0.30$ mmol) mixture in THF $(0.4 \mathrm{~mL})$ was added $p$-toluenesulfonic acid monohydrate $(1.7 \mathrm{mg}$, $0.02 \mathrm{mmol}$ ), stirring for $0.5 \mathrm{~h}$. The solvent was removed under reduced pressure and residue was dissolved in $1.5 \mathrm{ml} \mathrm{THF} / \mathrm{H}_{2} \mathrm{O}(1: 1, \mathrm{v} / \mathrm{v})$ solution. Then $p$-toluenesulfonic acid (18.0 $\mathrm{mg} 0.15 \mathrm{mmol})$ was added. Stirring was continued until hydrolysis was complete as seen by TLC. The reaction was quenched with adding EtOAc and satd. $\mathrm{NaHCO}$. The organic layer was separated, dried $\left(\mathrm{Na}_{2} \mathrm{SO}_{4}\right)$, concentrated under reduced pressure and purified by silica gel flash chromatography eluting with $75 \%$ EtOAc/hexane to give acetate $\mathbf{I I - 4 2}(83 \mathrm{mg}, 0.19 \mathrm{mmol}, 95 \%)$ as white foam. $R f=0.30(50 \%$ EtOAc/Hexane); $[\alpha]_{\mathrm{D}}^{26}=-141\left(c=0.5, \mathrm{CH}_{2} \mathrm{Cl}_{2}\right) ; \mathrm{IR}$ (thin film, $\left.\mathrm{cm}^{-1}\right) 3448,2937,1728$, 1712, 1611, 1368, 1234, 1067, 990, 851; ${ }^{1} \mathrm{H}$ NMR $\left(600 \mathrm{MHz}, \mathrm{CDCl}_{3}\right) \delta 8.60(\mathrm{~s}, 1 \mathrm{H})$, $7.99(\mathrm{~s}, 1 \mathrm{H}), 7.28(\mathrm{~d}, J=8.4 \mathrm{~Hz}, 1 \mathrm{H}), 7.09(\mathrm{~d}, J=8.4 \mathrm{~Hz}, 1 \mathrm{H}), 5.52(\mathrm{~d}, J=1.8 \mathrm{~Hz}, 1 \mathrm{H})$, $5.31(\mathrm{dd}, J=3.6,1.8 \mathrm{~Hz}, 1 \mathrm{H}), 4.23(\mathrm{ddd}, J=9.0,5.4,3.0 \mathrm{~Hz}, 1 \mathrm{H}), 3.70(\mathrm{ddd}, J=12.0$, 9.6, $6.0 \mathrm{~Hz}, 1 \mathrm{H}), 3.61(\mathrm{~s}, 3 \mathrm{H}), 3.16(\mathrm{dd}, J=9.6,9.6 \mathrm{~Hz}, 1 \mathrm{H}), 2.33(\mathrm{~s}, 1 \mathrm{H}), 2.29(\mathrm{~s}, 3 \mathrm{H})$, $2.22(\mathrm{~s}, 3 \mathrm{H}), 2.20(\mathrm{~s}, 3 \mathrm{H}), 1.30(\mathrm{~d}, J=6.6 \mathrm{~Hz}, 3 \mathrm{H}) ;{ }^{13} \mathrm{C} \mathrm{NMR}\left(150 \mathrm{MHz}, \mathrm{CDCl}_{3}\right) \delta 170.6$, $169.2,158.9,155.2,149.0,125.6,124.0,121.9,114.5,111.4,95.6,83.0,79.9,72.1,69.8$, 
68.8, 61.0, 24.6, 20.9, 17.9, 8.2; CIHRMS: Calculated for $\left[\mathrm{C}_{21} \mathrm{H}_{25} \mathrm{NO}_{9} \mathrm{H}^{+}\right]$: 436.1607 . Found: 436.1604 .

\section{2-(N-(7-hydroxy-8-methyl-2-oxo-2H-chromen-3-yl)acetamide)-tetrahydro-3-}

hydroxy-5-methoxy-6-methyl-2H-pyran-4-yl carbamate (II-43).

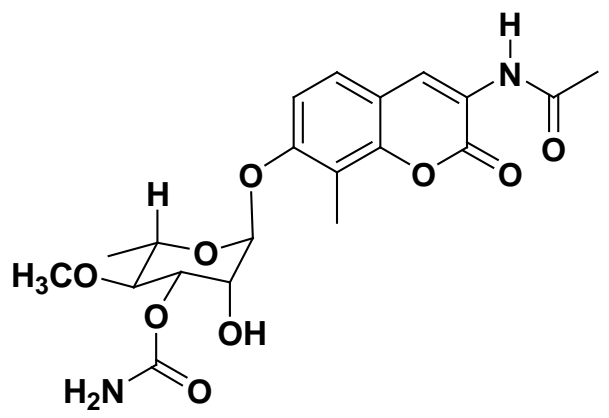

To a $\mathrm{CH}_{2} \mathrm{Cl}_{2}(0.9 \mathrm{~mL}, 0.1 \mathrm{M})$ solution of acetate alcohol II-42 (40 mg, $\left.0.10 \mathrm{mmol}\right)$ at 0 ${ }^{\circ} \mathrm{C}$ was added trichloro acetyl isocyanate $(20 \mu \mathrm{L}, 0.46 \mathrm{mmol})$ and the reaction was stirred for $1 \mathrm{~h}$. The solvent was removed under reduced pressure and residue was dissolved in $1.0 \mathrm{ml} \mathrm{MeOH} / \mathrm{H}_{2} \mathrm{O}(2: 1, \mathrm{v} / \mathrm{v})$ solution. Then potassium carbonate $(38 \mathrm{mg} 0.28 \mathrm{mmol})$ was added at $0{ }^{\circ} \mathrm{C}$. Stirring was continued until hydrolysis was complete as seen by TLC. The reaction was quenched with adding EtOAc and satd. NaHCO3. The organic layer was separated, dried $\left(\mathrm{Na}_{2} \mathrm{SO}_{4}\right)$, concentrated under reduced pressure and purified by silica gel flash chromatography eluting with MeOH/EtOAc/Hexane (10:40:50). Pure fractions were combined and concentrated to afford carbamate II-43 (31 mg, $0.07 \mathrm{mmol}$, $70 \%)$ as viscous oil. $R_{f}(70 \%$ EtOAc/Hexane $)=0.35 ;[\alpha]_{\mathrm{D}}^{26}=-42\left(c 0.5, \mathrm{CH}_{2} \mathrm{Cl}_{2}\right) ; \mathrm{IR}$ (thin film, $\mathrm{cm}^{-1}$ ) 3397, 3291, 2932, 1718, 1667, 1529, 1111, 1065, 990, 771; ${ }^{1} \mathrm{H} \mathrm{NMR}$ $\left(600 \mathrm{MHz}, \mathrm{CDCl}_{3}\right) \delta 8.40(\mathrm{~s}, 1 \mathrm{H}), 7.33(\mathrm{~d}, J=9.0 \mathrm{~Hz}, 1 \mathrm{H}), 7.14(\mathrm{~d}, J=8.4 \mathrm{~Hz}, 1 \mathrm{H}), 5.48$ $(\mathrm{d}, J=1.8 \mathrm{~Hz}, 1 \mathrm{H}), 5.09(\mathrm{dd}, J=9.6,3.0 \mathrm{~Hz}, 1 \mathrm{H}), 4.50(\mathrm{br} \mathrm{s}, 1 \mathrm{H}), 4.19(\mathrm{dd}, J=3.0,2.4$ 
Hz, 1H), $3.63(\mathrm{~m}, 1 \mathrm{H}), 3.55$ (s, 3H), 3.33 (dd, $J=9.6,9.6 \mathrm{~Hz}, 1 \mathrm{H}), 2.28$ (s, 3H), $2.16(\mathrm{~s}$, 3H), $2.11(\mathrm{br} \mathrm{s}, 1 \mathrm{H}), 1.21(\mathrm{~d}, J=6.0 \mathrm{~Hz}, 3 \mathrm{H})$; NMR (150 MHz, $\left.\mathrm{CDCl}_{3}\right) \delta$ 172.6, 159.1, 157.0, 126.9, 123.4, 115.8, 112.8, 99.9, 97.7, 84.2, 81.5, 75.6, 74.5, 70.4, 61.1, 45.9, 23.9, 23.1, 18.2, 8.4; CIHRMS Calcd for $\left[\mathrm{C}_{20} \mathrm{H}_{24} \mathrm{~N}_{2} \mathrm{O}_{9} \mathrm{Na}^{+}\right]$: 459.1379. Found 459.1376.

\section{$N$-(8-methyl-2-oxo-7-(tetrahydro-5-methoxy-6-methyl-2H-pyran-2-yloxy)-2H-} chromen-3-yl)acetamide (II-41).

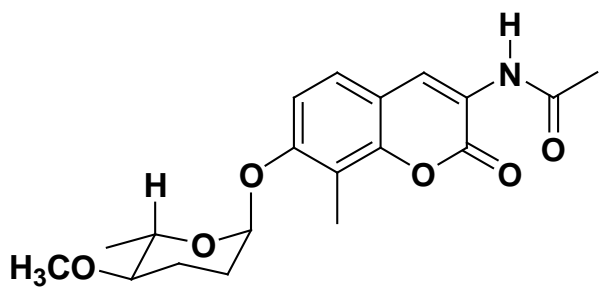

The ene compound II-39 (10 mg, $0.03 \mathrm{mmol})$ and $o-\mathrm{NO}_{2} \mathrm{C}_{6} \mathrm{H}_{4} \mathrm{SO}_{2} \mathrm{NHNH}_{2}(33 \mathrm{mg}, 0.16$ mmol) were dissolved in $0.2 \mathrm{~mL}$ of $\mathrm{CH}_{2} \mathrm{Cl}_{2}$ in a round bottom flask and cooled $0{ }^{\circ} \mathrm{C}$ under nitrogen atmosphere then triethylamine ( $39 \mu \mathrm{L}, 0.27 \mathrm{mmol}$ ) was added and the reaction mixture was stirred at $0{ }^{\circ} \mathrm{C}$ for 12 hours and on completion, as monitored by TLC. The reaction mixture was concentrated and was pipetted directly on to a silica gel column using $\mathrm{CH}_{2} \mathrm{Cl}_{2}(1 \mathrm{~mL})$ in three portions. The crude product was purified using silica gel flash chromatography eluting with $40 \%$ EtOAc/hexanes to give deoxy compound II-41 $(9.5 \mathrm{mg}, 0.02 \mathrm{mmol}, 95 \%)$ as viscous oil. $R_{f}(50 \%$ EtOAc/Hexane $)=$ $0.35 ;[\alpha]_{\mathrm{D}}^{26}=-23\left(c 1, \mathrm{CH}_{2} \mathrm{Cl}_{2}\right)$; IR (thin film, $\left.\mathrm{cm}^{-1}\right)$ 3352, 2951, 2929, 1712, 1677, 1374, 1138, 1086, 977, 783; ${ }^{1} \mathrm{H}$ NMR (600 MHz, $\left.\mathrm{CDCl}_{3}\right) \delta 8.61(\mathrm{~s}, 1 \mathrm{H}), 7.96(\mathrm{~s}, 1 \mathrm{H})$, $7.28(\mathrm{~d}, J=8.4 \mathrm{~Hz}, 1 \mathrm{H}), 7.15(\mathrm{~d}, J=8.4 \mathrm{~Hz}, 1 \mathrm{H}), 5.56(\mathrm{br} \mathrm{s}, 1 \mathrm{H}), 3.65(\mathrm{ddd}, J=12.0$, 9.0, 6.6 Hz, 1H), $3.40(\mathrm{~s}, 3 \mathrm{H}), 2.94(\mathrm{ddd}, J=10.8,9.0,4.8 \mathrm{~Hz}, 1 \mathrm{H}), 2.33(\mathrm{~s}, 3 \mathrm{H}), 2.22(\mathrm{~s}$, 
3H), $2.14(\mathrm{~m}, 2 \mathrm{H}), 1.90(\mathrm{~m}, 2 \mathrm{H}), 1.19(\mathrm{~d}, J=6.0 \mathrm{~Hz}, 3 \mathrm{H}) ;{ }^{13} \mathrm{C} \mathrm{NMR}\left(150 \mathrm{MHz}, \mathrm{CDCl}_{3}\right) \delta$ 169.1, 159.2, 156.2, 149.1, 125.5, 124.4, 121.4, 114.7, 113.8, 111.6, 94.8, 80.4, 69.2, 56.5, 29.3, 24.6, 23.2, 18.2, 8.2; CIHRMS Calcd for $\left[\mathrm{C}_{19} \mathrm{H}_{23} \mathrm{NO}_{6} \mathrm{Na}^{+}\right]$: 384.1423. Found 384.1418.

\section{$N$-(7-(5,6-dihydro-5-methoxy-6-methyl-2H-pyran-2-yloxy)-8-methyl-2-oxo-2H-} chromen-3-yl)-4-methoxy-3-3'-methoxyphenylbenzamide (II-45).

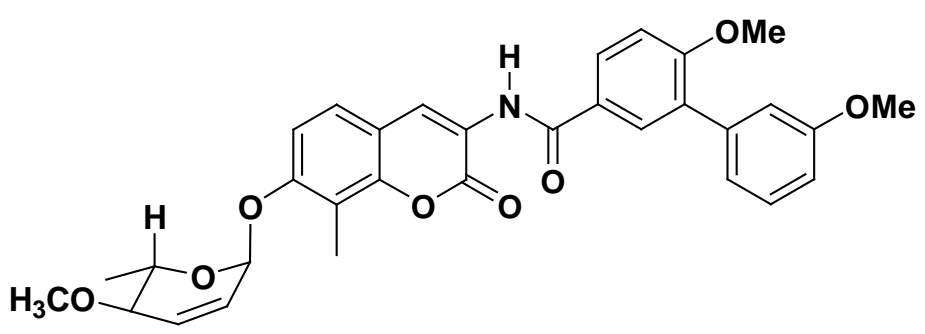

A THF (0.5 mL) solution of Piv-enol II-32 $(47 \mathrm{mg}, 0.28 \mathrm{mmol})$ and coumarin alcohol II$44(60 \mathrm{mg}, 0.14 \mathrm{mmol})$ was cooled to $0{ }^{\circ} \mathrm{C}$. A THF $(0.5 \mathrm{~mL})$ solution of $\mathrm{Pd}_{2}\left(\mathrm{dba}_{3}{ }_{3} \mathrm{CHCl}_{3}\right.$ (7.2 $\mathrm{mg}, 2.5 \mathrm{~mol} \%)$ and $\mathrm{PPh}_{3}(7.3 \mathrm{mg}, 10 \mathrm{~mol} \%)$ was added to the reaction mixture at 0 ${ }^{\circ} \mathrm{C}$. The reaction mixture was stirred at $0{ }^{\circ} \mathrm{C}$ for 5 hours. The reaction mixture was quenched with $5 \mathrm{~mL}$ of satd. aq. $\mathrm{NaHCO}_{3}$, extracted $(3 \times 5 \mathrm{~mL})$ with $\mathrm{Et}_{2} \mathrm{O}$, dried $\left(\mathrm{Na}_{2} \mathrm{SO}_{4}\right)$, and concentrated under reduced pressure. The crude product was purified using silica gel flash chromatography eluting with $35 \%$ EtOAc/hexanes to give coumarin-enol II-45 $(64 \mathrm{mg}, 0.12 \mathrm{mmol}, 83 \%)$ as viscous oil. $R_{f}(50 \%$ EtOAc/hexane $)=$ $0.30 ;[\alpha]^{25}=-26\left(c=1.0, \mathrm{CH}_{2} \mathrm{Cl}_{2}\right) ; \mathrm{IR}\left(\right.$ thin film, $\left.\mathrm{cm}^{-1}\right) 2934,1706,1669,1603,1364$, 1088, 939, 817; ${ }^{1} \mathrm{H}$ NMR (600 MHz, $\left.\mathrm{CDCl}_{3}\right) \delta 8.80$ (s, 1H), 8.70 (s, 1H), $7.92(\mathrm{dd}, J=$ 8.4, $2.4 \mathrm{~Hz}, 1 \mathrm{H}), 7.89(\mathrm{~d}, J=2.4 \mathrm{~Hz}, 1 \mathrm{H}), 7.36(\mathrm{~d}, J=8.4 \mathrm{~Hz}, 1 \mathrm{H}), 7.33(\mathrm{~d}, J=9.0 \mathrm{~Hz}$, 1H), $7.20(\mathrm{~d}, J=9.0 \mathrm{~Hz}, 1 \mathrm{H}), 7.12(\mathrm{~d}, J=7.8 \mathrm{~Hz}, 1 \mathrm{H}), 7.09(\mathrm{~d}, J=2.4,1.2 \mathrm{~Hz}, 1 \mathrm{H}), 7.06$ 
(d, $J=8.4 \mathrm{~Hz}, 1 \mathrm{H}), 6.93(\mathrm{dd}, J=7.8,3.0 \mathrm{~Hz}, 1 \mathrm{H}), 6.23(\mathrm{~d}, J=10.2 \mathrm{~Hz}, 1 \mathrm{H}), 5.94(\mathrm{td}, J=$ 12.6, $2.4 \mathrm{~Hz}, 1 \mathrm{H}), 5.66(\mathrm{br} \mathrm{s}, 1 \mathrm{H}), 3.93(\mathrm{~m}, 1 \mathrm{H}), 3.89(\mathrm{~s}, 3 \mathrm{H}), 3.85(\mathrm{~s}, 3 \mathrm{H}), 3.54(\mathrm{dd}, J=$ 10.2, $1.8 \mathrm{~Hz}, 1 \mathrm{H}), 3.46(\mathrm{~s}, 3 \mathrm{H}), 2.33(\mathrm{~s}, 3 \mathrm{H}), 1.30(\mathrm{~d}, J=6.0 \mathrm{~Hz}, 3 \mathrm{H}) ;{ }^{13} \mathrm{C}$ NMR $(150$ $\left.\mathrm{MHz}, \mathrm{CDCl}_{3}\right) \delta 165.4,159.7,159.4,159.3,157.0,149.0,138.6,131.5,131.0,129.9$, 129.1, 128.1, 126.1, 125.6, 125.4, 124.1, 122.0 (2C), 115.5, 115.2, 114.3, 113.1, 112.6, 111.0, 93.6, 77.8, 67.0, 56.7, 55.8, 55.3, 18.1, 8.3; CIHRMS: Calculated for $\left[\mathrm{C}_{32} \mathrm{H}_{31} \mathrm{NO}_{8} \mathrm{H}^{+}\right]:$558.2127, Found: 558.2126.

$N$-(7-(tetrahydro-3,4-dihydroxy-5-methoxy-6-methyl-2H-pyran-2-yloxy)-8-methyl-2oxo-2H-chromen-3-yl)-4-methoxy-3-3'-methoxyphenylbenzamide (II-46).

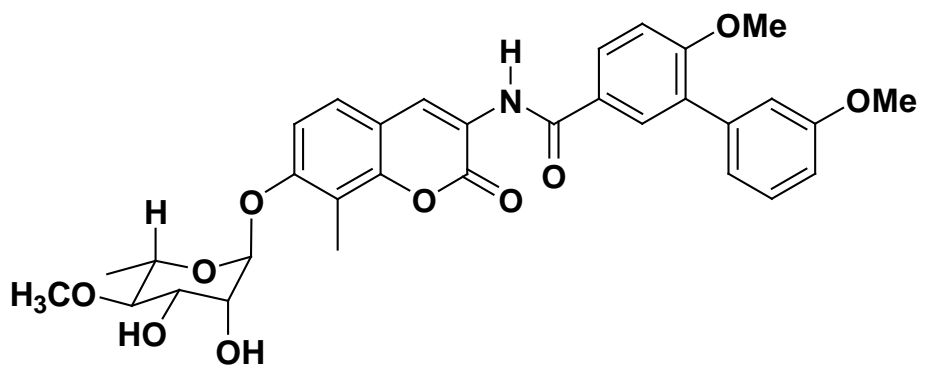

To a $\mathrm{CH}_{2} \mathrm{Cl}_{2}(0.8 \mathrm{~mL})$ solution of ene compound $\mathbf{I I}-45(50 \mathrm{mg}, 0.09 \mathrm{mmol})$ at $0{ }^{\circ} \mathrm{C}$ was added a solution of $(50 \% \mathrm{w} / \mathrm{v})$ of $N$-methyl morpholine $N$-oxide / water $(45 \mu \mathrm{L})$. Crystalline $\mathrm{OsO}_{4}(2 \mathrm{mg}, 5 \mathrm{~mol} \%)$ was added and the reaction was stirred for $12 \mathrm{~h}$. The reaction mixture was concentrated and was pipetted directly on to a silica gel column using $\mathrm{CH}_{2} \mathrm{Cl}_{2}(1 \mathrm{~mL})$ in three portions. Impurities were eluted with ether and the product was eluted with $\mathrm{MeOH} / \mathrm{EtOAc} / \mathrm{Hexane}$ (10:40:50). Pure fractions were combined and concentrated to afford diol II-46 (43 mg, $0.07 \mathrm{mmol}, 80 \%)$ as viscous oil. $R_{f}(80 \%$ EtOAc/Hexane $)=0.20 ;[\alpha]_{\mathrm{D}}^{26}=-25\left(c=1, \mathrm{CH}_{2} \mathrm{Cl}_{2}\right) ;$ IR (thin film, $\left.\mathrm{cm}^{-1}\right) 3465,2984$, 1737, 1670, 1371, 1291, 1140, 1091, 984; ${ }^{1} \mathrm{H}$ NMR $\left(600 \mathrm{MHz}, \mathrm{CDCl}_{3}\right) \delta 8.80(\mathrm{~s}, 1 \mathrm{H})$, 
$8.70(\mathrm{~s}, 1 \mathrm{H}), 7.92(\mathrm{dd}, J=6.0,2.4 \mathrm{~Hz}, 1 \mathrm{H}), 7.89(\mathrm{~d}, J=2.4 \mathrm{~Hz}, 1 \mathrm{H}), 7.35(\mathrm{~m}, 2 \mathrm{H}), 7.17$ $(\mathrm{d}, J=9.0 \mathrm{~Hz}, 1 \mathrm{H}), 7.12(\mathrm{~d}, J=9.0 \mathrm{~Hz}, 1 \mathrm{H}), 7.09(\mathrm{dd}, J=2.4,1.8 \mathrm{~Hz}, 1 \mathrm{H}), 7.07(\mathrm{~d}, J=$ $9.0 \mathrm{~Hz}, 1 \mathrm{H}), 6.93(\mathrm{dd}, J=8.4,1.8 \mathrm{~Hz}, 1 \mathrm{H}), 5.58(\mathrm{~d}, J=1.2 \mathrm{~Hz}, 1 \mathrm{H}), 4.22(\mathrm{dd}, J=3.6,1.2$ Hz, 1H), 4.09 (dd, $J=9.0,3.6 \mathrm{~Hz}, 1 \mathrm{H}), 3.90$ (s, 3H), 3.86 (s, 3H), 3.68 (ddd, $J$ = 9.6, 6.0, $3.6 \mathrm{~Hz}, 1 \mathrm{H}), 3.60(\mathrm{~s}, 3 \mathrm{H}), 3.20(\mathrm{dd}, J=9.6,9.6 \mathrm{~Hz}, 1 \mathrm{H}), 2.55(\mathrm{br} \mathrm{s}, 2 \mathrm{H}), 2.31(\mathrm{~s}, 3 \mathrm{H})$, $1.30(\mathrm{~d}, J=6.0 \mathrm{~Hz}, 3 \mathrm{H}) ;{ }^{13} \mathrm{C} \mathrm{NMR}\left(150 \mathrm{MHz}, \mathrm{CDCl}_{3}\right) \delta 165.5,159.8,159.3,155.3$, 149.0, 138.6, 131.1, 129.9, 129.1, 128.1, 126.0, 125.7, 124.0, 122.1, 122.0 (2C), 115.2, $114.7,114.5,113.1,111.4,111.0,97.4,82.9,71.3,70.5,68.5,61.0,55.8,55.3,18.0,8.2$; CIHRMS Calcd for $\left[\mathrm{C}_{32} \mathrm{H}_{33} \mathrm{NO}_{10} \mathrm{Na}^{+}\right]$: 614.2002. Found 614.2002.

\section{$N$-(7-(tetrahydro-3-acetoxy,4-hydroxy-5-methoxy-6-methyl-2H-pyran-2-yloxy)-8-} methyl-2-oxo-2H-chromen-3-yl)-4-methoxy-3-3'-methoxyphenylbenzamide (II-48).

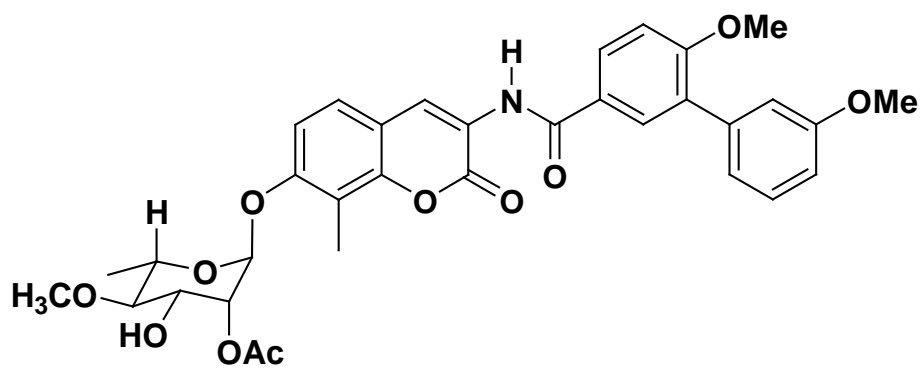

To a solution of diol II $\mathbf{I}-\mathbf{4 6}(65 \mathrm{mg}, 0.11 \mathrm{mmol})$ and trimethyl orthoacetate $(71 \mu \mathrm{L}, 0.55$ mmol) mixture in THF $(0.3 \mathrm{ml})$ was added $p$-toluenesulfonic acid monohydrate $(1.0 \mathrm{mg}$, $0.01 \mathrm{mmol}$ ), stirring for $0.5 \mathrm{~h}$. The solvent was removed under reduced pressure and residue was dissolved in $1.0 \mathrm{ml} \mathrm{THF} / \mathrm{H}_{2} \mathrm{O}(1: 1, \mathrm{v} / \mathrm{v})$ solution. Then $p$-toluenesulfonic acid $(10.0 \mathrm{mg} 0.05 \mathrm{mmol})$ was added. Stirring was continued until hydrolysis was complete as seen by TLC. The reaction was quenched with adding EtOAc and satd. NaHCO3. The organic layer was separated, dried $\left(\mathrm{Na}_{2} \mathrm{SO}_{4}\right)$, concentrated under reduced 
pressure and purified by silica gel flash chromatography eluting with $65 \%$ EtOAc/hexane to give acetate II-48 (64 mg, $0.10 \mathrm{mmol}, 92 \%)$ as white foam. $R f=0.37(10: 40: 50 \%$ $\mathrm{MeOH} /$ EtOAc/Hexane); $[\alpha]_{\mathrm{D}}^{26}=-6\left(c=1.0, \mathrm{CH}_{2} \mathrm{Cl}_{2}\right) ; \mathrm{IR}$ (thin film, $\left.\mathrm{cm}^{-1}\right) 3455,2980$, $1735,1693,1382,1290,1128,1042,978 ;{ }^{1} \mathrm{H}$ NMR $\left(600 \mathrm{MHz}, \mathrm{CDCl}_{3}\right) \delta 8.80(\mathrm{~d}, J=3.0$ Hz, 1H), $8.71(\mathrm{~s}, 1 \mathrm{H}), 7.92(\mathrm{dd}, J=9.0,2.4 \mathrm{~Hz}, 1 \mathrm{H}), 7.89(\mathrm{~d}, J=1.8 \mathrm{~Hz}, 1 \mathrm{H}), 7.36(\mathrm{~m}$, $1 \mathrm{H}), 7.16(\mathrm{~d}, J=8.4 \mathrm{~Hz}, 1 \mathrm{H}), 7.12(\mathrm{~m}, 1 \mathrm{H}), 7.09(\mathrm{~m}, 1 \mathrm{H}), 6.92(\mathrm{ddd}, J=8.4,2.4,1.2 \mathrm{~Hz}$, 1H), $5.54(\mathrm{br} \mathrm{s}, 1 \mathrm{H}), 5.37(\mathrm{dd}, J=9.0,3.0 \mathrm{~Hz}, 1 \mathrm{H}), 4.32(\mathrm{~m}, 1 \mathrm{H}), 4.12(\mathrm{dd}, J=13.8,7.2$ Hz, 1H), 3.90 (s, 3H), $3.86(\mathrm{~s}, 3 \mathrm{H}), 3.78(\mathrm{~m}, 1 \mathrm{H}), 3.62$ (br s, 1H), 3.51 (s, 3H), 3.69 (m, 1H), $2.36(\mathrm{~s}, 3 \mathrm{H}), 2.20(\mathrm{~s}, 3 \mathrm{H}), 2.17(\mathrm{dd}, J=13.8,3.6 \mathrm{~Hz}, 1 \mathrm{H}), 1.32(\mathrm{~d}, J=6.0 \mathrm{~Hz}, 1 \mathrm{H})$; ${ }^{13} \mathrm{C}$ NMR $\left(150 \mathrm{MHz}, \mathrm{CDCl}_{3}\right) \delta 170.6,169.8,165.5,159.8,159.3,155.2,149.1,138.6$, 131.1, 129.9, 129.1, 128.1, 125.6, 123.9, 122.0, 115.2, 113.1, 111.4, 111.0, 97.6, 65.7, 83.0, 79.9, 73.7, 69.5, 68.8, 60.5, 60.3, 55.8, 55.3, 21.1, 17.8, 14.1, 8.3; CIHRMS: Calculated for $\left[\mathrm{C}_{34} \mathrm{H}_{35} \mathrm{NO}_{11} \mathrm{Na}^{+}\right]$: 656.2107. Found: 656.2105 .

$N$-(7-(tetrahydro-3-hydroxy,4-carbamoyl-5-methoxy-6-methyl-2H-pyran-2-yloxy)-8methyl-2-oxo-2H-chromen-3-yl)-4-methoxy-3-3'-methoxyphenylbenzamide (II-49).

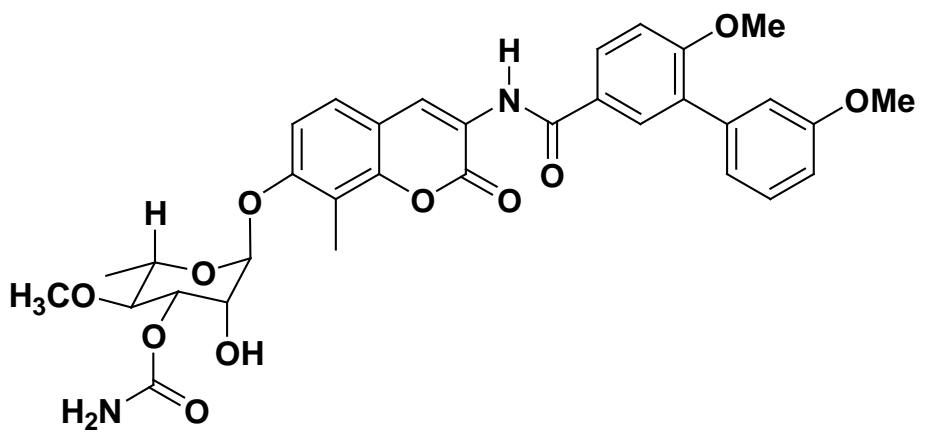

To a $\mathrm{CH}_{2} \mathrm{Cl}_{2}(0.5 \mathrm{~mL}, 0.1 \mathrm{M})$ solution of acetate alcohol II-48 $(35 \mathrm{mg}, 0.06 \mathrm{mmol})$ at $0{ }^{\circ} \mathrm{C}$ was added trichloro acetyl isocyanate $(11 \mu \mathrm{L}, 0.08 \mathrm{mmol})$ and the reaction was stirred for 
$1 \mathrm{~h}$. The solvent was removed under reduced pressure and residue was dissolved in 1.0 $\mathrm{ml} \mathrm{MeOH} / \mathrm{H}_{2} \mathrm{O}(2: 1, \mathrm{v} / \mathrm{v})$ solution. Then potassium carbonate $(22.9 \mathrm{mg} 0.17 \mathrm{mmol})$ was added at $0{ }^{\circ} \mathrm{C}$. Stirring was continued until hydrolysis was complete as seen by TLC. The reaction was quenched with adding EtOAc and satd. NaHCO3. The organic layer was separated, dried $\left(\mathrm{Na}_{2} \mathrm{SO}_{4}\right)$, concentrated under reduced pressure and purified by silica gel flash chromatography eluting with $\mathrm{MeOH} / \mathrm{EtOAc} / \mathrm{Hexane}$ (10:40:50). Pure fractions were combined and concentrated to afford carbamate II-49 (23 $\mathrm{mg}, 0.04 \mathrm{mmol}, 78 \%)$ as viscous oil. $R_{f}(70 \% \mathrm{EtOAc} / \mathrm{Hexane})=0.30 ;[\alpha]_{\mathrm{D}}^{26}=-43\left(c 0.5, \mathrm{CH}_{2} \mathrm{Cl}_{2}\right)$; IR (thin film, $\left.\mathrm{cm}^{-1}\right) 3434,2930,2852,1712,1606,1366,1265,1099,987,810 ;{ }^{1} \mathrm{H}$ NMR $(600 \mathrm{MHz}$, $\left.\mathrm{CDCl}_{3}\right) \delta 8.80(\mathrm{~s}, 1 \mathrm{H}), 7.92(\mathrm{dd}, J=6.6,2.4 \mathrm{~Hz}, 1 \mathrm{H}), 7.89(\mathrm{~d}, J=2.4 \mathrm{~Hz}, 1 \mathrm{H}), 7.35(\mathrm{~m}$, $3 \mathrm{H}), 7.15(\mathrm{~d}, J=8.4 \mathrm{~Hz}, 1 \mathrm{H}), 7.12(\mathrm{~d}, J=7.8 \mathrm{~Hz}, 1 \mathrm{H}), 7.09(\mathrm{dd}, J=2.4,1.8 \mathrm{~Hz}, 1 \mathrm{H})$, $6.93(\mathrm{dd}, J=8.4,2.4 \mathrm{~Hz}, 1 \mathrm{H}), 5.55(\mathrm{~d}, J=1.8 \mathrm{~Hz}, 1 \mathrm{H}), 5.26(\mathrm{dd}, J=9.0,3.6 \mathrm{~Hz}, 1 \mathrm{H})$, 4.36 (br s, 1H), 3.90 (s, 3H), 3.86 (s, 3H), $3.80(\mathrm{ddd}, J=9.6,6.0,5.4 \mathrm{~Hz}, 1 \mathrm{H}), 3.52$ (s, 3H), $3.36(\mathrm{~m}, 2 \mathrm{H}), 2.36(\mathrm{~s}, 3 \mathrm{H}), 2.17(\mathrm{~s}, 1 \mathrm{H}), 1.32(\mathrm{~d}, J=6.0 \mathrm{~Hz}, 3 \mathrm{H})$; NMR (150 MHz, $\left.\mathrm{CDCl}_{3}\right) \delta 160.0,159.5,155.6,149.2,138.8,131.2,130.1,129.3,128.3,126.1,125.9$ $125.8,124.3,122.2$ (2C), 115.4, 115.3, 114.7, 113.3, 111.7, 111.2, 98.1, 96.2, 80.2, 74.6, 69.4, 69.0, 60.5, 56.0, 55.5, 29.8, 18.0, 8.4; CIHRMS Calcd for $\left[\mathrm{C}_{33} \mathrm{H}_{34} \mathrm{~N}_{2} \mathrm{O}_{11} \mathrm{Na}^{+}\right]$: 657.2060. Found 657.2057.

\section{$N$-(7-(tetrahydro-5-methoxy-6-methyl-2H-pyran-2-yloxy)-8-methyl-2-oxo-2H- chromen-3-yl)-4-methoxy-3-3'-methoxyphenylbenzamide (II-47).}




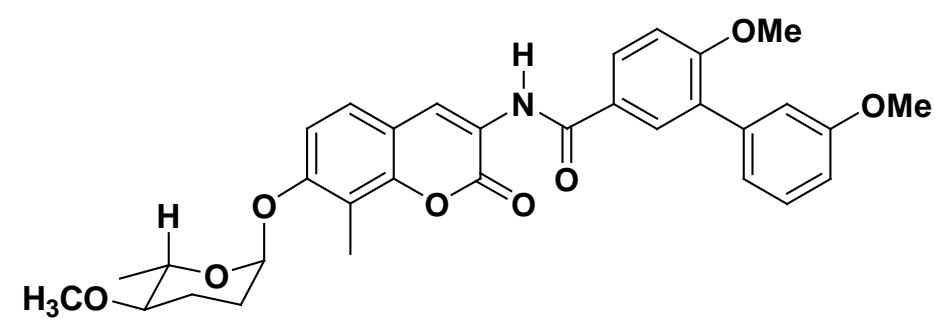

The ene compound II-45 (20 mg, $0.03 \mathrm{mmol})$ and $o-\mathrm{NO}_{2} \mathrm{C}_{6} \mathrm{H}_{4} \mathrm{SO}_{2} \mathrm{NHNH}_{2}(59 \mathrm{mg}, 0.29$ mmol) were dissolved in $0.3 \mathrm{~mL}$ of $\mathrm{CH}_{2} \mathrm{Cl}_{2}$ in a round bottom flask and cooled $0{ }^{\circ} \mathrm{C}$ under nitrogen atmosphere then triethylamine $(53 \mu \mathrm{L}, 0.36 \mathrm{mmol})$ was added and the reaction mixture was stirred at $0{ }^{\circ} \mathrm{C}$ for 12 hours and on completion, as monitored by TLC. The reaction mixture was concentrated and was pipetted directly on to a silica gel column using $\mathrm{CH}_{2} \mathrm{Cl}_{2}(1 \mathrm{~mL})$ in three portions. The crude product was purified using silica gel flash chromatography eluting with $30 \%$ EtOAc/hexanes to give deoxy compound II-47 (19 mg, $0.03 \mathrm{mmol}, 92 \%)$ as viscous oil. $R_{f}(50 \%$ EtOAc/Hexane $)=$ $0.35 ;[\alpha]_{\mathrm{D}}^{26}=-31\left(c 1, \mathrm{CH}_{2} \mathrm{Cl}_{2}\right) ; \mathrm{IR}\left(\right.$ thin film, $\left.\mathrm{cm}^{-1}\right)$ 2927, 2853, 1708, 1670, 1605, 1392, 1239, 1090, 979, 786; ${ }^{1} \mathrm{H}$ NMR $\left(600 \mathrm{MHz}, \mathrm{CDCl}_{3}\right) \delta 8.80(\mathrm{~s}, 1 \mathrm{H}), 8.70(\mathrm{~s}, 1 \mathrm{H})$, $7.92(\mathrm{dd}, J=8.4,2.4 \mathrm{~Hz}, 1 \mathrm{H}), 7.89(\mathrm{~d}, J=1.8 \mathrm{~Hz}, 1 \mathrm{H}), 7.34(\mathrm{~m}, 2 \mathrm{H}), 7.18(\mathrm{~d}, J=9.0 \mathrm{~Hz}$, 1H), $7.13(\mathrm{~d}, J=7.8 \mathrm{~Hz}, 1 \mathrm{H}), 7.09$ (dd, $J=2.4,1.8 \mathrm{~Hz}, 1 \mathrm{H}), 7.06(\mathrm{~s}, 1 \mathrm{H}), 6.93(\mathrm{dd}, J=$ 7.8, 2.4 Hz, 1H), 5.57 (br s, 1H), 3.89 (s, 3H), 3.86 (s, 3H), 3.67 (ddd, $J=9.6,6.0,4.8$ $\mathrm{Hz}, 1 \mathrm{H}), 3.50$ (s, 3H), 2.94 (ddd, $J=10.2,9.0,4.8 \mathrm{~Hz}, 1 \mathrm{H}), 2.35(\mathrm{~s}, 3 \mathrm{H}), 2.16(\mathrm{~m}, 2 \mathrm{H})$, $1.91(\mathrm{~m}, 2 \mathrm{H}), 1.19(\mathrm{~d}, J=6.0 \mathrm{~Hz}, 3 \mathrm{H}) ;{ }^{13} \mathrm{C} \mathrm{NMR}\left(150 \mathrm{MHz}, \mathrm{CDCl}_{3}\right) \delta 165.4,159.7$, $159.5,156.2,149.1,138.6,131.0,129.9,129.1,128.1,126.1,125.5,124.3,122.0,121.8$, $115.2,114.7,113.9,113.1,111.6,111.0,94.8,80.4,69.2,56.5,55.8,55.3,29.6,29.3$, 23.2, 18.2, 8.2; CIHRMS Calcd for $\left[\mathrm{C}_{32} \mathrm{H}_{33} \mathrm{NO}_{8} \mathrm{H}^{+}\right]$: 560.2284. Found 560.2281 


\section{5,6-dihydro-5-oxo-2H-pyran-2-yl pivalate (II-52).}

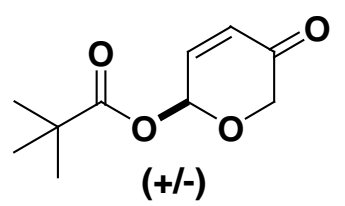

The furfuryl alcohol II-50 (20.0 g, $203.8 \mathrm{mmol}), 326 \mathrm{~mL}$ of THF, and $81 \mathrm{~mL}$ of $\mathrm{H}_{2} \mathrm{O}$ were added to a round bottom flask and cooled to $0{ }^{\circ} \mathrm{C}$. Solid $\mathrm{NaHCO}_{3}(34.2 \mathrm{~g}, 407.6$ mmol), $\mathrm{NaOAc} \cdot 3 \mathrm{H}_{2} \mathrm{O}(27.7 \mathrm{~g}, 203.8 \mathrm{mmol})$, and NBS (36.3 g, $\left.203.8 \mathrm{mmol}\right)$ were added to the solution and the mixture was stirred for $1 \mathrm{~h}$ at $0{ }^{\circ} \mathrm{C}$. The reaction was quenched with satd aq $\mathrm{NaHCO}_{3}(200 \mathrm{~mL})$, extracted $(3 \times 100 \mathrm{~mL})$ with $\mathrm{Et}_{2} \mathrm{O}$, dried $\left(\mathrm{Na}_{2} \mathrm{SO}_{4}\right)$, concentrated under reduced pressure and purified by silica gel chromatography eluting with 20\% EtOAc/hexanes to give hemiacetal II-51. The hemiacetal II-51 (16.0 g, 140.3 mmol) was dissolved in $\mathrm{CH}_{2} \mathrm{Cl}_{2}(350 \mathrm{~mL})$ and the solution was cooled to $-78{ }^{\circ} \mathrm{C}$. A $\mathrm{CH}_{2} \mathrm{Cl}_{2}(50 \mathrm{~mL})$ solution of Piv-Cl $(19.2 \mathrm{~mL}, 154.3 \mathrm{mmol})$, a catalytic amount of DMAP (856 mg, $7.0 \mathrm{mmol})$ and $\mathrm{Et}_{3} \mathrm{~N}(21 \mathrm{~mL}, 154.3 \mathrm{mmol})$ were added to the reaction mixture. The reaction was stirred for $6 \mathrm{~h}$ at $-78^{\circ} \mathrm{C}$. The reaction was quenched with $200 \mathrm{~mL}$ of satd. aq $\mathrm{NaHCO}_{3}$, extracted (3 x $150 \mathrm{~mL}$ ) with $\mathrm{Et}_{2} \mathrm{O}$, dried $\left(\mathrm{Na}_{2} \mathrm{SO}_{4}\right)$, and concentrated under reduced pressure. The crude product was purified using silica gel flash chromatography eluting with $10 \%$ EtOAc/hexanes to give Piv-enone II-52 (33.5 g, 169.1 mmol, $83 \%) . R_{f}(50 \%$ EtOAc/hexanes $)=0.52 ;$ IR $\left(\right.$ thin film, $\left.\mathrm{cm}^{-1}\right) 2955,2928,2859$, $1765,1713,1417,1379,1265,950 ;{ }^{1} \mathrm{H}$ NMR $\left(600 \mathrm{MHz}, \mathrm{CDCl}_{3}\right) \delta 6.92(\mathrm{dd}, J=10.2,3.6$ $\mathrm{Hz}, 1 \mathrm{H}), 6.46(\mathrm{~d}, J=3.6 \mathrm{~Hz}, 1 \mathrm{H}), 6.25(\mathrm{~d}, J=10.8 \mathrm{~Hz}, 1 \mathrm{H}), 4.47(\mathrm{~d}, J=16.8 \mathrm{~Hz}, 1 \mathrm{H})$, $4.20(\mathrm{~d}, J=16.8 \mathrm{~Hz}, 1 \mathrm{H}), 1.22(\mathrm{~s}, 9 \mathrm{H}) ;{ }^{13} \mathrm{C} \mathrm{NMR}\left(150 \mathrm{MHz}, \mathrm{CDCl}_{3}\right) \delta 193.4,176.9$, 142.4, 128.6, 86.5, 67.3, 39.2, 26.9 (3C); CIHRMS Calcd for $\left[\mathrm{C}_{10} \mathrm{H}_{14} \mathrm{O}_{4} \mathrm{Na}\right]^{+}: 221.0789$. Found 221.0784. 


\section{5,6-dihydro-5-hydroxy-2H-pyran-2-yl pivalate (II-52).}

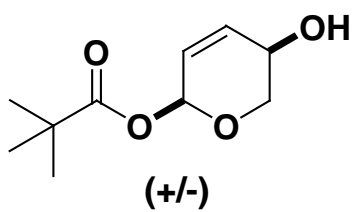

A solution of pyranone II-52 $(1.17 \mathrm{~g}, 5.46 \mathrm{mmol})$ in dry $\mathrm{CH}_{2} \mathrm{Cl}_{2}(4.5 \mathrm{~mL})$ and $0.4 \mathrm{M}$ $\mathrm{CeCl}_{3} / \mathrm{MeOH}(4.5 \mathrm{~mL})$ was cooled to $-78{ }^{\circ} \mathrm{C} . \mathrm{NaBH}_{4}(258 \mathrm{mg}, 5.46 \mathrm{mmol})$ was added and the reaction mixture was stirred for $2 \mathrm{~h}$ at $-0{ }^{\circ} \mathrm{C}$. The resulting solution was diluted with ether $(50 \mathrm{~mL})$ and was quenched with $25 \mathrm{~mL}$ of saturated $\mathrm{NaHCO}_{3}$, extracted $(3 \mathrm{x}$ $25 \mathrm{~mL})$ with $\mathrm{Et}_{2} \mathrm{O}$, dried $\left(\mathrm{Na}_{2} \mathrm{SO}_{4}\right)$, and concentrated under reduced pressure. The crude product was purified using silica gel chromatography eluting with $30 \%$ EtOAc/hexane to give enol II-53 (1.08 g, $5.02 \mathrm{mmol}, 92 \%)$ as a semi solid; $R f=0.38(50 \%$ EtOAc/Hexane); IR (thin film, $\mathrm{cm}^{-1}$ ) 3395 2965, 2938, 1674, 1519, 1245, 1054, 987; ${ }^{1} \mathrm{H}$ NMR $\left(600 \mathrm{MHz}, \mathrm{CDCl}_{3}\right) \delta 6.56$ (br s, 1H), 6.11 (dd, $\left.J=10.2,2.4 \mathrm{~Hz}, 1 \mathrm{H}\right), 5.75$ (ddd, $J$ $=10.2,2.4,2.4 \mathrm{~Hz}, 1 \mathrm{H}), 4.35(\mathrm{~m}, 1 \mathrm{H}), 3.90(\mathrm{~m}, 1 \mathrm{H}), 3.61(\mathrm{~m}, 1 \mathrm{H}), 2.05(\mathrm{~d}, J=7.2 \mathrm{~Hz}$, 1H), $1.21(\mathrm{~s}, 9 \mathrm{H}) ;{ }^{13} \mathrm{C}$ NMR $\left(150 \mathrm{MHz}, \mathrm{CDCl}_{3}\right) \delta 177.6,134.6,125.1,87.7,64.3,62.5$, 38.9, 26.9 (3C); CIHRMS Calcd for $\left[\mathrm{C}_{10} \mathrm{H}_{16} \mathrm{O}_{4} \mathrm{Na}\right]^{+}$: 223.0946. Found 223.0942.

\section{7-((2S,5R)-5,6-dihydro-5-hydroxy-2H-pyran-2-yloxy)-4-methyl-2H-chromen-2-one} (II-54).<smiles>Cc1cc(=O)oc2cc(OC3C=CC(O)CO3)ccc12</smiles> 
Method 1: A THF (1.0 mL) solution of Piv-enol II-53 (150 mg, $0.69 \mathrm{mmol})$ and 4methyl coumarin alcohol $5(61.1 \mathrm{mg}, 0.35 \mathrm{mmol})$ was cooled to $0{ }^{\circ} \mathrm{C}$. A THF $(0.3 \mathrm{~mL})$ solution of $\mathrm{Pd}_{2}(\mathrm{dba})_{3} \cdot \mathrm{CHCl}_{3}(18 \mathrm{mg}, 2.5 \mathrm{~mol} \%)$ and $R, R$-Trost ligand (27.5 $\left.\mathrm{mg}, 5 \mathrm{~mol} \%\right)$ was added to the reaction mixture at $0{ }^{\circ} \mathrm{C}$. The reaction mixture was stirred at $0{ }^{\circ} \mathrm{C}$ for 4 hours. The reaction mixture was quenched with $3 \mathrm{~mL}$ of satd. aq. $\mathrm{NaHCO}_{3}$, extracted (3 x $5 \mathrm{~mL})$ with $\mathrm{Et}_{2} \mathrm{O}$, dried $\left(\mathrm{Na}_{2} \mathrm{SO}_{4}\right)$, and concentrated under reduced pressure. The crude product was purified using silica gel flash chromatography eluting with 50\% EtOAc/hexanes to give coumarin-enol II-54 (143 mg, $0.52 \mathrm{mmol}, 75 \%)$ as viscous oil. Method 2: A THF (1.0 mL) solution of Piv-enol II-53 (130 mg, $0.60 \mathrm{mmol})$ and 4methyl coumarin alcohol $5(53 \mathrm{mg}, 0.30 \mathrm{mmol})$ was cooled to $0{ }^{\circ} \mathrm{C}$. A THF $(0.6 \mathrm{~mL})$ solution of $\mathrm{Pd}_{2}(\mathrm{dba})_{3} \cdot \mathrm{CHCl}_{3}(15 \mathrm{mg}, 2.5 \mathrm{~mol} \%)$ and $\mathrm{PPh}_{3}(15.7 \mathrm{mg}, 10 \mathrm{~mol} \%)$ was added to the reaction mixture at $0{ }^{\circ} \mathrm{C}$. The reaction mixture was stirred at $0{ }^{\circ} \mathrm{C}$ for 3 hours. The reaction mixture was quenched with $3 \mathrm{~mL}$ of satd. aq. $\mathrm{NaHCO}_{3}$, extracted $(3 \times 5 \mathrm{~mL})$ with $\mathrm{Et}_{2} \mathrm{O}$, dried $\left(\mathrm{Na}_{2} \mathrm{SO}_{4}\right)$, and concentrated under reduced pressure. The crude product was purified using silica gel flash chromatography eluting with 50-60\% EtOAc/hexanes to give coumarin-enol II-54 (140 $\mathrm{mg}, 0.51 \mathrm{mmol}, 85 \%)$ as viscous oil. $R_{f}(50 \%$ EtOAc/hexane $)=0.42 ;[\alpha]_{\mathrm{D}}^{26}=-145\left(c=1.0, \mathrm{CH}_{2} \mathrm{Cl}_{2}\right) ; \mathrm{IR}\left(\right.$ thin film, $\left.\mathrm{cm}^{-1}\right) 3440,2950$, 2942, 1731, 1613, 1389, 1134, 1067, 961; ${ }^{1} \mathrm{H}$ NMR $\left(600 \mathrm{MHz}, \mathrm{CDCl}_{3}\right) \delta 7.50(\mathrm{~d}, J=$ $10.2 \mathrm{~Hz}, 1 \mathrm{H}), 7.03$ (d, $J=2.4,1.2 \mathrm{~Hz}, 1 \mathrm{H}), 6.98(\mathrm{ddd}, J=7.2,3.6,1.2 \mathrm{~Hz}, 1 \mathrm{H}), 6.19$ (m, 1H), $6.14(\mathrm{dd}, J=7.2,2.4 \mathrm{~Hz}, 1 \mathrm{H}), 5.89$ (m, 1H), 5.67 (br s, 1H), 4.34 (m, 1H), 3.88 (dd, $J=10.8,6.0 \mathrm{~Hz}, 1 \mathrm{H}), 3.69(\mathrm{ddd}, J=18.6,8.4,1.8 \mathrm{~Hz}, 1 \mathrm{H}), 2.38(\mathrm{~d}, J=1.2 \mathrm{~Hz}, 3 \mathrm{H}), 2.06$ (br s, $1 \mathrm{H}) ;{ }^{13} \mathrm{C}$ NMR $\left(150 \mathrm{MHz}, \mathrm{CDCl}_{3}\right) \delta 161.4,160.0,154.8,152.5,135.2,125.5$, 
125.0, 114.6, 113.6, 112.4, 104.2, 92.4, 63.7, 62.3, 18.5; CIHRMS: Calculated for $\left[\mathrm{C}_{15} \mathrm{H}_{14} \mathrm{O}_{5} \mathrm{Na}^{+}\right]: 297.0739$, Found: 297.0736.

\section{7-((2S,3R,4R,5S)-tetrahydro-3,4,5-trihydroxy-2H-pyran-2-yloxy)-4-methyl-2H- chromen-2-one (II-55).}

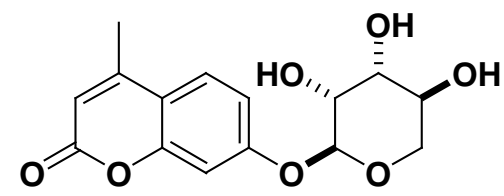

To a $\mathrm{CH}_{2} \mathrm{Cl}_{2}(3.6 \mathrm{~mL})$ solution of diene $\mathbf{I I}-\mathbf{5 4}(100 \mathrm{mg}, 0.36 \mathrm{mmol})$ at $0{ }^{\circ} \mathrm{C}$ was added a solution of $(50 \% \mathrm{w} / \mathrm{v})$ of $N$-methyl morpholine $N$-oxide / water $(0.2 \mathrm{~mL})$. Crystalline $\mathrm{OsO}_{4}(4.6 \mathrm{mg}, 5 \mathrm{~mol} \%)$ was added and the reaction was stirred for $12 \mathrm{~h}$. The reaction mixture was concentrated and was pipetted directly on to a silica gel column using $\mathrm{CH}_{2} \mathrm{Cl}_{2}(1 \mathrm{~mL})$ in three portions. Impurities were eluted with ether and the product was eluted with $\mathrm{MeOH} / \mathrm{EtOAc} / \mathrm{Hexane}$ (5:45:50). Pure fractions were combined and concentrated to afford diol II-55 (95 $\mathrm{mg}, 0.30 \mathrm{mmol}, 85 \%)$ as viscous oil. $R_{f}(60 \%$ EtOAc/Hexane $)=0.25 ;[\alpha]_{\mathrm{D}}^{26}=-50\left(c=1, \mathrm{CH}_{2} \mathrm{Cl}_{2}\right) ; \mathrm{IR}\left(\right.$ thin film, $\left.\mathrm{cm}^{-1}\right) 3420,2927$, $2939,1728,1655,1559,1371,1285,1117,1071,862 ;{ }^{1} \mathrm{H} \mathrm{NMR}\left(600 \mathrm{MHz}, \mathrm{CDCl}_{3}\right) \delta$ $7.70(\mathrm{~d}, J=9.0 \mathrm{~Hz}, 1 \mathrm{H}), 7.07(\mathrm{dd}, J=9.0,2.4 \mathrm{~Hz}, 1 \mathrm{H}), 7.03(\mathrm{~d}, J=2.4 \mathrm{~Hz}, 1 \mathrm{H}), 6.18(\mathrm{~d}$, $J=1.2 \mathrm{~Hz}, 1 \mathrm{H}), 5.49(\mathrm{~d}, J=3.0 \mathrm{~Hz}, 1 \mathrm{H}), 4.52(\mathrm{~s}, 1 \mathrm{H}), 3.98(\mathrm{t}, J=3.0 \mathrm{~Hz}, 1 \mathrm{H}), 3.86(\mathrm{~m}$, 2H), $3.77(\mathrm{ddd}, J=11.5,3.9,1.8 \mathrm{~Hz}, 1 \mathrm{H}), 3.48(\mathrm{~m}, 1 \mathrm{H}), 2.43(\mathrm{~d}, J=1.2 \mathrm{~Hz}, 3 \mathrm{H}) ;{ }^{13} \mathrm{C}$ NMR $\left(150 \mathrm{MHz}, \mathrm{CDCl}_{3}\right) \delta 163.3,161.1,156.0,155.4,127.3,116.0,114.8,112.8,104.8$, 100.2, 72.5, 71.0, 68.4, 65.1, 18.6; CIHRMS Calcd for $\left[\mathrm{C}_{15} \mathrm{H}_{16} \mathrm{O}_{7} \mathrm{Na}^{+}\right]$: 331.0793. Found 331.0791. 
(2S,6R)-6-[(2'S)-2'- $N$-Carbobenzyloxy-D-tyrosine methoxycarbonyl)-2-tert-butyldimethyl-silanyloxymethyl-6H-pyran-3-one (III-20).

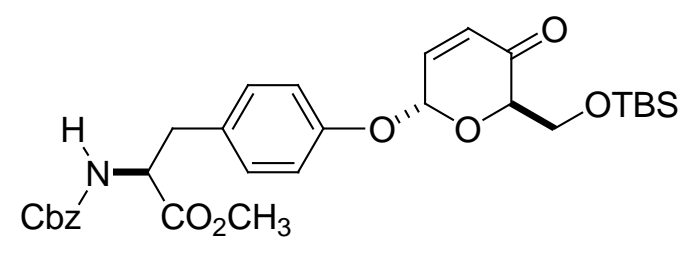

A $\mathrm{CH}_{2} \mathrm{Cl}_{2}(0.7 \mathrm{~mL})$ solution of compound III-18 $(260 \mathrm{mg}, 0.72 \mathrm{mmol})$ and Cbz-Dtyrosine methyl ester III-17 (238 mg, $0.726 \mathrm{mmol})$ was cooled to $0{ }^{\circ} \mathrm{C} . \mathrm{A} \mathrm{CH}_{2} \mathrm{Cl}_{2}(0.7$ $\mathrm{mL}$ ) solution of $\mathrm{Pd}_{2}(\mathrm{dba})_{3}{ }^{\cdot} \mathrm{CHCl}_{3}(18 \mathrm{mg}, 2.5 \mathrm{~mol} \%)$ and $\mathrm{PPh}_{3}(16 \mathrm{mg}, 10 \mathrm{~mol} \%)$ was added to the reaction mixture at $0{ }^{\circ} \mathrm{C}$. The reaction mixture was stirred at $0{ }^{\circ} \mathrm{C}$ for 3 hours. The reaction mixture was quenched with $5 \mathrm{~mL}$ of satd. aq. $\mathrm{NaHCO}_{3}$, extracted (3 x $5 \mathrm{~mL}$ ) with $\mathrm{Et}_{2} \mathrm{O}$, dried $\left(\mathrm{Na}_{2} \mathrm{SO}_{4}\right)$, and concentrated under reduced pressure. The crude product was purified using silica gel flash chromatography eluting with $14 \%$ EtOAc/hexanes to give enone III-20 (380 mg, $0.66 \mathrm{mmol}, 92 \%)$ as viscous oil. $R_{f}(30 \%$ EtOAc/hexanes $)=0.42 ;[\alpha]_{\mathrm{D}}^{26}=+0.8\left(c=1, \mathrm{CH}_{2} \mathrm{Cl}_{2}\right) ; \mathrm{IR}\left(\right.$ thin film, $\left.\mathrm{cm}^{-1}\right) 3351,2952$, $1752,1725,1509,1256,1219,1151,995,836 ;{ }^{1} \mathrm{H}$ NMR $\left(600 \mathrm{MHz}, \mathrm{CDCl}_{3}\right) \delta 7.35(\mathrm{~m}$, $5 \mathrm{H}), 7.05(\mathrm{~m}, 4 \mathrm{H}), 6.97(\mathrm{dd}, J=10.2,3.6 \mathrm{~Hz}, 1 \mathrm{H}), 6.22(\mathrm{~d}, J=10.8 \mathrm{~Hz}, 1 \mathrm{H}), 5.93(\mathrm{~d}, J=$ $3.6 \mathrm{~Hz}, 1 \mathrm{H}), 5.30(\mathrm{~d}, J=7.8 \mathrm{~Hz}, 1 \mathrm{H}), 5.10(\mathrm{~d}, J=12.6 \mathrm{~Hz}, 1 \mathrm{H}), 5.07(\mathrm{~d}, J=12.6 \mathrm{~Hz}$, $1 \mathrm{H}), 4.62(\mathrm{dd}, J=13.8,6.0 \mathrm{~Hz}, 1 \mathrm{H}), 4.54(\mathrm{dd}, J=4.8,3.0 \mathrm{~Hz}, 1 \mathrm{H}), 4.05(\mathrm{dd}, J=11.4$, $4.8 \mathrm{~Hz}, 1 \mathrm{H}), 4.02(\mathrm{dd}, J=11.4,2.4 \mathrm{~Hz}, 1 \mathrm{H}), 3.71(\mathrm{~s}, 3 \mathrm{H}), 3.10(\mathrm{dd}, J=13.2,5.4 \mathrm{~Hz}, 1 \mathrm{H})$, $3.04(\mathrm{dd}, J=14.4,6.0 \mathrm{~Hz}, 1 \mathrm{H}), 0.84(\mathrm{~s}, 9 \mathrm{H}), 0.05(\mathrm{~s}, 3 \mathrm{H}), 0.04(\mathrm{~s}, 3 \mathrm{H}) ;{ }^{13} \mathrm{C}$ NMR $(150$ $\left.\mathrm{MHz}, \mathrm{CDCl}_{3}\right) \delta 194.2,174.8,156.1,155.4,151.6,150.0,142.6,136.0,130.3(2 \mathrm{C}), 130.0$, 
128.6, 128.4(2C), 128.0, 127.9, 117.0, 91.9, 76.6, 66.8, 62.5, 54.7, 52.2, 37.2, 25.7(3C), 18.1, -5.44, -5.48; CIHRMS Calcd for $\left[\mathrm{C}_{30} \mathrm{H}_{39} \mathrm{NO}_{8} \mathrm{SiNa}^{+}\right]$: 592.2337. Found 592.2290.

\section{3-\{4-[6-(tert-Butyl-dimethylsilanyloxymethyl)-5-hydroxy-5,6-dihydro-2H-pyran-2-} yloxy]-phenyl\}-2- $N$-carbobenzyloxy-D-tyrosine methyl ester (III-21).

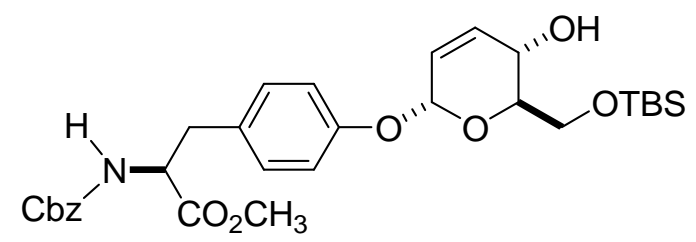

The enone compound III-20 (320 mg, $0.562 \mathrm{mmol}$ ) was dissolved in $0.5 \mathrm{~mL}$ of $\mathrm{CH}_{2} \mathrm{Cl}_{2}$ and $0.5 \mathrm{~mL} \mathrm{MeOH}$ in round bottom flask and cooled $-78{ }^{\circ} \mathrm{C}$ then $\mathrm{NaBH}_{4}(21 \mathrm{mg}, 0.562$ mmol) was added and the reaction mixture was stirred at $-78{ }^{\circ} \mathrm{C}$ for 3 hours and on completion, monitored by TLC, reaction mixture was diluted with ether and was quenched with $5 \mathrm{~mL}$ of satd. aq. $\mathrm{NaHCO}_{3}$, extracted $(3 \times 5 \mathrm{~mL})$ with $\mathrm{Et}_{2} \mathrm{O}$, dried $\left(\mathrm{Na}_{2} \mathrm{SO}_{4}\right)$, and concentrated under reduced pressure. The crude product was purified using silica gel flash chromatography eluting with $18 \%$ EtOAc/hexanes to give III-21 $(300 \mathrm{mg}, 0.525 \mathrm{mmol}, 87 \%)$ as viscous oil. $R_{f}(30 \%$ EtOAc/hexanes $)=0.30 ;[\alpha]_{\mathrm{D}}^{26}=+$ $29.0\left(c=1, \mathrm{CH}_{2} \mathrm{Cl}_{2}\right) ; \mathrm{IR}$ (thin film, $\left.\mathrm{cm}^{-1}\right) 3386,2925,1459,1255,1069,1027,838 ;{ }^{1} \mathrm{H}$ NMR (600 MHz, $\left.\mathrm{CDCl}_{3}\right) \delta 7.32(\mathrm{~m}, 5 \mathrm{H}), 6.98(\mathrm{~m}, 4 \mathrm{H}), 6.08(\mathrm{~d}, J=10.2 \mathrm{~Hz}, 1 \mathrm{H}), 5.88$ (ddd, $J=10.2,2.4,2.4 \mathrm{~Hz}, 1 \mathrm{H}), 5.57(\mathrm{~d}, J=1.8 \mathrm{~Hz}, 1 \mathrm{H}), 5.21(\mathrm{~d}, J=7.8 \mathrm{~Hz}, 1 \mathrm{H}), 5.11$ $(\mathrm{d}, J=13.2 \mathrm{~Hz}, 1 \mathrm{H}), 5.08(\mathrm{~d}, J=12.0 \mathrm{~Hz}, 1 \mathrm{H}), 4.62(\mathrm{dd}, J=13.2,5.4 \mathrm{~Hz}, 1 \mathrm{H}), 4.28(\mathrm{dd}$, $J=9.0,1.8 \mathrm{~Hz}, 1 \mathrm{H}), 3.89(\mathrm{dd}, J=10.2,4.8 \mathrm{~Hz}, 1 \mathrm{H}), 3.84(\mathrm{dqd}, J=12.0,7.2,4.8 \mathrm{~Hz}$, $1 \mathrm{H}), 3.75(\mathrm{dd}, J=9.6,6.6 \mathrm{~Hz}, 1 \mathrm{H}), 3.32(\mathrm{~s}, 3 \mathrm{H}), 3.08(\mathrm{dd}, J=13.8,5.4 \mathrm{~Hz}, 1 \mathrm{H}), 3.04$ (dd, $J=13.8,5.4 \mathrm{~Hz}, 1 \mathrm{H}), 3.02(\mathrm{~d}, J=3.0 \mathrm{~Hz}, 1 \mathrm{H}), 0.88(\mathrm{~s}, 9 \mathrm{H}), 0.08(\mathrm{~s}, 3 \mathrm{H}), 0.07$ (s, 
$3 \mathrm{H}) ;{ }^{13} \mathrm{C}$ NMR $\left(150 \mathrm{MHz}, \mathrm{CDCl}_{3}\right) \delta 171.9,156.5,133.3,130.3,130.2(2 \mathrm{C}), 128.5(2 \mathrm{C})$, $128.4,128.1,128.0,124.8,116.9(2 \mathrm{C}), 115.5,92.8,70.7,66.9,66.5,65.1,54.8,52.3$, 52.2, 37.2, 25.7(3C), 18.1, -5.5, -5.6; CIHRMS Calcd for $\left[\mathrm{C}_{30} \mathrm{H}_{41} \mathrm{NO}_{8} \mathrm{SiNa}^{+}\right]: 594.2493$ Found 594.2467.

$\left(1 ' S, 4^{\prime} S, 5 ' R, 1 S, 5 R\right)-1-\left[1^{\prime}-N\right.$-carbobenzyloxy-D-tyrosine methoxycarbonyl -5-(tertbutyl-dimethylsilanyloxymethyl)-1',4'-dihydro-5'H-pyran-4'-yloxy]-5-(tert-butyldimethylsilanyloxymethyl) -1H-pyran-4-one (III-16).

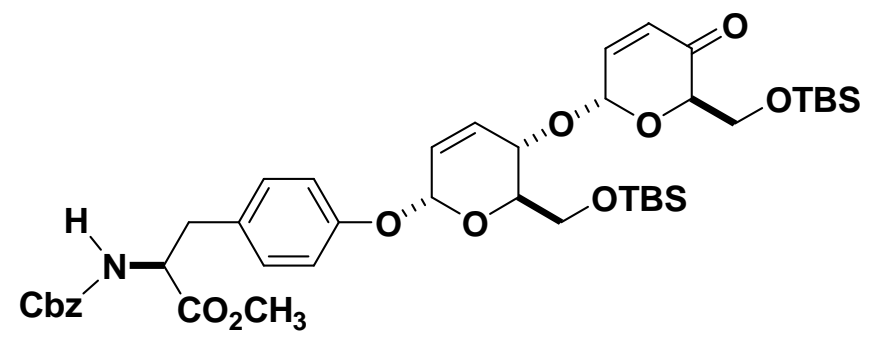

A $\mathrm{CH}_{2} \mathrm{Cl}_{2}(0.5 \mathrm{~mL})$ solution of compound III-16 $(280 \mathrm{mg}, 0.49 \mathrm{mmol})$ and alcohol III-21 (175 mg, $0.49 \mathrm{mmol})$ was cooled to $0{ }^{\circ} \mathrm{C}$. $\mathrm{A} \mathrm{CH}_{2} \mathrm{Cl}_{2}(0.5 \mathrm{~mL})$ solution of $\mathrm{Pd}_{2}\left(\mathrm{dba}_{3^{-}}\right.$ . $\mathrm{CHCl}_{3}(12 \mathrm{mg}, 2.5 \mathrm{~mol} \%)$ and $\mathrm{PPh}_{3}(11 \mathrm{mg}, 10 \mathrm{~mol} \%)$ was added to the reaction mixture at $0{ }^{\circ} \mathrm{C}$. The reaction mixture was stirred at $0{ }^{\circ} \mathrm{C}$ for 2 hours. The reaction mixture was quenched with $5 \mathrm{~mL}$ of satd. aq. $\mathrm{NaHCO}_{3}$, extracted $(3 \times 5 \mathrm{~mL})$ with $\mathrm{Et}_{2} \mathrm{O}$, dried $\left(\mathrm{Na}_{2} \mathrm{SO}_{4}\right)$, and concentrated under reduced pressure. The crude product was purified using silica gel flash chromatography eluting with $16 \%$ EtOAc/hexanes to give dienone III-16 (328 mg, $0.40 \mathrm{mmol}, 82 \%)$ as viscous oil. $R_{f}(30 \%$ EtOAc/hexanes $)=0.44 ;[\alpha]_{\mathrm{D}}^{26}$ $=+0.15\left(c=1, \mathrm{CH}_{2} \mathrm{Cl}_{2}\right) ; \mathrm{IR}\left(\right.$ thin film, $\left.\mathrm{cm}^{-1}\right) 3351,2952,1752,1725,1509,1256,1219$, 1151, 995, 836; ${ }^{1} \mathrm{H}$ NMR (600 MHz, $\left.\mathrm{CDCl}_{3}\right) \delta 7.35(\mathrm{~m}, 5 \mathrm{H}), 7.05(\mathrm{~m}, 4 \mathrm{H}), 6.84(\mathrm{dd}, J=$ $10.2,3.6 \mathrm{~Hz}, 1 \mathrm{H}), 6.23(\mathrm{~d}, J=10.8 \mathrm{~Hz}, 1 \mathrm{H}), 6.14(\mathrm{~d}, J=10.2 \mathrm{~Hz}, 1 \mathrm{H}), 5.95(\mathrm{ddd}, J=$ 
$10.2,2.4,1.8 \mathrm{~Hz}, 1 \mathrm{H}), 5.62(\mathrm{~d}, J=3.6 \mathrm{~Hz}, 1 \mathrm{H}), 5.60(\mathrm{~d}, J=1.8 \mathrm{~Hz}, 1 \mathrm{H}), 5.26(\mathrm{~d}, J=8.4$ $\mathrm{Hz}, 1 \mathrm{H}), 5.10(\mathrm{~d}, J=13.2 \mathrm{~Hz}, 1 \mathrm{H}), 5.07(\mathrm{~d}, J=12.6 \mathrm{~Hz}, 1 \mathrm{H}), 4.62(\mathrm{dd}, J=13.8,6.0 \mathrm{~Hz}$, $1 \mathrm{H}), 4.59(\mathrm{~d}, J=9.0 \mathrm{~Hz}, 1 \mathrm{H}), 4.44(\mathrm{dd}, J=3.6,2.4 \mathrm{~Hz}, 1 \mathrm{H}), 4.13(\mathrm{~d}, J=3.6 \mathrm{~Hz}, 1 \mathrm{H})$, $4.10(\mathrm{dd}, J=10.2,4.2 \mathrm{~Hz}, 1 \mathrm{H}), 3.96(\mathrm{dd}, J=10.2,2.4 \mathrm{~Hz}, 1 \mathrm{H}), 3.86(\mathrm{dq}, J=9.6,1.8 \mathrm{~Hz}$, $1 \mathrm{H}), 3.81(\mathrm{dd}, J=10.2,1.8 \mathrm{~Hz}, 1 \mathrm{H}), 3.70(\mathrm{~s}, 3 \mathrm{H}), 3.08(\mathrm{dd}, J=13.8,5.4 \mathrm{~Hz}, 1 \mathrm{H}), 3.03$ (dd, $J=13.8,6.0 \mathrm{~Hz}, 1 \mathrm{H}), 0.86(\mathrm{~s}, 9 \mathrm{H}), 0.85$ (s, 9H), 0.06 (s, 3H), 0.04 (s, 3H), 0.03 (s, 3H), $0.01(\mathrm{~s}, 3 \mathrm{H}) ;{ }^{13} \mathrm{C} \mathrm{NMR}\left(150 \mathrm{MHz}, \mathrm{CDCl}_{3}\right) \delta$ 194.5, 171.8, 156.4, 155.5, 143.8, 136.1, 130.1(2C), 130.0, 129.0, 128.5, 128.4(2C), 128.0, 127.9(2C), 126.4, 116.9, 92.8, $89.7,76.5,71.1,66.8,66.3,62.8,62.4,60.2,54.7,52.1,37.2,25.78(3 \mathrm{C}), 25.76(3 \mathrm{C})$, 18.28, 18.23, -5.4, -5.3, -5.4, -5.2; CIHRMS Calcd for $\left[\mathrm{C}_{42} \mathrm{H}_{61} \mathrm{NO}_{11} \mathrm{Si}_{2} \mathrm{Na}^{+}\right]:$834.3675. Found 834.3638.

$(1 ' S, 4 ' S, 5 ' R, 1 S, 4 S, 5 R)$-1-[ $N$-carbobenzyloxy-D-tyrosine methoxycarbonyl -5'-(tertbutyl-dimethylsilanyloxymethyl)-1',4'-dihydro-5'H-pyran-4'-yloxy]-5-(tert-butyldimethylsilanyloxymethyl)-1,4-dihydro-5H-pyran-4-ol (III-22).

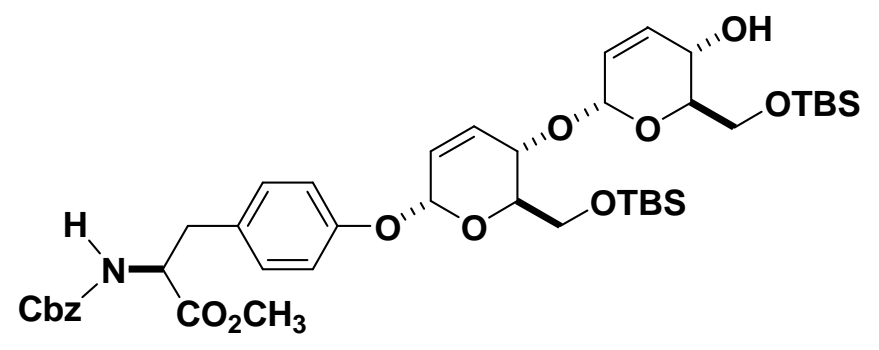

The enone compound III-16 (200 mg, $0.246 \mathrm{mmol}$ ) was dissolved in $0.3 \mathrm{~mL}$ of $\mathrm{CH}_{2} \mathrm{Cl}_{2}$ and $0.3 \mathrm{~mL} \mathrm{MeOH}$ were added to a round bottom flask and cooled to $-78{ }^{\circ} \mathrm{C}$ then $(9 \mathrm{mg}$, 
$0.246 \mathrm{mmol}) \mathrm{NaBH}_{4}$ was added and the reaction mixture was stirred at $-78^{\circ} \mathrm{C}$ for 6 hours and on completion, as monitored by TLC, reaction mixture is diluted with ether and was quenched with $5 \mathrm{~mL}$ of satd aq $\mathrm{NaHCO}_{3}$, extracted $(3 \times 5 \mathrm{~mL})$ with $\mathrm{Et}_{2} \mathrm{O}$, dried $\left(\mathrm{Na}_{2} \mathrm{SO}_{4}\right)$, and concentrated under reduced pressure. The crude product was purified using silica gel flash chromatography eluting with $20 \%$ EtOAc/hexanes to give enol III$22(182 \mathrm{mg}, 0.223 \mathrm{mmol}, 91 \%)$ as viscous oil. $R_{f}(40 \%$ EtOAc/hexanes $)=0.44 ;[\alpha]_{\mathrm{D}}^{26}=$ + $49\left(c=1, \mathrm{CH}_{2} \mathrm{Cl}_{2}\right)$; IR (thin film, $\left.\mathrm{cm}^{-1}\right) 3420,2930,2855,1725,1717,1510,1256$, 1219, 1151, 995, 836; ${ }^{1} \mathrm{H}$ NMR (600 MHz, $\left.\mathrm{CDCl}_{3}\right) \delta 7.35(\mathrm{~m}, 5 \mathrm{H}), 7.05(\mathrm{~m}, 4 \mathrm{H}), 6.15(\mathrm{~d}$, $J=10.2 \mathrm{~Hz}, 1 \mathrm{H}), 5.96(\mathrm{~d}, J=10.2 \mathrm{~Hz}, 1 \mathrm{H}), 5.92(\mathrm{dq}, J=10.2,1.2 \mathrm{~Hz}, 1 \mathrm{H}), 5.68(\mathrm{dd}, J=$ $10.2,2.4 \mathrm{~Hz}, 1 \mathrm{H}), 5.59(\mathrm{~d}, J=1.8 \mathrm{~Hz}, 1 \mathrm{H}), 5.25(\mathrm{~d}, J=7.8 \mathrm{~Hz}, 1 \mathrm{H}), 5.18(\mathrm{~d}, J=1.8 \mathrm{~Hz}$, $1 \mathrm{H}), 5.10(\mathrm{~d}, J=12.6 \mathrm{~Hz}, 1 \mathrm{H}), 5.08(\mathrm{~d}, J=12.6 \mathrm{~Hz}, 1 \mathrm{H}), 4.62(\mathrm{dd}, J=13.2,5.4 \mathrm{~Hz}, 1 \mathrm{H})$, $4.37(\mathrm{~d}, J=9.0 \mathrm{~Hz}, 1 \mathrm{H}), 4.24(\mathrm{~d}, J=8.4 \mathrm{~Hz}, 1 \mathrm{H}), 3.97(\mathrm{dd}, J=9.6,4.2 \mathrm{~Hz}, 1 \mathrm{H}), 3.89$ (ddd, $J=10.2,5.4,2.4 \mathrm{~Hz}, 1 \mathrm{H}), 3.85(\mathrm{dd}, J=10.2,1.8 \mathrm{~Hz}, 1 \mathrm{H}), 3.81(\mathrm{dd}, J=12.0,5.4$ Hz, 1H), 3.75 (dd, $J=10.2,7.2 \mathrm{~Hz}, 1 \mathrm{H}), 3.70(\mathrm{~s}, 3 \mathrm{H}), 3.71(\mathrm{~m}, 1 \mathrm{H}), 3.08(\mathrm{~d}, J=3.0 \mathrm{~Hz}$, 1H), 3.05 (dd, $J=8.4,6.0 \mathrm{~Hz}, 1 \mathrm{H}), 3.02(\mathrm{~d}, J=6.0 \mathrm{~Hz}, 1 \mathrm{H}), 0.91$ (s, 9H), $0.84(\mathrm{~s}, 9 \mathrm{H})$, $0.12(\mathrm{~s}, 3 \mathrm{H}), 0.11(\mathrm{~s}, 3 \mathrm{H}), 0.03(\mathrm{~s}, 3 \mathrm{H}), 0.02(\mathrm{~s}, 3 \mathrm{H}) ;{ }^{13} \mathrm{C} \mathrm{NMR}\left(150 \mathrm{MHz}, \mathrm{CDCl}_{3}\right) \delta$ $174.7,171.8,156.5,155.5,136.1,133.1,130.5,130.0(2 \mathrm{C}), 128.4(2 \mathrm{C}), 128.0,127.9(2 \mathrm{C})$, 126.0, 125.3, 117.1(2C), 92.7, 91.0, 71.3, 70.1, 66.8, 66.6, 66.5, 65.1, 62.6, 54.8, 52.1, 37.2, 25.8(3C), 25.7(3C), 18.3, 18.1, -5.1, -5.2, -5.5, -5.6; CIHRMS Calcd for $\left[\mathrm{C}_{42} \mathrm{H}_{63} \mathrm{NO}_{11} \mathrm{Si}_{2} \mathrm{Na}^{+}\right]:$836.3831. Found 836.3818.

(1'S,4'S,5'R,1S,4S,5R)-1-[1'- $N$-carbobenzyloxy-D-tyrosine methoxycarbonyl -5'(tert-butyl-dimethylsilanyloxymethyl)-1',4'-dihydro-5'H-pyran-4'-yloxy] -5-(tert- 


\section{butyl-dimethylsilanyloxymethyl)-1,4-dihydro-5H-pyran-O-4'-isovalaric ester (III-}

23).

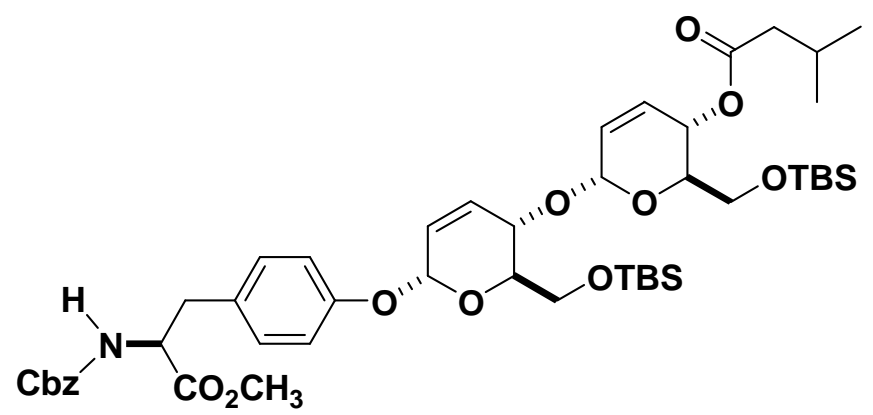

The alcohol compound III-22 (140 mg, $0.171 \mathrm{mmol})$, isovaleric acid (21 mg, 0.206 mmol) and DCC (42 mg, $0.206 \mathrm{mmol}$ ) were dissolved in $0.3 \mathrm{~mL}$ of $\mathrm{CH}_{2} \mathrm{Cl}_{2}$ in a round bottom flask and cooled to $0{ }^{\circ} \mathrm{C}$ then DMAP ( $\left.2 \mathrm{mg}, 0.01 \mathrm{mmol}\right)$ was added and the reaction mixture was stirred at $0{ }^{\circ} \mathrm{C}$ for 6 hours and on completion, as monitored by TLC, the reaction mixture was diluted with ether and was quenched with $5 \mathrm{~mL}$ of satd. aq. $\mathrm{NaHCO}_{3}$, extracted $(3 \times 5 \mathrm{~mL})$ with $\mathrm{Et}_{2} \mathrm{O}$, dried $\left(\mathrm{Na}_{2} \mathrm{SO}_{4}\right)$, and concentrated under reduced pressure. The crude product was purified using silica gel flash chromatography eluting with $15 \%$ EtOAc/hexanes to give III-23 (148 $\mathrm{mg}, 0.164 \mathrm{mmol}, 96 \%)$ as viscous oil. $R_{f}(30 \%$ EtOAc/hexanes $)=0.62 ;[\alpha]_{\mathrm{D}}^{26}=+90\left(c=1, \mathrm{CH}_{2} \mathrm{Cl}_{2}\right) ; \mathrm{IR}$ (thin film, $\left.\mathrm{cm}^{-1}\right)$ 2956, 2928, 1738, 1733, 1510, 1253, 1219, 1123, 986, 835; ${ }^{1} \mathrm{H}$ NMR $(600 \mathrm{MHz}$, $\left.\mathrm{CDCl}_{3}\right) \delta 7.34(\mathrm{~m}, 5 \mathrm{H}), 7.00(\mathrm{~m}, 4 \mathrm{H}), 6.20(\mathrm{dd}, J=10.2,2.4 \mathrm{~Hz}, 1 \mathrm{H}), 5.93(\mathrm{~m}, 2 \mathrm{H}), 5.74$ $(\mathrm{dd}, J=10.2,1.8 \mathrm{~Hz}, 1 \mathrm{H}), 5.59(\mathrm{~d}, J=1.2 \mathrm{~Hz}, 1 \mathrm{H}), 5.42(\mathrm{dd}, J=10.2,1.8 \mathrm{~Hz}, 1 \mathrm{H}), 5.28$ $(\mathrm{d}, J=1.2 \mathrm{~Hz}, 1 \mathrm{H}), 5.24(\mathrm{~d}, J=8.4 \mathrm{~Hz}, 1 \mathrm{H}), 5.10(\mathrm{~d}, J=12.6 \mathrm{~Hz}, 1 \mathrm{H}), 5.08(\mathrm{~d}, J=12.6$ $\mathrm{Hz}, 1 \mathrm{H}), 4.62(\mathrm{dd}, J=13.2,5.4 \mathrm{~Hz}, 1 \mathrm{H}), 4.42(\mathrm{~d}, J=9.0 \mathrm{~Hz}, 1 \mathrm{H}), 3.89(\mathrm{ddd}, J=10.2$, 5.4, $2.4 \mathrm{~Hz}, 1 \mathrm{H}), 3.85$ (dd, $J=10.2,1.8 \mathrm{~Hz}, 1 \mathrm{H}), 3.81(\mathrm{dd}, J=11.4,5.4 \mathrm{~Hz}, 1 \mathrm{H}), 3.78$ $(\mathrm{dd}, J=10.2,1.8 \mathrm{~Hz}, 1 \mathrm{H}), 3.75(\mathrm{~m}, 1 \mathrm{H}), 3.70(\mathrm{~s}, 3 \mathrm{H}), 3.71(\mathrm{~m}, 1 \mathrm{H}), 3.08(\mathrm{dd}, J=13.8$, 
$5.4 \mathrm{~Hz}, 1 \mathrm{H}), 3.04(\mathrm{dd}, J=13.8,6.0 \mathrm{~Hz}, 1 \mathrm{H}), 2.20(\mathrm{~d}, J=1.2 \mathrm{~Hz}, 2 \mathrm{H}), 2.10(\mathrm{~m}, 1 \mathrm{H}), 0.96$ (d, $J=6.6 \mathrm{~Hz}, 6 \mathrm{H}), 0.89$ (s, 9H), $0.85(\mathrm{~s}, 9 \mathrm{H}), 0.05(\mathrm{~s}, 6 \mathrm{H}), 0.03(\mathrm{~s}, 3 \mathrm{H}), 0.02(\mathrm{~s}, 3 \mathrm{H}),{ }^{13} \mathrm{C}$ NMR $\left(150 \mathrm{MHz}, \mathrm{CDCl}_{3}\right) \delta 172.1,171.8,156.6,155.5,136.2,130.3,130.0(2 \mathrm{C}), 129.8$, 128.4(2C), 128.0(2C), 127.9, 127.2, 126.1, 117.2(2C), 92.9, 90.9, 71.4, 69.6, 66.8, 66.3, 64.6, 62.8, 62.1, 54.7, 52.1, 43.3, 37.2, 25.9, 25.8(6C), 25.6, 22.3, 22.2 18.38, 18.36, -5.1, -5.2, -5.41, -5.45; CIHRMS Calcd for $\left[\mathrm{C}_{47} \mathrm{H}_{71} \mathrm{NO}_{12} \mathrm{Si}_{2} \mathrm{Na}^{+}\right]$: 920.4407. Found 920.4385.

\section{1'- $N$-carbobenzyloxy-D-tyrosine methoxycarbonyl -5',5-(tert-butyl- dimethylsilanyloxymethyl)-di-1,4- $\alpha$-D-mannose $-O-4$ ' isovalaric ester (III-3a).}

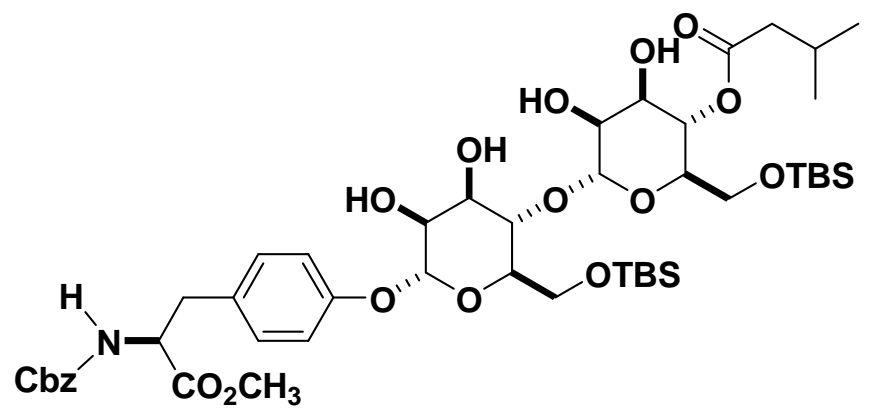

To a $t$-butanol-acetone $(0.2 \mathrm{~mL}, 1: 1)$ solution of diene ester III-23 (100 mg, $0.11 \mathrm{mmol})$ at $0{ }^{\circ} \mathrm{C}$ was added a solution of $(50 \%$ w/v) of $N$-methyl morpholine $N$-oxide / water $(0.1$ $\mathrm{mL})$. Crystalline $\mathrm{OsO}_{4}(1.4 \mathrm{mg}, 5 \mathrm{~mol} \%)$ was added and the reaction was stirred for 12 h. The reaction mixture was concentrated and was pipetted directly on to a silica gel column using $\mathrm{CH}_{2} \mathrm{Cl}_{2}(1 \mathrm{~mL})$ in three portions. Impurities were eluted with ether and the product was eluted with $\mathrm{MeOH} /$ ether (2:98 to 4:96). Pure fractions were combined and concentrated to afford bis- $\alpha$-D-manno-tetrol III-3a (91 $\mathrm{mg}, 0.094 \mathrm{mmol}, 85 \%$ ) as viscous oil. $R_{f}(90 \% \mathrm{EtOAc} / \mathrm{MeOH})=0.56 ;[\alpha]_{\mathrm{D}}^{26}=+61.9\left(c=2, \mathrm{CH}_{2} \mathrm{Cl}_{2}\right)$; IR (thin film, $\left.\mathrm{cm}^{-1}\right)$ 2956, 2928, 1738, 1733, 1510, 1253, 1219, 1123, 986, 835; ${ }^{1} \mathrm{H}$ NMR (600 
$\left.\mathrm{MHz}, \mathrm{CDCl}_{3}\right) \delta 7.31(\mathrm{~m}, 5 \mathrm{H}), 6.99(\mathrm{~m}, 4 \mathrm{H}), 5.45(\mathrm{~d}, J=1.2 \mathrm{~Hz}, 1 \mathrm{H}), 5.36(\mathrm{~d}, J=1.8 \mathrm{~Hz}$, $1 \mathrm{H}), 5.26(\mathrm{~d}, J=8.4 \mathrm{~Hz}, 1 \mathrm{H}), 5.10(\mathrm{~d}, J=12.6 \mathrm{~Hz}, 1 \mathrm{H}), 5.07(\mathrm{~d}, J=12.6 \mathrm{~Hz}, 1 \mathrm{H}), 5.02$ (dd, $J=10.2,9.0 \mathrm{~Hz}, 1 \mathrm{H}), 4.62(\mathrm{dd}, J=6.0,1.8 \mathrm{~Hz}, 1 \mathrm{H}), 4.22(\mathrm{ddd}, J=10.2,6.0,3.6$, $\mathrm{Hz}, 1 \mathrm{H}), 4.13(\mathrm{dd}, J=10.2,6.6 \mathrm{~Hz}, 1 \mathrm{H}), 4.03(\mathrm{dd}, J=4.2,1.8 \mathrm{~Hz}, 1 \mathrm{H}), 3.97$ (ddd, $J=$ 10.8, 7.2, $2.4 \mathrm{~Hz}, 1 \mathrm{H}), 3.93(\mathrm{dd}, J=10.2,4.2 \mathrm{~Hz}, 1 \mathrm{H}), 3.89(\mathrm{dd}, J=10.2,9.6 \mathrm{~Hz}, 1 \mathrm{H})$, $3.86(\mathrm{dd}, J=11.4,10.2 \mathrm{~Hz}, 1 \mathrm{H}), 3.78(\mathrm{dd}, J=10.2,4.8 \mathrm{~Hz}, 1 \mathrm{H}), 3.72(\mathrm{dd}, J=10.2,4.8$ $\mathrm{Hz}, 1 \mathrm{H}), 3.70(\mathrm{~s}, 3 \mathrm{H}), 3.70(\mathrm{~m}, 2 \mathrm{H}), 3.45(\mathrm{~d}, J=3.6 \mathrm{~Hz}, 1 \mathrm{H}), 3.25(\mathrm{~d}, J=7.2 \mathrm{~Hz}, 1 \mathrm{H})$, $3.10(\mathrm{~d}, J=1.2 \mathrm{~Hz}, 1 \mathrm{H}), 3.06(\mathrm{dd}, J=13.8,6.0 \mathrm{~Hz}, 1 \mathrm{H}), 3.02(\mathrm{dd}, J=15.0,5.4 \mathrm{~Hz}, 1 \mathrm{H})$, $2.24(\mathrm{dd}, J=15.0,7.2 \mathrm{~Hz}, 1 \mathrm{H}), 2.20(\mathrm{dd}, J=13.8 .0,7.2 \mathrm{~Hz}, 1 \mathrm{H}), 2.16(\mathrm{~d}, J=1.8 \mathrm{~Hz}$, 1H), $2.08(\mathrm{~m}, 1 \mathrm{H}), 0.96(\mathrm{~d}, J=6.6 \mathrm{~Hz}, 6 \mathrm{H}), 0.89$ (s, 9H), 0.84 (s, 9H), 0.07 (s, 3H), 0.06 (s, 3H), $0.02(\mathrm{~s}, 3 \mathrm{H}), 0.02(\mathrm{~s}, 3 \mathrm{H}) ;{ }^{13} \mathrm{C}$ NMR $\left(150 \mathrm{MHz}, \mathrm{CDCl}_{3}\right) \delta$ 173.5, 171.9, 155.6, 155.4, 136.1, 130.2(2C), 128.4(3C), 128.1(2C), 128.0, 116.8(2C), 98.3, 97.7, 73.7, 71.6, $71.2,70.8,70.1,70.0,69.8,66.9,62.8,54.8,52.2,43.4(2 \mathrm{C}), 37.2,25.9,25.9(3 \mathrm{C})$, 25.8(3C), 25.7, 22.3, 22.2, 18.4, 18.3, -5.2, -5.3, -5.40, -5.47; CIHRMS Calcd for $\left[\mathrm{C}_{47} \mathrm{H}_{75} \mathrm{NO}_{16} \mathrm{Si}_{2} \mathrm{Na}^{+}\right]:$988.4516. Found 988.4459.

\section{1'- $N$-carbobenzyloxy-D-tyrosine methoxycarbonyl -5',5-(tert-butyl-}

dimethylsilanyloxymethyl)-2,3,2',3'-di acetonide-bis-1,4-a-D-mannose- $O-$ 4' $^{-}$ isovalaric ester (III-3b).

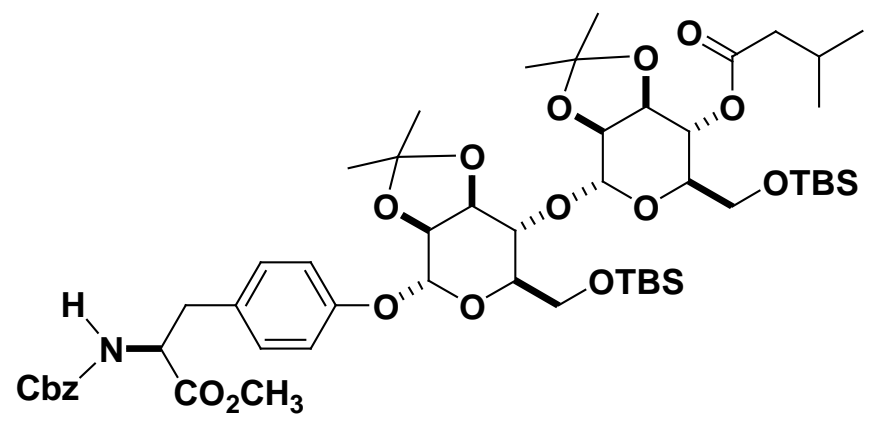


To a $\mathrm{CH}_{2} \mathrm{Cl}_{2}(0.1 \mathrm{~mL}, 1.0 \mathrm{M})$ solution of D-manno-tetrol III-3a $(20 \mathrm{mg}, 0.02 \mathrm{mmol})$ and 2,2-dimethoxypropane $(4.6 \mathrm{mg}, 0.04 \mathrm{mmol})$ at $0{ }^{\circ} \mathrm{C}$ was added CSA $(0.50 \mathrm{mg}, 10 \mathrm{~mol} \%)$ and the reaction was stirred for $6 \mathrm{~h}$. The reaction mixture was concentrated and was pipetted directly on to a silica gel column using $\mathrm{CH}_{2} \mathrm{Cl}_{2}(1 \mathrm{~mL})$ in three portions. Impurities were eluted with ether and the product was eluted with EtOAc/Hexane (40:60). Pure fractions were combined and concentrated to afford III-3b (17 mg, 0.02 mmol, $80 \%)$ as viscous oil. $R_{f}(50 \%$ EtOAc/Hexane $)=0.45 ;[\alpha]_{\mathrm{D}}^{26}=+49(c 1$, $\mathrm{CH}_{2} \mathrm{Cl}_{2}$ ); IR (thin film, $\mathrm{cm}^{-1}$ ) 2955, 2935, 1728, 1511, 1226, 1101, 1017, 833; ${ }^{1} \mathrm{H} \mathrm{NMR}$ $\left(600 \mathrm{MHz}, \mathrm{CDCl}_{3}\right) \delta 7.35(\mathrm{~m}, 5 \mathrm{H}), 7.29(\mathrm{~m}, 4 \mathrm{H}), 5.97(\mathrm{~d}, J=10.2 \mathrm{~Hz}, 1 \mathrm{H}), 5.92$ (ddd, $J$ $=10.2,3.01 .8 \mathrm{~Hz}, 1 \mathrm{H}), 5.40(\mathrm{br} \mathrm{s}, 1 \mathrm{H}), 5.04(\mathrm{br} \mathrm{s}, 2 \mathrm{H}), 4.94(\mathrm{dd}, J=9.6,7.8 \mathrm{~Hz}, 1 \mathrm{H})$, $4.79(\mathrm{~d}, J=12.0 \mathrm{~Hz}, 1 \mathrm{H}), 4.56(\mathrm{~d}, J=12.0 \mathrm{~Hz}, 1 \mathrm{H}), 4.38(\mathrm{ddd}, J=9.0,4.2,4.2 \mathrm{~Hz}, 1 \mathrm{H})$, $4.26(\mathrm{~d}, J=6.0 \mathrm{~Hz}, 1 \mathrm{H}), 4.21(\mathrm{dd}, J=13.2,5.4 \mathrm{~Hz}, 1 \mathrm{H}), 4.09(\mathrm{dd}, J=11.4,5.4 \mathrm{~Hz}, 1 \mathrm{H})$, $4.01(\mathrm{~m}, 1 \mathrm{H}), 3.78(\mathrm{~d}, J=5.4 \mathrm{~Hz}, 1 \mathrm{H}), 3.74(\mathrm{dd}, J=10.2,2.4 \mathrm{~Hz}, 1 \mathrm{H}), 3.72(\mathrm{br} \mathrm{s}, 1 \mathrm{H})$, $3.68(\mathrm{~d}, J=6.0 \mathrm{~Hz}, 1 \mathrm{H}), 3.67(\mathrm{~s}, 3 \mathrm{H}), 3.61(\mathrm{~d}, J=10.2 \mathrm{~Hz}, 1 \mathrm{H}), 3.06(\mathrm{dd}, J=7.2,6.0 \mathrm{~Hz}$, 1H), $2.95(\mathrm{~s}, 1 \mathrm{H}), 2.88(\mathrm{~s}, 1 \mathrm{H}), 2.26(\mathrm{dd}, J=15.0,6.6 \mathrm{~Hz}, 1 \mathrm{H}), 2.18(\mathrm{dd}, J=15.0,7.2 \mathrm{~Hz}$, 1H), $1.93(\mathrm{~m}, 3 \mathrm{H}), 1.85(\mathrm{~m}, 3 \mathrm{H}), 1.63(\mathrm{~m}, 3 \mathrm{H}), 1.50(\mathrm{~m}, 3 \mathrm{H}), 0.97(\mathrm{~s}, 6 \mathrm{H}), 0.95(\mathrm{~s}, 9 \mathrm{H})$, $0.90(\mathrm{~s}, 9 \mathrm{H}), 0.10(\mathrm{~s}, 3 \mathrm{H}), 0.09(\mathrm{~s}, 6 \mathrm{H}), 0.06(\mathrm{~s}, 3 \mathrm{H}) ;{ }^{13} \mathrm{C} \mathrm{NMR}\left(150 \mathrm{MHz}, \mathrm{CDCl}_{3}\right) \delta$ $173.2,172.6,154.2,137.5,130.3,128.7,128.6(2 \mathrm{C}), 128.5,128.4(2 \mathrm{C}), 128.1,128.0(2 \mathrm{C})$, 127.8, 116.7, 110.0, 92.9(2C), 75.1, 69.9(3C), 69.5(2C), 66.6, 62.8(2C), 58.8(2C), 53.2, 50.4, 40.1, 38.1, 29.3, 28.7(2C), 28.4, 26.0(3C), 25.9(3C), 22.3(2C), 18.6, 18.3(2C), -5.0, -5.2(2C), -5.3; CIHRMS Calcd for $\left[\mathrm{C}_{53} \mathrm{H}_{83} \mathrm{NO}_{16} \mathrm{Si}_{2} \mathrm{Na}^{+}-\mathrm{C}_{3} \mathrm{H}_{6}{ }^{2+}\right]$ : 1028.5142. Found 1028.4585 . 
1'- $N$-carbobenzyloxy-D-tyrosine methoxycarbonyl -5',5-(hydroxy methyl)-di-1,4- $\alpha$ D-mannose $-O-4$ ' isovaleric ester (III-2a).

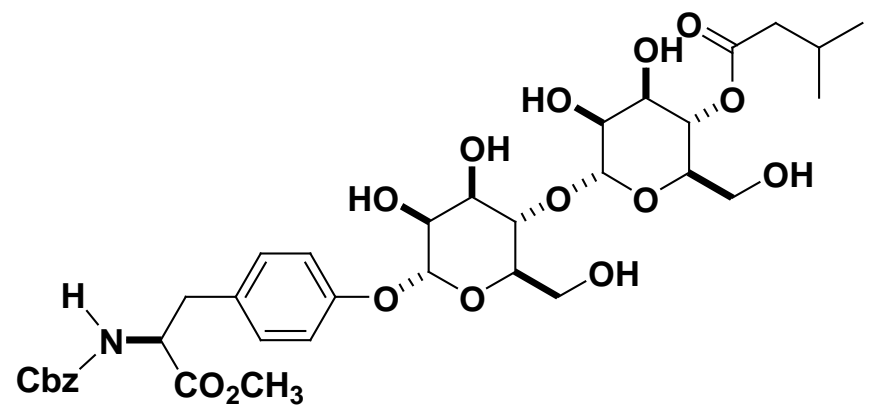

To a THF $(0.5 \mathrm{~mL}, 0.1 \mathrm{M})$ solution of D-manno-tetrol III-3a (50 mg, $0.05 \mathrm{mmol})$ at $0{ }^{\circ} \mathrm{C}$ was added a solution of TBAF in THF $(50 \mu \mathrm{L}, 1.0 \mathrm{M})$ and the reaction was stirred for 1 h. The reaction mixture was concentrated and was pipetted directly on to a silica gel column using $\mathrm{CH}_{2} \mathrm{Cl}_{2}(1 \mathrm{~mL})$ in three portions. Impurities were eluted with ether and the product was eluted with $\mathrm{MeOH} / \mathrm{EtOAc} / \mathrm{Hexane}$ (10:40:50). Pure fractions were combined and concentrated to afford III-2a $(31 \mathrm{mg}, 0.04 \mathrm{mmol}, 80 \%)$ as viscous oil. $R_{f}$ $(10: 50: 40 \% \mathrm{MeOH} / \mathrm{EtOAc} / \mathrm{Hexane})=0.20 ;[\alpha]_{\mathrm{D}}^{26}=+71\left(c 0.5, \mathrm{CH}_{3} \mathrm{OH}\right) ; \mathrm{IR}$ (thin film, $\left.\mathrm{cm}^{-1}\right) 3337,2956,2926,1738,1611,1510,1228,1217,1094,976,834 ;{ }^{1} \mathrm{H}$ NMR (600 $\left.\mathrm{MHz}, \mathrm{CDCl}_{3}\right) \delta 7.36(\mathrm{~m}, 5 \mathrm{H}), 7.17(\mathrm{~m}, 4 \mathrm{H}), 5.48(\mathrm{~d}, J=1.8 \mathrm{~Hz}, 1 \mathrm{H}), 5.42(\mathrm{~d}, J=1.8 \mathrm{~Hz}$, 1H), 5.09 (br s, 2H), $4.59($ br s, 1H), $4.45(\mathrm{ddd}, J=7.8,6.6,1.8 \mathrm{~Hz}, 1 \mathrm{H}), 4.08(\mathrm{dd}, J=$ 10.2, $1.8 \mathrm{~Hz}, 1 \mathrm{H}), 4.05(\mathrm{~m}, 3 \mathrm{H}), 4.00(\mathrm{br} \mathrm{s}, 1 \mathrm{H}), 3.89$ (dd, $J=9.6,2.4 \mathrm{~Hz}, 1 \mathrm{H}), 3.80$ (dd, $J=10.2,2.4 \mathrm{~Hz}, 1 \mathrm{H}), 3.74(\mathrm{~m}, 4 \mathrm{H}), 3.68(\mathrm{~m}, 1 \mathrm{H}), 3.62(\mathrm{~m}, 1 \mathrm{H}), 3.36(\mathrm{~m}, 3 \mathrm{H}), 3.15$ (ddd, $J=10.2,4.8,4.8 \mathrm{~Hz}, 1 \mathrm{H}), 2.93(\mathrm{~m}, 2 \mathrm{H}), 2.29(\mathrm{~m}, 1 \mathrm{H}), 2.13(\mathrm{~m}, 1 \mathrm{H}), 1.34(\mathrm{~m}, 1 \mathrm{H}), 1.03$ $(\mathrm{d}, J=6.6 \mathrm{~Hz}, 6 \mathrm{H}) ; \mathrm{NMR}\left(150 \mathrm{MHz}, \mathrm{CDCl}_{3}\right) \delta 172.1,155.8,155.3,136.4,130.5(2 \mathrm{C})$, 129.9, 128.4(2C), 128.2(2C), 116.6, 103.2, 102.4, 98.1, 71.8, 71.3, 67.1(2C), 61.1, 55.1, 
52.5(2C), 43.6, 43.1, 37.5, 36.6, 31.6, 30.5, 29.8, 28.6, 25.9, 23.1, 22.5(2C); CIHRMS Calcd for $\left[\mathrm{C}_{35} \mathrm{H}_{47} \mathrm{NO}_{16} \mathrm{Na}^{+}\right]$: 760.2787. Found 760.2798.

$(1 ' S, 4 ' S, 5 ' R, 1 S, 4 S, 5 R)-1-[1 '-N$-carbobenzyloxy-D-tyrosine methoxycarbonyl -5'-(tertbutyl-dimethylsilanyloxymethyl)-tetrahydro-pyran-4'-yloxy]-5-(tert-butyldimethylsilanyloxymethyl) -tetrahydro-pyran-O-4'-isovalaric ester (III-2b).

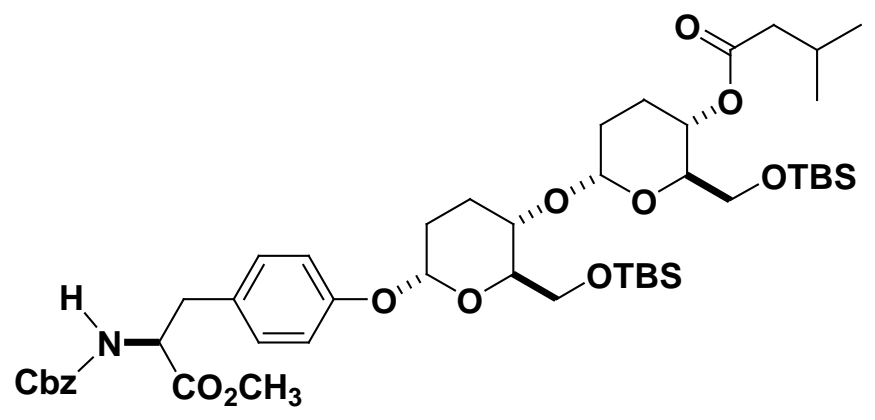

The dienone ester compound III-23 $(27 \mathrm{mg}, 0.03 \mathrm{mmol})$ and $o-\mathrm{NO}_{2} \mathrm{C}_{6} \mathrm{H}_{4} \mathrm{SO}_{2} \mathrm{NHNH}_{2}(20$ $\mathrm{mg}, 0.02 \mathrm{mmol}$ ) were dissolved in $0.2 \mathrm{~mL}$ of $\mathrm{CH}_{2} \mathrm{Cl}_{2}$ in a round bottom flask and cooled $0{ }^{\circ} \mathrm{C}$ under nitrogen atmosphere then triethylamine $(17 \mathrm{mg}, 0.17 \mathrm{mmol})$ was added and the reaction mixture was stirred at $0{ }^{\circ} \mathrm{C}$ for 12 hours and on completion, as monitored by TLC. The reaction mixture was concentrated and was pipetted directly on to a silica gel column using $\mathrm{CH}_{2} \mathrm{Cl}_{2}(1 \mathrm{~mL})$ in three portions. The crude product was purified using silica gel flash chromatography eluting with 15\% EtOAc/hexanes to give III-2b (18 mg, $0.02 \mathrm{mmol}, 95 \%)$ as viscous oil. $R_{f}(30 \%$ EtOAc/Hexane $)=0.46 ;[\alpha]_{\mathrm{D}}^{26}=+35(c 1$, $\mathrm{CH}_{2} \mathrm{Cl}_{2}$ ); IR (thin film, $\mathrm{cm}^{-1}$ ) 2952, 2929, 1735, 1510, 1254, 1220, 1123, 996, 836; ${ }^{1} \mathrm{H}$ NMR (600 MHz, $\left.\mathrm{CDCl}_{3}\right) \delta 7.34(\mathrm{~m}, 5 \mathrm{H}), 6.98(\mathrm{~m}, 4 \mathrm{H}), 5.45(\mathrm{br} \mathrm{s}, 1 \mathrm{H}), 5.24(\mathrm{~d}, J=7.8$ Hz, 1H), 5.09 (br s, 2H), 5.08 (d, $J=2.4 \mathrm{~Hz}, 1 \mathrm{H}), 4.73$ (ddd, $J=10.2,9.6,4.8 \mathrm{~Hz}, 1 \mathrm{H}$ ), $4.60(\mathrm{ddd}, J=7.8,5.4,5.4 \mathrm{~Hz}, 1 \mathrm{H}), 3.84(\mathrm{~d}, J=11.4 \mathrm{~Hz}, 1 \mathrm{H}), 3.79-3.67(\mathrm{~m}, 6 \mathrm{H}), 3.70$ (s, 
3H), $3.05(\mathrm{dd}, J=13.2,6.0 \mathrm{~Hz}, 1 \mathrm{H}), 3.01(\mathrm{dd}, J=13.2,5.4 \mathrm{~Hz}, 1 \mathrm{H}), 2.15(\mathrm{~m}, 3 \mathrm{H}), 2.06$ (m, 1H), $2.00(\mathrm{~m}, 2 \mathrm{H}), 1.80(\mathrm{~m}, 3 \mathrm{H}), 1.71(\mathrm{~m}, 2 \mathrm{H}), 0.89(\mathrm{~d}, J=6.6 \mathrm{~Hz}, 6 \mathrm{H}), 0.86(\mathrm{~s}, 9 \mathrm{H})$ 0.84 (s, 9H), 0.03 (s, 6H), $0.01(\mathrm{~s}, 6 \mathrm{H}) ;{ }^{13} \mathrm{C}$ NMR $\left(67.5 \mathrm{MHz}, \mathrm{CDCl}_{3}\right) \delta$ 172.0, 172.2, 156.4, 136.5, 130.2, 128.4(2C), 128.2(2C), 128.1, 128.0(2C), 116.7(2C), 94.8, 90.5, 75.1, $73.4,71.5,67.7,66.8,66.4,63.1,62.6,54.6,52.0,43.6,37.5,28.6,25.8(3 \mathrm{C}), 25.7(3 \mathrm{C})$, 25.7, 23.6, 22.2(3C), 22.0, 18.4, 18.2, -5.1, -5.3, -5.40, -5.44; CIHRMS Calcd for $\left[\mathrm{C}_{47} \mathrm{H}_{75} \mathrm{NO}_{12} \mathrm{Si}_{2} \mathrm{Na}^{+}\right]:$924.4720. Found 924.4702.

\section{3-\{4-[6-(tert-Butyl-dimethylsilanyloxymethyl)-5-oxo-5,6-dihydro-2H-pyran-2-yloxy]-} phenyl\}-2- $N$-carbobenzyloxy-D-tyrosine methyl ester (III-24a).

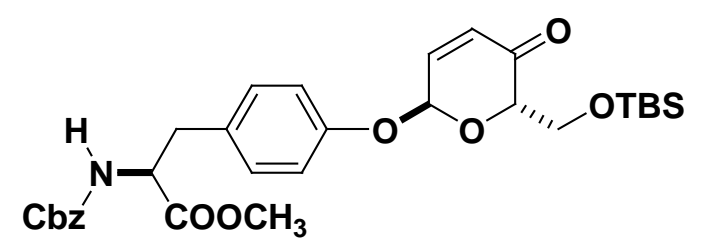

A $\mathrm{CH}_{2} \mathrm{Cl}_{2}(0.6 \mathrm{~mL})$ solution of compound (ent)- III-18 (200 mg, $\left.0.55 \mathrm{mmol}\right)$ and Cbz-D-tyrosine methyl ester III-17 (220 mg, $0.67 \mathrm{mmol})$ was cooled to $0{ }^{\circ} \mathrm{C} . \mathrm{A} \mathrm{CH}_{2} \mathrm{Cl}_{2}$ (0.6 mL) solution of $\mathrm{Pd}_{2}\left(\mathrm{dba}_{3} . \mathrm{CHCl}_{3}(14 \mathrm{mg}, 2.5 \mathrm{~mol} \%)\right.$ and $\mathrm{PPh}_{3}(14 \mathrm{mg}, 10 \mathrm{~mol} \%)$ was added to the reaction mixture at $0{ }^{\circ} \mathrm{C}$. The reaction mixture was stirred at $0{ }^{\circ} \mathrm{C}$ for 3 hours. The reaction mixture was quenched with $5 \mathrm{~mL}$ of satd. aq. $\mathrm{NaHCO}_{3}$, extracted ( 3 x $5 \mathrm{~mL}$ ) with $\mathrm{Et}_{2} \mathrm{O}$, dried over $\mathrm{Na}_{2} \mathrm{SO}_{4}$, and concentrated under reduced pressure. The crude product was purified using silica gel flash chromatography eluting with $20 \%$ EtOAc/hexanes to give III-24a (278 $\mathrm{mg}, 0.50 \mathrm{mmol}, 90 \%)$ as viscous oil. $R_{f}(40 \%$ EtOAc/hexanes) $=0.40 ;[\alpha]_{\mathrm{D}}^{26}=\left(c 0.64, \mathrm{CH}_{2} \mathrm{Cl}_{2}\right) ; \mathrm{IR}$ (thin film, $\left.\mathrm{cm}^{-1}\right) 3351,2952$, 
$1752,1725,1509,1256,1219,1151,995,836 ;{ }^{1} \mathrm{H}$ NMR $\left(270 \mathrm{MHz}, \mathrm{CDCl}_{3}\right) \delta 7.35(\mathrm{~m}$, $5 \mathrm{H}), 7.05(\mathrm{~m}, 4 \mathrm{H}), 6.97(\mathrm{dd}, J=10.2,3.5 \mathrm{~Hz}, 1 \mathrm{H}), 6.22(\mathrm{~d}, J=10.2 \mathrm{~Hz}, 1 \mathrm{H}), 5.94(\mathrm{~d}, J=$ $3.3 \mathrm{~Hz}, 1 \mathrm{H}), 5.33$ (dd, $J=8.5,4.2 \mathrm{~Hz}, 1 \mathrm{H}), 5.09$ (br s, 2H), $4.62(\mathrm{dd}, J=13.2,5.7 \mathrm{~Hz}$, $1 \mathrm{H}), 4.53(\mathrm{dd}, J=4.1,3.1 \mathrm{~Hz}, 1 \mathrm{H}), 4.15-4.00(\mathrm{~m}, 2 \mathrm{H}), 3.71(\mathrm{~s}, 3 \mathrm{H}), 3.69(\mathrm{~m}, 2 \mathrm{H}), 0.84$ (s, 9H), 0.05 (s, 3H), 0.04 (s, 3H); ${ }^{13} \mathrm{C} \mathrm{NMR}\left(67.5 \mathrm{MHz}, \mathrm{CDCl}_{3}\right) \delta$ 194.2, 177.7, 156.1, 155.4, 151.6, 150.0, 142.6, 136.0, 133.1, 130.2, 130.0, 128.5, 128.3, 128.0, 127.9, 121.2, $116.9,91.8,83.3,66.8,62.4,54.7,52.2,37.1,25.1(3 \mathrm{C}), 18.1,-5.46,-5.49$; CIHRMS Calcd for $\left[\mathrm{C}_{30} \mathrm{H}_{39} \mathrm{NO}_{8} \mathrm{SiNa}^{+}\right]$: 592.2343 . Found 592.2369.

\section{3-\{4-[6-(tert-Butyl-dimethylsilanyloxymethyl)-5-hydroxy-5,6-dihydro-2H-pyran-2-} yloxy]-phenyl\}-2- $N$-carbobenzyloxy-D-tyrosine methyl ester (III-24b).

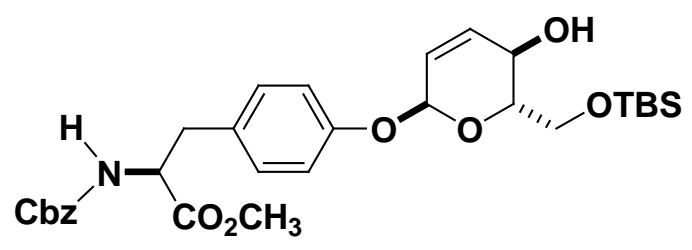

The enone compound III-24a (300 mg, $0.527 \mathrm{mmol}$ ), dissolved in $0.5 \mathrm{~mL}$ of $\mathrm{CH}_{2} \mathrm{Cl}_{2}$ and $0.5 \mathrm{~mL} \mathrm{MeOH}$, was added to a round bottom flask and cooled to $-24{ }^{\circ} \mathrm{C}$ then $(20 \mathrm{mg}$, $0.527 \mathrm{mmol}) \mathrm{NaBH}_{4}$ was added and the reaction mixture was stirred at $-24^{\circ} \mathrm{C}$ for 1 hour and on completion, as monitored by TLC, the reaction mixture was diluted with ether and was quenched with $5 \mathrm{~mL}$ of satd. aq. $\mathrm{NaHCO}_{3}$, extracted $(3 \times 5 \mathrm{~mL})$ with $\mathrm{Et}_{2} \mathrm{O}$, dried over $\mathrm{Na}_{2} \mathrm{SO}_{4}$, and concentrated under reduced pressure. The crude product was purified using silica gel flash chromatography eluting with $25 \%$ EtOAc/hexanes to give III-24b $(270 \mathrm{mg}, 0.47 \mathrm{mmol}, 89 \%)$ as viscous oil. $R_{f}(30 \%$ EtOAc/hexanes $)=0.30 ;[\alpha]_{\mathrm{D}}^{26}=-$ 
$82\left(c 1, \mathrm{CH}_{2} \mathrm{Cl}_{2}\right.$ ); IR (thin film, $\left.\mathrm{cm}^{-1}\right) 3357,2928,1722,1611,1439,1225,1054,985$, 835; ${ }^{1} \mathrm{H}$ NMR $\left(600 \mathrm{MHz}, \mathrm{CDCl}_{3}\right) \delta 7.33(\mathrm{~m}, 5 \mathrm{H}), 6.97(\mathrm{~m}, 4 \mathrm{H}), 6.07(\mathrm{dd}, J=10.2,1.2$ $\mathrm{Hz}, 1 \mathrm{H}), 5.85(\mathrm{ddd}, J=10.2,2.4,2.4 \mathrm{~Hz}, 1 \mathrm{H}), 5.56(\mathrm{dd}, J=2.4,1.2 \mathrm{~Hz}, 1 \mathrm{H}), 5.20(\mathrm{~d}, J=$ $7.8 \mathrm{~Hz}, 1 \mathrm{H}), 5.09(\mathrm{~d}, J=12.0 \mathrm{~Hz}, 1 \mathrm{H}), 5.07(\mathrm{~d}, J=12.0 \mathrm{~Hz}, 1 \mathrm{H}), 4.61(\mathrm{dd}, J=13.2,5.4$ Hz, 1H), 4.26 (dd, $J=9.0,1.8 \mathrm{~Hz}, 1 \mathrm{H}), 3.89$ (dd, $J=10.2,4.8 \mathrm{~Hz}, 1 \mathrm{H}), 3.83$ (ddd, $J=$ 9.0, 7.2, $4.8 \mathrm{~Hz}, 1 \mathrm{H}), 3.73(\mathrm{dd}, J=9.6,6.6 \mathrm{~Hz}, 1 \mathrm{H}), 3.31(\mathrm{~s}, 3 \mathrm{H}), 3.08(\mathrm{dd}, J=13.8,5.4$ Hz, 1H), 3.04 (dd, $J=13.8,5.4 \mathrm{~Hz}, 1 \mathrm{H}), 3.01$ (d, $J=3.0 \mathrm{~Hz}, 1 \mathrm{H}), 0.87$ (s, 9H), 0.07 (s, 3H), $0.05(\mathrm{~s}, 3 \mathrm{H}) ;{ }^{13} \mathrm{C} \mathrm{NMR}\left(150 \mathrm{MHz}, \mathrm{CDCl}_{3}\right) \delta 171.9,156.6,136.2,133.3,130.2$, 130.2(2C), 128.5(2C), 128.1, 128.0, 124.8, 116.9(2C), 116.6, 92.8, 70.7, 66.9, 66.8, 65.3, 54.8, 52.3, 52.2, 37.3, 25.7(3C), 18.1, -5.5, -5.6; CIHRMS Calcd for $\left[\mathrm{C}_{30} \mathrm{H}_{41} \mathrm{NO}_{8} \mathrm{SiNa}^{+}\right]$: 594.2493 Found 594.2500.

$\left(1 ' R, 4^{\prime} R, 5 ' S, 1 R, 5 S\right)-1-\left[1^{\prime}-N\right.$-carbobenzyloxy-D-tyrosine methoxycarbonyl -5-(tertbutyl-dimethylsilanyloxymethyl)-1',4'-dihydro-5'H-pyran-4'-yloxy]-5-(tert-butyldimethylsilanyloxymethyl) -1H-pyran-4-one (III-24).

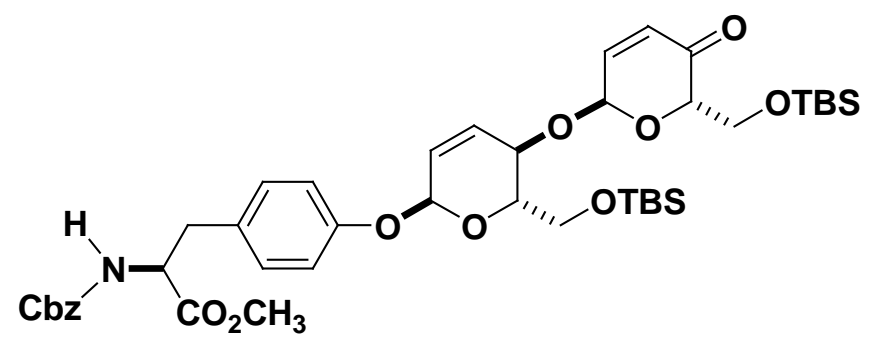

A $\mathrm{CH}_{2} \mathrm{Cl}_{2}(0.5 \mathrm{~mL})$ solution of compound (ent)-III-18 (163 mg, $\left.0.45 \mathrm{mmol}\right)$ and alcohol III-24b $(260 \mathrm{mg}, 0.45 \mathrm{mmol})$ was cooled to $0{ }^{\circ} \mathrm{C}$. $\mathrm{A} \mathrm{CH}_{2} \mathrm{Cl}_{2}(0.5 \mathrm{~mL})$ solution of $\mathrm{Pd}_{2}(\mathrm{dba})_{3} \cdot \mathrm{CHCl}_{3}(11.8 \mathrm{mg}, 2.5 \mathrm{~mol} \%)$ and $\mathrm{PPh}_{3}(12 \mathrm{mg}, 10 \mathrm{~mol} \%)$ was added to the reaction mixture at $0{ }^{\circ} \mathrm{C}$. The reaction mixture was stirred at $0{ }^{\circ} \mathrm{C}$ for 2 hours. The 
reaction mixture was quenched with $5 \mathrm{~mL}$ of satd. aq. $\mathrm{NaHCO}_{3}$, extracted $(3 \times 5 \mathrm{~mL})$ with $\mathrm{Et}_{2} \mathrm{O}$, dried over $\mathrm{Na}_{2} \mathrm{SO}_{4}$, and concentrated under reduced pressure. The crude product was purified using silica gel flash chromatography eluting with $16 \%$ EtOAc/hexanes to give dienone III-24 $(313 \mathrm{mg}, 0.38 \mathrm{mmol}, 85 \%)$ as viscous oil. $R_{f}$ $(30 \%$ EtOAc/hexanes $)=0.44 ;[\alpha]_{\mathrm{D}}^{26}=-63\left(c 1, \mathrm{CH}_{2} \mathrm{Cl}_{2}\right) ; \mathrm{IR}$ (thin film, $\left.\mathrm{cm}^{-1}\right) 3341$, $2952,1725,1700,1509,1252,1226,1123,984,836 ;{ }^{1} \mathrm{H}$ NMR $\left(600 \mathrm{MHz}, \mathrm{CDCl}_{3}\right) \delta 7.34$ (m, 5H), $7.00(\mathrm{~m}, 4 \mathrm{H}), 6.84(\mathrm{dd}, J=10.2,3.6 \mathrm{~Hz}, 1 \mathrm{H}), 6.23(\mathrm{~d}, J=10.2 \mathrm{~Hz}, 1 \mathrm{H}), 6.14(\mathrm{~d}$, $J=10.8 \mathrm{~Hz}, 1 \mathrm{H}), 5.95(\mathrm{ddd}, J=10.2,3.0,1.2 \mathrm{~Hz}, 1 \mathrm{H}), 5.62(\mathrm{~d}, J=3.6 \mathrm{~Hz}, 1 \mathrm{H}), 5.61(\mathrm{~d}$, $J=1.8 \mathrm{~Hz}, 1 \mathrm{H}), 5.18(\mathrm{~d}, J=7.8 \mathrm{~Hz}, 1 \mathrm{H}), 5.10(\mathrm{br} \mathrm{s}, 2 \mathrm{H}), 4.62(\mathrm{dd}, J=13.8,6.0 \mathrm{~Hz}, 1 \mathrm{H})$, $4.59(\mathrm{~d}, J=9.0 \mathrm{~Hz}, 1 \mathrm{H}), 4.44(\mathrm{dd}, J=3.6,2.4 \mathrm{~Hz}, 1 \mathrm{H}), 4.13(\mathrm{~d}, J=3.6 \mathrm{~Hz}, 1 \mathrm{H}), 4.10$ (dd, $J=10.2,4.2 \mathrm{~Hz}, 1 \mathrm{H}), 3.96(\mathrm{dd}, J=10.2,2.4 \mathrm{~Hz}, 1 \mathrm{H}), 3.86(\mathrm{dq}, J=12.0,3.6 \mathrm{~Hz}$, $1 \mathrm{H}), 3.80(\mathrm{dd}, J=12.0,4.2 \mathrm{~Hz}, 1 \mathrm{H}), 3.72(\mathrm{~s}, 3 \mathrm{H}), 3.07(\mathrm{dd}, J=13.8,5.4 \mathrm{~Hz}, 1 \mathrm{H}), 3.03$ (dd, $J=13.8,6.0 \mathrm{~Hz}, 1 \mathrm{H}), 0.86(\mathrm{~s}, 9 \mathrm{H}), 0.84(\mathrm{~s}, 9 \mathrm{H}), 0.06(\mathrm{~s}, 3 \mathrm{H}), 0.04$ (s, 3H), 0.02 (s, 3H), $0.01(\mathrm{~s}, 3 \mathrm{H}) ;{ }^{13} \mathrm{C}$ NMR $\left(150 \mathrm{MHz}, \mathrm{CDCl}_{3}\right) \delta$ 194.6, 171.9, 156.4, 155.6, 143.9, 136.2, 133.3, 130.2(2C), 130.1, 129.1, 128.6, 128.5(2C), 128.1, 128.0, 126.6(2C), 117.0, $92.9,89.8,76.7,71.3,66.9,66.5,62.9,62.5,54.8,52.2,37.3,25.9(3 \mathrm{C}), 25.8(3 \mathrm{C}), 18.4$, 18.3, -5.1, -5.3(2C), -5.4; CIHRMS Calcd for $\left[\mathrm{C}_{42} \mathrm{H}_{61} \mathrm{NO}_{11} \mathrm{Si}_{2} \mathrm{Na}^{+}\right]$: 834.3675. Found 834.3648.

$(1 ' R, 4 ' R, 5 ' S, 1 R, 4 R, 5 S)-1-[N$-carbobenzyloxy-D-tyrosine methoxycarbonyl -5'-(tertbutyl-dimethylsilanyloxymethyl)-1',4'-dihydro-5'H-pyran-4'-yloxy]-5-(tert-butyldimethylsilanyloxymethyl)-1,4-dihydro-5H-pyran-4-ol (III-25a). 


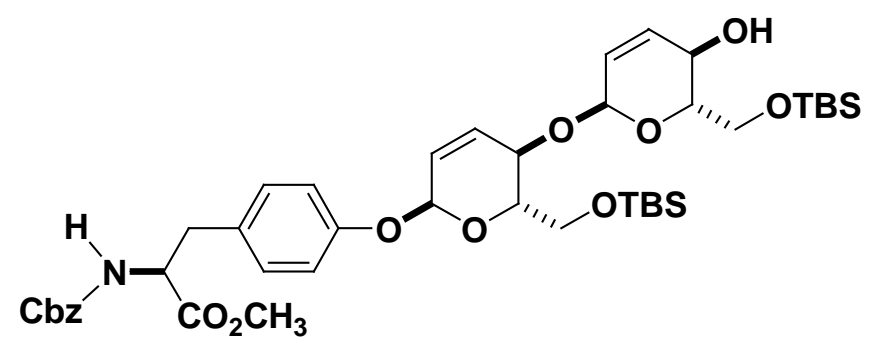

The dienone compound III-24 (300 mg, $0.37 \mathrm{mmol}$ ), dissolved in $0.4 \mathrm{~mL}$ of $\mathrm{CH}_{2} \mathrm{Cl}_{2}$ and $0.4 \mathrm{~mL} \mathrm{MeOH}$, was added to a round bottom flask and cooled to $-24{ }^{\circ} \mathrm{C}$ then $\mathrm{NaBH}_{4}(14$ $\mathrm{mg}, 0.37 \mathrm{mmol}$ ) was added and the reaction mixture was stirred at $-24{ }^{\circ} \mathrm{C}$ for 1 hour and on completion, as monitored by TLC, the reaction mixture is diluted with ether and was quenched with $5 \mathrm{~mL}$ of satd. aq. $\mathrm{NaHCO}_{3}$, extracted $(3 \times 5 \mathrm{~mL})$ with $\mathrm{Et}_{2} \mathrm{O}$, dried over $\mathrm{Na}_{2} \mathrm{SO}_{4}$, and concentrated under reduced pressure. The crude product was purified using silica gel flash chromatography eluting with $25 \%$ EtOAc/hexanes to give III-25a (271 $\mathrm{mg}, 0.333 \mathrm{mmol}, 90 \%)$ as viscous oil. $R_{f}(30 \%$ EtOAc/hexanes $)=0.30 ;[\alpha]_{\mathrm{D}}^{26}=-72(c$ 1, $\mathrm{CH}_{2} \mathrm{Cl}_{2}$ ); IR (thin film, $\mathrm{cm}^{-1}$ ) 3433, 2928, 2857, 1724, 1509, 1253, 1226, 1123, 1044, 981, 836; ${ }^{1} \mathrm{H}$ NMR (600 MHz, $\left.\mathrm{CDCl}_{3}\right) \delta 7.34(\mathrm{~m}, 5 \mathrm{H}), 7.00(\mathrm{~m}, 4 \mathrm{H}), 6.15(\mathrm{~d}, J=10.8$ $\mathrm{Hz}, 1 \mathrm{H}), 5.97(\mathrm{dd}, J=10.2,1.8 \mathrm{~Hz}, 1 \mathrm{H}), 5.92(\mathrm{ddd}, J=10.2,3.0,1.8 \mathrm{~Hz}, 1 \mathrm{H}), 5.68$ (ddd, $J=10.2,2.4,1.8 \mathrm{~Hz}, 1 \mathrm{H}), 5.59(\mathrm{~d}, J=1.8 \mathrm{~Hz}, 1 \mathrm{H}), 5.18(\mathrm{br} \mathrm{s}, 2 \mathrm{H}), 5.09$ (br s, 2H), 4.61 $(\mathrm{dd}, J=13.2,5.4 \mathrm{~Hz}, 1 \mathrm{H}), 4.36(\mathrm{~d}, J=8.4 \mathrm{~Hz}, 1 \mathrm{H}), 4.24(\mathrm{~d}, J=8.4 \mathrm{~Hz}, 1 \mathrm{H}), 3.97(\mathrm{dd}, J$ = 9.6, $4.2 \mathrm{~Hz}, 1 \mathrm{H}), 3.89$ (dddd, $J=5.4,3.6,1.8,1.8 \mathrm{~Hz}, 1 \mathrm{H}), 3.85(\mathrm{dd}, J=11.4,2.4 \mathrm{~Hz}$, 1H), $3.80(\mathrm{dd}, J=11.4,5.4 \mathrm{~Hz}, 1 \mathrm{H}), 3.73(\mathrm{dd}, J=10.2,1.8 \mathrm{~Hz}, 1 \mathrm{H}), 3.72(\mathrm{~s}, 3 \mathrm{H}), 3.69$ (m, 1H), $3.06(\mathrm{~d}, J=6.0 \mathrm{~Hz}, 1 \mathrm{H}), 3.05(\mathrm{~d}, J=5.4 \mathrm{~Hz}, 1 \mathrm{H}), 3.02(\mathrm{~d}, J=2.4 \mathrm{~Hz}, 1 \mathrm{H}), 0.92$ $(\mathrm{s}, 9 \mathrm{H}), 0.84(\mathrm{~s}, 9 \mathrm{H}), 0.12(\mathrm{~s}, 3 \mathrm{H}), 0.11(\mathrm{~s}, 3 \mathrm{H}), 0.02(\mathrm{~s}, 3 \mathrm{H}), 0.01(\mathrm{~s}, 3 \mathrm{H}) ;{ }^{13} \mathrm{C}$ NMR $(150$ $\left.\mathrm{MHz}, \mathrm{CDCl}_{3}\right) \delta 171.9,156.7,155.6,136.2,133.1,130.5,130.1(2 \mathrm{C}), 130.0,129.0$ 128.4(2C), 128.1, 128.0, 126.1, 125.4, 117.2, 116.9, 92.8, 91.1, 71.4, 70.0, 66.9, 66.9, 
66.7, 65.3, 62.6, 54.8, 52.2, 37.3, 25.8(3C), 25.8(3C), 18.3, 18.2, -5.0, -5.1, -5.4, -5.6;

CIHRMS Calcd for $\left[\mathrm{C}_{42} \mathrm{H}_{63} \mathrm{NO}_{11} \mathrm{Si}_{2} \mathrm{Na}^{+}\right]$: 836.3831. Found 836.3844.

$\left(1 ' R, 4^{\prime} R, 5^{\prime} S, 1 R, 4 R, 5 S\right)-1$-[1'- $N$-carbobenzyloxy-D-tyrosine methoxycarbonyl -5'(tert-butyl-dimethylsilanyloxymethyl)-1',4'-dihydro-5'H-pyran-4'-yloxy] -5-(tertbutyl-dimethylsilanyloxymethyl)-1,4-dihydro-5H-pyran-O-4'-isovalaric ester (III25).

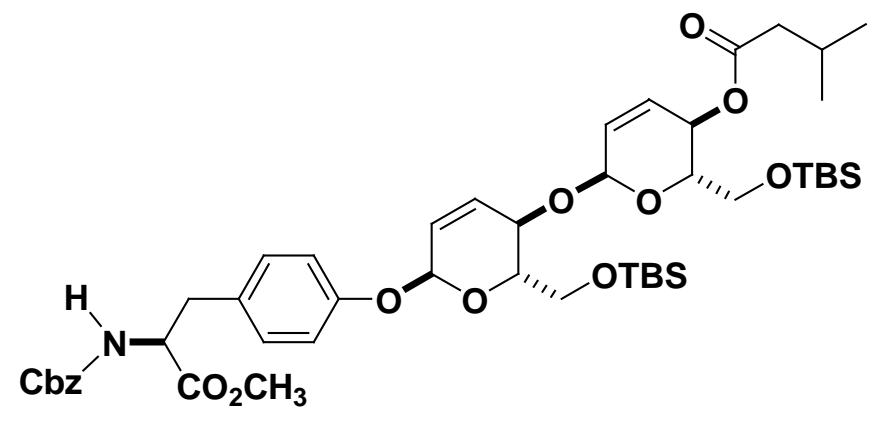

The alcohol compound III-25a (135 mg, $0.17 \mathrm{mmol})$, isovaleric acid (20 mg, $0.20 \mathrm{mmol})$ and DCC (41 mg, $0.20 \mathrm{mmol})$ were dissolved in $0.3 \mathrm{~mL}$ of $\mathrm{CH}_{2} \mathrm{Cl}_{2}$ in a round bottom flask and cooled to $0{ }^{\circ} \mathrm{C}$ then DMAP ( $2 \mathrm{mg}, 0.016 \mathrm{mmol}$ ) was added and the reaction mixture was stirred at $0{ }^{\circ} \mathrm{C}$ for 6 hours and on completion, as monitored by TLC, the reaction mixture was diluted with ether and was quenched with $5 \mathrm{~mL}$ of satd. aq. $\mathrm{NaHCO}_{3}$, extracted ( 3 x $5 \mathrm{~mL}$ ) with $\mathrm{Et}_{2} \mathrm{O}$, dried over $\mathrm{Na}_{2} \mathrm{SO}_{4}$, and concentrated under reduced pressure. The crude product was purified using silica gel flash chromatography eluting with $15 \%$ EtOAc/hexanes to give III-25 (141.5mg, $0.16 \mathrm{mmol}, 95 \%)$ as viscous oil. $R_{f}(30 \%$ EtOAc/hexanes $)=0.60 ;[\alpha]_{\mathrm{D}}^{26}=-99\left(c 1, \mathrm{CH}_{2} \mathrm{Cl}_{2}\right) ; \mathrm{IR}$ (thin film, $\left.\mathrm{cm}^{-1}\right)$ 
2956, 2929, 1737, 1509, 1253, 1219, 1123, 986, 835; ${ }^{1} \mathrm{H}$ NMR (600 MHz, $\left.\mathrm{CDCl}_{3}\right) \delta 7.34$ (m, 5H), $7.00(\mathrm{~m}, 4 \mathrm{H}), 6.20(\mathrm{~d}, J=10.2 \mathrm{~Hz}, 1 \mathrm{H}), 5.94(\mathrm{dd}, J=2.4,1.8 \mathrm{~Hz}, 1 \mathrm{H})), 5.92$ $(\mathrm{dd}, J=2.4,1.8 \mathrm{~Hz}, 1 \mathrm{H})), 5.76(\mathrm{dd}, J=3.0,1.8 \mathrm{~Hz}, 1 \mathrm{H}), 5.74(\mathrm{ddd}, J=10.2,2.4,2.4 \mathrm{~Hz}$, $1 \mathrm{H}), 5.59(\mathrm{~d}, J=1.8 \mathrm{~Hz}, 1 \mathrm{H}), 5.41(\mathrm{dd}, J=10.2,1.8 \mathrm{~Hz}, 1 \mathrm{H}), 5.28(\mathrm{~d}, J=1.8 \mathrm{~Hz}, 1 \mathrm{H}))$, $5.17(\mathrm{~d}, J=7.8 \mathrm{~Hz}, 1 \mathrm{H})) 5.10(\mathrm{br} \mathrm{s}, 2 \mathrm{H}), 4.62(\mathrm{dd}, J=13.2,5.4 \mathrm{~Hz}, 1 \mathrm{H}), 4.41(\mathrm{~d}, J=9.0$ Hz, 1H), 3.90 (dddd, $J=10.2,5.4,2.4,2.4 \mathrm{~Hz}, 1 \mathrm{H}), 3.85$ (dd, $J=11.4,2.4 \mathrm{~Hz}, 1 \mathrm{H}), 3.80$ (dd, $J=11.4,5.4 \mathrm{~Hz}, 1 \mathrm{H}), 3.77(\mathrm{dd}, J=6.0,2.4 \mathrm{~Hz}, 1 \mathrm{H}), 3.75(\mathrm{~m}, 1 \mathrm{H}), 3.72(\mathrm{~s}, 3 \mathrm{H})$, $3.06(\mathrm{dd}, J=9.6,4.2 \mathrm{~Hz}, 1 \mathrm{H}), 3.04(\mathrm{dd}, J=9.6,5.4 \mathrm{~Hz}, 1 \mathrm{H}), 2.20(\mathrm{~d}, J=1.8 \mathrm{~Hz}, 2 \mathrm{H})$, $2.10(\mathrm{~m}, 1 \mathrm{H}), 0.96(\mathrm{~d}, J=7.6 \mathrm{~Hz}, 6 \mathrm{H}), 0.89$ (s, 9H), 0.84 (s, 9H), 0.05 (s, 6H), 0.02 (s, $3 \mathrm{H}), 0.01(\mathrm{~s}, 3 \mathrm{H}) ;{ }^{13} \mathrm{C} \mathrm{NMR}\left(150 \mathrm{MHz}, \mathrm{CDCl}_{3}\right) \delta 172.2,171.9,156.7,136.2,130.4$, 130.1(2C), 129.9, 128.5(2C), 128.1(2C), 128.0, 127.3, 126.2, 117.3, 117.2(2C), 92.9, 91.0, 71.5, 69.7, 66.9, 66.4, 64.7, 62.9, 62.2, 54.8, 52.2, 43.4, 37.3, 25.94(3C), 25.92(3C), 25.7, 22.4, 22.3, 20.2, 18.45(2C), -5.1, -5.2, -5.4, -5.3; CIHRMS Calcd for $\left[\mathrm{C}_{47} \mathrm{H}_{71} \mathrm{NO}_{12} \mathrm{Si}_{2} \mathrm{Na}^{+}\right]:$920.4407. Found 920.4401 .

1'- $N$-carbobenzyloxy-D-tyrosine methoxycarbonyl -5',5-(tert-butyl-

dimethylsilanyloxymethyl)-di-1,4- $\alpha$-1-mannose-O-4'-isovalaric ester (III-26a):

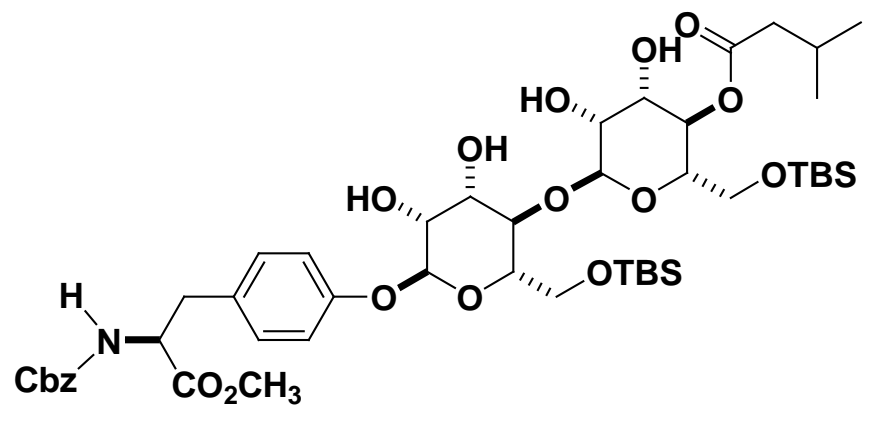


To a $\mathrm{CH}_{2} \mathrm{Cl}_{2}(1.6 \mathrm{~mL}, 0.1 \mathrm{M})$ solution of dienone III-25 (148 $\left.\mathrm{mg}, 0.16 \mathrm{mmol}\right)$ at $0{ }^{\circ} \mathrm{C}$ was added a solution of $(50 \% \mathrm{w} / \mathrm{v})$ of $N$-methyl morpholine $N$-oxide / water $(0.1 \mathrm{~mL})$. Crystalline $\mathrm{OsO}_{4}(4.2 \mathrm{mg}, 10 \mathrm{~mol} \%)$ was added and the reaction was stirred for $12 \mathrm{~h}$. The reaction mixture was concentrated and was pipetted directly on to a silica gel column using $\mathrm{CH}_{2} \mathrm{Cl}_{2}(1 \mathrm{~mL})$ in three portions. Impurities were eluted with ether and the product was eluted with $\mathrm{MeOH} / \mathrm{EtOAc} / \mathrm{Hexane}$ (10:40:50). Pure fractions were combined and concentrated to afford manno-tetrol III-26a (137 mg, $0.142 \mathrm{mmol}, 86 \%)$ as viscous oil. $R_{f}(60 \%$ EtOAc/Hexane $)=0.20 ;[\alpha]_{\mathrm{D}}^{26}=-20\left(c 1, \mathrm{CH}_{3} \mathrm{OH}\right) ; \mathrm{IR}$ (thin film, $\left.\mathrm{cm}^{-1}\right) 3441$, 2928, 2855, 1726, 1611, 1510, 1252, 1227, 1107, 1020, 835; ${ }^{1} \mathrm{H}$ NMR $(600 \mathrm{MHz}$, $\left.\mathrm{CDCl}_{3}\right) \delta 7.36(\mathrm{~m}, 5 \mathrm{H}), 7.10(\mathrm{~m}, 4 \mathrm{H}), 5.47(\mathrm{~d}, J=1.8 \mathrm{~Hz}, 1 \mathrm{H}), 5.44(\mathrm{~d}, J=1.8 \mathrm{~Hz}, 1 \mathrm{H})$, $5.20(\mathrm{dd}, J=10.2,9.6 \mathrm{~Hz}, 1 \mathrm{H}), 5.10(\mathrm{br} \mathrm{s}, 2 \mathrm{H}), 4.87$ (br s, 4H), 4.59 (m, 1H), 4.45 (dd, $J$ $=9.0,5.4 \mathrm{~Hz}, 1 \mathrm{H}), 4.08(\mathrm{dd}, J=9.0,3.0 \mathrm{~Hz}, 1 \mathrm{H}), 4.02(\mathrm{dd}, J=3.0,2.4 \mathrm{~Hz}, 1 \mathrm{H}), 4.00(\mathrm{~d}$, $J=1.8 \mathrm{~Hz}, 1 \mathrm{H}), 3.98(\mathrm{dd}, J=9.0,1.8 \mathrm{~Hz}, 1 \mathrm{H}), 3.90(\mathrm{dd}, J=10.2,9.0 \mathrm{~Hz}, 1 \mathrm{H}), 3.88$ (ddd, $J=10.2,6.6,2.4 \mathrm{~Hz}, 1 \mathrm{H}), 3.87(\mathrm{dd}, J=10.2,9.6 \mathrm{~Hz}, 1 \mathrm{H}), 3.84(\mathrm{dd}, J=4.8,1.8 \mathrm{~Hz}, 1 \mathrm{H})$, $3.83(\mathrm{~m}, 1 \mathrm{H}), 3.75(\mathrm{~m}, 3 \mathrm{H}), 3.74(\mathrm{dd}, J=4.2,1.8 \mathrm{~Hz}, 1 \mathrm{H}), 3.69(\mathrm{ddd}, J=9.6,6.6,1.8 \mathrm{~Hz}$, $1 \mathrm{H}), 3.13(\mathrm{dd}, J=13.8,5.4 \mathrm{~Hz}, 1 \mathrm{H}), 2.92(\mathrm{dd}, J=13.8,9.0 \mathrm{~Hz}, 1 \mathrm{H}), 2.29(\mathrm{dd}, J=15.0$, $7.2 \mathrm{~Hz}, 1 \mathrm{H}), 2.24(\mathrm{dd}, J=15.0,7.2 \mathrm{~Hz}, 1 \mathrm{H}), 2.13(\mathrm{~m}, 1 \mathrm{H}), 1.03(\mathrm{~d}, J=7.2 \mathrm{~Hz}, 6 \mathrm{H}), 0.95$ (s, 9H), 0.89 (s, 9H), 0.12 (s, 3H), 0.11 (s, 3H), 0.06 (s, 3H), 0.05 (s, 3H); ${ }^{13} \mathrm{C}$ NMR (150 $\left.\mathrm{MHz}, \mathrm{CDCl}_{3}\right) \delta 173.8,171.9,155.6,136.2,130.3(2 \mathrm{C}), 128.5(3 \mathrm{C}), 128.2,128.0$ 116.6(2C), 97.5, 96.9, 73.9, 71.49, 71.40, 71.0, 70.8, 70.3(2C), 68.8, 66.9, 63.0, 62.9, $54.8,52.3,43.4(2 \mathrm{C}), 37.4,25.9(3 \mathrm{C}), 25.8(3 \mathrm{C}), 25.8,22.3(2 \mathrm{C}), 22.3,18.4,18.3,-5.2,-5.3$, -5.40, -5.46; CIHRMS Calcd for $\left[\mathrm{C}_{47} \mathrm{H}_{75} \mathrm{NO}_{16} \mathrm{Si}_{2} \mathrm{Na}^{+}\right]$: 988.4516. Found 988.4485. 

mannose - $O-4$ ' isovaleric ester (III-26).

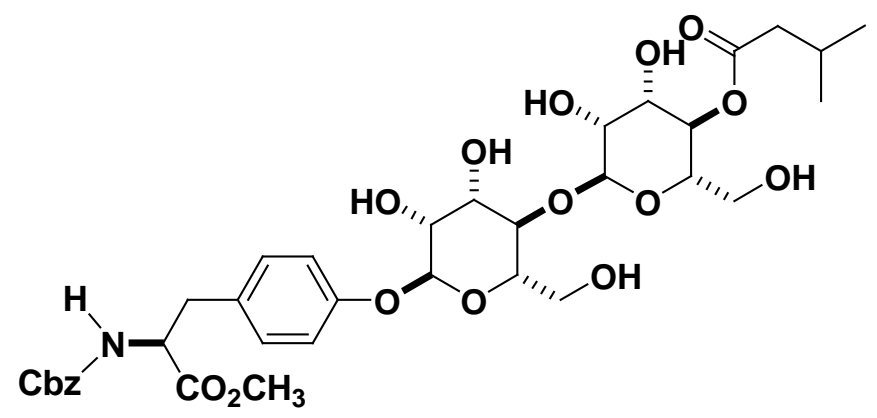

To a THF $(0.8 \mathrm{~mL}, 0.1 \mathrm{M})$ solution of manno-tetrol III-26a $(67 \mathrm{mg}, 0.16 \mathrm{mmol})$ at $0{ }^{\circ} \mathrm{C}$ was added a solution of TBAF in THF $(138 \mu \mathrm{L}, 1.0 \mathrm{M})$ and the reaction was stirred for 1 h. The reaction mixture was concentrated and was pipetted directly on to a silica gel column using $\mathrm{CH}_{2} \mathrm{Cl}_{2}(1 \mathrm{~mL})$ in three portions. Impurities were eluted with ether and the product was eluted with $\mathrm{MeOH} / \mathrm{EtOAc} / \mathrm{Hexane}$ (10:40:50). Pure fractions were combined and concentrated to afford III-26 (40 mg, $0.054 \mathrm{mmol}, 78 \%$ ) as viscous oil. $R_{f}(10: 50: 40 \% \mathrm{MeOH} /$ EtOAc/Hexane $)=0.20 ;[\alpha]_{\mathrm{D}}^{26}=-110\left(c 0.5, \mathrm{CH}_{3} \mathrm{OH}\right) ; \mathrm{IR}$ (thin film, $\left.\mathrm{cm}^{-1}\right) 3337,2956,2926,1738,1611,1510,1228,1217,1094,976,834 ;{ }^{1} \mathrm{H}$ NMR $\left(600 \mathrm{MHz}, \mathrm{CDCl}_{3}\right) \delta 7.35(\mathrm{~m}, 5 \mathrm{H}), 7.10(\mathrm{~m}, 4 \mathrm{H}), 5.48(\mathrm{~d}, J=1.8 \mathrm{~Hz}, 1 \mathrm{H}), 5.42(\mathrm{~d}, J=$ $2.4 \mathrm{~Hz}, 1 \mathrm{H}), 5.12$ (dd, $J=9.6,9.6 \mathrm{~Hz}, 1 \mathrm{H}), 5.07$ (br s, 2H), 4.59 (s, 1H), 4.45 (ddd, $J=$ 12.6, 9.6, $1.2 \mathrm{~Hz}, 1 \mathrm{H}), 4.10(\mathrm{dd}, J=9.0,3.6 \mathrm{~Hz}, 1 \mathrm{H}), 4.02-4.08(\mathrm{~m}, 4 \mathrm{H}), 4.00(\mathrm{dd}, J=$ 3.6, $1.8 \mathrm{~Hz}, 1 \mathrm{H}), 3.89(\mathrm{dd}, J=9.6,3.0 \mathrm{~Hz}, 1 \mathrm{H}), 3.83(\mathrm{dd}, J=5.4,3.0 \mathrm{~Hz}, 1 \mathrm{H}), 3.80(\mathrm{dd}, J$ $=7.2,5.4 \mathrm{~Hz}, 1 \mathrm{H}), 3.77-3.72(\mathrm{~m}, 1 \mathrm{H}), 3.75(\mathrm{~s}, 3 \mathrm{H}), 3.67(\mathrm{~s}, 1 \mathrm{H}), 3.62(\mathrm{dd}, J=6.0,3.0$ Hz, 1H), 3.35 (br s, 4H), 3.14 (dd, $J=13.8,4.2 \mathrm{~Hz}, 1 \mathrm{H}), 2.92(\mathrm{dd}, J=13.8,9.0 \mathrm{~Hz}, 1 \mathrm{H}$ ), $2.31(\mathrm{dd}, J=14.4,7.2 \mathrm{~Hz}, 1 \mathrm{H}), 2.30(\mathrm{dd}, J=7.2,2.4 \mathrm{~Hz}, 1 \mathrm{H}), 2.27(\mathrm{dd}, J=14.4,7.2 \mathrm{~Hz}$, 
1H), $2.14(\mathrm{~m}, 1 \mathrm{H}), 1.02(\mathrm{~d}, J=6.6 \mathrm{~Hz}, 6 \mathrm{H}) ; \mathrm{NMR}\left(150 \mathrm{MHz}, \mathrm{CDCl}_{3}\right) \delta$ 173.6, 172.0, 155.7, 136.1, 130.3(2C), 128.4(3C), 128.1, 127.9, 116.3(2C), 101.6, 97.9, 73.9, 71.9, 71.6(2C), 71.1, 70.6, 69.3, 66.9, 60.9, 60.6, 60.4, 54.8, 52.2, 43.3, 42.9, 37.2, 29.5, 25.6, 22.23, 22.20; CIHRMS Calcd for $\left[\mathrm{C}_{35} \mathrm{H}_{47} \mathrm{NO}_{16} \mathrm{Na}^{+}\right]$: 760.2787. Found 760.2750.

1'-N-carbobenzyloxy-D-tyrosine methoxycarbonyl -5',5-(tert-butyldimethylsilanyloxymethyl)-2,3,2',3'-bis acetonide-di-1,4- $\alpha$-l-mannose- $O-\mathbf{4}^{\prime}$ '-isovalaric ester (III-27).

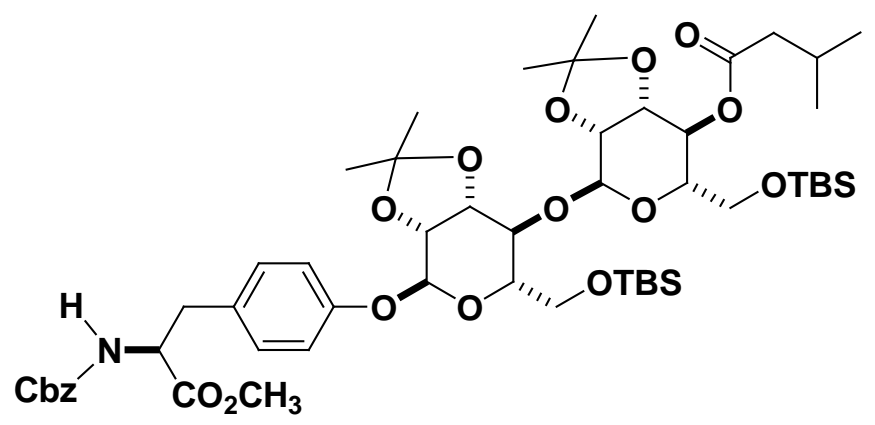

To a $\mathrm{CH}_{2} \mathrm{Cl}_{2}(0.1 \mathrm{~mL}, 1.0 \mathrm{M})$ solution of manno-tetrol III-26a $(25 \mathrm{mg}, 0.02 \mathrm{mmol})$ and 2,2-dimethoxypropane $(5.7 \mathrm{mg}, 0.05 \mathrm{mmol})$ at $0{ }^{\circ} \mathrm{C}$ was added CSA (0.60 mg, $\left.10 \mathrm{~mol} \%\right)$ and the reaction was stirred for $6 \mathrm{~h}$. The reaction mixture was concentrated and was pipetted directly on to a silica gel column using $\mathrm{CH}_{2} \mathrm{Cl}_{2}(1 \mathrm{~mL})$ in three portions. Impurities were eluted with ether and the product was eluted with EtOAc/Hexane (40:60). Pure fractions were combined and concentrated to afford III-27 (22 mg, 0.02 mmol, $81 \%)$ as viscous oil. $R_{f}(50 \%$ EtOAc/Hexane $)=0.44 ;[\alpha]_{\mathrm{D}}^{26}=-31(c 1$, $\mathrm{CH}_{2} \mathrm{Cl}_{2}$ ); IR (thin film, $\mathrm{cm}^{-1}$ ) 2953, 2928, 1726, 1510, 1220, 1091, 1019, 833; ${ }^{1} \mathrm{H}$ NMR $\left(600 \mathrm{MHz}, \mathrm{CDCl}_{3}\right) \delta 7.31(\mathrm{~m}, 5 \mathrm{H}), 6.96(\mathrm{~m}, 4 \mathrm{H}), 5.51(\mathrm{~d}, J=1.2 \mathrm{~Hz}, 1 \mathrm{H}), 5.42(\mathrm{~d}, J=$ 
$1.8 \mathrm{~Hz}, 1 \mathrm{H}), 5.06(\mathrm{br} \mathrm{s}, 2 \mathrm{H}), 4.96(\mathrm{dd}, J=10.2,7.8 \mathrm{~Hz}, 2 \mathrm{H}), 4.62(\mathrm{dd}, J=13.8,5.4 \mathrm{~Hz}$, $1 \mathrm{H}), 4.25(\mathrm{dd}, J=9.6,3.6, \mathrm{~Hz}, 1 \mathrm{H}), 4.16(\mathrm{dd}, J=12.6,5.4 \mathrm{~Hz}, 1 \mathrm{H}), 4.10(\mathrm{~d}, J=5.4 \mathrm{~Hz}$, 1H), $4.00(\mathrm{~m}, 1 \mathrm{H}), 3.91(\mathrm{~m}, 1 \mathrm{H}), 3.89(\mathrm{dd}, J=10.2,6.0 \mathrm{~Hz}, 1 \mathrm{H}), 3.86(\mathrm{dd}, J=9.6,4.8$ $\mathrm{Hz}, 1 \mathrm{H}), 3.84(\mathrm{~d}, J=9.0 \mathrm{~Hz}, 1 \mathrm{H}), 3.75(\mathrm{~m}, 2 \mathrm{H}), 3.69(\mathrm{~s}, 3 \mathrm{H}), 3.64(\mathrm{~d}, J=5.4 \mathrm{~Hz}, 1 \mathrm{H})$, $3.02(\mathrm{~m}, 2 \mathrm{H}), 3.03(\mathrm{~d}, J=6.0 \mathrm{~Hz}, 1 \mathrm{H}), 2.73(\mathrm{~s}, 1 \mathrm{H}), 2.56(\mathrm{~s}, 1 \mathrm{H}), 2.24(\mathrm{dd}, J=14.4,7.2$ Hz, 1H), 2.20 (m, 1H), 2.16 (s, 1H), $2.12(\mathrm{dd}, J=13.2,6.0 \mathrm{~Hz}, 1 \mathrm{H}), 0.94(\mathrm{~d}, J=6.6 \mathrm{~Hz}$, $6 \mathrm{H}), 0.87(\mathrm{~s}, 9 \mathrm{H}), 0.81(\mathrm{~s}, 9 \mathrm{H}), 0.06(\mathrm{~s}, 3 \mathrm{H}), 0.04(\mathrm{~s}, 3 \mathrm{H}),-0.08(\mathrm{~s}, 3 \mathrm{H}),-0.01(\mathrm{~s}, 3 \mathrm{H}) ;{ }^{13} \mathrm{C}$ NMR (150 MHz, $\left.\mathrm{CDCl}_{3}\right) \delta 172.0,171.9,155.5,155.4,130.3(2 \mathrm{C}), 128.4(3 \mathrm{C}), 128.1(2 \mathrm{C})$, 128.0, 116.8(2C), 109.9 (2C), 97.7, 95.0, 76.0, 75.8, 73.8, 71.2, 70.8, 69.8, 69.1(2C), $66.9,63.1,62.9,54.8,54.7,52.2,43.3(2 \mathrm{C}), 37.2,29.6,27.4,26.3,25.85(3 \mathrm{C}), 25.81(3 \mathrm{C})$, 25.6, 22.3, 22.2, $18.4, \quad 18.3, \quad-5.3(2 \mathrm{C}), \quad-5.47, \quad-5.57$; CIHRMS Calcd for $\left[\mathrm{C}_{53} \mathrm{H}_{83} \mathrm{NO}_{16} \mathrm{Si}_{2} \mathrm{Na}^{+}-\mathrm{C}_{3} \mathrm{H}_{6}{ }^{2+}\right]:$ 1028.5142. Found 1028.4585.

$\left(1^{\prime} R, 4^{\prime} R, 5 ' S, 1 R, 4 R, 5 S\right)-1-\left[1^{\prime}-N\right.$-carbobenzyloxy-D-tyrosine methoxycarbonyl -5'(tert-butyl-dimethylsilanyloxymethyl)-tetrahydro-pyran-4' -yloxy]-5-(tert-butyldimethylsilanyloxymethyl)-tetrahydro-pyran-O-4'-isovalaric ester (III-28):

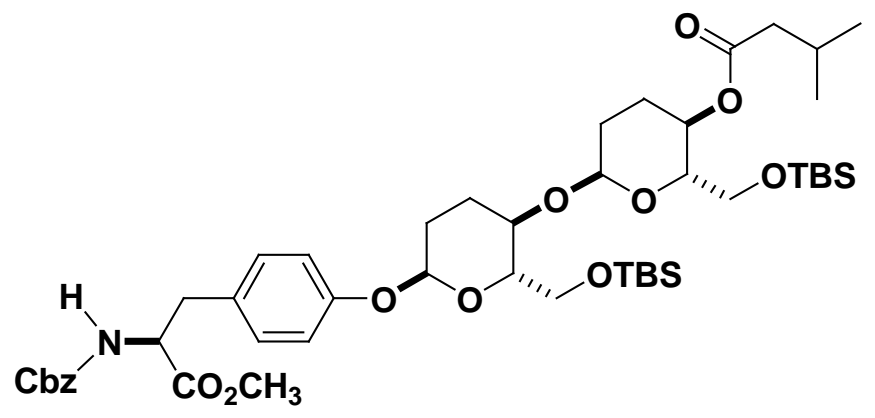

The diene ester III-25 (27 mg, $0.03 \mathrm{mmol})$ and $o-\mathrm{NO}_{2} \mathrm{C}_{6} \mathrm{H}_{4} \mathrm{SO}_{2} \mathrm{NHNH}_{2}(91.6 \mathrm{mg}, 0.45$ mmol) were dissolved in $0.3 \mathrm{~mL}$ of $\mathrm{CH}_{2} \mathrm{Cl}_{2}$ in a round bottom flask and cooled to $0{ }^{\circ} \mathrm{C}$ under nitrogen atmosphere then triethylamine $(60.6 \mathrm{mg}, 0.60 \mathrm{mmol})$ was added and the 
reaction mixture was stirred at $0{ }^{\circ} \mathrm{C}$ for 12 hours and on completion, as monitored by TLC. The reaction mixture was concentrated and was pipetted directly on to a silica gel column using $\mathrm{CH}_{2} \mathrm{Cl}_{2}(1 \mathrm{~mL})$ in three portions. The crude product was purified using silica gel flash chromatography eluting with $15 \%$ EtOAc/hexanes to give III-28 (26.2 $\mathrm{mg}, 0.03 \mathrm{mmol}, 97 \%)$ as viscous oil. $R_{f}(30 \%$ EtOAc/Hexane $)=0.46 ;[\alpha]_{\mathrm{D}}^{26}=-59(c 2$, $\mathrm{CH}_{2} \mathrm{Cl}_{2}$ ); IR (thin film, $\mathrm{cm}^{-1}$ ) 2955, 2928, 1738, 1510, 1252, 1219, 1122, 996, 835; ${ }^{1} \mathrm{H}$ NMR (600 MHz, CDCl $) \delta 7.33(\mathrm{~m}, 5 \mathrm{H}), 6.97(\mathrm{~m}, 4 \mathrm{H}), 5.44(\mathrm{br} \mathrm{s}, 1 \mathrm{H}), 5.24(\mathrm{~d}, J=7.8$ Hz, 1H), 5.08 (br s, 2H), 5.07 (d, $J=2.4 \mathrm{~Hz}, 1 \mathrm{H}), 4.73$ (ddd, $J=10.2,9.6,4.8 \mathrm{~Hz}, 1 \mathrm{H}$ ), 4.60 (ddd, $J=7.8,5.4,5.4 \mathrm{~Hz}, 1 \mathrm{H}), 3.85(\mathrm{~d}, J=11.4 \mathrm{~Hz}, 1 \mathrm{H}), 3.78-3.64(\mathrm{~m}, 6 \mathrm{H}), 3.70$ (s, 3H), $3.05(\mathrm{dd}, J=13.2,6.0 \mathrm{~Hz}, 1 \mathrm{H}), 3.01(\mathrm{dd}, J=13.2,5.4 \mathrm{~Hz}, 1 \mathrm{H}), 2.13(\mathrm{~m}, 3 \mathrm{H}), 2.06$ (ddd, $J=13.2,6.6,6.6 \mathrm{~Hz}, 1 \mathrm{H}), 2.00(\mathrm{~m}, 2 \mathrm{H}), 1.80(\mathrm{~m}, 3 \mathrm{H}), 1.71(\mathrm{~m}, 2 \mathrm{H}), 0.93(\mathrm{~d}, J=$ $6.6 \mathrm{~Hz}, 6 \mathrm{H}), 0.88$ (s, 9H), 0.84 (s, 9H), 0.02 (s, 6H), 0.018 (s, 3H), 0.004 (s, 3H); ${ }^{13} \mathrm{C}$ NMR $\left(150 \mathrm{MHz}, \mathrm{CDCl}_{3}\right) \delta$ 172.1, 172.0, 156.2, 136.2, 130.1(2C), 128.6, 128.4(2C), 128.1, 128.0, 116.9(2C), 94.9, 90.5, 75.0, 73.3, 71.5, 67.5, 66.9, 66.3, 63.2, 62.6, 54.8, 52.2, 43.6, 37.3, 28.7, 25.92(3C), 25.91(3C), 25.7, 23.6, 22.3(2C), 22.2(2C), 22.0, 18.4, 18.3, -5.1, -5.3, -5.43, -5.46; CIHRMS Calcd for $\left[\mathrm{C}_{47} \mathrm{H}_{75} \mathrm{NO}_{12} \mathrm{Si}_{2} \mathrm{Na}^{+}\right]$: 924.4720. Found 924.4717.

(1'S,4'S,5'R,1S,4S,5R)-1-[1'- $N$-carbobenzyloxy-D-tyrosine methoxycarbonyl -5'(tert-butyl-dimethylsilanyloxymethyl)-1',4'-dihydro-5'H-pyran-4'-yloxy] -5-(tertbutyl-dimethylsilanyloxymethyl)-1,4-dihydro-5H-pyran- $O$-4-methyl carbonate (III31). 


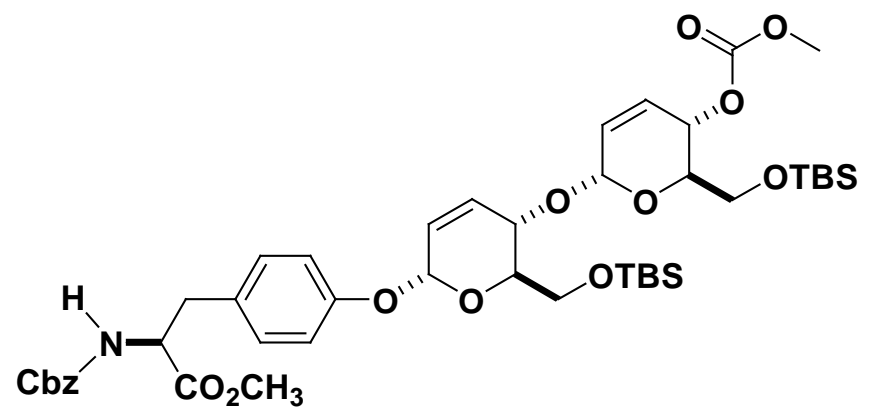

To a solution of allylic alcohol III-22 $(400 \mathrm{mg}, 0.50 \mathrm{mmol})$ in $\operatorname{dry} \mathrm{CH}_{2} \mathrm{Cl}_{2}(1.0 \mathrm{ml})$ at 0 ${ }^{\circ} \mathrm{C}$, was added pyridine (237 mg, $3.00 \mathrm{mmol}$ ), DMAP (30 mg), and methyl chloroformate (283 $\mathrm{mg}, 3.00 \mathrm{mmol})$. After stirring $24 \mathrm{~h}$ at room temperature, water $(3 \mathrm{ml})$ was added and then the mixture was extracted with EtOAc $(3 \times 10 \mathrm{ml})$, dried $\left(\mathrm{Na}_{2} \mathrm{SO}_{4}\right)$, concentrated under reduced pressure. The crude product was purified using silica gel flash chromatography eluting with 15\% EtOAc/hexane to give carbonate III-31 (370.6 mg, $0.43 \mathrm{mmol}, 85 \%)$ as viscous oil. $R_{f}(30 \%$ EtOAc/hexane $)=0.65 ;[\alpha]_{\mathrm{D}}^{26}=+50(c=1$, $\mathrm{CH}_{2} \mathrm{Cl}_{2}$ ); IR (thin film, $\mathrm{cm}^{-1}$ ) 2928, 2885, 1748, 1441, 1509, 1266, 1090, 981, 865; ${ }^{1} \mathrm{H}$ NMR (600 MHz, $\left.\mathrm{CDCl}_{3}\right) \delta 7.34(\mathrm{~m}, 5 \mathrm{H}), 7.00(\mathrm{~m}, 4 \mathrm{H}), 6.18(\mathrm{~d}, J=10.2 \mathrm{~Hz}, 1 \mathrm{H}), 6.01$ (d, $J=10.2 \mathrm{~Hz}, 1 \mathrm{H}), 5.94(\mathrm{dd}, J=2.4,1.8 \mathrm{~Hz}, 1 \mathrm{H}), 5.92(\mathrm{ddd}, J=10.8,2.4,1.8 \mathrm{~Hz}, 1 \mathrm{H})$, $5.77(\mathrm{ddd}, J=10.2,3.0,1.8 \mathrm{~Hz}, 1 \mathrm{H}), 5.59(\mathrm{~d}, J=1.8 \mathrm{~Hz}, 1 \mathrm{H}), 5.31(\mathrm{dd}, J=3.0,1.8 \mathrm{~Hz}$, $1 \mathrm{H}), 5.29(\mathrm{dd}, J=3.0,2.4 \mathrm{~Hz}, 1 \mathrm{H}), 5.11(\mathrm{~d}, J=12.6 \mathrm{~Hz}, 1 \mathrm{H}), 5.08(\mathrm{~d}, J=12.6 \mathrm{~Hz}, 1 \mathrm{H})$, $4.61(\mathrm{ddd}, J=8.4,6.0,5.4 \mathrm{~Hz}, 1 \mathrm{H}), 4.43(\mathrm{~d}, J=9.6 \mathrm{~Hz}, 1 \mathrm{H}), 3.88(\mathrm{dd}, J=5.4,2.4 \mathrm{~Hz}$, $1 \mathrm{H}), 3.86(\mathrm{dd}, J=6.0,2.4 \mathrm{~Hz}, 1 \mathrm{H}), 3.84(\mathrm{dd}, J=4.8,1.8 \mathrm{~Hz}, 1 \mathrm{H}), 3.83(\mathrm{dd}, J=4.8,2.4$ Hz, 1H), 3.81 (dd, $J=4.8,1.8 \mathrm{~Hz}, 1 \mathrm{H}), 3.79$ (s, 3H), 3.76 (s, 1H), 3.71 (s, 3H), 3.07 (ddd, $J=8.4,5.4,5.4 \mathrm{~Hz}, 1 \mathrm{H}), 3.05$ (ddd, $J=7.8,6.0,5.4 \mathrm{~Hz}, 1 \mathrm{H}), 0.89$ (s, 9H), $0.84(\mathrm{~s}, 9 \mathrm{H})$, $0.06(\mathrm{~s}, 3 \mathrm{H}), 0.05(\mathrm{~s}, 3 \mathrm{H}), 0.02(\mathrm{~s}, 3 \mathrm{H}), 0.01(\mathrm{~s}, 3 \mathrm{H}) ;{ }^{13} \mathrm{C} \mathrm{NMR}\left(150 \mathrm{MHz}, \mathrm{CDCl}_{3}\right) \delta$ $178.3,171.9,156.6,155.6,155.0,136.2,130.3,130.1,129.4,129.0,128.5$ (2C), 128.1, 
128.0, 127.6, 126.2, 117.2, 117.0, 109.9, 92.9, 91.0, 71.4, 69.3, 68.4, 66.9, 66.4, 62.7, $62.0,54.9,54.8,52.2,37.4,25.93(3 \mathrm{C}), 25.91$ (3C), 18.43, 18.42, -5.0, -5.2, -5.4 (2C); CIHRMS Calcd for $\left[\mathrm{C}_{44} \mathrm{H}_{65} \mathrm{NO}_{13} \mathrm{Si}_{2} \mathrm{Na}^{+}\right]$: 894.3892. Found 894.3890.

$(1 ' S, 4$ 'S, 5' $R, 1 S, 4 S, 5 R)-1$-[1'- $N$-carbobenzyloxy-D-tyrosine methoxycarbonyl -5'-(tertbutyl-dimethylsilanyloxymethyl)-1',4'-dihydro-5'H-pyran-4'-yloxy]-5-(tert-butyldimethylsilanyloxymethyl)-4-azido-1,4-dihydro-5H-pyran (III-32).

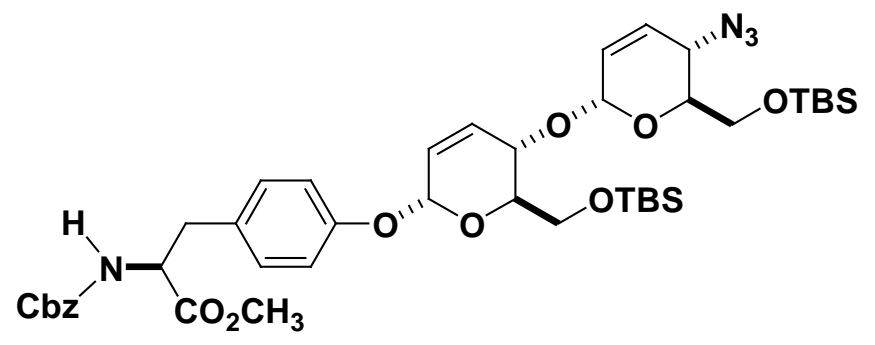

To a mixture of carbonate III-31 (210 mg, $0.24 \mathrm{mmol})$, allylpalladium chloride dimer (13.2 $\mathrm{mg}, 0.03 \mathrm{mmol})$ and 1,4-bis(diphenylphosphino)butane $(61.6 \mathrm{mg}, 0.13 \mathrm{mmol})$ in dry THF $(0.25 \mathrm{ml})$ was added TMSN $_{3}(277 \mathrm{mg}, 1.20 \mathrm{mmol})$ under argon atmosphere. The solution was stirred at room temperature for $3 \mathrm{~h}$. Then the mixture was evaporated with a amount of silica gel under reduced pressure, purified using silica gel flash chromatography eluting with 15\% EtOAc/hexane to give allylic azide III-32 (162 mg, $0.19 \mathrm{mmol}, 80 \%)$ as viscous oil. $R_{f}(30 \%$ EtOAc/hexane $)=0.70 ;[\alpha]_{\mathrm{D}}^{26}=+65(c=1$, $\mathrm{CH}_{2} \mathrm{Cl}_{2}$ ); IR (thin film, $\mathrm{cm}^{-1}$ ) 2952, 2929, 2856, 2103, 1726, 1510, 1253, 1043, 993, 830; ${ }^{1} \mathrm{H}$ NMR $\left(600 \mathrm{MHz}, \mathrm{CDCl}_{3}\right) \delta 7.35(\mathrm{~m}, 5 \mathrm{H}), 6.99(\mathrm{~m}, 4 \mathrm{H}), 6.18(\mathrm{~d}, J=10.2 \mathrm{~Hz}, 1 \mathrm{H})$, $6.00(\mathrm{~d}, J=10.2 \mathrm{~Hz}, 1 \mathrm{H}), 5.94(\mathrm{ddd}, J=10.8,2.4,1.8 \mathrm{~Hz}, 1 \mathrm{H}), 5.83(\mathrm{ddd}, J=10.2,3.0$, $2.4 \mathrm{~Hz}, 1 \mathrm{H}), 5.59(\mathrm{~d}, J=2.4 \mathrm{~Hz}, 1 \mathrm{H}), 5.26(\mathrm{~d}, J=1.8 \mathrm{~Hz}, 1 \mathrm{H}), 5.11(\mathrm{~d}, J=12.6 \mathrm{~Hz}, 1 \mathrm{H})$, $5.08(\mathrm{~d}, J=12.6 \mathrm{~Hz}, 1 \mathrm{H}), 4.62(\mathrm{ddd}, J=7.8,6.0,5.4 \mathrm{~Hz}, 1 \mathrm{H}), 4.42(\mathrm{~d}, J=9.0 \mathrm{~Hz}, 1 \mathrm{H})$, 
$4.14(\mathrm{dd}, J=3.8,1.8 \mathrm{~Hz}, 1 \mathrm{H}), 4.12(\mathrm{dd}, J=10.2,1.8 \mathrm{~Hz}, 1 \mathrm{H}), 3.92(\mathrm{~d}, J=3.0 \mathrm{~Hz}, 1 \mathrm{H})$, $3.90(\mathrm{~d}, J=3.0 \mathrm{~Hz}, 1 \mathrm{H}), 3.88(\mathrm{ddd}, J=4.2,3.6,3.0 \mathrm{~Hz}, 1 \mathrm{H}), 3.86(\mathrm{~d}, J=1.8 \mathrm{~Hz}, 1 \mathrm{H})$, $3.80(\mathrm{dd}, J=4.2,2.4 \mathrm{~Hz}, 1 \mathrm{H}), 3.71(\mathrm{~s}, 3 \mathrm{H}), 3.65(\mathrm{ddd}, J=9.0,3.0,1.8 \mathrm{~Hz}, 1 \mathrm{H}), 3.08$ (ddd, $J=8.4,6.0,5.4 \mathrm{~Hz}, 1 \mathrm{H}), 3.04$ (ddd, $J=8.4,6.0,5.4 \mathrm{~Hz}, 1 \mathrm{H}), 0.91(\mathrm{~s}, 9 \mathrm{H}), 0.85$ (s, 9H), 0.099 (s, 3H), 0.094 (s, 3H), 0.03 (s, 3H), 0.02 (s, 3H); ${ }^{13} \mathrm{C} \mathrm{NMR}\left(150 \mathrm{MHz}, \mathrm{CDCl}_{3}\right)$ $\delta 171.9,156.6,155.6,136.2,130.3,130.1(2 \mathrm{C}), 129.0(2 \mathrm{C}), 128.5(2 \mathrm{C}), 128.1,128.0$ (2C), 126.2, $117.2(2 \mathrm{C}), 109.9,92.9,90.8,71.3,70.5,66.9,66.2,62.6,62.4,54.8,53.4$, 52.2, 37.3, 25.9 (6C), 18.4 (2C), $-5.0,-5.2$ (2C), -5.3 ; CIHRMS Calcd for $\left[\mathrm{C}_{42} \mathrm{H}_{62} \mathrm{~N}_{4} \mathrm{O}_{10} \mathrm{Si}_{2} \mathrm{Na}^{+}\right]:$861.3902. Found 861.3910.

$\left(1 ' S, 4^{\prime} S, 5^{\prime} R, 1 S, 4 S, 5 R\right)-1-\left[1^{\prime}-N\right.$-carbobenzyloxy-D-tyrosine methoxycarbonyl -5'(tert-butyl-dimethylsilanyloxymethyl)-1',4'-dihydro-5'H-pyran-4'-yloxy]-5-(tertbutyl-dimethylsilanyloxymethyl)-4-amino-1,4-dihydro-5H-pyran-( III-30).

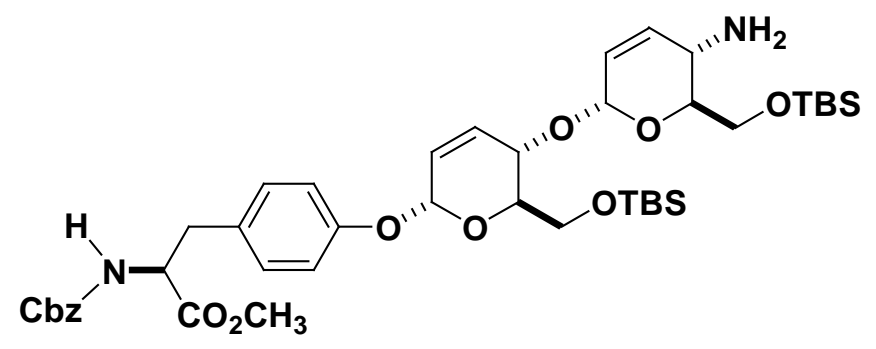

To a solution of Azide III-32 (140 mg, $0.16 \mathrm{mmol})$ in THF/ $\mathrm{H}_{2} \mathrm{O}(9: 1, \mathrm{v} / \mathrm{v}, 0.3 \mathrm{ml})$ was added $(n-\mathrm{Bu})_{3} \mathrm{P}(84 \mathrm{mg}, 0.41 \mathrm{mmol})$, the mixture was stirred at room temperature for $1 \mathrm{~h}$. Then the reaction mixture was dried with a little silica gel under reduced pressure and the crude product was purified using silica gel flash chromatography eluting with $\mathrm{MeOH} / \mathrm{EtOAc} / \mathrm{hexane}$ (10:40:50) to give allylic amine III-30 (100 mg, $0.12 \mathrm{mmol}, 70 \%)$ as a colorless oil, $R_{f}=0.50(10: 40: 50 \% \mathrm{MeOH} / \mathrm{EtOAc} / \mathrm{Hexane}) ;[\alpha]_{\mathrm{D}}^{26}=+24(c=1$, 
$\mathrm{CH}_{2} \mathrm{Cl}_{2}$ ); IR (thin film, $\mathrm{cm}^{-1}$ ) 3361, 2944, 2833, 1740, 1448, 1374, 1240, 1120, 1040, 981, 847; ${ }^{1} \mathrm{H}$ NMR $\left(600 \mathrm{MHz}, \mathrm{CD}_{3} \mathrm{OD}\right) \delta 7.27(\mathrm{~m}, 5 \mathrm{H}), 6.99(\mathrm{~m}, 4 \mathrm{H}), 6.21(\mathrm{~d}, J=9.6$ $\mathrm{Hz}, 1 \mathrm{H}), 5.91(\mathrm{~d}, J=10.2 \mathrm{~Hz}, 1 \mathrm{H}), 5.84(\mathrm{ddd}, J=10.2,10.2,8.4 \mathrm{~Hz}, 1 \mathrm{H}), 5.69$ (ddd, $J=$ $11.4,10.2,10.2 \mathrm{~Hz}, 1 \mathrm{H}), 5.59$ (d, $J=2.4 \mathrm{~Hz}, 1 \mathrm{H}), 5.22$ (br s, $1 \mathrm{H}), 5.02$ (d, $J=12.6 \mathrm{~Hz}$, 1H), $4.95(\mathrm{~d}, J=12.6 \mathrm{~Hz}, 1 \mathrm{H}), 4.36(\mathrm{~m}, 1 \mathrm{H}), 4.27(\mathrm{~d}, J=9.0 \mathrm{~Hz}, 1 \mathrm{H}), 3.91(\mathrm{dd}, J=10.8$, $4.2 \mathrm{~Hz}, 1 \mathrm{H}), 3.86(\mathrm{~m}, 1 \mathrm{H}), 3.83(\mathrm{dd}, J=6.0,3.0 \mathrm{~Hz}, 1 \mathrm{H}), 3.80(\mathrm{dd}, J=10.2,4.2 \mathrm{~Hz}, 1 \mathrm{H})$, $3.75(\mathrm{dd}, J=10.8,6.0 \mathrm{~Hz}, 1 \mathrm{H}), 3.65(\mathrm{~s}, 3 \mathrm{H}), 3.50(\mathrm{ddd}, J=9.6,4.2,3.6 \mathrm{~Hz}, 1 \mathrm{H}), 3.46$ (ddd, $J=9.0,4.8,4.2 \mathrm{~Hz}, 1 \mathrm{H}), 3.36$ (dd, $J=11.4,9.6 \mathrm{~Hz}, 1 \mathrm{H}$ ), 3.03 (ddd, $J=8.4,6.0,5.4$ Hz, 1H), 2.85 (ddd, $J=8.4,6.0,5.4 \mathrm{~Hz}, 1 \mathrm{H}), 2.11(\mathrm{~s}, 2 \mathrm{H}), 0.89$ (d, $J=4.2 \mathrm{~Hz}, 9 \mathrm{H}), 0.79$ $(\mathrm{d}, J=5.4 \mathrm{~Hz}, 9 \mathrm{H}), 0.77(\mathrm{~d}, J=4.2 \mathrm{~Hz}, 6 \mathrm{H}),-0.00(\mathrm{~s}, 3 \mathrm{H}),-0.02(\mathrm{~d}, J=2.4 \mathrm{~Hz}, 3 \mathrm{H}) ;{ }^{13} \mathrm{C}$ NMR (150 MHz, $\left.\mathrm{CDCl}_{3}\right) \delta$ 172.3, 172.0, 156.6, 155.7, 136.2, 131.7, 130.1 (2C), 129.1 (2C), 128.4 (2C), $128.1(2 \mathrm{C}), 126.4,117.3$ (2C), 92.9, 90.7, 71.6, 66.9, 63.6, 63.2, 54.8, 52.2, 46.1, 44.0, 37.2, 26.1, 25.9 (3C), 25.8 (3C), 22.3, 18.4, 18.3, -5.1, -5.2, -5.3, -5.4; CIHRMS Calcd for $\left[\mathrm{C}_{42} \mathrm{H}_{64} \mathrm{~N}_{2} \mathrm{O}_{10} \mathrm{Si}_{2} \mathrm{H}^{+}\right]$: 813.4177. Found 813.4177.

$(1 ' S, 4 ' S, 5 ' R, 1 S, 4 S, 5 R)-1-[1 '-N$-carbobenzyloxy-D-tyrosine methoxycarbonyl -5'(tert-butyl-dimethylsilanyloxymethyl)-1',4' -dihydro-5'H-pyran-4'-yloxy] -5-(tertbutyl-dimethylsilanyloxymethyl)-1,4-dihydro-5H-pyran- $\mathrm{N}$-4-isovalaric amide (III33).

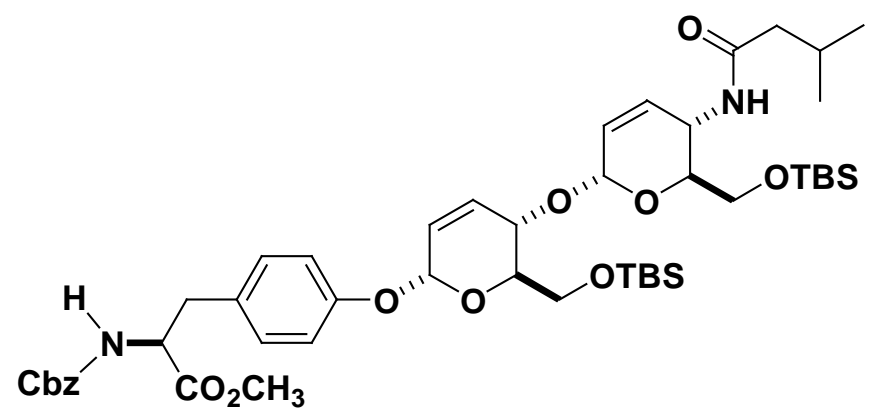


The amine compound III-30 (100 mg, $0.12 \mathrm{mmol})$, isovaleric acid (15 mg, $0.15 \mathrm{mmol})$ and DCC (30 mg, $0.14 \mathrm{mmol}$ ) were dissolved in $0.3 \mathrm{~mL}$ of $\mathrm{CH}_{2} \mathrm{Cl}_{2}$ in a round bottom flask and cooled to $0{ }^{\circ} \mathrm{C}$ then DMAP (2 mg, $0.01 \mathrm{mmol}$ ) was added and the reaction mixture was stirred at $0{ }^{\circ} \mathrm{C}$ for 6 hours and on completion, as monitored by TLC, the reaction mixture was diluted with ether and was quenched with $5 \mathrm{~mL}$ of satd. aq. $\mathrm{NaHCO}_{3}$, extracted $(3 \times 5 \mathrm{~mL})$ with $\mathrm{Et}_{2} \mathrm{O}$, dried $\left(\mathrm{Na}_{2} \mathrm{SO}_{4}\right)$, and concentrated under reduced pressure. The crude product was purified using silica gel flash chromatography eluting with 30\% EtOAc/hexanes to give amide III-33 (94 mg, $0.10 \mathrm{mmol}, 85 \%$ ) as viscous oil. $R_{f}(30 \%$ EtOAc/hexanes $)=0.70 ;[\alpha]_{\mathrm{D}}^{26}=+4\left(c=1, \mathrm{CH}_{2} \mathrm{Cl}_{2}\right)$; IR (thin film, $\left.\mathrm{cm}^{-1}\right) 2985,2927,1736,1447,1372,1253,1098,1043,938,846 ;{ }^{1} \mathrm{H}$ NMR $(600 \mathrm{MHz}$, $\left.\mathrm{CDCl}_{3}\right) \delta 7.33(\mathrm{~m}, 5 \mathrm{H}), 7.00(\mathrm{~m}, 4 \mathrm{H}), 6.20(\mathrm{~d}, J=10.8 \mathrm{~Hz}, 1 \mathrm{H}), 5.92(\mathrm{ddd}, J=10.2,3.0$, $1.8 \mathrm{~Hz}, 1 \mathrm{H}), 5.81(\mathrm{~d}, J=10.2 \mathrm{~Hz}, 1 \mathrm{H}), 5.71(\mathrm{ddd}, J=10.2,3.0,2.4 \mathrm{~Hz}, 1 \mathrm{H}), 5.56(\mathrm{~d}, J=$ $2.4 \mathrm{~Hz}, 1 \mathrm{H}), 5.21(\mathrm{~m}, 1 \mathrm{H}), 5.07$ (br s, 2H), $4.58(\mathrm{dd}, J=10.2,5.4 \mathrm{~Hz}, 1 \mathrm{H}), 4.52(\mathrm{~d}, J=$ $8.4 \mathrm{~Hz}, 1 \mathrm{H}), 4.32(\mathrm{~d}, J=9.0 \mathrm{~Hz}, 1 \mathrm{H}), 3.90(\mathrm{~m}, 1 \mathrm{H}), 3.86(\mathrm{~d}, J=11.4 \mathrm{~Hz}, 1 \mathrm{H}), 3.82(\mathrm{~m}$, 2H), $3.76(\mathrm{ddd}, J=11.4,5.4,4.8 \mathrm{~Hz}, 1 \mathrm{H}), 3.72(\mathrm{dd}, J=11.4,3.0 \mathrm{~Hz}, 1 \mathrm{H}), 3.69(\mathrm{~s}, 3 \mathrm{H})$, $3.66(\mathrm{dd}, J=3.0,2.4 \mathrm{~Hz}, 1 \mathrm{H}), 3.64(\mathrm{dd}, J=4.2,2.4 \mathrm{~Hz}, 1 \mathrm{H}), 3.03(\mathrm{~m}, 2 \mathrm{H}), 2.11(\mathrm{~m}, 1 \mathrm{H})$, $2.00(\mathrm{~d}, J=7.8 \mathrm{~Hz}, 2 \mathrm{H}), 0.93(\mathrm{~d}, J=6.6 \mathrm{~Hz}, 6 \mathrm{H}), 0.87(\mathrm{~s}, 9 \mathrm{H}), 0.80(\mathrm{~s}, 9 \mathrm{H}), 0.04(\mathrm{~s}, 3 \mathrm{H})$, $0.03(\mathrm{~s}, 3 \mathrm{H}), 0.00(\mathrm{~s}, 3 \mathrm{H}),-0.02(\mathrm{~s}, 3 \mathrm{H}) ;{ }^{13} \mathrm{C} \mathrm{NMR}\left(150 \mathrm{MHz}, \mathrm{CDCl}_{3}\right) \delta$ 171.9, 156.6, 155.6, 136.2, 133.1, 130.5, 130.1, 129.0, 128.4 (2C), 128.1 (2C), $128.0(2 \mathrm{C}), 126.1,125.4$ (2C), 117.2 (2C), 92.8, 91.1, 71.4, 70.0, 66.9 (2C), 66.8, 66.7, $65.3(2 \mathrm{C}), 62.6,54.8,52.2$, 37.3, 27.3, 25.9 (3C), 25.8 (3C), 24.0, 18.3, 18.2, -5.0, -5.1, -5.4, -5.6; CIHRMS Calcd for $\left[\mathrm{C}_{47} \mathrm{H}_{72} \mathrm{~N}_{2} \mathrm{O}_{11} \mathrm{Si}_{2} \mathrm{Na}^{+}\right]$: 919.4572. Found 919.4543. 


\section{1'- $N$-carbobenzyloxy-D-tyrosine methoxycarbonyl -5',5-(tert-butyl-}

\section{dimethylsilanyloxymethyl)-di-1,4- $\alpha$-D-mannose $-N-4$ isovalaric amide (III-34):}

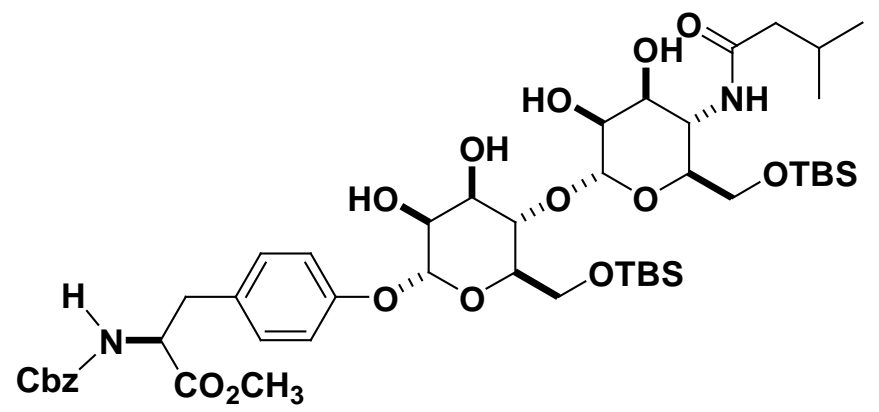

To a $\mathrm{CH}_{2} \mathrm{Cl}_{2}(1.3 \mathrm{~mL})$ solution of diene amide III-33 $(120 \mathrm{mg}, 0.13 \mathrm{mmol})$ at $0{ }^{\circ} \mathrm{C}$ was added a solution of $(50 \% \mathrm{w} / \mathrm{v})$ of $N$-methyl morpholine $N$-oxide / water $(0.1 \mathrm{~mL})$. Crystalline $\mathrm{OsO}_{4}(3.4 \mathrm{mg}, 10 \mathrm{~mol} \%)$ was added and the reaction was stirred for $12 \mathrm{~h}$. The reaction mixture was concentrated and was pipetted directly on to a silica gel column using $\mathrm{CH}_{2} \mathrm{Cl}_{2}(1 \mathrm{~mL})$ in three portions. Impurities were eluted with ether and the product was eluted with $\mathrm{MeOH} / \mathrm{EtOAc} /$ hexanes (10:40:50). Pure fractions were combined and concentrated to afford bis-manno-amido-tetrol III-34 (101 $\mathrm{mg}, 0.10 \mathrm{mmol}, 78 \%)$ as viscous oil. $R_{f}(90 \% \mathrm{EtOAc} / \mathrm{MeOH})=0.40 ;[\alpha]_{\mathrm{D}}^{26}=+12.5\left(c=1, \mathrm{CH}_{2} \mathrm{Cl}_{2}\right)$; IR (thin film, $\left.\mathrm{cm}^{-1}\right) 2956,2928,1738,1733,1510,1253,1219,1123,986,835 ;{ }^{1} \mathrm{H}$ NMR $(600$ $\left.\mathrm{MHz}, \mathrm{CDCl}_{3}\right) \delta 7.30(\mathrm{~m}, 5 \mathrm{H}), 6.98(\mathrm{~m}, 4 \mathrm{H}), 5.45(\mathrm{~d}, J=1.2 \mathrm{~Hz}, 1 \mathrm{H}), 5.34(\mathrm{~d}, J=1.8 \mathrm{~Hz}$, $1 \mathrm{H}), 5.22(\mathrm{~d}, J=1.8 \mathrm{~Hz}, 1 \mathrm{H}), 5.01(\mathrm{~d}, J=12.6 \mathrm{~Hz}, 1 \mathrm{H}), 5.00(\mathrm{~d}, J=12.6 \mathrm{~Hz}, 1 \mathrm{H}), 4.50$ (br s, 1H), $4.37(\mathrm{dd}, J=8.4,5.4 \mathrm{~Hz}, 1 \mathrm{H}), 4.34(\mathrm{dd}, J=9.0,4.8 \mathrm{~Hz}, 1 \mathrm{H}), 4.06(\mathrm{dd}, J=$ 14.4, 7.2 Hz, 1H), $4.02(\mathrm{dd}, J=6.6,6.6 \mathrm{~Hz}, 1 \mathrm{H}), 3.99(\mathrm{dd}, J=9.0,4.2 \mathrm{~Hz}, 1 \mathrm{H}), 3.93(\mathrm{dd}$, $J=6.0,4.2 \mathrm{~Hz}, 1 \mathrm{H}), 3.91(\mathrm{~m}, 1 \mathrm{H}), 3.85(\mathrm{~d}, J=11.4 \mathrm{~Hz}, 1 \mathrm{H}), 3.82(\mathrm{dd}, J=6.0,5.4 \mathrm{~Hz}$, $1 \mathrm{H}), 3.81(\mathrm{dd}, J=9.6,5.4 \mathrm{~Hz}, 1 \mathrm{H}), 3.73(\mathrm{dd}, J=6.6,3.0 \mathrm{~Hz}, 1 \mathrm{H}), 3.72(\mathrm{~d}, J=3.0 \mathrm{~Hz}$, $1 \mathrm{H}), 3.70(\mathrm{dd}, J=3.0,1.8 \mathrm{~Hz}, 1 \mathrm{H}), 3.68(\mathrm{dd}, J=6.0,1.8 \mathrm{~Hz}, 1 \mathrm{H}), 3.65(\mathrm{~s}, 3 \mathrm{H}), 3.60(\mathrm{~m}$, 
$1 \mathrm{H}), 3.03(\mathrm{dd}, J=9.6,4.8 \mathrm{~Hz}, 1 \mathrm{H}), 3.02(\mathrm{dd}, J=9.0,4.8 \mathrm{~Hz}, 1 \mathrm{H}), 2.86(\mathrm{dd}, J=9.0,4.2$ $\mathrm{Hz}, 1 \mathrm{H}), 2.84(\mathrm{dd}, J=9.6,4.2 \mathrm{~Hz}, 1 \mathrm{H}), 2.15(\mathrm{~m}, 1 \mathrm{H}), 2.04(\mathrm{dd}, J=5.4,1.8 \mathrm{~Hz}, 1 \mathrm{H}), 2.02$ $(\mathrm{dd}, J=4.8,1.2 \mathrm{~Hz}, 1 \mathrm{H}), 0.87(\mathrm{~d}, J=6.0 \mathrm{~Hz}, 6 \mathrm{H}), 0.81(\mathrm{~s}, 9 \mathrm{H}), 0.80(\mathrm{~s}, 9 \mathrm{H}), 0.05(\mathrm{~s}, 3 \mathrm{H})$, $0.03(\mathrm{~s}, 3 \mathrm{H}), 0.00(\mathrm{~s}, 3 \mathrm{H}),-0.01(\mathrm{~s}, 3 \mathrm{H}) ;{ }^{13} \mathrm{C} \mathrm{NMR}\left(150 \mathrm{MHz}, \mathrm{CDCl}_{3}\right) \delta$ 173.0, 172.9, 157.3, 155.9, 137.1, 131.1, 130.3 (2C), 128.4 (2C), 127.9, 127.6 (2C), 116.9 (2C), 101.1, $99.0,73.7,73.5,72.5,72.1,71.3(2 \mathrm{C}), 69.7,69.4,66.5,64.1,63.3,56.0,51.6,43.5(2 \mathrm{C})$, 36.81, 30.9, 28.8, 25.8 (2C), 25.5 (3C), 21.8, 18.3, 18.2, -5.7, -5.9, -6.1, -6.3; CIHRMS Calcd for $\left[\mathrm{C}_{47} \mathrm{H}_{76} \mathrm{~N}_{2} \mathrm{O}_{15} \mathrm{Si}_{2} \mathrm{Na}^{+}\right]$: 987.4682. Found 987.4680.

\section{1 '- $N$-carbobenzyloxy-D-tyrosine methoxycarbonyl -5',5-(hydroxy methyl)-di-1,4- $\alpha$ - D-mannose $-N-4$ isovaleric amide (III-29).}

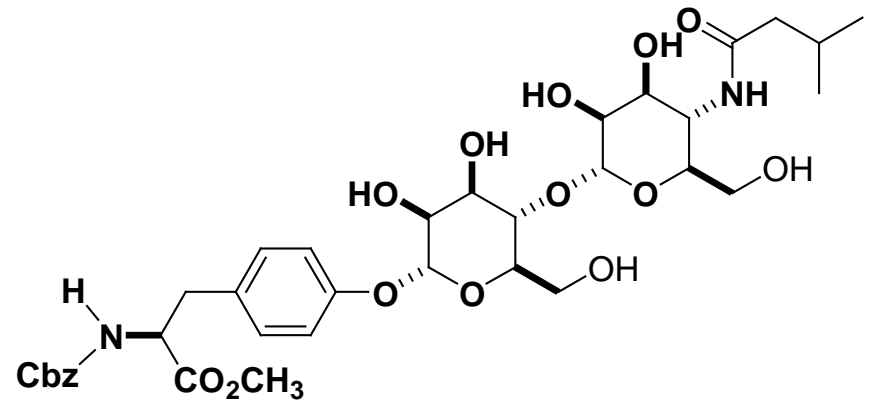

To a THF $(0.4 \mathrm{~mL}, 0.1 \mathrm{M})$ solution of D-manno-tetrol III-34 (40 mg, $0.04 \mathrm{mmol})$ at $0{ }^{\circ} \mathrm{C}$ was added a solution of TBAF in THF $(85 \mu \mathrm{L}, 0.08 \mathrm{mmol})$ and the reaction was stirred for $1 \mathrm{~h}$. The reaction mixture was concentrated and was pipetted directly on to a silica gel column using $\mathrm{CH}_{2} \mathrm{Cl}_{2}(1 \mathrm{~mL})$ in three portions. Impurities were eluted with ether and the product was eluted with $\mathrm{MeOH} / \mathrm{EtOAc} / \mathrm{Hexane}$ (20:40:40). Pure fractions were combined and concentrated to afford hexol III-29 (31 mg, $0.04 \mathrm{mmol}, 80 \%)$ as viscous oil. $R_{f}(10: 50: 40 \% \mathrm{MeOH} / \mathrm{EtOAc} /$ Hexane $)=0.20 ;[\alpha]_{\mathrm{D}}^{26}=+15\left(c 0.5, \mathrm{CH}_{3} \mathrm{OH}\right) ; \mathrm{IR}$ 
(thin film, $\mathrm{cm}^{-1}$ ) 3330, 2960, 2928, 1735, 1621, 1511, 1232, 1092, 983, 829; ${ }^{1} \mathrm{H}$ NMR $\left(600 \mathrm{MHz}, \mathrm{CDCl}_{3}\right) \delta 7.30(\mathrm{~m}, 5 \mathrm{H}), 6.99(\mathrm{~m}, 4 \mathrm{H}), 5.39(\mathrm{~d}, J=1.2 \mathrm{~Hz}, 1 \mathrm{H}), 5.33(\mathrm{~d}, J=$ $1.8 \mathrm{~Hz}, 1 \mathrm{H}), 5.23(\mathrm{~d}, J=1.8 \mathrm{~Hz}, 1 \mathrm{H}), 5.00(\mathrm{~m}, 2 \mathrm{H}), 4.37(\mathrm{dd}, J=5.4,4.8 \mathrm{~Hz}, 1 \mathrm{H}), 4.35$ (dd, $J=5.4,4.2 \mathrm{~Hz}, 1 \mathrm{H}), 4.16(\mathrm{dd}, J=11.4,6.6 \mathrm{~Hz}, 1 \mathrm{H}), 4.01(\mathrm{dd}, J=9.0,3.6 \mathrm{~Hz}, 1 \mathrm{H})$, $3.96(\mathrm{dd}, J=9.6,6.6 \mathrm{~Hz}, 1 \mathrm{H}), 3.91(\mathrm{dd}, J=3.6,1.8 \mathrm{~Hz}, 1 \mathrm{H}), 3.80(\mathrm{dd}, J=9.6,3.0 \mathrm{~Hz}$, $1 \mathrm{H}), 3.74(\mathrm{dd}, J=8.4,4.8 \mathrm{~Hz}, 1 \mathrm{H}), 3.71(\mathrm{dd}, J=8.4,4.2 \mathrm{~Hz}, 1 \mathrm{H}), 3.67(\mathrm{~d}, J=3.0 \mathrm{~Hz}$, $1 \mathrm{H}), 3.65(\mathrm{~m}, 3 \mathrm{H}), 3.60(\mathrm{dd}, J=9.0,4.2 \mathrm{~Hz}, 1 \mathrm{H}), 3.58(\mathrm{dd}, J=9.6,4.2 \mathrm{~Hz}, 1 \mathrm{H}), 3.53(\mathrm{dd}$, $J=5.4,3.0 \mathrm{~Hz}, 1 \mathrm{H}), 3.15(\mathrm{dd}, J=5.4,2.4 \mathrm{~Hz}, 1 \mathrm{H}), 3.05(\mathrm{dd}, J=9.0,4.8 \mathrm{~Hz}, 1 \mathrm{H}), 3.04$ (dd, $J=9.6,4.8 \mathrm{~Hz}, 1 \mathrm{H}), 2.84(\mathrm{~m}, 2 \mathrm{H}), 2.22(\mathrm{dd}, J=4.8,1.8 \mathrm{~Hz}, 1 \mathrm{H}), 2.19(\mathrm{dd}, J=5.4$, $1.8 \mathrm{~Hz}, 1 \mathrm{H}), 2.04(\mathrm{~m}, 1 \mathrm{H}), 0.92(\mathrm{~d}, J=6.6 \mathrm{~Hz}, 6 \mathrm{H}) ; \mathrm{NMR}\left(150 \mathrm{MHz}, \mathrm{CDCl}_{3}\right) \delta 174.5$, 174.0, 158.4, 156.8, 138.2, 132.2, 131.4 (2C), 129.5 (2C), 129.0, 128.7 (2C), 117.8, $102.8,103.4,100.1,75.7,74.9,74.0,73.8,73.6,73.0,72.5,70.6,67.6,65.2,62.8,62.4$, 57.1, 52.8, 44.4, 37.9, 26.8, 22.9; CIHRMS Calcd for $\left[\mathrm{C}_{35} \mathrm{H}_{48} \mathrm{~N}_{2} \mathrm{O}_{15} \mathrm{Na}^{+}\right]$: 759.2947 . Found 759.2940.

\section{1'- $N$-carbobenzyloxy-D-tyrosine methoxycarbonyl -5',5-(tert-butyl- \\ dimethylsilanyloxymethyl)-2,3,2',3'-di acetonide-bis-1,4- $\alpha$-D-mannose- $N$-4- isovalaric amide (III-35).}

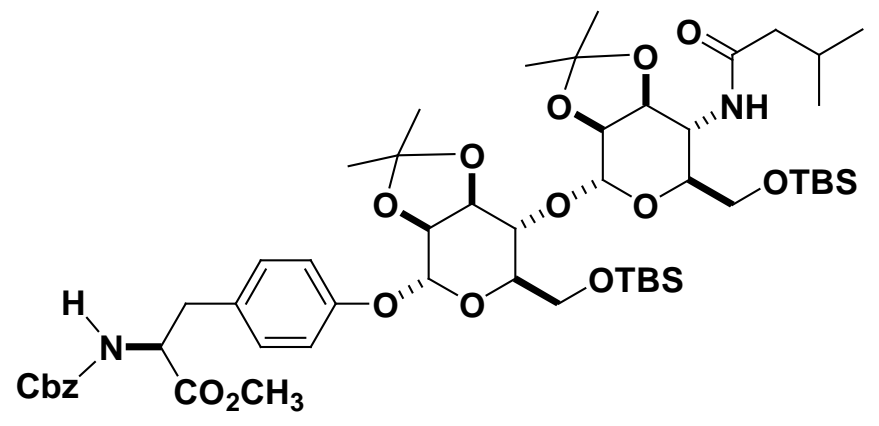


To a $\mathrm{CH}_{2} \mathrm{Cl}_{2}(0.1 \mathrm{~mL}, 1.0 \mathrm{M})$ solution of D-manno-amido-tetrol III-34 (10 mg, 0.01 mmol) and 2,2-dimethoxypropane $(2.4 \mathrm{mg}, 0.02 \mathrm{mmol})$ at $0{ }^{\circ} \mathrm{C}$ was added CSA $(0.23 \mathrm{mg}$, $10 \mathrm{~mol} \%$ ) and the reaction was stirred for $3 \mathrm{~h}$. The reaction mixture was concentrated and was pipetted directly on to a silica gel column using $\mathrm{CH}_{2} \mathrm{Cl}_{2}(1 \mathrm{~mL})$ in three portions. Impurities were eluted with ether and the product was eluted with EtOAc/Hexane (50:50). Pure fractions were combined and concentrated to afford di-acetonide III-35 (9 $\mathrm{mg}, 0.01 \mathrm{mmol}, 80 \%)$ as viscous oil. $R_{f}(50 \%$ EtOAc/Hexane $)=0.40 ;[\alpha]_{\mathrm{D}}^{26}=+65(c$ 1, $\mathrm{CH}_{2} \mathrm{Cl}_{2}$ ); IR (thin film, $\mathrm{cm}^{-1}$ ) 2951, 2930, 1731, 1498, 1228, 1009, 831; ${ }^{1} \mathrm{H}$ NMR (600 $\left.\mathrm{MHz}, \mathrm{CDCl}_{3}\right) \quad 7.34(\mathrm{~m}, 5 \mathrm{H}), 6.98(\mathrm{~m}, 4 \mathrm{H}), 5.77(\mathrm{~d}, J=10.8 \mathrm{~Hz}, 1 \mathrm{H}), 5.39(\mathrm{~d}, J=8.4$ $\mathrm{Hz}, 1 \mathrm{H}), 5.20(\mathrm{~d}, J=7.8 \mathrm{~Hz}, 1 \mathrm{H}), 5.09(\mathrm{br} \mathrm{s}, 2 \mathrm{H}), 4.62(\mathrm{dd}, J=7.2,4.8 \mathrm{~Hz}, 1 \mathrm{H}), 4.44$ $(\mathrm{dd}, J=6.0,1.2 \mathrm{~Hz}, 1 \mathrm{H}), 4.32(\mathrm{~d}, J=5.4 \mathrm{~Hz}, 1 \mathrm{H}), 4.16(\mathrm{~d}, J=5.4 \mathrm{~Hz}, 1 \mathrm{H}), 4.09(\mathrm{~d}, J=$ $4.8 \mathrm{~Hz}, 1 \mathrm{H}), 4.08(\mathrm{dd}, J=10.2,4.8 \mathrm{~Hz}, 1 \mathrm{H}), 3.95(\mathrm{~m}, \mathrm{H}), 3.72(\mathrm{~s}, 3 \mathrm{H}), 3.69(\mathrm{~m}, 2 \mathrm{H}), 3.69$ (m, 1H), $3.62(\mathrm{~d}, J=3.6 \mathrm{~Hz}, 1 \mathrm{H}), 3.55(\mathrm{dd}, J=10.2,3.0 \mathrm{~Hz}, 1 \mathrm{H}), 3.09(\mathrm{dd}, J=13.2,6.0$ $\mathrm{Hz}, 1 \mathrm{H}), 3.03(\mathrm{dd}, J=12.8,6.0 \mathrm{~Hz}, 1 \mathrm{H}), 2.16(\mathrm{~s}, 1 \mathrm{H}), 2.15(\mathrm{~m}, 1 \mathrm{H}), 2.12(\mathrm{dd}, J=4.8,1.2$ Hz, 1H), $2.09(\mathrm{~m}, 1 \mathrm{H}), 2.05(\mathrm{~d}, J=3.6 \mathrm{~Hz}, 1 \mathrm{H}), 1.65(\mathrm{~s}, 3 \mathrm{H}), 1.50(\mathrm{~s}, 3 \mathrm{H}), 1.38(\mathrm{~s}, 3 \mathrm{H})$, 1.35 (s, 3H), 0.95 (d, J = 6.6 Hz, 6H), 0.87 (s, 9H), 0.84 (s, 9H), 0.42 (s, 3H), 0.03 (s, $6 \mathrm{H}), 0.02(\mathrm{~s}, 3 \mathrm{H}) ;{ }^{13} \mathrm{C}$ NMR $\left(150 \mathrm{MHz}, \mathrm{CDCl}_{3}\right) 173.1,171.8,155.5,137.5,130.3,128.7$ (2C), $128.6(2 \mathrm{C}), 128.5,128.4$ (2C), 128.0 (2C), 127.8, 116.7, 109.9, 97.5, 92.9 (2C), 76.1, 75.7, 71.4, 70.0, 69.9 (2C), 69.5 (2C), 69.3, 62.7 (2C), 58.8(2C), 56.4, 52.2, 43.3, 38.5, 34.5, 28.8(2C), 28.7 (2C), 27.5, 26.4, 25.9 (2C), 25.7, 18.6, 18.3, -5.1, -5.2 (2C), 5.3; CIHRMS Calcd for $\left[\mathrm{C}_{53} \mathrm{H}_{84} \mathrm{~N}_{2} \mathrm{O}_{15} \mathrm{Si}_{2} \mathrm{H}^{+}\right]$: 1045.5488. Found 1045.5503. 
$(1 ' S, 4 ' S, 5 ' R, 1 S, 4 S, 5 R)-1-[1 '-N$-carbobenzyloxy-D-tyrosine methoxycarbonyl -5'-(tertbutyl-dimethylsilanyloxymethyl)-tetrahydro-pyran-4'-yloxy]-5-(tert-butyldimethylsilanyloxymethyl) -tetrahydro-pyran- $N$-4-isovalaric amide (III-36).

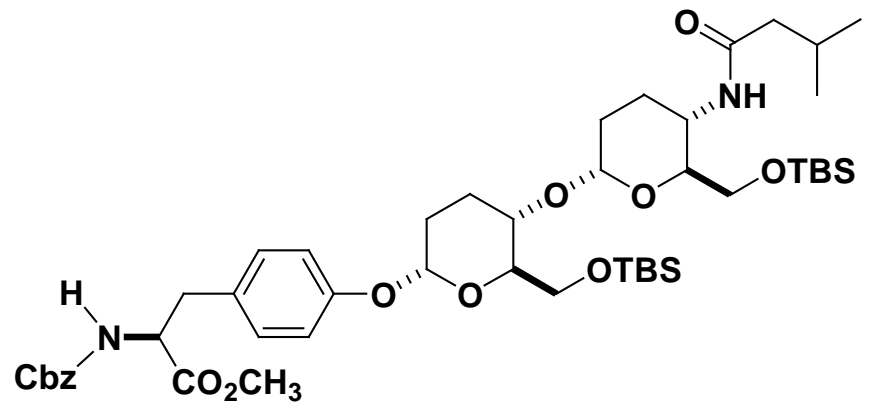

The dieno-amide compound III-33 (70 mg, $0.07 \mathrm{mmol})$ and $o-\mathrm{NO}_{2} \mathrm{C}_{6} \mathrm{H}_{4} \mathrm{SO}_{2} \mathrm{NHNH}_{2}(170$ $\mathrm{mg}, 1.17 \mathrm{mmol}$ ) were dissolved in $0.8 \mathrm{~mL}$ of $\mathrm{CH}_{2} \mathrm{Cl}_{2}$ in a round bottom flask and cooled $0{ }^{\circ} \mathrm{C}$ under nitrogen atmosphere then triethylamine $(112 \mathrm{mg}, 1.56 \mathrm{mmol})$ was added and the reaction mixture was stirred at $0{ }^{\circ} \mathrm{C}$ for 12 hours and on completion, as monitored by TLC. The reaction mixture was concentrated and was pipetted directly on to a silica gel column using $\mathrm{CH}_{2} \mathrm{Cl}_{2}(1 \mathrm{~mL})$ in three portions. The crude product was purified using silica gel flash chromatography eluting with $25 \%$ EtOAc/hexanes to give di-deoxy pyranoside III-36 $(68 \mathrm{mg}, 0.07 \mathrm{mmol}, 95 \%)$ as viscous oil. $R_{f}(30 \%$ EtOAc/Hexane $)=$ $0.50 ;[\alpha]_{\mathrm{D}}^{26}=+37\left(c 1, \mathrm{CH}_{2} \mathrm{Cl}_{2}\right) ;$ IR (thin film, $\left.\mathrm{cm}^{-1}\right)$ 2932, 2927, 1725, 1509, 1220, 1055, 965, 836; ${ }^{1} \mathrm{H}$ NMR $\left(600 \mathrm{MHz}, \mathrm{CDCl}_{3}\right) \delta 7.33(\mathrm{~m}, 5 \mathrm{H}), 6.98(\mathrm{~m}, 4 \mathrm{H}), 5.43$ (br s, 1H), $5.26(\mathrm{~d}, J=7.8 \mathrm{~Hz}, 1 \mathrm{H}), 5.07(\mathrm{br} \mathrm{s}, 2 \mathrm{H}), 4.58(\mathrm{dd}, J=12.8,5.4 \mathrm{~Hz}, 1 \mathrm{H}), 3.87(\mathrm{dd}, J$ $=9.0,7.2 \mathrm{~Hz}, 1 \mathrm{H}), 3.73(\mathrm{~d}, J=3.0 \mathrm{~Hz}, 1 \mathrm{H}), 3.72(\mathrm{~m}, 2 \mathrm{H}), 3.69(\mathrm{~m}, 3 \mathrm{H}), 3.65(\mathrm{dd}, J=$ 6.0, $5.4 \mathrm{~Hz}, 1 \mathrm{H}), 3.63(\mathrm{dd}, J=6.0,4.8 \mathrm{~Hz}, 1 \mathrm{H}), 3.50(\mathrm{dd}, J=10.2,4.2 \mathrm{~Hz}, 1 \mathrm{H}), 3.47(\mathrm{~m}$, 1H), $3.03(\mathrm{dd}, J=14.4,5.4 \mathrm{~Hz}, 1 \mathrm{H}), 3.01(\mathrm{dd}, J=14.4,5.4 \mathrm{~Hz}, 1 \mathrm{H}), 2.13(\mathrm{~m}, 1 \mathrm{H}), 2.01$ $(\mathrm{dd}, J=13.2,7.8 \mathrm{~Hz}, 1 \mathrm{H}), 1.99(\mathrm{dd}, J=13.2,7.8 \mathrm{~Hz}, 1 \mathrm{H}), 1.95(\mathrm{~m}, 1 \mathrm{H}), 1.85(\mathrm{dd}, J=$ 
8.4, $4.2 \mathrm{~Hz}, 1 \mathrm{H}), 1.82(\mathrm{~m}, 1 \mathrm{H}), 1.79(\mathrm{dd}, J=8.4,3.6 \mathrm{~Hz}, 1 \mathrm{H}), 1.76(\mathrm{dd}, J=7.8,3.6 \mathrm{~Hz}$, $1 \mathrm{H}), 1.74(\mathrm{dd}, J=7.8,4.2 \mathrm{~Hz}, 1 \mathrm{H}), 1.68(\mathrm{~m}, 2 \mathrm{H}), 1.06(\mathrm{~m}, 2 \mathrm{H}), 0.88(\mathrm{~d}, J=6.0 \mathrm{~Hz}, 6 \mathrm{H})$, $0.81(\mathrm{~s}, 9 \mathrm{H}), 0.80(\mathrm{~s}, 9 \mathrm{H}), 0.06(\mathrm{~s}, 3 \mathrm{H}), 0.03(\mathrm{~s}, 3 \mathrm{H}),-0.02(\mathrm{~s}, 3 \mathrm{H}),-0.03(\mathrm{~s}, 3 \mathrm{H}) ;{ }^{13} \mathrm{C}$ NMR $\left(150 \mathrm{MHz}, \mathrm{CDCl}_{3}\right) \delta 172.0,156.2,136.2,130.0(2 \mathrm{C}), 128.6(2 \mathrm{C}), 128.4(2 \mathrm{C})$, 128.1 (2C), 128.0 (2C), 116.9 (2C), 94.9, 90.8, 74.0, 73.5, 72.1, 67.2, 66.9, 64.3, 63.5, 54.8, 54.7, 52.2 (2C), 47.3, 47.2, 37.3, 29.4, 28.7, 26.0 (3C), 25.9 (3C), 25.8, 22.2, 18.36, 18.33, -5.0, -5.1, -5.2, -5.3; CIHRMS Calcd for $\left[\mathrm{C}_{47} \mathrm{H}_{76} \mathrm{~N}_{2} \mathrm{O}_{11} \mathrm{Si}_{2} \mathrm{Na}^{+}\right]$: 923.4885. Found 923.4886.

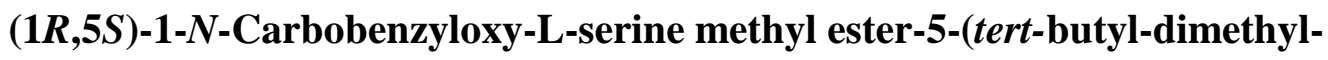
silanyloxymethyl)-1H-pyran-2-one (III-39a).<smiles>COC(=O)[C@H](COC1C=CC(=O)C(CO[SbH2])O1)NC(=O)O</smiles>

A $\mathrm{CH}_{2} \mathrm{Cl}_{2}(1 \mathrm{~mL})$ solution of compound (ent)-III-18 (358 mg, $\left.1 \mathrm{mmol}\right)$ and $N$ carbobenyloxy-L-serine methyl ester 9 h $(506 \mathrm{mg}, 2 \mathrm{mmol})$ was cooled to $0{ }^{\circ} \mathrm{C}$. A $\mathrm{CH}_{2} \mathrm{Cl}_{2}(1 \mathrm{~mL})$ solution of $\mathrm{Pd}_{2}(\mathrm{DBA})_{3} \cdot{ }^{\cdot} \mathrm{CHCl}_{3}(25 \mathrm{mg}, 2.5 \mathrm{~mol} \%)$ and $\mathrm{PPh}_{3}(26 \mathrm{mg}, 10$ mol\%) was added to the reaction mixture at $0{ }^{\circ} \mathrm{C}$. The reaction mixture was stirred at 0 ${ }^{\circ} \mathrm{C}$ for 3 hours. The reaction mixture was quenched with $5 \mathrm{~mL}$ of satd aq $\mathrm{NaHCO}_{3}$, extracted $(3 \times 5 \mathrm{~mL})$ with $\mathrm{Et}_{2} \mathrm{O}$, dried $\left(\mathrm{Na}_{2} \mathrm{SO}_{4}\right)$, and concentrated under reduced pressure. The crude product was purified using silica gel flash chromatography eluting with $14 \%$ EtOAc/hexanes to give $430 \mathrm{mg}(0.87 \mathrm{mmol}, 87 \%)$ of III-39a: $R_{f}(40 \%$ 
EtOAc/hexanes $)=0.46 ;[\alpha]_{\mathrm{D}}^{26}=-2\left(c=1.5, \mathrm{CH}_{2} \mathrm{Cl}_{2}\right) ; \mathrm{IR}\left(\right.$ thin film, $\left.\mathrm{cm}^{-1}\right) 2956,1726$, 1701, 1518, 1213, 1021, 836; ${ }^{1} \mathrm{H}$ NMR $\left(270 \mathrm{MHz}, \mathrm{CDCl}_{3}\right) \delta 7.34(\mathrm{~m}, 5 \mathrm{H}), 6.77(\mathrm{dd}, J=$ 10.0, $3.5 \mathrm{~Hz}, 1 \mathrm{H}), 6.06(\mathrm{~d}, J=10.2 \mathrm{~Hz}, 1 \mathrm{H}), 5.70(\mathrm{~d}, J=8.7 \mathrm{~Hz}, 1 \mathrm{H}), 5.26(\mathrm{~d}, J=3.3$ $\mathrm{Hz}, 1 \mathrm{H}), 5.12(\mathrm{br} \mathrm{s}, 2 \mathrm{H}), 4.60(\mathrm{ddd}, J=8.7,3.1,3.1 \mathrm{~Hz}, 1 \mathrm{H}), 4.26(\mathrm{~m}, 2 \mathrm{H}), 4.24(\mathrm{~d}, J=$ $3.5 \mathrm{~Hz}, 1 \mathrm{H}), 3.99$ (m, 2H) $3.84(\mathrm{dd}, J=9.8,3.1 \mathrm{~Hz}, 1 \mathrm{H}), 3.72(\mathrm{~s}, 3 \mathrm{H}), 0.85(\mathrm{~s}, 9 \mathrm{H}), 0.05$ (s, 6H); ${ }^{13} \mathrm{C}$ NMR $\left(67.5 \mathrm{MHz}, \mathrm{CDCl}_{3}\right) \delta 194.4,170.2,156,143.1,136,128.6,128.5$, 128.3, 128.2, 93.1, 76.2, 68.5, 67.2, 62.6, 54.0, 52.7, 25.8 (3C), 18.3, -5.3(2C); CIHRMS Calcd for $\left[\mathrm{C}_{24} \mathrm{H}_{35} \mathrm{NO}_{8} \mathrm{Si}+\mathrm{H}\right]^{+}:$494.2210. Found 494.2244.

\section{3-\{4-[6-(tert-Butyl-dimethyl-silanyloxymethyl)-5-hydroxy-5,6-dihydro-2H-pyran-2- yloxy]-phenyl\}-2- N-Carbobenzyloxy-L-serine methyl ester (III-39b).}

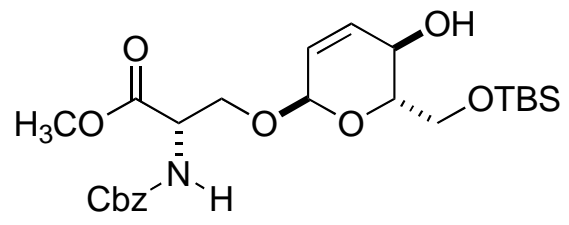

A $\mathrm{CH}_{2} \mathrm{Cl}_{2}(0.7 \mathrm{~mL})$ solution of compound III-39a $(352 \mathrm{mg}, 0.713 \mathrm{mmol})$ and $\mathrm{MeOH}(0.7$ $\mathrm{mL}$ ) was cooled to $-78{ }^{\circ} \mathrm{C}$. $\mathrm{NaBH}_{4}(26 \mathrm{mg}, 0.713 \mathrm{mmol})$ was added and the reaction mixture was stirred at $-78^{\circ} \mathrm{C}$ for 3 hours. The reaction mixture was diluted with ether $(10$ $\mathrm{mL})$ and was quenched with $5 \mathrm{~mL}$ of satd aq $\mathrm{NaHCO}_{3}$, extracted $(3 \times 5 \mathrm{~mL})$ with $\mathrm{Et}_{2} \mathrm{O}$, dried $\left(\mathrm{Na}_{2} \mathrm{SO}_{4}\right)$, and concentrated under reduced pressure. The crude product was purified using silica gel flash chromatography eluting with $25 \%$ EtOAc/hexanes to give $310 \mathrm{mg}(0.685 \mathrm{mmol}, 87 \%)$ of III-39b: $R_{f}(50 \%$ EtOAc/hexanes $)=0.34 ;[\alpha]_{\mathrm{D}}^{26}=+1(c$ $=0.7, \mathrm{CH}_{2} \mathrm{Cl}_{2}$ ); IR (thin film, $\mathrm{cm}^{-1}$ ) $3437,2953,1725,1517,1213,1076,1009,838 ;{ }^{1} \mathrm{H}$ 
NMR (270 MHz, $\left.\mathrm{CDCl}_{3}\right) \delta 7.36(\mathrm{~m}, 5 \mathrm{H}), 5.90(\mathrm{~d}, J=10.1 \mathrm{~Hz}, 1 \mathrm{H}), 5.73(\mathrm{~d}, J=8.6 \mathrm{~Hz}$, $1 \mathrm{H}), 5.64(\mathrm{ddd}, J=10.1,2.4,2.4 \mathrm{~Hz}, 1 \mathrm{H}), 5.10(\mathrm{br} \mathrm{s}, 2 \mathrm{H}), 4.87(\mathrm{~s}, 1 \mathrm{H}), 4.55(\mathrm{ddd}, J=$ 6.6, 3.2, 3.2 Hz, 1H), 4.15 (ddd, $J=9.8,3.2,3.2 \mathrm{~Hz}, 2 \mathrm{H}), 3.86(\mathrm{dd}, J=10.3,5.2 \mathrm{~Hz}, 1 \mathrm{H})$, 3.78-3.66 (m, 5H), $3.54(\mathrm{dd}, J=8.9,5.9 \mathrm{~Hz}, 1 \mathrm{H}), 3.12(\mathrm{~s}, 1 \mathrm{H}), 0.89$ (s, 9H), 0.89 (s, 6H);

${ }^{13} \mathrm{C}$ NMR $\left(67.5 \mathrm{MHz}, \mathrm{CDCl}_{3}\right) \delta 170.4,155.9,136.0,133.1,128.4(2 \mathrm{C}), 128.1,128.0$ (2C), 124.9, 93.8, 70.3, 67.6, 66.9, 66.1, 64.7, 54.1, 52.4, 25.7 (3C), 18.1, -5.6 (2C); CIHRMS Calcd for $\left[\mathrm{C}_{24} \mathrm{H}_{37} \mathrm{NO}_{8} \mathrm{SiNa}^{+}\right]$: 518.2186 Found 518.2187.

$\left(1 ' R, 4^{\prime} R, 5^{\prime} S, 1 R, 5 S\right)$-1-[1'-N-Carbobenzyloxy-L-serine methyl ester -5-(tert-butyldimethyl-silanyloxymethyl)-1',4'-dihydro-5' $H$-pyran-4'-yloxy]-5-(tert-butyldimethyl-silanyloxymethyl) -1H-pyran-4-one (III-39).

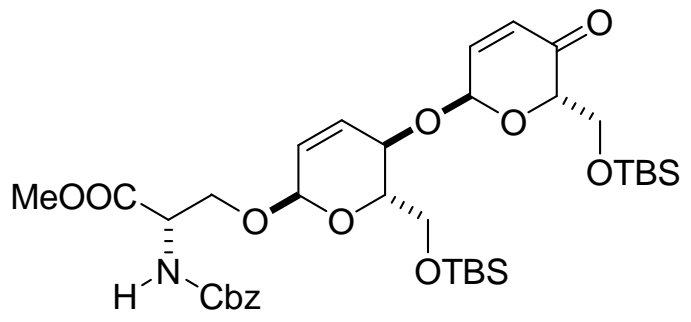

A CH $\mathrm{Cl}_{2}(0.3 \mathrm{~mL})$ solution of compound (ent)-III-18 $(120 \mathrm{mg}, 0.23 \mathrm{mmol})$ and alcohol III-39b $(101 \mathrm{mg}, 0.28 \mathrm{mmol})$ was cooled to $0{ }^{\circ} \mathrm{C}$. $\mathrm{A} \mathrm{CH}_{2} \mathrm{Cl}_{2}(0.3 \mathrm{~mL})$ solution of $\mathrm{Pd}_{2}(\mathrm{DBA})_{3} \mathrm{CHCl}_{3}(6 \mathrm{mg}, 2.5 \mathrm{~mol} \%)$ and $\mathrm{PPh}_{3}(6.1 \mathrm{mg}, 10 \mathrm{~mol} \%)$ was added to the reaction mixture at $0{ }^{\circ} \mathrm{C}$. The reaction mixture was stirred at $0{ }^{\circ} \mathrm{C}$ for 2 hours. The reaction mixture was quenched with $5 \mathrm{~mL}$ of satd aq $\mathrm{NaHCO}_{3}$, extracted $(3 \times 5 \mathrm{~mL})$ with $\mathrm{Et}_{2} \mathrm{O}$, dried $\left(\mathrm{Na}_{2} \mathrm{SO}_{4}\right)$, and concentrated under reduced pressure. The crude product was purified using silica gel flash chromatography eluting with 20\% EtOAc/hexanes to give 
III-39 $(149 \mathrm{mg}, 0.20 \mathrm{mmol}, 85 \%)$ as viscous oil. $R_{f}(30 \% \mathrm{EtOAc} / \mathrm{hexanes})=0.30 ;[\alpha]_{\mathrm{D}}^{26}$ $=+19\left(c=1, \mathrm{CH}_{2} \mathrm{Cl}_{2}\right) ; \mathrm{IR}\left(\right.$ thin film, $\left.\mathrm{cm}^{-1}\right)$ 3350, 2927, 1723, 1700, 1507, 1251, 1015, 830; ${ }^{1} \mathrm{H}$ NMR $\left(600 \mathrm{MHz}, \mathrm{CDCl}_{3}\right) \delta 7.36(\mathrm{~m}, 5 \mathrm{H}), 6.80(\mathrm{dd}, J=10.2,3.6 \mathrm{~Hz}, 1 \mathrm{H}), 6.23$ $(\mathrm{d}, J=10.2 \mathrm{~Hz}, 1 \mathrm{H}), 6.06(\mathrm{~d}, J=10.2 \mathrm{~Hz}, 1 \mathrm{H}), 5.76(\mathrm{ddd}, J=10.2,2.4,2.4 \mathrm{~Hz}, 1 \mathrm{H}), 5.62$ $(\mathrm{d}, J=8.4 \mathrm{~Hz}, 1 \mathrm{H}), 5.55(\mathrm{~d}, J=3.0 \mathrm{~Hz}, 1 \mathrm{H}), 5.12(\mathrm{br} \mathrm{s}, 1 \mathrm{H}), 4.96(\mathrm{~d}, J=7.2 \mathrm{~Hz}, 1 \mathrm{H})$, $4.56(\mathrm{ddd}, J=7.8,3.0,3.0 \mathrm{~Hz}, 1 \mathrm{H}), 4.43(\mathrm{dd}, J=9.6,1.8 \mathrm{~Hz}, 1 \mathrm{H}), 4.40(\mathrm{dd}, J=4.2,2.4$ $\mathrm{Hz}, 1 \mathrm{H}), 4.19(\mathrm{dd}, J=9.6,3.6 \mathrm{~Hz}, 1 \mathrm{H}), 4.12(\mathrm{dd}, J=13.8,6.6 \mathrm{~Hz}, 1 \mathrm{H}), 4.09$ (dd, $J=11.4$, $4.2 \mathrm{~Hz}, 1 \mathrm{H}), 3.95(\mathrm{dd}, J=10.8,2.4 \mathrm{~Hz}, 1 \mathrm{H}), 3.80(\mathrm{dd}, J=11.4,1.8 \mathrm{~Hz}, 1 \mathrm{H}), 3.75(\mathrm{dd}, J=$ $11.4,4.8 \mathrm{~Hz}, 1 \mathrm{H}), 3.73(\mathrm{~s}, 3 \mathrm{H}), 3.63(\mathrm{dd}, J=4.8,1.8 \mathrm{~Hz}, 1 \mathrm{H}), 3.62(\mathrm{dd}, J=5.4,2.4 \mathrm{~Hz}$, 1H), $0.88(\mathrm{~s}, 9 \mathrm{H}), 0.85(\mathrm{~s}, 9 \mathrm{H}), 0.06(\mathrm{~s}, 6 \mathrm{H}), 0.05(\mathrm{~s}, 3 \mathrm{H}), 0.03(\mathrm{~s}, 3 \mathrm{H}) ;{ }^{13} \mathrm{C}$ NMR (150 $\left.\mathrm{MHz}, \mathrm{CDCl}_{3}\right) \delta 194.6,170.4,155.9,143.9(2 \mathrm{C}), 136.1,129.2,128.5(2 \mathrm{C}), 128.5,128.2$ $128.1,126.8,94.0,89.7,70.5,67.6,67.0,66.8,62.9,62.7,60.3,54.1,52.5,25.9$ (3C), 25.8 (3C), 18.4, 18.3, -5.1, -5.3, -5.3, -5.4; CIHRMS Calcd for $\left[\mathrm{C}_{36} \mathrm{H}_{57} \mathrm{NO}_{11} \mathrm{Si}_{2} \mathrm{Na}^{+}\right]$: 758.3362. Found 758.3378.

(1'R,4'R,5'S,1R,4R,5S)-1-[ N-Carbobenzyloxy-L-serine methyl ester -5'-(tert-butyldimethyl-silanyloxymethyl)-1',4'-dihydro-5'H-pyran-4'-yloxy]-5-(tert-butyldimethyl-silanyloxymethyl)-1,4-dihydro-5H-pyran-4-ol (III-40a).<smiles>COC(=O)[C@H](COC1C=CC(OC2C=CC(O)[C@@H](C[OH+])O2)[C@@H](CO[SbH2])O1)NC(=O)O</smiles> 
The ketone compound III-39 (328 mg, $0.439 \mathrm{mmol}$ ), was dissolved in $0.4 \mathrm{~mL}$ of $\mathrm{CH}_{2} \mathrm{Cl}_{2}$ and $0.4 \mathrm{~mL} \mathrm{MeOH}$ were added to a round bottom flask and cooled $-78{ }^{\circ} \mathrm{C}$ then $(16.6 \mathrm{mg}$, $0.439 \mathrm{mmol}) \mathrm{NaBH}_{4}$ was added and the reaction mixture was stirred at $-78^{\circ} \mathrm{C}$ for 6 hours and on completion monitored by TLC, reaction mixture is diluted with ether and was quenched with $5 \mathrm{~mL}$ of satd. aq $\mathrm{NaHCO}_{3}$, extracted $(3 \times 5 \mathrm{~mL})$ with $\mathrm{Et}_{2} \mathrm{O}$, dried $\left(\mathrm{Na}_{2} \mathrm{SO}_{4}\right)$, and concentrated under reduced pressure. The crude product was purified using silica gel flash chromatography eluting with $25 \%$ EtOAc/hexanes to give III-40a $(279.6 \mathrm{mg}, 0.37 \mathrm{mmol}, 85 \%)$ as viscous oil. $R_{f}(30 \%$ EtOAc/hexanes $)=0.25 ;[\alpha]_{\mathrm{D}}^{26}=-$ $5\left(c=1, \mathrm{CH}_{2} \mathrm{Cl}_{2}\right)$; IR (thin film, $\left.\mathrm{cm}^{-1}\right) 3450,2929,17.16,1500,1249,1039,829,777 ;{ }^{1} \mathrm{H}$ NMR (600 MHz, $\left.\mathrm{CDCl}_{3}\right) \delta 7.36(\mathrm{~m}, 5 \mathrm{H}), 5.98(\mathrm{~d}, J=10.2 \mathrm{~Hz}, 1 \mathrm{H}), 5.95(\mathrm{dd}, J=10.2$, $1.2 \mathrm{~Hz}, 1 \mathrm{H}), 5.73$ (ddd, $J=9.6,3.0,1.8 \mathrm{~Hz}, 1 \mathrm{H}), 5.65(\mathrm{dd}, J=2.4,1.8 \mathrm{~Hz}, 1 \mathrm{H}), 5.64(\mathrm{dd}$, $J=2.4,1.8 \mathrm{~Hz}, 1 \mathrm{H}), 5.13($ br s, $2 \mathrm{H}), 5.11($ br s, $1 \mathrm{H}), 4.91(\mathrm{~d}, J=1.2 \mathrm{~Hz}, 1 \mathrm{H}), 4.56$ (ddd, $J=7.2,3.0,3.0 \mathrm{~Hz}, 1 \mathrm{H}), 4.23(\mathrm{dd}, J=9.0,2.4 \mathrm{~Hz}, 1 \mathrm{H}), 4.23(\mathrm{dd}, J=9.6,1.8 \mathrm{~Hz}$, $1 \mathrm{H}), 4.20(\mathrm{ddd}, J=10.8,1.8,1.8 \mathrm{~Hz}, 1 \mathrm{H}), 3.94(\mathrm{dd}, J=9.6,4.2 \mathrm{~Hz}, 1 \mathrm{H}), 3.87(\mathrm{dd}, J=$ 10.2, $1.8 \mathrm{~Hz}, 1 \mathrm{H}), 3.74(\mathrm{~s}, 3 \mathrm{H}), 3.73(\mathrm{~d}, J=1.8 \mathrm{~Hz}, 1 \mathrm{H}), 3.71(\mathrm{dd}, J=6.0,2.4 \mathrm{~Hz}, 1 \mathrm{H})$, $3.69(\mathrm{dd}, J=9.6,4.2 \mathrm{~Hz}, 1 \mathrm{H}), 3.66(\mathrm{dd}, J=7.8,4.2 \mathrm{~Hz}, 1 \mathrm{H}), 3.64(\mathrm{~d}, J=1.8 \mathrm{~Hz}, 1 \mathrm{H})$, $3.03(\mathrm{~d}, J=2.4 \mathrm{~Hz}, 1 \mathrm{H}), 0.91(\mathrm{~s}, 9 \mathrm{H}), 0.90(\mathrm{~s}, 9 \mathrm{H}), 0.11(\mathrm{~s}, 3 \mathrm{H}), 0.10(\mathrm{~s}, 3 \mathrm{H}), 0.08$ (s, 3H), $0.07(\mathrm{~s}, 3 \mathrm{H}) ;{ }^{13} \mathrm{C} \mathrm{NMR}\left(150 \mathrm{MHz}, \mathrm{CDCl}_{3}\right) \delta 170.4,156.0,136.2,133.0(2 \mathrm{C}), 129.8$, 128.5 (2C), 128.2, 128.1, 126.3, 125.4, 93.8, 91.0, 70.5, 69.9, 67.5, 67.1, 67.0, 66.9, 65.4, 62.9, 54.1, 52.5, 25.9 (3C), 25.8 (3C), 18.4, 18.2, -5.1, -5.2, -5.4, -5.6; CIHRMS Calcd for $\left[\mathrm{C}_{36} \mathrm{H}_{59} \mathrm{NO}_{11} \mathrm{Si}_{2} \mathrm{Na}^{+}\right]:$760.3518. Found 760.3543 . 
1'(2' $N$-Carbobenzyloxy-L-serine methyl ester -1'-yloxy)- -5',5-(tert-butyl-dimethylsilanyloxymethyl)-bis-1,4- $\alpha$-L-mannose (III-40b).

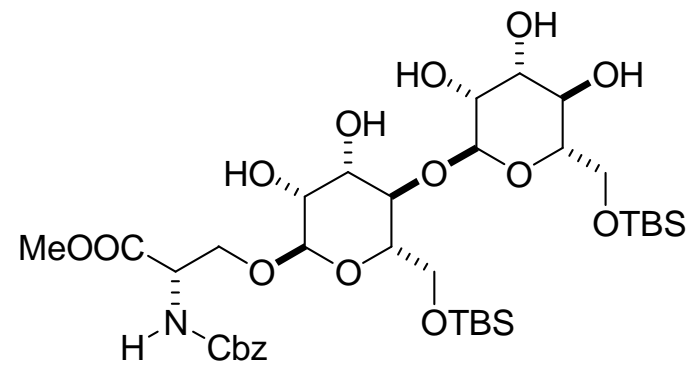

A $\mathrm{CH}_{2} \mathrm{Cl}_{2}(0.7 \mathrm{~mL}, 1 \mathrm{~mol})$ solution of compound of allyl alcohol III-40a (55 mg, 0.07 mmol) at $0{ }^{\circ} \mathrm{C}$ was added a solution of $(50 \% \mathrm{w} / \mathrm{v})$ of $\mathrm{N}$-methyl morpholine $\mathrm{N}$-oxide / water $(0.05 \mathrm{~mL})$. Crystalline $\mathrm{OsO}_{4}(1.0 \mathrm{mg}, 5 \mathrm{~mol} \%)$ was added and the reaction was stirred for $12 \mathrm{~h}$. The reaction mixture was concentrated and was pipetted directly on to a silica gel column using a small amount of $\mathrm{CH}_{2} \mathrm{Cl}_{2}(1 \mathrm{~mL})$ in three portions. The crude product was purified using silica gel flash chromatography eluting with $100 \%$ EtOAc to give III-40b $(50 \mathrm{mg}, \quad 0.06 \mathrm{mmol}, \quad 86 \%)$ as viscous oil. $R_{f} \quad(40: 10: 50$ EtOAc/MeOH/Hexane $)=0.20 ;[\alpha]_{\mathrm{D}}^{26}=-45\left(c=1, \mathrm{CH}_{2} \mathrm{Cl}_{2}\right) ;$ IR $\left(\right.$ thin film, $\left.\mathrm{cm}^{-1}\right) 3419$, 2929, 2856, 1720, 1508, 1264, 1094, 1028, 970, 836; ${ }^{1} \mathrm{H}$ NMR (600 MHz, $\left.\mathrm{CDCl}_{3}\right) \delta 7.33$ (m, 5H), $5.82(\mathrm{~d}, 8.4 \mathrm{~Hz}, 1 \mathrm{H}), 5.16(\mathrm{~s}, 1 \mathrm{H}), 5.09$ (br s, 2H ), $4.72(\mathrm{~d}, 1.2 \mathrm{~Hz}, 1 \mathrm{H}), 4.55$ (dd, $J=5.4,2.4 \mathrm{~Hz}, 1 \mathrm{H}), 4.09$ (ddd, $J=10.2,2.4,1.8 \mathrm{~Hz}, 1 \mathrm{H}), 3.96(\mathrm{~d}, J=2.4 \mathrm{~Hz}, 1 \mathrm{H})$, $3.84(\mathrm{~d}, J=6.0 \mathrm{~Hz}, 1 \mathrm{H}), 3.81(\mathrm{dd}, J=9.6,4.8 \mathrm{~Hz}, 1 \mathrm{H}), 3.79(\mathrm{~d}, J=4.8 \mathrm{~Hz}, 1 \mathrm{H}), 3.75(\mathrm{br}$ s, $1 \mathrm{H}), 3.74(\mathrm{~d}, J=2.4 \mathrm{~Hz}, 1 \mathrm{H}), 3.72(\mathrm{~s}, 3 \mathrm{H}), 3.67(\mathrm{~d}, J=6.0 \mathrm{~Hz}, 1 \mathrm{H}), 3.64(\mathrm{dd}, J=9.0$, $5.4 \mathrm{~Hz}, 1 \mathrm{H}), 3.58(\mathrm{dd}, J=10.2,2.4 \mathrm{~Hz}, 1 \mathrm{H}), 3.43(\mathrm{~d}, J=3.0 \mathrm{~Hz}, 1 \mathrm{H}), 3.38(\mathrm{dd}, J=15.6$, $7.2 \mathrm{~Hz}, 1 \mathrm{H}), 3.13(\mathrm{~d}, \quad J=2.4 \mathrm{~Hz}, 1 \mathrm{H}), 0.88(\mathrm{~s}, 9 \mathrm{H}), 0.87$ (s, 9H), 0.08 (s, 3H), 0.07 (s, $3 \mathrm{H}), 0.05(\mathrm{~s}, 6 \mathrm{H}) ;{ }^{13} \mathrm{C} \mathrm{NMR}\left(67.5 \mathrm{MHz}, \mathrm{CDCl}_{3}\right) \delta 172.0,158.4,138.0,129.4(2 \mathrm{C})$, 129.0, 128.8 (2C), 102.5, 100.9, 75.4, 75.3, 73.9, 72.9, 72.5, 72.2, 72.0, 68.4, 67.7, 67.6, 
64.8, 64.6, 55.4, 52.9, 26.6 (3C), 26.5 (3C), 19.4, 19.2, -4.8 (2C), -4.9, -5.0; CIHRMS Calcd for $\left[\mathrm{C}_{36} \mathrm{H}_{63} \mathrm{NO}_{15} \mathrm{Si}_{2} \mathrm{Na}^{+}\right]$: 828.3628. Found 828.3646.

$(1 ' R, 4 ' R, 5 ' S, 1 R, 4 R, 5 S)-1$-[1'-N-Carbobenzyloxy-L-serine methyl ester -5'-(tertbutyl-dimethyl-silanyloxymethyl)-1',4'-dihydro-5'H-pyran-4'-yloxy] -5-(tert-butyldimethyl-silanyloxymethyl)-1,4-dihydro-5H-pyran-4-isovalaric ester (III-40).

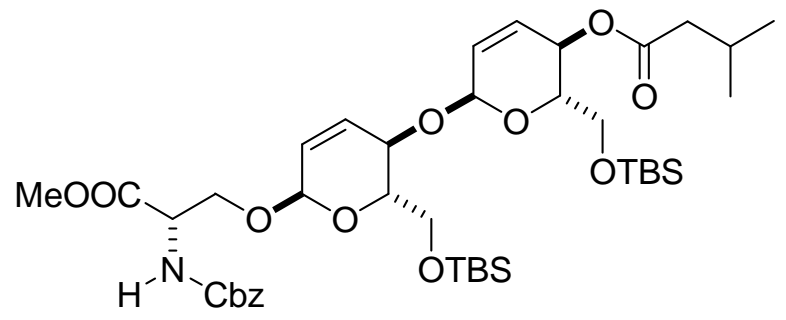

The alcohol compound III-40a (278 mg, $0.371 \mathrm{mmol})$, isovaleric acid $(45.2 \mathrm{mg}, 0.445$ mmol) and DCC (91.7 mg, $0.445 \mathrm{mmol})$ were dissolved in $0.7 \mathrm{~mL}$ of $\mathrm{CH}_{2} \mathrm{Cl}_{2}$ in a round bottom flask and cooled $0{ }^{\circ} \mathrm{C}$ then $(4.5 \mathrm{mg}, 0.1 \mathrm{mmol})$ DMAP was added and the reaction mixture was stirred at $0^{\circ} \mathrm{C}$ for 6 hours and on completion monitored by TLC, reaction mixture is diluted with ether and was quenched with $5 \mathrm{~mL}$ of satd aq $\mathrm{NaHCO}_{3}$, extracted (3 x $5 \mathrm{~mL}$ ) with $\mathrm{Et}_{2} \mathrm{O}$, dried $\left(\mathrm{Na}_{2} \mathrm{SO}_{4}\right)$, and concentrated under reduced pressure. The crude product was purified using silica gel flash chromatography eluting with $20 \%$ EtOAc/hexanes to give III-40 (280 mg, $0.34 \mathrm{mmol}, 91 \%)$ as viscous oil. $R_{f}(30 \%$ EtOAc/hexanes $)=0.50 ;[\alpha]_{\mathrm{D}}^{26}=-76\left(c=1, \mathrm{CH}_{2} \mathrm{Cl}_{2}\right) ; \mathrm{IR}\left(\right.$ thin film, $\left.\mathrm{cm}^{-1}\right) 2928,1738$, 1464, 1510, 1254, 1092, 1005, 836; ${ }^{1} \mathrm{H}$ NMR $\left(600 \mathrm{MHz}, \mathrm{CDCl}_{3}\right) \delta 7.34(\mathrm{~m}, 5 \mathrm{H}), 6.03(\mathrm{~d}$, $J=10.2 \mathrm{~Hz}, 1 \mathrm{H}), 5.92(\mathrm{~d}, J=10.2 \mathrm{~Hz}, 1 \mathrm{H}), 5.74(\mathrm{dd}, J=10.2,3.0 \mathrm{~Hz}, \mathrm{H}), 5.71(\mathrm{dd}, J=$ $10.8,2.4 \mathrm{~Hz}, 1 \mathrm{H}), 5.39$ (dd, $J=9.0,1.2 \mathrm{~Hz}, 1 \mathrm{H}), 5.21(\mathrm{~d}, J=2.4 \mathrm{~Hz}, 1 \mathrm{H}), 5.13(\mathrm{br} \mathrm{s}, 2 \mathrm{H})$, 
$4.92(\mathrm{br} \mathrm{s}, 1 \mathrm{H}), 4.55(\mathrm{dd}, J=6.6,3.0 \mathrm{~Hz}, 1 \mathrm{H}), 4.26(\mathrm{~d}, J=9.6 \mathrm{~Hz}, 1 \mathrm{H}), 4.20(\mathrm{dd}, J=9.6$, $3.6 \mathrm{~Hz}, 1 \mathrm{H}), 3.88(\mathrm{~d}, J=1.8 .0 \mathrm{~Hz}, 1 \mathrm{H}), 3.86(\mathrm{dd}, J=3.0,1.8 \mathrm{~Hz}, 1 \mathrm{H}), 3.84(\mathrm{ddd}, J=7.2$, 2.4, $2.4 \mathrm{~Hz}, 1 \mathrm{H}), 3.75(\mathrm{~d}, J=3.0 \mathrm{~Hz}, 1 \mathrm{H}), 3.73(\mathrm{~s}, 3 \mathrm{H}), 3.72(\mathrm{dd}, J=10.2,3.6 \mathrm{~Hz}, 1 \mathrm{H})$, $3.71(\mathrm{dd}, J=6.6,3.6 \mathrm{~Hz}, 1 \mathrm{H}), 3.68(\mathrm{~d}, J=1.8 \mathrm{~Hz}, 1 \mathrm{H}), 3.67(\mathrm{dd}, J=6.0,3.0 \mathrm{~Hz}, 1 \mathrm{H})$, $2.21(\mathrm{~d}, J=1.8 \mathrm{~Hz}, 1 \mathrm{H}), 2.19(\mathrm{~d}, J=1.8 \mathrm{~Hz}, 1 \mathrm{H}), 2.10(\mathrm{~m}, 1 \mathrm{H}), 0.96(\mathrm{~d}, J=7.2 \mathrm{~Hz}, 6 \mathrm{H})$, 0.90 (s, 9H), 0.88 (s, 9H), 0.08 (s, 3H), 0.07 (s, 3H), 0.04 (s, 3H), 0.04 (s, 3H); ${ }^{13} \mathrm{C}$ NMR $\left(150 \mathrm{MHz}, \mathrm{CDCl}_{3}\right) \delta 173.7,172.1,158.4,138.1,130.5,130.4,129.4(2 \mathrm{C}), 129.0,128.9$ (2C), 128.8, 127.7, 95.3, 92.3, 72.0, 70.9, 68.4, 68.1, 67.7, 65.9, 64.4, 63.4, 55.6, 52.8, 44.2, 26.9, 26.6 (3C), 26.4 (5C), 22.7, 22.7, 19.4, 19.2, -4.8, -4.9, -5.0, -5.1; CIHRMS Calcd for $\left[\mathrm{C}_{41} \mathrm{H}_{67} \mathrm{NO}_{12} \mathrm{Si}_{2} \mathrm{Na}^{+}\right]$: 844.4094. Found 844.4116.

\section{1'- $N$-Carbobenzyloxy-L-serine methyl ester -5',5-(tert-butyl-dimethyl-}

silanyloxymethyl)-bis-1,4- $\alpha$-L-mannose - isovalaric ester (III-41).

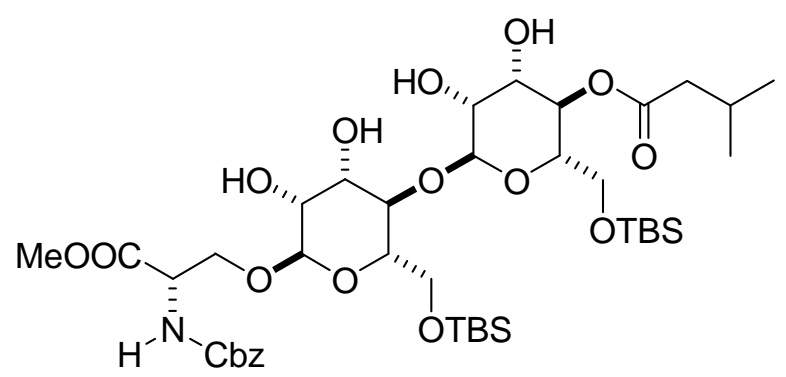

A $\mathrm{CH}_{2} \mathrm{Cl}_{2}(0.3 \mathrm{~mL}, 1 \mathrm{Mol})$ solution of compound of allyl alcohol III-40 (106 mg, 0.127 mmol) at $0{ }^{\circ} \mathrm{C}$ was added a solution of $(50 \% \mathrm{w} / \mathrm{v})$ of $\mathrm{N}$-methyl morpholine $\mathrm{N}$-oxide / water $(0.10 \mathrm{~mL})$. Crystalline $\mathrm{OsO}_{4}(1.6 \mathrm{mg}, 5 \mathrm{~mol} \%)$ was added and the reaction was stirred for $12 \mathrm{~h}$. The reaction mixture was concentrated and was pipetted directly on to a silica gel column using a small amount of $\mathrm{CH}_{2} \mathrm{Cl}_{2}(1 \mathrm{~mL})$ in three portions. The crude product was purified using silica gel flash chromatography eluting with 50:30:20 ( EtOAc 
: Hexane : Methanol) to give III-41 (140 mg, $0.16 \mathrm{mmol}, 94 \%)$ as viscous oil. $R_{f}(60: 40$ EtOAc/Hexane $)=0.20 ;[\alpha]_{\mathrm{D}}^{26}=-46\left(c=1, \mathrm{CH}_{2} \mathrm{Cl}_{2}\right) ; \mathrm{IR}\left(\right.$ thin film, $\left.\mathrm{cm}^{-1}\right) 3448,2954$, 2928, 1725, 1517, 1463, 1252, 1210, 1097, 834; ${ }^{1} \mathrm{H}$ NMR (600 MHz, $\left.\mathrm{CDCl}_{3}\right) \delta 7.35(\mathrm{~m}$, $5 \mathrm{H}), 5.70(\mathrm{~d}, J=9.0 \mathrm{~Hz}, 1 \mathrm{H}), 5.28(\mathrm{br} \mathrm{s}, 1 \mathrm{H}), 5.13(\mathrm{br} \mathrm{s}, 2 \mathrm{H}), 4.98(\mathrm{dd}, J=9.0,9.0 \mathrm{~Hz}$, $1 \mathrm{H}), 4.76(\mathrm{~d}, J=3.0 \mathrm{~Hz}, 1 \mathrm{H}), 4.57(\mathrm{dd}, J=5.4,3.0 \mathrm{~Hz}, 1 \mathrm{H}), 4.12(\mathrm{ddd}, J=10.2,3.6,3.6$ Hz, 1H), $3.91(\mathrm{~d}, J=4.8 \mathrm{~Hz}, 1 \mathrm{H}), 3.88(\mathrm{dd}, J=9.6,4.8 \mathrm{~Hz}, 1 \mathrm{H}), 3.87(\mathrm{dd}, J=9.6,4.2$ Hz, 1H), $3.84(\mathrm{~d}, J=10.2,3.0 \mathrm{~Hz}, 1 \mathrm{H}), 3.82(\mathrm{~d}, J=6.0,3.0 \mathrm{~Hz}, 1 \mathrm{H}), 3.74(\mathrm{~s}, 3 \mathrm{H}), 3.69$ (d, $J=4.2 \mathrm{~Hz}, 1 \mathrm{H}), 3.68$ (br s, 1H), $3.63(\mathrm{dd}, J=9.6,2.4 \mathrm{~Hz}, 1 \mathrm{H}), 3.46$ (ddd, $J=8.4,6.0$, $3.0 \mathrm{~Hz}, 1 \mathrm{H}), 3.28(\mathrm{~d}, J=3.0 \mathrm{~Hz}, 1 \mathrm{H}), 3.15(\mathrm{~d}, J=7.2 \mathrm{~Hz}, 1 \mathrm{H}), 2.82(\mathrm{~d}, J=3.0 \mathrm{~Hz}, 1 \mathrm{H})$, $2.24(\mathrm{dd}, J=14.4,7.2 \mathrm{~Hz}, 1 \mathrm{H}), 2.19(\mathrm{dd}, J=14.4,7.2 \mathrm{~Hz}, 1 \mathrm{H}), 2.11(\mathrm{~m}, 1 \mathrm{H}), 0.96(\mathrm{~d}, J$ $=7.2 \mathrm{~Hz}, 6 \mathrm{H}), 0.90(\mathrm{~s}, 9 \mathrm{H}), 0.88(\mathrm{~s}, 9 \mathrm{H}), 0.07(\mathrm{~s}, 6 \mathrm{H}), 0.06(\mathrm{~s}, 3 \mathrm{H}), 0.05(\mathrm{~s}, 3 \mathrm{H}) ;{ }^{13} \mathrm{C}$ NMR (150 MHz, $\left.\mathrm{CDCl}_{3}\right) \delta 173.5,170.4,155.9,136.0,128.5$ (3C), 128.2, 128.1, 98.9, 98.0, 73.6, 71.7, 71.4, 70.9, 70.8, 70.1, 70.0, 69.8, 67.1, 67.0, 63.1, 62.9, 53.9, 52.6, 43.4, 25.8 (6C), 25.7, 22.3, 22.3, 18.4, 18.3, -5.2, -5.3, -5.4, -5.5; CIHRMS Calcd for $\left[\mathrm{C}_{41} \mathrm{H}_{71} \mathrm{NO}_{16} \mathrm{Si}_{2} \mathrm{Na}^{+}\right]:$912.4203. Found 912.4209.

(1'R,4'R,5'S,1R,4R,5S)-1-[1'-N-Carbobenzyloxy-L-serine methyl ester -5'-(tertbutyl-dimethyl-silanyloxymethyl)-tetrahydro-pyran-4'-yloxy]-5-(tert-butyldimethyl-silanyloxymethyl) -tetrahydro-pyran-4-isovalaric ester (III-43). 


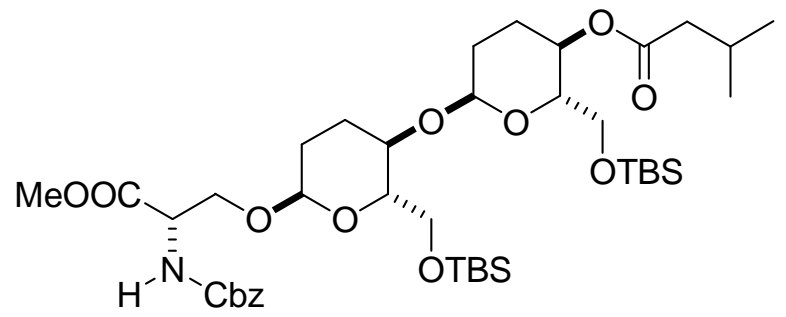

The allyl alcohol compound III-40 (49 mg, $0.058 \mathrm{mmol}$ ), $O-\mathrm{NO}_{2} \mathrm{ArSO}_{2} \mathrm{NHNH}_{2}$ (71.6 $\mathrm{mg}, 0.353 \mathrm{mmol}$ ) were dissolved in $0.6 \mathrm{~mL}$ of $\mathrm{CH}_{2} \mathrm{Cl}_{2}$ in a round bottom flask and cooled $0{ }^{\circ} \mathrm{C}$ under nitrogen condition then triethylamine $(47.6 \mathrm{mg}, 0.471 \mathrm{mmol})$ was added and the reaction mixture was stirred at $0{ }^{\circ} \mathrm{C}$ for 12 hours and on completion monitored by TLC, reaction mixture is three portions. The crude product was purified using silica gel flash chromatography eluting with $20 \%$ EtOAc/hexanes to give III-43 (48.3 mg, 0.057 mmol, $98 \%)$ as viscous oil. $R_{f}(30 \%$ EtOAc/Hexane $)=0.60 ;[\alpha]_{\mathrm{D}}^{26}=-69(c=1$, $\mathrm{CH}_{2} \mathrm{Cl}_{2}$ ); IR (thin film, $\mathrm{cm}^{-1}$ ) 2953, 2928, 1734, 1508, 1462, 1251, 1205, 1122, 993, 833; ${ }^{1} \mathrm{H}$ NMR (600 MHz, $\left.\mathrm{CDCl}_{3}\right) \delta 7.35(\mathrm{~m}, 5 \mathrm{H}), 5.61(\mathrm{~d}, J=9.0 \mathrm{~Hz}, 1 \mathrm{H}), 5.14(\mathrm{br} \mathrm{s}, 2 \mathrm{H})$, $5.00(\mathrm{~d}, J=2.4 \mathrm{~Hz}, 1 \mathrm{H}), 4.74(\mathrm{~d}, J=2.4 \mathrm{~Hz}, 1 \mathrm{H}), 4.70(\mathrm{ddd}, J=10.2,9.6,4.8 \mathrm{~Hz}, 1 \mathrm{H})$, $4.57(\mathrm{ddd}, J=6.6,3.6,3.0 \mathrm{~Hz}, 1 \mathrm{H}), 4.13(\mathrm{dd}, J=10.2,3.6 \mathrm{~Hz}, 1 \mathrm{H}), 3.92(\mathrm{dd}, J=10.2$, $1.8 \mathrm{~Hz}, 1 \mathrm{H}), 3.75(\mathrm{~s}, 3 \mathrm{H}), 3.72(\mathrm{dd}, J=10.8,5.4 \mathrm{~Hz}, 1 \mathrm{H}), 3.69(\mathrm{~d}, J=2.4 \mathrm{~Hz}, 1 \mathrm{H}), 3.68$ $(\mathrm{dd}, J=4.8,1.8 \mathrm{~Hz}, 1 \mathrm{H}), 3.67(\mathrm{~d}, J=2.4 \mathrm{~Hz}, 1 \mathrm{H}), 3.64(\mathrm{~m}, 1 \mathrm{H}), 3.60(\mathrm{dd}, J=10.2,3.0$ $\mathrm{Hz}, 1 \mathrm{H}), 3.55$ (ddd, $J=10.8,10.2,4.2 \mathrm{~Hz}, 1 \mathrm{H}), 3.44(\mathrm{~m}, 1 \mathrm{H}), 2.15(\mathrm{~d}, J=2.4 \mathrm{~Hz}, 1 \mathrm{H})$, $2.14(\mathrm{~d}, J=1.2 \mathrm{~Hz}, 1 \mathrm{H}), 2.12-2.04(\mathrm{~m}, 4 \mathrm{H}), 1.79-1.42(\mathrm{~m}, 4 \mathrm{H}), 0.95(\mathrm{~d}, J=6.6 \mathrm{~Hz}, 6 \mathrm{H})$, $0.91(\mathrm{~s}, 9 \mathrm{H}), 0.87(\mathrm{~s}, 9 \mathrm{H}), 0.08(\mathrm{~s}, 3 \mathrm{H}), 0.07(\mathrm{~s}, 3 \mathrm{H}), 0.02(\mathrm{~s}, 3 \mathrm{H}), 0.02(\mathrm{~s}, 3 \mathrm{H}) ;{ }^{13} \mathrm{C} \mathrm{NMR}$ $\left(150 \mathrm{MHz}, \mathrm{CDCl}_{3}\right) \delta 172.0,170.0,155.9,136.1,128.5$ (3C), 128.2, 128.1, 95.9, 90.3, 73.1, 71.6, 67.6, 67.0, 66.4 (2C), 63.4, 62.7, 54.0, 52.3, 43.6 (3C), 28.7, 28.2, 26.0 (3C), 
25.9 (3C), 25.7, 23.7, 22.4, 22.3, 22.1, 18.4, 18.3, -5.1, -5.3, -5.3, -5.4; CIHRMS Calcd for $\left[\mathrm{C}_{41} \mathrm{H}_{71} \mathrm{NO}_{12} \mathrm{Si}_{2} \mathrm{Na}^{+}\right]$: 848.4407. Found 848.4392.

$(1 R, 5 S)$-1-N-Carbobenzyloxy-L-threonine methyl ester-5-(tert-butyl-dimethylsilanyloxymethyl)-1H-pyran-2-one (III-45a).

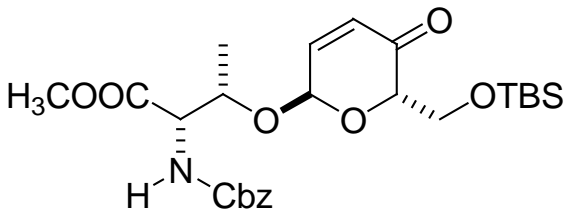

A $\mathrm{CH}_{2} \mathrm{Cl}_{2}(1 \mathrm{~mL})$ solution of compound (ent)-III-18 $(716 \mathrm{mg}, 1.396 \mathrm{mmol})$ and $\mathrm{N}$ Carbobenyloxy-L-threonine methyl ester III-44 (447 mg, $1.675 \mathrm{mmol})$ was cooled to 0 ${ }^{\circ} \mathrm{C} . \quad \mathrm{A} \mathrm{CH}_{2} \mathrm{Cl}_{2}(0.4 \mathrm{~mL})$ solution of $\mathrm{Pd}_{2}(\mathrm{DBA}){ }_{3}{ }^{\cdot} \mathrm{CHCl}_{3}(36 \mathrm{mg}, 2.5 \mathrm{~mol} \%)$ and $\mathrm{PPh}_{3}(33$ $\mathrm{mg}, 10 \mathrm{~mol} \%$ ) was added to the reaction mixture at $0{ }^{\circ} \mathrm{C}$. The reaction mixture was stirred at $0{ }^{\circ} \mathrm{C}$ for 3 hours. The reaction mixture was quenched with $5 \mathrm{~mL}$ of satd aq $\mathrm{NaHCO}_{3}$, extracted $(3 \times 5 \mathrm{~mL})$ with $\mathrm{Et}_{2} \mathrm{O}$, dried $\left(\mathrm{Na}_{2} \mathrm{SO}_{4}\right)$, and concentrated under reduced pressure. The crude product was purified using silica gel flash chromatography eluting with $16 \%$ EtOAc/hexanes to give $914 \mathrm{mg}(1.802 \mathrm{mmol}, 90 \%)$ of III-45a: $R_{f}$ $(20 \%$ EtOAc/hexanes $)=0.34 ;[\alpha]_{\mathrm{D}}^{26}=-45\left(c=1.5, \mathrm{CH}_{2} \mathrm{Cl}_{2}\right) ; \mathrm{IR}\left(\right.$ thin film, $\left.\mathrm{cm}^{-1}\right) 2953$, $1727,1702,1514,1215,1022,836 ;{ }^{1} \mathrm{H}$ NMR $\left(270 \mathrm{MHz}, \mathrm{CDCl}_{3}\right) \delta 7.33(\mathrm{~m}, 5 \mathrm{H}), 6.72$ (dd, $J=10.3,3.5 \mathrm{~Hz}, 1 \mathrm{H}), 6.07(\mathrm{~d}, J=10.3 \mathrm{~Hz}, 1 \mathrm{H}), 5.47(\mathrm{~d}, J=9.7 \mathrm{~Hz}, 1 \mathrm{H}), 5.36(\mathrm{~d}, J$ $=3.6 \mathrm{~Hz}, 1 \mathrm{H}), 5.14(\mathrm{br} \mathrm{s}, 2 \mathrm{H}), 4.58(\mathrm{qd}, J=6.3,2.4 \mathrm{~Hz}, 1 \mathrm{H}), 4.44(\mathrm{dd}, J=9.7,2.4 \mathrm{~Hz}$, $1 \mathrm{H}), 4.22(\mathrm{dd}, J=4.1,2.7 \mathrm{~Hz}, 1 \mathrm{H}), 4.04(\mathrm{dd}, J=11.0,4.3 \mathrm{~Hz}, 1 \mathrm{H}), 3.92(\mathrm{dd}, J=11.0$, $2.5 \mathrm{~Hz}, 1 \mathrm{H}), 3.70(\mathrm{~s}, 3 \mathrm{H}), 1.29$ (d, $J=6.3,3 \mathrm{H}), 0.85$ (s, 9H), 0.05 (s, 3H), 0.04 (s, 3H);

${ }^{13} \mathrm{C}$ NMR $\left(67.5 \mathrm{MHz}, \mathrm{CDCl}_{3}\right) \delta 194.2,171.1,156.6,143.4,136.0,128.5$ (2C), 128.3, 
128.2, 128.1, 89.7, 76.0, 76.1, 72.1, 67.2, 62.5, 58.5, 52.5, 25.8 (3C), 18.3, 15.9, -5.3, 5.4; CIHRMS Calcd for $\left[\mathrm{C}_{25} \mathrm{H}_{37} \mathrm{NO}_{8} \mathrm{SiNa}^{+}\right]$: 530.2186. Found 530.2195.

\section{3-\{4-[6-(tert-Butyl-dimethyl-silanyloxymethyl)-5-hydroxy-5,6-dihydro-2H-pyran-2- yloxy]-phenyl\}-2- N-Carbobenzyloxy-L-threonine methyl ester (III-45b).}

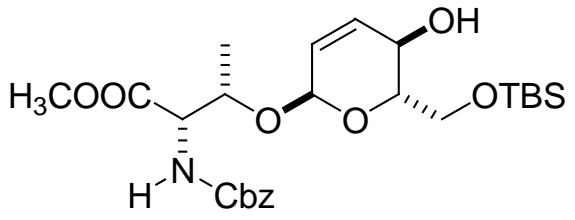

A $\mathrm{CH}_{2} \mathrm{Cl}_{2}(0.3 \mathrm{~mL})$ solution of compound III-45a $(120 \mathrm{mg}, 0.236 \mathrm{mmol})$ and $\mathrm{MeOH}(0.3$ $\mathrm{mL})$ was cooled to $-78{ }^{\circ} \mathrm{C}$. $\mathrm{NaBH}_{4}(8.5 \mathrm{mg}, 0.236 \mathrm{mmol})$ was added and the reaction mixture was stirred at $-78^{\circ} \mathrm{C}$ for 3 hours. The reaction mixture was diluted with ether $(10$ $\mathrm{mL})$ and was quenched with $5 \mathrm{~mL}$ of satd aq $\mathrm{NaHCO}_{3}$, extracted $(3 \times 5 \mathrm{~mL})$ with $\mathrm{Et}_{2} \mathrm{O}$, dried $\left(\mathrm{Na}_{2} \mathrm{SO}_{4}\right)$, and concentrated under reduced pressure. The crude product was purified using silica gel flash chromatography eluting with 20\% EtOAc/hexanes to give

$111 \mathrm{mg}(0.218 \mathrm{mmol}, 91 \%)$ of III-45b: $R_{f}(40 \%$ EtOAc/hexanes $)=0.32 ;[\alpha]_{\mathrm{D}}^{26}=+2(\mathrm{c}$ $=1, \mathrm{CH}_{2} \mathrm{Cl}_{2}$ ); IR (thin film, $\mathrm{cm}^{-1}$ ) 3437, 2953, 1725, 1517, 1213, 1076, 1009, 838; ${ }^{1} \mathrm{H}$ NMR (270 MHz, $\left.\mathrm{CDCl}_{3}\right) \delta 7.35(\mathrm{~m}, 5 \mathrm{H}), 5.90(\mathrm{~d}, J=10.2 \mathrm{~Hz}, 1 \mathrm{H}), 5.58(\mathrm{dd}, J=10.2$, $2.3 \mathrm{~Hz}, 1 \mathrm{H}), 5.50$ (d, $J=9.7 \mathrm{~Hz}, 1 \mathrm{H}), 5.13(\mathrm{br} \mathrm{s}, 2 \mathrm{H}), 4.96(\mathrm{~s}, 1 \mathrm{H}), 4.46$ (qd, $J=6.3,2.3$, $1 \mathrm{H}), 4.38(\mathrm{dd}, J=9.7,2.3 \mathrm{~Hz}, 1 \mathrm{H}), 4.17(\mathrm{~d}, J=9.3,1 \mathrm{H}), 3.95(\mathrm{dd}, J=9.7,4.5 \mathrm{~Hz}, 1 \mathrm{H})$, $3.73(\mathrm{~s}, 3 \mathrm{H}), 3.67(\mathrm{~m}, 1 \mathrm{H}), 3.45(\mathrm{ddd}, J=12.8,12.8,4.5 \mathrm{~Hz}, 1 \mathrm{H}), 3.08(\mathrm{~s}, 1 \mathrm{H}), 1.22(\mathrm{~d}, J$ $=6.3 \mathrm{~Hz}, 3 \mathrm{H}), 0.90(\mathrm{~s}, 9 \mathrm{H}), 0.11(\mathrm{~s}, 6 \mathrm{H}) ;{ }^{13} \mathrm{C} \mathrm{NMR}\left(67.5 \mathrm{MHz}, \mathrm{CDCl}_{3}\right) \delta 170.9,156.6$, 136.2, 132.9, 128.5 (2C), $128.1(2 \mathrm{C}), 128.0,125.2,90.7,71.3,69.4,67.0(2 \mathrm{C}), 65.3,58.8$, 
52.3, 25.7 (3C), 18.1, 15.9, -5.6, -5.6; CIHRMS Calcd for $\left[\mathrm{C}_{25} \mathrm{H}_{39} \mathrm{NO}_{8} \mathrm{SiNa}^{+}\right]: 532.2343$ Found 532.2356.

$\left(1^{\prime} R, 4^{\prime} R, 5 ' S, 1 R, 5 S\right)-1-\left[1^{\prime}-N\right.$-Carbobenzyloxy-L-threonine methyl ester -5-(tert-butyldimethyl-silanyloxymethyl)-1',4'-dihydro-5' $H$-pyran-4'-yloxy]-5-(tert-butyldimethyl-silanyloxymethyl) -1H-pyran-4-one (III-45).

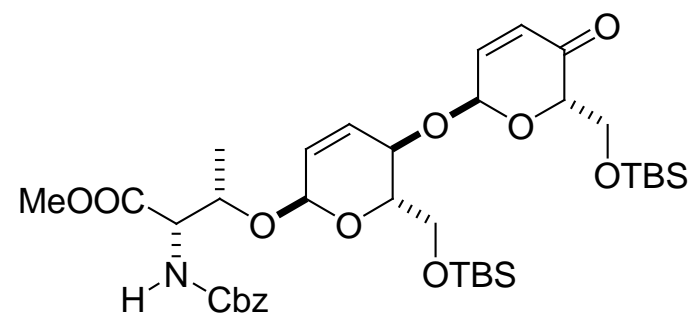

A $\mathrm{CH}_{2} \mathrm{Cl}_{2}(0.7 \mathrm{~mL})$ solution of compound (ent)-III-18 $(574 \mathrm{mg}, 1.60 \mathrm{mmol})$ and alcohol III-45b $(700 \mathrm{mg}, 1.34 \mathrm{mmol})$ was cooled to $0{ }^{\circ} \mathrm{C} . \mathrm{A} \mathrm{CH}_{2} \mathrm{Cl}_{2}(0.7 \mathrm{~mL})$ solution of $\mathrm{Pd}_{2}(\mathrm{DBA})_{3} \mathrm{CHCl}_{3}(34.5 \mathrm{mg}, 2.5 \mathrm{~mol} \%)$ and $\mathrm{PPh}_{3}(35 \mathrm{mg}, 10 \mathrm{~mol} \%)$ was added to the reaction mixture at $0{ }^{\circ} \mathrm{C}$. The reaction mixture was stirred at $0{ }^{\circ} \mathrm{C}$ for 2 hours. The reaction mixture was quenched with $25 \mathrm{~mL}$ of satd aq $\mathrm{NaHCO}_{3}$, extracted $(3 \times 25 \mathrm{~mL})$ with $\mathrm{Et}_{2} \mathrm{O}$, dried $\left(\mathrm{Na}_{2} \mathrm{SO}_{4}\right)$, and concentrated under reduced pressure. The crude product was purified using silica gel flash chromatography eluting with $20 \%$ EtOAc/hexanes to give 10j $\alpha$ (924 mg, $0.82 \mathrm{mmol}, 91 \%)$ as viscous oil. $R_{f}(30 \%$ EtOAc/hexanes $)=0.50$; $[\alpha]_{\mathrm{D}}^{26}=+14\left(c=1, \mathrm{CH}_{2} \mathrm{Cl}_{2}\right) ; \mathrm{IR}\left(\right.$ thin film, $\left.\mathrm{cm}^{-1}\right) 3444,2930,2857,1729,1701,1511$, $1254,1013,835 ;{ }^{1} \mathrm{H} \mathrm{NMR}\left(600 \mathrm{MHz}, \mathrm{CDCl}_{3}\right) \delta 7.35(\mathrm{~m}, 5 \mathrm{H}), 6.81(\mathrm{dd}, J=10.2,3.6 \mathrm{~Hz}$, 1H), $6.12(\mathrm{~d}, J=10.2 \mathrm{~Hz}, 1 \mathrm{H}), 6.07(\mathrm{~d}, J=10.2 \mathrm{~Hz}, 1 \mathrm{H}), 5.69$ (ddd, $J=10.2,2.4,2.4 \mathrm{~Hz}$, $1 \mathrm{H}), 5.56(\mathrm{~d}, J=3.6 \mathrm{~Hz}, 1 \mathrm{H}), 5.54(\mathrm{~d}, J=10.2 \mathrm{~Hz}, 1 \mathrm{H}), 5.13(\mathrm{br} \mathrm{s}, 2 \mathrm{H}), 5.01(\mathrm{~d}, J=2.4$ Hz, 1H), 4.50 (dd, $J=9.0,1.2 \mathrm{~Hz}, 1 \mathrm{H}), 4.44$ (dddd, $J=9.6,6.6,3.0,3.0 \mathrm{~Hz}, 1 \mathrm{H}), 4.39$ 
$(\mathrm{dd}, J=6.6,3.0 \mathrm{~Hz}, 1 \mathrm{H}), 4.37(\mathrm{~d}, J=3.0 \mathrm{~Hz}, 1 \mathrm{H}), 4.10(\mathrm{dd}, J=11.4,3.6 \mathrm{~Hz}, 1 \mathrm{H}), 3.93$ $(\mathrm{dd}, J=11.4,2.4 \mathrm{~Hz}, 1 \mathrm{H}), 3.78(\mathrm{dd}, J=7.8,2.4 \mathrm{~Hz}, 1 \mathrm{H}), 3.76(\mathrm{dd}, J=6.6,2.4 \mathrm{~Hz}, 1 \mathrm{H})$, 3.69 (s, 3H), 3.51 (ddd, $J=10.2,2.4,2.4 \mathrm{~Hz}, 1 \mathrm{H}), 1.23$ (d, $J=6.0 \mathrm{~Hz}, 3 \mathrm{H}), 0.88$ (s, 9H), 0.84 (s, 9H), 0.06 (s, 3H), 0.05 (s, 3H), 0.04 (s, 3H), 0.03 (s, 3H); ${ }^{13} \mathrm{C}$ NMR (150 MHz, $\left.\mathrm{CDCl}_{3}\right) \delta 194.8,170.9,156.6,144.0,136.2,129.2,128.6,128.5(2 \mathrm{C}), 128.1,128.0(2 \mathrm{C})$ 127.0, 90.4, 89.6, 76.6, 70.8, 70.1, 67.1, 66.2, 62.9, 62.2, 58.8, 52.3, 25.9 (3C), $25.8(3 \mathrm{C})$, 18.4, 18.3, 15.7, -5.1, -5.3 (2C), -5.4; CIHRMS Calcd for $\left[\mathrm{C}_{37} \mathrm{H}_{59} \mathrm{NO}_{11} \mathrm{Si}_{2} \mathrm{Na}^{+}\right]$: 772.3518 . Found 772.3543.

$(1 ' R, 4 ' R, 5 ' S, 1 R, 4 R, 5 S)$-1-[N-Carbobenzyloxy-L-threonine methyl ester -5'-(tertbutyl-dimethyl-silanyloxymethyl)-1',4'-dihydro-5'H-pyran-4' -yloxy]-5-(tert-butyldimethyl-silanyloxymethyl)-1,4-dihydro-5H-pyran-4-ol (III-46a).

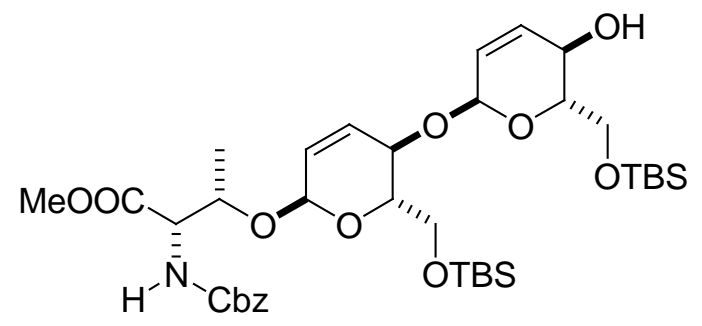

The ketone compound III-45 (600 mg, $0.79 \mathrm{mmol}$ ), was dissolved in $0.8 \mathrm{~mL}$ of $\mathrm{CH}_{2} \mathrm{Cl}_{2}$ and $0.8 \mathrm{~mL} \mathrm{MeOH}$ were added to a round bottom flask and cooled $-78{ }^{\circ} \mathrm{C}$ then $(44.7 \mathrm{mg}$, $1.18 \mathrm{mmol}) \mathrm{NaBH}_{4}$ was added and the reaction mixture was stirred at $-78^{\circ} \mathrm{C}$ for 6 hours and on completion monitored by TLC, reaction mixture is diluted with ether and was quenched with $10 \mathrm{~mL}$ of satd. aq $\mathrm{NaHCO}_{3}$, extracted $(3 \times 10 \mathrm{~mL})$ with $\mathrm{Et}_{2} \mathrm{O}$, dried $\left(\mathrm{Na}_{2} \mathrm{SO}_{4}\right)$, and concentrated under reduced pressure. The crude product was purified using silica gel flash chromatography eluting with $25 \%$ EtOAc/hexanes to give III-46a 
$(529 \mathrm{mg}, 0.69 \mathrm{mmol}, 88 \%)$ as viscous oil. $R_{f}(30 \%$ EtOAc/hexanes $)=0.40 ;[\alpha]_{\mathrm{D}}^{26}=-$ $16\left(c=1, \mathrm{CH}_{2} \mathrm{Cl}_{2}\right)$; IR (thin film, $\mathrm{cm}^{-1}$ ) 3445, 2953, 2930, 1729, 1514, 1254, 1036, 837, 779; ${ }^{1} \mathrm{H}$ NMR (600 MHz, $\left.\mathrm{CDCl}_{3}\right) \delta 7.36(\mathrm{~m}, 5 \mathrm{H}), 5.99(\mathrm{dd}, J=10.2,2.4 \mathrm{~Hz}, 1 \mathrm{H}), 5.95$ (dd, $J=10.2,1.8 \mathrm{~Hz}, 1 \mathrm{H}), 5.66(\mathrm{ddd}, J=10.2,2.4,2.4 \mathrm{~Hz}, 1 \mathrm{H}), 5.63(\mathrm{dd}, J=4.8,2.4 \mathrm{~Hz}$, $1 \mathrm{H}), 5.58(\mathrm{~d}, J=9.6 \mathrm{~Hz}, 1 \mathrm{H}), 5.13(\mathrm{dd}, J=9.0,1.8 \mathrm{~Hz}, 1 \mathrm{H}), 5.12(\mathrm{br} \mathrm{s}, 2 \mathrm{H}), 5.00(\mathrm{~d}, J=$ $2.4 \mathrm{~Hz}, 1 \mathrm{H}), 4.45(\mathrm{dddd}, J=9.0,3.6,3.0,3.0 \mathrm{~Hz}, 1 \mathrm{H}), 4.38(\mathrm{dd}, J=10.2,3.0 \mathrm{~Hz}, 1 \mathrm{H})$, $4.27(\mathrm{dd}, J=10.2,1.8 \mathrm{~Hz}, 1 \mathrm{H}), 4.23(\mathrm{dd}, J=10.8,3.0 \mathrm{~Hz}, 1 \mathrm{H}), 3.96(\mathrm{dd}, J=10.2,4.2$ $\mathrm{Hz}, 1 \mathrm{H}), 3.83(\mathrm{dd}, J=6.0,1.8 \mathrm{~Hz}, 1 \mathrm{H}), 3.81(\mathrm{~d}, J=1.8 \mathrm{~Hz}, 1 \mathrm{H}), 3.75(\mathrm{dd}, J=10.8,4.8$ $\mathrm{Hz}, 1 \mathrm{H}), 3.71(\mathrm{~s}, 3 \mathrm{H}), 3.54(\mathrm{ddd}, J=9.0,5.4,1.8 \mathrm{~Hz}, 1 \mathrm{H}), 3.08(\mathrm{~d}, J=3.6 \mathrm{~Hz}, 1 \mathrm{H}), 2.03$ (br s, 1H), 1.25 (d, J = 6.6 Hz, 3H), $0.91(\mathrm{~s}, 9 \mathrm{H}), 0.89$ (s, 9H), $0.11(\mathrm{~s}, 3 \mathrm{H}), 0.10(\mathrm{~s}, 3 \mathrm{H})$, 0.07 (s, 3H), 0.06 (s, 3H); ${ }^{13} \mathrm{C}$ NMR (150 MHz, $\left.\mathrm{CDCl}_{3}\right) \delta 170.9,156.6,136.2,132.9$, $129.7,128.4$ (2C), 128.1, 128.0 (2C), 126.5, 125.5, 90.9, 90.0, 70.5, 70.1, 69.8, 67.0, $66.5,65.3,62.5,60.3,58.9,52.3,25.9(3 \mathrm{C}), 25.8(3 \mathrm{C}), 18.5,18.1,15.5,-5.1,-5.2,-5.4,-$ 5.6; CIHRMS Calcd for $\left[\mathrm{C}_{37} \mathrm{H}_{61} \mathrm{NO}_{11} \mathrm{Si}_{2} \mathrm{Na}^{+}\right]$: 774.3675. Found 774.3655.

1'(2' N-Carbobenzyloxy-L-threonine methyl ester -1'-yloxy)- -5',5-(tert-butyldimethyl-silanyloxymethyl)-bis-1,4- $\alpha$-L-mannose (III-46b).

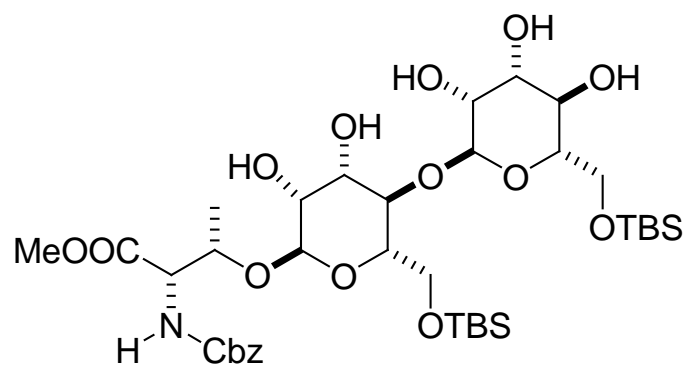

A $\mathrm{CH}_{2} \mathrm{Cl}_{2}(0.8 \mathrm{~mL}, 1 \mathrm{~mol})$ solution of compound of allyl alcohol III-46a $(60 \mathrm{mg}, 0.08$ mmol) at $0{ }^{\circ} \mathrm{C}$ was added a solution of $(50 \% \mathrm{w} / \mathrm{v})$ of $\mathrm{N}$-methyl morpholine $\mathrm{N}$-oxide / 
water $(0.05 \mathrm{~mL})$. Crystalline $\mathrm{OsO}_{4}(1.0 \mathrm{mg}, 5 \mathrm{~mol} \%)$ was added and the reaction was stirred for $12 \mathrm{~h}$. The reaction mixture was concentrated and was pipetted directly on to a silica gel column using a small amount of $\mathrm{CH}_{2} \mathrm{Cl}_{2}(1 \mathrm{~mL})$ in three portions. The crude product was purified using silica gel flash chromatography eluting with $100 \%$ EtOAc to give III-46b (54 mg, 0.07 mmol, $83 \%)$ as viscous oil. $R_{f} \quad$ (40:10:50 EtOAc/MeOH/Hexane $)=0.25 ;[\alpha]_{\mathrm{D}}^{26}=-30\left(c=1, \mathrm{CH}_{2} \mathrm{Cl}_{2}\right) ; \mathrm{IR}\left(\right.$ thin film, $\left.\mathrm{cm}^{-1}\right) 3356$, 2929, 2857, 1719, 1529, 1252, 1046, 968, 838; ${ }^{1} \mathrm{H}$ NMR (600 MHz, $\left.\mathrm{CDCl}_{3}\right) \delta 7.36(\mathrm{~m}$, $5 \mathrm{H}), 5.68(\mathrm{~d}, J=9.6 \mathrm{~Hz}, 1 \mathrm{H}), 5.19(\mathrm{~d}, J=10.2 \mathrm{~Hz}, 1 \mathrm{H}), 5.14(\mathrm{br} \mathrm{s}, 2 \mathrm{H}), 4.85(\mathrm{~d}, J=10.8$ $\mathrm{Hz}, 1 \mathrm{H}), 4.44(\mathrm{dd}, J=10.2,2.4 \mathrm{~Hz}, 1 \mathrm{H}), 4.40(\mathrm{dd}, J=6.0,2.4 \mathrm{~Hz}, 1 \mathrm{H}), 4.06(\mathrm{~d}, J=6.0$ $\mathrm{Hz}, 1 \mathrm{H}), 3.97$ (br s, 1H), $3.84(\mathrm{~d}, J=5.4 \mathrm{~Hz}, 1 \mathrm{H}), 3.81$ (br s, 2H), $3.78(\mathrm{dd}, J=9.0,2.4$ Hz, 1H), $3.74(\mathrm{~d}, J=3.6 \mathrm{~Hz}, 1 \mathrm{H}), 3.71(\mathrm{~s}, 3 \mathrm{H}), 3.69$ (br s, 1H), 3.68 (br s, 1H), 3.38 (m, $3 \mathrm{H}), 3.33(\mathrm{dd}, J=8.4,6.0 \mathrm{~Hz}, 1 \mathrm{H}), 3.08(\mathrm{br} \mathrm{s}, 1 \mathrm{H}), 1.97(\mathrm{~m}, 4 \mathrm{H}), 1.20(\mathrm{~d}, J=6.0 \mathrm{~Hz}, 3 \mathrm{H}$ $0.90(\mathrm{~s}, 9 \mathrm{H}), 0.89(\mathrm{~s}, 9 \mathrm{H}), 0.10(\mathrm{~s}, 3 \mathrm{H}), 0.09(\mathrm{~s}, 3 \mathrm{H}), 0.06(\mathrm{~s}, 6 \mathrm{H}) ;{ }^{13} \mathrm{C} \mathrm{NMR}(150 \mathrm{MHz}$ $\left.\mathrm{CDCl}_{3}\right) \delta 171.3,156.6,136.1,128.5(2 \mathrm{C}), 128.2,128.1(2 \mathrm{C}), 99.8,94.6,74.4,71.9,71.8$ 71.4, 71.3, 70.6, 70.4, 69.8, 69.7, 67.2, 64.6, 62.8, 58.2, 52.5, 25.9 (3C), 25.8 (3C), 18.3, 18.3, 14.3, -5.2, -5.3, -5.4, -5.5; CIHRMS Calcd for $\left[\mathrm{C}_{37} \mathrm{H}_{65} \mathrm{NO}_{15} \mathrm{Si}_{2} \mathrm{Na}^{+}\right]: \quad 842.3784$. Found 842.3751.

$(1 ' R, 4 ' R, 5 ' S, 1 R, 4 R, 5 S)-1-\left[1^{\prime}-N\right.$-Carbobenzyloxy-L-threonine methyl ester -5 '-(tertbutyl-dimethyl-silanyloxymethyl)-1',4'-dihydro-5' H-pyran-4'-yloxy] -5-(tert-butyldimethyl-silanyloxymethyl)-1,4-dihydro-5H-pyran-4-isovalaric ester (III-46). 


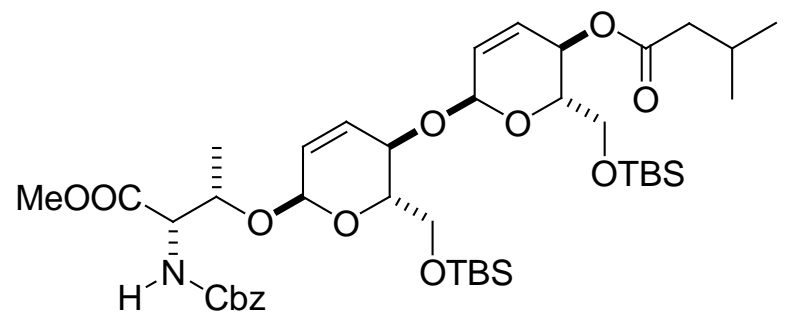

The alcohol compound III-46a $(540 \mathrm{mg}, 0.71 \mathrm{mmol})$, isovaleric acid $(86.6 \mathrm{mg}, 0.85$ mmol) and DCC (175 mg, $0.85 \mathrm{mmol})$ were dissolved in $1.5 \mathrm{~mL}$ of $\mathrm{CH}_{2} \mathrm{Cl}_{2}$ in a round bottom flask and cooled $0{ }^{\circ} \mathrm{C}$ then $(8.6 \mathrm{mg}, 0.1 \mathrm{mmol})$ DMAP was added and the reaction mixture was stirred at $0{ }^{\circ} \mathrm{C}$ for 6 hours and on completion monitored by TLC, reaction mixture is diluted with ether and was quenched with $5 \mathrm{~mL}$ of satd aq $\mathrm{NaHCO}_{3}$, extracted (3 x $5 \mathrm{~mL}$ ) with $\mathrm{Et}_{2} \mathrm{O}$, dried $\left(\mathrm{Na}_{2} \mathrm{SO}_{4}\right)$, and concentrated under reduced pressure. The crude product was purified using silica gel flash chromatography eluting with $20 \%$ EtOAc/hexanes to give III-46 (557 mg, $0.66 \mathrm{mmol}, 93 \%)$ as viscous oil. $R_{f}(30 \%$ EtOAc/hexanes $)=0.60 ;[\alpha]_{\mathrm{D}}^{26}=-54\left(c=1, \mathrm{CH}_{2} \mathrm{Cl}_{2}\right) ; \mathrm{IR}\left(\right.$ thin film, $\left.\mathrm{cm}^{-1}\right) 3448,2931$, $1736,1511,1461,1254,1123,1008,836 ;{ }^{1} \mathrm{H} \mathrm{NMR}\left(600 \mathrm{MHz}, \mathrm{CDCl}_{3}\right) \delta 7.36(\mathrm{~m}, 5 \mathrm{H})$, $6.04(\mathrm{~d}, J=10.8 \mathrm{~Hz}, 1 \mathrm{H}), 5.91(\mathrm{~d}, J=10.2 \mathrm{~Hz}, 1 \mathrm{H}), 5.71(\mathrm{ddd}, J=10.2,2.4,2.4 \mathrm{~Hz}, 1 \mathrm{H})$, $5.67(\mathrm{ddd}, J=10.2,2.4,1.8 \mathrm{~Hz}, 1 \mathrm{H}), 5.60(\mathrm{~d}, J=10.2 \mathrm{~Hz}, 1 \mathrm{H}), 5.42(\mathrm{dd}, J=9.6,1.2 \mathrm{~Hz}$, $1 \mathrm{H}), 5.22(\mathrm{~d}, J=2.4 \mathrm{~Hz}, 1 \mathrm{H}), 5.12(\mathrm{br} \mathrm{s}, 2 \mathrm{H}), 5.01(\mathrm{~d}, J=1.8 \mathrm{~Hz}, 1 \mathrm{H}), 4.44(\mathrm{dd}, J=6.0$, $3.6 \mathrm{~Hz}, 1 \mathrm{H}), 4.38(\mathrm{dd}, J=9.0,3.6 \mathrm{~Hz}, 1 \mathrm{H}), 4.34(\mathrm{dd}, J=9.0,1.8 \mathrm{~Hz}, 1 \mathrm{H}), 3.84(\mathrm{dd}, J=$ $4.2,1.8 \mathrm{~Hz}, 1 \mathrm{H}), 3.81(\mathrm{dd}, J=11.4,1.8 \mathrm{~Hz}, 1 \mathrm{H}), 3.78(\mathrm{~d}, J=4.8 \mathrm{~Hz}, 1 \mathrm{H}), 3.76(\mathrm{~d}, J=4.2$ Hz, 1H), 3.73 (d, $J=7.8 \mathrm{~Hz}, 1 \mathrm{H}), 3.71(\mathrm{~s}, 3 \mathrm{H}), 3.58$ (dddd, $J=7.2,5.4,1.8,1.8 \mathrm{~Hz}, 1 \mathrm{H}$ ), $2.21(\mathrm{~d}, J=1.2 \mathrm{~Hz}, 1 \mathrm{H}), 2.19(\mathrm{~d}, J=1.2 \mathrm{~Hz}, 1 \mathrm{H}), 2.11(\mathrm{~m}, 1 \mathrm{H}), 1.20(\mathrm{~d}, J=6.6 \mathrm{~Hz}, 3 \mathrm{H})$, $0.96(\mathrm{~d}, J=6.8 \mathrm{~Hz}, 6 \mathrm{H}), 0.89$ (s, 9H), $0.88(\mathrm{~s}, 9 \mathrm{H}), 0.07$ (s, 3H), $0.06(\mathrm{~s}, 3 \mathrm{H}), 0.03(\mathrm{~s}, 3 \mathrm{H})$, 
$0.03(\mathrm{~s}, 3 \mathrm{H}) ;{ }^{13} \mathrm{C} \mathrm{NMR}\left(150 \mathrm{MHz}, \mathrm{CDCl}_{3}\right) \delta 172.5,170.9,156.7,136.3,130.0,129.6$, 128.4 (2C), 128.0 (2C), 127.4, 126.6, 90.8, 90.2, 70.7, 70.6, 70.2, 69.6, 67.0, 66.3, 64.7, 62.8, 62.1, 58.9, 52.3, 43.6, 43.4, 26.0, 25.9 (3C), 25.7 (3C), 22.4, 22.3, 18.5, 18.4, 15.6, 5.1, -5.3 (2C), -5.4; CIHRMS Calcd for $\left[\mathrm{C}_{42} \mathrm{H}_{69} \mathrm{NO}_{12} \mathrm{Si}_{2} \mathrm{Na}^{+}\right]$: 858.4250. Found 858.4267.

1 '- $N$-Carbobenzyloxy-L-threonine methyl ester -5',5-(tert-butyl-dimethylsilanyloxymethyl)-bis-1,4- $\alpha$-L-mannose - isovalaric ester (III-47).

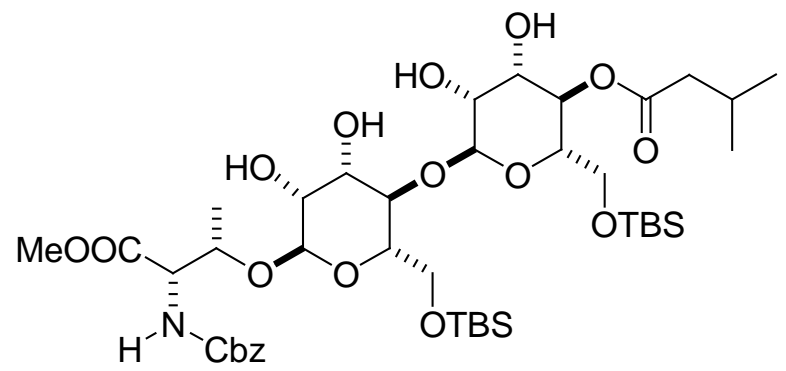

A $\mathrm{CH}_{2} \mathrm{Cl}_{2}(2.5 \mathrm{~mL}, 0.5 \mathrm{Mol})$ solution of compound of diene III-46 (430 mg, $\left.0.51 \mathrm{mmol}\right)$ at $0{ }^{\circ} \mathrm{C}$ was added a solution of $(50 \% \mathrm{w} / \mathrm{v})$ of $\mathrm{N}$-methyl morpholine $\mathrm{N}$-oxide / water $(0.25$ $\mathrm{mL})$. Crystalline $\mathrm{OsO}_{4}(13 \mathrm{mg}, 10 \mathrm{~mol} \%)$ was added and the reaction was stirred for 12 h. The reaction mixture was concentrated and was pipetted directly on to a silica gel column using a small amount of $\mathrm{CH}_{2} \mathrm{Cl}_{2}(3 \mathrm{~mL})$ in three portions. The crude product was purified using silica gel flash chromatography eluting with 50:30:20 ( EtOAc : Hexane : Methanol) to give III-47 (394 mg, $0.43 \mathrm{mmol}, 85 \%)$ as viscous oil. $R_{f}(60: 40$ EtOAc/Hexane $)=0.20 ;[\alpha]_{\mathrm{D}}^{26}=-63\left(c=1, \mathrm{CH}_{2} \mathrm{Cl}_{2}\right) ; \mathrm{IR}\left(\right.$ thin film, $\left.\mathrm{cm}^{-1}\right) 3443,2931$, $2857,1730,1517,1462,1100,1031,836 ;{ }^{1} \mathrm{H}$ NMR $\left(600 \mathrm{MHz}, \mathrm{CDCl}_{3}\right) \delta 7.36(\mathrm{~m}, 5 \mathrm{H})$, $5.69(\mathrm{~d}, J=10.2 \mathrm{~Hz}, 1 \mathrm{H}), 5.18(\mathrm{br} \mathrm{s}, 1 \mathrm{H}), 5.14(\mathrm{br} \mathrm{s}, 2 \mathrm{H}), 4.85(\mathrm{~d}, J=10.2 \mathrm{~Hz}, 1 \mathrm{H}), 4.44$ (d, $J=10.2,2.4 \mathrm{~Hz}, 1 \mathrm{H}), 4.40(\mathrm{dd}, J=6.0,2.4 \mathrm{~Hz}, 1 \mathrm{H}), 4.06(\mathrm{~d}, J=6.6 \mathrm{~Hz}, 1 \mathrm{H}), 3.97$ (s, $1 \mathrm{H}), 3.84(\mathrm{~d}, J=5.6 \mathrm{~Hz}, 1 \mathrm{H}), 3.82(\mathrm{dd}, J=10.2 \mathrm{~Hz}, 1 \mathrm{H}), 3.81-3.73(\mathrm{~m}, 6 \mathrm{H}), 3.74(\mathrm{~d}, J=$ 
$3.6 \mathrm{~Hz}, 1 \mathrm{H}), 3.71(\mathrm{~s}, 3 \mathrm{H}), 3.68(\mathrm{~d}, J=10.2 \mathrm{~Hz}, 1 \mathrm{H}), 3.38(\mathrm{~d}, J=6.0 \mathrm{~Hz}, 1 \mathrm{H}), 3.33(\mathrm{dd}, J$ $=9.6,6.0 \mathrm{~Hz}, 1 \mathrm{H}), 3.10(\mathrm{~d}, J=7.8 \mathrm{~Hz}, 1 \mathrm{H}), 2.75(\mathrm{br} \mathrm{s}, 1 \mathrm{H}), 2.23(\mathrm{dd}, J=15.0,7.8 \mathrm{~Hz}$, $1 \mathrm{H}), 2.18(\mathrm{dd}, J=14.2,7.2 \mathrm{~Hz}, 1 \mathrm{H}), 2.10(\mathrm{~m}, 1 \mathrm{H}), 1.20(\mathrm{~d}, J=6.0 \mathrm{~Hz}, 3 \mathrm{H}), 0.94(\mathrm{~d}, J=$ $7.2 \mathrm{~Hz}, 6 \mathrm{H}), 0.91$ (s, 9H), 0.88 (s, 9H), 0.10 (s, 3H), 0.09 (s, 3H), 0.06 (s, 6H); ${ }^{13} \mathrm{C}$ NMR $\left(150 \mathrm{MHz}, \mathrm{CDCl}_{3}\right) \delta$ 173.5, 171.2, 156.6, 136.0, 128.5 (2C), 128.2, 128.1 (2C), 97.5, $94.6,73.5,71.6,71.4,71.3,71.1,70.9,70.1,69.9,69.8,69.3,67.2,62.9,58.5,52.4,43.4$, 25.9 (3C), 25.8 (3C), 25.7, 22.4, 22.3, 18.4, 18.3, 14.5, -5.2, -5.3, -5.4, -5.5; CIHRMS Calcd for $\left[\mathrm{C}_{42} \mathrm{H}_{73} \mathrm{NO}_{16} \mathrm{Si}_{2} \mathrm{Na}^{+}\right]$: 926.4360 . Found 926.4373 .

$\left(1 ' R, 4^{\prime} R, 5 ' S, 1 R, 4 R, 5 S\right)-1-\left[1^{\prime}-N\right.$-Carbobenzyloxy-L-threonine methyl ester -5'-(tertbutyl-dimethyl-silanyloxymethyl)-tetrahydro-pyran-4'-yloxy]-5-(tert-butyl-dimethylsilanyloxymethyl) -tetrahydro-pyran-4-isovalaric ester (III-49).

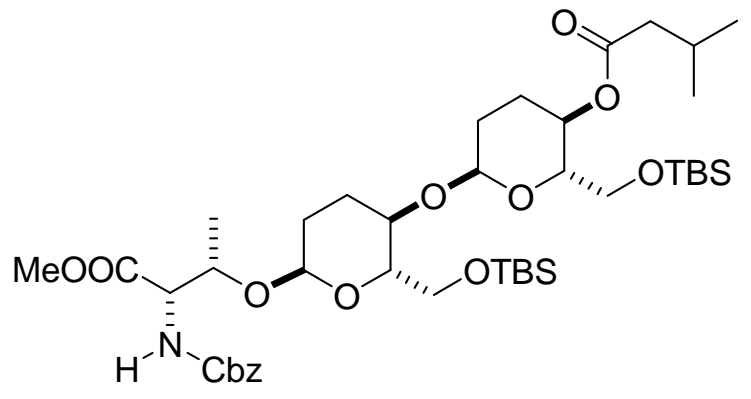

The allyl alcohol compound III-46 (85 mg, $0.10 \mathrm{mmol}), \mathrm{O}-\mathrm{NO}_{2} \mathrm{ArSO}_{2} \mathrm{NHNH}_{2}(305 \mathrm{mg}$, $1.50 \mathrm{mmol}$ ) were dissolved in $1.0 \mathrm{~mL}$ of $\mathrm{CH}_{2} \mathrm{Cl}_{2}$ in a round bottom flask and cooled $0{ }^{\circ} \mathrm{C}$ under nitrogen condition then triethylamine $(203 \mathrm{mg}, 2.00 \mathrm{mmol}$ ) was added and the reaction mixture was stirred at $0{ }^{\circ} \mathrm{C}$ for 12 hours and on completion monitored by TLC, reaction mixture is three portions. The crude product was purified using silica gel flash chromatography eluting with 20\% EtOAc/hexanes to give III-49 (76 mg, $0.09 \mathrm{mmol}, 90$ $\%)$ as viscous oil. $R_{f}(30 \%$ EtOAc/Hexane $)=0.60 ;[\alpha]_{\mathrm{D}}^{26}=-102\left(c=1, \mathrm{CH}_{2} \mathrm{Cl}_{2}\right) ; \mathrm{IR}$ 
(thin film, $\mathrm{cm}^{-1}$ ) $3450,2955,2857,1735,1510,1461,1253,1124,995,836 ;{ }^{1} \mathrm{H}$ NMR $\left(600 \mathrm{MHz}, \mathrm{CDCl}_{3}\right) \delta 7.35(\mathrm{~m}, 5 \mathrm{H}), 5.50(\mathrm{~d}, J=9.6 \mathrm{~Hz}, 1 \mathrm{H}), 5.14(\mathrm{br} \mathrm{s}, 2 \mathrm{H}), 5.00(\mathrm{~d}, J=$ $2.4 \mathrm{~Hz}, 1 \mathrm{H}), 4.86(\mathrm{~d}, J=3.0 \mathrm{~Hz}, 1 \mathrm{H}), 4.72(\mathrm{dd}, J=10.2,6.0,2.4 \mathrm{~Hz}, 1 \mathrm{H}), 4.41(\mathrm{dd}, J=$ 8.4, $2.4 \mathrm{~Hz}, 1 \mathrm{H}), 4.40(\mathrm{dd}, J=4.2,2.4 \mathrm{~Hz}, 1 \mathrm{H}), 3.84(\mathrm{dd}, J=10.8,1.8 \mathrm{~Hz}, 1 \mathrm{H}), 3.75(\mathrm{dd}$, $J=11.4,4.8 \mathrm{~Hz}, 1 \mathrm{H}), 3.72(\mathrm{~s}, 3 \mathrm{H}), 3.70(\mathrm{dd}, J=10.2,2.4 \mathrm{~Hz}, 1 \mathrm{H}), 3.69(\mathrm{dd}, J=4.2,1.8$ Hz, 1H), 3.57 (ddd, $J=10.2,1.8,1.8 \mathrm{~Hz}, 1 \mathrm{H}), 3.34(\mathrm{~m}, 2 \mathrm{H}), 2.14(\mathrm{dd}, J=8.4,3.6 \mathrm{~Hz}$, $1 \mathrm{H}), 2.13(\mathrm{dd}, J=8.4,3.6 \mathrm{~Hz}, 1 \mathrm{H}), 2.08(\mathrm{~m}, 1 \mathrm{H}), 1.21(\mathrm{~d}, J=6.0 \mathrm{~Hz}, 3 \mathrm{H}), 1.75(\mathrm{ddd}, J=$ 12.6, 4.2, 4.2 Hz, 1H), $1.71(\mathrm{dd}, J=10.8,2.4 \mathrm{~Hz}, 1 \mathrm{H}), 1.69(\mathrm{dd}, J=12.6,3.0 \mathrm{~Hz}, 1 \mathrm{H})$, $1.63(\mathrm{dd}, J=12.0,6.0 \mathrm{~Hz}, 1 \mathrm{H}), 1.59(\mathrm{~m}, 2 \mathrm{H}), 1.46(\mathrm{dd}, J=10.2,6.0 \mathrm{~Hz}, 1 \mathrm{H}), 1.42(\mathrm{dd}, J$ $=10.8,6.0 \mathrm{~Hz}, 1 \mathrm{H}), 0.90(\mathrm{~d}, J=6.0 \mathrm{~Hz}, 6 \mathrm{H}), 0.88(\mathrm{~s}, 9 \mathrm{H}), 0.87(\mathrm{~s}, 9 \mathrm{H}), 0.08(\mathrm{~s}, 3 \mathrm{H}), 0.07$ (s, 3H), 0.04 (s, 3H), 0.02 (s, 3H); ${ }^{13} \mathrm{C}$ NMR (150 MHz, $\left.\mathrm{CDCl}_{3}\right) \delta$ 172.0, 171.1, 156.7, 136.2, 128.5 (2C), 128.2, 128.1, 91.3, 90.2, 72.9, 71.5, 68.8, 67.6, 67.1, 66.1, 63.1, 62.6, 58.8, 52.1, 43.6, 28.8, 28.4, 26.3, 26.0 (3C), 25.9 (3C), 25.7, 23.7, 22.4, 22.3, 21.9, 18.4, 18.3, 14.8, -5.1, -5.3, -5.4 (2C); CIHRMS Calcd for $\left[\mathrm{C}_{42} \mathrm{H}_{73} \mathrm{NO}_{12} \mathrm{Si}_{2} \mathrm{Na}^{+}\right]$: 862.4563 . Found 862.4522.

1'- $N$-Carbobenzyloxy-L-threonine methyl ester -5',5-(tert-butyl-dimethylsilanyloxymethyl)-2,3,2',3'-bis acetonide-bis-1,4-a-L-mannose-isovalaric ester (III48). 


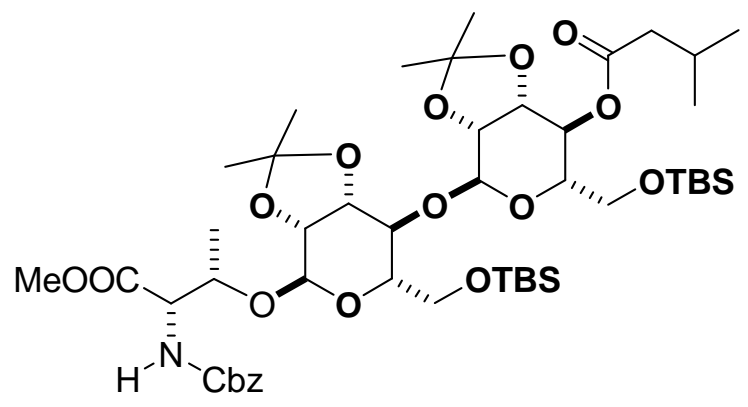

To a $\mathrm{CH}_{2} \mathrm{Cl}_{2}(0.1 \mathrm{~mL}, 1.0 \mathrm{M})$ solution of tris- $\alpha$-D-manno-tetrol III-46 (70 mg, $\left.0.07 \mathrm{mmol}\right)$ and 2,2 dimethoxy propane $(9.5 \mathrm{mg}, 0.17 \mathrm{mmol})$ at $0{ }^{\circ} \mathrm{C}$ add CSA $(1.8 \mathrm{mg}, 10 \mathrm{~mol} \%)$ and the reaction was stirred for $6 \mathrm{~h}$. The reaction mixture was concentrated and was pipetted directly on to a silica gel column using a small amount of $\mathrm{CH}_{2} \mathrm{Cl}_{2}(2 \mathrm{~mL})$ in three portions. Impurities were eluted with ether and the product was eluted with EtOAc/Hexane (40:60). Pure fractions were combined and concentrated to afford III-48 $(66 \mathrm{mg}, 0.06 \mathrm{mmol}, 82 \%): R_{f}(50 \% \mathrm{EtOAc} / \mathrm{Hexane})=0.50 ;[\alpha]_{\mathrm{D}}^{26}=-38\left(c 1, \mathrm{CH}_{2} \mathrm{Cl}_{2}\right) ;$ IR (thin film, $\mathrm{cm}^{-1}$ ) 2953, 2934, 1735, 1510, 1253, 1090, 1019, 835; ${ }^{1} \mathrm{H}$ NMR $(600 \mathrm{MHz}$, $\left.\mathrm{CDCl}_{3}\right) \delta 7.34(\mathrm{~m}, 5 \mathrm{H}), 6.61(\mathrm{~d}, J=10.2 \mathrm{~Hz}, 1 \mathrm{H}), 5.41(\mathrm{~d}, J=10.2,4.8 \mathrm{~Hz}, 1 \mathrm{H}), 5.10(\mathrm{br}$ s, $2 \mathrm{H}), 4.51(\mathrm{dd}, J=12.0,3.6 \mathrm{~Hz}, 1 \mathrm{H}), 4.38(\mathrm{ddd}, J=12.6,10.2,3.6 \mathrm{~Hz}, 1 \mathrm{H}), 4.12(\mathrm{dd}, J$ $=13.2,2.4 \mathrm{~Hz}, 1 \mathrm{H}), 4.09(\mathrm{dd}, J=6.6,2.4 \mathrm{~Hz}, 1 \mathrm{H}), 4.06(\mathrm{~d}, J=10.2,6.0 \mathrm{~Hz}, 1 \mathrm{H}), 4.01(\mathrm{~d}$, $J=7.2,6.0 \mathrm{~Hz}, 1 \mathrm{H}), 3.96(\mathrm{dd}, J=12.6,6.0 \mathrm{~Hz}, 1 \mathrm{H}), 3.90(\mathrm{dd}, J=10.2,7.8 \mathrm{~Hz}, 1 \mathrm{H}), 3.71$ $(\mathrm{dd}, J=8.4,2.4 \mathrm{~Hz}, 1 \mathrm{H}), 3.67(\mathrm{~d}, J=16.2 \mathrm{~Hz}, 1 \mathrm{H}), 3.62(\mathrm{~m}, 1 \mathrm{H}), 3.57(\mathrm{~s}, 3 \mathrm{H}), 3.56(\mathrm{~d}, J$ $=4.2 \mathrm{~Hz}, 1 \mathrm{H}), 3.45(\mathrm{ddd}, J=10.2,6.6,3.6 \mathrm{~Hz}, 1 \mathrm{H}), 3.28(\mathrm{ddd}, J=10.2,3.6,3.6 \mathrm{~Hz}, 1 \mathrm{H})$, $3.23(\mathrm{ddd}, J=10.2,3.0,2.4 \mathrm{~Hz}, 1 \mathrm{H}), 2.21(\mathrm{dd}, J=10.2,3.6 \mathrm{~Hz}, 1 \mathrm{H}), 2.21(\mathrm{~d}, J=10.2$, 3.6 Hz, 1H), 2.19 (dd, $J=10.8,3.6 \mathrm{~Hz}, 1 \mathrm{H}), 2.05(\mathrm{~m}, 1 \mathrm{H}), 1.49$ (d, $J=6.0 \mathrm{~Hz}, 3 \mathrm{H}), 1.46$ $(\mathrm{d}, J=7.8 \mathrm{~Hz}, 3 \mathrm{H}), 1.30(\mathrm{~d}, J=5.4 \mathrm{~Hz}, 3 \mathrm{H}), 1.26(\mathrm{~d}, J=8.4 \mathrm{~Hz}, 3 \mathrm{H}), 1.20(\mathrm{~d}, J=5.4 \mathrm{~Hz}$, $3 \mathrm{H}), 0.98(\mathrm{~d}, J=6.6 \mathrm{~Hz}, 6 \mathrm{H}), 0.89(\mathrm{~s}, 9 \mathrm{H}), 0.87(\mathrm{~s}, 9 \mathrm{H}), 0.03(\mathrm{~s}, 3 \mathrm{H}), 0.02(\mathrm{~s}, 3 \mathrm{H}), 0.01$ 
(s, 3H), -0.00 (s, 3H); ${ }^{13} \mathrm{C}$ NMR (150 MHz, $\left.\mathrm{CDCl}_{3}\right) \delta 172.7,170.8,137.3,128.5(2 \mathrm{C})$, 128.2(2C), 128.1(2C), 114.2, 113.4, 109.5, 97.0, 92.9, 78.6, 78.3, 75.8, 70.9, 70.7, 70.0, 69.7, 69.0, 67.2 (2C), 62.3, 62.0, 58.5, 52.3, 43.5, 27.7(2C), 26.3 (2C), 25.9 (3C), 25.8 (3C), 22.3, 22.2, 18.4, 18.3, 14.5, $-5.1,-5.3,-5.5$ (2C); CIHRMS Calcd for $\left[\mathrm{C}_{48} \mathrm{H}_{81} \mathrm{NO}_{16} \mathrm{Si}_{2} \mathrm{Na}^{+}-\mathrm{C}_{3} \mathrm{H}_{6}{ }^{2+}\right]$ : 966.4486 . Found 966.4441.

\section{Benzyl (S)-1-(methoxycarbonyl)-2-phenylethylcarbamate (III-64).}

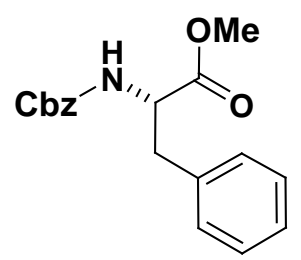

To a solution of acid III-58 (8.6 g, $28.7 \mathrm{mmol})$ in $\mathrm{MeOH}(56 \mathrm{~mL})$ was added TMSCl

$(9.2 \mathrm{~mL}, 71.5 \mathrm{mmol})$ at room temperature. After stirring for $24 \mathrm{hr}$ at room temperature, the reaction was quenched by addition of water. The $\mathrm{MeOH}$ was removed in vacuo and then the mixture was extracted with EtOAc $(3 \times 25 \mathrm{ml})$, dried $\left(\mathrm{Na}_{2} \mathrm{SO}_{4}\right)$, concentrated under reduced pressure. The crude product was purified using silica gel flash chromatography eluting with 20\% EtOAc/hexane to give ester III-64 (8.6 g, $27.32 \mathrm{mmol}$, $95 \%)$ as viscous oil. $R_{f}(50 \%$ EtOAc/hexane $)=0.71 ;[\alpha]_{\mathrm{D}}^{26}=+7\left(c=1, \mathrm{CH}_{2} \mathrm{Cl}_{2}\right)$; IR (thin film, $\mathrm{cm}^{-1}$ ) 3336, 2953, 2880, 1755, 1516, 1212, 1054, 1038, 744; ${ }^{1} \mathrm{H}$ NMR (600 $\left.\mathrm{MHz}, \mathrm{CDCl}_{3}\right) \delta 7.34(\mathrm{~m}, 5 \mathrm{H}), 7.27(\mathrm{~m}, 5 \mathrm{H}), 5.22(\mathrm{~d}, J=7.8 \mathrm{~Hz}, 1 \mathrm{H}), 5.11(\mathrm{~d}, J=12.6$ Hz, 1H), 5.09 (d, $J=12.6 \mathrm{~Hz}, 1 \mathrm{H}), 4.66(\mathrm{dd}, J=12.0,6.6 \mathrm{~Hz}, 1 \mathrm{H}), 3.72(\mathrm{~s}, 3 \mathrm{H}), 3.15(\mathrm{dd}$, $J=13.8,5.4 \mathrm{~Hz}, 1 \mathrm{H}), 3.09(\mathrm{dd}, J=13.8,5.4 \mathrm{~Hz}, 1 \mathrm{H}) ;{ }^{13} \mathrm{C} \mathrm{NMR}\left(150 \mathrm{MHz}, \mathrm{CDCl}_{3}\right) \delta$ 171.9, 155.5, 136.2, 135.6, 129.2 (2C), 128.5 (2C), 128.4 (2C), 128.1 (2C), 128.0, 127.1, 66.9, 54.7, 52.2, 38.2.; CIHRMS Calcd for $\left[\mathrm{C}_{18} \mathrm{H}_{19} \mathrm{NO}_{4} \mathrm{H}^{+}\right]$: 314.1392. Found 314.1386. 


\section{(S)-methyl 2-amino-3-phenylpropanoate (III-65).}

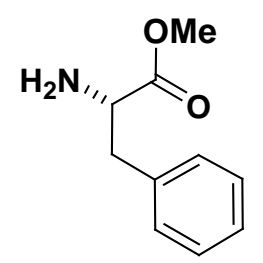

To a solution of ester III-64 (3.8 g, $12.1 \mathrm{mmol})$ in dry $\mathrm{MeOH}(24 \mathrm{~mL})$ was added $\mathrm{Pd} / \mathrm{C}$ (129 mg) and the mixture was stirred under $\mathrm{H}_{2}$ at a 100-psi pressure for $12 \mathrm{~h}$ at room temperature. The catalyst was filtered off through a short pad of Celite, concentrated under reduced pressure. The resulting crude product was pipetted directly on to a silica gel column using $\mathrm{CH}_{2} \mathrm{Cl}_{2}(1 \mathrm{~mL})$ in three portions. Impurities were eluted with ether and the product was eluted with EtOAc/hexanes (40:60). Pure fractions were combined and concentrated to afford amine III-65 $(5.14 \mathrm{mg}, 28.9 \mathrm{mmol}, 97 \%)$ as viscous oil. $R_{f}(50 \%$

EtOAc/hexane $)=0.30 ;[\alpha]_{\mathrm{D}}^{26}=+17\left(c=1, \mathrm{CH}_{2} \mathrm{Cl}_{2}\right) ; \mathrm{IR}$ (thin film, $\left.\mathrm{cm}^{-1}\right) 3345,3028$, 1745, 1662, 1496, 1204, 1016, 700; ${ }^{1} \mathrm{H}$ NMR (600 MHz, $\left.\mathrm{CDCl}_{3}\right) \delta 7.31(\mathrm{~m}, 5 \mathrm{H}), 3.73$ $(\mathrm{dd}, J=7.8,5.4 \mathrm{~Hz}, 1 \mathrm{H}), 3.70(\mathrm{~s}, 3 \mathrm{H}), 3.07(\mathrm{dd}, J=13.2,5.4 \mathrm{~Hz}, 1 \mathrm{H}), 2.86(\mathrm{dd}, J=13.8$, $7.8 \mathrm{~Hz}, 1 \mathrm{H}), 1.45(\mathrm{~s}, 2 \mathrm{H}) ;{ }^{13} \mathrm{C} \mathrm{NMR}\left(150 \mathrm{MHz}, \mathrm{CDCl}_{3}\right) \delta 175.3,137.2,129.2(2 \mathrm{C}), 128.5$ (2C), 126.7, 55.8, 51.8, 41.0; CIHRMS Calcd for $\left[\mathrm{C}_{10} \mathrm{H}_{13} \mathrm{NO}_{2} \mathrm{H}^{+}\right]$: 180.1024. Found 180.1019.

(S)-benzyl (methylcarbamoyl)-methyl-2-amino-3-phenylpropanoate (III-66). 


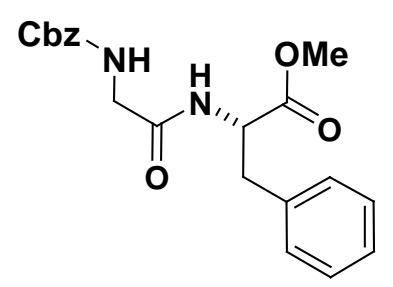

To a solution of amine III-65 (1.28 g, $7.15 \mathrm{mmol})$, Cbz-glycin acid III-57 (1.65 g, 7.86 mmol) in $\mathrm{CH}_{3} \mathrm{CN}(15 \mathrm{ml})$ was added $\mathrm{HBTU}(2.98 \mathrm{~g}, 7.86 \mathrm{mmol})$ at $0{ }^{\circ} \mathrm{C}$, and after $5 \mathrm{~min}$, triethylamine $(2.0 \mathrm{~mL}, 15.3 \mathrm{mmol})$ was added and stirring was continued for $8 \mathrm{~h}$ at $\mathrm{rt}$. The mixture was taken up in EtOAc $(50 \mathrm{~mL})$ and washed with $5 \%$ aqueous $\mathrm{KHSO}_{4}$ solution, 5\% aqueous $\mathrm{NaHCO}_{3}$ solution, and saturated aqueous $\mathrm{NaCl}$ solution. Then the reaction mixture was dried $\left(\mathrm{Na}_{2} \mathrm{SO}_{4}\right)$ and concentrated under reduced pressure. The crude product was purified using silica gel flash chromatography eluting with

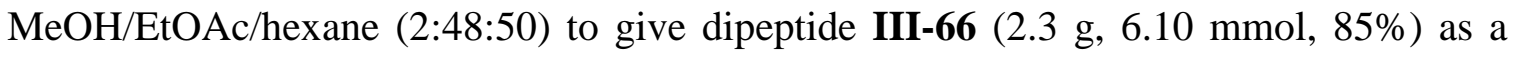
colorless oil, $R_{f}=0.41\left(50 \%\right.$ EtOAc/Hexane); $[\alpha]_{\mathrm{D}}^{26}=+49\left(c=1, \mathrm{CH}_{2} \mathrm{Cl}_{2}\right) ; \mathrm{IR}$ (thin film, $\left.\mathrm{cm}^{-1}\right) 3311,2953,1718,1664,1516,1213,1047,732 ;{ }^{1} \mathrm{H}$ NMR $(600 \mathrm{MHz}$, $\left.\mathrm{CD}_{3} \mathrm{OD}\right) \delta 7.36(\mathrm{~m}, 5 \mathrm{H}), 7.25-7.06(\mathrm{~m}, 5 \mathrm{H}), 6.38$ (br s, $\left.1 \mathrm{H}\right), 5.33$ (br s, 1H), 5.12 (br s, 2H), $4.88(\mathrm{dd}, J=13.2,5.4 \mathrm{~Hz}, 1 \mathrm{H}), 3.88(\mathrm{dd}, J=10.2,5.4 \mathrm{~Hz}, 1 \mathrm{H}), 3.81(\mathrm{dd}, J=10.2$, $5.4 \mathrm{~Hz}, 1 \mathrm{H}), 3.71(\mathrm{~s}, 3 \mathrm{H}), 3.12(\mathrm{~d}, J=5.4 \mathrm{~Hz}, 1 \mathrm{H}), 3.10(\mathrm{~d}, J=5.4 \mathrm{~Hz}, 1 \mathrm{H}) \mathrm{d} ;{ }^{13} \mathrm{C} \mathrm{NMR}$ $\left(150 \mathrm{MHz}, \mathrm{CDCl}_{3}\right) \delta$ 171.6, 168.5, 136.1, 135.5, 129.2, $128.6(2 \mathrm{C}), 128.5$ (2C), 128.2 (2C), 128.0 (2C), 127.1 (2C), 67.1, 53.0, 52.3, 44.3, 37.8; CIHRMS Calcd for $\left[\mathrm{C}_{20} \mathrm{H}_{22} \mathrm{~N}_{2} \mathrm{O}_{5} \mathrm{H}^{+}\right]$: 371.1607. Found 371.1601.

$(1 ' S, 4 ' S, 5 ' R, 1 S, 4 S, 5 R)-1-[1 '-N$-carbobenzyloxy-D-tyrosine methoxycarbonyl -5'(III-67). 


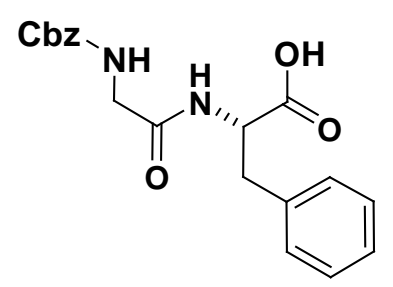

To a solution of methyl ester III-66 (3.7 g, $9.60 \mathrm{mmol})$ in $\mathrm{CH}_{3} \mathrm{OH}(18 \mathrm{~mL})$ was added $\mathrm{LiOH}(0.46 \mathrm{~g}, 19.26 \mathrm{mmol})$ at $0{ }^{\circ} \mathrm{C}$, and the reaction mixture was stirred at $\mathrm{rt}$ for 2 hours and on completion, as monitored by TLC, the reaction mixture was diluted with ether and was quenched with $5 \mathrm{~mL}$ of satd. aq. $\mathrm{NaHCO}_{3}$, extracted $(3 \times 25 \mathrm{~mL})$ with AcOEt. The combined aqueous phases were taken to $\mathrm{pH} 3$ by addition of $2 \mathrm{M}$ aqueous $\mathrm{KHSO}_{4}$ and extracted with AcOEt $(3 \times 25 \mathrm{~mL})$. The combined organic phases were washed with saturated aqueous $\mathrm{NaCl}$ solution $(3 \times 10 \mathrm{~mL})$, dried, and dried $\left(\mathrm{Na}_{2} \mathrm{SO}_{4}\right)$, and concentrated under reduced pressure. The crude product was purified using silica gel flash chromatography eluting with 100\% EtOAc to give acid III-67 $(2.84 \mathrm{~g}, 7.68 \mathrm{mmol}$, $80 \%)$ as viscous oil. $R_{f}(10: 40: 50 \% \mathrm{MeOH} / \mathrm{EtOAc/hexanes})=0.20 ;[\alpha]_{\mathrm{D}}^{26}=+24(c=1$, $\mathrm{CH}_{2} \mathrm{Cl}_{2}$ ); IR (thin film, $\mathrm{cm}^{-1}$ ) 3312, 2982, 2935, 1737, 1655, 1556, 1259, 1048, 940, 743; ${ }^{1} \mathrm{H}$ NMR $\left(600 \mathrm{MHz}, \mathrm{CDCl}_{3}\right) \delta 7.30(\mathrm{~m}, 5 \mathrm{H}), 7.16(\mathrm{~m}, 5 \mathrm{H}), 5.05(\mathrm{br} \mathrm{s}, 2 \mathrm{H}), 5.64(\mathrm{dd}, J=$ 7.8, $5.4 \mathrm{~Hz}, 1 \mathrm{H}), 3.74(\mathrm{~d}, J=17.4 \mathrm{~Hz}, 1 \mathrm{H}), 3.70(\mathrm{~d}, J=17.4 \mathrm{~Hz}, 1 \mathrm{H}), 3.14(\mathrm{dd}, J=14.4$, $4.8 \mathrm{~Hz}, 1 \mathrm{H}), 2.96(\mathrm{dd}, J=13.8,8.4 \mathrm{~Hz}, 1 \mathrm{H}) ;{ }^{13} \mathrm{C} \mathrm{NMR}\left(150 \mathrm{MHz}, \mathrm{CDCl}_{3}\right) 173.1,170.6$, 157.7, 136.9, 129.1 (2C), 128.27 (2C), 128.29, 127.8 (2C), 127.6 (2C), 126.4, 66.6, 53.7, 53.6, 43.5, 37.1; CIHRMS Calcd for $\left[\mathrm{C}_{19} \mathrm{H}_{20} \mathrm{~N}_{2} \mathrm{O}_{5} \mathrm{H}^{+}\right]$: 357.1444. Found 357.1450.

\section{Benzyl (S)-1-(methoxycarbonyl)-2-(4-tert-butyl-dimethylsilanyloxy phenyl) ethylcarbamate (III-53).}




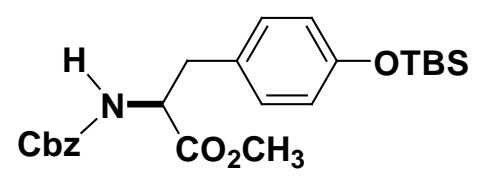

D-tyrosine III-68 was first protected as its methyl ester. Thionyl chloride $(2.3 \mathrm{~mL})$ was added drop wise to dry methanol $(30 \mathrm{~mL})$ at $-15^{\circ} \mathrm{C}$, following by addition of $\mathrm{D}$ tyrosine $(2.8 \mathrm{~g}, 16.2 \mathrm{mmol})$. The resulting mixture was warmed to room temperature for $15 \mathrm{hrs}$. The solvent was removed under reduced pressure, and the residue was further dried under high vacuum to provide the methyl ester as a white solid. The above methyl ester and $\mathrm{Na}_{2} \mathrm{CO}_{3}(2.2 \mathrm{~g}, 15.7 \mathrm{mmol})$ was dissolved in $1: 1$ mixture $60 \mathrm{~mL}$ of acetone $/ \mathrm{H}_{2} \mathrm{O}$. Benzyl chloroformate $(\mathrm{CbzCl})(4.7 \mathrm{~mL}, 34.7 \mathrm{mmol})$ was added drop wise. After $2 \mathrm{hrs}$ at room temperature, the reaction solution was diluted with EtOAc $(250 \mathrm{~mL})$. The layers were separated, and the organic layer was washed with $\mathrm{H}_{2} \mathrm{O}$, brine, dried $\left(\mathrm{MgSO}_{4}\right)$, filtered and concentrated under reduced pressure. The residue was purified by flash chromatography (silica, 3:1 to 1:1 hexane/EtOAc) to give N-Cbz protected tyrosine methyl ester III-68a (5.0 g, 95\%). Phenol III-68a (2.47 g, $7.50 \mathrm{mmol}), 15 \mathrm{~mL}$ of $\mathrm{CH}_{2} \mathrm{Cl}_{2}$, and $4.2 \mathrm{~mL}$ of $\mathrm{Et}_{3} \mathrm{~N}$ were added to a round bottom flask and cooled to $0{ }^{\circ} \mathrm{C}$. A catalytic amount $(92 \mathrm{mg}, 0.75 \mathrm{mmol})$ of DMAP was added followed by addition of tertbutyldimethylsilyl chloride $(1.35 \mathrm{~g}, 9.00 \mathrm{mmol})$ and the solution was stirred at $0{ }^{\circ} \mathrm{C}$ for 6 h. The reaction was quenched with $1 \mathrm{M} \mathrm{NaHSO}_{4}$ and extracted $(3 \times 50 \mathrm{~mL})$ with $\mathrm{Et}_{2} \mathrm{O}$, washed with satd aq $\mathrm{NaHCO}_{3}(2 \times 50 \mathrm{~mL})$, and dried $\left(\mathrm{Na}_{2} \mathrm{SO}_{4}\right)$. The reaction mixture was concentrated and was pipetted directly on to a silica gel column using $\mathrm{CH}_{2} \mathrm{Cl}_{2}(1 \mathrm{~mL})$ in three portions. Impurities were eluted with ether and the product was eluted with EtOAc/Hexane (15:85). Pure fractions were combined and concentrated to afford protected tyrosine III-53 (2.89 $\mathrm{g}, 6.52 \mathrm{mmol}, 87 \%)$ as viscous oil. $R_{f}(50 \%$ 
EtOAc/Hexane $)=0.80 ;[\alpha]_{\mathrm{D}}^{26}=-75\left(c 1, \mathrm{CH}_{2} \mathrm{Cl}_{2}\right) ; \mathrm{IR}\left(\right.$ thin film, $\left.\mathrm{cm}^{-1}\right)$ 3337, 2954, 2930, 1720, 1508, 1438, 1225, 1055, 837; ${ }^{1} \mathrm{H}$ NMR $\left(600 \mathrm{MHz}, \mathrm{CDCl}_{3}\right) \delta 7.34(\mathrm{~m}, 5 \mathrm{H})$, $6.95(\mathrm{~d}, J=7.8 \mathrm{~Hz}, 1 \mathrm{H}), 6.75(\mathrm{~d}, J=8.4 \mathrm{~Hz}, 2 \mathrm{H}), 5.21(\mathrm{br} \mathrm{s}, 1 \mathrm{H}), 5.12(\mathrm{~d}, J=12.0 \mathrm{~Hz}$, 1H), $5.09(\mathrm{~d}, J=12.0 \mathrm{~Hz}, 1 \mathrm{H}), 4.62(\mathrm{dd}, J=13.2,6.0 \mathrm{~Hz}, 1 \mathrm{H}), 3.69(\mathrm{~s}, 3 \mathrm{H}), 3.04(\mathrm{~m}$, 2H), $1.63(\mathrm{~m}, 1 \mathrm{H}), 0.98(\mathrm{~s}, 9 \mathrm{H}), 0.18(\mathrm{~s}, 6 \mathrm{H}) ;{ }^{13} \mathrm{C} \mathrm{NMR}\left(150 \mathrm{MHz}, \mathrm{CDCl}_{3}\right) \delta$ 172.2, 155.8, 155.0, 136.5, 130.4 (2C), 128.7 (2C), 128.4, 128.3 (2C), $128.2(2 \mathrm{C}), 120.3,67.1$, 55.1, 52.4, 37.7, 25.8 (3C), 18.3, -4.1, -4.2; CIHRMS Calcd for $\left[\mathrm{C}_{24} \mathrm{H}_{33} \mathrm{NO}_{5} \mathrm{H}^{+}\right]$: 444.2206. Found 444.2201.

(S)-methyl 2-amino-3-(4-tert-butyl-dimethylsilanyloxyphenyl)propanoate (III-69).

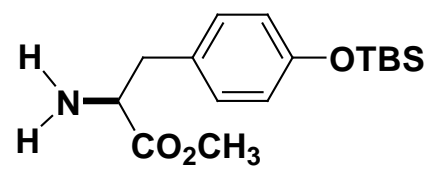

To a solution of Cbz-ester III-53 (3.3 g, $7.45 \mathrm{mmol})$ in dry MeOH $(15 \mathrm{~mL})$ was added $\mathrm{Pd} / \mathrm{C}(79 \mathrm{mg})$ and the mixture was stirred under $\mathrm{H}_{2}$ at a 100-psi pressure for $12 \mathrm{~h}$ at room temperature. The catalyst was filtered off through a short pad of Celite, concentrated under reduced pressure. The resulting crude product was pipetted directly on to a silica gel column using $\mathrm{CH}_{2} \mathrm{Cl}_{2}(1 \mathrm{~mL})$ in three portions. Impurities were eluted with ether and the product was eluted with EtOAc/hexanes (40:60). Pure fractions were combined and concentrated to afford amine III-69 $(2.50 \mathrm{~g}, 6.63 \mathrm{mmol}, 89 \%)$ as viscous oil. $R_{f}(50 \%$ EtOAc/Hexane $)=0.33 ;[\alpha]_{\mathrm{D}}^{26}=+8\left(c 1, \mathrm{CH}_{2} \mathrm{Cl}_{2}\right) ;$ IR (thin film, $\left.\mathrm{cm}^{-1}\right) 3365,2953$, $2930,1738,1509,1211,1217,1015,914,839 ;{ }^{1} \mathrm{H}$ NMR $\left(600 \mathrm{MHz}, \mathrm{CDCl}_{3}\right) \delta 7.95(\mathrm{~s}$, 1H), $7.01(\mathrm{~d}, J=8.4 \mathrm{~Hz}, 2 \mathrm{H}), 7.75(\mathrm{~d}, J=8.4 \mathrm{~Hz}, 2 \mathrm{H}), 3.68(\mathrm{~s}, 3 \mathrm{H}), 3.66(\mathrm{~m}, 2 \mathrm{H}), 2.97$ 
$(\mathrm{dd}, J=13.8,5.4 \mathrm{~Hz}, 1 \mathrm{H}), 2.78(\mathrm{dd}, J=13.2,7.8 \mathrm{~Hz}, 1 \mathrm{H}), 0.95(\mathrm{~s}, 9 \mathrm{H}), 0.16(\mathrm{~s}, 6 \mathrm{H}) ;{ }^{13} \mathrm{C}$ NMR $\left(150 \mathrm{MHz}, \mathrm{CDCl}_{3}\right) \delta$ 175.4, 154.5, 130.3, $130.1(2 \mathrm{C}), 120.1(2 \mathrm{C}), 115.5,55.8$, 51.8, 40.3, $25.6(3 \mathrm{C}), 18.1,-4.4$; CIHRMS Calcd for $\left[\mathrm{C}_{16} \mathrm{H}_{27} \mathrm{NO}_{3} \mathrm{H}^{+}\right]$: 310.1838. Found 310.1833.

1'- $N$-carbobenzyloxy-D-tyrosine methoxycarbonyl -5',5-(tert-butyldimethylsilanyloxymethyl)-di-1,4- $\alpha$-D-mannose $-N-4$ isovalaric amide (III-52a).

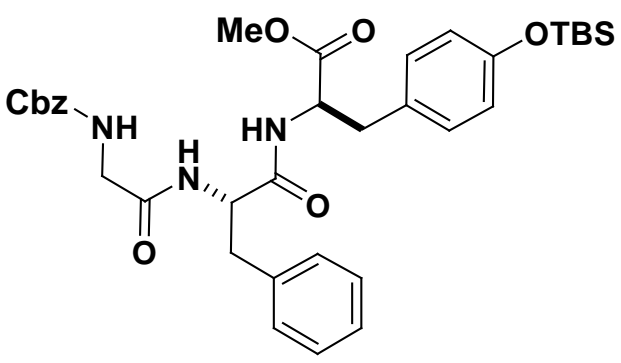

To a solution of amine III-69 $(83.5 \mathrm{mg}, 0.27 \mathrm{mmol})$, acid III-67 (100 mg, $0.27 \mathrm{mmol})$ in $\mathrm{CH}_{3} \mathrm{CN}(0.5 \mathrm{~mL})$ was added $\mathrm{HBTU}(112.5 \mathrm{mg}, 0.29 \mathrm{mmol})$ at $0{ }^{\circ} \mathrm{C}$, and after $5 \mathrm{~min}$, triethylamine $(76 \mu \mathrm{L}, 0.54 \mathrm{mmol})$ was added and stirring was continued for $5 \mathrm{~h}$ at $\mathrm{rt}$. The mixture was taken up in EtOAc $(10 \mathrm{~mL})$ and washed with 5\% aqueous KHSO4 solution, $5 \%$ aqueous $\mathrm{NaHCO} 3$ solution, and saturated aqueous $\mathrm{NaCl}$ solution. Then the reaction mixture was dried $\left(\mathrm{Na}_{2} \mathrm{SO}_{4}\right)$ and concentrated under reduced pressure. The crude product was purified using silica gel flash chromatography eluting with EtOAc/hexane (60:40) to give tripeptide III-52a $(140 \mathrm{mg}, 0.22 \mathrm{mmol}, 80 \%)$ as a colorless oil, $R_{f}=0.60(100 \%$ EtOAc); $[\alpha]_{\mathrm{D}}^{26}=+35\left(c=1, \mathrm{CH}_{2} \mathrm{Cl}_{2}\right) ; \mathrm{IR}$ (thin film, $\left.\mathrm{cm}^{-1}\right) 3275,2954,1739,1696$, 1508, 1241, 1218, 915, 838; ${ }^{1} \mathrm{H}$ NMR $\left(600 \mathrm{MHz}, \mathrm{CDCl}_{3}\right) \delta 7.33(\mathrm{~m}, 5 \mathrm{H}), 7.23(\mathrm{~m}, 4 \mathrm{H})$, $7.10(\mathrm{~d}, J=7.2 \mathrm{~Hz}, 2 \mathrm{H}), 6.76(\mathrm{~d}, J=7.2 \mathrm{~Hz}, 2 \mathrm{H}), 6.69(\mathrm{~m}, 3 \mathrm{H}), 6.50(\mathrm{br} \mathrm{s}, 1 \mathrm{H}), 5.45(\mathrm{br}$ s, 1H), $5.08($ br s, $2 \mathrm{H}), 4.70(\mathrm{~m}, 2 \mathrm{H}), 3.80(\mathrm{~d}, J=5.4 \mathrm{~Hz}, 1 \mathrm{H}), 3.79(\mathrm{~d}, J=5.4 \mathrm{~Hz}, 1 \mathrm{H})$, 
$3.59(\mathrm{~s}, 3 \mathrm{H}), 2.98(\mathrm{~m}, 1 \mathrm{H}), 2.88(\mathrm{dd}, J=13.8,6.6 \mathrm{~Hz}, 1 \mathrm{H}), 2.79(\mathrm{dd}, J=13.8,6.0 \mathrm{~Hz}$, 1H), $0.94(\mathrm{~s}, 9 \mathrm{H}), 0.14(\mathrm{~s}, 6 \mathrm{H}) ;{ }^{13} \mathrm{C}$ NMR $\left(150 \mathrm{MHz}, \mathrm{CDCl}_{3}\right) \delta \quad 171.6,170.1,168.8$, $156.5,154.78,154.73,136.2,136.0,130.1(2 \mathrm{C}), 129.3$ (2C), $128.6(2 \mathrm{C}), 128.5(2 \mathrm{C})$, $128.2(2 \mathrm{C}), 128.1(2 \mathrm{C}), 127.0,120.1,67.2,54.2,53.3,52.2,44.4,38.3,37.0,25.6(3 \mathrm{C})$, 18.1, -4.4 (2C); CIHRMS Calcd for $\left[\mathrm{C}_{35} \mathrm{H}_{45} \mathrm{~N}_{3} \mathrm{O}_{7} \mathrm{SiH}^{+}\right]$: 648.3105. Found 648.3102.

1'- $N$-carbobenzyloxy-D-tyrosine methoxycarbonyl -5',5-(hydroxy methyl)-di-1,4- D-mannose $-N-4$ isovaleric amide (III-70).

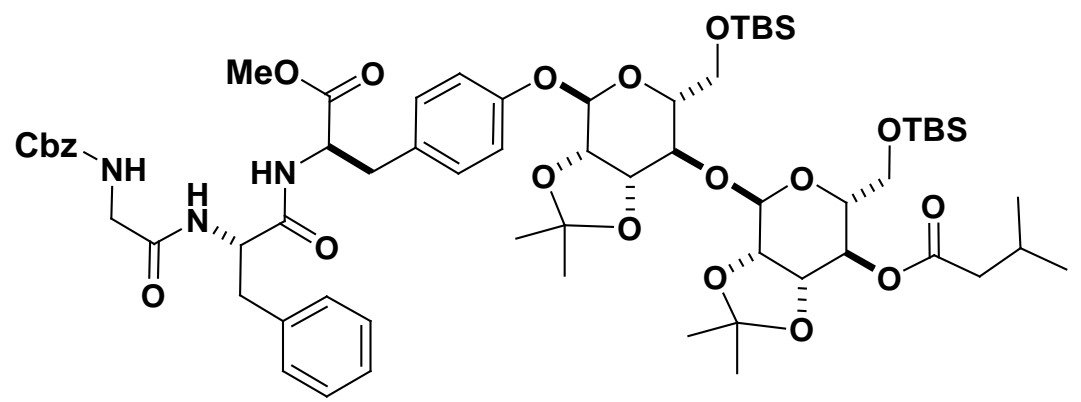

To a solution of tyrosine ester III-3b $(600 \mathrm{mg}, 0.62 \mathrm{mmol})$ in dry $\mathrm{MeOH}(3.0 \mathrm{~mL})$ was added $\mathrm{Pd} / \mathrm{C}(6.5 \mathrm{mg})$ and the mixture was stirred under $\mathrm{H}_{2}$ at a 100-psi pressure for $6 \mathrm{~h}$ at room temperature and checked against the completion of the reaction with TLC analysis. The catalyst was filtered off through a short pad of Celite, concentrated under reduced pressure as amine III-70, $R_{f}=0.35$ (70\% EtOAc). To a solution of crude amine III-70 (100 mg, $0.11 \mathrm{mmol})$, acid III-67 (58.6 mg, $0.17 \mathrm{mmol})$ in $\mathrm{CH}_{3} \mathrm{CN}(0.2 \mathrm{~mL})$ was added HBTU ( $45.7 \mathrm{mg}, 0.12 \mathrm{mmol})$ at $0{ }^{\circ} \mathrm{C}$, and after $5 \mathrm{~min}, i \operatorname{Pr}_{2} \mathrm{NEt}(31 \mu \mathrm{L}, 0.22 \mathrm{mmol})$ was added and stirring was continued for $4 \mathrm{~h}$ at $\mathrm{rt}$. The mixture was taken up in EtOAc (10 $\mathrm{mL}$ ) and washed with $5 \%$ aqueous $\mathrm{KHSO}_{4}$ solution, $5 \%$ aqueous $\mathrm{NaHCO}_{3}$ solution, and saturated aqueous $\mathrm{NaCl}$ solution. Then the reaction mixture was dried $\left(\mathrm{Na}_{2} \mathrm{SO}_{4}\right)$ and concentrated under reduced pressure. The crude product was purified using silica gel 
flash chromatography eluting with EtOAc/hexane (40:60) to give tripeptide III-52b (96 $\mathrm{mg}, 0.07 \mathrm{mmol}, 70 \%)$ as a colorless oil, $R_{f}=0.47(70 \%$ EtOAc $) ;[\alpha]_{\mathrm{D}}^{26}=+35(c=1$, $\mathrm{CH}_{2} \mathrm{Cl}_{2}$ ); IR (thin film, $\mathrm{cm}^{-1}$ ) 3296, 2930, 2857, 1741, 1649, 1510, 1372, 1225, 1140, 986, 835; ${ }^{1} \mathrm{H}$ NMR (600 MHz, $\left.\mathrm{CDCl}_{3}\right) \delta 7.35(\mathrm{~m}, 5 \mathrm{H}), 7.22(\mathrm{~m}, 5 \mathrm{H}), 7.13(\mathrm{~m}, 1 \mathrm{H}), 6.91$ (m, 1H), $6.63(\mathrm{~d}, J=7.2 \mathrm{~Hz}, 1 \mathrm{H}), 6.29(\mathrm{~m}, 1 \mathrm{H}), 5.75(\mathrm{~s}, 1 \mathrm{H}), 5.69(\mathrm{~s}, 1 \mathrm{H}), 5.48(\mathrm{br} \mathrm{s}$, 1H), $5.10(\mathrm{~m}, 2 \mathrm{H}), 4.97(\mathrm{dd}, J=10.2,7.2 \mathrm{~Hz}, 1 \mathrm{H}), 4.70(\mathrm{dd}, J=13.2,7.8 \mathrm{~Hz}, 1 \mathrm{H}), 4.60$ (d, $J=7.8 \mathrm{~Hz}, 1 \mathrm{H}), 4.40(\mathrm{dd}, J=6.6,6.0 \mathrm{~Hz}, 1 \mathrm{H}), 4.30(\mathrm{~d}, J=5.4 \mathrm{~Hz}, 1 \mathrm{H}), 4.13(\mathrm{~m}, 2 \mathrm{H})$, $4.01(\mathrm{dd}, J=10.2,7.2 \mathrm{~Hz}, 1 \mathrm{H}), 3.83(\mathrm{~d}, J=6.0 \mathrm{~Hz}, 1 \mathrm{H}), 3.76(\mathrm{~s}, 3 \mathrm{H}), 3.66$ (br s, $1 \mathrm{H})$, $3.65(\mathrm{~d}, J=1.2 \mathrm{~Hz}, 1 \mathrm{H}), 3.65(\mathrm{br} \mathrm{s}, 1 \mathrm{H}), 3.62(\mathrm{~m}, 3 \mathrm{H}), 3.05(\mathrm{dd}, J=13.8,5.4 \mathrm{~Hz}, 1 \mathrm{H})$, $2.98(\mathrm{~m}, 2 \mathrm{H}), 2.64(\mathrm{~m}, 1 \mathrm{H}), 2.25(\mathrm{dd}, J=15.0,6.6 \mathrm{~Hz}, 1 \mathrm{H}), 2.16(\mathrm{dd}, J=14.4,7.8 \mathrm{~Hz}$, 1H), $2.09(\mathrm{~m}, 1 \mathrm{H}), 1.16(\mathrm{~s}, 3 \mathrm{H}), 1.54(\mathrm{~s}, 3 \mathrm{H}), 1.35(\mathrm{~s}, 3 \mathrm{H}), 1.34(\mathrm{~s}, 3 \mathrm{H}), 1.24(\mathrm{~m}, 1 \mathrm{H})$, $0.95(\mathrm{~d}, J=1.8 \mathrm{~Hz}, 3 \mathrm{H}), 0.94(\mathrm{~d}, J=1.8 \mathrm{~Hz}, 3 \mathrm{H}), 0.86(\mathrm{~s}, 18 \mathrm{H}), 0.04(\mathrm{~s}, 6 \mathrm{H}) 0.03(\mathrm{~s}, 6 \mathrm{H})$; ${ }^{13} \mathrm{C}$ NMR $\left(150 \mathrm{MHz}, \mathrm{CDCl}_{3}\right) \delta 171.8,171.2,169.9,169.0,154.6,136.2,130.3$ (2C), 129.8, 129.1, 129.2 (2C), 128.69, 128.65 (2C), 128.5 (2C), 128.2 (2C), 128.1 (2C), 127.0, $116.8,110.0,109.7,95.6,95.4,78.7,76.0,75.87,75.80,71.1,70.0,69.4,69.3,67.2,62.8$, $61.4,54.1,53.3,52.3,44.4,43.3,37.6,37.0,37.1,29.6,27.8,27.5,26.4,26.3,25.9$ (3C), 25.7 (3C), 27.3, 18.5 (2C), -5.3 (4C); CIHRMS Calcd for $\left[\mathrm{C}_{64} \mathrm{H}_{95} \mathrm{~N}_{3} \mathrm{O}_{18} \mathrm{Si}_{2} \mathrm{Na}^{+}\right]$: 1272.6227. Found 1272.6400.

\section{Benzyl (R)-1-(methoxycarbonyl)-2-(4-tert-butyl-dimethylsilanyloxyl) ethylcarbamate (III-60).}




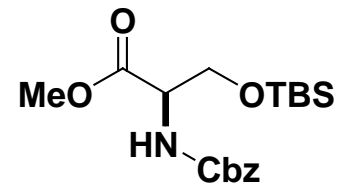

Alcohol III-59 (5.0 g, $19.76 \mathrm{mmol}), 40 \mathrm{~mL}$ of $\mathrm{CH}_{2} \mathrm{Cl}_{2}$, and $7.9 \mathrm{~mL}$ of $\mathrm{Et}_{3} \mathrm{~N}$ were added to a round bottom flask and cooled to $0{ }^{\circ} \mathrm{C}$. A catalytic amount $(241 \mathrm{mg}, 1.97$ mmol) of DMAP was added followed by addition of tert-butyldimethylsilyl chloride (3.57 g, $23.71 \mathrm{mmol}$ ) and the solution was stirred at $0{ }^{\circ} \mathrm{C}$ for $10 \mathrm{~h}$. The reaction was quenched with $1 \mathrm{M} \mathrm{NaHSO}_{4}$ and extracted $(3 \times 50 \mathrm{~mL})$ with $\mathrm{Et}_{2} \mathrm{O}$, washed with satd aq $\mathrm{NaHCO}_{3}(2 \times 50 \mathrm{~mL})$, and dried $\left(\mathrm{Na}_{2} \mathrm{SO}_{4}\right)$. The reaction mixture was concentrated and was pipetted directly on to a silica gel column using $\mathrm{CH}_{2} \mathrm{Cl}_{2}(1 \mathrm{~mL})$ in three portions. Impurities were eluted with ether and the product was eluted with EtOAc/Hexane (15:85). Pure fractions were combined and concentrated to afford protected tyrosine III$\mathbf{6 0}(6.1 \mathrm{~g}, 16.9 \mathrm{mmol}, 90 \%)$ as viscous oil. $R_{f}(40 \%$ EtOAc/Hexane $)=0.75 ;[\alpha]_{\mathrm{D}}^{26}=-$ $43\left(c 1, \mathrm{CH}_{2} \mathrm{Cl}_{2}\right.$ ); IR (thin film, $\left.\mathrm{cm}^{-1}\right) 3443,2953,2857,1721,1504,1203,1063,870$, 775; ${ }^{1} \mathrm{H}$ NMR (600 MHz, $\left.\mathrm{CDCl}_{3}\right) \delta 7.35(\mathrm{~m}, 5 \mathrm{H}), 5.57(\mathrm{~d}, J=8.4 \mathrm{~Hz}, 1 \mathrm{H}), 5.14(\mathrm{~d}, J=$ $12.0 \mathrm{~Hz}, 1 \mathrm{H}), 5.10(\mathrm{~d}, J=12.0 \mathrm{~Hz}, 1 \mathrm{H}), 4.41(\mathrm{~d}, J=8.4 \mathrm{~Hz}, 1 \mathrm{H}), 4.04(\mathrm{dd}, J=10.2,1.8$ Hz, 1H), $3.83(\mathrm{dd}, J=10.2,2.4 \mathrm{~Hz}, 1 \mathrm{H}), 3.73$ (s, 3H), 0.83 (s, 9H), 0.002 (s, 3H), -0.006 $(\mathrm{s}, 3 \mathrm{H}) ;{ }^{13} \mathrm{C}$ NMR $\left(150 \mathrm{MHz}, \mathrm{CDCl}_{3}\right) \delta 170.8,155.9,136.2,128.4(2 \mathrm{C}), 128.1(2 \mathrm{C})$, 128.0, 69.9, 63.6, 55.9, 52.3, 25.6 (3C), 18.1, -5.5, -5.7; CIHRMS Calcd for $\left[\mathrm{C}_{18} \mathrm{H}_{29} \mathrm{NO}_{5} \mathrm{SiH}^{+}\right]:$368.1893. Found 368.1884.

\section{Benzyl (R)-1-((R)-1,2-di-tert-butoxyethylcarbamoyl)-2-(4-tert-butyl-dimethyl silanyloxy) ethylcarbamate (III-61).}




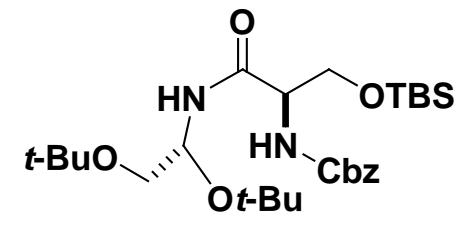

To a solution of amine III-56 (1.68 g, $7.71 \mathrm{mmol})$, acid III-55 (2.88 g, $8.84 \mathrm{mmol})$ in $\mathrm{CH}_{3} \mathrm{CN}(15 \mathrm{ml})$ was added $\mathrm{HBTU}(3.20 \mathrm{~g}, 8.84 \mathrm{mmol})$ at $0{ }^{\circ} \mathrm{C}$, and after $5 \mathrm{~min}$, triethylamine $(2.1 \mathrm{~mL}, 15.4 \mathrm{mmol})$ was added and stirring was continued for $12 \mathrm{~h}$ at $\mathrm{rt}$. The mixture was taken up in EtOAc $(50 \mathrm{~mL})$ and washed with $5 \%$ aqueous $\mathrm{KHSO}_{4}$ solution, 5\% aqueous $\mathrm{NaHCO}_{3}$ solution, and saturated aqueous $\mathrm{NaCl}$ solution. Then the reaction mixture was dried $\left(\mathrm{Na}_{2} \mathrm{SO}_{4}\right)$ and concentrated under reduced pressure. The crude product was purified using silica gel flash chromatography eluting with EtOAc/hexane (20:80) to give dipeptide III-61 $(3.45 \mathrm{~g}, 6.39 \mathrm{mmol}, 83 \%)$ as a colorless oil, $R_{f}=0.65$ $\left(50 \%\right.$ EtOAc/Hexane); $[\alpha]_{\mathrm{D}}^{26}=+6\left(c=4, \mathrm{CH}_{2} \mathrm{Cl}_{2}\right) ; \mathrm{IR}\left(\right.$ thin film, $\left.\mathrm{cm}^{-1}\right) 3348,2930$, $2857,1732,1670,1497,1249,1099,875,732 ;{ }^{1} \mathrm{H}$ NMR $\left(600 \mathrm{MHz}, \mathrm{CD}_{3} \mathrm{OD}\right) \delta 7.33(\mathrm{~m}$, $5 \mathrm{H}), 7.15(\mathrm{~d}, J=7.8 \mathrm{~Hz}, 1 \mathrm{H}), 5.69(\mathrm{br} \mathrm{s}, 1 \mathrm{H}), 5.10(\mathrm{br} \mathrm{s}, 2 \mathrm{H}), 4.58(\mathrm{ddd}, J=5.4,3.0,3.0$ Hz, 1H), $4.24($ br s, 1H), $4.04(\mathrm{~d}, J=6.6 \mathrm{~Hz}, 1 \mathrm{H}), 3.75(\mathrm{dd}, J=8.4,3.0 \mathrm{~Hz}, 1 \mathrm{H}), 3.69$ (dd, $J=10.2,6.0 \mathrm{~Hz}, 1 \mathrm{H}), 3.48(\mathrm{~m}, 1 \mathrm{H}), 1.43(\mathrm{~s}, 9 \mathrm{H}), 1.10(\mathrm{~s}, 9 \mathrm{H}), 0.87$ (s, 9H), $0.06(\mathrm{~s}$, $6 \mathrm{H}) ;{ }^{13} \mathrm{C} \mathrm{NMR}\left(150 \mathrm{MHz}, \mathrm{CDCl}_{3}\right) \delta$ 169.6, 169.4, 168.8, 136.0, $128.3(2 \mathrm{C}), 128.0,127.9$ (2C), 81.5, 72.8, 66.8, 63.1, 62.0, 56.1, 53.2, 27.8 (3C), 27.1 (3C), 25.7 (3C), 18.1, -5.5, 5.6; CIHRMS Calcd for $\left[\mathrm{C}_{28} \mathrm{H}_{48} \mathrm{~N}_{2} \mathrm{O}_{7} \mathrm{H}^{+}\right]$: 553.3309. Found 555.3305.

(2R)-N-((R)-1,2-di-tert-butoxyethyl)-2-amino-3-(4-tert-butyl-dimethylsilanyloxy) propanamide (III-62). 


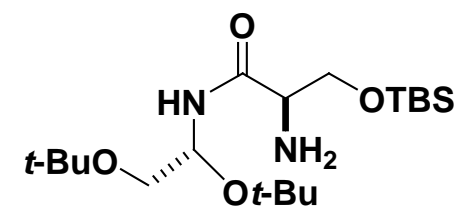

To a solution of Cbz-ester III-61 (1.89 g, $3.50 \mathrm{mmol})$ in dry MeOH $(7.0 \mathrm{~mL})$ was added $\mathrm{Pd} / \mathrm{C}(37 \mathrm{mg})$ and the mixture was stirred under $\mathrm{H}_{2}$ at a 100-psi pressure for $8 \mathrm{~h}$ at room temperature. The catalyst was filtered off through a short pad of Celite, concentrated under reduced pressure. The resulting crude product was pipetted directly on to a silica gel column using $\mathrm{CH}_{2} \mathrm{Cl}_{2}(1 \mathrm{~mL})$ in three portions. Impurities were eluted with ether and the product was eluted with EtOAc/hexanes (50:50). Pure fractions were combined and concentrated to afford amine III-62 $(1.27 \mathrm{~g}, 3.15 \mathrm{mmol}, 90 \%)$ as viscous oil. $R_{f}(50 \%$ EtOAc/Hexane $)=0.21 ;[\alpha]_{\mathrm{D}}^{26}=+7\left(c 1, \mathrm{CH}_{2} \mathrm{Cl}_{2}\right) ;$ IR (thin film, $\left.\mathrm{cm}^{-1}\right)$ 3392, 2971, 2930, 1729, 1672, 1509, 1365, 1230, 837; ${ }^{1} \mathrm{H}$ NMR $\left(600 \mathrm{MHz}, \mathrm{CDCl}_{3}\right) \delta 7.93(\mathrm{~d}, J=8.4$ Hz, 1H), 4.45 (ddd, $J=8.4,3.6,3.0 \mathrm{~Hz}, 1 \mathrm{H}), 3.86(\mathrm{dd}, J=9.6,4.8 \mathrm{~Hz}, 1 \mathrm{H}), 3.77$ (dd, $J=$ 9.0, $3.0 \mathrm{~Hz}, 1 \mathrm{H}), 3.71(\mathrm{dd}, J=9.6,7.2 \mathrm{~Hz}, 1 \mathrm{H}), 3.51(\mathrm{dd}, J=8.4,3.6 \mathrm{~Hz}, 1 \mathrm{H}), 3.46(\mathrm{dd}, J$ $=6.6,4.8 \mathrm{~Hz}, 1 \mathrm{H}), 1.65(\mathrm{br} \mathrm{s}, 2 \mathrm{H}), 1.44(\mathrm{~s}, 9 \mathrm{H}), 1.33(\mathrm{~s}, 9 \mathrm{H}), 0.87(\mathrm{~s}, 9 \mathrm{H}), 0.59(\mathrm{~s}, 3 \mathrm{H})$, 0.53 (s, 3H); ${ }^{13} \mathrm{C}$ NMR $\left(150 \mathrm{MHz}, \mathrm{CDCl}_{3}\right) \delta 172.5,169.3,81.5,72.9,65.3,62.3,56.9$, 52.9, 27.9 (3C), 27.3 (3C), 25.8 (3C), 18.2, -5.40, -5.44; CIHRMS Calcd for $\left[\mathrm{C}_{20} \mathrm{H}_{42} \mathrm{NO}_{5} \mathrm{SiH}^{+}\right]:$419.2941. Found 419.2936.

(2R)- $N$-((R)-1,2-di-tert-butoxyethyl)-2-amido-3-(4-tert-butyl-dimethylsilanyloxy)5amino-fluorenylmethoxycarbonyl-6-(benzyloxy)hexamide (III-63). 


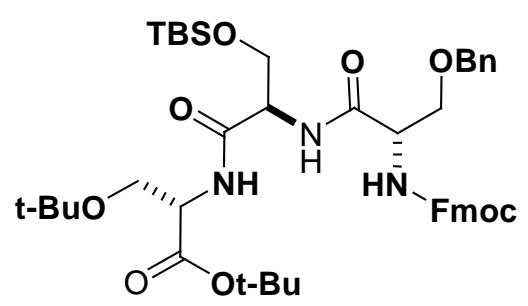

To a solution of dipeptide amine III-62 (860 mg, $2.13 \mathrm{mmol})$, acid III-54 (976 mg, 2.34 mmol) in $\mathrm{CH}_{3} \mathrm{CN}(4.5 \mathrm{~mL})$ was added $\mathrm{HBTU}(887 \mathrm{mg}, 2.34 \mathrm{mmol})$ at $0{ }^{\circ} \mathrm{C}$, and after 5 min, triethylamine $(0.6 \mathrm{~mL}, 15.3 \mathrm{mmol})$ was added and stirring was continued for $4 \mathrm{~h}$ at rt. The mixture was taken up in EtOAc $(50 \mathrm{~mL})$ and washed with $5 \%$ aqueous $\mathrm{KHSO}_{4}$ solution, 5\% aqueous $\mathrm{NaHCO}_{3}$ solution, and saturated aqueous $\mathrm{NaCl}$ solution. Then the reaction mixture was dried $\left(\mathrm{Na}_{2} \mathrm{SO}_{4}\right)$ and concentrated under reduced pressure. The crude product was purified using silica gel flash chromatography eluting with EtOAc/hexane (35:65) to give tripeptide III-63 $(1.48 \mathrm{~g}, 1.81 \mathrm{mmol}, 85 \%)$ as a colorless oil, $R_{f}=0.65$ $\left(50 \%\right.$ EtOAc/Hexane); $[\alpha]_{\mathrm{D}}^{26}=+8\left(c=3, \mathrm{CH}_{2} \mathrm{Cl}_{2}\right) ; \mathrm{IR}$ (thin film, $\left.\mathrm{cm}^{-1}\right) 3296,2954$, $2929,1731,1645,1520,1249,1152,1102,838,737 ;{ }^{1} \mathrm{H}$ NMR $\left(600 \mathrm{MHz}, \mathrm{CD}_{3} \mathrm{OD}\right) \delta$ $7.67(\mathrm{~m}, 2 \mathrm{H}), 7.58(\mathrm{~m}, 2 \mathrm{H}), 7.39(\mathrm{~m}, 4 \mathrm{H}), 7.33(\mathrm{~m}, 5 \mathrm{H}), 7.19(\mathrm{~d}, J=8.4 \mathrm{~Hz}, 1 \mathrm{H}), 5.71(\mathrm{br}$ s, 1H), $4.65(\mathrm{~d}, J=6.6 \mathrm{~Hz}, 1 \mathrm{H}), 4.61(\mathrm{~m}, 2 \mathrm{H}), 4.51(\mathrm{br} \mathrm{s}, 1 \mathrm{H}), 4.90(\mathrm{dd}, J=10.2,4.8 \mathrm{~Hz}$, $1 \mathrm{H}), 4.41(\mathrm{~d}, J=7.8 \mathrm{~Hz}, 1 \mathrm{H}), 4.39(\mathrm{~d}, J=7.8 \mathrm{~Hz}, 1 \mathrm{H}), 4.36(\mathrm{~d}, J=7.2 \mathrm{~Hz}, 1 \mathrm{H}), 4.21(\mathrm{dd}$, $J=7.2,6.6 \mathrm{~Hz}, 1 \mathrm{H}), 4.06(\mathrm{dd}, J=8.4,3.0 \mathrm{~Hz}, 1 \mathrm{H}), 3.90(\mathrm{br} \mathrm{s}, 1 \mathrm{H}), 3.77(\mathrm{dd}, J=9.0,3.0$ Hz, 1H), $3.62(\mathrm{~m}, 2 \mathrm{H}), 3.52$ (d, $J=8.4,3.6 \mathrm{~Hz}, 1 \mathrm{H}), 1.45$ (s, 9H), $1.12(\mathrm{~s}, 9 \mathrm{H}), 0.90$ (s, 9H), 0.11 (s, 3H), 0.10 (s, 3H); ${ }^{13} \mathrm{C}$ NMR (150 MHz, $\left.\mathrm{CDCl}_{3}\right) \delta$ 169.7, 169.3, 168.9, $143.8,143.6$ (2C), 141.25, 141.23 (2C), 137.3, 128.4 (2C), 127.9 (2C), 127.8 (2C), 128.6 (2C), 127.0, 125.1, 119.9 (2C), 81.7, 73.3, 70.0, 69.3, 67.3, 62.9, 62.1, 54.6, 53.4, 47.0, 
27.9 (3C), 27.2 (3C), 25.86, 25.81 (3C), 18.1, -5.4, -5.5; CIHRMS Calcd for $\left[\mathrm{C}_{45} \mathrm{H}_{63} \mathrm{~N}_{3} \mathrm{O}_{9} \mathrm{SiH}^{+}\right]:$818.4399. Found 818.4419.

(1'S,4'S,5' $R, 1 S, 4 S, 5 R)-1-[1 '-N$-carbobenzyloxy-D-tyrosine methoxycarbonyl -5'-(tertdimethylsilanyloxymethyl) -tetrahydro-pyran- $N$-4-isovaleric amide (IV-7).

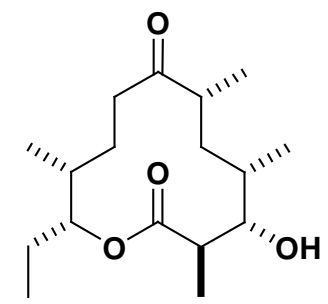

The enone compound IV-2 (100 mg, $0.34 \mathrm{mmol})$ and $o-\mathrm{NO}_{2} \mathrm{C}_{6} \mathrm{H}_{4} \mathrm{SO}_{2} \mathrm{NHNH}_{2}(414 \mathrm{mg}$, $2.04 \mathrm{mmol}$ ) were dissolved in $3.4 \mathrm{~mL}$ of $\mathrm{CH}_{2} \mathrm{Cl}_{2}$ in a round bottom flask and cooled $0{ }^{\circ} \mathrm{C}$ under nitrogen atmosphere then triethylamine $(393 \mu \mathrm{L}, 2.72 \mathrm{mmol})$ was added and the reaction mixture was stirred at $0{ }^{\circ} \mathrm{C}$ for 12 hours and on completion, as monitored by TLC. The reaction mixture was concentrated and was pipetted directly on to a silica gel column using $\mathrm{CH}_{2} \mathrm{Cl}_{2}(1 \mathrm{~mL})$ in three portions. The crude product was purified using silica gel flash chromatography eluting with EtOAc/hexanes (15:85) to give deoxy ketone IV-7 (96 mg, $0.32 \mathrm{mmol}, 95 \%)$ as viscous oil. $R_{f}(50 \%$ EtOAc/Hexane $)=0.70 ;[\alpha]_{\mathrm{D}}^{26}$ $=-19.4\left(c 1, \mathrm{CH}_{2} \mathrm{Cl}_{2}\right)$; IR (thin film, $\left.\mathrm{cm}^{-1}\right) 3481,2965,2934,1726,1703,1458,1170$, 992, 898; ${ }^{1} \mathrm{H}$ NMR $\left(600 \mathrm{MHz}, \mathrm{CDCl}_{3}\right) \delta 4.69(\mathrm{ddd}, J=7.8,5.4,2.4 \mathrm{~Hz}, 1 \mathrm{H}), 3.50(\mathrm{dd}, J$ $=10.2,5.4 \mathrm{~Hz}, 1 \mathrm{H}), 3.10(\mathrm{ddd}, J=18.6,12.6,1.2 \mathrm{~Hz}, 1 \mathrm{H}), 2.70(\mathrm{~m}, 1 \mathrm{H}), 2.54(\mathrm{~m}, 1 \mathrm{H})$, $2.12(\mathrm{ddd}, J=19.2,5.4,2.4 \mathrm{~Hz}, 1 \mathrm{H}), 1.87(\mathrm{~m}, 3 \mathrm{H}), 1.55(\mathrm{~d}, J=5.4 \mathrm{~Hz}, 1 \mathrm{H}), 1.53(\mathrm{~m}$, 2H), $1.44(\mathrm{~m}, 1 \mathrm{H}), 1.36(\mathrm{dd}, J=12.6,3.6 \mathrm{~Hz}, 1 \mathrm{H}), 1.26(\mathrm{~d}, J=6.6 \mathrm{~Hz}, 3 \mathrm{H}), 1.17(\mathrm{~d}, J=$ $7.2 \mathrm{~Hz}, 3 \mathrm{H}), 1.03(\mathrm{~d}, J=6.6 \mathrm{~Hz}, 3 \mathrm{H}), 0.94(\mathrm{~d}, J=7.2 \mathrm{~Hz}, 3 \mathrm{H}), 0.90(\mathrm{t}, J=7.2 \mathrm{~Hz}, 3 \mathrm{H})$; 
${ }^{13} \mathrm{C}$ NMR $\left(150 \mathrm{MHz}, \mathrm{CDCl}_{3}\right) \delta 214.2,174.5,80.9,79.0,45.9,43.8,33.97,33.94,33.5$, 32.2, 24.2, 20.6, 19.4, 17.9, 16.9, 15.3, 10.8; CIHRMS Calcd for $\left[\mathrm{C}_{17} \mathrm{H}_{30} \mathrm{O}_{4} \mathrm{Na}^{+}\right]$: 321.2041. Found 321.2037.

\section{(S)-1-(2-Furyl)-ethanol (IV-6a).}

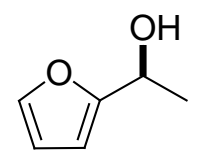

To a $25 \mathrm{ml}$ flask was added furan ketone II-24 (800 mg, $7.27 \mathrm{mmol}), \mathrm{CH}_{2} \mathrm{Cl}_{2}(3.7 \mathrm{ml})$, formic acid/triethylamine $(1: 1,9.71 \mathrm{ml})$ and Noyori asymmetric transfer hydrogenation catalyst $(R)-\mathrm{Ru}\left(\eta^{6}\right.$-mesitylene)-(S, $\left.S\right)$-TsDPEN $(22.2 \mathrm{mg}, 0.5 \mathrm{~mol} \%)$. The resulting solution was stirred at room temperature for $24 \mathrm{~h}$. The reaction mixture was diluted with water $(10 \mathrm{ml})$ and extracted with EtOAc $(3 \times 25 \mathrm{ml})$. The combined organic layers were washed with saturated $\mathrm{NaHCO}_{3}$, dried over $\mathrm{Na}_{2} \mathrm{SO}_{4}$, and concentrated under reduced pressure. The crude product was purified by silica gel flash chromatography eluting with 30\% EtOAc/hexane to give furan alcohol IV-6a (745 mg, $6.76 \mathrm{mmol}$, 93\%): colorless oil; $R_{f}(30 \%$ EtOAc/hexane $)=0.41 ;[\alpha]^{25}{ }_{\mathrm{D}}=+21\left(c=1.0, \mathrm{CH}_{2} \mathrm{Cl}_{2}\right) ; \mathrm{IR}$ (thin film, $\left.\mathrm{cm}^{-1}\right)$ $3360,2980,2935,1668,1505,1467,1370,1229,1149,1007,877,734 ;{ }^{1} \mathrm{H}$ NMR $(600$ $\left.\mathrm{MHz}_{\mathrm{CDCl}}\right) \delta 7.30(\mathrm{~d}, J=1.8,1 \mathrm{H}), 6.26(\mathrm{dd}, J=3.0,1.8 \mathrm{~Hz}, 1 \mathrm{H}), \quad 6.15(\mathrm{~d}, J=3.0$, $1 \mathrm{H}), 4.78(\mathrm{dq}, J=6.6,6.6 \mathrm{~Hz}, 1 \mathrm{H}), 3.11(\mathrm{~s}, 1 \mathrm{H}), 1.46(\mathrm{~d}, J=6.6 \mathrm{~Hz}, 3 \mathrm{H}) ;{ }^{13} \mathrm{C}$ NMR $(150$ $\left.\mathrm{MHz}, \mathrm{CDCl}_{3}\right) \delta$ 157.7, 141.6, 109.9, 104.9, 63.3, 21.1.

(2S)-6-Hydroxy-2- methyl-2H-Pyran-3 (6H)-one (II-26). 


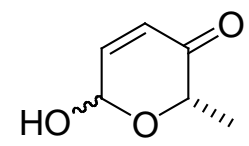

Compound furan alcohol IV-6a $(8.30 \mathrm{~g}, 74.1 \mathrm{mmol}), 184.5 \mathrm{~mL}$ of THF, and $61.5 \mathrm{~mL}$ of $\mathrm{H}_{2} \mathrm{O}$ were added to a round bottom flask and cooled to $0{ }^{\circ} \mathrm{C}$. Solid $\mathrm{NaHCO}_{3}(12.4 \mathrm{~g}$, $148.2 \mathrm{mmol}), \mathrm{NaOAc} \cdot 3 \mathrm{H}_{2} \mathrm{O}(10.1 \mathrm{~g}, 74.1 \mathrm{mmol})$, and NBS (13.1 g, $\left.74.1 \mathrm{mmol}\right)$ were added to the solution and the mixture was stirred for $1 \mathrm{~h}$ at $0{ }^{\circ} \mathrm{C}$. The reaction was quenched with saturated $\mathrm{NaHCO}_{3}(200 \mathrm{~mL})$, extracted $(3 \times 200 \mathrm{~mL})$ with $\mathrm{Et}_{2} \mathrm{O}$, dried $\left(\mathrm{Na}_{2} \mathrm{SO}_{4}\right)$, concentrated under reduced pressure and purified by silica gel chromatography eluting with 25\% EtOAc/hexane to give pyranone II-26 (8.56 g, $67.4 \mathrm{mmol}, 91 \%): R_{f}$ $(60 \%$ EtOAc/hexane $)=0.29 ;[\alpha]^{25}=+44\left(c=1.0, \mathrm{CH}_{2} \mathrm{Cl}_{2}\right) ; \mathrm{IR}\left(\right.$ thin film, $\left.\mathrm{cm}^{-1}\right) 3381$, 2988, 2942, 1692, 1447, 1373, 1232, 1021, 937; ${ }^{1} \mathrm{H}$ NMR (600 MHz, $\left.\mathrm{CDCl}_{3}\right)$ major isomer $\delta 6.82(\mathrm{dd}, J=10.2,3.0 \mathrm{~Hz}, 1 \mathrm{H}), 5.96(\mathrm{~d}, J=10.2,1 \mathrm{H}), 5.48(\mathrm{~d}, J=3.0 \mathrm{~Hz}, 1 \mathrm{H})$, $3.99(\mathrm{q}, J=7.2 \mathrm{~Hz}, 1 \mathrm{H}), 1.23(\mathrm{~d}, J=7.2 \mathrm{~Hz}, 3 \mathrm{H}) ;{ }^{13} \mathrm{C} \mathrm{NMR}\left(150 \mathrm{MHz}, \mathrm{CDCl}_{3}\right)$ major isomer $\delta 197.6,145.3,126.6,87.2,74.8,15.1$.

(2S, 6S)-tert-butyl -5,6-dihydro-6-methyl-5-oxo-2H-pyran-2-yl carbonate (IV-6 $\alpha$ ).

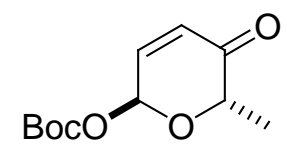

Pyronone alcohol II-26 (5.5 g, $42.9 \mathrm{mmol})$ was dissolved in $\mathrm{CH}_{2} \mathrm{Cl}_{2}(42 \mathrm{~mL})$ and the solution was cooled to $-78{ }^{\circ} \mathrm{C}$. $\quad \mathrm{A} \mathrm{CH}_{2} \mathrm{Cl}_{2}(2 \mathrm{~mL})$ solution of $(\mathrm{Boc})_{2} \mathrm{O}(13.46 \mathrm{~g}, 51.6$ mmol) and a catalytic amount of DMAP $(524.2 \mathrm{mg}, 4.76 \mu \mathrm{mol})$ was added to the reaction mixture. The reaction was stirred for $1 \mathrm{~h}$ at $-78{ }^{\circ} \mathrm{C}$, and quenched with $200 \mathrm{~mL}$ of saturated $\mathrm{NaHCO}_{3}$, extracted with $\mathrm{Et}_{2} \mathrm{O}(3 \times 200 \mathrm{~mL})$, dried $\left(\mathrm{Na}_{2} \mathrm{SO}_{4}\right)$, and concentrated 
under reduced pressure. The crude product was purified using silica gel flash chromatography eluting with $6 \% \mathrm{EtOAc} / \mathrm{hexane}$ to give $7.62 \mathrm{~g}(33.5 \mathrm{mmol}, 78 \%)$ of two diastereomers of Boc-protected pyranone IV-6 $\alpha$ and IV-6 $\beta$ in 3:1: $R_{f}\left(20 \% \mathrm{Et}_{2} \mathrm{O} / \mathrm{hexane}\right)$ $=0.58 ;[\alpha]_{D}^{25}=+98\left(c=1.0, \mathrm{CH}_{2} \mathrm{Cl}_{2}\right) ; \mathrm{IR}\left(\right.$ thin film, $\left.\mathrm{cm}^{-1}\right) 2984,2942,1752,1703$, 1371, 1273, 1254, 1153, 938, 838; ${ }^{1} \mathrm{H}$ NMR (600 MHz, $\left.\mathrm{CDCl}_{3}\right) \delta 6.78(\mathrm{dd}, J=10.2,3.6$ $\mathrm{Hz}, 1 \mathrm{H}), 6.22(\mathrm{~d}, J=3.6 \mathrm{~Hz}, 1 \mathrm{H}), 6.09(\mathrm{~d}, J=10.2 \mathrm{~Hz}, 1 \mathrm{H}), 4.53(\mathrm{q}, J=6.6 \mathrm{~Hz}, 1 \mathrm{H})$, $1.40(\mathrm{~s}, 9 \mathrm{H}), 1.28(\mathrm{~d}, J=6.6 \mathrm{~Hz}, 3 \mathrm{H}) ;{ }^{13} \mathrm{C} \mathrm{NMR}\left(150.8 \mathrm{MHz}, \mathrm{CDCl}_{3}\right) \delta 195.5,151.7$ 140.9, 128.2, 89.1, 83.3, 72.0, 27.5 (3C), 15.1; ClHRMS Calculated for $\left[\mathrm{C}_{11} \mathrm{H}_{16} \mathrm{O}_{5} \mathrm{Na}^{+}\right]$: 251.0890, Found: 251.0883.

(2S, 6S)-tert-butyl -5,6-dihydro-6-methyl-5-oxo-2H-pyran-2-yl carbonate (IV-6 $\beta$ ).

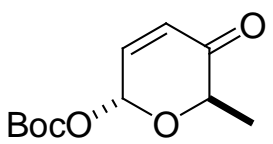

To a $1000 \mathrm{ml}$ flask was added furan ketone II-26 (40 g, $363.5 \mathrm{mmol}), \mathrm{CH}_{2} \mathrm{Cl}_{2}(240 \mathrm{ml})$, formic acid/triethylamine $(5: 3,480 \mathrm{ml})$ and Noyori asymmetric transfer hydrogenation catalyst $(R)-\mathrm{Ru}\left(\eta^{6}\right.$-mesitylene $)-(R, \quad R)$-TsDPEN (222 $\left.\mathrm{mg}, 0.1 \mathrm{~mol} \%\right)$. The resulting solution was stirred at room temperature for $24 \mathrm{~h}$. The reaction mixture was diluted with water $(500 \mathrm{ml})$ and extracted with EtOAc $(3 \times 700 \mathrm{ml})$. The combined organic layers were washed with saturated $\mathrm{NaHCO}_{3}$, dried over $\mathrm{Na}_{2} \mathrm{SO}_{4}$, and concentrated under reduced pressure. The resulted crude compound furan alcohol was dissolve in $603 \mathrm{~mL}$ of $\mathrm{THF} / \mathrm{H}_{2} \mathrm{O}$ (3:1) and cooled down to $0{ }^{\circ} \mathrm{C}$. Solid $\mathrm{NaHCO}_{3}(60.9 \mathrm{~g}, 727.8 \mathrm{mmol})$, $\mathrm{NaOAc} \cdot 3 \mathrm{H}_{2} \mathrm{O}(49.6 \mathrm{~g}, 363.9 \mathrm{mmol})$, and NBS $(64.3 \mathrm{~g}, 363.7 \mathrm{mmol})$ were added to the solution and the mixture was stirred for $1 \mathrm{~h}$ at $0{ }^{\circ} \mathrm{C}$. The reaction was quenched with 
saturated $\mathrm{NaHCO}_{3}(600 \mathrm{~mL})$, extracted $(3$ x $800 \mathrm{~mL})$ with $\mathrm{Et}_{2} \mathrm{O}$, dried $\left(\mathrm{Na}_{2} \mathrm{SO}_{4}\right)$, concentrated under reduced pressure. The crude mixture was dissolved in $\mathrm{CH}_{2} \mathrm{Cl}_{2}(500$ $\mathrm{mL})$ and the solution was cooled to $-78{ }^{\circ} \mathrm{C}$. (Boc) $)_{2} \mathrm{O}(93 \mathrm{~g}, 400 \mathrm{mmol})$ and a catalytic amount of DMAP ( $4.0 \mathrm{~g}$ ) was added to the reaction mixture. The reaction was stirred for $12 \mathrm{~h}$ at -78 to $-30{ }^{\circ} \mathrm{C}$, and quenched with saturated $\mathrm{NaHCO}_{3}$, extracted with $\mathrm{Et}_{2} \mathrm{O}$, dried $\left(\mathrm{Na}_{2} \mathrm{SO}_{4}\right)$, and concentrated under reduced pressure. The crude product was purified using silica gel flash chromatography eluting with $6 \%$ EtOAc/hexane to give $61.4 \mathrm{~g}$ (269.2 mmol, 80\%) of two diastereomers of Boc-protected pyranone II-26 $\boldsymbol{\beta}$ in $3: 1: R_{f}$ $\left(20 \% \mathrm{Et}_{2} \mathrm{O} /\right.$ hexane $)=0.58 ;[\alpha]_{\mathrm{D}}^{25}=-98\left(c=1.0, \mathrm{CH}_{2} \mathrm{Cl}_{2}\right) ; \mathrm{IR}\left(\right.$ thin film, $\left.\mathrm{cm}^{-1}\right) 2984$, 2942, 1752, 1703, 1371, 1273, 1254, 1153, 938, 838; ${ }^{1} \mathrm{H}$ NMR $\left(600 \mathrm{MHz}, \mathrm{CDCl}_{3}\right) \delta 6.78$ $(\mathrm{dd}, J=10.2,3.6 \mathrm{~Hz}, 1 \mathrm{H}), 6.22(\mathrm{~d}, J=3.6 \mathrm{~Hz}, 1 \mathrm{H}), 6.09(\mathrm{~d}, J=10.2 \mathrm{~Hz}, 1 \mathrm{H}), 4.53(\mathrm{q}, J$ $=6.6 \mathrm{~Hz}, 1 \mathrm{H}), 1.40(\mathrm{~s}, 9 \mathrm{H}), 1.28(\mathrm{~d}, J=6.6 \mathrm{~Hz}, 3 \mathrm{H}) ;{ }^{13} \mathrm{C} \mathrm{NMR}\left(150.8 \mathrm{MHz}, \mathrm{CDCl}_{3}\right) \delta$ 195.5, 151.7, 140.9, 128.2, 89.1, 83.3, 72.0, 27.5 (3C), 15.1; ClHRMS Calculated for $\left[\mathrm{C}_{11} \mathrm{H}_{16} \mathrm{O}_{5} \mathrm{Na}^{+}\right]: 251.0890$, Found: 251.0883 .

\section{6-(12-ethyl-3,5,7,11-tetramethyl-oxacyclododec-2,8-dione)-2-methyl-6H-pyran-3-one} (IV-8).

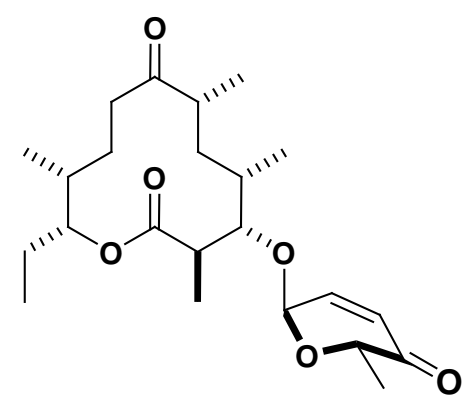

A $\mathrm{CH}_{2} \mathrm{Cl}_{2}(0.6 \mathrm{~mL})$ solution of Boc-enone IV-6 $(93 \mathrm{mg}, 0.40 \mathrm{mmol})$ and 10deoxymethynolide alcohol $5(60 \mathrm{mg}, 0.20 \mathrm{mmol})$ was cooled to $0{ }^{\circ} \mathrm{C} . \mathrm{A} \mathrm{CH}_{2} \mathrm{Cl}_{2}(0.2 \mathrm{~mL})$ 
solution of $\mathrm{Pd}_{2}(\mathrm{dba})_{3}{ }^{\cdot} \mathrm{CHCl}_{3}(21 \mathrm{mg}, 2.5 \mathrm{~mol} \%)$ and $\mathrm{PPh}_{3}(22 \mathrm{mg}, 10 \mathrm{~mol} \%)$ was added to the reaction mixture at $0{ }^{\circ} \mathrm{C}$. The reaction mixture was stirred at $0{ }^{\circ} \mathrm{C}$ for 5 hours. The reaction mixture was quenched with $5 \mathrm{~mL}$ of satd. aq. $\mathrm{NaHCO}_{3}$, extracted $(3 \times 5 \mathrm{~mL})$ with $\mathrm{Et}_{2} \mathrm{O}$, dried $\left(\mathrm{Na}_{2} \mathrm{SO}_{4}\right)$, and concentrated under reduced pressure. The crude product was purified using silica gel flash chromatography eluting with $20 \%$ EtOAc/hexanes to give enone IV-8 (75 mg, $0.18 \mathrm{mmol}, 90 \%)$ as viscous oil. $R_{f}(50 \%$ EtOAc/hexanes $)=$ $0.50 ;[\alpha]_{\mathrm{D}}^{26}=-65(c=0.3, \mathrm{MeOH}) ; \mathrm{IR}\left(\right.$ thin film, $\left.\mathrm{cm}^{-1}\right) 2965,2930,1724,1701,1459$ 1171, 1019, 974; ${ }^{1} \mathrm{H}$ NMR $\left(600 \mathrm{MHz}, \mathrm{CDCl}_{3}\right) \delta 6.85(\mathrm{dd}, J=10.8,3.0 \mathrm{~Hz}, 1 \mathrm{H}), 6.07(\mathrm{~d}$, $J=10.2 \mathrm{~Hz}, 1 \mathrm{H}), 5.24(\mathrm{~d}, J=3.6 \mathrm{~Hz}, 1 \mathrm{H}), 4.70(\mathrm{ddd}, J=7.8,5.4,2.4 \mathrm{~Hz}, 1 \mathrm{H}), 4.60$ (dd, $J=13.8,6.6 \mathrm{~Hz}, 1 \mathrm{H}), 3.65(\mathrm{dd}, J=10.2,1.2 \mathrm{~Hz}, 1 \mathrm{H}), 3.09$ (ddd, $J=19.6,11.4,2.4 \mathrm{~Hz}$, 1H), 2.87 (ddd, $J=16.8,7.2,6.6 \mathrm{~Hz}, 1 \mathrm{H}), 2.56(\mathrm{~m}, 1 \mathrm{H}), 2.13(\mathrm{ddd}, J=18.6,5.4,3.0 \mathrm{~Hz}$, $1 \mathrm{H}), 1.88(\mathrm{~d}, J=13.2 \mathrm{~Hz}, 1 \mathrm{H}), 1.83(\mathrm{~m}, 1 \mathrm{H}), 1.51(\mathrm{~m}, 3 \mathrm{H}), 1.47(\mathrm{~m}, 1 \mathrm{H}), 1.36(\mathrm{dd}, J=$ 7.2, $2.4 \mathrm{~Hz}, 1 \mathrm{H}), 1.42(\mathrm{~m}, 1 \mathrm{H}), 1.36(\mathrm{~d}, J=6.6 \mathrm{~Hz}, 3 \mathrm{H}), 1.24(\mathrm{~d}, J=7.2 \mathrm{~Hz}, 3 \mathrm{H}), 1.14(\mathrm{~d}$, $J=7.2 \mathrm{~Hz}, 3 \mathrm{H}), 1.09(\mathrm{~d}, J=6.6 \mathrm{~Hz}, 3 \mathrm{H}), 0.95(\mathrm{~d}, J=7.2 \mathrm{~Hz}, 3 \mathrm{H}), 0.89(\mathrm{t}, J=7.2 \mathrm{~Hz}$, $3 \mathrm{H}) ;{ }^{13} \mathrm{C} \mathrm{NMR}\left(150 \mathrm{MHz}, \mathrm{CDCl}_{3}\right) \delta 214.1,196.8,174.1,142.5,126.9,109.9,95.8,88.5$, $81.1,70.6,45.8,44.1,34.7,34.3,33.8,32.3,24.2,20.6,17.9,17.1,15.3,14.9,10.8$; CIHRMS Calcd for $\left[\mathrm{C}_{23} \mathrm{H}_{36} \mathrm{O}_{6} \mathrm{Na}^{+}\right]$: 431.2409. Found 431.2405.

\section{6-(12-ethyl-3,5,7,11-tetramethyl-oxacyclododec-2,8-dione)-2-methyl-3, 6-dihydro-}

\section{H-pyran-3-ol (IV-9).}




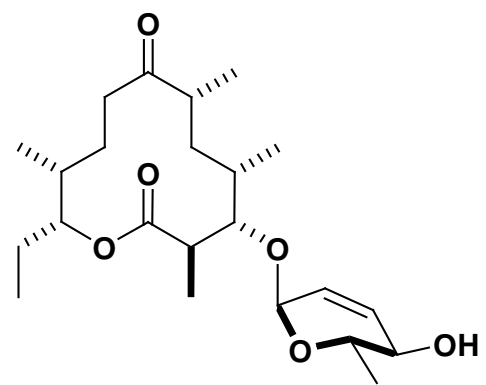

The enone compound IV-8 (60 mg, $0.15 \mathrm{mmol}$ ) was dissolved in $0.2 \mathrm{~mL}$ of $\mathrm{CH}_{2} \mathrm{Cl}_{2}$ and $0.2 \mathrm{~mL} \mathrm{MeOH}$ in round bottom flask and cooled $-78{ }^{\circ} \mathrm{C}$ then $\mathrm{NaBH}_{4}(6.1 \mathrm{mg}, 0.15$ mmol) was added and the reaction mixture was stirred at $-78{ }^{\circ} \mathrm{C}$ for 3 hours and on completion, monitored by TLC, reaction mixture was diluted with ether and was quenched with $2 \mathrm{~mL}$ of satd. aq. $\mathrm{NaHCO}_{3}$, extracted $(3 \times 5 \mathrm{~mL})$ with $\mathrm{Et}_{2} \mathrm{O}$, dried $\left(\mathrm{Na}_{2} \mathrm{SO}_{4}\right)$, and concentrated under reduced pressure. The crude product was purified using silica gel flash chromatography eluting with $25 \%$ EtOAc/hexanes to give allylic alcohol IV-9 (56 mg, $0.14 \mathrm{mmol}, 92 \%)$ as viscous oil. $R_{f}(50 \%$ EtOAc/hexanes $)=0.35$; $[\alpha]_{\mathrm{D}}^{26}=-14(c=0.25, \mathrm{MeOH}) ; \mathrm{IR}\left(\right.$ thin film, $\left.\mathrm{cm}^{-1}\right) 3444,2968,2934,1726,1704$, 1460, 1171, 1039, 899; ${ }^{1} \mathrm{H}$ NMR (600 MHz, $\left.\mathrm{CDCl}_{3}\right) \delta 5.94(\mathrm{~d}, J=10.2 \mathrm{~Hz}, 1 \mathrm{H}), 5.78(\mathrm{td}$, $J=10.2,2.4 \mathrm{~Hz}, 1 \mathrm{H}), 4.98($ br s, $1 \mathrm{H}), 4.68(\mathrm{ddd}, J=7.2,4.8,2.4 \mathrm{~Hz}, 1 \mathrm{H}), 3.81(\mathrm{~d}, J=8.4$ Hz, 1H), 3.67 (ddd, $J=12.6,9.0,6.0 \mathrm{~Hz}, 1 \mathrm{H}), 3.57$ (d, $J=10.2 \mathrm{~Hz}, 1 \mathrm{H}), 3.10$ (ddd, $J=$ 18.6, 12.6, $2.4 \mathrm{~Hz}, 1 \mathrm{H}), 2.83(\mathrm{~m}, 1 \mathrm{H}), 2.55(\mathrm{~m}, 1 \mathrm{H}), 2.14(\mathrm{ddd}, J=18.6,5.4,2.4 \mathrm{~Hz}, 1 \mathrm{H})$, $2.06(\mathrm{~m}, 1 \mathrm{H}), 1.88(\mathrm{~m}, 2 \mathrm{H}), 1.83(\mathrm{~m}, 2 \mathrm{H}), 1.66(\mathrm{br} \mathrm{s}, 1 \mathrm{H}), 1.51(\mathrm{td}, J=14.4,7.2 \mathrm{~Hz}, 1 \mathrm{H})$, $1.29(\mathrm{~d}, J=6.0 \mathrm{~Hz}, 3 \mathrm{H}), 1.24(\mathrm{br} \mathrm{s}, 2 \mathrm{H}), 1.21(\mathrm{~d}, J=7.2 \mathrm{~Hz}, 3 \mathrm{H}), 1.14(\mathrm{~d}, J=7.2 \mathrm{~Hz}$, $3 \mathrm{H}), 1.07(\mathrm{~d}, J=6.6 \mathrm{~Hz}, 3 \mathrm{H}), 0.95(\mathrm{~d}, J=6.6 \mathrm{~Hz}, 3 \mathrm{H}), 0.88(\mathrm{t}, J=7.2 \mathrm{~Hz}, 3 \mathrm{H}) ;{ }^{13} \mathrm{C} \mathrm{NMR}$ $\left(150 \mathrm{MHz}, \mathrm{CDCl}_{3}\right) \delta 214.4,174.4,133.4,126.0,96.4,86.8,80.9,69.2,68.0,45.9,44.3$, 
34.8, 34.0, 33.8, 32.2, 29.6, 24.2, 20.5, 17.9, 17.6, 17.1, 15.3, 10.8; CIHRMS Calcd for $\left[\mathrm{C}_{23} \mathrm{H}_{38} \mathrm{O}_{6} \mathrm{Na}^{+}\right]:$433.2566. Found 433.2560.

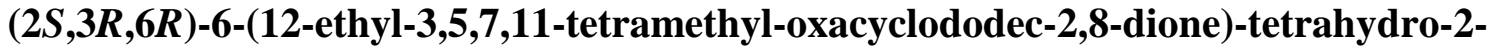
methyl-2H-pyran-3-ol (IV-10).

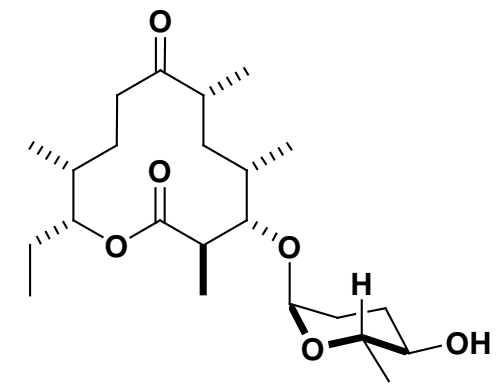

The enol compound IV-9 (40 mg, $0.10 \mathrm{mmol})$ and $o-\mathrm{NO}_{2} \mathrm{C}_{6} \mathrm{H}_{4} \mathrm{SO}_{2} \mathrm{NHNH}_{2}(120 \mathrm{mg}, 0.60$ mmol) were dissolved in $1.0 \mathrm{~mL}$ of $\mathrm{CH}_{2} \mathrm{Cl}_{2}$ in a round bottom flask and cooled $0{ }^{\circ} \mathrm{C}$ under nitrogen atmosphere then triethylamine $(114 \mu \mathrm{L}, 0.80 \mathrm{mmol})$ was added and the reaction mixture was stirred at $0{ }^{\circ} \mathrm{C}$ for 12 hours and on completion, as monitored by TLC. The reaction mixture was concentrated and was pipetted directly on to a silica gel column using $\mathrm{CH}_{2} \mathrm{Cl}_{2}(1 \mathrm{~mL})$ in three portions. The crude product was purified using silica gel flash chromatography eluting with EtOAc/hexanes (30:70) to give deoxy alcohol IV-10 (40 mg, $0.09 \mathrm{mmol}, 96 \%)$ as viscous oil. $R_{f}(50 \% \mathrm{EtOAc} / \mathrm{Hexane})=0.26$; $[\alpha]_{\mathrm{D}}^{26}=-34\left(c 0.25, \mathrm{CH}_{2} \mathrm{Cl}_{2}\right) ;$ IR (thin film, $\left.\mathrm{cm}^{-1}\right) 3449,2963,2928,1726,1703,1459$, 1170, 1019, 940, 898; ${ }^{1} \mathrm{H}$ NMR $\left(600 \mathrm{MHz}, \mathrm{CDCl}_{3}\right) \delta 4.74(\mathrm{~d}, J=2.4 \mathrm{~Hz}, 1 \mathrm{H}), 4.67$ (ddd, $J=8.4,5.4,1.8 \mathrm{~Hz}, 1 \mathrm{H}), 3.70(\mathrm{ddd}, J=12.6,9.0,6.0 \mathrm{~Hz}, 1 \mathrm{H}), 3.48(\mathrm{~d}, J=10.2 \mathrm{~Hz}, 1 \mathrm{H})$, 3.28 (ddd, $J=10.2,9.0,4.2 \mathrm{~Hz}, 1 \mathrm{H}), 3.10(\mathrm{ddd}, J=18.6,12.6,2.4 \mathrm{~Hz}, 1 \mathrm{H}), 2.79$ (ddd, $J$ $=13.2,10.2,7.2 \mathrm{~Hz}, 1 \mathrm{H}), 2.55(\mathrm{~m}, 1 \mathrm{H}), 2.13(\mathrm{~m}, 1 \mathrm{H}), 1.93(\mathrm{~m}, 1 \mathrm{H}), 1.91(\mathrm{~m}, 1 \mathrm{H}), 1.89$ 
(d, $J=2.4 \mathrm{~Hz}, 1 \mathrm{H}), 1.87(\mathrm{~d}, J=1.8 \mathrm{~Hz}, 1 \mathrm{H}), 1.84(\mathrm{~m}, 1 \mathrm{H}), 1.82(\mathrm{dd}, J=4.2,2.4 \mathrm{~Hz}, 1 \mathrm{H})$, $1.75(\mathrm{ddd}, J=10.2,7.2,3.6 \mathrm{~Hz}, 1 \mathrm{H}), 1.73(\mathrm{~m}, 1 \mathrm{H}), 1.69(\mathrm{dd}, J=10.2,3.6 \mathrm{~Hz}, 1 \mathrm{H}), 1.43$ (m, 3H), $1.37(\mathrm{~d}, J=4.8 \mathrm{~Hz}, 1 \mathrm{H}), 1.21(\mathrm{~d}, J=6.6 \mathrm{~Hz}, 3 \mathrm{H}), 1.17(\mathrm{~d}, J=7.2 \mathrm{~Hz}, 3 \mathrm{H}), 1.15$ (d, $J=7.2 \mathrm{~Hz}, 3 \mathrm{H}), 1.05(\mathrm{~d}, J=6.6 \mathrm{~Hz}, 3 \mathrm{H}), 0.91(\mathrm{~d}, J=7.2 \mathrm{~Hz}, 3 \mathrm{H}), 0.87(\mathrm{t}, J=7.2 \mathrm{~Hz}$, $3 \mathrm{H}) ;{ }^{13} \mathrm{C} \mathrm{NMR}\left(150 \mathrm{MHz}, \mathrm{CDCl}_{3}\right) \delta 214.3,174.5,99.2,80.8,72.0,70.2,45.9,44.3,34.7$, 34.3, 33.8, 32.2, 29.9, 29.6, 27.6, 24.2, 20.6, 19.2, 18.0, 17.5, 17.0, 15.5, 10.7; CIHRMS Calcd for $\left[\mathrm{C}_{23} \mathrm{H}_{40} \mathrm{O}_{6} \mathrm{Na}^{+}\right]$: 435.2722. Found 435.2717.

$(2 R, 5 R, 6 R)$-2-(12-ethyl-3,5,7,11-tetramethyl-oxacyclododec-2,8-dione)-tetrahydro-6methyl-2H-pyran-3,4,5-triol (IV-11).

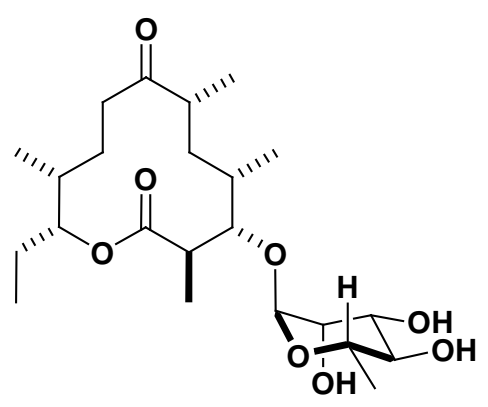

To a $\mathrm{CH}_{2} \mathrm{Cl}_{2}(0.2 \mathrm{~mL})$ solution of allylic alcohol $\mathbf{I V - 9}(10 \mathrm{mg}, 0.02 \mathrm{mmol})$ at $0{ }^{\circ} \mathrm{C}$ was added a solution of $(50 \% \mathrm{w} / \mathrm{v})$ of $N$-methyl morpholine $N$-oxide / water $(10 \mu \mathrm{L})$. Crystalline $\mathrm{OsO}_{4}(2.0 \mathrm{mg}, 10 \mathrm{~mol} \%)$ was added and the reaction was stirred for $12 \mathrm{~h}$. The reaction mixture was concentrated and was pipetted directly on to a silica gel column using $\mathrm{CH}_{2} \mathrm{Cl}_{2}(1 \mathrm{~mL})$ in three portions. Impurities were eluted with ether and the product was eluted with $\mathrm{MeOH} / \mathrm{EtOAc} /$ hexanes (10:40:50). Pure fractions were combined and concentrated to afford triol IV-11 $(9.7 \mathrm{mg}, 0.02 \mathrm{mmol}, 91 \%)$ as viscous oil. $R_{f}(90 \%$ EtOAc $/ \mathrm{MeOH})=0.30 ;[\alpha]_{\mathrm{D}}^{26}=-39(c=0.3, \mathrm{MeOH}) ; \mathrm{IR}\left(\right.$ thin film, $\left.\mathrm{cm}^{-1}\right) 3390,2965$, 
$2929,1723,1702,1458,1173,1062,899,735 ;{ }^{1} \mathrm{H}$ NMR $\left(600 \mathrm{MHz}, \mathrm{CDCl}_{3}\right) \delta 4.80(\mathrm{~d}, J$ $=1.2 \mathrm{~Hz}, 1 \mathrm{H}), 4.67(\mathrm{ddd}, J=8.4,4.8,1.8 \mathrm{~Hz}, 1 \mathrm{H}), 4.02(\mathrm{br} \mathrm{s}, 1 \mathrm{H}), 3.78(\mathrm{ddd}, J=12.6$, 9.6, 6.0 Hz, 1H), $3.71(\mathrm{~m}, 1 \mathrm{H}), 3.51(\mathrm{~d}, J=10.2 \mathrm{~Hz}, 1 \mathrm{H}), 3.47(\mathrm{dd}, J=10.2,9.0 \mathrm{~Hz}, 1 \mathrm{H})$, 3.09 (ddd, $J=12.0,9.2,2.4 \mathrm{~Hz}, 1 \mathrm{H}), 2.81(\mathrm{ddd}, J=13.8,10.2,6.6 \mathrm{~Hz}, 1 \mathrm{H}), 2.54(\mathrm{~m}$, $1 \mathrm{H}), 2.42(\mathrm{~m}, 1 \mathrm{H}), 2.23(\mathrm{~m}, 1 \mathrm{H}), 2.15(\mathrm{dd}, J=5.4,3.0 \mathrm{~Hz}, 1 \mathrm{H}), 1.85(\mathrm{~m}, 2 \mathrm{H}), 1.52(\mathrm{dd}, J$ $=12.6,7.2 \mathrm{~Hz}, 1 \mathrm{H}), 1.55(\mathrm{~m}, 3 \mathrm{H}), 1.44(\mathrm{~m}, 2 \mathrm{H}), 1.29(\mathrm{~d}, J=6.0 \mathrm{~Hz}, 3 \mathrm{H}), 1.25(\mathrm{~d}, J=1.2$ $\mathrm{Hz}, 1 \mathrm{H}), 1.22(\mathrm{~d}, J=6.6 \mathrm{~Hz}, 3 \mathrm{H}), 1.16(\mathrm{~d}, J=6.6 \mathrm{~Hz}, 3 \mathrm{H}), 1.06(\mathrm{~d}, J=6.6 \mathrm{~Hz}, 3 \mathrm{H}), 0.93$ $(\mathrm{d}, J=7.2 \mathrm{~Hz}, 3 \mathrm{H}), 0.89(\mathrm{t}, J=7.2 \mathrm{~Hz}, 3 \mathrm{H}) ;{ }^{13} \mathrm{C} \mathrm{NMR}\left(150 \mathrm{MHz}, \mathrm{CDCl}_{3}\right) \delta 214.1,174.2$, 102.6, 81.1, 73.4, 71.8, 71.2, 68.4, 45.8, 44.1, 34.6, 34.2, 33.8, 32.3, 29.6, 24.2, 20.6, 19.2, 18.0, 17.1, 16.9, 15.5, 10.7; CIHRMS Calcd for $\left[\mathrm{C}_{23} \mathrm{H}_{40} \mathrm{O}_{8} \mathrm{Na}^{+}\right]$: 467.2620. Found 467.2616.

$(2 R, 3 R, 6 R)$-6-(12-ethyl-3,5,7,11-tetramethyl-oxacyclododec-2,8-dione)-3,6-dihydro2-methyl-2H-pyran-3-yl methyl carbonate (IV-12).

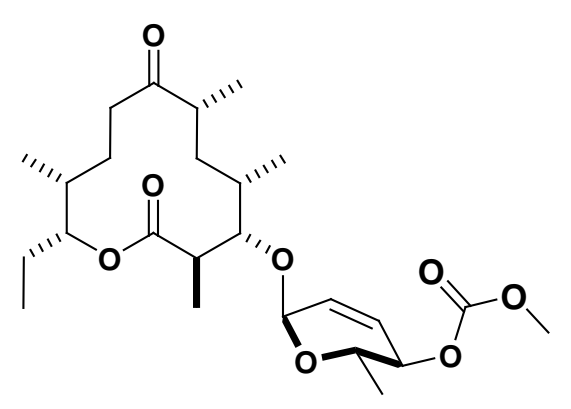

To a solution of allylic alcohol IV-9 $(60 \mathrm{mg}, 0.15 \mathrm{mmol})$ in dry $\mathrm{CH}_{2} \mathrm{Cl}_{2}(1.0 \mathrm{~mL})$ at $0{ }^{\circ} \mathrm{C}$, was added pyridine ( $59 \mu \mathrm{L}, 0.73 \mathrm{mmol}$ ), DMAP (3.6 mg), and methyl chloroformate (57 $\mathrm{mg}, 0.73 \mathrm{mmol})$. After stirring $24 \mathrm{~h}$ at room temperature, water $(1 \mathrm{~mL})$ was added and then the mixture was extracted with EtOAc $(3 \times 5 \mathrm{~mL})$, dried $\left(\mathrm{Na}_{2} \mathrm{SO}_{4}\right)$, concentrated under reduced pressure. The crude product was purified using silica gel flash 
chromatography eluting with EtOAc/hexane (20:80) to give carbonate IV-12 $(60.2 \mathrm{mg}$, $0.13 \mathrm{mmol}, 88 \%)$ as viscous oil. $R_{f}(50 \%$ EtOAc/hexane $)=0.50 ;[\alpha]_{\mathrm{D}}^{26}=-12(c=0.5$, $\mathrm{CH}_{2} \mathrm{Cl}_{2}$ ); IR (thin film, $\mathrm{cm}^{-1}$ ) 2962, 2924, 1752, 1701, 1459, 1261, 1045, 970; ${ }^{1} \mathrm{H}$ NMR $\left(600 \mathrm{MHz}, \mathrm{CDCl}_{3}\right) \delta 5.94(\mathrm{~d}, J=10.2 \mathrm{~Hz}, 1 \mathrm{H}), 5.85(\mathrm{ddd}, J=7.8,4.8,2.4 \mathrm{~Hz}, 1 \mathrm{H}), 5.01$ (br s, $1 \mathrm{H}$ ), 4.85 (dd, $J=9.0,1.8 \mathrm{~Hz}, 1 \mathrm{H}), 4.69$ (ddd, $J=8.4,5.4,1.8 \mathrm{~Hz}, 1 \mathrm{H}$ ), 3.95 (ddd, $J=12.0,9.6,6.0 \mathrm{~Hz}, 1 \mathrm{H}), 3.81(\mathrm{~s}, 3 \mathrm{H}), 3.65(\mathrm{dd}, J=10.2,1.2 \mathrm{~Hz}, 1 \mathrm{H}), 3.10(\mathrm{ddd}, J=$ 18.6, 12.6, $2.4 \mathrm{~Hz}, 1 \mathrm{H}), 2.84$ (ddd, $J=13.8,10.2,6.6 \mathrm{~Hz}, 1 \mathrm{H}), 2.54$ (ddd, $J=13.2,7.2$, $1.8 \mathrm{~Hz}, 1 \mathrm{H}), 2.12$ (ddd, $J=18.6,6.0,2.4 \mathrm{~Hz}, 1 \mathrm{H}), 1.89(\mathrm{dd}, J=6.0,1.2 \mathrm{~Hz}, 1 \mathrm{H}), 1.85$ (br s, $1 \mathrm{H}), 1.83(\mathrm{~m}, 2 \mathrm{H}), 1.52(\mathrm{dd}, J=7.2,6.0 \mathrm{~Hz}, 1 \mathrm{H}), 1.50(\mathrm{dd}, J=7.2,6.6 \mathrm{~Hz}, 1 \mathrm{H}), 1.43$ (m, 1H), $1.36(\mathrm{ddd}, J=16.8,9.6,4.2 \mathrm{~Hz}, 1 \mathrm{H}), 1.24(\mathrm{~d}, J=6.0 \mathrm{~Hz}, 3 \mathrm{H}), 1.21(\mathrm{~d}, J=7.2$ $\mathrm{Hz}, 3 \mathrm{H}), 1.15(\mathrm{~d}, J=7.2 \mathrm{~Hz}, 3 \mathrm{H}), 1.05(\mathrm{~d}, J=6.6 \mathrm{~Hz}, 3 \mathrm{H}), 0.92(\mathrm{~d}, J=7.2 \mathrm{~Hz}, 3 \mathrm{H}), 0.88$ $(\mathrm{t}, J=7.8 \mathrm{~Hz}, 3 \mathrm{H}) ;{ }^{13} \mathrm{C} \mathrm{NMR}\left(150 \mathrm{MHz}, \mathrm{CDCl}_{3}\right) \delta 214.5,174.5,155.5,129.3,127.8$, $96.5,87.2,81.1,74.5,64.9,55.2,46.1,44.4,34.9,34.2,34.0,32.4,24.4,20.8,19.5,18.1$, 17.8, 17.3, 15.4, 11.0; CIHRMS Calcd for $\left[\mathrm{C}_{25} \mathrm{H}_{40} \mathrm{O}_{8} \mathrm{Na}^{+}\right]$: 491.2620. Found 491.2615.

(2R,3R,6R)-3-azido-6-(12-ethyl-3,5,7,11-tetramethyl-oxacyclododec-2,8-dione)-3,6dihydro-2-methyl-2H-pyran (IV-13).

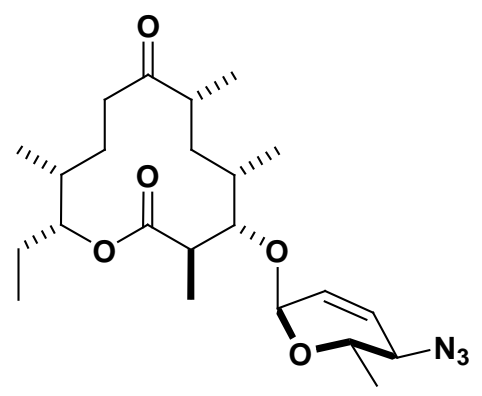


To a mixture of carbonate $\mathbf{~ I V - 1 2 ~ ( 5 5 ~ m g , ~} 0.12 \mathrm{mmol}$ ), allylpalladium chloride dimer (6.4 $\mathrm{mg}, 0.02 \mathrm{mmol}$ ) and 1,4-bis(diphenylphosphino)butane (30 $\mathrm{mg}, 0.08 \mathrm{mmol})$ in anhydrous THF $(0.20 \mathrm{~mL})$ was added $\mathrm{TMSN}_{3}(77 \mu \mathrm{g}, 0.59 \mathrm{mmol})$ under argon atmosphere. The solution was stirred at room temperature for $3 \mathrm{~h}$. Then the mixture was evaporated under reduced pressure, purified using silica gel flash chromatography eluting with EtOAc/hexane (15:85) to give allylic azide IV-13 (43 $\mathrm{mg}, 0.10 \mathrm{mmol}, 85 \%)$ as viscous oil. $R_{f}(30 \%$ EtOAc/hexane $)=0.60 ;[\alpha]_{\mathrm{D}}^{26}=-45\left(c=1, \mathrm{CH}_{2} \mathrm{Cl}_{2}\right)$; IR (thin film, $\left.\mathrm{cm}^{-1}\right)$ 2968, 2934, 2103, 1725, 1703, 1459, 1170, 1041, 984; ${ }^{1} \mathrm{H}$ NMR (600 MHz, $\left.\mathrm{CDCl}_{3}\right) \delta$ $5.96(\mathrm{~d}, J=10.8 \mathrm{~Hz}, 1 \mathrm{H}), 5.93(\mathrm{ddd}, J=9.6,2.4,2.4 \mathrm{~Hz}, 1 \mathrm{H}), 5.00(\mathrm{br} \mathrm{s}, 1 \mathrm{H}), 4.69$ (ddd, $J=8.4,6.0,2.4 \mathrm{~Hz}, 1 \mathrm{H}), 3.74(\mathrm{ddd}, J=12.6,9.0,6.6 \mathrm{~Hz}, 1 \mathrm{H}), 3.51(\mathrm{~d}, J=10.2 \mathrm{~Hz}, 1 \mathrm{H})$, $3.55(\mathrm{~d}, J=9.6 \mathrm{~Hz}, 1 \mathrm{H}), 3.10(\mathrm{ddd}, J=18.6,12.6,2.4 \mathrm{~Hz}, 1 \mathrm{H}), 2.84(\mathrm{ddd}, J=13.2,10.2$, $7.2 \mathrm{~Hz}, 1 \mathrm{H}), 2.55(\mathrm{~m}, 1 \mathrm{H}), 2.13(\mathrm{ddd}, J=18.6,6.0,1.8 \mathrm{~Hz}, 1 \mathrm{H}), 1.88(\mathrm{~m}, 2 \mathrm{H}), 1.83(\mathrm{~m}$, 1H), $1.51(\mathrm{~m}, 2 \mathrm{H}), 1.43(\mathrm{~m}, 1 \mathrm{H}), 1.35(\mathrm{~m}, 2 \mathrm{H}), 1.30(\mathrm{~d}, J=6.6 \mathrm{~Hz}, 3 \mathrm{H}), 1.21(\mathrm{~d}, J=7.2$ $\mathrm{Hz}, 3 \mathrm{H}), 1.15(\mathrm{~d}, J=6.6 \mathrm{~Hz}, 3 \mathrm{H}), 1.05(\mathrm{~d}, J=6.6 \mathrm{~Hz}, 3 \mathrm{H}), 0.93(\mathrm{~d}, J=7.2 \mathrm{~Hz}, 3 \mathrm{H}), 0.88$ (t, $J=7.2 \mathrm{~Hz}, 3 \mathrm{H}) ;{ }^{13} \mathrm{C} \mathrm{NMR}\left(150 \mathrm{MHz}, \mathrm{CDCl}_{3}\right) \delta 214.5,174.5,128.6,128.2,94.4,87.3$, 81.1, 66.3, 60.2, 46.1, 44.4, 34.9, 34.2, 34.0, 32.4, 24.4, 20.8, 19.5, 18.3, 18.1, 17.4, 15.5, 11.0; CIHRMS Calcd for $\left[\mathrm{C}_{23} \mathrm{H}_{37} \mathrm{~N}_{3} \mathrm{O}_{5} \mathrm{Na}^{+}\right]$: 458.2630. Found 458.2625.

(2R,3R,6R)-3-azido-6-(12-ethyl-3,5,7,11-tetramethyl-oxacyclododec-2,8-dione)tetrahydro-2-methyl-2H-pyran (IV-14). 


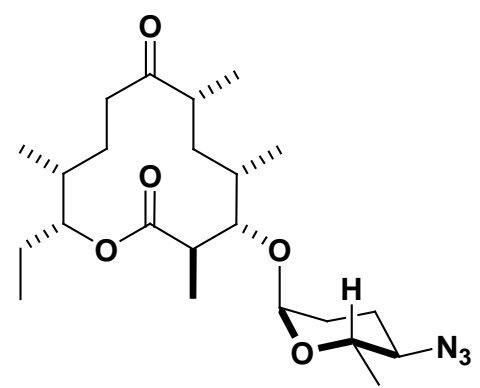

The allylic azide compound IV-13 $(20 \mathrm{mg}, 0.04 \mathrm{mmol})$ and $o-\mathrm{NO}_{2} \mathrm{C}_{6} \mathrm{H}_{4} \mathrm{SO}_{2} \mathrm{NHNH}_{2}(56$ $\mathrm{mg}, 0.27 \mathrm{mmol}$ ) were dissolved in $0.4 \mathrm{~mL}$ of $\mathrm{CH}_{2} \mathrm{Cl}_{2}$ in a round bottom flask and cooled $0{ }^{\circ} \mathrm{C}$ under nitrogen atmosphere then triethylamine $(60 \mu \mathrm{L}, 0.36 \mathrm{mmol})$ was added and the reaction mixture was stirred at $0{ }^{\circ} \mathrm{C}$ for 12 hours and on completion, as monitored by TLC. The reaction mixture was concentrated and was pipetted directly on to a silica gel column using $\mathrm{CH}_{2} \mathrm{Cl}_{2}(1 \mathrm{~mL})$ in three portions. The crude product was purified using silica gel flash chromatography eluting with EtOAc/hexanes (15:85) to give deoxy azide IV-14 (18 mg, $0.04 \mathrm{mmol}, 90 \%)$ as viscous oil. $R_{f}(50 \%$ EtOAc/Hexane $)=0.65 ;[\alpha]_{\mathrm{D}}^{26}$ $=-13\left(c=0.5, \mathrm{CH}_{2} \mathrm{Cl}_{2}\right)$; IR (thin film, $\left.\mathrm{cm}^{-1}\right)$ 2965, 2930, 1724, 1701, 1459, 1171, 1019, 974; ${ }^{1} \mathrm{H}$ NMR $\left(600 \mathrm{MHz}, \mathrm{CDCl}_{3}\right) \delta 4.78(\mathrm{~d}, J=3.0 \mathrm{~Hz}, 1 \mathrm{H}), 4.68(\mathrm{ddd}, J=8.4$, 4.2, $2.4 \mathrm{~Hz}, 1 \mathrm{H}), 3.73(\mathrm{ddd}, J=12.6,9.6,6.6 \mathrm{~Hz}, 1 \mathrm{H}), 3.48(\mathrm{~d}, J=10.8 \mathrm{~Hz}, 1 \mathrm{H}), 3.10(\mathrm{~m}$, 1H), $3.04(\mathrm{~m}, 1 \mathrm{H}), 2.80(\mathrm{ddd}, J=13.2,10.2,7.2 \mathrm{~Hz}, 1 \mathrm{H}), 2.56(\mathrm{ddd}, J=11.4,7.8,4.2$ Hz, 1H), $2.14(\mathrm{~m}, 1 \mathrm{H}), 1.97(\mathrm{~m}, 1 \mathrm{H}), 1.95(\mathrm{dd}, J=4.2,1.8 \mathrm{~Hz}, 1 \mathrm{H}), 1.88(\mathrm{br} \mathrm{s}, 1 \mathrm{H}), 1.86$ (m, 2H), $1.84(\mathrm{~m}, 1 \mathrm{H}), 1.80(\mathrm{~d}, J=2.4 \mathrm{~Hz}, 1 \mathrm{H}), 1.78(\mathrm{dd}, J=9.0,2.4 \mathrm{~Hz}, 1 \mathrm{H}), 1.75(\mathrm{~d}, J$ $=3.6 \mathrm{~Hz}, 1 \mathrm{H}), 1.51(\mathrm{~d}, J=7.2 \mathrm{~Hz}, 3 \mathrm{H}), 1.44(\mathrm{~m}, 1 \mathrm{H}), 1.35(\mathrm{~m}, 1 \mathrm{H}), 1.21(\mathrm{~d}, J=6.6 \mathrm{~Hz}$, $3 \mathrm{H}), 1.17(\mathrm{t}, J=6.0 \mathrm{~Hz}, 3 \mathrm{H}), 1.03(\mathrm{~d}, J=6.6 \mathrm{~Hz}, 3 \mathrm{H}), 0.93(\mathrm{~d}, J=7.2 \mathrm{~Hz}, 3 \mathrm{H}), 0.88(\mathrm{t}, J$ $=7.2 \mathrm{~Hz}, 3 \mathrm{H}) ;{ }^{13} \mathrm{C}$ NMR $\left(150 \mathrm{MHz}, \mathrm{CDCl}_{3}\right) \delta 214.3,174.4,99.1,88.3,80.9,68.2,62.6$, 
$45.9,44.3,34.7,34.5,33.8,32.2,29.5,24.5,23.9,20.6,19.2,18.3,18.0,17.0,15.5,10.7$; CIHRMS Calcd for $\left[\mathrm{C}_{23} \mathrm{H}_{39} \mathrm{~N}_{3} \mathrm{O}_{5} \mathrm{Na}^{+}\right]$: 460.2787. Found 460.2783.

\section{(2R,5R,6R)-5-azido-2-(12-ethyl-3,5,7,11-tetramethyl-oxacyclododec-2,8-dione)-} tetrahydro-6-methyl-2H-pyran-3,4-diol (IV-15).

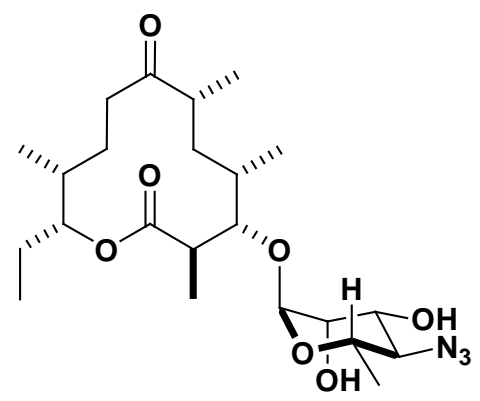

To a $\mathrm{CH}_{2} \mathrm{Cl}_{2}(0.3 \mathrm{~mL})$ solution of allylic azide $\mathbf{~ I V - 1 3 ~}(15 \mathrm{mg}, 0.3 \mathrm{mmol})$ at $0{ }^{\circ} \mathrm{C}$ was added a solution of $(50 \% \mathrm{w} / \mathrm{v})$ of $N$-methyl morpholine $N$-oxide / water $(17 \mu \mathrm{L})$. Crystalline $\mathrm{OsO}_{4}(1.0 \mathrm{mg}, 10 \mathrm{~mol} \%)$ was added and the reaction was stirred for $12 \mathrm{~h}$. The reaction mixture was concentrated and was pipetted directly on to a silica gel column using $\mathrm{CH}_{2} \mathrm{Cl}_{2}(1 \mathrm{~mL})$ in three portions. Impurities were eluted with ether and the product was eluted with $\mathrm{MeOH} / \mathrm{EtOAc/hexanes} \mathrm{(2:48:50).} \mathrm{Pure} \mathrm{fractions} \mathrm{were} \mathrm{combined} \mathrm{and}$ concentrated to afford diol IV-15 (15 mg, $0.03 \mathrm{mmol}, 90 \%)$ as viscous oil. $R_{f}(50 \%$ EtOAc/hexanes $)=0.20 ;[\alpha]_{\mathrm{D}}^{26}=-62(c=0.5, \mathrm{MeOH}) ; \mathrm{IR}\left(\right.$ thin film, $\left.\mathrm{cm}^{-1}\right) 3411,2968$, $2933,2108,1725,1703,1459,1174,1051,997,900 ;{ }^{1} \mathrm{H}$ NMR $\left(600 \mathrm{MHz}, \mathrm{CDCl}_{3}\right) \delta 4.81$ $(\mathrm{d}, J=1.2 \mathrm{~Hz}, 1 \mathrm{H}), 4.67(\mathrm{ddd}, J=7.8,5.4,2.4 \mathrm{~Hz}, 1 \mathrm{H}), 4.11(\mathrm{dd}, J=14.4,7.2 \mathrm{~Hz}, 1 \mathrm{H})$, $4.00(\mathrm{ddd}, J=4.8,3.0,1.8 \mathrm{~Hz}, 1 \mathrm{H}), 3.78(\mathrm{ddd}, J=10.2,7.2,3.0 \mathrm{~Hz}, 1 \mathrm{H}), 3.69(\mathrm{~m}, 1 \mathrm{H})$, $3.51(\mathrm{~d}, J=10.2 \mathrm{~Hz}, 1 \mathrm{H}), 3.31(\mathrm{~d}, J=10.2 \mathrm{~Hz}, 1 \mathrm{H}), 3.07(\mathrm{ddd}, J=17.4,6.6,2.4 \mathrm{~Hz}, 1 \mathrm{H})$, $2.80(\mathrm{ddd}, J=13.2,10.2,6.6 \mathrm{~Hz}, 1 \mathrm{H}), 2.55(\mathrm{~m}, 2 \mathrm{H}), 2.35(\mathrm{br} \mathrm{s}, 1 \mathrm{H}), 1.16(\mathrm{br} \mathrm{s}, 1 \mathrm{H}), 2.13$ 
$(\mathrm{m}, 1 \mathrm{H}), 1.84(\mathrm{~m}, 3 \mathrm{H}), 1.60(\mathrm{~d}, J=3.0 \mathrm{~Hz}, 1 \mathrm{H}), 1.51(\mathrm{dd}, J=14.4,7.8 \mathrm{~Hz}, 1 \mathrm{H}), 1.44(\mathrm{~m}$, 1H), $1.32(\mathrm{~d}, J=6.0 \mathrm{~Hz}, 3 \mathrm{H}), 1.20(\mathrm{~d}, J=6.6 \mathrm{~Hz}, 3 \mathrm{H}), 1.15(\mathrm{~d}, J=6.0 \mathrm{~Hz}, 3 \mathrm{H}), 1.03(\mathrm{~d}$, $J=6.6 \mathrm{~Hz}, 3 \mathrm{H}), 0.93(\mathrm{~d}, J=7.2 \mathrm{~Hz}, 3 \mathrm{H}), 0.88(\mathrm{t}, J=7.2 \mathrm{~Hz}, 3 \mathrm{H}) ;{ }^{13} \mathrm{C} \mathrm{NMR}(150 \mathrm{MHz}$, $\left.\mathrm{CDCl}_{3}\right) \delta 214.1,174.1,102.4,89.6,81.1,70.5,67.4,65.8,60.3,45.8,44.1,34.6,34.2$, 33.7, 32.3, 24.2, 20.5, 19.2, 18.0, 17.0, 15.6, 14.1, 10.7; CIHRMS Calcd for $\left[\mathrm{C}_{23} \mathrm{H}_{39} \mathrm{~N}_{3} \mathrm{O}_{7} \mathrm{Na}^{+}\right]$: 492.2685. Found 492.2684.

(2S,3R,6R)-6-(12-ethyl-3,5,7,11-tetramethyl-oxacyclododec-2,8-dione)-tetrahydro-2methyl-2H-pyran-3-amine (IV-17).

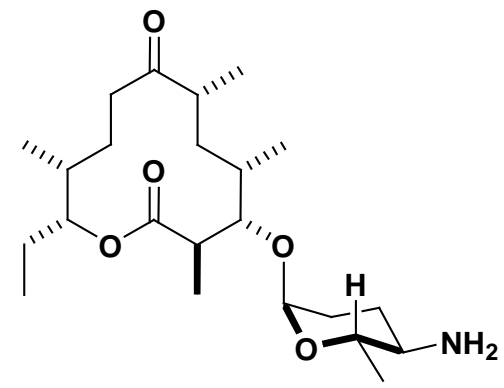

To a solution of allylic azide IV-13 $(15 \mathrm{mg}, 0.04 \mathrm{mmol})$ in dry $\mathrm{MeOH}(0.3 \mathrm{~mL})$ was added $\mathrm{Pd} / \mathrm{C}(10 \mathrm{mg})$ and the mixture was stirred under $\mathrm{H}_{2}$ at an $100 \mathrm{psi}$ pressure for $8 \mathrm{~h}$ at room temperature. The catalyst was filtered off through a short pad of Celite, concentrated under reduced pressure. The resulting crude product was pipetted directly on to a silica gel column using $\mathrm{CH}_{2} \mathrm{Cl}_{2}(1 \mathrm{~mL})$ in three portions. Impurities were eluted with ether and the product was eluted with $\mathrm{MeOH} / \mathrm{EtOAc} /$ hexanes (200:40:40). Pure fractions were combined and concentrated to afford deoxy amine IV-17 (13.2 $\mathrm{mg}, 0.03$ mmol, $92 \%)$ as viscous oil. $R_{f}(90 \% \mathrm{EtOAc} / \mathrm{MeOH})=0.30 ;[\alpha]_{\mathrm{D}}^{26}=-38(c=0.5$, MeOH); IR (thin film, $\mathrm{cm}^{-1}$ ) 3345, 2964, 2929, 1728, 1705, 1457, 1167, 1016, 983, 897; 
${ }^{1} \mathrm{H}$ NMR $\left(600 \mathrm{MHz}, \mathrm{CDCl}_{3}\right) \delta 4.71(\mathrm{~d}, J=3.0 \mathrm{~Hz}, 1 \mathrm{H}), 4.61(\mathrm{ddd}, J=9.0,4.2,1.8 \mathrm{~Hz}$, 1H), $3.92(\mathrm{ddd}, J=12.6,9.6,6.6 \mathrm{~Hz}, 1 \mathrm{H}), 3.40(\mathrm{~d}, J=10.2 \mathrm{~Hz}, 1 \mathrm{H}), 3.12(\mathrm{~m}, 1 \mathrm{H}), 2.79$ (ddd, $J=13.2,10.2,7.2 \mathrm{~Hz}, 1 \mathrm{H}), 2.48(\mathrm{~m}, 1 \mathrm{H}), 1.95(\mathrm{~d}, J=10.2 \mathrm{~Hz}, 1 \mathrm{H}), 1.91(\mathrm{~d}, J=$ $10.2 \mathrm{~Hz}, 1 \mathrm{H}), 1.79(\mathrm{~m}, 2 \mathrm{H}), 1.76$ (br s, 1H), $1.68(\mathrm{~m}, 3 \mathrm{H}), 1.53(\mathrm{~m}, 3 \mathrm{H}), 1.50(\mathrm{~m}, 1 \mathrm{H})$, $1.48(\mathrm{dd}, J=6.0,2.4 \mathrm{~Hz}, 1 \mathrm{H}), 1.36(\mathrm{ddd}, J=15.6,10.2,4.2 \mathrm{~Hz}, 1 \mathrm{H}), 1.25(\mathrm{br} \mathrm{s}, 2 \mathrm{H}), 1.15$ $(\mathrm{d}, J=7.2 \mathrm{~Hz}, 3 \mathrm{H}), 1.14(\mathrm{~d}, J=6.0 \mathrm{~Hz}, 3 \mathrm{H}), 1.12(\mathrm{~d}, J=7.2 \mathrm{~Hz}, 3 \mathrm{H}), 1.03(\mathrm{~d}, J=6.0 \mathrm{~Hz}$, $3 \mathrm{H}), 0.90(\mathrm{~d}, J=7.2 \mathrm{~Hz}, 3 \mathrm{H}), 0.89(\mathrm{t}, J=7.2 \mathrm{~Hz}, 3 \mathrm{H}) ;{ }^{13} \mathrm{C} \mathrm{NMR}\left(150 \mathrm{MHz}, \mathrm{CDCl}_{3}\right)$ $\delta 217.1,176.6,101.2,82.6,68.9,66.8,49.7,47.4,41.0,36.3,35.9,35.2,33.7,31.5,25.4$, 21.7, 19.5, 19.2, 18.5, 17.8, 16.2, 16.1, 11.3; CIHRMS Calcd for $\left[\mathrm{C}_{23} \mathrm{H}_{41} \mathrm{NO}_{5} \mathrm{H}^{+}\right]$: 412.3063. Found 412.3061.

$(2 R, 3 R, 4 R, 5 R, 6 S)$-5-amino-2-(12-ethyl-3,5,7,11-tetramethyl-oxacyclododec-2,8dione)-tetrahydro-6-methyl-2H-pyran-3,4-diol (IV-16).

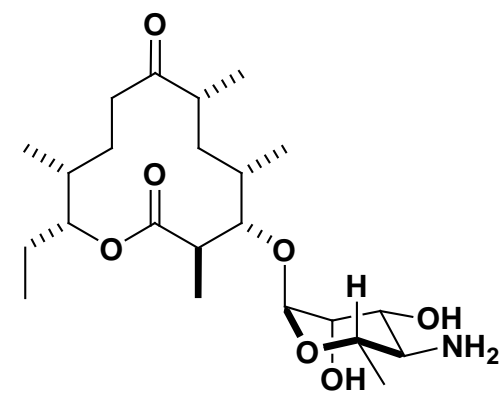

To a solution of dihydroxy azide IV-15 $(10 \mathrm{mg}, 0.02 \mathrm{mmol})$ in dry $\mathrm{MeOH}(0.2 \mathrm{~mL})$ was added $\mathrm{Pd} / \mathrm{C}(2 \mathrm{mg})$ and the mixture was stirred under $\mathrm{H}_{2}$ at an $100 \mathrm{psi}$ pressure for $6 \mathrm{~h}$ at room temperature. The catalyst was filtered off through a short pad of Celite, concentrated under reduced pressure. The resulting crude product was pipetted directly on to a silica gel column using $\mathrm{CH}_{2} \mathrm{Cl}_{2}(1 \mathrm{~mL})$ in three portions. Impurities were eluted with ether and the product was eluted with $\mathrm{MeOH} / \mathrm{EtOAc/hexanes} \mathrm{(20:40:40).} \mathrm{Pure}$ 
fractions were combined and concentrated to afford dihydroxy amine IV-16 $(8.5 \mathrm{mg}$, $0.02 \mathrm{mmol}, 90 \%)$ as viscous oil. $R_{f}(90 \% \mathrm{EtOAc} / \mathrm{MeOH})=0.20 ;[\alpha]_{\mathrm{D}}^{26}=-130(c=$ 0.5, MeOH); IR (thin film, $\mathrm{cm}^{-1}$ ) 3373, 2967, 2933, 1721, 1702, 1458, 1366, 1173, 1032, 984; ${ }^{1} \mathrm{H}$ NMR $\left(600 \mathrm{MHz}, \mathrm{CDCl}_{3}\right) \delta 4.71(\mathrm{~d}, J=2.4 \mathrm{~Hz}, 1 \mathrm{H}), 4.63(\mathrm{ddd}, J=8.4,4.8,1.8$ $\mathrm{Hz}, 1 \mathrm{H}), 3.85(\mathrm{dd}, J=3.0,1.8 \mathrm{~Hz}, 1 \mathrm{H}), 3.69(\mathrm{ddd}, J=12.6,9.6,5.4 \mathrm{~Hz}, 1 \mathrm{H}), 3.48$ (dd, $J$ $=10.2,3.0 \mathrm{~Hz}, 1 \mathrm{H}), 3.42(\mathrm{~d}, J=10.8 \mathrm{~Hz}, 1 \mathrm{H}), 3.13(\mathrm{ddd}, J=19.2,12.0,2.4 \mathrm{~Hz}, 1 \mathrm{H})$, $2.83(\mathrm{ddd}, J=13.8,10.2,6.6 \mathrm{~Hz}, 1 \mathrm{H}), 2.76(\mathrm{t}, J=10.2 \mathrm{~Hz}, 1 \mathrm{H}), 2.49(\mathrm{~m}, 1 \mathrm{H}), 2.21(\mathrm{~m}$, 2H), $1.88(\mathrm{ddd}, J=12.6,7.8,4.8 \mathrm{~Hz}, 1 \mathrm{H}), 1.81(\mathrm{dd}, J=14.4,2.4 \mathrm{~Hz}, 1 \mathrm{H}), 1.57(\mathrm{~m}, 1 \mathrm{H})$, $1.55(\mathrm{~m}, 3 \mathrm{H}), 1.45(\mathrm{~d}, J=4.2 \mathrm{~Hz}, 1 \mathrm{H}), 1.43(\mathrm{dd}, J=4.2,1.8 \mathrm{~Hz}, 1 \mathrm{H}), 1.40(\mathrm{~d}, J=4.2 \mathrm{~Hz}$, 1H), 1.27 (br s, 1H), $1.24(\mathrm{~m}, 1 \mathrm{H}), 1.20(\mathrm{~d}, J=7.2 \mathrm{~Hz}, 3 \mathrm{H}), 1.19(\mathrm{~d}, J=6.0 \mathrm{~Hz}, 3 \mathrm{H}), 1.16$ $(\mathrm{d}, J=7.2 \mathrm{~Hz}, 3 \mathrm{H}), 1.04(\mathrm{~d}, J=6.6 \mathrm{~Hz}, 3 \mathrm{H}), 0.92(\mathrm{~d}, J=6.6 \mathrm{~Hz}, 3 \mathrm{H}), 0.88(\mathrm{t}, J=7.2 \mathrm{~Hz}$, $3 \mathrm{H}) ;{ }^{13} \mathrm{C}$ NMR $\left(150 \mathrm{MHz}, \mathrm{CDCl}_{3}\right) \delta 217.1,176.3,105.5,82.7,72.1,71.5,71.1,55.3$, $47.4,45.8,35.9,35.8,35.1,33.6,31.4,25.4,21.7,19.5,18.3,18.1,17.6,16.0,11.3$; CIHRMS Calcd for $\left[\mathrm{C}_{23} \mathrm{H}_{41} \mathrm{NO}_{7} \mathrm{H}^{+}\right]$: 444.2961. Found 444.2955.

(2R,3S,6R)-3-azido-6-(12-ethyl-3,5,7,11-tetramethyl-oxacyclododec-2,8-dione))-3,6dihydro-2-methyl-2H-pyran (IV-19).

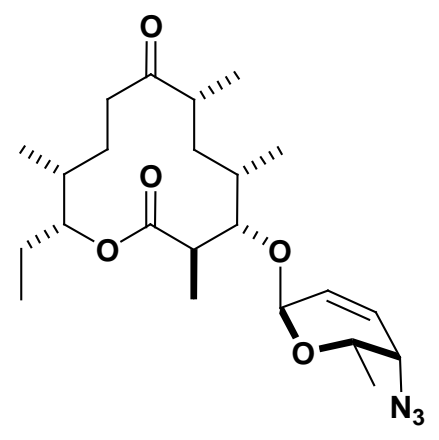


To a solution of allylic alcohol IV-9 (15 mg, $0.03 \mathrm{mmol})$ and methanesulphonyl chloride $(4 \mu \mathrm{L}, 0.05 \mathrm{mmol})$ in dry $\mathrm{CH}_{2} \mathrm{Cl}_{2}(0.3 \mathrm{~mL})$ at $0{ }^{\circ} \mathrm{C}$, was added triethylamine $(6 \mu \mathrm{L}, 0.04$ mmol). After stirring $8 \mathrm{~h}$ at room temperature, water $(1 \mathrm{~mL})$ was added and then the mixture was extracted with EtOAc $(3 \times 5 \mathrm{~mL})$, dried $\left(\mathrm{Na}_{2} \mathrm{SO}_{4}\right)$, concentrated under reduced pressure. The crude product was purified using silica gel flash chromatography eluting with EtOAc/hexane (15:85) to give mesylate IV-18. To a solution mesylate IV-18 (15 mg, $0.12 \mathrm{mmol})$ in THF $(0.3 \mathrm{~mL})$ was added $\mathrm{NaN}_{3}(3.0 \mathrm{mg}, 0.05 \mathrm{mmol})$ under argon atmosphere. The solution was stirred at room temperature for $2 \mathrm{~h}$. Then the mixture was evaporated under reduced pressure, purified using silica gel flash chromatography eluting with EtOAc/hexane (20:80) to give allylic azide IV-19 (10 mg, $0.02 \mathrm{mmol}, 80 \%)$ as viscous oil. $R_{f}(30 \%$ EtOAc/hexane $)=0.55 ;[\alpha]_{\mathrm{D}}^{26}=+73\left(c=0.3, \mathrm{CH}_{2} \mathrm{Cl}_{2}\right) ; \mathrm{IR}$ (thin film, $\left.\mathrm{cm}^{-1}\right)$ 2967, 2932, 2100, 1725, 1702, 1460, 1170, 1041, 972; ${ }^{1} \mathrm{H}$ NMR $(600 \mathrm{MHz}$, $\left.\mathrm{CDCl}_{3}\right) \delta 6.16(\mathrm{dd}, J=10.2,3.0 \mathrm{~Hz}, 1 \mathrm{H}), 6.10(\mathrm{dd}, J=10.2,6.0 \mathrm{~Hz}, 1 \mathrm{H}), 5.06(\mathrm{~d}, J=2.4$ Hz, 1H), 4.69 (ddd, $J=7.8,5.4,2.4 \mathrm{~Hz}, 1 \mathrm{H}), 4.22(\mathrm{ddd}, J=13.2,6.0,2.4 \mathrm{~Hz}, 1 \mathrm{H}), 3.62$ (d, $J=10.2 \mathrm{~Hz}, 1 \mathrm{H}), 3.13(\mathrm{dd}, J=5.4,2.4 \mathrm{~Hz}, 1 \mathrm{H}), 3.09(\mathrm{ddd}, J=12.6,6.0,2.4 \mathrm{~Hz}, 1 \mathrm{H})$, $2.84(\mathrm{~m}, 1 \mathrm{H}), 2.54(\mathrm{~m}, 1 \mathrm{H}), 2.13(\mathrm{~m}, 1 \mathrm{H}), 1.91(\mathrm{~d}, J=13.2 \mathrm{~Hz}, 1 \mathrm{H}), 1.87(\mathrm{~d}, J=13.2 \mathrm{~Hz}$ $1 \mathrm{H}), 1.83(\mathrm{~m}, 1 \mathrm{H}), 1.53(\mathrm{dd}, J=8.6,6.0 \mathrm{~Hz}, 1 \mathrm{H}), 1.50(\mathrm{~d}, J=7.2,6.6 \mathrm{~Hz}, 1 \mathrm{H}), 1.43(\mathrm{~m}$, $2 \mathrm{H}), 1.32(\mathrm{~d}, J=6.0 \mathrm{~Hz}, 3 \mathrm{H}), 1.25(\mathrm{~d}, J=6.6 \mathrm{~Hz}, 3 \mathrm{H}), 1.17(\mathrm{~m}, 1 \mathrm{H}), 1.14(\mathrm{~d}, J=7.2 \mathrm{~Hz}$, $3 \mathrm{H}), 1.07(\mathrm{~d}, J=6.6 \mathrm{~Hz}, 3 \mathrm{H}), 1.07(\mathrm{~d}, J=7.2 \mathrm{~Hz}, 3 \mathrm{H}), 0.89(\mathrm{t}, J=7.8 \mathrm{~Hz}, 3 \mathrm{H}) ;{ }^{13} \mathrm{C}$ NMR $\left(150 \mathrm{MHz}, \mathrm{CDCl}_{3}\right) \delta 214.2,174.2,129.8,124.9,96.3,86.7,80.9,66.4,55.1,45.9,44.2$, 34.7, 34.1, 33.8, 32.2, 24.2, 20.6, 19.3, 17.9, 17.2, 16.9, 15.3, 10.8; CIHRMS Calcd for $\left[\mathrm{C}_{23} \mathrm{H}_{37} \mathrm{~N}_{3} \mathrm{O}_{5} \mathrm{Na}^{+}\right]:$458.2630. Found 458.2631. 


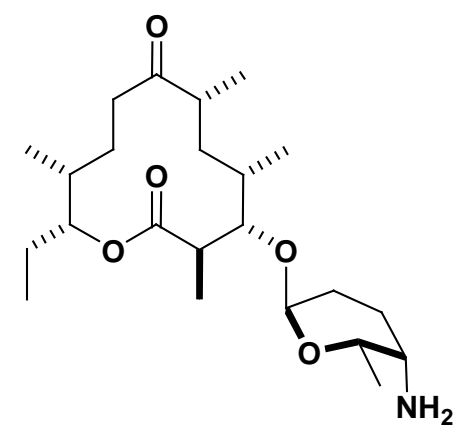

To a solution of allylic azide IV-19 $(10 \mathrm{mg}, 0.02 \mathrm{mmol})$ in dry $\mathrm{MeOH}(0.2 \mathrm{~mL})$ was added $\mathrm{Pd} / \mathrm{C}(5 \mathrm{mg})$ and the mixture was stirred under $\mathrm{H}_{2}$ at an $100 \mathrm{psi}$ pressure for $7 \mathrm{~h}$ at room temperature. The catalyst was filtered off through a short pad of Celite, concentrated under reduced pressure. The resulting crude product was pipetted directly on to a silica gel column using $\mathrm{CH}_{2} \mathrm{Cl}_{2}(1 \mathrm{~mL})$ in three portions. Impurities were eluted with ether and the product was eluted with $\mathrm{MeOH} / \mathrm{EtOAc/hexanes} \mathrm{(20:60:20).} \mathrm{Pure}$ fractions were combined and concentrated to afford deoxy amine IV-20 (7.1 mg, 0.02 mmol, $87 \%)$ as viscous oil. $R_{f}(90 \%$ EtOAc/MeOH $)=0.35 ;[\alpha]_{\mathrm{D}}^{26}=+39(c=0.3$, $\mathrm{CH}_{2} \mathrm{Cl}_{2}$ ); IR (thin film, $\mathrm{cm}^{-1}$ ) 3329, 2942, 2832, 1728, 1705, 1449, 1112, 1021, 856; ${ }^{1} \mathrm{H}$ NMR (600 MHz, $\left.\mathrm{CDCl}_{3}\right) \delta 4.62(\mathrm{dd}, J=6.0,2.4 \mathrm{~Hz}, 1 \mathrm{H}), 4.60(\mathrm{br} \mathrm{s}, 1 \mathrm{H}), 4.30(\mathrm{~m}, 1 \mathrm{H})$, $4.18(\mathrm{dd}, J=6.0,2.4 \mathrm{~Hz}, 1 \mathrm{H}), 4.12(\mathrm{~d}, J=9.6 \mathrm{~Hz}, 1 \mathrm{H}), 3.41(\mathrm{~d}, J=10.8 \mathrm{~Hz}, 1 \mathrm{H}), 3.38$ (m, 1H), $3.15(\mathrm{dd}, J=3.6,1.8 \mathrm{~Hz}, 1 \mathrm{H}), 3.13(\mathrm{dd}, J=6.0,1.8 \mathrm{~Hz}, 1 \mathrm{H}), 3.10(\mathrm{~d}, J=2.4$ $\mathrm{Hz}, 1 \mathrm{H}), 2.84(\mathrm{dd}, J=9.0,3.6 \mathrm{~Hz}, 1 \mathrm{H}), 2.80(\mathrm{dd}, J=10.2,6.6 \mathrm{~Hz}, 1 \mathrm{H}), 2.61(\mathrm{br} \mathrm{s}, 1 \mathrm{H})$, $2.47(\mathrm{~m}, 1 \mathrm{H}), 2.46(\mathrm{dd}, J=6.0,5.4 \mathrm{~Hz}, 1 \mathrm{H}), 2.39(\mathrm{~s}, 1 \mathrm{H}), 2.24(\mathrm{~s}, 1 \mathrm{H}), 2.22(\mathrm{dd}, J=8.4$, $3.0 \mathrm{~Hz}, 1 \mathrm{H}), 2.19(\mathrm{dd}, J=5.4,3.0 \mathrm{~Hz}, 1 \mathrm{H}), 1.74(\mathrm{~m}, 1 \mathrm{H}), 1.53(\mathrm{~m}, 1 \mathrm{H}), 1.24(\mathrm{br} \mathrm{s}, 1 \mathrm{H})$, $1.15(\mathrm{~d}, J=6.6 \mathrm{~Hz}, 3 \mathrm{H}), 1.14(\mathrm{~d}, J=6.6 \mathrm{~Hz}, 3 \mathrm{H}), 1.12(\mathrm{~d}, J=6.6 \mathrm{~Hz}, 3 \mathrm{H}), 1.01(\mathrm{~d}, J=$ 
$6.0 \mathrm{~Hz}, 3 \mathrm{H}), 0.89(\mathrm{~d}, J=6.6 \mathrm{~Hz}, 3 \mathrm{H}), 0.89(\mathrm{t}, J=7.2 \mathrm{~Hz}, 3 \mathrm{H}) ;{ }^{13} \mathrm{C} \mathrm{NMR}(150 \mathrm{MHz}$, $\left.\mathrm{CDCl}_{3}\right) \delta 216.0,175.4,100.3,81.5,56.5,84.5,48.5,46.3,34.9,34.7,34.5,34.0,32.5$, 29.8, 27.5, 24.3, 20.8, 20.6, 18.4, 17.2, 16.5, 14.9, 10.1; CIHRMS Calcd for $\left[\mathrm{C}_{23} \mathrm{H}_{41} \mathrm{NO}_{5} \mathrm{H}^{+}\right]$: 412.3063. Found 412.3061.

\section{6-(12-ethyl-3,5,7,11-tetramethyl-oxacyclododec-2,8-dione )-2-methyl-2H-pyran-} 3(6H)-one (IV-22).

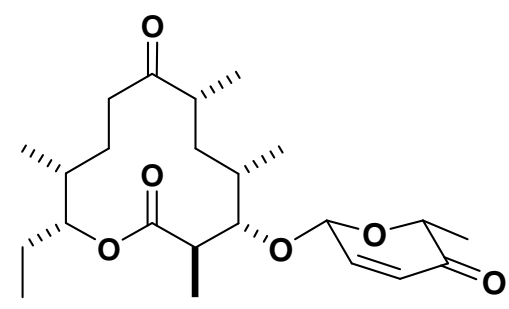

A $\mathrm{CH}_{2} \mathrm{Cl}_{2}(1.5 \mathrm{~mL})$ solution of Boc-enone IV-21 $(366 \mathrm{mg}, 1.60 \mathrm{mmol})$ and 10 deoxymethynolide alcohol IV-7 $(120 \mathrm{mg}, 0.41 \mathrm{mmol})$ was cooled to $0{ }^{\circ} \mathrm{C} . \mathrm{A} \mathrm{CH}_{2} \mathrm{Cl}_{2}(1.5$ $\mathrm{mL}$ ) solution of $\mathrm{Pd}_{2}(\mathrm{dba})_{3}{ }^{\cdot} \mathrm{CHCl}_{3}(41 \mathrm{mg}, 2.5 \mathrm{~mol} \%)$ and $\mathrm{PPh}_{3}(42 \mathrm{mg}, 10 \mathrm{~mol} \%)$ was added to the reaction mixture at $0{ }^{\circ} \mathrm{C}$. The reaction mixture was stirred at $0{ }^{\circ} \mathrm{C}$ for 2 hours. The reaction mixture was quenched with $10 \mathrm{~mL}$ of satd. aq. $\mathrm{NaHCO}_{3}$, extracted (3 x $10 \mathrm{~mL})$ with $\mathrm{Et}_{2} \mathrm{O}$, dried $\left(\mathrm{Na}_{2} \mathrm{SO}_{4}\right)$, and concentrated under reduced pressure. The crude product was purified using silica gel flash chromatography eluting with $20 \%$ EtOAc/hexanes to give enone IV-22 $(131 \mathrm{mg}, 0.32 \mathrm{mmol}, 80 \%)$ as viscous oil. $R_{f}(50 \%$ EtOAc/hexanes $)=0.45 ;[\alpha]_{\mathrm{D}}^{26}=-12\left(c=1.0, \mathrm{CH}_{2} \mathrm{Cl}_{2}\right) ; \mathrm{IR}\left(\right.$ thin film, $\left.\mathrm{cm}^{-1}\right) 2967,2934$, $1725,1703,1459,1171,1018,899 ;{ }^{1} \mathrm{H}$ NMR $\left(600 \mathrm{MHz}, \mathrm{CDCl}_{3}\right) \delta 6.92(\mathrm{dd}, J=10.2,1.2$ Hz, 1H), 6.12 (dd, $J=10.2,1.8 \mathrm{~Hz}, 1 \mathrm{H}), 5.34$ (d, $J=1.2 \mathrm{~Hz}, 1 \mathrm{H}), 4.70$ (ddd, $J=9.0,4.8$, $1.8 \mathrm{~Hz}, 1 \mathrm{H}), 4.11(\mathrm{~m}, 1 \mathrm{H}), 3.68(\mathrm{dd}, J=10.2,1.2 \mathrm{~Hz}, 1 \mathrm{H}), 3.11$ (ddd, $J=18.6,6.6,2.4$ 
Hz, 1H), $2.92(\mathrm{ddd}, J=13.2,10.2,7.2 \mathrm{~Hz}, 1 \mathrm{H}), 2.56(\mathrm{~m}, 1 \mathrm{H}), 2.14(\mathrm{~m}, 1 \mathrm{H}), 1.89(\mathrm{dd}, J=$ 6.0, $3.0 \mathrm{~Hz}, 1 \mathrm{H}), 1.86($ br s, 1H), $1.84(\mathrm{dd}, J=3.0,2.4 \mathrm{~Hz}, 1 \mathrm{H}), 1.53(\mathrm{dd}, J=13.8,7.2$ $\mathrm{Hz}, 1 \mathrm{H}), 1.45(\mathrm{~m}, 2 \mathrm{H}), 1.42(\mathrm{~d}, J=6.6 \mathrm{~Hz}, 3 \mathrm{H}), 1.39(\mathrm{~d}, J=4.2 \mathrm{~Hz}, 1 \mathrm{H}), 1.26(\mathrm{~d}, J=6.6$ Hz, 3H), $1.17(\mathrm{~m}, 1 \mathrm{H}), 1.13$ (d, $J=6.6 \mathrm{~Hz}, 3 \mathrm{H}), 1.09$ (d, $J=6.6 \mathrm{~Hz}, 3 \mathrm{H}), 0.94$ (d, $J=7.2$ $\mathrm{Hz}, 3 \mathrm{H}), 0.90(\mathrm{t}, J=7.2 \mathrm{~Hz}, 3 \mathrm{H}) ;{ }^{13} \mathrm{C} \mathrm{NMR}\left(150 \mathrm{MHz}, \mathrm{CDCl}_{3}\right) \delta 214.5,196.6,174.4$, $147.5,129.2,98.6,88.2,81.3,75.3,46.0,44.1,34.6,34.3,34.0,32.4,24.4,20.8,19.4$, 18.1, 17.1, 15.9, 15.8, 11.0; CIHRMS Calcd for $\left[\mathrm{C}_{23} \mathrm{H}_{36} \mathrm{O}_{6} \mathrm{Na}^{+}\right]$: 431.2409. Found 431.2403.

6-(12-ethyl-3,5,7,11-tetramethyl-oxacyclododec-2,8-dione)-3,6-dihydro-2-methyl-2Hpyran-3-ol (IV-23).

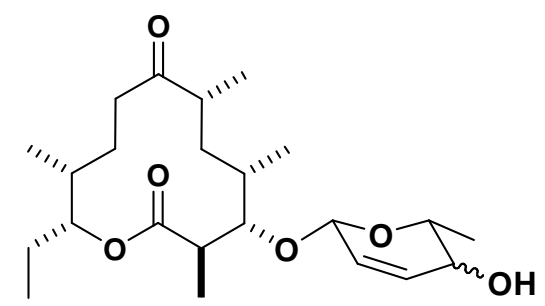

The enone compound $8 \mathbf{~ I V - 2 2 ~ ( 9 0 ~ m g , ~} 0.22 \mathrm{mmol})$ was dissolved in $0.3 \mathrm{~mL}$ of $\mathrm{CH}_{2} \mathrm{Cl}_{2}$ and $0.3 \mathrm{~mL} \mathrm{MeOH}$ in round bottom flask and cooled $-78{ }^{\circ} \mathrm{C}$ then $\mathrm{NaBH}_{4}(9.2 \mathrm{mg}, 0.24$ mmol) was added and the reaction mixture was stirred at $-78{ }^{\circ} \mathrm{C}$ for 3 hours and on completion, monitored by TLC, reaction mixture was diluted with ether and was quenched with $2 \mathrm{~mL}$ of satd. aq. $\mathrm{NaHCO}_{3}$, extracted $(3 \times 5 \mathrm{~mL})$ with $\mathrm{Et}_{2} \mathrm{O}$, dried $\left(\mathrm{Na}_{2} \mathrm{SO}_{4}\right)$, and concentrated under reduced pressure. The crude product was purified using silica gel flash chromatography eluting with $25 \%$ EtOAc/hexanes to give allylic alcohol IV-23 (77 mg, $0.19 \mathrm{mmol}, 85 \%)$ as viscous oil. $R_{f}(50 \%$ EtOAc/hexanes $)=0.35$; 
$[\alpha]_{\mathrm{D}}^{26}=+7\left(c=1.0, \mathrm{CH}_{2} \mathrm{Cl}_{2}\right) ; \mathrm{IR}$ (thin film, $\left.\mathrm{cm}^{-1}\right) 3329,2939,22834,1721,1697$, $1456,1172,1032,900 ;{ }^{1} \mathrm{H} \mathrm{NMR}\left(600 \mathrm{MHz}, \mathrm{CDCl}_{3}\right) \delta 5.92(\mathrm{td}, J=12.0,2.4 \mathrm{~Hz}, 1 \mathrm{H})$, $5.82(\mathrm{td}, J=11.4,1.2 \mathrm{~Hz}, 1 \mathrm{H}), 5.06(\mathrm{br} \mathrm{s}, 1 \mathrm{H}), 4.70(\mathrm{dd}, J=10.8,2.4 \mathrm{~Hz}, 1 \mathrm{H}), 3.93$ (ddd, $J=10.2,6.0,1.8 \mathrm{~Hz}, 1 \mathrm{H}), 3.60(\mathrm{~d}, J=7.2 \mathrm{~Hz}, 1 \mathrm{H}), 3.50(\mathrm{~m}, 1 \mathrm{H}), 3.10(\mathrm{ddd}, J=15.6$, 12.6, $2.4 \mathrm{~Hz}, 1 \mathrm{H}), 2.85(\mathrm{~m}, 1 \mathrm{H}), 2.55(\mathrm{~m}, 1 \mathrm{H}), 2.10(\mathrm{~m}, 1 \mathrm{H}), 2.04(\mathrm{~s}, 1 \mathrm{H}), 1.86(\mathrm{br} \mathrm{s}, 1 \mathrm{H})$, $1.84(\mathrm{~m}, 2 \mathrm{H}), 1.54(\mathrm{dd}, J=8.4,5.4 \mathrm{~Hz}, 1 \mathrm{H}), 1.50(\mathrm{dd}, J=5.4,1.2 \mathrm{~Hz}, 1 \mathrm{H}), 1.43(\mathrm{~m}, 2 \mathrm{H})$, $1.33(\mathrm{~d}, J=6.0 \mathrm{~Hz}, 3 \mathrm{H}), 1.26(\mathrm{dd}, J=7.2,2.4 \mathrm{~Hz}, 1 \mathrm{H}), 1.23(\mathrm{~d}, J=6.6 \mathrm{~Hz}, 3 \mathrm{H}), 1.13(\mathrm{~d}$, $J=7.2 \mathrm{~Hz}, 3 \mathrm{H}), 1.09(\mathrm{~d}, J=6.0 \mathrm{~Hz}, 3 \mathrm{H}), 0.93(\mathrm{~d}, J=7.2 \mathrm{~Hz}, 3 \mathrm{H}), 0.90(\mathrm{t}, J=7.2 \mathrm{~Hz}$, $3 \mathrm{H}) ;{ }^{13} \mathrm{C} \mathrm{NMR}\left(150 \mathrm{MHz}, \mathrm{CDCl}_{3}\right) \delta 214.5,174.5,132.8,128.9,99.4,87.5,80.9,74.5$, $69.0,64.5,45.9,44.0,34.5,34.2,33.8,32.2,24.2,20.6,19.2,18.1,16.9,15.5,10.7$; CIHRMS Calcd for $\left[\mathrm{C}_{23} \mathrm{H}_{38} \mathrm{O}_{6} \mathrm{Na}^{+}\right]$: 433.2566. Found 433.2559.

\section{2-(12-ethyl-3,5,7,11-tetramethyl-oxacyclododec-2,8-dione)-3,6-dihydro-6-methyl-2H-} pyran (IV-24).

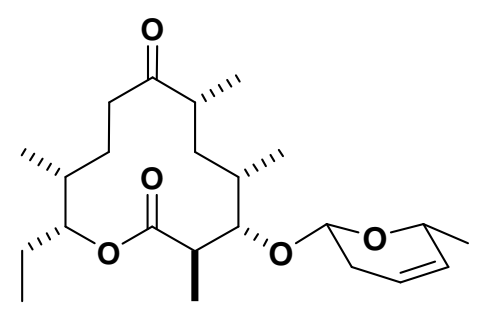

A flask was charged with dry $\mathrm{N}$-methyl morpholine (NMM) $0.8 \mathrm{~mL}$, triphenyl phosphine (126 mg, $0.48 \mathrm{mmol}$ ) and was cooled to $-30{ }^{\circ} \mathrm{C}$ under $\mathrm{Ar}$ atmosphere. Diethylazodicarboxylate $(69 \mu \mathrm{L}, 0.44 \mathrm{mmol})$ was added and the reaction was stirred for $25 \mathrm{~min}$, Allylic alcohol IV-23 (60 mg, $0.15 \mathrm{mmol}$ ) was added in a 1M solution of NMM and the reaction mixture was stirred for $10 \mathrm{~min}$, followed by addition of o- 
nitrobenzenesulfonyl hydrazide (NBSH) $(89 \mathrm{mg}, 0.44 \mathrm{mmol})$. The reaction was stirred at $-30{ }^{\circ} \mathrm{C}$ for $2 \mathrm{~h}$ and was monitored by TLC, upon consumption of starting material, warm up to room temperature and stirred for another $4 \mathrm{~h}$. The reaction mixture was diluted with ether $(10 \mathrm{~mL})$ and was quenched with $5 \mathrm{~mL}$ of satd aq $\mathrm{NaHCO}_{3}$, extracted $(3 \mathrm{x} 5 \mathrm{~mL})$ with $\mathrm{Et}_{2} \mathrm{O}$, dried $\left(\mathrm{Na}_{2} \mathrm{SO}_{4}\right)$, and concentrated under reduced pressure. The crude product was purified using silica gel flash chromatography eluting with $30 \%$ EtOAc/hexanes to give IV-24 (29 mg, $0.07 \mathrm{mmol}, 40 \%)$ of viscous product: $R_{f}(50 \%$ EtOAc/hexanes $)=$

$0.60 ;[\alpha]_{\mathrm{D}}^{26}=+20\left(c=0.5, \mathrm{CH}_{2} \mathrm{Cl}_{2}\right) ; \mathrm{IR}$ (thin film, $\left.\mathrm{cm}^{-1}\right) 2959,2932,1756,1700$ 1429, 1131, 1017, 985; ${ }^{1} \mathrm{H}$ NMR $\left(600 \mathrm{MHz}, \mathrm{CDCl}_{3}\right) \delta 5.64(\mathrm{~m}, 1 \mathrm{H}), 5.57(\mathrm{td}, J=11.4$, $1.8 \mathrm{~Hz}, 1 \mathrm{H}), 4.69$ (ddd, $J=7.8,5.4,2.4 \mathrm{~Hz}, 1 \mathrm{H}), 4.60(\mathrm{dd}, J=8.4,3.6 \mathrm{~Hz}, 1 \mathrm{H}), 4.27$ (m, 1H), $3.54(\mathrm{~d}, J=10.2 \mathrm{~Hz}, 1 \mathrm{H}), 3.12(\mathrm{ddd}, J=19.2,12.0,2.4 \mathrm{~Hz}, 1 \mathrm{H}), 2.85(\mathrm{~m}, 1 \mathrm{H}), 2.57$ $(\mathrm{m}, 1 \mathrm{H}), 2.18(\mathrm{dd}, J=5.4,3.6 \mathrm{~Hz}, 1 \mathrm{H}), 2.13(\mathrm{dd}, J=5.4,3.0 \mathrm{~Hz}, 1 \mathrm{H}), 2.10(\mathrm{dd}, J=5.4$, $3.0 \mathrm{~Hz}, 1 \mathrm{H}), 1.86$ (br s, 1H), $1.84(\mathrm{~m}, 1 \mathrm{H}), 1.53(\mathrm{~d}, J=1.8 \mathrm{~Hz}, 1 \mathrm{H}), 1.52(\mathrm{~d}, J=2.4 \mathrm{~Hz}$, 1H), 1.51 (br s, 1H), $1.49(\mathrm{dd}, J=4.2,2.4 \mathrm{~Hz}, 1 \mathrm{H}), 1.44(\mathrm{~m}, 2 \mathrm{H}), 1.24(\mathrm{~d}, J=7.2 \mathrm{~Hz}$, $3 \mathrm{H}), 1.22(\mathrm{~d}, J=6.6 \mathrm{~Hz}, 3 \mathrm{H}), 1.13(\mathrm{~d}, J=6.6 \mathrm{~Hz}, 3 \mathrm{H}), 1.10(\mathrm{br} \mathrm{s}, 3 \mathrm{H}), 0.94(\mathrm{~d}, J=7.2$ $\mathrm{Hz}, 3 \mathrm{H}), 0.89(\mathrm{~d}, J=7.2 \mathrm{~Hz}, 3 \mathrm{H}) ;{ }^{13} \mathrm{C} \mathrm{NMR}\left(150 \mathrm{MHz}, \mathrm{CDCl}_{3}\right) \delta 214.6,174.6,131.3$, $122.7,100.9,86.4,80.8,71.1,45.9,44.1,34.5,34.1,33.8,32.1,31.0,24.2,20.7,20.6$, 19.2, 18.0, 16.9, 15.4, 10.7; CIHRMS Calcd for $\left[\mathrm{C}_{23} \mathrm{H}_{38} \mathrm{O}_{5} \mathrm{Na}^{+}\right]$: 417.2617. Found 417.2613.

(3S,4R,6R)-6-(12-ethyl-3,5,7,11-tetramethyl-oxacyclododec-2,8-dione)-tetrahydro-2methyl-2H-pyran-3,4-diol (IV-25). 


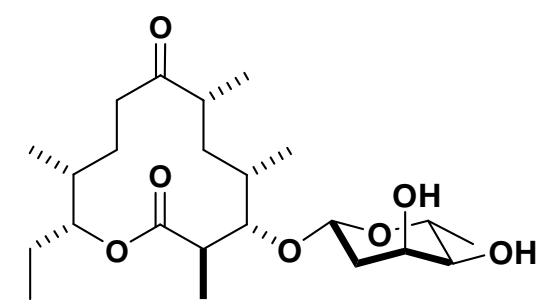

To a $\mathrm{CH}_{2} \mathrm{Cl}_{2}(0.5 \mathrm{~mL})$ solution of ene $\mathbf{I V - 2 4}(10 \mathrm{mg}, 0.03 \mathrm{mmol})$ at $0{ }^{\circ} \mathrm{C}$ was added a solution of $(50 \% \mathrm{w} / \mathrm{v})$ of $N$-methyl morpholine $N$-oxide / water $(10 \mu \mathrm{L})$. Crystalline $\mathrm{OsO}_{4}(0.3 \mathrm{mg}, 10 \mathrm{~mol} \%)$ was added and the reaction was stirred for $12 \mathrm{~h}$. The reaction mixture was concentrated and was pipetted directly on to a silica gel column using $\mathrm{CH}_{2} \mathrm{Cl}_{2}(1 \mathrm{~mL})$ in three portions. Impurities were eluted with ether and the product was eluted with $\mathrm{MeOH} / \mathrm{EtOAc} /$ hexanes (10:40:50). Pure fractions were combined and concentrated to afford diol IV-25 (8 mg, $0.02 \mathrm{mmol}, 70 \%)$ as viscous oil. $R_{f}(90 \%$ EtOAc $/ \mathrm{MeOH})=0.20 ;[\alpha]_{\mathrm{D}}^{26}=-12\left(c=0.5, \mathrm{CH}_{2} \mathrm{Cl}_{2}\right) ;$ IR (thin film, $\left.\mathrm{cm}^{-1}\right) 3442,2928$, $2919,1741,1711,1459,1167,1080,989,734 ;{ }^{1} \mathrm{H}$ NMR $\left(600 \mathrm{MHz}, \mathrm{CDCl}_{3}\right) \delta 4.78(\mathrm{dd}, J$ $=10.2,1.8 \mathrm{~Hz}, 1 \mathrm{H}), 4.69(\mathrm{ddd}, J=12.0,7.8,1.8 \mathrm{~Hz}, 1 \mathrm{H}), 4.10(\mathrm{dd}, J=6.6,3.0 \mathrm{~Hz}, 1 \mathrm{H})$, $3.68(\mathrm{~m}, 1 \mathrm{H}), 3.52(\mathrm{~d}, J=10.8 \mathrm{~Hz}, 1 \mathrm{H}), 3.31(\mathrm{dd}, J=10.2,3.0 \mathrm{~Hz}, 1 \mathrm{H}), 3.11(\mathrm{ddd}, J=$ 16.8, 12.6, $1.8 \mathrm{~Hz}, 1 \mathrm{H}), 2.81(\mathrm{~m}, 1 \mathrm{H}), 2.56(\mathrm{~m}, 1 \mathrm{H}), 2.25(\mathrm{~m}, 1 \mathrm{H}), 2.12(\mathrm{~m}, 2 \mathrm{H}), 1.96(\mathrm{~m}$, $1 \mathrm{H}), 1.88(\mathrm{~d}, J=2.4 \mathrm{~Hz}, 1 \mathrm{H}), 1.84(\mathrm{~m}, 2 \mathrm{H}), 1.69(\mathrm{ddd}, J=13.8,10.2,2.4 \mathrm{~Hz}, 1 \mathrm{H}), 1.55$ (br s, 2H), $1.46(\mathrm{~d}, J=6.0 \mathrm{~Hz}, 1 \mathrm{H}), 1.26(\mathrm{~d}, J=6.0 \mathrm{~Hz}, 3 \mathrm{H}), 1.21(\mathrm{~d}, J=6.6 \mathrm{~Hz}, 3 \mathrm{H})$, $1.12(\mathrm{~d}, J=7.2 \mathrm{~Hz}, 3 \mathrm{H}), 1.06(\mathrm{~d}, J=6.0 \mathrm{~Hz}, 3 \mathrm{H}), 0.93(\mathrm{~d}, J=6.6 \mathrm{~Hz}, 3 \mathrm{H}), 0.89(\mathrm{t}, J=$ $7.2 \mathrm{~Hz}, 3 \mathrm{H}) ;{ }^{13} \mathrm{C} \mathrm{NMR}\left(150 \mathrm{MHz}, \mathrm{CDCl}_{3}\right) \delta 214.6,174.6,99.4,86.9,80.8,73.0,69.2$, $68.3,45.9,44.0,37.8,34.5,34.1,33.8,32.1,29.6,24.2,20.6,19.2,17.9,16.9,15.4,10.7$; CIHRMS Calcd for $\left[\mathrm{C}_{23} \mathrm{H}_{40} \mathrm{O}_{7} \mathrm{Na}^{+}\right]$: 451.2671. Found 451.2665. 
6-(11-hydroxy-12-ethyl-3,5,7,11-tetramethyl-oxacyclododec-2,8-dione)-2-methyl-6Hpyran-3-one (IV-28).

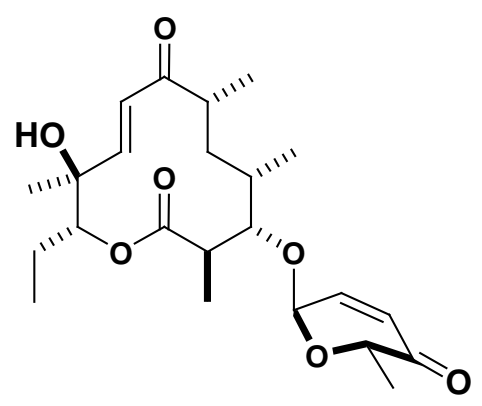

$\mathrm{A} \mathrm{CH}_{2} \mathrm{Cl}_{2}(0.1 \mathrm{~mL})$ solution of Boc-enone IV-27 (17 mg, $\left.0.07 \mathrm{mmol}\right)$ and 11-hydroxy10-deoxymethynolide alcohol IV-26 $(20 \mathrm{mg}, 0.06 \mathrm{mmol})$ was cooled to $0{ }^{\circ} \mathrm{C} . \mathrm{A} \mathrm{CH}_{2} \mathrm{Cl}_{2}$ (0.1 mL) solution of $\mathrm{Pd}_{2}(\mathrm{dba})_{3} \cdot \mathrm{CHCl}_{3}(2 \mathrm{mg}, 2.5 \mathrm{~mol} \%)$ and $\mathrm{PPh}_{3}(2 \mathrm{mg}, 10 \mathrm{~mol} \%)$ was added to the reaction mixture at $0{ }^{\circ} \mathrm{C}$. The reaction mixture was stirred at $0{ }^{\circ} \mathrm{C}$ for 2 hours. The reaction mixture was quenched with $3 \mathrm{~mL}$ of satd. aq. $\mathrm{NaHCO}_{3}$, extracted (3 x $3 \mathrm{~mL}$ ) with $\mathrm{Et}_{2} \mathrm{O}$, dried $\left(\mathrm{Na}_{2} \mathrm{SO}_{4}\right)$, and concentrated under reduced pressure. The crude product was purified using silica gel flash chromatography eluting with $35 \%$ EtOAc/hexanes to give enone $\mathbf{I V - 2 8}(21 \mathrm{mg}, 0.05 \mathrm{mmol}, 80 \%)$ as viscous oil. $R_{f}(50 \%$ EtOAc/hexanes $)=0.30 ;[\alpha]_{\mathrm{D}}^{26}=+44\left(c=0.5, \mathrm{CH}_{2} \mathrm{Cl}_{2}\right) ; \mathrm{IR}\left(\right.$ thin film, $\left.\mathrm{cm}^{-1}\right) 3401,2970$ 2936, 1725, 1686, 1458, 1280, 1153, 1082, 977; ${ }^{1} \mathrm{H}$ NMR (600 MHz, $\left.\mathrm{CDCl}_{3}\right) \delta 6.85(\mathrm{dd}$, $J=10.2,3.6 \mathrm{~Hz}, 1 \mathrm{H}), 5.59(\mathrm{~d}, J=16.2 \mathrm{~Hz}, 1 \mathrm{H}), 6.32(\mathrm{~d}, J=16.2 \mathrm{~Hz}, 1 \mathrm{H}), 6.07$ (d, $J=$ $10.2 \mathrm{~Hz}, 1 \mathrm{H}), 5.26(\mathrm{~d}, J=3.0 \mathrm{~Hz}, 1 \mathrm{H}), 4.79(\mathrm{dd}, J=10.8,1.8 \mathrm{~Hz}, 1 \mathrm{H}), 4.58(\mathrm{dd}, J=13.2$ $7.2 \mathrm{~Hz}, 1 \mathrm{H}), 3.78(\mathrm{~d}, J=10.2 \mathrm{~Hz}, 1 \mathrm{H}), 2.74(\mathrm{~m}, 1 \mathrm{H}), 2.57(\mathrm{~m}, 1 \mathrm{H}), 1.95(\mathrm{ddd}, J=16.2$, 5.4, $1.8 \mathrm{~Hz}, 1 \mathrm{H}), 1.66(\mathrm{~m}, 1 \mathrm{H}), 1.51(\mathrm{~m}, 2 \mathrm{H}), 1.38(\mathrm{~d}, J=6.6 \mathrm{~Hz}, 3 \mathrm{H}), 1.37(\mathrm{~s}, 3 \mathrm{H}), 1.35$ $(\mathrm{d}, J=7.2 \mathrm{~Hz}, 3 \mathrm{H}), 1.25(\mathrm{~m}, 2 \mathrm{H}), 1.21(\mathrm{~d}, J=7.2 \mathrm{~Hz}, 3 \mathrm{H}), 1.05(\mathrm{~d}, J=6.6 \mathrm{~Hz}, 3 \mathrm{H}), 0.92$ $(\mathrm{t}, J=7.2 \mathrm{~Hz}, 3 \mathrm{H}) ;{ }^{13} \mathrm{C} \mathrm{NMR}\left(150 \mathrm{MHz}, \mathrm{CDCl}_{3}\right) \delta 203.6,196.6,174.7,148.8,142.5$, 
$126.9,125.4,95.8,87.4,76.5,74.4,70.7,44.9,42.8,34.2,33.8,21.2,19.4,18.1,17.5$, 16.5, 14.9, 10.6; CIHRMS Calcd for $\left[\mathrm{C}_{23} \mathrm{H}_{34} \mathrm{O}_{7} \mathrm{Na}^{+}\right]$: 445.2202. Found 445.2201.

\section{6-(12-ethyl-3,5,7,11-tetramethyl-oxacyclododec-9-ene-2,8-dione)-2-methyl-6H-} pyran-3-one.

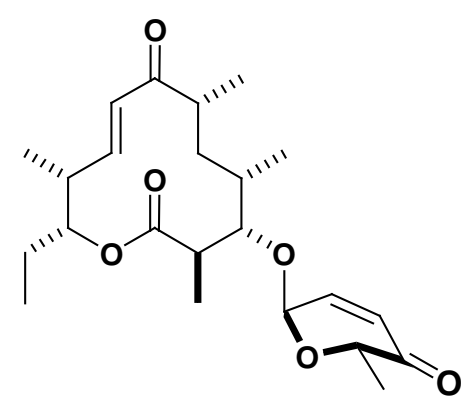

A $\mathrm{CH}_{2} \mathrm{Cl}_{2}(0.2 \mathrm{~mL})$ solution of Boc-enone $(31 \mathrm{mg}, 0.14 \mathrm{mmol})$ and 10-methynolide alcohol (20 mg, $0.07 \mathrm{mmol})$ was cooled to $0{ }^{\circ} \mathrm{C}$. $\mathrm{A} \mathrm{CH}_{2} \mathrm{Cl}_{2}(0.2 \mathrm{~mL})$ solution of $\mathrm{Pd}_{2}(\mathrm{dba})_{3} \cdot \mathrm{CHCl}_{3}(7 \mathrm{mg}, 2.5 \mathrm{~mol} \%)$ and $\mathrm{PPh}_{3}(7 \mathrm{mg}, 10 \mathrm{~mol} \%)$ was added to the reaction mixture at $0{ }^{\circ} \mathrm{C}$. The reaction mixture was stirred at $0{ }^{\circ} \mathrm{C}$ for 5 hours. The reaction mixture was quenched with $3 \mathrm{~mL}$ of satd. aq. $\mathrm{NaHCO}_{3}$, extracted $(3 \times 5 \mathrm{~mL})$ with $\mathrm{Et}_{2} \mathrm{O}$, dried $\left(\mathrm{Na}_{2} \mathrm{SO}_{4}\right)$, and concentrated under reduced pressure. The crude product was purified using silica gel flash chromatography eluting with 15\% EtOAc/hexanes to give enone $(24 \mathrm{mg}, 0.06 \mathrm{mmol}, 88 \%)$ as viscous oil. $R_{f}(50 \%$ EtOAc/hexanes $)=0.60 ;[\alpha]_{\mathrm{D}}^{26}$ $=-32(c=0.5, \mathrm{MeOH}) ; \mathrm{IR}\left(\right.$ thin film, $\left.\mathrm{cm}^{-1}\right) 2979,2949,1718,1705,1458,1171,1018$, 969; ${ }^{1} \mathrm{H}$ NMR $\left(600 \mathrm{MHz}, \mathrm{CDCl}_{3}\right) \delta 6.83(\mathrm{dd}, J=10.2,3.6 \mathrm{~Hz}, 1 \mathrm{H}), 6.75(\mathrm{dd}, J=16.2$, $5.4 \mathrm{~Hz}, 1 \mathrm{H}), 6.42(\mathrm{dd}, J=16.2,1.2 \mathrm{~Hz}, 1 \mathrm{H}), 6.06(\mathrm{~d}, J=10.2 \mathrm{~Hz}, 1 \mathrm{H}), 5.19(\mathrm{~d}, J=2.4$ Hz, 1H), 4.79 (ddd, $J=8.4,5.4,2.4 \mathrm{~Hz}, 1 \mathrm{H}), 4.60(\mathrm{dd}, J=13.8,6.6 \mathrm{~Hz}, 1 \mathrm{H}), 3.68(\mathrm{~d}, J=$ $10.8 \mathrm{~Hz}, 1 \mathrm{H}), 2.77(\mathrm{~m}, 1 \mathrm{H}), 2.63(\mathrm{~m}, 1 \mathrm{H}), 2.54(\mathrm{~m}, 1 \mathrm{H}), 1.71(\mathrm{~m}, 3 \mathrm{H}), 1.57(\mathrm{~m}, 2 \mathrm{H}), 1.36$ 
$(\mathrm{d}, J=6.6 \mathrm{~Hz}, 3 \mathrm{H}), 1.27(\mathrm{~d}, J=7.2 \mathrm{~Hz}, 3 \mathrm{H}), 1.20(\mathrm{~d}, J=7.2 \mathrm{~Hz}, 3 \mathrm{H}), 1.11(\mathrm{~d}, J=6.6 \mathrm{~Hz}$, $3 \mathrm{H}), 1.05(\mathrm{~d}, J=6.0 \mathrm{~Hz}, 3 \mathrm{H}), 0.91(\mathrm{t}, J=7.2 \mathrm{~Hz}, 3 \mathrm{H}) ;{ }^{13} \mathrm{C} \mathrm{NMR}\left(150 \mathrm{MHz}, \mathrm{CDCl}_{3}\right)$ $\delta 204.5,196.8,174.3,147.0,142.0,126.9,125.7,95.8,88.1,74.0,70.6,45.0,43.7,37.8$, 34.1, 33.5, 25.1, 17.6, 17.5, 16.1, 14.9, 10.2, 9.5; CIHRMS Calcd for $\left[\mathrm{C}_{23} \mathrm{H}_{34} \mathrm{O}_{6} \mathrm{Na}^{+}\right]$: 429.2253. Found 429.2249.

\section{6-(12-ethyl-3,5,7,11-tetramethyl-oxacyclododec-9-ene-2,8-dione)-2-methyl-3, 6-}

\section{dihydro-2H-pyran-3-ol.}

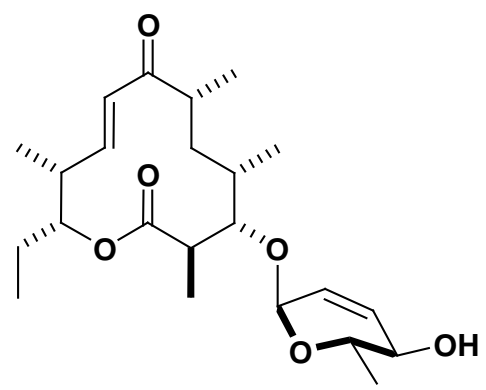

The enone compound (20 mg, $0.05 \mathrm{mmol}$ ) was dissolved in $0.2 \mathrm{~mL}$ of $\mathrm{CH}_{2} \mathrm{Cl}_{2}$ and 0.2 $\mathrm{mL} \mathrm{MeOH}$ in round bottom flask and cooled $-78{ }^{\circ} \mathrm{C}$ then $\mathrm{NaBH}_{4}(2.0 \mathrm{mg}, 0.05 \mathrm{mmol})$ was added and the reaction mixture was stirred at $-78{ }^{\circ} \mathrm{C}$ for 3 hours and on completion, monitored by TLC, reaction mixture was diluted with ether and was quenched with $2 \mathrm{~mL}$ of satd. aq. $\mathrm{NaHCO}_{3}$, extracted ( $\left.3 \times 5 \mathrm{~mL}\right)$ with $\mathrm{Et}_{2} \mathrm{O}$, dried $\left(\mathrm{Na}_{2} \mathrm{SO}_{4}\right)$, and concentrated under reduced pressure. The crude product was purified using silica gel flash chromatography eluting with $25 \%$ EtOAc/hexanes to give allylic alcohol $(18 \mathrm{mg}, 0.04$

mmol, $90 \%)$ as viscous oil. $R_{f}(50 \%$ EtOAc/hexanes $)=0.40 ;[\alpha]_{\mathrm{D}}^{26}=-62(c=0.33$, $\mathrm{MeOH}$ ); IR (thin film, $\mathrm{cm}^{-1}$ ) 3470, 2951, 2923, 1725, 1711, 1478, 1179, 1045, 890; ${ }^{1} \mathrm{H}$ NMR $\left(600 \mathrm{MHz}, \mathrm{CDCl}_{3}\right) \delta 6.74(\mathrm{dd}, J=15.6,6.0 \mathrm{~Hz}, 1 \mathrm{H}), 6.42(\mathrm{~d}, J=15.6 \mathrm{~Hz}, 1 \mathrm{H})$, 
$5.94(\mathrm{~d}, J=10.2 \mathrm{~Hz}, 1 \mathrm{H}), 5.79(\mathrm{dd}, J=10.2,2.4 \mathrm{~Hz}, 1 \mathrm{H}), 4.97(\mathrm{~m}, 1 \mathrm{H}), 4.12(\mathrm{dd}, J=$ 14.4, $7.2 \mathrm{~Hz}, 1 \mathrm{H}), 3.82(\mathrm{~m}, 1 \mathrm{H}), 3.69(\mathrm{~m}, 1 \mathrm{H}), 3.62(\mathrm{~d}, J=10.8 \mathrm{~Hz}, 1 \mathrm{H}), 2.75(\mathrm{ddd}, J=$ 14.4, 10.8, 7.2 Hz, 1H), $2.63(\mathrm{dd}, J=6.6,6.6 \mathrm{~Hz}, 1 \mathrm{H}), 2.55(\mathrm{~m}, 1 \mathrm{H}), 1.90(\mathrm{~m}, 1 \mathrm{H}), 1.73$ (dd, $J=14.4,6.6 \mathrm{~Hz}, 1 \mathrm{H}), 1.68(\mathrm{~m}, 1 \mathrm{H}), 1.55(\mathrm{br} \mathrm{s}, 2 \mathrm{H}), 1.33(\mathrm{dd}, J=13.8,4.2 \mathrm{~Hz}, 1 \mathrm{H})$, $1.30(\mathrm{~d}, J=6.0 \mathrm{~Hz}, 3 \mathrm{H}), 1.25(\mathrm{~d}, J=6.0 \mathrm{~Hz}, 3 \mathrm{H}), 1.20(\mathrm{~d}, J=6.6 \mathrm{~Hz}, 3 \mathrm{H}), 1.10(\mathrm{~d}, J=$ $6.6 \mathrm{~Hz}, 3 \mathrm{H}), 1.04(\mathrm{~d}, J=6.6 \mathrm{~Hz}, 3 \mathrm{H}), 0.91(\mathrm{t}, J=7.2 \mathrm{~Hz}, 3 \mathrm{H}) ;{ }^{13} \mathrm{C} \mathrm{NMR}(150 \mathrm{MHz}$, $\left.\mathrm{CDCl}_{3}\right) \delta 204.8,174.6,146.9,133.3,126.1,125.9,96.4,86.4,73.8,69.5,68.4,68.0,60.3$, 45.0, 43.9, 37.8, 34.2, 33.3, 25.1, 17.6, 16.2, 10.2, 9.5; CIHRMS Calcd for $\left[\mathrm{C}_{23} \mathrm{H}_{36} \mathrm{O}_{6} \mathrm{Na}^{+}\right]$: 431.2409. Found 431.2405. 


\section{References:}

${ }^{1}$ Gijsen, H. J. M.; Qiao, L.; Fitz, W.; Wong, C.-H. Chem. Rev. 1996, 96, 443-73..

${ }^{2}$ Ko, S. Y.; Lee, A. W. M.; Masamune, S.; Reed, L. A.; Sharpless, K. B. Science 1983, 220, 949-951.

3 a) Zamoiski, A., Banaszek, A.; Grynkiewicz, G. 1982, 40, 1. b) Achmatowicz, O.; Bielski, R. Carbohydr. Res. 1977, 55, 165-176. c) Grapsas, I., K.; Couladouros, E. A.; Georgiadis, M. P. Pol. J. Chem. 1990, 64, 823-826.

4 (a) Johnson, C. R.; Golebiowski, A.; Steensma, D. H.; Scialdone, M. A. J. Org. Chem. 1993, 58, 7185-7194. (b) Hudlicky, T.; Pitzer, K. K.; Stabile, M. R.; Thorpe, A. J.;

Whited, G. M. J. Org. Chem. 1996, 61, 4151-4153.

5 a) Timmer, M. S. M.; Adibekian, A.; Seeberger, P. H. Angew. Chem. 2005, 117, 77777780; Angew. Chem. Int. Ed. 2005, 44, 7605-7607. b) Schmidt, R. R. Pure Appl. Chem. 1987, 59, 415- 424. c) Kirschning, A.; Jesberger, M.; Schçning, K. U. Synthesis 2001, 507-540.

${ }^{6}$ a) Danishefsky, S. J. Chemtracts 1989, 273. For improved catalysis see: b) Schaus, S. E.; Branalt, J.; Jacobsen, E. N. J. Org. Chem. 1998, 63, 403-405.

7 Henderson, I.; Sharpless, K. B.; Wong, C.-H. J. Am. Chem. Soc. 1994, 116, 558-561.

${ }^{8}$ Gijsen, H. J. M.; Qiao, L.; Fitz, W.; Wong, C.-H. Chem. Rev. 1996, 96, 443-73.

9 Northrup, A. B.; MacMillan, D. W. C. Science 2004, 305, 1752-1755.

10 Covell, D. J.; Vermeulen, N. A.; Labenz, N. A.; White, M. C. Angew. Chem. Int. Ed. 2006, 45, 8217-8220.

${ }^{11}$ Harris, J. M.; Keranen, M. D.; Nguyen, H.; Young, V. G.; O’Doherty, G. A. Carbohydr. Res. 2000, 328, 17-36.

12 (a) Fujii, A.; Hashiguchi, S.; Uematsu, N.; Ikariya, T.; Noyori, R. J. Am. Chem. Soc. 1996, 118, 2521-2522. (b) Noyori, R.; Ohkuma, T. Angew. Chem., Int. Ed. 2001, 40, 4073.

${ }^{13}$ Li, M.; Scott, J. G. and G. A. O'Doherty, Tetrahedron Lett. 2004, 45, 1005-1009.

14 (a) Achmatowicz, O. ; Bielski, R. Carbohydr. Res. 1977, 55, 165-176. (b) Grapsas, I., K.; Couladouros, E. A.; Georgiadis, M. P. Pol. J. Chem. 1990, 64, 823-826. (c) Harris, J. M.; Keranen, M. D.; O’Doherty, G. A. J. Org. Chem. 1999, 64, 2982-2983. (d) Harris, J. M.; Keranen, M. D.; Nguyen, H.; Young, V. G.; O’Doherty, G. A. Carbohydrate Res. 2000, 328, 17-36. 
${ }^{15}$ (a) Hooper, D. C.; Wolfson, J. S.; McHugh, G. L.; Winters, M. B.; Swartz, M. N. Antimicrob. Agents Chemother. 1982, 22, 662-671. (b) Maxwell, A. Mol. Microbiol. 1993, 9, 681-686. (c) Maxwell, A. Trends Microbiol. 1997, 5, 102-109. (d) Maxwell, A. Biochem. Soc. Trans. 1999, 27, 48-53.

${ }^{16}$ (a) Tsai, F. T.; Singh, O. M.; Skarzynski, T.; Wonacott, A. J.; Weston, S.; Tucker, A.; Pauptit, R. A.; Breeze, A. L.; Poyser, J. P.; O’Brien, R.; Ladbury, J. E.; Wigley, D. B. Proteins 1997, 28, 41-52. (b) Lewis, R. J.; Singh, O, M,; Smith, C. V.; Skarzynski, T.; Maxwell, A.; Wonacott, A. J.; Wigley, D. B. EMBO J. 1996, 15, 1412-1420.

${ }^{17}$ (a) Stackman, R.W.; Eckenstein, F.; Frei, B.; Kulhanek, D.; Nowlin, J.; Quinn, J.F.; Exp Neurol, 2003, 184, 510-520. (b) Goh, L.M.; Barlow, P.J.; Yong, C.S.; Food Chem, 2003, 82, 275-282.

${ }^{18}$ Babu, R. S.; Zhou, M.; O’Doherty, G. A. J. Am. Chem. Soc. 2004, 126, 3428-3429

${ }^{19}$ Babu, R. S.; O’Doherty, G. A. J. Am. Chem. Soc. 2003, 125, 12406-12408.

${ }^{20}$ VanRheenen, V.; Kelly, R. C.; Cha, D. Y. Tetrahedron Lett. 1976, 17, 1973-1976.

${ }^{21}$ Onuma, S.; N., Y.; Saito, Y. Bull. Chem. Soc. Jpn. 1966, 39, 1091. Nomoto, S.; Shimoyama, A. Tetrahedron Lett. 2001, 42, 1753-1755.

22 (a) Fox, J. J.; Kuwada, Y.; Watanabe, K. A.; Ueda, T.; Whipple, E. B. Antimicrob. Agents Chemother. 1964, 518-529. (b) Watanabe, K. A.; Falco, E. A.; Fox, J. J. J. Am. Chem. Soc. 1972, 94, 3272-3274.

23 (a) Ennifar, S.; Das, B. C.; Nash, S. M.; Nagarajan, R. J. Chem. Soc., Chem. Commun. 1977, 41-42. For its synthesis, see: (b) Ikemoto, N.; Schreiber, S. L. J. Am. Chem. Soc. 1990, 112, 9657-9659. (c) Ikemoto, N.; Schreiber, S. L. J. Am. Chem. Soc. 1992, 114, 2524-2536.

${ }^{24}$ Harada, S.; Mizuta, E.; Kishi, T. J. Am. Chem. Soc. 1978, 100, 4895-4897.

25 Takahashi, A.; Ikeda, D.; Naganawa, H.; Okami, Y.; Umezawa, H. J. Antibiot. 1986, 39, 1041-1046.

${ }^{26}$ (a) Ito, T.; Ohba, K.; Koyama, M.; Sezaki, M.; Tohyama, H.; Shomura, T.; Fukuyasu, H.; Kazuno, Y.; Niwa, T.; Kojima, M.; Niida, T. J. Antibiot. 1984, 37, 931-934. (b) For its synthesis, see: Buchanan, J. G.; Stoddart, J.; Wightman, R. H. J. Chem. Soc., Perkin Trans. 1 1994, 11, 1417-1426.

27 (a) Watanabe, K. A.; Matsuda, A.; Itoh, T. Can. J. Chem. 1981, 59, 468-472. (b) Watanabe, K. A.; Matsuda, A.; Itoh, T. Can. J. Chem. 1981, 59, 468-72. 
28 (a) Seto, H.; Koyama, M.; Ogino, H.; Tsuruoka, T.; Inouye, S.; Otake, N. Tetrahedron Lett. 1983, 24, 1805-1808. (b) Czernecki, S.; Franco, S.; Horns, S.; Valery, J.-M. Tetrahedron Lett. 1996, 37, 4003-4006.

29 Recent AdVances in Nucleosides: Chemistry and Chemotherapy; Chu, C. K., Ed.; Elsevier Science: Amsterdam, 2002.

30 (a) Nord, L. D.; Dalley, N. K.; McKernan, P. A.; Robins, R. K. J.Med. Chem. 1987, 30, 1044-54. (b) Zorbach, W. W.; Saeki, S. J. Org. Chem. 1964, 29, 2018-2019.

31 Groebke, K.; Hunziker, J.; Fraser, W.; Peng, L.; Diederichsen, U.; Zimmermann, K.; Holzner, A.; Leumann, C.; Eschenmoser, A. HelV. Chim. Acta. 1998, 81, 375-474.

32 (a) Leutzinger, E. E.; Meguro, T.; Townsend, L. B.; Shuman, D. A.; Schweizer, M. P.; Stewart, C. M.; Robins, R. K. J. Org. Chem. 1972, 37, 3695-3703. (b) Boehringer, M.; Roth, H. J.; Hunziker, J.; Goebel, M.; Krishnan, R.; Giger, A.; Schweizer, B.; Schreiber, J.; Leumann, C.; Eschenmoser, A. HelV. Chim. Acta. 1992, 75, 1416-1477.

33 For the Pd-catalyzed $N$-glycosylation of indolocarboazoles, see: Trost, B. M.; Krische, M. J.; Berl, V.; Grenzer, E. M. Org. Lett. 2002, 4, 2005-2008.

${ }^{34}$ While our approach has the flexibility to prepare either D- or L-pyranonosides, we targeted the L-enantiopodes of both 46 and 47 (i.e., (ent)-46 and (ent)-47), because of our interest in comparing the antitumor activity of (ent)-46 to 46.

35 The differences in the stability and post-glycosylation modification in the a- and bbenzimidazole glycosides as well as the comparison to the $O$-glycosides are not without precedent. Similar phenomena for anomeric imidazoles have been described as a reverse anomeric effect; see: (a) Randell, K. D.; Johnston, B. D.; Green, D. F. and Pinto, B. M. J. Org. Chem. 2000, 65, 220-226. (b) Vaino, A. R.; Szarek, W. A. J. Org. Chem. 2001, 66, 1097-1102.

36 (a) Myers, A. G.; Zheng, B. J. Am. Chem. Soc. 1996, 118, 4492-4493. (b) Myers, A. G.; Zheng, B. Tetrahedron Lett. 1996, 37, 4841-4844. (c) Myers, A. G.; Zheng, B.; Movassaghi, M. J. Org. Chem. 1997, 62, 7507.

36 (a) Hooper, D. C.; Wolfson, J. S.; McHugh, G. L.; Winters, M. B.; Swartz, M. N. Antimicrob. Agents Chemother. 1982, 22, 662-671. (b) Maxwell, A. Mol. Microbiol. 1993, 9, 681-686. (c) Maxwell, A. Trends Microbiol. 1997, 5, 102-109. (d) Maxwell, A. Biochem. Soc. Trans. 1999, 27, 48-53.

38 (a) Tsai, F. T.; Singh, O. M.; Skarzynski, T.; Wonacott, A. J.; Weston, S.; Tucker, A.; Pauptit, R. A.; Breeze, A. L.; Poyser, J. P.; O’Brien, R.; Ladbury, J. E.; Wigley, D. B. 
Proteins 1997, 28, 41-52. (b) Lewis, R. J.; Singh, O, M,; Smith, C. V.; Skarzynski, T.; Maxwell, A.; Wonacott, A. J.; Wigley, D. B. EMBO J. 1996, 15, 1412-1420.

${ }^{39}$ Hanahan, D.; Weinberg, R. A. Cell 2000, 100, 57.

${ }^{40}$ Gang Shen; Xiao Ming Yu; Brian S. J. Blagg. Bioorg. Med. Chem. Lett. 2004, 14, 5903.

${ }^{41}$ Spencer, C. F.; Rodin, J. O.; Walton, E.; Holly, F. W.; Folkers, K. J. Am. Chem. Soc. 1958, $80,140$.

${ }^{42}$ Peixoto, C.; Laurin, P.; Klich, M.; Dupuis-Hamelin, C.; Mauvais, P.; Lassaigne, P.; Bonnefoy, A.; Musicki, B. Tetrahedron Lett. 2000, 41, 1741.

${ }^{43}$ Vaterlaus, B. P.; Kiss, J.; Spiegelberg, H. Helv. Chim. Acta 1964, 47, 381.

${ }^{44}$ X Ming Yu, G Shen, L Neckers, H Blake, J Holzbeierlein, B Cronk, and B S. J. Blagg. J. Am. Chem. Soc. 2005, 127, 12778-12779.

45 Burlison, J. A.; Neckers, L. M.; Smith, A.; Maxwell, A.; Blagg,* B. S. J. Novobiocin. Redesigning DNA Gyrase Inhibitors for Selective Inhibition of Hsp90. J. Am. Chem. Soc. 2006, 128, 15529-15536.

46 Albermann, C.; Soriano, A.; Jiang, J.; Vollmer, H.; Biggins, J. B.; Barton, W. A.; Lesniak, J.; Nikolov, D. B.; Thorson, J. S. Org. Lett. 2003, 5, 933.

${ }^{47}$ (a) Griffith, B. R.; Langenhan, J. M.; Thorson, J. S. Curr. Opin. Biotechnol. 2005, 16, 622-630. (b) Langenhan, J. M.; Peters, N. R.; Guzei, I. A.; Hoffman, F. M.; Thorson, J. S. Proc. Natl. Acad. Sci. U.S.A. 2005, 102, 12305-12310. (c) Ahmed, A.; Peters, N. R.; Fitzgerald, M. K.; Watson, J. A., Jr.; Hoffman, F. M.; Thorson, J. S. J. Am. Chem. Soc. 2006, 128, 14224-14225.

${ }^{48}$ (a) Babu, R. S.; Zhou, M.; O’Doherty, G. A. J. Am. Chem. Soc. 2004, 126, 3428-3429. (b) Guppi, S. R.; Zhou, M.; O’Doherty, G. A. Org. Lett. 2006, 8, 293-296. (c) Babu, R. S.; O'Doherty, G. A. J. Am. Chem. Soc. 2003, 125, 12406-12408.

49 (a) Fujii, A.; Hashiguchi, S.; Uematsu, N.; Ikariya, T.; Noyori, R. J. Am. Chem. Soc. 1996, 118, 2521-2522. (b) Li, M.; Scott, J. G.; O’Doherty, G. A. Tetrahedron Lett. 2004, 45, 1005-1009. (c) Li, M.; O'Doherty, G. A. Tetrahedron Lett. 2004, 45, 6407-6411.

${ }^{50}$ See ref 11. (a) Babu, R. S.; O’Doherty, G. A. J. Carb. Chem. 2005, 24, 169-177. (b) Guo, H.; O’Doherty, G. A. Org. Lett. 2005, 7, 3921-3924.

${ }^{51}$ For the Luche reduction, see: (a) Luche, J. L. J. Am. Chem. Soc. 1978, 100, 2226-2227. (b) Haukaas, M. H.; O’Doherty, G. A. Org. Lett. 2001, 3, 401-404. 
52 a) Kunz, H.; Unverzagt, C. Angew. Chem., Int. Ed. Engl. 1988, 27, 1697-1699. (b) Unverzagt, C.; Kunz, H. Bioorg. Med. Chem. 1994, 2, 1189-1201. (c) McDonald, F. E.; Danishefsky, S. J. J. Org. Chem. 1992, 57, 7001-7002.

53 Kocovsky, P. Tetrahedron Lett. 1986, 27, 5521.

${ }^{54}$ Haukaas, M. H.; O’Doherty, G. A. Org. Lett. 2002, 4, 1771-1774.

${ }^{55}$ Walsh, C. T. Nature 2000, 406, 775-781.

56 He, H.; Williamson, R. T.; Shen, B.; Grazaini, E. I., Yang, H. Y.; Sakya, S. M.; Petersen, P. J.; Carter, G. T. J. Am. Chem. Soc. 2002, 124, 9729-9736.

57 (a) Petersen, P. J.; Wang, T. Z.; Dushin, R. G.; Bradford, P. A. Antimicrob. Agents Chemother. 2004, 48, 739-746. (b) Sum, P. E.; How, D.; Torres, N.; Petersen, P. J.; Lenoy, E. B.; Weiss, W. J.; Mansour, T. S. Bioorg. Med. Chem. Lett. 2003, 13, 11511155. (c) He, H.; Shen, B.; Petersen, P. J.; Weiss, W. J.; Yang, H. Y.; Wang, T.-Z.; Dushin, R. G.; Koehn, F. E.; Carter, G. T. Bioorg. Med. Chem. Lett. 2004, 14, 279-282.

58 (a) Wang, T.-Z.; Wheless, K. L.; Sutherland, A. G.; Dushin, R. G. Heterocycles 2004, 62, 131-135. (b) Dushin, R. G.; Wang, T. Z.; Sum, P. E.; He, H.; Sutherland, A. G.; Ashcroft, J. S.; Graziani, E. I.; Koehn, F. E.; Bradford, P. A.; Petersen, P. J.; Wheless, K. L.; How, D.; Torres, N.; Lenoy, E. B.; Weiss, W. J.; Lang, S. A.; Projan, S. J.; Shlaes, D. M.; Mansour, T. S. J. Med. Chem. 2004, 47, 3487-3490.

59 Singh, M. P.; Petersen, P. J.; Weiss, W. J.; Janso, J. E.; Luckman, S. W.; Lenoy, E. B.; Bradford, P. A.; Testa, R. T.; Greenstein, M. Antimicrob. Agents Chemother. 2003, 47, 62-69.

60 We were mindful of Kahne's discovery of simple disaccharide fragments of vancomycin with significant activity toward vancomycin resistance bacteria, see: Sun, B.; Chen, Z.; Eggert, U. S.; Shaw, S. J.; LaTour, J. V.; Kahne, D. J. Am. Chem. Soc. 2001, $123,12722-12723$.

61 Babu, R. S.; O'Doherty, G. A. J. Am. Chem. Soc. 2003, 125, 12406-12407.

62 (a) Babu, R. S.; Zhou, M.; O'Doherty, G. A. J. Am. Chem. Soc. 2004, 126, 3428-3429. (b) Babu, R. S.; O'Doherty, G. A. J. Carbohydr. Chem. 2005, 24, 169-177. (c) VanRheenen, V.; Cha, D. Y.; Hartley, W. M. Organic Syntheses; Wiley \& Sons: New York, 1988; Collect. Vol. VI, p 342.

63 Presumably, the L,L-diastereomer and the bis-2,3-dideoxy analogues of mannopeptimycin-E would have improved stability. 
64 Pyranones such as $\mathbf{6}$ can be prepared in three steps from achiral acylfurans such as $\mathbf{7}$ in either enantiomeric form (D/L). The pyranone asymmetry is derived from a Noyori reduction; see ref 7 and: Li, M.; Scott, J. G.; O'Doherty, G. A. Tetrahedron Lett. 2004, 45, 1005-1009

65 The relative stereochemistry of $\mathbf{3 a}$ and $\mathbf{1 4 a}$ was determined by analysis of various coupling constants from their ${ }^{1} \mathrm{H}$ NMR spectra; see the Supporting Information.

${ }^{66}$ We have found $o$-nitrophenylsulfonylhydrazide/triethylamine to be an excellent diimide precursor, ideal for reducing pyrans of this type; see ref 8 and: Haukaas, M. H.; O'Doherty, G. A. Org. Lett. 2002, 4, 1771-1774.

67 To achieve complete conversion, occasionally the crude reaction mixture may need to be resubjected to the diimide conditions.

${ }^{68}$ Guo, H.; O’Doherty, G. A. Org. Lett. 2006, 8, 1609-1612.

69 (a) Babu, R. S.; Zhou, M.; O’Doherty, G. A. J. Am. Chem. Soc. 2004, 126, 3428-3429. (b) Babu, R. S.; O’Doherty, G. A. J. Carbohydr. Chem. 2005, 24, 169-177. (c) Guppi, S. R.; Zhou, M.; O’Doherty, G. A. Org. Lett. 2006, 8, 293-296.

70 de Oliveira, R. N.; Cottier, L.; Sinou, D.; Srivastava, R. M. Tetrahedron 2005, 61, 8271-8281.

71 (a) Staudinger, H.; Meyer, J. HelV. Chim. Acta 1919, 2, 635-646. (b) Chen, J.; Forsyth, C. J. Org. Lett. 2003, 5, 1281-1283.

${ }^{72}$ (a) Castro, B.; Dormoy, J. R.; Evin, G.; Selve, C. Tetrahedron Lett.1975, 1219-1222. (b) Dourtoglou, V.; Ziegler, J.-C.; Gross, B. Tetrahedron Lett. 1978, 1269-1272. (c) Fre'rot, E.; Coste, J.; Pantaloni, A.; Dufour, M.-N.; Jouin, P. Tetrahedron 1991, 47, 259270. (d) Carpino, L. A.; El-Fahan, A.; Minor, C. A.; Albericio, F. J. Chem. Soc., Chem. Commun. 1994, 201-203.

${ }^{73}$ Lin, S.; Yang, Z.-Q.;.Kowk, B. H. B.; Koldobskiy, M. A.; Crews, C. M.; Danishefsky, S. J. J. Chem. Soc., Chem. Commun. 2004, 6347-6355.

74 (a) Rawlings, B. J. Nat. Prod. Rep. 2001, 18, 190-227. (b) Rawlings, B. J. ibid. 231281. (c) Staunton, J.; Weissman, K. J. Nat. Prod. Rep. 2001, 18, 380-416. (d) Walsh, C. Antibiotics: Actions, Origins, Resistance; ASM Press: Washington, D.C., 2003. (e) Weissman, K. J. Philos. Trans. R. Soc. London, Ser. A 2004, 362, 2671-2690.

75 Xue, Y.; Zhao, L.; Liu, H.-w.; Sherman, D. H. Proc. Natl. Acad. Sci. U.S.A. 1998, 95, 12111-12116. 
${ }^{76}$ D. O'Hagan (1991) The polyketide metabolites, Ellis Horwood, Chichester.

77 Staunton, J. and Weissman, K.J. Polyketide biosynthesis: a millennium review. Nat. Prod. Rep. 2001, 18, 380-416.

78 (a) Me'ndez, C. and Salas, J.A. Trends Biotechnol. 2001, 11, 449-456. (b) Rix, U., Fischer, C., Remsing, L.L. and Rohr, J. Nat. Prod. Rep. 2002, 19, 542-580.

79 (a) Borisova, S. A.; Zhao, L.; Melancüon, C. E., III; Kao, C.-L.; Liu, H.-w. J.Am. Chem. Soc. 2004, 126, 6534-6535. (b) Borisova, S. A.; Zhao, L.; Sherman, D. H.; Liu, H.-w. Org. Lett. 1999, 1, 133-136. (c) Kao, C.-L.; Borisova, S. A.; Kim, H. J.; Liu, H.-w. J. Am. Chem. Soc. 2006, 128, 5606-5607.

80 (a) Rodriguez, E.; McDaniel, R. Curr. Opin. Microbiol. 2001, 4, 526-534. (b) Rix, U.; Fischer, C.; Remsing, L. L.; Rohr, J. Nat. Prod. Rep. 2002, 19, 542-580. (c) Walsh, C.; Freel Meyers, C. L.; Losey, H. C. J. Med. Chem. 2003, 46, 3425-3436.

${ }^{81}$ (a) He, X. M.; Liu, H.-w. Annu. ReV. Biochem. 2002, 71, 701-754. (b) Johnson, D. A.; Liu, H.-w. Curr. Opin. Chem. Biol. 1998, 2, 642-649. (c) Liu, H.-w.; Thorson, J. S. Annu. ReV. Microbiol. 1994, 48, 223-256.

82 (a) Thorson, J. S.; Hosted, T. J., Jr.; Jiang, J.; Biggins, J. B.; Ahlert, J. Curr. Org. Chem. 2001, 5, 139-167. (b) Kren, V.; Martı́nkova', L. Curr. Med. Chem. 2001, 8, 1303 1328. (c) Weymouth-Wilson, A. C. Nat. Prod. Rep. 1997, 14, 99-110.

83 (a) Hong, J. S. J.; Park, S. H.; Choi, C. Y.; Sohng, J. K.; Yoon, Y. J. FEMS Microbiology Letters, 2004, 238, 391-399. (b) see the reference: 7

${ }^{84}$ (a) Babu, R. S.; O’Doherty, G. A. J. Am. Chem. Soc. 2003, 125, 12406-12407. (b) Comely, A. C.; Eelkema, R.; Minnaard, A. J.; Feringa, B. L. J. Am. Chem. Soc. 2003, 125, 8714-8715. (c) Kim, H.; Men, H.; Lee, C. J. Am. Chem. Soc. 2004, 126, 1336-1337. (d) Babu, R. S.; Zhou, M.; O’Doherty, G. A. J. Am. Chem. Soc. 2004, 126, 3428-3429.

85 (a) Fujii, A.; Hashiguchi, S.; Uematsu, N.; Ikariya, T.; Noyori, R. J. Am. Chem. Soc. 1996, 118, 2521-2522. (b) Li, M.; Scott, J. G.; O’Doherty, G. A. Tetrahedron Lett. 2004, 45, 1005-1009. (c) Li, M.; O’Doherty, G. A. Tetrahedron Lett. 2004, 45, 6407-6411.

${ }^{86}$ (a) Babu, R. S.; O’Doherty, G. A. J. Carbohydr. Chem. 2005, 24, 169-177. (b) Li, M.; Scott, J. G.; O’Doherty, G. A. Tetrahedron Lett. 2004, 45, 1005-1009.

${ }^{87}$ Haukaas, M. H.; O’Doherty, G. A. Org. Lett. 2002, 4, 1771-1774.

88 (a) Luche, J. L. J. Am. Chem. Soc. 1978, 100, 2226-2227. (b) Haukaas, M. H.; O’Doherty, G. A. Org. Lett. 2001, 3, 401-404. 
89 (a) Myers, A. G.; Zheng, B. J. Am. Chem. Soc. 1996, 118, 4492-4493. (b) Myers, A. G.; Zheng, B. Tetrahedron Lett. 1996, 37, 4841-4844. (c) Myers, A. G.; Zheng, B.;

Movassaghi, M. J. Org. Chem. 1997, 62, 7507. (d) Haukaas, M. H.; O’Doherty, G. A. Org. Lett. 2002, 4, 1771-1774. 